Old Dominion University

ODU Digital Commons

Fall 2019

\title{
Validation of Neutrino Energy Estimation Using Electron Scattering Data
}

Mariana Khachatryan

Old Dominion University, marishnem@gmail.com

Follow this and additional works at: https://digitalcommons.odu.edu/physics_etds

Part of the Nuclear Commons

\section{Recommended Citation}

Khachatryan, Mariana. "Validation of Neutrino Energy Estimation Using Electron Scattering Data" (2019). Doctor of Philosophy (PhD), Dissertation, Physics, Old Dominion University, DOI: 10.25777/dm57-7q54 https://digitalcommons.odu.edu/physics_etds/123

This Dissertation is brought to you for free and open access by the Physics at ODU Digital Commons. It has been accepted for inclusion in Physics Theses \& Dissertations by an authorized administrator of ODU Digital Commons. For more information, please contact digitalcommons@odu.edu. 


\section{VALIDATION OF NEUTRINO ENERGY ESTIMATION USING ELECTRON SCATTERING DATA}

by

Mariana Khachatryan

B.S. May 2011, Yerevan State University, Armenia

M.S. May 2013, Yerevan State University, Armenia

M.S. December 2015, Old Dominion University

A Dissertation Submitted to the Faculty of

Old Dominion University in Partial Fulfillment of the

Requirements for the Degree of

DOCTOR OF PHILOSOPHY

PHYSICS

OLD DOMINION UNIVERSITY

December 2019

Approved by:

Lawrence Weinstein (Director)

Rocco Schiavilla (Member)

Stepan Stepanyan (Member)

Mark Havey (Member)

John Adam (Member) 


\title{
ABSTRACT
}

\section{VALIDATION OF NEUTRINO ENERGY ESTIMATION USING ELECTRON SCATTERING DATA}

\author{
Mariana Khachatryan \\ Old Dominion University, 2019 \\ Director: Dr. Lawrence Weinstein
}

To study neutrino oscillations, the knowledge of the initial neutrino energy is required. This energy cannot be determined directly because neutrino beams have a broad energy distribution. Instead, the initial energy for each event is estimated from the final state particles of a neutrino-nucleus interaction using two main approaches. It can be determined either from the total energy of all the final state particles or, if the neutrino scatters quasielastically from a bound nucleon, then the initial energy can be calculated approximately using the scattered angle and energy of the outgoing charged lepton. This requires a detailed understanding of neutrino-nucleus interaction cross section for various interaction channels, for different atomic nuclei, and for a wide range of neutrino energies.

None of these energy reconstruction techniques have been tested experimentally using beams of known energy. We exploited the similarity of electron-nucleus and neutrino-nucleus interactions, and applied the methods of neutrino energy estimation to Jefferson Lab CLAS electron scattering data for 1.1, 2.2 and $4.4 \mathrm{GeV}$ electrons incident on ${ }^{3} \mathrm{He},{ }^{4} \mathrm{He}, \mathrm{C}$ and $\mathrm{Fe}$ targets. We show that the energy reconstruction from the scattered electron plus proton provides a better description of the beam energy than the energy reconstruction from the scattered electron alone, however only $16-55 \%$ of events reconstruct to within $5 \%$ of the beam energy. The energy reconstruction works better for lighter nuclei and lower energies. The tails in the reconstructed energy distributions, corresponding to low (compared to the beam energy) reconstructed energy values, are almost identical for the two reconstruction methods and the consistency between the results from two reconstruction methods does not imply accuracy. We show that energy reconstruction is improved by restricting the transverse momentum of the scattered electron plus proton to be smaller than the nuclear Fermi momentum.

We have also compared the results from data to GENIE neutrino event generator results running in electron scattering mode, using the most up to date version. We show that GENIE fails to fully describe the data. We also looked at the potential effects of incorrect energy reconstruction for the proposed DUNE experiment, by generating events with GENIE but 
reconstructing them using our data. The difference is far greater than the needed DUNE precision. 
Copyright, 2019, by Mariana Khachatryan, All Rights Reserved. 


\section{ACKNOWLEDGEMENTS}

I would like to thank my supervisor Larry Weinstein for all his help and guidance throughout my PhD program. He has both encouraged and challenged me to learn sharing my work in a competitive atmosphere in a successful way. He not only gave me knowledge on nuclear physics, but also taught me skills on writing and presenting scientific work. He has been very helpful and prompt in assisting me with the questions that I got throughout my research and has been a great example and motivator for me.

I am grateful to Stepan Stepanyan for his additional supervision, for his support in all questions that I got and for many hours of patient explanations. I am also thankful to everyone from e2 run group that have provided me data and tools for my research.

I am thankful to Or Hen, Adi Ashkenazi, Afrodite Papadopoulou from MIT, Florian Hauenstein (ODU) and Eli Piastzky from Tel Aviv University for their guidance and support in making this project successful.

I would also like to thank my dissertation committee members, my professors from ODU physics department and everyone who made this journey possible.

Finally, I would like to thank my husband Rafayel for his love and support and my parents for teaching me the importance of education from a young age and encouraging and supporting me in my decisions.

I dedicate this thesis to my daughter Ariana Anna Paremuzyan for motivating me to be a good example for her. 


\section{TABLE OF CONTENTS}

Page

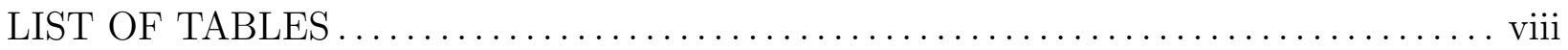

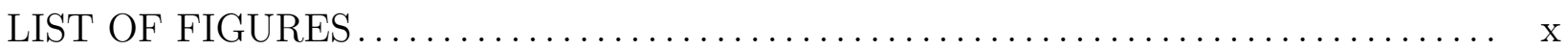

Chapter

1. INTRODUCTION AND THEORY $\ldots \ldots \ldots \ldots \ldots \ldots \ldots \ldots \ldots \ldots \ldots \ldots \ldots \ldots \ldots \ldots$

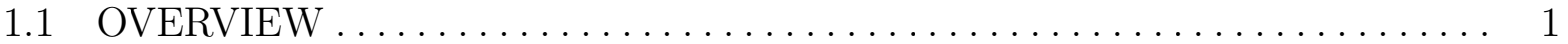

1.2 ACCELERATOR BASED NEUTRINO EXPERIMENTS . . . . . . . . . 10

1.3 DETERMINATION OF NEUTRINO FLUX IN LONG BASELINE NEUTRINO OSCILLATION EXPERIMENTS $\ldots \ldots \ldots \ldots \ldots \ldots \ldots \ldots \ldots \ldots$

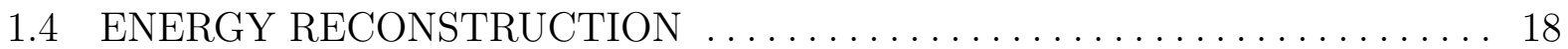

1.5 ELECTRON AND NEUTRINO INTERACTION WITH MATTER . . . . . . 19

1.6 NEUTRINO INTERACTION AND ENERGY RECONSTRUCTION SYS-

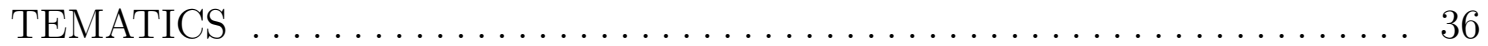

2. EXPERIMENT ............................................ 40

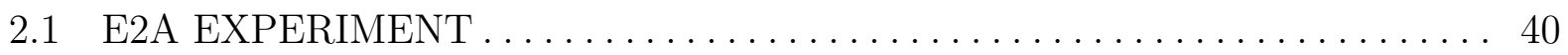

2.2 CONTINUOUS ELECTRON BEAM ACCELERATOR FACILITY $\ldots \ldots \ldots . .42$

2.3 CEBAF LARGE ACCEPTANCE SPECTROMETER (CLAS) . . . . . . . . 43

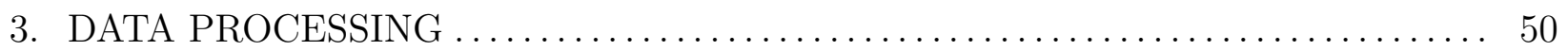

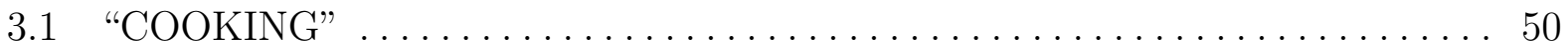

3.2 RE-CALIBRATION OF TOF TIMING $\ldots \ldots \ldots \ldots \ldots \ldots \ldots \ldots \ldots \ldots \ldots$

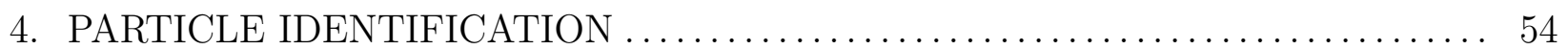

4.1 ELECTRON IDENTIFICATION $\ldots \ldots \ldots \ldots \ldots \ldots \ldots \ldots \ldots \ldots \ldots \ldots \ldots$

4.2 PROTON IDENTIFICATION $\ldots \ldots \ldots \ldots \ldots \ldots \ldots \ldots \ldots \ldots \ldots \ldots \ldots \ldots$

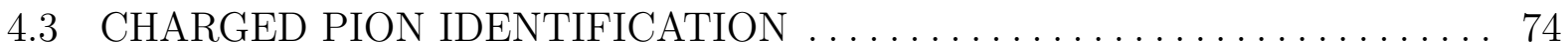

4.4 PHOTON IDENTIFICATION $\ldots \ldots \ldots \ldots \ldots \ldots \ldots \ldots \ldots \ldots \ldots \ldots \ldots \ldots$

4.5 PROTON ENERGY LOSS CORRECTION . . . . . . . . . . . . . . . . . . . 84

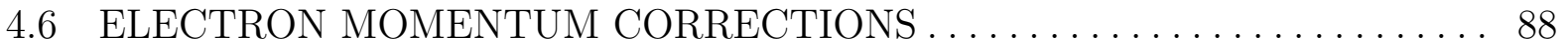

4.7 ELECTRON FIDUCIAL CUTS $\ldots \ldots \ldots \ldots \ldots \ldots \ldots \ldots \ldots \ldots \ldots \ldots$

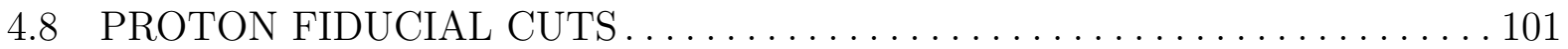

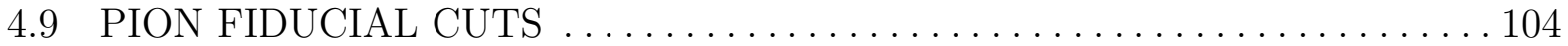

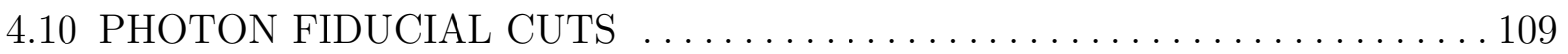

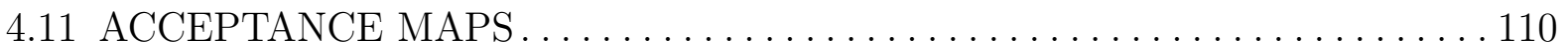

4.12 Z-VERTEX CORRECTION AND CUTS OF PROTONS, ELECTRONS

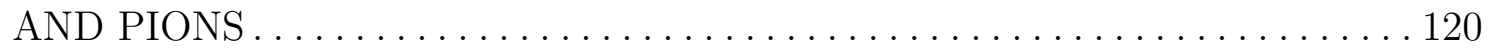




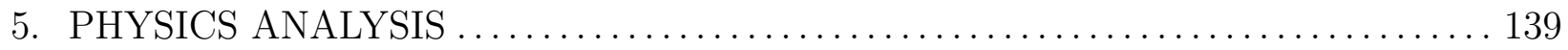

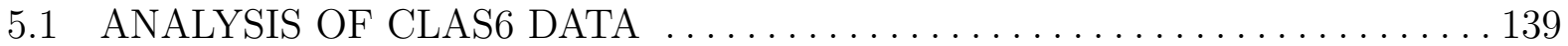

5.2 COMPARISON OF 1.1, 2.2 AND 4.4 GEV $A\left(e, e^{\prime}\right)$ AND $A\left(e, e^{\prime} p\right)$ ANALYSIS 156

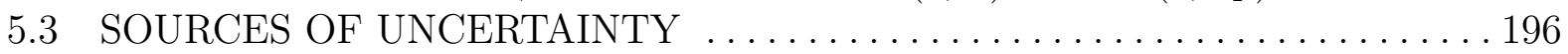

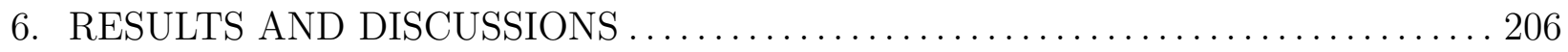

6.1 SUMMARY OF DATA ANALYSIS RESULTS ..................... 206

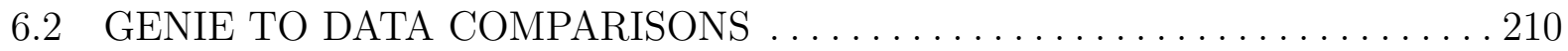

6.3 PROJECTED IMPACT ON NEUTRINO UNCERTAINTIES $\ldots \ldots \ldots \ldots \ldots 213$

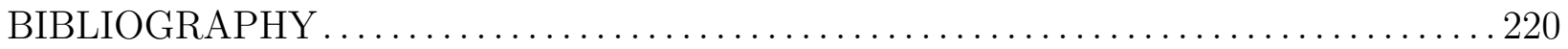

APPENDICES

A. Z-VERTEX CORRECTION AND CUTS OF PROTONS, PIONS AND ELECTRONS FOR ${ }^{4} \mathrm{He},{ }^{12} \mathrm{C}$ AND ${ }^{56} \mathrm{Fe}$ TARGETS FOR 2.2 GEV AND

4.4 GEV BEAM ENERGIES. . . . . . . . . . . . . . . . . . . . . . 221

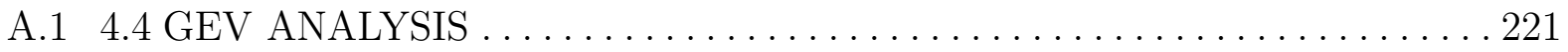

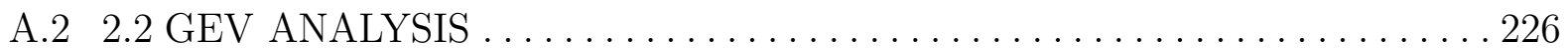

B. THE $A\left(e, e^{\prime}\right)$ AND $A\left(e, e^{\prime} p\right)$ ANALYSIS PLOTS FOR ${ }^{4} \mathrm{He}$ AND ${ }^{12} \mathrm{C}$ TAR-

GETS FOR 2.2 GEV AND 4.4 GEV BEAM ENERGIES. . . . . . . . . . . . . 234

B.1 4.4 GEV $A\left(e, e^{\prime}\right)$ AND $A\left(e, e^{\prime} p\right)$ ANALYSIS $\ldots \ldots \ldots \ldots \ldots \ldots \ldots \ldots \ldots \ldots \ldots \ldots \ldots \ldots \ldots$

B.2 $2.2 \mathrm{GEV} A\left(e, e^{\prime}\right)$ AND $A\left(e, e^{\prime} p\right)$ ANALYSIS $\ldots \ldots \ldots \ldots \ldots \ldots \ldots \ldots \ldots \ldots \ldots \ldots \ldots$ 


\section{LIST OF TABLES}

Table

Page

1 The best-fit values and $3 \sigma$ allowed ranges of the 3 neutrino oscillation parameters. 7

2 The decay of $\pi^{\prime}$ 's and $K$ ' into neutrinos. $\ldots \ldots \ldots \ldots \ldots \ldots \ldots \ldots \ldots$

3 The table of uncertainties from different sources in $\mathrm{T} 2 \mathrm{~K}$ experiment. . . . . . . 38

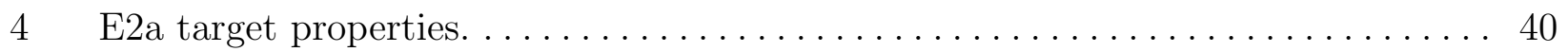

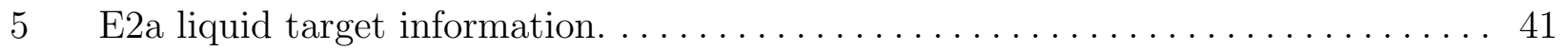

$6 \quad$ List of E2a runs used in current analysis. $\ldots \ldots \ldots \ldots \ldots \ldots \ldots \ldots \ldots \ldots \ldots$

7 Cut values on the time difference between hits of the electron candidate in $\mathrm{CC}$ and SC, corrected for the distance traveled from CC to SC for different sectors

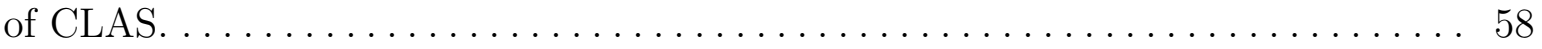

8 The EC timing offsets for different sectors in ns and the cuts applied on the velocity of neutral particles to select photons for all targets at 2.2 and $4.4 \mathrm{GeV} . \quad$. 85

$9 \quad$ The energy and momentum thresholds for different particles. . . . . . . . . 85

10 Electron momentum correction factors for different sectors at $1.1,2.2$ and $4.4 \mathrm{GeV}$

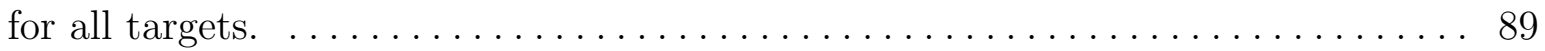

11 The table of cut values on the $u, v, w$ EC local coordinates of an electron for different sectors of CLAS, used to select the initial fiducial region used for the

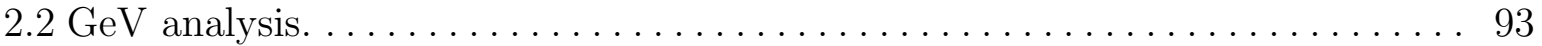

12 The simulated target positions were shifted from their default positions in order to better match the reconstructed target positions in data. $\ldots \ldots \ldots \ldots \ldots \ldots$

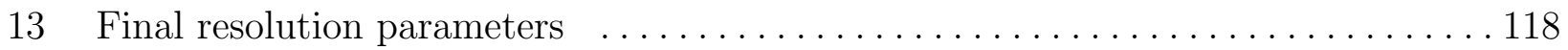

14 The cut values on the electron $z$ vertex, and the difference between the vertexes of the electron and the proton for different targets and beam energies. These cut values are used to select particles that are coming from the target region and

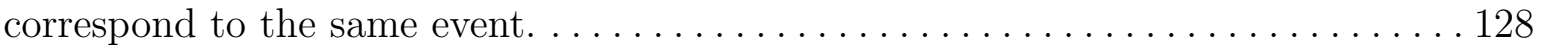

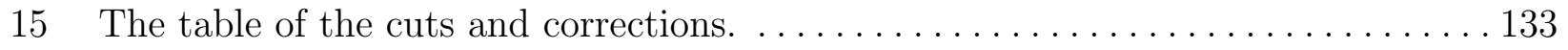

16 Number of good $\left(e, e^{\prime}\right)$ and $\left(e, e^{\prime} p\right)$ events in millions. . . . . . . . . . . 141 
17 Offsets to correct the binding energies in reconstructed energy calculations for

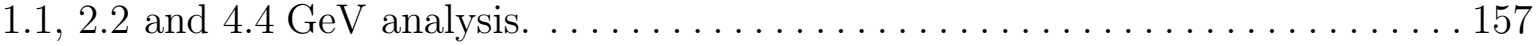

18 Fraction of events reconstructed within $5 \%$ of beam energy at 1.1, 2.2 and $4.4 \mathrm{GeV}$

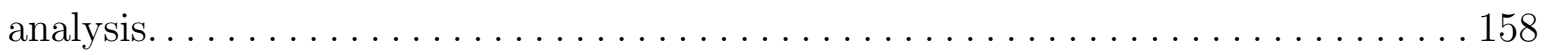

19 Fraction of events reconstructed within $10 \%$ of beam energy at 1.1, 2.2 and

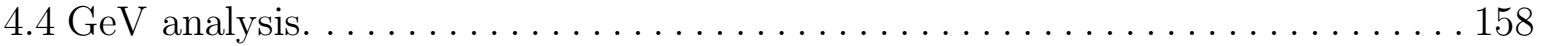

20 Fraction of events reconstructed within $20 \%$ of beam energy at 1.1, 2.2 and

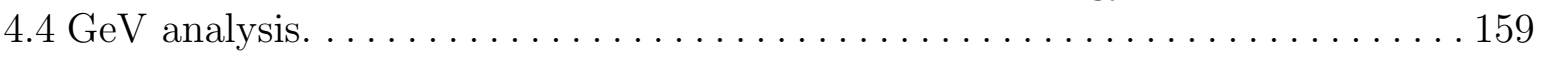

21 The summary of statistical and systematic uncertainty sources in our analysis. . . 205 


\section{LIST OF FIGURES}

Figure

1 Impact on extracted neutrino oscillation parameters if the neutrino event generator does not describe the underlying physics accurately. . . . . . . . 2

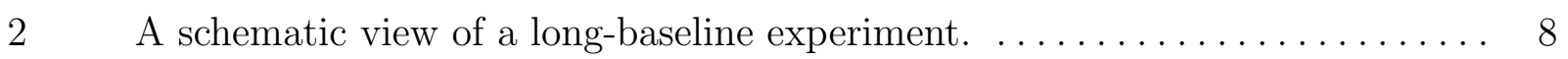

3 The neutrino oscillation signal from the $\mathrm{T} 2 \mathrm{~K}$ experiment. ......... 9

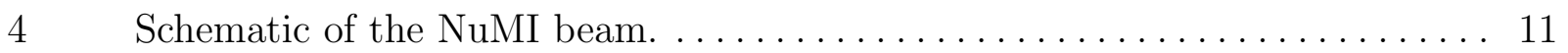

$5 \quad$ Variation of neutrino spectra for different focusing schemes at NuMI. . . . . . 13

6 Current neutrino sources as a function of neutrino energy. . . . . . . . 16

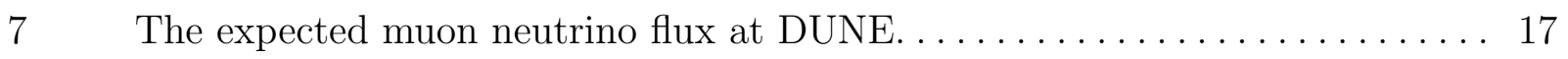

8 The diagrams of QE scattering of an electron on a nucleon and CC QE

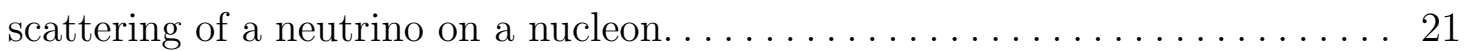

9 Schematic representation of the electron-nucleus inclusive cross section at

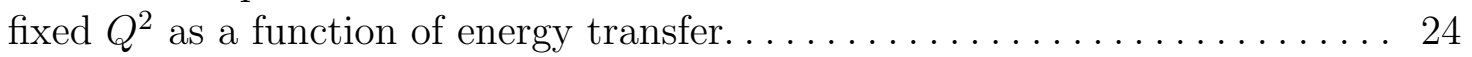

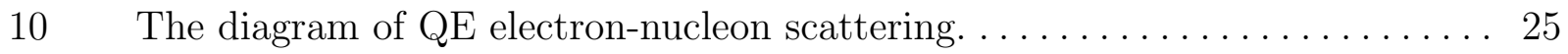

11 The diagrams of different two-body interactions of electrons in a nucleus. . . 26

12 The missing energy spectra for semi inclusive ${ }^{12} \mathrm{C}\left(e, e^{\prime} p\right)$ reaction in the $\mathrm{QE}$

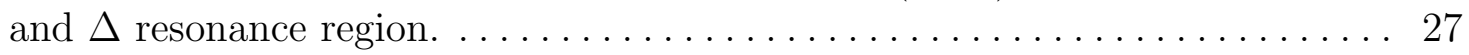

13 The missing energy spectra for semi inclusive ${ }^{12} \mathrm{C}\left(e, e^{\prime} p\right)$ reaction for three

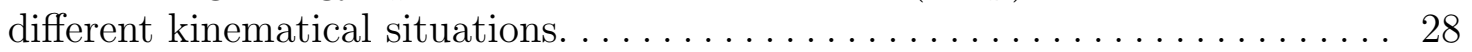

14 Spectroscopic factors for various nuclei extracted by comparing $A\left(e, e^{\prime} p\right)$ valence knockout cross-sections to mean field theory $[1] \ldots \ldots \ldots \ldots \ldots \ldots \ldots .29$

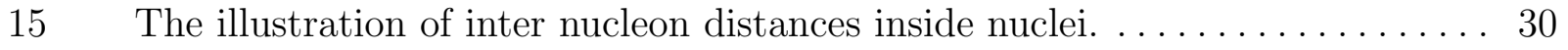

16 Per nucleon momentum distributions calculated by Schiavilla et al. [2] in

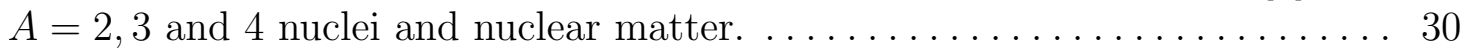

17 Charged current neutrino-nucleus inclusive scattering cross section. . . . . . . 33 
18 Flux-unfolded MiniBooNE $\nu_{\mu}$ CCQE cross section per neutron as a function

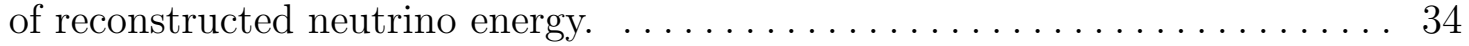

19 CCQE cross section for a $\mathrm{CH}_{2}$ target obtained in the MiniBooNE experiment. 35

20 The $\nu_{e}$ appearance probability in a $\nu_{\mu}$ beam at a distance of $1300 \mathrm{~km}$, calcu-

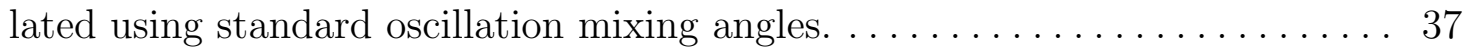

$21 \delta_{\mathrm{CP}}$ sensitivity for $50 \%$ of possible $\delta_{\mathrm{CP}}$ values as a function of exposure at DUNE experiment. . . . . . . . . . . . . . . . . . . . . . . . . 37

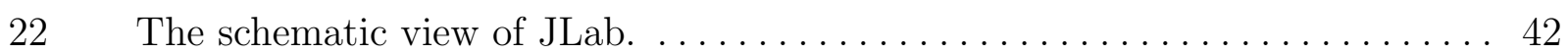

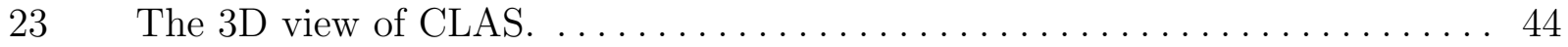

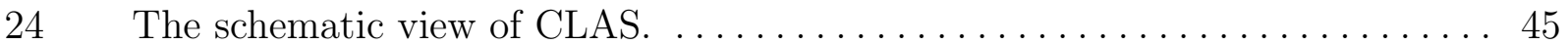

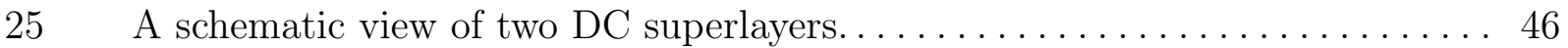

26 A 3D view of CLAS Cherenkov Detector. .................... 46

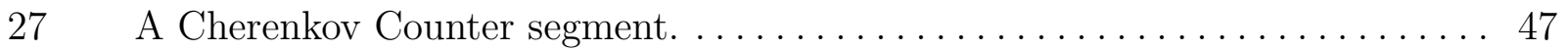

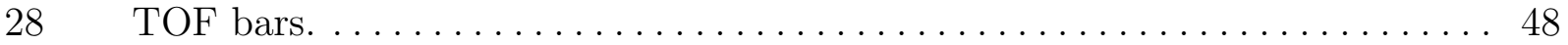

29 The view of EC module layers. $\ldots \ldots \ldots \ldots \ldots \ldots \ldots \ldots \ldots \ldots \ldots \ldots$

30 The timing of forward paddles with respect to the RF time for electrons detected in the forward TOF paddles of different sectors. . . . . . . . . . 51

31 The $\pi^{+}$and $e^{-}$TOF time difference plotted versus $\pi^{+}$TOF paddle number

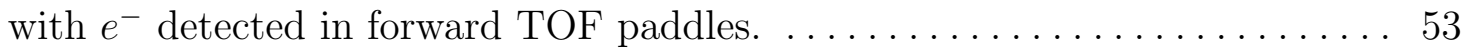

32 The ratio of the total energy deposited in the calorimeter over the momentum

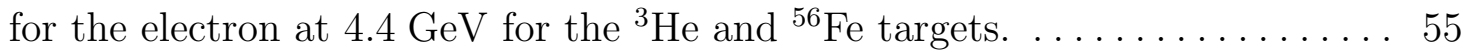

33 The energy deposited in the inner layer of the calorimeter for the electron

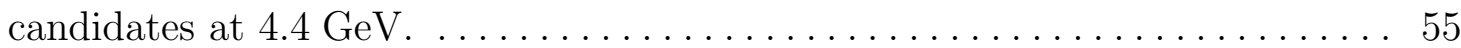

34 The total energy deposited in the calorimeter for the electron candidates at

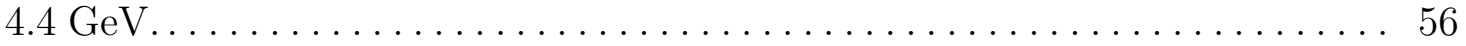

35 The ratio of the total energy deposited in the calorimeter over the momentum

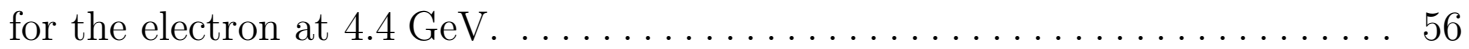

36 The distribution of the number of photo-electrons detected in the CC for

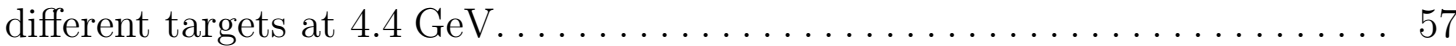


37 The time difference between hits of the electron candidate in $\mathrm{CC}$ and SC, corrected for the distance traveled from $\mathrm{CC}$ to $\mathrm{SC}$ at $4.4 \mathrm{GeV} \ldots \ldots \ldots \ldots 60$

38 The distribution of the angle between a CC hit and the nearest SC hit at $4.4 \mathrm{GeV}$.

39 The distribution of the number of photo-electrons detected in the CC for electron candidates at $4.4 \mathrm{GeV}$ after different cuts................. 61

40 The ratio of the total energy deposited in the calorimeter over the momentum for the electron candidates at $2.2 \mathrm{GeV}$.

41 The energy deposited in the inner layer of calorimeter with the cut for the electron candidates at $2.2 \mathrm{GeV}$. .

42 The total energy deposited in the calorimeter for the electron candidates at

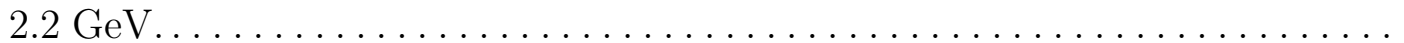

43 The distribution of the angle between a $\mathrm{CC}$ hit and the nearest $\mathrm{SC}$ hit at

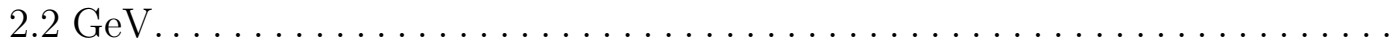

44 The time difference between hits of the electron candidate in $\mathrm{CC}$ and SC, corrected for the distance traveled from $\mathrm{CC}$ to $\mathrm{SC}$ at $2.2 \mathrm{GeV} \ldots \ldots \ldots \ldots 64$

45 The distribution of the number of photo-electrons detected in the $\mathrm{CC}$ at

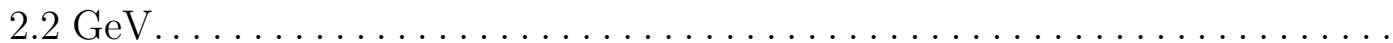

46 The ratio of the total energy deposited in the calorimeter over the momentum for the electron candidates at $1.1 \mathrm{GeV}$ for all targets. ............... 66

47 The energy deposited in the inner layer of calorimeter with the cut for the

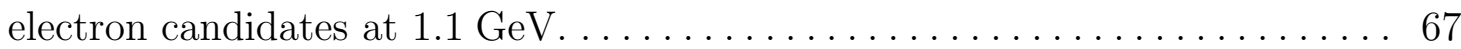

48 The time difference between hits of the electron candidate in $\mathrm{CC}$ and SC, corrected for the distance traveled from $\mathrm{CC}$ to $\mathrm{SC}$ at $1.1 \mathrm{GeV} \ldots \ldots \ldots \ldots 68$

49 The distribution of the angle between a CC hit and the nearest SC hit at $2.2 \mathrm{GeV}$

50 The distribution of the number of photo-electrons detected in the $\mathrm{CC}$ at

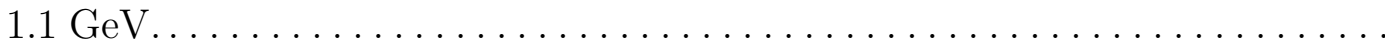

51 Beta from the $\mathrm{SC}$ vs momentum from the $\mathrm{DC}$ for positively charged particles

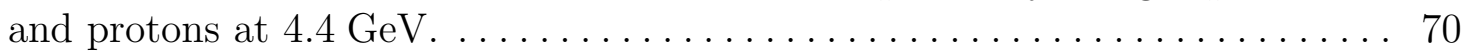

52 The $\Delta t$ plot as a function of momentum for positive particles at $4.4 \mathrm{GeV}$ for the ${ }^{3} \mathrm{He}$ and ${ }^{56} \mathrm{Fe}$ targets. ............................... 70 
53 Beta from the SC vs momentum from the DC for positively charged particles

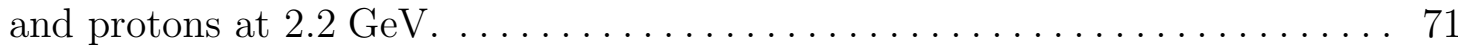

54 The plot of $\Delta t$ as a function of momentum from the DC for positive particles

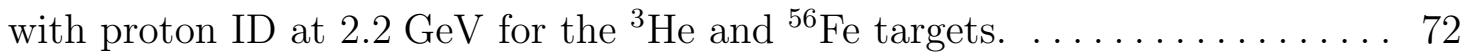

55 Beta from the SC vs momentum from the DC for positively charged particles

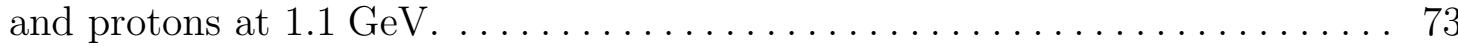

56 The plot of $\Delta t$ as a function of momentum from the DC for positive particles with proton ID at $1.1 \mathrm{GeV}$ for the ${ }^{3} \mathrm{He}$ and ${ }^{12} \mathrm{C}$ targets. . . . . . . . . . 74

57 The plot of $\Delta t$ as a function of momentum from the DC for $\pi^{-}$candidates

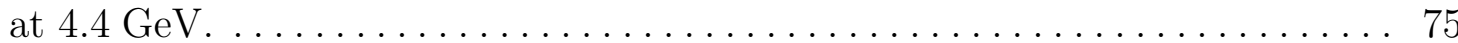

58 The plot of $\Delta t$ as a function of momentum from the DC for $\pi^{+}$candidates

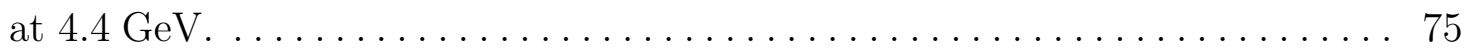

59 The plot of $\Delta t$ as a function of momentum from the DC for $\pi^{-}$candidates

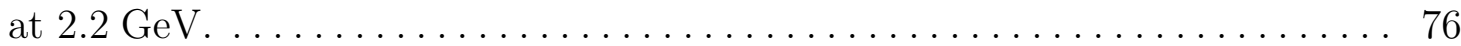

60 The plot of $\Delta t$ as a function of momentum from the DC for or $\pi^{+}$candidates

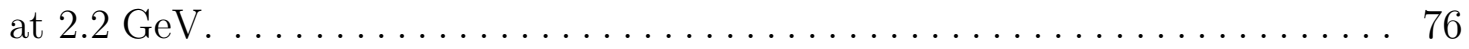

61 The plot of $\Delta t$ as a function of momentum from the DC for $\pi^{-}$candidates

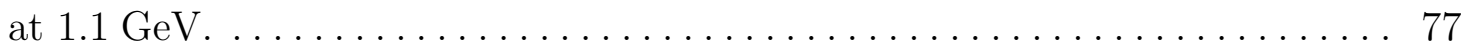

62 The plot of $\Delta t$ as a function of momentum from the DC for or $\pi^{+}$candidates

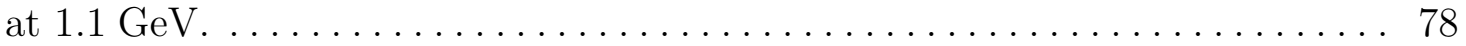

63 The $\beta$ of neutral particles in EC before and after corrections at 2.2 and $4.4 \mathrm{GeV} .79$

64 The $\beta$ of neutral particles in EC before and after corrections at $1.1 \mathrm{GeV} \ldots . .80$

65 The energy spectra of photon candidates at different beam energies for ${ }^{12} \mathrm{C} \ldots \quad 80$

66 The $\Delta t_{\mathrm{EC}}$ as a function of SC paddles in different CLAS sectors for electrons at $4.4 \mathrm{GeV}$.

67 The $\Delta t_{\mathrm{EC}}$ as a function of $\mathrm{SC}$ paddles in different CLAS sectors for electrons at $2.2 \mathrm{GeV}$.

68 The $\Delta t_{\mathrm{EC}}$ as a function of $\mathrm{SC}$ paddles in different CLAS sectors for electrons

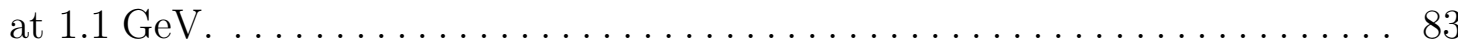

69 The $\Delta t_{\mathrm{EC}}$ for electrons before and after corrections at $4.4 \mathrm{GeV}$. 
$70 \quad$ The $\Delta t_{\mathrm{EC}}$ for electrons before and after corrections at $2.2 \mathrm{GeV} \ldots \ldots 86$

71 The $\Delta t_{\mathrm{EC}}$ for electrons before and after corrections at $1.1 \mathrm{GeV} \ldots \ldots 86$

72 The difference between reconstructed and generated proton momenta vs reconstructed momentum for ${ }^{3} \mathrm{He} . \ldots \ldots \ldots \ldots \ldots \ldots \ldots \ldots \ldots$

73 The distributions of electron momentum corrections factors for sectors 1 and

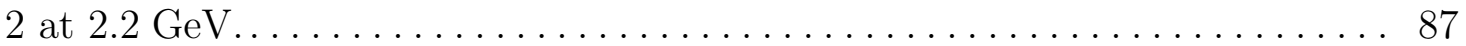

$74 \quad$ The ${ }^{3} \mathrm{He}\left(e, e^{\prime} p p\right) n$ missing mass calculated with obtained electron momentum corrections vs electron $\phi$ angle at $4.4 \mathrm{GeV} \ldots \ldots \ldots \ldots \ldots \ldots \ldots$

75 The ${ }^{3} \mathrm{He}\left(e, e^{\prime} p p\right) n$ missing mass distributions before and after electron mo-

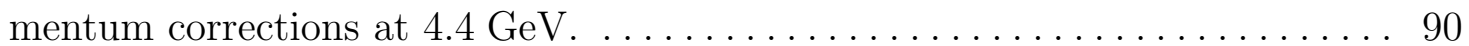

76 The ${ }^{3} \mathrm{He}\left(e, e^{\prime} p p\right) n$ missing mass calculated with obtained electron momentum corrections vs electron $\phi$ angle at $2.2 \mathrm{GeV} \ldots \ldots \ldots \ldots \ldots \ldots \ldots \ldots \ldots \ldots \ldots \ldots$

77 The ${ }^{3} \mathrm{He}\left(e, e^{\prime} p p\right) n$ missing mass distributions before and after electron mo-

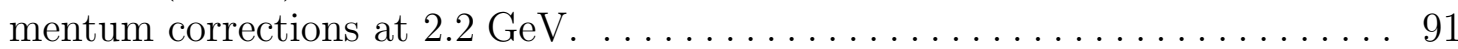

78 The ${ }^{3} \mathrm{He}\left(e, e^{\prime} p p\right) n$ missing mass calculated with obtained electron momentum corrections vs electron $\phi$ angle at $1.1 \mathrm{GeV} \ldots \ldots \ldots \ldots \ldots \ldots \ldots \ldots \ldots \ldots$

79 The ${ }^{3} \mathrm{He}\left(e, e^{\prime} p p\right) n$ missing mass distributions before and after electron mo-

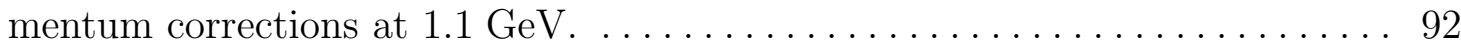

80 The distributions for the electron EC local coordinates for sector 1 at $4.4 \mathrm{GeV} .94$

81 The $\theta$ vs $\phi$ distributions for electrons for two momentum bins before and

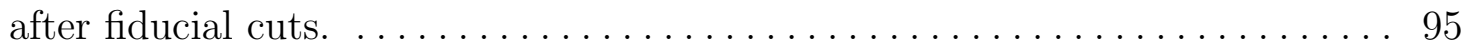

82 The $\theta$ vs $\phi$ distributions for electrons for different sectors before and after

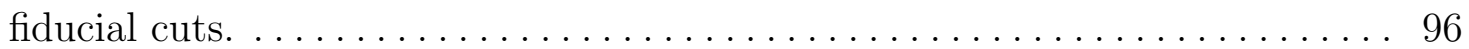

83 The distributions of the electron EC local coordinates for sector 5 at $2.2 \mathrm{GeV} .97$

84 The $\theta$ vs $\phi$ distributions of the electron for two momentum bins before and

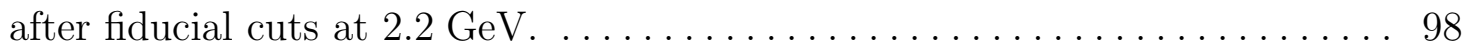

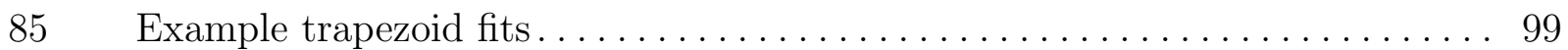

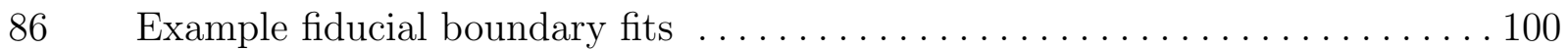

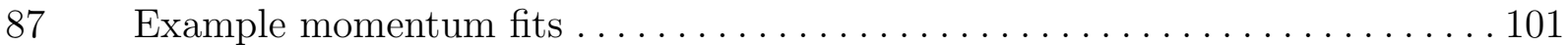


88 The $\theta$ vs $\phi$ distributions for electrons with $p=1 \mathrm{GeV} / \mathrm{c}$ before and after fiducial cuts. . . . . . . . . . . . . . . . . . . . . . . . . . . . . . 102

89 The $\theta$ vs $\phi$ distributions for the proton before and after fiducial cuts at $4.4 \mathrm{GeV} .103$

90 The $\theta$ vs $\phi$ distributions for the proton before and after fiducial cuts at $2.2 \mathrm{GeV} .103$

91 The $\theta$ vs $\phi$ distributions for protons with $p=0.6 \mathrm{GeV} / \mathrm{c}$ before and after

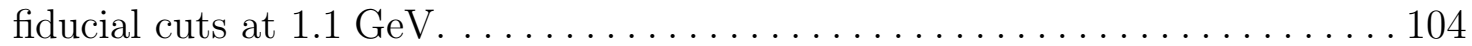

92 The $\theta$ vs $\phi$ distributions for the $\pi^{+}$before and after fiducial cuts at $4.4 \mathrm{GeV} .104$

93 The $\theta$ vs $\phi$ distributions for the $\pi^{-}$before and after fiducial cuts at $4.4 \mathrm{GeV} .105$

94 The $\theta$ vs $\phi$ distributions for $\pi^{-}$with $p=0.25 \mathrm{GeV} / \mathrm{c}$ before and after fiducial

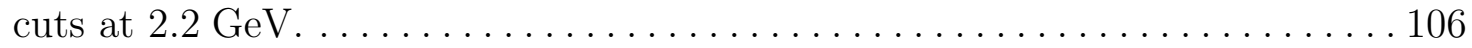

95 The $\theta$ vs $\phi$ distributions for the $\pi^{+}$before and after fiducial cuts at $2.2 \mathrm{GeV} .107$

96 The $\theta$ vs $\phi$ distributions for the $\pi^{-}$before and after fiducial cuts at $2.2 \mathrm{GeV} .107$

97 The $\theta$ vs $\phi$ distributions for $\pi^{-}$with $p=0.3 \mathrm{GeV} / \mathrm{c}$ before and after fiducial

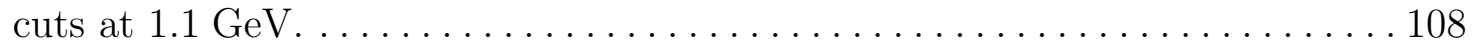

98 The $\theta$ vs $\phi$ distributions for $\pi^{+}$with $p=0.3 \mathrm{GeV} / \mathrm{c}$ before and after fiducial

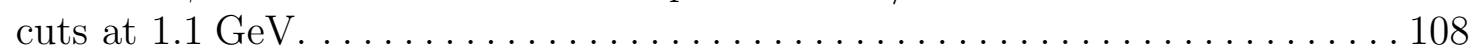

99 The $\cos \theta$ vs $\phi$ distributions for photons at $4.4 \mathrm{GeV} \ldots \ldots \ldots \ldots \ldots$

100 The $\cos \theta$ vs $\phi$ distributions for photons with fiducial cut outline. . . . . . . 110

101 The acceptance for protons calculated with acceptance maps. . . . . . . . . 112

102 The acceptance for electrons calculated with acceptance maps. . . . . . . . 113

103 Three momentum slices through a single sector of the electron acceptance are shown. The acceptance was calculated from the acceptance map corresponding to the solid foil target position, 2250 A torus current, and PID and fiducial cuts from the $4.461 \mathrm{GeV}$ beam energy analysis. Red dashed lines are shown at $20^{\circ}$ to illustrate how the detector covers more forward angles as the electron momentum increases, due to reduced in-bending. . . . . . . . . . . 114

104 Events were generated with a constant number per bin, as illustrated by the green points in the right cartoon. If events were generated randomly over the entire phase space, as shown by the red points in the left cartoon, some bins would be underpopulated, or even completely empty. . . . . . . . . 115 
105 For hadrons generated in sector 5 (illustrated by green dots), an accompanying electron was generated in the high-acceptance region of sector 2 (illustrated by the blue dot) to satisfy the simulation trigger requirements. . . 116

106 Our vertex shift for the carbon foil target takes the original simulated vertex distribution in green and moves it to the blue distribution in order to match

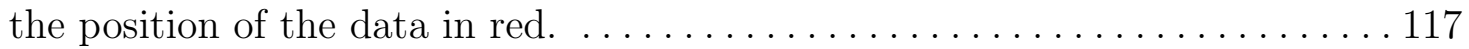

107 The simulated vertex resolution as a function of scattering angle was tuned

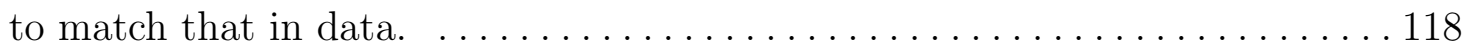

108 The simulated mass-squared distribution for protons from Eq. 59 matched data best when we set $f$ to zero. The simulated distribution shown here has been scaled to show the shape agreement. . . . . . . . . . . . . . . 119

109 Map histograms for $2 \mathrm{GeV} / c$ protons: the only reason there are not 10 generated events in every bin is because of the occasional loss of the trigger

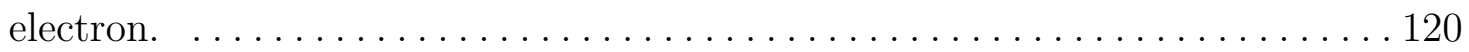

$110 \quad$ Vertex correction function for ${ }^{3} \mathrm{He}$ at $4.4 \mathrm{GeV} \ldots \ldots \ldots \ldots \ldots \ldots \ldots \ldots \ldots$

111 The $\phi$ angle of the electrons plotted vs the $z$ component of the vertex for the

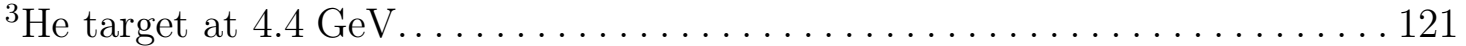

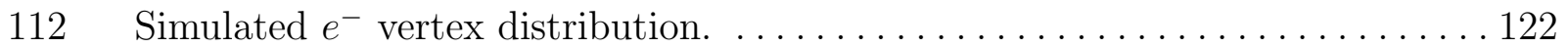

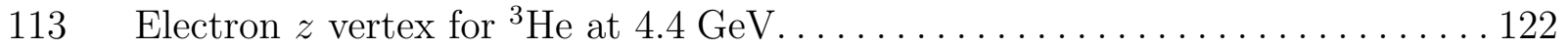

114 The electron and proton $z$ vertex difference for ${ }^{3} \mathrm{He}$ at $4.4 \mathrm{GeV} \ldots \ldots \ldots 123$

115 The electron and charged pion $z$ vertex difference for ${ }^{3} \mathrm{He}$ at $4.4 \mathrm{GeV} \ldots \ldots 123$

116 Vertex correction function for ${ }^{3} \mathrm{He}$ at $2.2 \mathrm{GeV} \ldots \ldots \ldots \ldots \ldots \ldots \ldots \ldots \ldots$

117 The $\phi$ angle of the electrons plotted vs the $z$ component of the vertex for the

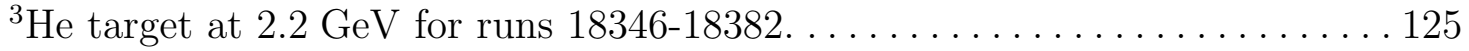

118 Electron $z$ vertex for ${ }^{3} \mathrm{He}$ at $2.2 \mathrm{GeV}$ for runs $18346-18382 \ldots \ldots \ldots \ldots \ldots$

119 The electron and proton $z$ vertex difference for ${ }^{3} \mathrm{He}$ at $2.2 \mathrm{GeV}$ for runs

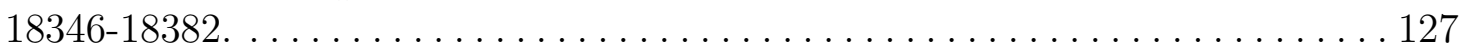

120 The electron and charged pion $z$ vertex difference for ${ }^{3} \mathrm{He}$ at $2.2 \mathrm{GeV}$ for runs

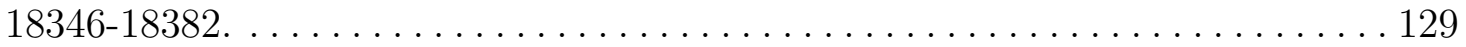

121 The $\phi$ angle of the electrons plotted vs the $z$ component of the vertex for the

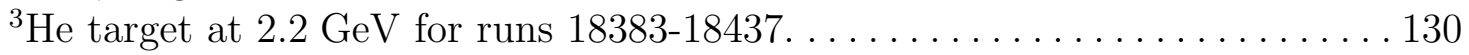


122 Electron $z$ vertex for ${ }^{3} \mathrm{He}$ at $2.2 \mathrm{GeV}$ for runs $18383-18437 \ldots \ldots \ldots \ldots \ldots 131$

123 The electron and proton $z$ vertex difference for ${ }^{3} \mathrm{He}$ at $2.2 \mathrm{GeV}$ for runs

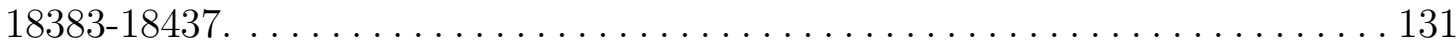

124 The electron and charged pion $z$ vertex difference for ${ }^{3} \mathrm{He}$ at $2.2 \mathrm{GeV}$ for runs

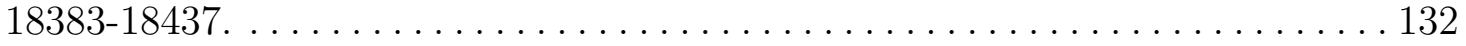

125 Vertex correction function for ${ }^{3} \mathrm{He}$ and ${ }^{12} \mathrm{C}$ at $1.1 \mathrm{GeV} \ldots \ldots \ldots \ldots \ldots \ldots$

126 The $\phi$ angle of the electrons plotted vs the $z$ component of the vertex for the ${ }^{3} \mathrm{He}$ and ${ }^{12} \mathrm{C}$ targets at $1.1 \mathrm{GeV}$ before and after vertex correction. . . . . . . 135

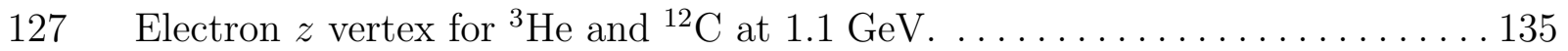

128 The electron and proton $z$ vertex difference for ${ }^{3} \mathrm{He}$ and ${ }^{12} \mathrm{C}$ targets at $1.1 \mathrm{GeV} .136$

129 The electron and charged pion $z$ vertex difference for ${ }^{3} \mathrm{He}$ at $1.1 \mathrm{GeV} \ldots \ldots 136$

130 The electron and charged pion $z$ vertex difference for ${ }^{12} \mathrm{C}$ at $1.1 \mathrm{GeV} \ldots \ldots 137$

131 Electron corrected $z$ vertex for ${ }^{12} \mathrm{C}$ and ${ }^{3} \mathrm{He}$ at $1.1 \mathrm{GeV} \ldots \ldots \ldots \ldots \ldots$

132 The electron $\theta$ angle distribution ${ }^{3} \mathrm{He}$ at $4.4 \mathrm{GeV} \ldots \ldots \ldots \ldots \ldots \ldots \ldots$

133 Vertex correction function for ${ }^{3} \mathrm{He}$ at $4.4 \mathrm{GeV}$ for different $\theta$ angle values. . . 138

134 Kinematic variables at $4.4 \mathrm{GeV} \ldots \ldots \ldots \ldots \ldots \ldots \ldots$. . . . . . . . . . . . . . . . . 142

135 Kinematic variables at $4.4 \mathrm{GeV}$ for ${ }^{56} \mathrm{Fe} \ldots \ldots \ldots \ldots \ldots \ldots \ldots \ldots \ldots$

136 The $\theta$ vs $\phi$ angular distribution of $e^{-}, \pi^{+}, \pi^{-}$and protons for ${ }^{3} \mathrm{He}$ at $4.4 \mathrm{GeV} .144$

137 The $\theta$ vs $\phi$ angular distribution of $\pi^{-}$and protons for ${ }^{3} \mathrm{He}$ at $4.4 \mathrm{GeV}$ plotted

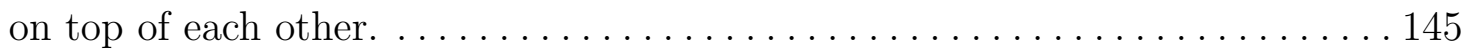

138 Multiplicity distributions of $\pi^{ \pm} / \gamma$ for ${ }^{3} \mathrm{He}$ and ${ }^{56} \mathrm{Fe}$ targets at $4.4 \mathrm{GeV} \ldots \ldots 145$

139 Multiplicity distributions of protons for ${ }^{3} \mathrm{He}$ and ${ }^{56} \mathrm{Fe}$ targets at $4.4 \mathrm{GeV} \ldots 146$

140 Reconstructed energy $E_{\mathrm{QE}}$ of $A\left(e, e^{\prime} p\right)$ after different cuts at $4.4 \mathrm{GeV} \ldots \ldots 147$

141 The diagrams for the $\pi$ and proton rotation around the three momentum transfer $(\vec{q})$ for the estimation of events with undetected $\pi$ in $A\left(e, e^{\prime}\right)$ and

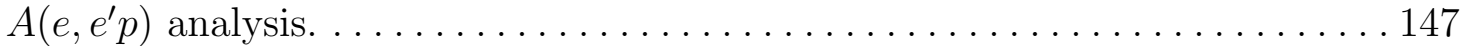

142 The number of events as a function of angle between the electron and the photon versus the difference of their azimuthal angles in logarithmic scale. . . 160 
143 The scheme of the subtraction for undetected few charged pion and photon

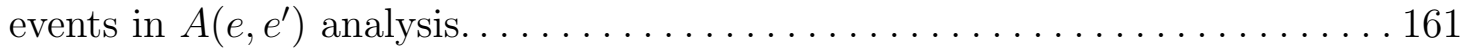

144 Reconstructed energy $E_{\mathrm{QE}}$ of $A\left(e, e^{\prime}\right) 0 \pi$ spectrum before and after subtraction for undetected pions for different targets at $4.4 \mathrm{GeV}$.

145 The plot of the number of protons as a function of number of charged pions and photons for ${ }^{56} \mathrm{Fe}$ at $4.4 \mathrm{GeV} \ldots \ldots \ldots \ldots \ldots \ldots \ldots \ldots \ldots \ldots \ldots \ldots \ldots \ldots \ldots \ldots$

146 The scheme of the subtraction for undetected four proton events in $A\left(e, e^{\prime} p\right)$

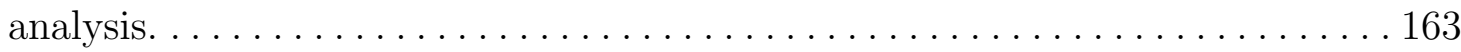

$147 E_{\mathrm{QE}}$ and $E_{\text {calor }}$ distributions of $A\left(e, e^{\prime} p\right) 0 \pi$ spectrum before subtraction for undetected pions, after subtraction for undetected pions and after subtraction for undetected pions and undetected protons for different targets at $4.4 \mathrm{GeV}$. 164

$148 E_{\mathrm{QE}}$ distributions of $A\left(e, e^{\prime}\right)$ and $A\left(e, e^{\prime} p\right) 0 \pi$ spectrum and $E_{\text {calor }}$ distribution of $A\left(e, e^{\prime} p\right) 0 \pi$ spectrum after subtraction for undetected pions and undetected protons for different targets at $4.4 \mathrm{GeV}$.

$149 E_{\text {calor versus }} E_{\mathrm{QE}}$ distributions of $A\left(e, e^{\prime} p\right) 0 \pi$ spectrum for different targets

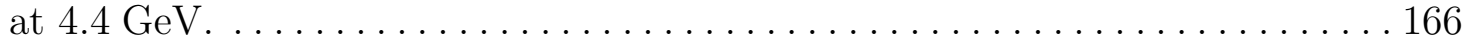

150 Reconstructed energy $E_{\mathrm{QE}}$ as a function of the total perpendicular momentum for the scattered electron and the knockout nucleon system before and after subtracting for undetected pions and protons at $4.4 \mathrm{GeV} \ldots \ldots \ldots \ldots 7$

$151 E_{\text {calor }}$ and $E_{\mathrm{QE}}$ distributions of $A\left(e, e^{\prime} p\right) 0 \pi$ spectrum after subtraction for undetected pions and undetected protons for different regions of $P_{\text {miss }}^{\perp}$ for

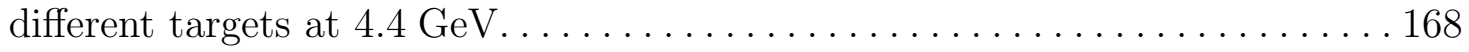

$152 E_{\text {calor }}$ distributions of $A\left(e, e^{\prime} p\right) 0 \pi$ spectrum after subtraction for undetected pions and undetected protons for different regions of $P_{\text {miss }}^{\perp}$ for different targets at $4.4 \mathrm{GeV}$ in full range. . . . . . . . . . . . . . . . . . . . . . 169

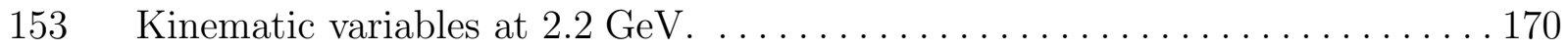

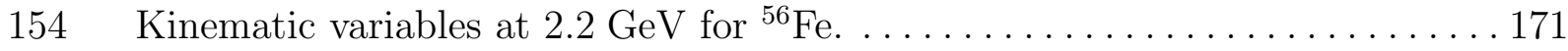

155 The $\theta$ vs $\phi$ angular distribution of $e^{-}, \pi^{+}, \pi^{-}$and protons for ${ }^{3} \mathrm{He}$ at $2.2 \mathrm{GeV} .172$

156 The $\theta$ vs $\phi$ angular distribution of $\pi^{-}$and protons for ${ }^{3} \mathrm{He}$ at $2.2 \mathrm{GeV}$ plotted

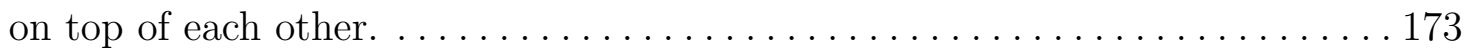

157 Multiplicity distributions of $\pi^{ \pm} / \gamma$ for ${ }^{3} \mathrm{He}$ and ${ }^{56} \mathrm{Fe}$ targets at $2.2 \mathrm{GeV} \ldots \ldots 173$

158 Multiplicity distributions of protons for ${ }^{3} \mathrm{He}$ and ${ }^{56} \mathrm{Fe}$ targets at $2.2 \mathrm{GeV} \ldots 174$ 
159 Reconstructed energy $E_{\mathrm{QE}}$ of $A\left(e, e^{\prime}\right) 0 \pi$ spectrum before and after subtraction for undetected pions for different targets at $2.2 \mathrm{GeV} . \ldots \ldots \ldots \ldots \ldots 175$

$160 \quad E_{\mathrm{QE}}$ and $E_{\text {calor }}$ of $A\left(e, e^{\prime} p\right) 0 \pi$ spectrum before and after subtraction for undetected pions and undetected protons for different targets at $2.2 \mathrm{GeV}$. . 176

$161 E_{\mathrm{QE}}$ distributions of $A\left(e, e^{\prime}\right)$ and $A\left(e, e^{\prime} p\right) 0 \pi$ spectrum and $E_{\text {calor }}$ distribution of $A\left(e, e^{\prime} p\right) 0 \pi$ spectrum after subtraction for undetected pions and undetected protons for different targets at $2.2 \mathrm{GeV} \ldots \ldots \ldots \ldots \ldots \ldots \ldots$

$162 E_{\text {calor versus }} E_{\mathrm{QE}}$ distributions of $A\left(e, e^{\prime} p\right) 0 \pi$ spectrum for different targets

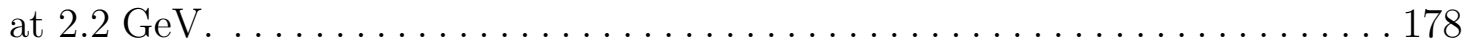

163 Reconstructed energy $E_{\mathrm{QE}}$ as a function of the total perpendicular momentum for the scattered electron and the knockout nucleon system before and after subtracting for undetected pions and protons at $2.2 \mathrm{GeV} \ldots \ldots \ldots \ldots \ldots 179$

$164 E_{\text {calor }}$ and $E_{\mathrm{QE}}$ distributions of $A\left(e, e^{\prime} p\right) 0 \pi$ spectrum after subtraction for undetected pions and undetected protons for different regions of $P_{\text {miss }}^{\perp}$ for

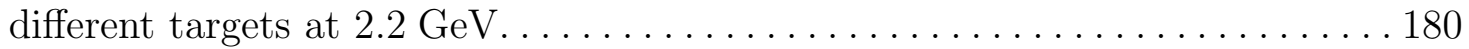

$165 E_{\text {calor }}$ distributions of $A\left(e, e^{\prime} p\right) 0 \pi$ spectrum after subtraction for undetected pions and undetected protons for different regions of $P_{\text {miss }}^{\perp}$ for different targets

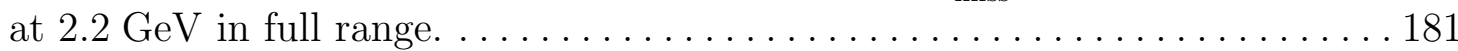

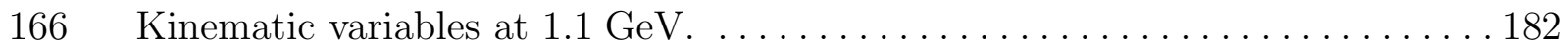

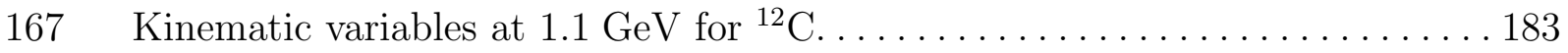

168 The $\theta$ vs $\phi$ angular distribution of $e^{-}, \pi^{+}, \pi^{-}$and protons for ${ }^{3} \mathrm{He}$ at $1.1 \mathrm{GeV} .184$

169 The $\theta$ vs $\phi$ angular distribution of $\pi^{-}$and protons for ${ }^{3} \mathrm{He}$ at $1.1 \mathrm{GeV}$ plotted

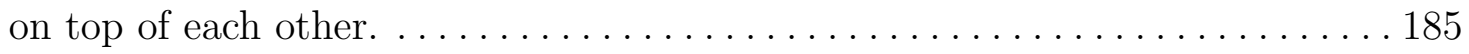

170 Multiplicity distributions of $\pi^{ \pm} \gamma$ for ${ }^{3} \mathrm{He}$ and ${ }^{12} \mathrm{C}$ targets at $1.1 \mathrm{GeV} \ldots \ldots 185$

171 Multiplicity distributions of protons for ${ }^{3} \mathrm{He}$ and ${ }^{12} \mathrm{C}$ targets at $1.1 \mathrm{GeV} \ldots \ldots 6$

172 Reconstructed energy $E_{\mathrm{QE}}$ of $A\left(e, e^{\prime}\right) 0 \pi$ spectrum before and after subtraction for undetected pions for different targets at $1.1 \mathrm{GeV} . \ldots \ldots \ldots \ldots \ldots$

$173 E_{\mathrm{QE}}$ and $E_{\text {calor }}$ of $A\left(e, e^{\prime} p\right) 0 \pi$ spectrum before and after subtraction for undetected pions and undetected protons for different targets at $1.1 \mathrm{GeV}$. . 188

$174 E_{\mathrm{QE}}$ distributions of $A\left(e, e^{\prime}\right)$ and $A\left(e, e^{\prime} p\right) 0 \pi$ spectrum and $E_{\text {calor }}$ distribution of $A\left(e, e^{\prime} p\right) 0 \pi$ spectrum after subtraction for undetected pions and undetected protons for different targets at $1.1 \mathrm{GeV}$. 


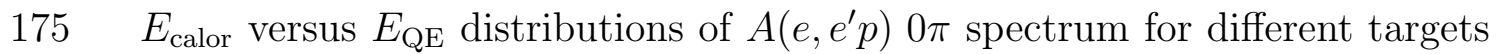

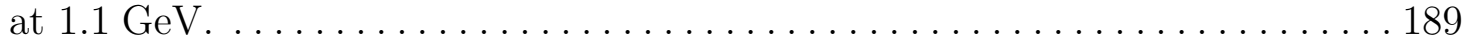

176 Reconstructed energy $E_{\mathrm{QE}}$ as a function of the total perpendicular momentum for the scattered electron and the knockout nucleon system before and after subtracting for undetected pions and protons at $1.1 \mathrm{GeV} \ldots \ldots \ldots \ldots 190$

$177 E_{\text {calor }}$ and $E_{\mathrm{QE}}$ distributions of $A\left(e, e^{\prime} p\right) 0 \pi$ spectrum after subtraction for undetected pions and undetected protons for different regions of $P_{\text {miss }}^{\perp}$ for

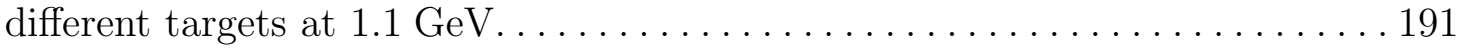

$178 E_{\mathrm{QE}}$ distributions of $A\left(e, e^{\prime}\right)$ and $A\left(e, e^{\prime} p\right) 0 \pi$ spectrum and $E_{\text {calor }}$ distribution of $A\left(e, e^{\prime} p\right) 0 \pi$ spectrum after subtraction for undetected pions and undetected protons for all targets at $2.2 \mathrm{GeV}$ and $4.4 \mathrm{GeV}$.

$179 E_{\mathrm{QE}}$ distributions of $A\left(e, e^{\prime}\right)$ and $A\left(e, e^{\prime} p\right) 0 \pi$ spectrum and $E_{\text {calor }}$ distribution of $A\left(e, e^{\prime} p\right) 0 \pi$ spectrum after subtraction for undetected pions and undetected protons for all targets at $1.1 \mathrm{GeV}, 2.2 \mathrm{GeV}$ and $4.4 \mathrm{GeV} \ldots \ldots . .193$

180 Fractional energy reconstruction "feed down" for $E_{\text {calor }}$ method and $A\left(e, e^{\prime} p\right)$ spectrum, for all targets at $1.1 \mathrm{GeV}, 2.2 \mathrm{GeV}$ and $4.4 \mathrm{GeV} \ldots \ldots \ldots \ldots \ldots . \ldots 194$

181 Fractional energy reconstruction "feed down" for $E_{\mathrm{QE}}$ method and $A\left(e, e^{\prime} p\right)$ spectrum, for all targets at $1.1 \mathrm{GeV}, 2.2 \mathrm{GeV}$ and $4.4 \mathrm{GeV} \ldots \ldots \ldots \ldots \ldots$

182 The neutrino oscillation signal from the T2K experiment with $\pm 5 \%$ error

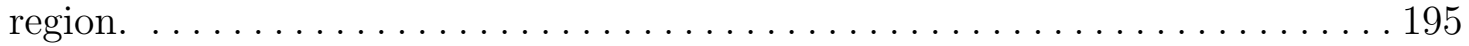

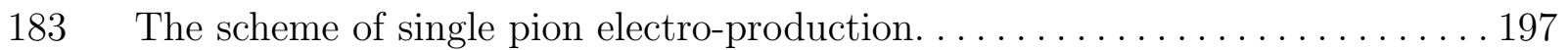

$184 E_{\mathrm{QE}}$ zero pi spectrum for $A\left(e, e^{\prime}\right)$ subtracted for undetected one charged pion events with and without accounting for $\phi_{\pi}$ dependence of the cross section. . 199

185 The ratio of the $E_{\mathrm{QE}} 0$ pi spectra for $A\left(e, e^{\prime}\right)$ subtracted for undetected 1 charged pion events with and without accounting for $\phi_{\pi}$ dependence of the

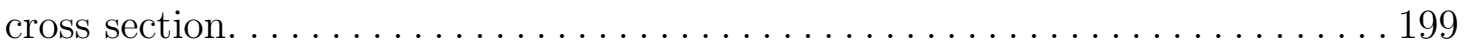

186 The fractional systematic uncertainty due to photon PID cut vs $E_{\text {calor }}\left(e, e^{\prime} p\right) .201$

187 The $\beta$ distribution of all neutral particles and the ones that have deposited

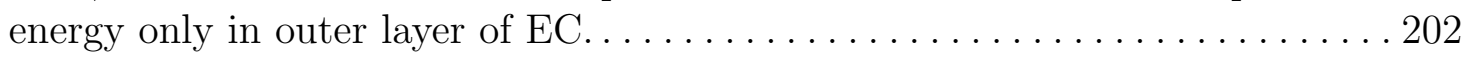

188 The $E_{\text {calor }}$ subtraction uncertainty due to fiducial cuts. . . . . . . . . . . 204

$189 E_{\mathrm{QE}}$ distributions of $A\left(e, e^{\prime}\right)$ and $A\left(e, e^{\prime} p\right) 0 \pi$ spectrum and $E_{\text {calor }}$ distribution of $A\left(e, e^{\prime} p\right) 0 \pi$ spectrum after subtractions for ${ }^{12} \mathrm{C}$ and ${ }^{56} \mathrm{Fe} \ldots \ldots \ldots \ldots \ldots$ 
$190 E_{\text {calor versus }} E_{\mathrm{QE}}$ distributions of $A\left(e, e^{\prime} p\right) 0 \pi$ spectrum for ${ }^{56} \mathrm{Fe}$ at $4.4 \mathrm{GeV} .208$

191 Reconstructed energy $E_{\mathrm{QE}}$ as a function of $P_{\mathrm{miss}}^{\perp}$ for the scattered electron and the knockout nucleon system before and after subtracting for undetected

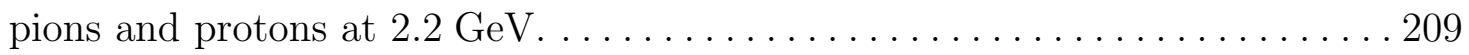

192 The distribution of $P_{\text {miss }}^{\perp}$ at $2.2 \mathrm{GeV}$ from data and GENIE. . . . . . . . 210

193 The $E_{\mathrm{QE}}$ distributions of ${ }^{12} \mathrm{C}\left(e, e^{\prime} p\right) 0 \pi$ spectrum for different regions of $P_{\text {miss }}^{\perp}$ at $2.2 \mathrm{GeV}$ from data and GENIE. . . . . . . . . . . . . . . . . . . . . . 211

194 The energy feed-down spectra for $E_{\mathrm{QE}}$ method from data and GENIE, for

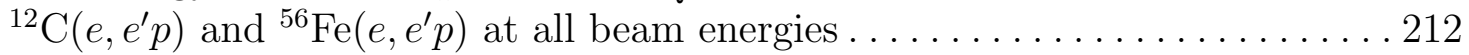

195 The energy feed-down spectra for $E_{\text {calor }}$ method from data and GENIE, for

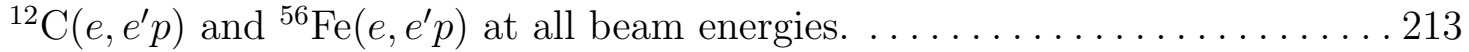

196 The reconstructed incident neutrino energy spectra for the DUNE far detector with oscillation included, simulated with GENIE and reconstructed with

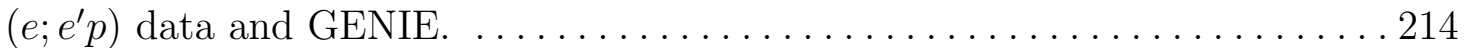

197 The $\phi$ angle of the electrons plotted vs z component of vertex for ${ }^{4} \mathrm{He}$ target

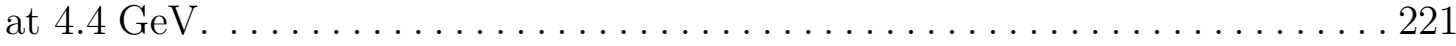

198 The distributions of electron $z$ vertex and electron and proton $z$ vertex dif-

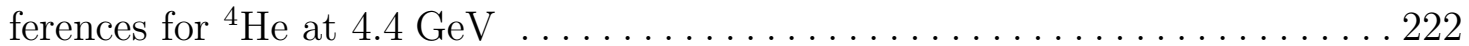

199 The electron and charged pion $z$ vertex difference for ${ }^{4} \mathrm{He}$ at $4.4 \mathrm{GeV} \ldots \ldots 222$

200 The $\phi$ angle of the electrons plotted vs z component of vertex for ${ }^{4} \mathrm{He}$ target

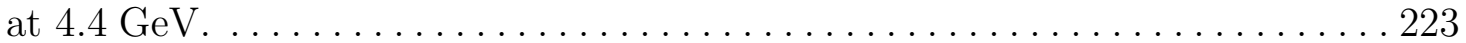

201 The distributions of electron $z$ vertex and electron and proton $z$ vertex dif-

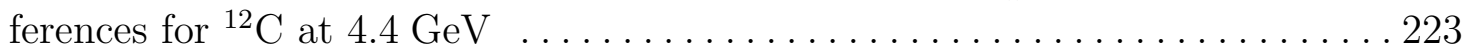

202 The electron and charged pion $z$ vertex difference for ${ }^{12} \mathrm{C}$ at $4.4 \mathrm{GeV} \ldots \ldots 224$

203 The $\phi$ angle of the electrons plotted vs z component of vertex for ${ }^{56} \mathrm{Fe}$ target

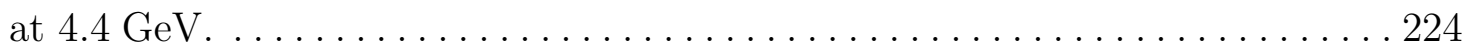

204 The distributions of electron $z$ vertex and electron and proton $z$ vertex dif-

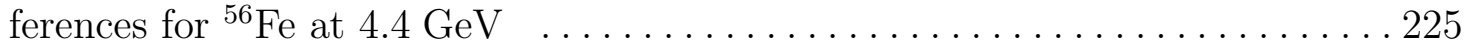

205 The electron and charged pion $z$ vertex difference for ${ }^{56} \mathrm{Fe}$ at $4.4 \mathrm{GeV} \ldots \ldots 225$

206 The $\phi$ angle of the electrons plotted vs z component of vertex for ${ }^{4} \mathrm{He}$ target

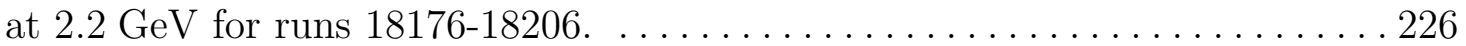


207 The distributions of electron $z$ vertex and electron and proton $z$ vertex differences for ${ }^{4} \mathrm{He}$ at $2.2 \mathrm{GeV}$ for runs $18176-18206 \ldots \ldots \ldots \ldots \ldots \ldots \ldots \ldots$

208 The electron and charged pion $z$ vertex difference for ${ }^{4} \mathrm{He}$ at $2.2 \mathrm{GeV}$ for runs 18176-18206. . . . . . . . . . . . . . . . . . . . . . . . . . 227

209 The $\phi$ angle of the electrons plotted vs z component of vertex for ${ }^{4} \mathrm{He}$ target

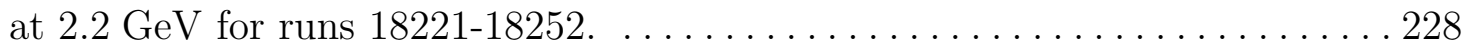

210 The distributions of electron $z$ vertex and electron and proton $z$ vertex differences for ${ }^{4} \mathrm{He}$ at $2.2 \mathrm{GeV}$ for runs $18221-18252 \ldots \ldots \ldots \ldots \ldots \ldots \ldots \ldots$

211 The electron and charged pion $z$ vertex difference for ${ }^{4} \mathrm{He}$ at $2.2 \mathrm{GeV}$ for runs 18221-18252. . . . . . . . . . . . . . . . . . . . . . . . . . . . . . . . . 229

212 The $\phi$ angle of the electrons plotted vs z component of vertex for ${ }^{4} \mathrm{He}$ target

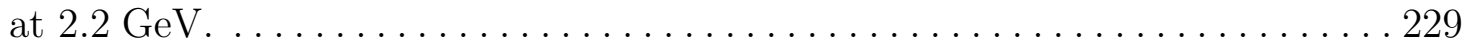

213 The distributions of electron $z$ vertex and electron and proton $z$ vertex dif-

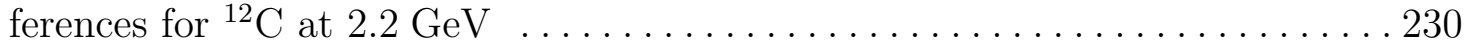

214 The electron and charged pion $z$ vertex difference for ${ }^{12} \mathrm{C}$ at $2.2 \mathrm{GeV} \ldots \ldots 230$

215 The $\phi$ angle of the electrons plotted vs $\mathrm{z}$ component of vertex for ${ }^{56} \mathrm{Fe}$ target

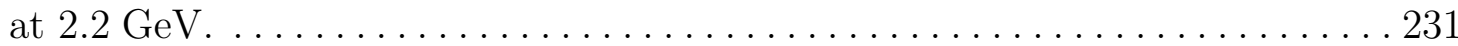

216 The distributions of electron $z$ vertex and electron and proton $z$ vertex dif-

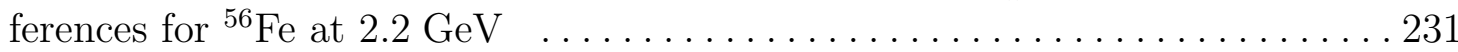

217 The electron and charged pion $z$ vertex difference for ${ }^{56} \mathrm{Fe}$ at $2.2 \mathrm{GeV} \ldots \ldots 232$

218 The $\phi$ angle of the electrons plotted vs $\mathrm{z}$ component of vertex for ${ }^{56} \mathrm{Fe}$ target

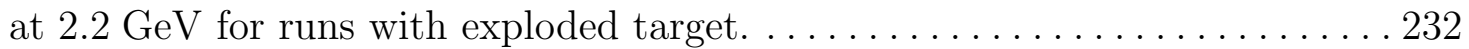

219 The distributions of electron $z$ vertex and electron and proton $z$ vertex differences for ${ }^{56} \mathrm{Fe}$ at $2.2 \mathrm{GeV}$ for runs with exploded target. . . . . . . . . 233

220 The electron and charged pion $z$ vertex difference for ${ }^{56} \mathrm{Fe}$ at $2.2 \mathrm{GeV} \ldots \ldots 233$

221 Multiplicity distributions of $\pi^{ \pm}$for ${ }^{4} \mathrm{He}$ and ${ }^{12} \mathrm{C}$ targets at $4.4 \mathrm{GeV} \ldots \ldots 234$

222 Multiplicity distributions of protons for ${ }^{4} \mathrm{He}$ and ${ }^{12} \mathrm{C}$ targets at $4.4 \mathrm{GeV} \ldots \ldots 235$

223 Reconstructed energy $E_{\mathrm{QE}}$ as a function of the total perpendicular momentum for the scattered electron and the knockout nucleon system before and after subtraction for undetected pions and protons $4.4 \mathrm{GeV} \ldots \ldots \ldots \ldots 236$ 
224 Reconstructed energy $E_{\mathrm{QE}}$ of $A\left(e, e^{\prime}\right) 0 \pi$ spectrum before and after subtraction for undetected pions for different targets at $4.4 \mathrm{GeV} . \ldots \ldots \ldots \ldots \ldots 237$

$225 E_{\mathrm{QE}}$ and $E_{\text {calor }}$ of $A\left(e, e^{\prime} p\right) 0 \pi$ spectrum before and after subtraction for undetected pions and undetected protons for different targets at $4.4 \mathrm{GeV}$. . . 238

226 Multiplicity distributions of $\pi^{ \pm}$for ${ }^{4} \mathrm{He}$ and ${ }^{12} \mathrm{C}$ targets at $2.2 \mathrm{GeV} \ldots \ldots 239$

227 Multiplicity distributions of protons for ${ }^{4} \mathrm{He}$ and ${ }^{12} \mathrm{C}$ targets at $2.2 \mathrm{GeV}$. . . 239

228 Reconstructed energy $E_{\mathrm{QE}}$ as a function of the total perpendicular momentum for the scattered electron and the knockout nucleon system before and after subtracting for undetected pions and protons at $2.2 \mathrm{GeV} \ldots \ldots \ldots \ldots 240$

229 Reconstructed energy $E_{\mathrm{QE}}$ of $A\left(e, e^{\prime}\right) 0 \pi$ spectrum before and after subtraction for undetected pions for different targets at $2.2 \mathrm{GeV} . \ldots \ldots \ldots \ldots \ldots 24$

$230 E_{\mathrm{QE}}$ and $E_{\text {calor }}$ of $A\left(e, e^{\prime} p\right) 0 \pi$ spectrum before and after subtraction for undetected pions and undetected protons for different targets at $2.2 \mathrm{GeV}$. . . 242 


\section{CHAPTER 1}

\section{INTRODUCTION AND THEORY}

\subsection{OVERVIEW}

\subsubsection{INTRODUCTION}

Experiments that study neutrino oscillations need to know the incident neutrino energy to be able to extract the distribution of the neutrino beam incident on the detector. The distributions (i.e., at the near and far detectors) of the neutrino beam before and after oscillations are then used to obtain the oscillation signal and to extract neutrino oscillation parameters.

Experiments that study neutrino oscillations as well as nucleon weak form factors are particularly interested in quasi-elastic (QE) scattering of a neutrino from a bound nucleon via the neutral current (NC) mediated by $Z$ boson exchange $\nu+N \rightarrow \nu+N$ or scattering via charged currents (CC) mediated by $W^{+}$or $W^{-}$boson exchange: $\nu_{1}+n \rightarrow l^{-}+p$ or $\bar{\nu}_{1}+p \rightarrow l^{+}+n$, as in these reactions the nucleon comes out intact and the two body nature of the interaction allows the kinematics to be fully reconstructed from the outgoing particles.

The neutrino initial energy cannot be measured directly because neutrino beams are not monoenergetic. High energy neutrino beams are made by scattering protons on nuclei to create mesons (pions, kaons) that then decay to a neutrino and antilepton or to an antineutrino and lepton. For example the $\pi^{+}$decays into $\mu^{+}$and $\nu_{\mu}$, while the $\pi^{-}$decays into $\mu^{-}$and $\bar{\nu}_{\mu}$. The mixed beam then passes through a thick absorber until the charged particles stop, leaving a pure neutrino plus anti-neutrino beam.

After the neutrino interacts with the material inside a detector the momenta, identities and energies of the detected outgoing particles are used to reconstruct the initial neutrino energy.

There are two methods that are used for energy reconstruction.

In the first "Calorimetric" method, the total energy of all the detected final state particles is summed to obtain the incident neutrino energy. This method is used in calorimetricdetector based neutrino experiments. The complications of this method arise from the fact that the detectors have limited acceptances and are not sensitive to certain types of particles. 
The second "QE" method reconstructs the initial neutrino energy using only the scattered angle and the energy of the outgoing charged lepton, assuming that the neutrino scatters from a bound nucleon at rest.

This is the method used in water Cherenkov-detectors, which can not detect nucleons. If we ignore the recoil momentum and approximate the energy of the residual nuclear system by a constant, we obtain the neutrino energy [3]:

$$
E_{\mathrm{QE}}=\frac{2 M \epsilon-m_{1}^{2}+2 M E_{1}}{2\left(M-E_{1}+\left|k_{1}\right| \cos \theta_{1}\right)}
$$

where $M$ is the nucleon mass, $\epsilon$ is the average single-nucleon separation energy, $m_{1}$ is the mass of the outgoing lepton, $k_{\mathrm{l}}$ is its momentum, $E_{\mathrm{l}}$ is its energy and $\theta_{\mathrm{l}}$ is the angle between the outgoing lepton and the direction of the neutrino beam.

If the neutrino scatters non quasi-elastically, then the reconstructed energy will be incorrect.

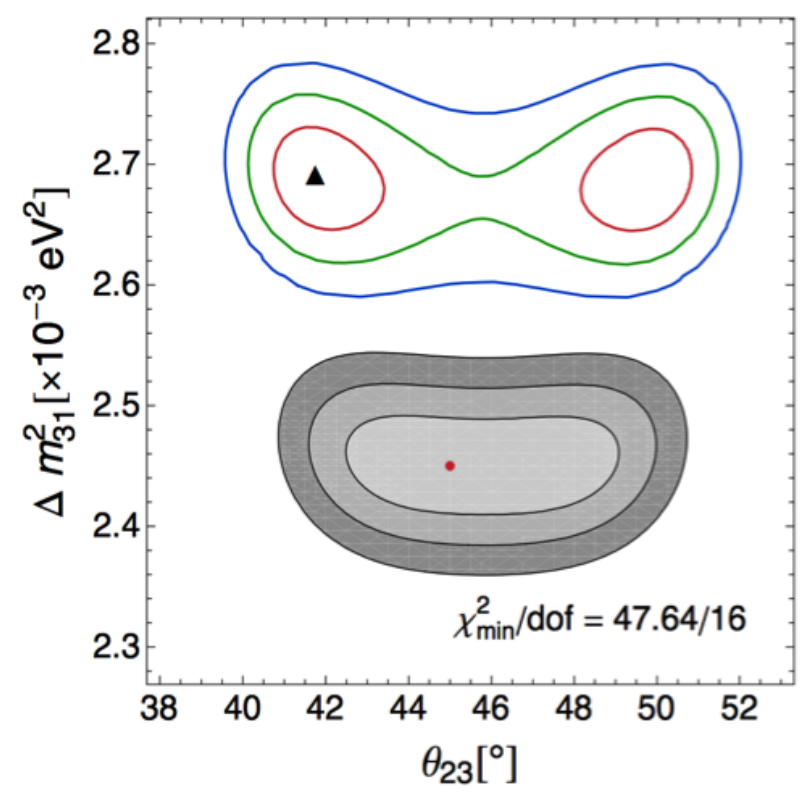

FIG. 1: Impact on extracted neutrino oscillation parameters if the neutrino event generator does not describe the underlying physics accurately [4]. The grey regions show the 1,2 and $3 \sigma$ confidence regions for the case when the data was generated and analyzed with the same event generator GIBUU, and red, green and blue lines show the same confidence regions for the case when oscillation parameters were obtained by generating data with GIBUU and analyzing with the GENIE event generator. The red dot indicates the true input value while the the black triangle shows the location of the best fit point. 
These energy reconstruction techniques have never been tested with a beam of known energy. They are usually tested using simulations, which use neutrino "event generators" to simulate neutrino interactions. These event generators include information on various neutrino interaction processes for different targets and beam energies and themselves need to be tested against real data. The neutrino event generators play an important role in long and short-baseline neutrino experiments, including separation of the signal from the background.

Neutrino event generators allow to connect the reconstructed energy with the real energy via so called "migration matrices". However these generators are not perfect because nuclear physics is complicated. Different generators have different migration matrices.

In order to see the impact that different event generators have on the determination of neutrino oscillation parameters, the oscillation parameters obtained by generating and analyzing the data with the same event generator GIBUU were compared to the oscillation parameters obtained by generating the measured "data", i.e. 'nature' with GIBUU and analyzing the data using GENIE event generator. The results are shown in Fig.1. The extracted mixing angle and the mass splitting depend on the generator used, and it is not clear which one of the generators is more accurate.

With the upcoming high impact experiments such as DUNE and Hyper-Kamiokande it is important to study and constrain the biases due to mis-modeling and incorrect energy reconstruction.

Since electrons and neutrinos are both leptons and thus interact with matter in similar ways, in the following analysis we use electron-nucleon scattering data to study neutrino beam energy reconstruction. As the electron beam energy is known, we can test energy reconstruction in selective kinematics and identify regions of phase space where the neutrino event generators running in the electron scattering mode describe the data well. This will

allow us to test and improve the existing neutrino event generators that are used in neutrino experiments and thus decrease the systematic uncertainties in current and future neutrino experiments.

\subsubsection{NEUTRINO OSCILLATIONS}

Neutrinos created by charged current weak interactions are in electron, muon or tau flavor eigenstates. The "flavor states" $\nu_{\alpha}$, where $\alpha$ corresponds to $e^{-}, \mu$ and $\tau$, do not have well defined masses, but instead are superpositions of mass eigenstates $\left(m_{1}, m_{2}, m_{3}\right)$. They are related via the "Pontecorvo-Maki-Nakagawa-Sakata" (PMNS) mixing matrix $\nu_{\alpha}=$ 


$$
\begin{aligned}
& \sum_{m_{i}} U_{\alpha m_{i}} \nu_{m_{i}} . \\
& U_{\mathrm{PMNS}}=\left(\begin{array}{ccc}
1 & 0 & 0 \\
0 & \cos \theta_{23} & \sin \theta_{23} \\
0 & -\sin \theta_{23} & \cos \theta_{23}
\end{array}\right) \times\left(\begin{array}{cccc}
\cos \theta_{13} & 0 & \sin \theta_{13} e^{-i \delta_{\mathrm{CP}}} \\
0 & 1 & 0 & \\
-\sin \theta_{13} e^{-i \delta_{\mathrm{CP}}} & 0 & \cos \theta_{13} &
\end{array}\right) \\
& \times\left(\begin{array}{ccc}
\cos \theta_{12} & \sin \theta_{12} & 0 \\
-\sin \theta_{12} & \cos \theta_{12} & 0 \\
0 & 0 & 1
\end{array}\right)
\end{aligned}
$$

where $\theta_{12}, \theta_{23}$ and $\theta_{13}$ are the mixing angles that describe how much the mass and flavor eigenstates are mixed. The $\delta_{\mathrm{CP}}$ phase, if found to be non zero, would be the source of leptonic-CP violation.

A flavor eigenstate can be written in terms of the mass eigenstates:

$$
\left|\psi(x=0)>=U_{\alpha 1}\right| \nu_{m_{1}}>+U_{\alpha 2}\left|\nu_{m_{2}}>+U_{\alpha 3}\right| \nu_{m_{3}}>
$$

As the neutrinos travel away from their source, the mass eigenstates have different frequencies, leading to flavor changing:

$$
\left|\psi(L)>=U_{\alpha 1}\right| \nu_{m_{1}}>e^{-i \phi_{1}}+U_{\alpha 2}\left|\nu_{m_{2}}>e^{-i \phi_{2}}+U_{\alpha 3}\right| \nu_{m_{3}}>e^{-i \phi_{3}}
$$

where the phase $\phi_{i}=p_{i} \cdot x=E_{i} t-\left|\overrightarrow{p_{i}}\right| L \approx\left(E_{i}-\left|\overrightarrow{p_{i}}\right|\right) L, p_{i}$ is the corresponding four momentum, $E_{i}$ energy, $L$ is the traveled distance after a time $t$, and $\overrightarrow{p_{i}}$ is the three momentum vector.

When a neutrino is created via the Charged Current Weak interaction it is in a pure flavor state $\alpha$, which is a superposition of mass eigenstates (Eq. 3). As it travels away from the source it becomes a mixture of three flavors, with the proportion of each varying during the travel. The wave function describing a neutrino that has traveled some distance from the source given by Eq. 4 can be used to obtain the probability of detecting a neutrino of a certain flavor by expressing the mass eigenstates in terms of flavor eigenstates using the inverted relation $\nu_{m_{i}}=\sum_{\alpha} U_{\alpha m_{i}}^{*} \nu_{\alpha}$.

$$
\begin{aligned}
P\left(\nu_{\alpha} \rightarrow \nu_{\beta}\right)=\delta_{\alpha \beta}- & 4 \sum_{i>j} \operatorname{Re}\left(U_{\alpha i}^{*} U_{\beta i} U_{\alpha j} U_{\beta j}^{*}\right) \sin ^{2}\left(\Delta m_{i j}^{2} \frac{L}{4 E}\right)+ \\
& +2 \sum_{i>j} \operatorname{Im}\left(U_{\alpha i}^{*} U_{\beta i} U_{\alpha j} U_{\beta j}^{*}\right) \sin \left(\Delta m_{i j}^{2} \frac{L}{2 E}\right)
\end{aligned}
$$


where $\delta_{\alpha \beta}$ is the Kronecker delta function and

$$
\Delta m_{i j}^{2}=m_{i}^{2}-m_{j}^{2}, \quad \Delta m_{31}^{2}=\Delta m_{32}^{2}+\Delta m_{21}^{2}
$$

This probability is given by Eq. 5 [5] and is a function of distance traveled by the neutrino, energy of the neutrino, mixing angles and the square of three mass splittings, which are given in Eq. 6.

Assuming $\delta_{\mathrm{CP}}=0$, which is a valid assumption for the resolution of the ongoing neutrino oscillation experiments, we can write the probability of $\nu_{\mu}$ changing to $\nu_{e}$ as [6]:

$$
\begin{array}{r}
P\left(\nu_{\mu} \rightarrow \nu_{e}\right)=\sin ^{2}\left(\theta_{23}\right) \sin ^{2}\left(2 \theta_{13}\right) \sin ^{2}\left(\Delta m_{32}^{2} \Phi\right)+\cos ^{2}\left(\theta_{23}\right) \sin ^{2}\left(2 \theta_{13}\right) \sin ^{2}\left(\Delta m_{21}^{2} \Phi\right)+ \\
+\cos \left(\theta_{13}\right) \sin \left(2 \theta_{12}\right) \sin \left(2 \theta_{13}\right) \sin \left(2 \theta_{23}\right) \sin \left(\Delta m_{32}^{2} \Phi\right) \sin \left(\Delta m_{21}^{2} \Phi\right) \cos \left(\Delta m_{32}^{2} \Phi\right)
\end{array}
$$

where $\Phi=\frac{L}{4 E}$.

For the appearance experiments, where we create $\nu$ in flavor state $\alpha$ and then look for $\nu$ in flavor state $\beta \neq \alpha$, Eq. 5 can be considered in two different limits of $L / E$.

For small $L / E, \Delta m_{12}^{2} L / E$ is very small and with the reasonable approximation $\Delta m_{13}^{2} \approx$ $\Delta m_{23}^{2}$ one obtains:

$$
P\left(\nu_{\alpha} \rightarrow \nu_{\beta}\right)=-4\left[U_{\alpha 1} U_{\beta 1} U_{\alpha 3} U_{\beta 3}+U_{\alpha 2} U_{\beta 2} U_{\alpha 3} U_{\beta 3}\right] \sin ^{2}\left(\Delta m_{23}^{2} \frac{L}{4 E}\right)
$$

For large $L / E$, the $\sin ^{2}\left(\Delta m_{23}^{2} L / 4 E\right)$ and $\sin ^{2}\left(\Delta m_{13}^{2} L / 4 E\right)$ terms average to $1 / 2$ over many oscillation periods and Eq. 5 reduces to:

$$
P\left(\nu_{\alpha} \rightarrow \nu_{\beta}\right)=-4\left[\left(U_{\alpha 1} U_{\beta 1} U_{\alpha 2} U_{\beta 2}\right) \sin ^{2}\left(\Delta m_{12}^{2} \frac{L}{4 E}\right)+\frac{1}{2}\left(U_{\alpha 1} U_{\beta 1} U_{\alpha 3} U_{\beta 3}+U_{\alpha 2} U_{\beta 2} U_{\alpha 3} U_{\beta 3}\right)\right]
$$

since $\Delta m_{12}^{2}$ is about 100 times smaller than $\Delta m_{13}^{2}$ and $\Delta m_{23}^{2}$.

Under the assumption $\theta_{13}=0$, Eq. 8 becomes:

$$
\begin{aligned}
& P\left(\nu_{\mu} \rightarrow \nu_{\tau}\right)=\sin ^{2}\left(2 \theta_{23}\right) \sin ^{2}\left(\Delta m_{23}^{2} \frac{L}{4 E}\right) \\
& P\left(\nu_{e} \rightarrow \nu_{\mu}\right)=0 \\
& P\left(\nu_{e} \rightarrow \nu_{\tau}\right)=0
\end{aligned}
$$

and Eq. 9 reduces to:

$$
P\left(\nu_{e} \rightarrow \nu_{\mu, \tau}\right)=\sin ^{2}\left(2 \theta_{12}\right) \sin ^{2}\left(\Delta m_{12}^{2} \frac{L}{4 E}\right)
$$


These two equations correspond to two flavor oscillations, and are used in the discussions of atmospheric and solar neutrino oscillations. Eq. 10 describes atmospheric oscillation, where the transition $\nu_{\mu}$ to $\nu_{\tau}$ dominates. That is why the oscillation parameters in Eq. 10 are called atmospheric oscillation parameters. In solar neutrino oscillation the dominant transition is $\nu_{e}$ to $\nu_{\mu}$ described by Eq. 11, and the associated oscillation parameters are called solar oscillation parameters [5].

The current knowledge of oscillation parameters suggests that $\theta_{13}$ is small, which means that in certain cases the transition probability for two flavor mixing obtained above can be applicable:

$$
P\left(\nu_{\alpha} \rightarrow \nu_{\beta}\right)=\sin ^{2}(2 \theta) \sin ^{2}\left(\Delta m^{2} \frac{L}{4 E}\right)
$$

where $\Delta m^{2}=m_{1}^{2}-m_{2}^{2}$ and $m_{1}, m_{2}$ are the two mass eigenstates and $\theta$ is the mixing angle.

To measure oscillation parameters, scientist often use neutrino beams from accelerators. For the measurement of $\Delta m_{23}^{2}$ that is of the order of $10^{-3} \mathrm{eV}^{2}$, we need to perform an experiment with $L / E$ combination that is of the same order. If we use neutrino beams from accelerators, which typically have energies of the order of $1 \mathrm{GeV}$, we will need a baseline of about $400 \mathrm{~km}$ to do such a measurement.

We have derived the formula for neutrino oscillations in vacuum. Neutrino oscillation patterns can change in the matter. Neutrinos traveling in matter experience an additional potential. Often neutrinos of different flavor experience different potentials, as some of them do not have enough energy to interact via the CC weak interaction and create corresponding leptons, and therefore interact with matter only via the $\mathrm{NC}$ weak interaction. The neutrino oscillations are caused by the phase difference between the wavepackets of different mass eigenstates in Eq. 4. In matter, these phase differences will be different than in vacuum due to the difference in the potentials experienced by neutrinos of different flavors, and the vacuum neutrino oscillation patterns will be distorted. Experiments where neutrinos travel long distances are sensitive to matter effects due to neutrinos traveling through the ground.

In fact the determination of the sign of the mass splittings via neutrino oscillation experiments is only possible due to matter effects.

All of the above mentioned parameters still lack precise measurements, due to the difficulties in performing neutrino experiments and the resulting uncertainties. The current knowledge of neutrino oscillation parameters in the three neutrino framework is shown in Table 1. The sign of $\Delta m_{32}^{2}$ and $\Delta m_{31}^{2}$ is not known yet. $\Delta m_{32}^{2}>0$ and $\Delta m_{31}^{2}>0$ corresponds to normal mass ordering (NO) $m_{1}<m_{2}<m_{3}$ and $\Delta m_{32}^{2}<0$ and $\Delta m_{31}^{2}<0$ to 


\begin{tabular}{|ccc|}
\hline Parameter & best-fit & $3 \sigma$ \\
\hline \hline$\Delta m_{21}^{2}\left[10^{-5} \mathrm{eV}^{2}\right]$ & 7.37 & $6.93-7.96$ \\
\hline$\Delta m_{31}^{2}\left[10^{-3} \mathrm{eV}^{2}\right]$ & 2.56 & $2.45-2.69$ \\
\hline$\Delta m_{23}^{2}\left[10^{-3} \mathrm{eV}^{2}\right]$ & 2.54 & $2.42-2.66$ \\
\hline $\sin ^{2} \theta_{12}$ & $0.297,\left(\theta_{12}=33.02^{\circ}\right)$ & $0.250-0.354$ \\
\hline $\sin ^{2} \theta_{23} \Delta m_{31(32)}^{2}>0$ & $0.425,\left(\theta_{23}=45.21^{\circ}\right)$ & $0.381-0.615$ \\
\hline $\sin ^{2} \theta_{23} \Delta m_{32(31)}^{2}<0$ & $0.589,\left(\theta_{23}=50.13^{\circ}\right)$ & $0.384-0.636$ \\
\hline $\sin ^{2} \theta_{13} \Delta m_{31(32)}^{2}>0$ & $0.0215,\left(\theta_{13}=8.432^{\circ}\right)$ & $0.0190-0.0240$ \\
\hline $\sin ^{2} \theta_{13} \Delta m_{32(31)}^{2}<0$ & $0.0216,\left(\theta_{13}=8.451^{\circ}\right)$ & $0.0190-0.0242$ \\
\hline$\delta_{\mathrm{CP}} / \pi$ & $1.38(1.31)$ & $2 \sigma(1.0-1.9)(2 \sigma(0.92-1.88))$ \\
\hline
\end{tabular}

TABLE 1: The best fit values and $3 \sigma$ allowed ranges of the 3 neutrino oscillation parameters, derived from a global fit of the current neutrino oscillation data [7]. The best fit value and the $2 \sigma$ allowed range for the Dirac phase $\delta_{\mathrm{CP}}$ are shown for the cases $m_{1}<m_{2}<m_{3}$ and $m_{3}<m_{2}<m_{1}$ (values in brackets).

inverted mass ordering (IO) $m_{3}<m_{2}<m_{1}$.

The current unknowns in the three neutrino framework:

- Precise values of the mass splittings and mixing angles.

- The value of $\delta_{C P}$ violation phase.

- The mass hierarchy (the sign of $\Delta m_{31}^{2}$ ).

- The sign of $\left(\theta_{23}-\pi / 4\right)$.

- Are the neutrinos and antineutrinos identical (Dirac/Majorana nature)?

- What is the absolute neutrino mass?

The last two questions can not be answered by neutrino oscillation experiments.

The mass hierarchy and $\delta_{C P}$ violation phase can be measured in $\nu_{\mu} \rightarrow \nu_{e}$ and $\bar{\nu}_{\mu} \rightarrow \bar{\nu}_{e}$ appearance studies.

The leptonic-CP violation could explain the observed Baryon Asymmetry of the Universe through Baryogenesis. In this scheme, due to $\mathrm{CP}$ violation in the leptonic sector, very 
heavy right-handed Majorana neutrinos (which do not conserve lepton number) could decay, producing more antileptons than leptons in the early universe. When the universe cools below the electroweak phase transition $(T \sim 250 \mathrm{GeV})$, the lepton asymmetry will generate a baryon asymmetry by a mechanism conserving the difference between baryon and lepton numbers $B$ and $L$ that is already present in the Standard Model.

\subsubsection{LONG BASELINE NEUTRINO EXPERIMENTS}

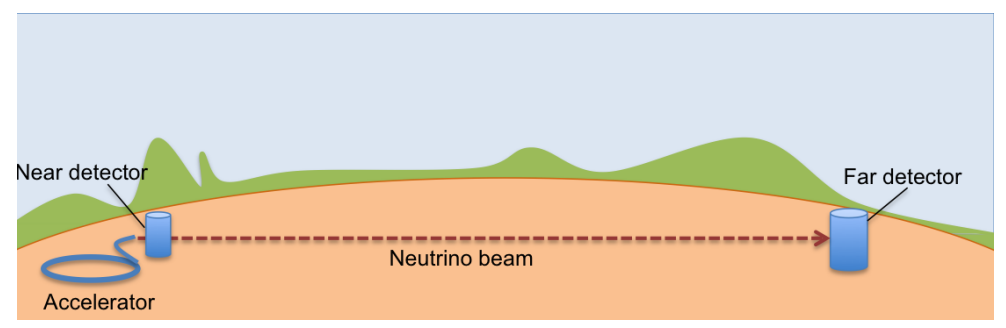

FIG. 2: A schematic view of a typical long-baseline experiment.

In order to study neutrino oscillations and determine the oscillation parameters, so called "Long baseline" experiments are performed (see Fig. 2), where the flavor composition of the neutrino beam is measured at the near detector before oscillation and then again at the far detector after traveling a long distance (from a few hundred meters to a few hundred $\mathrm{km}$ ). There are two signals in "Long baseline" experiments:

- Appearance experiments look for the appearance of neutrinos of a particular flavor from a neutrino beam created initially in a pure state of a different flavor. The oscillation signal is the observation of a non-zero number of neutrinos with a flavor different from that of the neutrinos in the initial beam.

- Disappearance experiments look for the disappearance of neutrinos with a particular flavor by comparing the energy spectra (see the top plot in Fig. 3) of certain flavor neutrinos before and after oscillations. The ratio of these spectra should have an oscillating pattern (see the bottom plot in Fig. 3) and is referred to as an "oscillation signal".

The two biggest ongoing long baseline neutrino oscillation experiments are No $\nu$ a in the US and the Tokai-to-Kamioka (T2K) disappearance experiment in Japan. T2K uses the $\nu_{\mu}$ beam from the JPARC facility to study the oscillation parameters $\Delta m_{23}^{2}, \theta_{23}$ and $\theta_{13} 295$ 


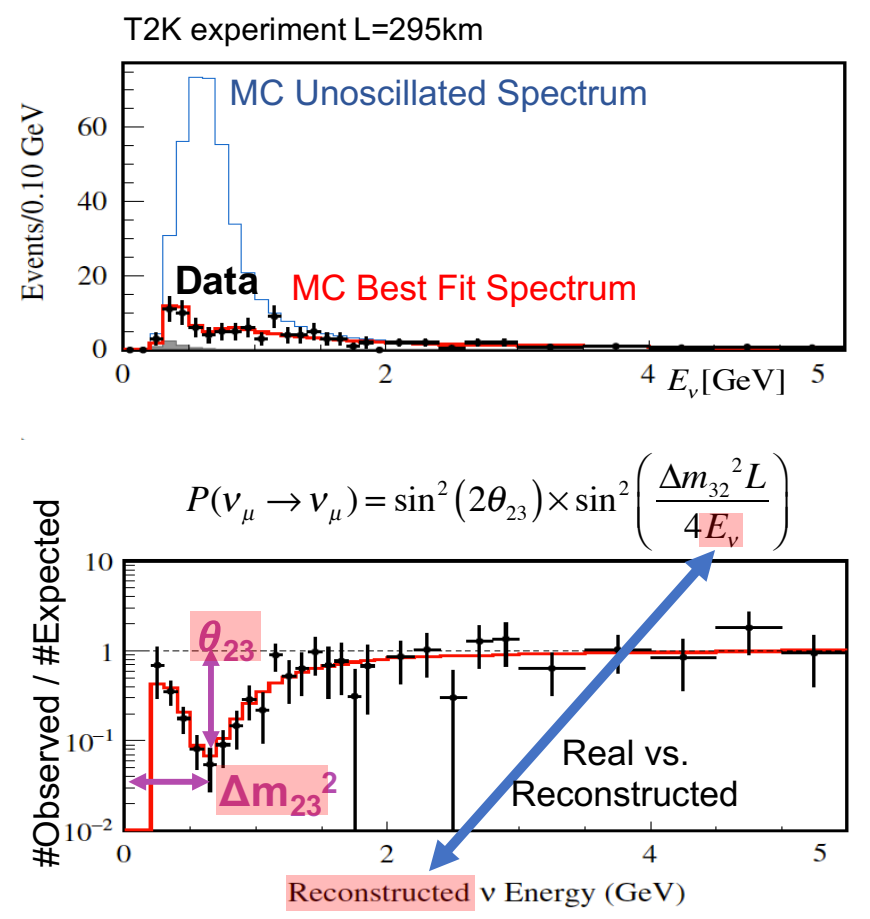

FIG. 3: Top: Reconstructed neutrino energy spectrum from data, best-fit prediction, and unoscilated prediction. Bottom: Ratio of oscillated to unoscillated events as a function of neutrino energy for the data and the best-fit spectrum [8].

km away from the source. The No $\nu$ a experiment uses the higher energy off-axis NuMI $\nu_{\mu}$ beam from Fermilab, to study the oscillation parameters $810 \mathrm{~km}$ away from the target

The two future flagship experiments will be DUNE and HyperK. DUNE (Deep Underground Neutrino Experiment) will use Fermilab. The beam will travel $1300 \mathrm{~km}$ to the detector at the Sanford Underground Research Laboratory. Hyper-Kamiokande (HK)(Japan) is going to be 10 times bigger than the Super-Kamiokande water Cherenkov detector, and will use a muon neutrino beam from J-PARC. Its construction will start in Japan in 2020. Both experiments have a rich physics program, including measurements of neutrino oscillation parameters and the $\mathrm{CP}$ violation phase.

To study neutrino oscillations the knowledge of the neutrino initial energy is required. The neutrino oscillation parameters are determined from the fit to the neutrino oscillation signal, which is usually plotted as a function of distance traveled divided by the energy of neutrino. We can see the oscillation signal as a function of neutrino energy for the T2K experiment in Fig. 3. Based on Eq. 12 the position of the peak in the oscillation signal 
is a measure of the square of the corresponding mass splitting and the height of the peak is a measure of the corresponding mixing angle. The position of the peak depends on the neutrino energy and the height of the peak depends on the neutrino event generators used to correct the neutrino flux for backgrounds. If the incoming neutrino energy is not precisely reconstructed, the oscillation signal can be washed out, or the extracted oscillation parameters can be biased. Similarly incorrect $\nu$-nucleus interaction modeling will affect the accuracy of the extracted oscillation parameters.

\subsection{ACCELERATOR BASED NEUTRINO EXPERIMENTS}

There are many sources of neutrinos. Those include solar neutrinos, atmospheric neutrinos, which are created in the upper levels of atmosphere after cosmic rays (p, He, etc.) interact with nuclei in the atmosphere, as well as neutrinos created during supernova explosions and other astrophysical sources. Various experiments have studied neutrinos coming from these sources. Raymond Davis shared a Nobel prize in Physics in 2002 for detection of solar neutrinos with the Homestake experiment in South Dakota in the late 1960s. The Homestake experiment was the first to see a deficit of electron neutrinos in the observed solar neutrino flux, which was known as the solar neutrino problem. Later on the solar neutrino problem was explained by the discovery of neutrino oscillations by the Super-Kamiokande water Cherenkov detector in Japan, by studying atmospheric neutrinos.

Nuclear reactors produce low energy (few MeV) electron neutrino beams from nuclear beta decay. These beams are suitable for solar neutrino oscillation parameter studies.

High energy (few $\mathrm{GeV}$ ) neutrino beams are made by scattering protons on nuclei to create mesons (pions, kaons) that then decay to a neutrino or anti-neutrino with the corresponding anti lepton or lepton. This way neutrino oscillation experiments have control over the neutrino flux and distance, unlike "natural" sources of neutrinos, where the flux and the distance that the neutrinos travel are fixed.

The decay of charged pions into neutrinos with corresponding branching ratios is shown in Table 2. The $\nu_{\mu}$ and $\bar{\nu}_{\mu}$ branching ratios are much bigger than those for $\nu_{e}$ and $\bar{\nu}_{e}$. Thus it is much easier to make a high energy $\nu_{\mu}$ beam than a high energy $\nu_{e}$ beam. After the mesons are produced in proton-target collisions, charged particles are directed along the decay pipe using electromagnetic devices called focusing horns. Positive mesons are used to create $\nu_{\mu}$ beams and negative mesons are used to obtain $\bar{\nu}_{\mu}$ beams. The mixed beam then passes through a thick absorber until the charged particles stop, leaving only neutrinos or anti-neutrinos in the beam. Some neutrinos can also be produced via muon 


\begin{tabular}{lcc} 
Neutrino beam & Anti-neutrino beam & Branching ratio \\
\hline$\pi^{+} \rightarrow \mu^{+} \nu_{\mu}$ & $\pi^{-} \rightarrow \mu^{-} \bar{\nu}_{\mu}$ & 0.999 \\
$\pi^{+} \rightarrow e^{+} \nu_{e}$ & $\pi^{-} \rightarrow e^{-} \bar{\nu}_{e}$ & $\approx 10^{-4}$ \\
$K^{+} \rightarrow \mu^{+} \nu_{\mu}$ & $K^{-} \rightarrow \mu^{-} \bar{\nu}_{\mu}$ & 0.63 \\
$K^{+} \rightarrow e^{+} \nu_{e}$ & $K^{-} \rightarrow e^{-} \bar{\nu}_{e}$ & $\approx 10^{-5}$ \\
$K^{+} \rightarrow \pi^{0} \mu^{+} \nu_{\mu}$ & $K^{-} \rightarrow \pi^{0} \mu^{-} \bar{\nu}_{\mu}$ & 0.03 \\
$K^{+} \rightarrow \pi^{0} e^{+} \nu_{e}$ & $K^{-} \rightarrow \pi^{0} e^{-} \bar{\nu}_{e}$ & 0.05 \\
$\mu^{+} \rightarrow e^{+} \nu_{e} \bar{\nu}_{\mu}$ & $\mu^{-} \rightarrow e^{-} \bar{\nu}_{e} \nu_{\mu}$ & $\approx 1$
\end{tabular}

TABLE 2: The decay of charged $\pi$ 's and kaons into neutrinos.

decay $\mu^{+} \rightarrow e^{+} \nu_{e} \bar{\nu}_{\mu}$ and $\mu^{-} \rightarrow e^{-} \nu_{\mu} \bar{\nu}_{e}$ that have $100 \%$ branching ratios. These are a source of $\nu_{e}$ and $\bar{\nu}_{e}$ background in the beam.

In order to describe the flux of the beam, Monte-Carlo simulations are used that describe the electromagnetic and hadronic interaction of the particles in the beam with the different beam line components with different materials and geometry. The simulation needs to accurately reproduce the hadronic interaction of the proton beam with the target material.

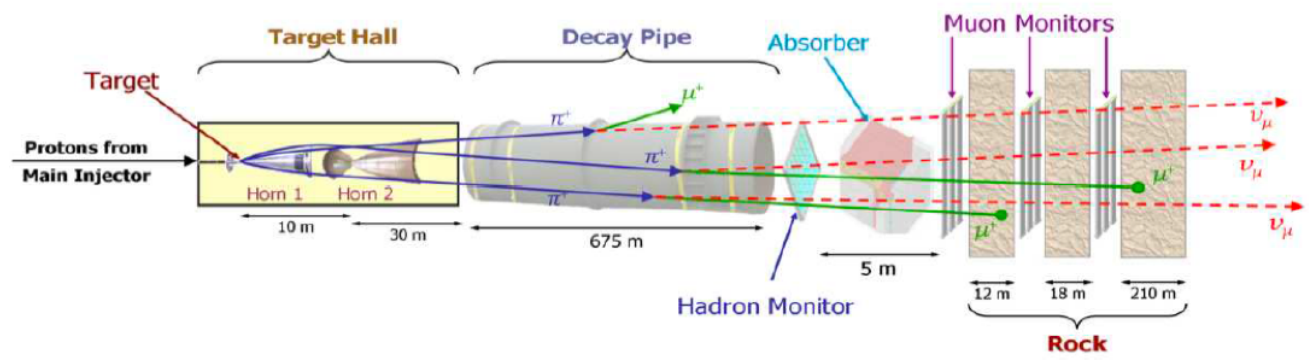

FIG. 4: Schematic of the NuMI beam [9].

The three most popular neutrino beam lines are:

- The J-PARC facility in Japan uses a $30 \mathrm{GeV}$ proton beam from the J-PARC Main 
Ring and directs it on a target composed of graphite rods [10]. Focusing magnets called magnetic horns focus the hadrons and preferentially select $\pi^{+}$or $\pi^{-}$. The produced hadrons travel in the $100 \mathrm{~m}$ long decay volume, where the produced pions can decay to mesons plus neutrinos. Then the particles pass through the beam dump made of large graphite walls, where all the undecayed pions, protons and other charged particles get absorbed. Only the neutrinos and a small fraction of the muons penetrate the beam dump and proceed to two near detectors located $280 \mathrm{~m}$ downstream of the target: the INGRID near detector monitors the intensity and the direction of the beam and is composed of iron and plastic scintillator layers. ND280 is placed $2.5^{\circ}$ off-axis and measures the purity and energy distribution of neutrino beam. It is magnetized and consists of several subdetectors, such as a time projection chamber and subdetectors composed of plastic scintillator layers sandwitched with layers of iron, lead, water and other material. J-PARC was the first to use an off-axis neutrino beam. The offaxis beam energy distribution is narrower. J-PARC has the world's highest intensity neutrino beam.

- The Booster Neutrino Beamline (BNB) at the Fermi National Accelerator Laboratory (FNAL) uses $8 \mathrm{GeV}$ protons from the booster synchrotron and directs them on a beryllium target to produce $\nu_{\mu}$ or $\bar{\nu}_{\mu}$ beams of $800 \mathrm{MeV}$ average energy. The magnetic horns focus the negative or positive pions depending on the type of neutrino beam to be produced.

- The NuMI neutrino beam at FNAL is obtained by directing the $120 \mathrm{GeV}$ proton beam on a graphite target (see Fig. 4). The different configurations of two magnetic horns allow to obtain low energy (LE), medium energy (ME) or high energy (HE) neutrino beams. The LE beam is peaked at $3.5 \mathrm{GeV}$, while HE the range is about few tens of $\mathrm{GeV}$. The energy distributions for different magnetic horn configurations are shown in Fig. 5.

Energy distributions for all these beams are shown in Fig. 5.

There are three main types of detectors used in accelerator based neutrino oscillation experiments. Those are Cherenkov detectors, liquid argon time projection chambers (LArTPC) and Scintillator detectors.

Neutrino oscillation experiments often use heavy nuclei such as argon, iron or carbon for detector material to have higher interaction rates. 


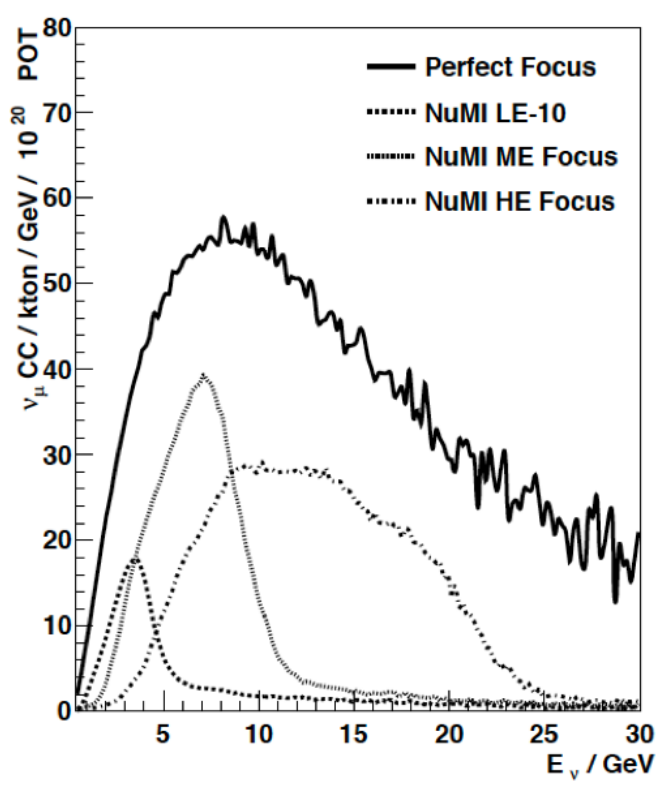

FIG. 5: The NuMI event rate (flux $\times$ cross-section) at the Soudan mine in Minnesota for different focusing configurations of magnetic horns [9]. "Perfect focusing" is when all the produced pions are directed in the beam line direction.

Cherenkov detectors detect leptons and pions produced after the neutrino interacts with detector material. Having sufficient velocity these particles produce Cherenkov light inside the detector volume. The rings of produced Cherenkov light are detected by the surrounding PMTs. Muons can be separated from electrons as muons make a sharp Cherenkov ring, while the electrons and photons shower, and the nearly parallel $e^{+}$and $e^{-}$in the shower combine to produce fuzzy rings. The particle production vertex can be determined from the position, diameter and the shape of the ring. After detecting the Cherenkov ring and associated cone, the velocity and thus the momentum of the particle can be determined using the relation between the velocity $\beta$ and the angle between the particle trajectory and the angle of emission of the Cherenkov radiation $\cos \theta=1 / \beta n$. Here $n$ is the refractive index of the material. The threshold momentum for particles to produce Cherenkov radiation is $p=m c / \sqrt{n^{2}-1}$, where $m$ is the mass of the particle, and $c$ is the speed of the light in vacuum. For refractive index of water $n=1.33$, the threshold kinetic energy for muons is $\approx 55 \mathrm{MeV}$, for charged pions is $75 \mathrm{MeV}, 0 \mathrm{MeV}$ for $\pi^{0}$ and $\approx 485 \mathrm{MeV}$ for protons. Detectors using mineral oil with $n=1.47$ have lower detection thresholds and also produce scintillation light [11]. The detection threshold of these detectors is about $1 \mathrm{MeV}$ for $e^{-}$. These experiments include T2K which uses Super-Kamiokande as a far detector, which is a 
Cherenkov detector using water. MiniBooNE used a Cherenkov detector filled with mineral oil. These detectors cannot detect protons or neutrons. Because of the huge sizes of these detectors, applying a magnetic field is impractical, and thus the charge of the interacting particle can not be determined. The signature of the CCQE interaction in these detectors is the detection of a lepton and no charged pions. The energy of the neutrino for this type of reaction is typically determined using the QE energy reconstruction method.

Experiments using LArTPCs include MicroBooNE, ArgoNeut, and the proposed DUNE experiment. The neutrino interacts with an argon nucleus producing charged particles. The charged particles ionize the liquid argon as they travel through it. Due to the applied electric field, the ionization electrons drift towards the anode wire planes, where they are collected. The detected charge is proportional to the deposited energy. They use events with fully contained particle tracks and using the known stopping power in liquid argon, they convert the track lengths into their energies. These detectors detect all the charged particles in the final state with their energies, only the detection of neutrons is challenging. The threshold kinetic energy is $\approx 35 \mathrm{MeV}$ for muons and charged pions, $\approx 60 \mathrm{MeV}$ for protons and $\approx 30 \mathrm{MeV}$ for electrons and photons. There is no practical way to determine the charge of the particle. The observation of $\mu^{-}$capture can help to differentiate between $\mu^{-}$and $\mu^{+}$. Particle identification for different charged particles can be done by analyzing the deposited energy $d E / d x$ as a function of distance along the track. The $e^{-}, e^{+}$and $\gamma$ create an electromagnetic shower. Analyzing the deposited energy $d E / d x$ for the first few $\mathrm{cm}$ of electromagnetic shower, one can separate between $e^{-}$and $\gamma$, as the latter becomes visible after decaying into an $e^{-} e^{+}$pair and then deposits double the energy. By detecting the full final state particles and determining the neutrino energy from the calorimetric method, the event can be fully reconstructed.

The third type of detectors used are scintillator detectors. These consist of scintillator bars stacked together with PMT readouts on the sides. The neutrino interacts with a nuclear target and the produced particles pass through the scintillator. Charged particles excite the atoms of scintillator which de-excite by emitting scintillation light. There are also additional detectors used that surround the scintillator region and detect the energies of final state particles. Here again the only challenge is neutron detection. By detecting the full final state particles, the energy of the incoming neutrino in the event can be determined from the calorimetric method. The usage of nuclear targets data on neutrino-nucleus scattering processes is important for understanding of weak nuclear structure. Scintillator detectors are used for example in the Miner $\nu$ a experiment as well as for the MicroBooNE far detector. 
The current neutrino experiments that use the NuMI neutrino beam to study neutrinonucleus interactions and neutrino oscillations include:

- Minerva studies neutrino interaction cross sections and nuclear effects. It uses a scintillator detector called an active tracker region, surrounded by electromagnetic and hadronic calorimeters to track and measure the energies of the outgoing particles after the neutrino interacts with the target material placed before the main body of the detector [12]. It is located in the cavern, at Fermilab, next to the MINOS detector. The experiment uses targets made of $\mathrm{He}, \mathrm{CH}, \mathrm{H}_{2} \mathrm{O}, \mathrm{Fe}$ and $\mathrm{Pb}$. It uses the NuMI LE beam peaked at $3.5 \mathrm{GeV}$ and the ME beam peaked at $5.5 \mathrm{GeV}$.

- MINOS long baseline neutrino oscillation experiment. It uses near and far detectors with similar structure [13]. The detectors consist of layers perpendicular to the beam direction. Each layer consists of scintillator layer used for charged particle detection followed by a Fe layer, which is magnetized for momentum measurements and charge discrimination. The near detector is placed at Fermilab and the far detector is $735 \mathrm{~km}$ away in the Soudan Mine Underground lab in northern Minnesota. It uses the NuMI LE beam peaked at $3.5 \mathrm{GeV}$.

- No $\nu$ a is focused on precision measurement of neutrino oscillation parameters [14]. Both detectors are sintillator detectors filled with mineral oil and are similar in structure. The near detector is located at Fermilab and the far detector is located $805 \mathrm{~km}$ away in Ash River, Minnesota. It uses the off-axis NuMI beam that is peaked at $2 \mathrm{GeV}$, where the oscillation of $\nu_{\mu}$ to $\nu_{e}$ should be maximum.

- ArgoNeut studied low energy neutrino interactions and underground detector operations, and served as a prototype for bigger LArTPCS for future neutrino experiments. It used a LArTPC placed in front of the MINOS near detector [15]. It finished data taking in March 2010. Its beam energy was peaked at $4.3 \mathrm{GeV}$.

The current neutrino experiments that use the BNB neutrino beam to study neutrino-nucleus interactions and neutrino oscillation include:

- MiniBooNE is focused on neutrino oscillation studies. Its beam travels $450 \mathrm{~m}$ before reaching the spherical Cherenkov detector at Fermilab. It was filled with mineral oil. It has completed its data taking with the neutrino beam peaked at $0.8 \mathrm{GeV}$. 
- MicroBooNE studies low energy neutrino cross sections using an LArTPC. It has the same baseline as MiniBooNE [16] and uses a neutrino beam with broad energy distribution from $0.5-3 \mathrm{GeV}$.

- SciBooNE was designed to measure neutrino and ant-neutrino cross sections. It had a baseline of $100 \mathrm{~m}$ and a detector consisting of a scintillator tracker region, an electromagnetic detector and a muon range detector (MRD). The MRD consisted of successive layers of iron and scintillator and measured the muon momentum. Its beam energy was peaked at $0.8 \mathrm{GeV}$

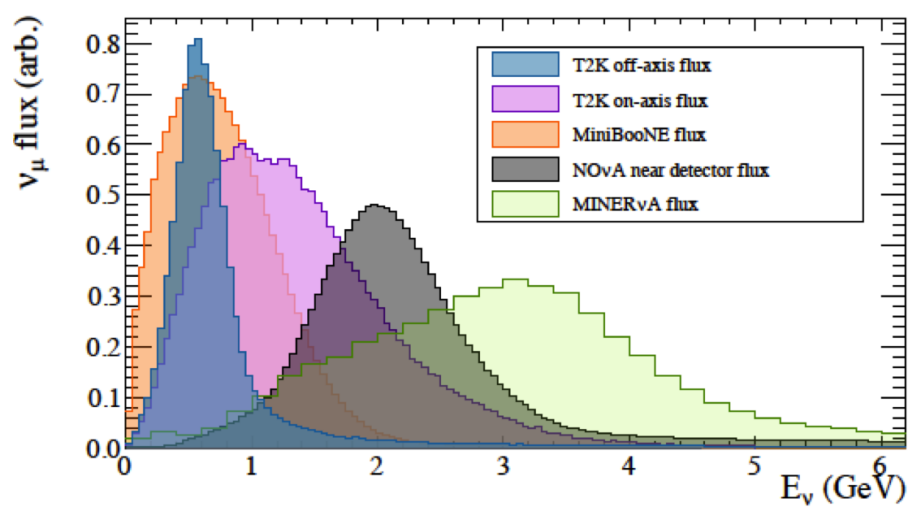

FIG. 6: Current neutrino sources as a function of neutrino energy.

$\mathrm{T} 2 \mathrm{~K}$ is focused on precision measurement of neutrino oscillation parameters. It uses the ND280 near detector at the J-PARC facility and the world's largest underground neutrino detector, Super-Kamiokande, $295 \mathrm{~km}$ away at the Kamioka mine as a far detector. ND280 is placed $2.5^{\circ}$ off-axis, $280 \mathrm{~m}$ away from target. It is a complex detector consisting of various detectors. Its beam energy is peaked at $0.6 \mathrm{GeV}$.

The incident energy spectra of different neutrino oscillation experiments are shown in Fig. 6 .

Upcoming high impact experiments include:

- DUNE will address questions such as the matter-antimatter asymmetry, the dynamics of supernova and the stability of matter. It is proposed to be a long baseline neutrino oscillation experiment using the world's most intense neutrino beam from the Long Baseline Facility (LBNF) located at Fermilab [18]. The DUNE far detector will be a LArTPC located $1300 \mathrm{~km}$ away in the Sanford Underground Research Facility (South Dakota) and the design of the near detector is not yet finalized. Possible neutrino beam energy distributions are shown in Fig. 7. 


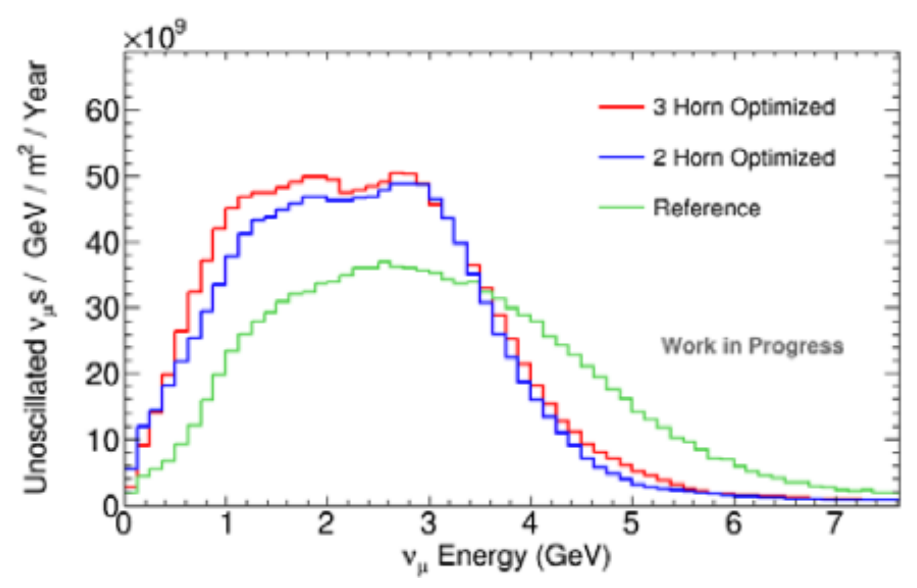

FIG. 7: Possible neutrino-mode fluxes of muon neutrinos as a function of neutrino energy for DUNE [17].

- Hyper-Kamiokande will measure neutrino oscillation parameters, including the $\mathrm{CP}$ violation phase, proton decay and atmospheric and astronomical neutrinos. It will be 10 times bigger than Super-Kamiokande and will also be located in the Kamioka mine, in Japan [19]. It will consist of two half megaton water Cherenkov detectors. The expected flux of the off-axis muon neutrino beam from the J-PARC facility will be peaked at $\sim 600 \mathrm{MeV}$.

\subsection{DETERMINATION OF NEUTRINO FLUX IN LONG BASELINE NEUTRINO OSCILLATION EXPERIMENTS}

In order to estimate the $\nu_{\mu}$ and $\nu_{e}$ appearance signals $N_{\mathrm{FD}}^{\text {expected }}\left(\nu_{\mu}\right)$ and $N_{\mathrm{FD}}^{\text {expected }}\left(\nu_{e}\right)$ at the far detector in long baseline neutrino oscillation experiments using near and far detectors, the measured $\nu_{\mu}$ spectrum at the near detector $N_{\mathrm{ND}}^{\text {data }}\left(\nu_{\mu}\right)$ is extrapolated to the far detector. Similarly the $\nu_{e}$ spectrum at the near detector $N_{\mathrm{ND}}^{\text {data }}\left(\nu_{e}\right)$, which consists mainly of $\nu_{e}$ events and NC $\pi^{0}$ misidentified events, is used to estimate the background in the $\nu_{e}$ appearance signal at the far detector.

The measured neutrino spectrum is the product of the neutrino flux $\Phi$, the detector efficiency and smearing $(\epsilon)$ and the neutrino interaction dynamics $(\sigma)$. When extrapolating the neutrino spectrum from near to far detector, one has to take into account:

- The difference between the neutrino fluxes at near and far detectors $\Phi_{\mathrm{ND}}$ and $\Phi_{\mathrm{FD}}$, as the near detector sees an extended source of neutrinos while the far detector sees a 
point source. A reliable simulation is needed to simulate the beam line and extrapolate the event rate at near detector to obtain the one at the far detector.

- Near and far detectors have different efficiencies and resolutions $\epsilon_{\mathrm{ND}}$ and $\epsilon_{\mathrm{FD}}$. If the near and far detectors are identical, this uncertainty is mostly canceled in the determination of the $\nu_{\mu}$ signal, but not in the determination of the $\nu_{e}$ signal, as that is extrapolated from $N_{\mathrm{ND}}^{\text {data }}\left(\nu_{\mu}\right)$, and so there are additional differences in the criteria used for $\nu_{e}$ and $\nu_{\mu}$ and detector responses.

- The difference in neutrino interactions in the near and far detectors $\sigma_{\mathrm{ND}}$ and $\sigma_{\mathrm{FD}}$. For identical detectors this cancels out in extrapolation of $\nu_{\mu}$ signal but not for extrapolation of $\nu_{e}$ and $\nu_{\tau}$ signals, due to differences in interactions. The $\sigma_{\mathrm{ND}}$ and $\sigma_{\mathrm{FD}}$ cross sections and associated uncertainties are estimated using neutrino event generators.

- The neutrino energy $E_{\nu}$ is not known. It is reconstructed from other kinematic observables.

All of the listed points contribute to the uncertainties of the neutrino oscillation signal in the long baseline neutrino oscillation experiments with near and far detectors.

\subsection{ENERGY RECONSTRUCTION}

Long baseline neutrino experiments compare the event rate for a given neutrino energy at the near detector with the one at the far detector. The neutrino energy is determined from the final state particles. For CCQE scattering the reconstructed energy can be determined from the outgoing lepton kinematics using Eq. 1.

This $E_{\mathrm{QE}}$ method is used by experiments using Cherenkov detectors, that are large tanks of mineral oil or water, surrounded by photo detectors to collect the light. The final state protons in neutrino QE interaction are below the Cherenkov threshold, and are not detected. Because of the Fermi motion of the bound nucleons, the energy reconstructed via the $E_{Q E}$ method is smeared around the true value.

The calorimetric method of energy reconstruction is used in the tracking detectors such as liquid argon time projection chambers. Tracking detectors can detect all the final state charged particles.

Neutrino oscillation experiments often use the CCQE scattering channel for neutrino energy reconstruction. 
The standard CCQE event selection in neutrino oscillation experiments is a detected charged lepton, no detected pions and any number of nucleons for experiments with Cherenkov detectors [11].

Experiments with tracking detectors select events with a detected charged lepton and a proton and no detected pions as CCQE sample [11].

These events are usually called QE-like as they include contributions from non-QE reaction mechanisms [11], [20], [21]. Incorrect identification of a QE scattering event introduces a bias in the reconstructed energy. In order to unfold the reconstructed energy and obtain the true energy spectrum, neutrino event generators are used. Here by unfolding we mean removing the effect of the measuring device from a measurement. Currently uncertainties in nuclear modeling in the description of neutrino-nucleus scattering represent the main source of systematic error for long baseline neutrino experiments.

\subsection{ELECTRON AND NEUTRINO INTERACTION WITH MATTER}

Electron and neutrino scattering from nuclei should be similar.

Electrons interact via the Electromagnetic interaction by exchanging virtual photons. The propagator for the electromagnetic interaction is $-i g_{\mu \nu} / q^{2}$, where $g_{\mu \nu}$ is the metric tensor. The current of the electromagnetic interaction is given by Eq. 13:

$$
j_{\mu}^{\mathrm{em}}=i g_{\mathrm{e}} \bar{u} \gamma_{\mu} u
$$

where $u$ and $\bar{u}$ are Dirac spinors, $\gamma^{\mu}$ are Dirac matrices and $g_{\mathrm{e}}=\sqrt{4 \pi \alpha}$ is the electromagnetic coupling constant, and $\alpha \cong 1 / 137$ is the fine structure constant. The parity operator for Dirac spinors is: $\hat{P}=\gamma^{0}$. The electromagnetic current is a vector current as it transforms under the parity operator the following way [22]:

$$
\hat{P}\left(\bar{u} \gamma_{\mu} u\right)=\left(\bar{u} \gamma_{0}\right) \gamma_{\mu}\left(\gamma_{0} u\right)
$$

For space and time coordinates separately we get:

$$
\begin{array}{r}
\hat{P}\left(\bar{u} \gamma_{0} u\right)=\left(\bar{u} \gamma_{0}\right) \gamma_{0}\left(\gamma_{0} u\right)=\bar{u} \gamma_{0} u \\
\hat{P}\left(\bar{u} \gamma_{\mu} u\right)=\left(\bar{u} \gamma_{0}\right) \gamma_{\mu}\left(\gamma_{0} u\right)=-\bar{u} \gamma_{\mu} u
\end{array}
$$

with $\mu=1,2,3$. The spatial components change the sign while the time component stays the same. The four-vectors that transform like this under parity are called vector like.

In Dirac theory the matrix element $M_{i f}$ corresponding to a transition probability is the product of two vector currents, so parity is conserved in Quantum Electrodynamics (QED). 
Similar to photons in QED, the mediators of weak interactions are $W^{ \pm}$and $Z^{o}$ bosons. There are two types of weak interactions, charged current weak interactions (CC) mediated by $W_{ \pm}$bosons and neutral current $(\mathrm{NC})$ weak interactions mediated by the $Z^{\circ}$ boson.

While the photon is massless, $W^{ \pm}$and $Z^{\circ}$ bosons are very heavy, with their masses equal to $80.379 \pm 0.012 \mathrm{GeV} / \mathrm{c}^{2}$ and $91.1876 \pm 0.0021 \mathrm{GeV} / \mathrm{c}^{2}$ correspondingly. As massive particles with spin $s=1$ they have three $(2 s+1)$ allowed polarization states $\left(m_{s}=1,0,-1\right)$.

The propagator for the weak interaction is $-i\left(g_{\mu \nu}-q_{\mu} q_{\nu} / M^{2}\right) /\left(q^{2}-M^{2}\right)$, where $M$ is the mass of $W^{ \pm}$and $Z^{\circ}$ bosons and $q$ is the four momentum transfer. In most cases $q^{2} \ll M$ so the propagator for weak interactions can be rewritten as $i g_{\mu \nu} / M$.

The current for the CC Weak interaction is given by [23]:

$$
j_{ \pm}^{\mu}=\bar{u} \frac{-i g_{\mathrm{w}}}{2 \sqrt{2}}\left(\gamma^{\mu}-\gamma^{\mu} \gamma^{5}\right) u
$$

where $\gamma^{5}=i \gamma^{0} \gamma^{1} \gamma^{2} \gamma^{3}$ and $g_{\mathrm{w}}$ is the weak coupling constant. As we have seen above $\gamma^{\mu}$ yields a vector coupling, and $\gamma^{\mu} \gamma^{5}$ transforms under parity operation the following way [22]:

$$
\hat{P}\left(\bar{u} \gamma^{\mu} \gamma^{5} u\right)=\left(\bar{u} \gamma_{0}\right) \gamma^{\mu} \gamma^{5}\left(\gamma_{0} u\right)
$$

For space and time coordinates separately we get:

$$
\begin{array}{r}
\hat{P}\left(\bar{u} \gamma^{0} \gamma^{5} u\right)=\left(\bar{u} \gamma^{0}\right) \gamma^{0} \gamma^{5}\left(\gamma^{0} u\right)=-\bar{u} \gamma^{0} \gamma^{5} u \\
\hat{P}\left(\bar{u} \gamma^{\mu} \gamma^{5} u\right)=\left(\bar{u} \gamma^{0}\right) \gamma^{\mu} \gamma^{5}\left(\gamma^{0} u\right)=\bar{u} \gamma^{\mu} \gamma^{5} u
\end{array}
$$

where $\mu=1,2,3$. It follow that the spatial component does not change but the time component changes the sign. The four-vectors that transform the following way under the parity operation are called axial-vectors. So the weak current is the sum of vector and axial currents, which means that parity is violated in the weak interaction.

It is traditional to express the formulas for weak interaction via the Fermi coupling constant $G_{\mathrm{F}}=\frac{\sqrt{2}}{8}\left(\frac{g_{\mathrm{w}}}{M_{W}}\right)^{2} \cdot G_{\mathrm{F}}=1.166 \times 10^{-5} \mathrm{GeV}^{-2}[24]$, and the weak coupling constant is $g_{\mathrm{w}}=0.66$. In analogy to QED, the "weak fine structure constant" can be calculated using the relation $\alpha_{\mathrm{w}}=g_{\mathrm{w}}^{2} / 4 \pi=1 / 29$. This is a few times larger than the QED fine structure constant. The weak interaction coupling constant is not small; the masses of the $W^{ \pm}$and $Z^{o}$ bosons are so large that for the $q^{2}$ range of most experiments $M^{2} c^{2}$ dominates in the denominator of propagator $q^{2}-M^{2} c^{2}$ making the interaction weak.

Electrons and neutrinos are both leptons, elementary particles with spin $1 / 2$, that do not interact via the strong interaction. They are point-like and do not have internal structure. They have similar interactions with nuclei and both interact by exchanging a single vector 
boson. The current for electromagnetic interactions is a vector current, the CC weak current is a sum of vector and axial currents. This means that we can test and improve the nuclear models and different reaction mechanisms implemented in neutrino event generators using electron scattering data, by turning off the axial response etc. (running in electron scattering mode).

Of particular interest to neutrino physics is the CC QE Weak interaction of a neutrino with a nucleon inside the nucleus, leading to a charged lepton and a nucleon in the final state. The analogy of the latter in electron scattering would be the quasi-elastic scattering of an electron on a moving nucleon inside the nucleus, with a final state consisting of the scattered electron and the knock-out nucleon (see Fig. 8). In this reaction the nucleon does not get excited and recoils elastically.

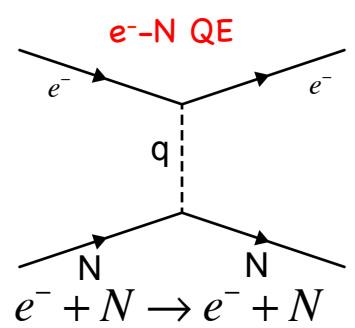

(a)

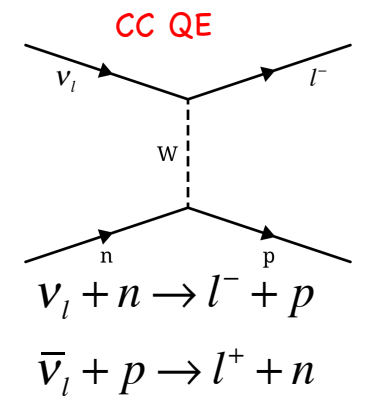

(b)

FIG. 8: The diagrams of (a) quasi-elastic scattering of an electron on a nucleon and (b) CC QE scattering of a neutrino on a nucleon.

To obtain the cross section for QE lepton nucleon scattering one needs to contract the hadronic current with the leptonic current. The hadronic current for QE lepton nucleon scattering is given in Section 1.5.1.

\subsubsection{ELECTRON AND NEUTRINO ELASTIC SCATTERING ON FREE NU- CLEONS}

The hadronic current for electron elastic scattering on a free nucleon is written in terms of well known Dirac and Pauli nucleon form factors $F_{1}(q)$ and $F_{2}(q)$,

$$
V^{\mu}=F_{1}\left(q^{2}\right) \gamma^{\mu}+\frac{i}{2 M} F_{2}\left(q^{2}\right) \sigma^{\mu \lambda} q_{\lambda}
$$

where $q$ is the four momentum transfer. The form factors describe the nucleon structure. They are directly related to the electric $G_{\mathrm{E}}(q)$ and magnetic $G_{\mathrm{M}}(q)$ form factors from the 
conserved vector current hypothesis (CVC).

$$
F_{1}(q)=\frac{G_{\mathrm{E}}(q)-\frac{q^{2}}{4 M^{2}} G_{\mathrm{M}}(q)}{1-\frac{q^{2}}{4 M^{2}}}, \quad \xi F_{2}(q)=\frac{G_{\mathrm{M}}(q)-G_{\mathrm{E}}(q)}{1-\frac{q^{2}}{4 M^{2}}}
$$

The electromagnetic form factors of the proton can be approximated by a dipole form,

$$
G_{\mathrm{E}}(q) \cong \frac{1}{\left(1-\frac{q^{2}}{M_{\mathrm{V}}^{2}}\right)^{2}}, \quad G_{\mathrm{M}}(q) \cong \frac{1+\xi}{\left(1-\frac{q^{2}}{M_{\mathrm{V}}^{2}}\right)^{2}}
$$

$\xi=\mu_{p}-\mu_{n}$, is the difference between the proton and neutron magnetic moments and $M_{\mathrm{V}}$ is the vector cut-off mass $\left(M_{\mathrm{V}}^{2} \approx 0.711 \mathrm{GeV}^{2}\right) . \mu_{p}=2.79 \mu_{N}, \mu_{n}=-1.91 \mu_{N}$, where $\mu_{N}=e / 2 M$ is the nuclear magneton, with $e$ being the elementary charge.

The nucleon weak current is a sum of the axial and vector currents,

$$
\begin{gathered}
\Gamma^{\mu}=V^{\mu}-A^{\mu} \\
A^{\mu}=-F_{A}\left(q^{2}\right) \gamma^{\mu} \gamma^{5}-\frac{F_{p}\left(q^{2}\right)}{M} q^{\mu} \gamma_{5}
\end{gathered}
$$

where $q^{\mu}$ are the components of four momentum transfer.

In neutrino scattering, in addition to the $F_{1}(q)$ and $F_{2}(q)$ vector form factors, there is the axial-vector form factor $F_{A}$ and the pseudo-scalar form factor $F_{P}$. The pseudo-scalar form factor enters the cross section in terms proportional to $m_{\text {lepton }}^{2} / M^{2}$ and thus is negligible for neutrino-nucleon elastic scattering. In analogy to $G_{\mathrm{E}}, F_{A}$ is approximated in dipole form for low energy transfers,

$$
F_{A}(q) \cong \frac{g_{A}}{\left(1+\frac{q^{2}}{M_{A}^{2}}\right)^{2}}
$$

where $g_{A}$ is the axial coupling constant and is determined by $F_{A}\left(q^{2}=0\right)=1.2671$ in $\beta^{-}$ decay experiments and $M_{A}$ is the axial mass. The axial mass has been determined using CCQE scattering data on deuterium as $M_{A} \approx 1 \mathrm{GeV}$, as it is less influenced by nuclear effects.

\subsubsection{ELECTRON-NUCLEUS INCLUSIVE SCATTERING CROSS SECTION}

The differential cross section for scattering of an electron with initial four-momentum $k_{e}=\left(E_{e}, \vec{k}_{e}\right)$ off a nuclear target $A$, without detecting the final state of the target $X$,

$$
e+A \rightarrow e^{\prime}+X
$$


in the one photon exchange approximation can be written as [25]:

$$
\frac{d^{2} \sigma}{d \Omega_{e^{\prime}} d E_{e^{\prime}}}=\frac{E_{e^{\prime}}}{E_{e}} \frac{\alpha^{2}}{Q^{4}} L_{\lambda \mu} W^{\lambda \mu}
$$

where $k_{e^{\prime}}=\left(E_{e^{\prime}}, \vec{k}_{e^{\prime}}\right)$ is the four momentum of the scattered electron, $\alpha \cong 1 / 137$ is the fine structure constant, $q=(\omega, \vec{q})=k_{e}-k_{e^{\prime}}$ is the four momentum transfer, $Q^{2}=-q^{2}$ and $d \Omega_{e^{\prime}}$ is the differential solid angle in the direction of $\vec{k}_{e^{\prime}}$ and $L_{\lambda \mu}$ is the electron response tensor:

$$
L^{\lambda \mu}=2\left(k_{e}^{\lambda} k_{e^{\prime}}^{\mu}+k_{e}^{\mu} k_{e^{\prime}}^{\lambda}-g^{\lambda \mu}\left(k_{e} \cdot k_{e^{\prime}}\right)\right)
$$

here the electron mass has been neglected and $g^{\lambda \mu}$ is diagonal with the following diagonal elements $(1,-1,-1,-1) . W_{\mu \nu}$ is the nuclear response tensor which describes the nuclear structure and is defined as:

$$
W^{\lambda \mu}=\sum_{X}\left\langle 0\left|J^{\lambda}\right| X\right\rangle\left\langle X\left|J^{\lambda}\right| 0\right\rangle \delta^{(4)}\left(P_{0}+q-P_{X}\right)
$$

Here $|0\rangle$ and $|X\rangle$ are the initial and final nuclear states with four momenta $P_{0}$ and $P_{X}$ correspondingly, $J^{\mu}=\left(J^{0}, \vec{J}\right)$ is the nuclear electromagnetic current and the sum includes all hadronic final states.

Taking into account the conservation of parity, gauge invariance and Lorentz covariance $W^{\lambda \mu}$ can be written in the following general form, via $W_{1}$ and $W_{2}$ structure functions:

$$
W^{\lambda \mu}=W_{1}\left(-g^{\lambda \mu}+\frac{q^{\lambda} q^{\mu}}{q^{2}}\right)+\frac{W_{2}}{M_{A}^{2}}\left(P_{0}^{\lambda}-\frac{\left(P_{0} \cdot q\right)}{q^{2}} q^{\lambda}\right)\left(P_{0}^{\mu}-\frac{\left(P_{0} \cdot q\right)}{q^{2}} q^{\mu}\right)
$$

where $M_{A}$ is the target mass, the $W_{1}$ and $W_{2}$ structure functions are functions of $Q^{2}$ and $\left(P_{0} \cdot q\right)$. In the target rest frame $\left(P_{0} \cdot q\right)=M_{A} \omega$ and $W_{1}$ and $W_{2}$ become functions of energy and momentum transfer. Using Eq. 29 we can write the electron inclusive scattering cross section of Eq. 26 as:

$$
\frac{d^{2} \sigma}{d \Omega_{e^{\prime}} d E_{e^{\prime}}}=\left(\frac{d \sigma}{d \Omega_{e^{\prime}}}\right)_{M}\left[W_{2}(|\vec{q}|, \omega)+2 W_{1}(|\vec{q}|, \omega) \tan ^{2} \frac{\theta_{e}}{2}\right]
$$

where

$$
\left(d \sigma d \Omega_{e^{\prime}}\right)_{M}=\alpha^{2} \cos ^{2}\left(\theta_{e} / 2\right) /\left[4 E_{e}^{2} \sin ^{4}\left(\theta_{e} / 2\right)\right]
$$

is the Mott cross section and $\theta_{e}$ is the scattering angle of the electron.

Eq. 30 can also be written in terms of contributions arising from scattering processes that involve longitudinally and transversely polarized virtual photons:

$$
\frac{d^{2} \sigma}{d \Omega_{e^{\prime}} d E_{e^{\prime}}}=\left(\frac{d \sigma}{d \Omega_{e^{\prime}}}\right)_{M}\left[\left(\frac{Q^{2}}{|\vec{q}|^{2}}\right)^{2} R_{\mathrm{L}}(|\vec{q}|, \omega)+\left(\tan ^{2} \frac{\theta_{e}}{2}+\frac{1}{2} \frac{Q^{2}}{|\vec{q}|^{2}}\right) R_{\mathrm{T}}(|\vec{q}|, \omega)\right]
$$


where $R_{\mathrm{L}}$ and $R_{\mathrm{T}}$ are the longitudinal and transverse structure functions and are connected to $W_{1}$ and $W_{2}$ structure functions via simple relations:

$$
\begin{array}{r}
R_{\mathrm{T}}=2 W_{1} \\
\left(\frac{Q^{2}}{|\vec{q}|^{2}}\right)^{2} R_{\mathrm{L}}=W_{2}-\frac{Q^{2}}{|\vec{q}|^{2}} W_{1}
\end{array}
$$

At relatively small momentum transfer $(|\vec{q}|<500 \mathrm{MeV})$ the nuclear response tensor $W_{\mu \nu}$ can be exactly calculated for few nucleon systems, with the nuclear wave functions of initial and final states obtained using a Hamiltonian with two and three nucleon interactions.

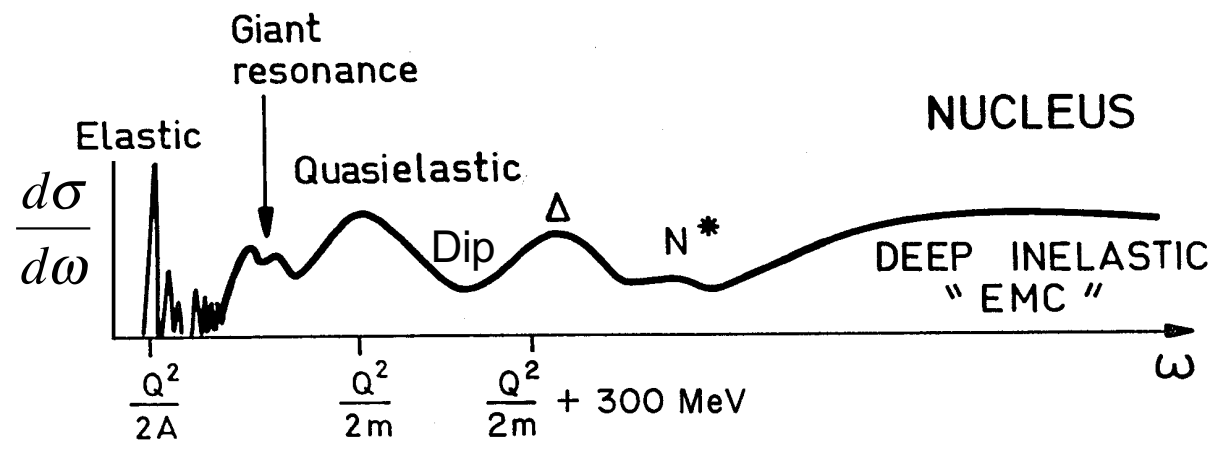

FIG. 9: Schematic representation of the electron-nucleus inclusive cross section at fixed $Q^{2}$ as a function of energy transfer.

Fig. 9 shows the schematic representation of the inclusive electron scattering $\left(e, e^{\prime}\right)$ cross section off a nucleus with mass $A$ as a function of energy loss $\omega$ at a fixed four momentum transfer $Q^{2}=\vec{q}^{2}-\omega^{2}$, where $\vec{q}^{2}$ is the transferred three momentum. The first sharp peak at $\omega=Q^{2} / 2 A$ corresponds to electron-nucleus elastic scattering. The sharp peaks next to the elastic peak correspond to nuclear excitations to discrete states, and are followed by the region corresponding to excitation of collective modes such as giant resonances. The large peak at $\omega=Q^{2} / 2 m$, where $m$ is the nucleon mass, corresponds to QE electron-nucleon scattering. The width of the peak is due to nucleon Fermi momentum, and its location corresponds to the kinetic energy transferred to the nucleon that was initially at rest in the lab frame. The bumps to the right of the QE peak correspond to the resonace region (RES), where the electron scatters on a nucleon and excites it to a $\Delta$ (peak at $Q^{2} / 2 m+300 \mathrm{MeV}$ ) and $N^{*}$ resonances. At even higher values of $\omega$, deep inelastic scattering (DIS) on quarks bound in nucleons dominates. In the so called "dip region" between the QE peak and $\Delta$ - resonance peak, meson exchange currents (MEC) come into play, where the virtual photon couples couples to an exchanged pion between two nucleons. 


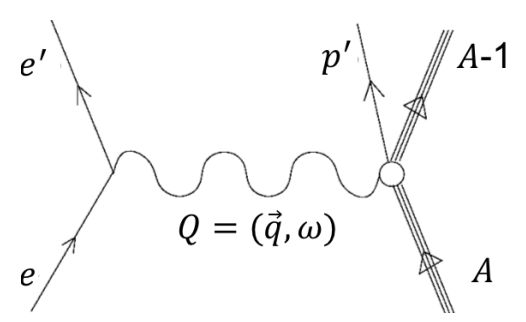

FIG. 10: The diagram of QE electron scattering on nucleon inside the nucleus with mass number $A$.

Electrons can interact via interactions involving one or two-body currents in the nucleus.

One body currents mean that electron interacts with a single nucleon inside the nucleus. That is the case for above mentioned QE scattering, given by a diagram in Fig 10, and quasi-free $\Delta$ production processes or when the electron scatters on a nucleon in nucleonnucleon Short Range Correlation pair (SRC) (see Fig. 11c), where the correlated partner is also ejected from the nucleus. SRCs are two-nucleon properties in the nuclear ground state, responsible for the high momentum part of the nuclear wave function.

Processes involving two nucleons are (1) MEC, (2) excitation of the nucleon into an intermediate $\Delta$ resonant state (Isobar Configuration (IC)), which is followed by de-excitation and pion emission that is absorbed by another nucleon $(\Delta N \rightarrow N N)$, (3) the electron can be scattered on a nucleon in a Short Range Correlation (SRC) pair, followed by ejection of both nucleons, (4) final state interactions (FSI), where the knocked nucleon re-scatters from other nucleons in the nucleus. The diagrams of these processes are shown in Fig. 11. These reaction mechanisms yield to the same final state and interfere with each other. Other reaction mechanisms can lead to final states with one or more pions.

The processes other than rescattering (FSI), that yield to two particles in the final state (including pion production and reabsorption) are sometimes referred to as 2 particle-2 hole $(2 \mathrm{p} 2 \mathrm{~h})$ reactions in neutrino event generators.

Depending on the lepton beam energy, different reaction mechanism dominate in the $\mathrm{A}\left(e, e^{\prime}\right)$ process. Neutrino experiments select events with zero detected pions in order to prefer $\mathrm{QE}$ scattering events for which the $E_{\mathrm{QE}}$ energy reconstruction method is applicable. However the other reaction channels still contribute.

GENIE is one of the most popular neutrino event generators used by neutrino community. Comparison of electron scattering data with GENIE neutrino event generator results obtained by turning off the axial response will improve the nuclear model used, as well as 


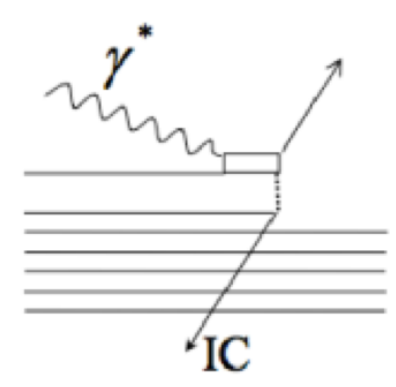

(a)

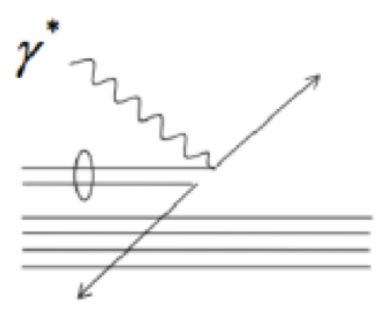

SRC

(c)

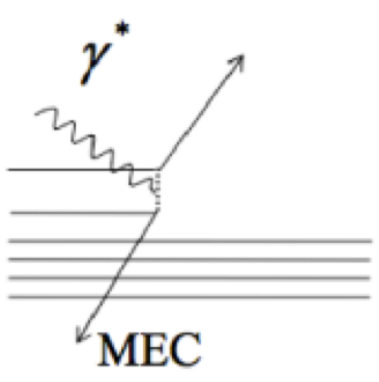

(b)

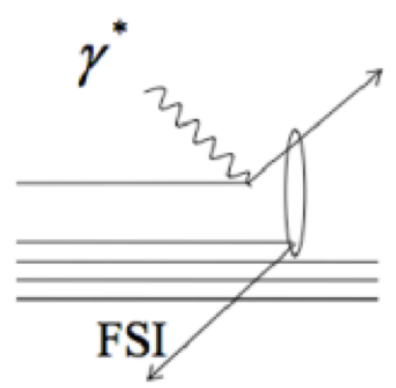

(d)

FIG. 11: The diagrams of different two-body interactions of electrons in a nucleus. (a) IC: excitation of the nucleon into an intermediate $\Delta$ resonant state (Isobar Configuration (IC)), which de-excites via $\Delta \mathrm{N} \rightarrow \mathrm{NN}$; (b) MEC: the virtual photon is absorbed on an exchanged meson between two nucleons; (c) SRC: the electron scatters on a nucleon of a Short Range Correlation (SRC) pair followed by ejection of both nucleons; (d) FSI: electron knocks out a nucleon which re-scatters from other nucleons in the nucleus.

different interaction channels implemented in GENIE, such as QE, MEC, RES, DIS. The nuclear model used in current version of GENIE (v3.0.6) is Local Fermi Gas Model, which means it does not include nucleon-nucleon SRC.

\subsubsection{DIFFERENT REACTION MECHANISMS IN $\left(e, e^{\prime} p\right)$ REACTION}

The semi-inclusive $\left(e, e^{\prime} p\right)$ reaction can distinguish between different reaction channels contributing to the $\left(e, e^{\prime}\right)$ process at specific $\omega$ and $Q^{2}=\vec{q}^{2}-\omega^{2}$, where $\vec{q}^{2}$ is the transferred three momentum. Depending on the missing energy $\epsilon_{\mathrm{m}}=\omega-T_{p}$, where $T_{p}$ is the proton final kinetic energy, different reaction mechanisms occur.

Semi inclusive $\left(e, e^{\prime} p\right)$ reaction studies on ${ }^{12} \mathrm{C}$ have been performed at the MIT-Bates Linear Accelerator at different kinematics. These show how different reaction mechanisms 
appear in $\left(e, e^{\prime} p\right)$ studies.
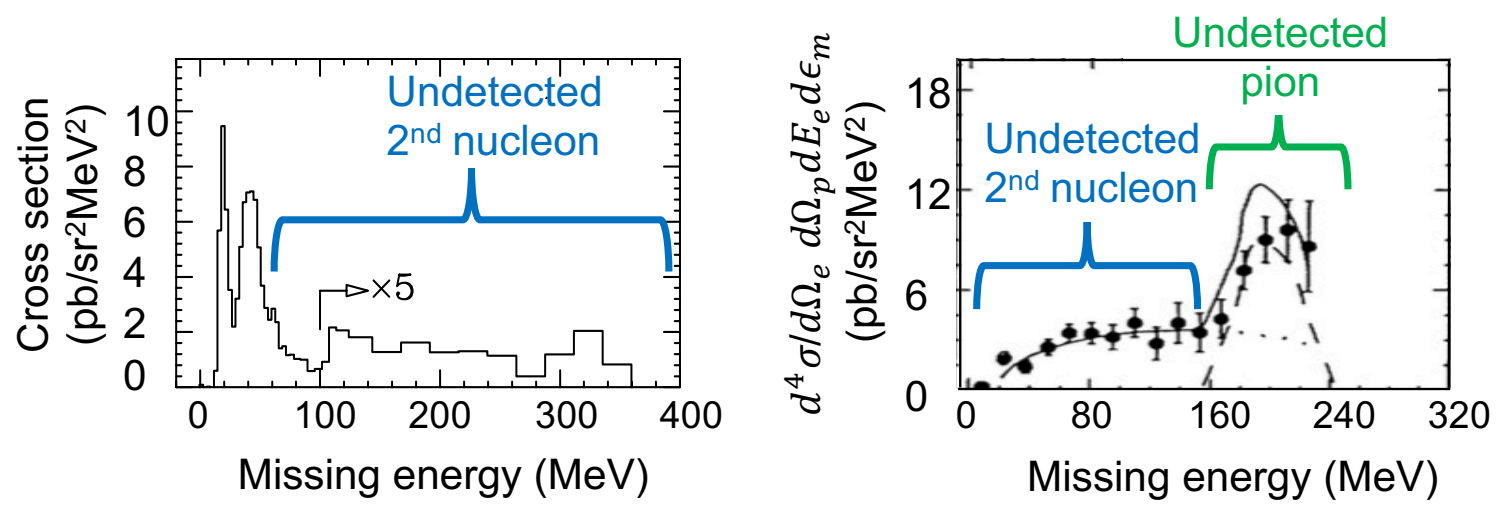

FIG. 12: The missing energy spectra for semi inclusive ${ }^{12} \mathrm{C}\left(e, e^{\prime} p\right)$ reaction for two different kinematical situations: QE (left plot)[26] and $\Delta$ resonance region (right plot)[27]. The dashed curve in the right plot corresponds to quasifree $\Delta$ production with harmonic oscillator momentum distributions, the dotted curve is the three-body phase space result for two nucleon knockout, and the solid curve is their sum.

The studies carried out by Baghaei[27] and Morrison[26] have probed $\Delta$ resonance and QE regions correspondingly and show the strengths in the ${ }^{12} \mathrm{C}\left(e, e^{\prime} p\right)$ reaction missing energy spectrum due to different undetected particles (see Fig. 12). The kinematics for QE region studies was the following: $E_{0}=796 \mathrm{MeV}$ beam energy, $\omega=475 \mathrm{MeV},|\vec{q}|=990 \mathrm{MeV} / \mathrm{c}$, and electron scattering angle $\theta_{e}=118.1^{\circ}$. Two main regions are observed in the cross section as a function of missing energy: (1) The first sharp peak from the left, at $\epsilon_{\mathrm{m}}=18 \mathrm{MeV}$ (below the two-particle emission threshold $\epsilon_{\mathrm{m}}=28 \mathrm{MeV}$ ), corresponds to QE knockout of ${ }^{1} p$ - shell protons. (2) The second peak region, from 28 to $50 \mathrm{MeV}$, contains contributions from both QE knockout of more deeply bound ${ }^{1} s-$ shell nucleons and from two nucleon knockout. (3) The region to the right of the second peak corresponds entirely to two nucleon knockout.

Baghaei has used the following kinematics: $E_{0}=460 \mathrm{MeV}$ beam energy, $\omega=275 \mathrm{MeV}$, $|\vec{q}|=401 \mathrm{MeV} / \mathrm{c}$, and electron scattering angle $\theta_{e}=60^{\circ}$, corresponding to a point roughly halfway between the dip region and the $\Delta$ resonance peak. The plot of the cross section as function of missing energy has the following features: (1) The region from $28 \mathrm{MeV}$ to $165 \mathrm{MeV}$ corresponds to pionless two or more nucleon knockout. (2) Above the real pion production threshold $(165 \mathrm{MeV})$ is dominated by $\Delta(1232)$ resonance production, that decays producing a pion in addition to the proton.

By choosing the kinematics such that the transferred energy stays the same and varying 

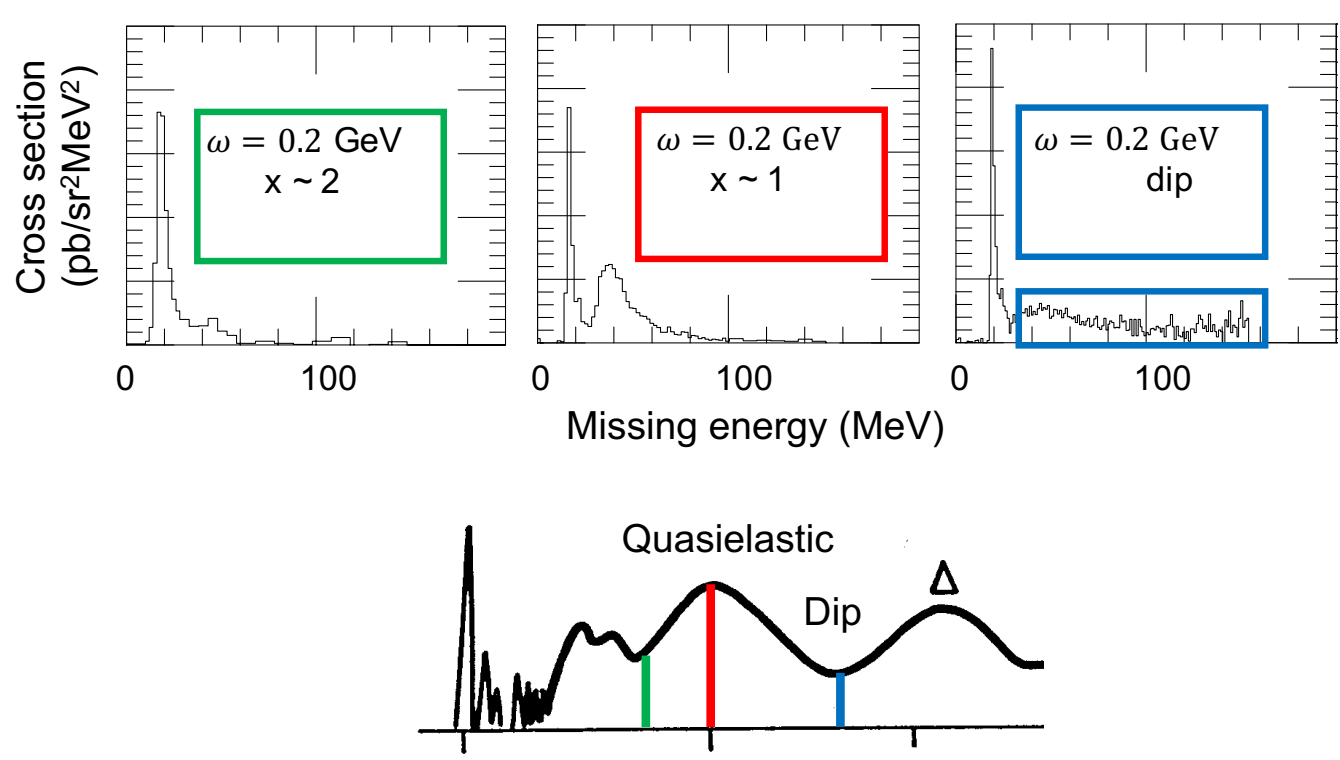

FIG. 13: The missing energy spectra for semi inclusive ${ }^{12} \mathrm{C}\left(e, e^{\prime} p\right)$ reaction for three different kinematical situations: QE (left[28] and middle[29] plots) and Dip region (right plot)[30]. Also shown is the cross section as a function of energy transfer for the $\left(e, e^{\prime}\right)$ reaction, with color coded vertical lines showing the reaction region probed at each of the kinematics settings.

the transferred momentum one can study electron-nucleus interaction regions where different reaction mechanisms dominate. Penn [28], Weinstein [29] and Lourie [30] have measured the regions corresponding to the $\mathrm{QE}$ electron nucleon scattering region and the dip region, where electron interactions via two body currents dominate.

The results are shown in Fig. 13.

For all three studies $\omega=0.2 \mathrm{GeV}$. Penn has used a kinematics with Bjorken variable $x=Q^{2} / 2 m \omega=2$, where $m$ is the nucleon mass, to probe the low energy loss side of the QE peak. Weinstein has used $x=1$, that corresponds to QE electron nucleon scattering, and Lourie has chosen kinematics with $E_{0}=459 \mathrm{MeV}, \omega=200 \mathrm{MeV},|\vec{q}|=400 \mathrm{MeV} / \mathrm{c}, \theta_{e}=60^{\circ}$ in order to probe the dip region.

The nuclear effects (MEC, IC, Correlations, Delta, FSI, etc.) are practically identical for electron and neutrino scattering. With electron scattering data we can choose kinematics to minimize the contribution of "uninteresting" reaction mechanisms and enhance the ones we want to study. We can then test different models implemented in the neutrino event 
generators running them in the electron scattering mode.

\subsubsection{LONG-RANGE AND SHORT-RANGE NUCLEAR CORRELATIONS}

According to the Independent Particle Model (IPM) of nucleon interaction, also known as the Hartree-Fock approximation, the motion of the nucleons inside the nuclei is not affected by other individual nucleons and they move in the average potential due to the rest of the nucleons. This produces the shell model, and provides a good description of some nuclear properties, such as charge distributions, spins, parities, ground state energies and more. However the electron scattering experiments $A\left(e, e^{\prime} p\right)$ show that the cross section of valence

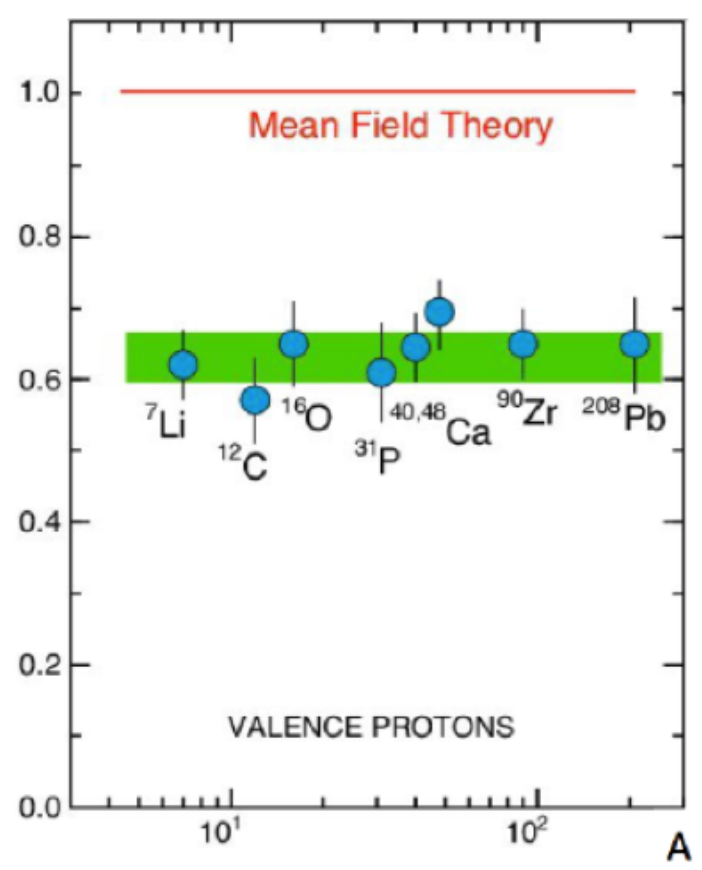

FIG. 14: Spectroscopic factors for various nuclei extracted by comparing $A\left(e, e^{\prime} p\right)$ valence knockout cross-sections to mean field theory[1]

proton (protons outside the closed shells) knockout is only $60-70 \%$ of that expected (see Fig. 14). This in turn means that only $60-70 \%$ of nucleons inside nucleus can be described by the IPM, while the rest are part of higher order configurations, such as Short Range Correlations.

We distinguish two type of nucleon correlations, Short Range Correlations (SRC) and Long Range Correlations (LRC). The strong short range $N N$ repulsion gives rise to the correlated motion of the interacting nucleons and prevents them from overlapping. The long range attractive $N N$ interaction, which is the most precisely known part of the $N N$ 
interaction, keeps the nuclei from falling apart and causes nucleon LRC. The latter is well described by single pion exchange.

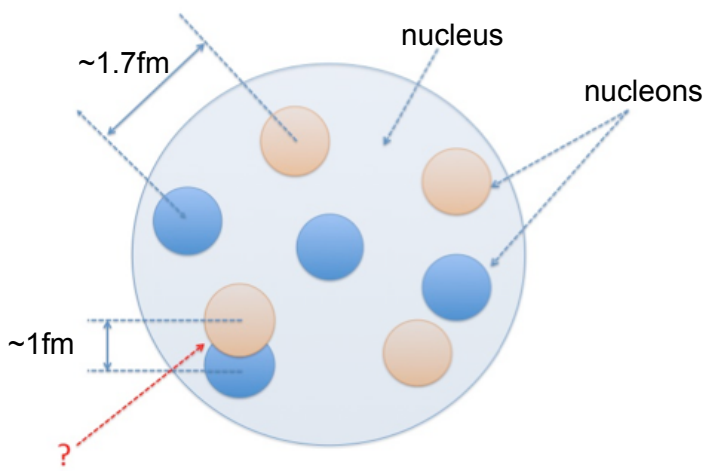

FIG. 15: The illustration of inter nucleon distances inside nuclei. The average distance between the nucleons in nuclei is $\sim 1.7 \mathrm{fm}$, while the distance between the nucleons in SRC pair is $\sim 1 \mathrm{fm}$.

SRCs are pairs of nucleons with high relative momentum (compared to the Fermi momentum) and smaller Center of Mass (CM) momentum, situated very close to each other ( $\sim 1 \mathrm{fm}$ ), less than the average distance between nucleons in nuclei of $1.7 \mathrm{fm}$ (see Fig. 15). This means that the nuclear densities inside SRCs significantly exceed the average nuclear densities and can approach the densities of neutron stars.

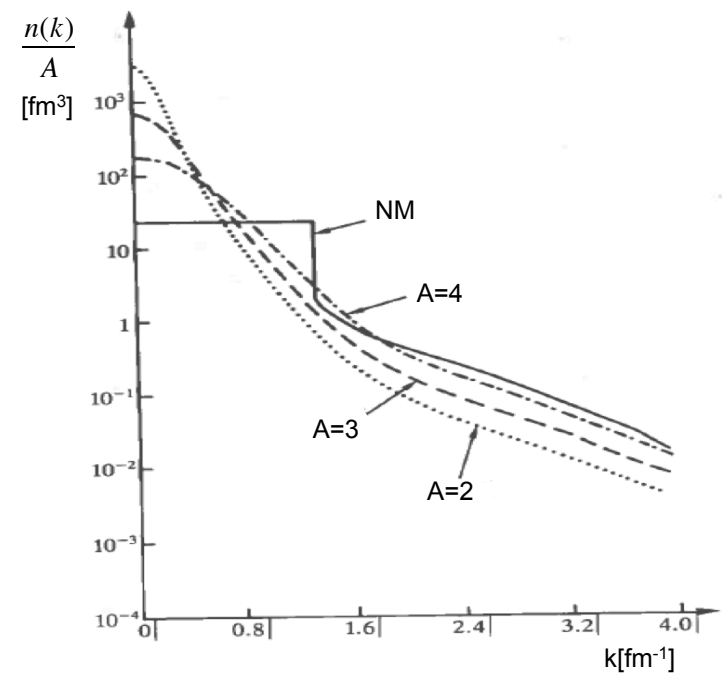

FIG. 16: Per nucleon momentum distributions calculated by Schiavilla et al. [2] in $A=2,3$ and 4 nuclei and nuclear matter.

In order to solve the many body problem one must find a solution to the Schrodinger 
equation, with a Hamiltonian that includes all types of $N N$ interactions. This can be done exactly for few nucleon systems (containing up to 12 nucleons) using Monte-Carlo techniques.

Fig. 16 shows the momentum distributions of protons and neutrons in nuclei with $A=2$ and $A=3,4$ calculated using a realistic Hamiltonian [2], which includes three nucleon interactions. If the SRC is due to Short Range Repulsive part of the $N N$ interaction then it should depend only on the nearest nucleon and the nucleon momentum distribution for momenta greater than the Fermi momentum $(\approx 250 \mathrm{MeV} / \mathrm{c})$ should be the same for all nuclei and that is what we see in Fig. 16.

\subsubsection{NEUTRINO-NUCLEUS INCLUSIVE SCATTERING CROSS SECTION}

The inclusive cross section for neutrino-nucleus scattering induced by charge-changing weak currents $(\mathrm{CC})$

$$
\nu_{l}+A \rightarrow l^{-}+X, \quad \bar{\nu}_{l}+A \rightarrow l^{+}+X
$$

and neutral weak currents $(\mathrm{NC})$

$$
\nu_{l}+A \rightarrow \nu_{l}+X, \quad \bar{\nu}_{l}+A \rightarrow \bar{\nu}_{l}+X
$$

can be obtained by summing over the final nuclear states and averaging over the initial states [31]. Here $A$ is the target nucleus, $l^{ \pm}$is the final state lepton and $X$ is the nuclear final state.

$$
\frac{d^{2} \sigma}{d \Omega d \epsilon^{\prime}}=\frac{1}{2 J_{M}+1} \sum_{M} \sum_{f} \frac{d \sigma_{f M}}{d \epsilon^{\prime} d \Omega}=\left(\frac{G}{2 \pi}\right)^{2} k^{\prime} \epsilon^{\prime} L_{\sigma \tau} W^{\sigma \tau}
$$

where $k^{\mu}=(\epsilon, \vec{k})$ and $k^{\mu^{\prime}}=\left(\epsilon^{\prime}, \vec{k}^{\prime}\right)$ are the four momenta of the initial and final leptons and $G$ is a constant. $M$ is the spin projection of the initial nuclear state $|A, M\rangle$ and $|f\rangle$ represents the final nuclear state with energy $E_{f} . L_{\sigma \tau}$ is the leptonic tensor:

$$
L_{\sigma \tau}=\frac{1}{\epsilon \epsilon^{\prime}}\left(k_{\sigma} k_{\tau}^{\prime}+k_{\sigma}^{\prime} k_{\tau}-g_{\sigma \tau} k k^{\prime} \pm i \epsilon_{\sigma \alpha \tau \beta} k^{\alpha} k^{\prime \beta}\right)
$$

where the + sign is for neutrino and $-\operatorname{sign}$ for anti-neutrino initiated processes and $\epsilon_{0123}=$ +1 . The nuclear tensor $W^{\sigma \tau}$ is the same as in the electron inclusive scattering given by Eq. 28, only the electromagnetic current should be replaced by weak NC or CC currents :

$$
W^{\sigma \tau}=\frac{1}{2 J_{M}+1} \sum_{M} \sum_{f} \delta\left(\omega+E_{M}-E_{f}\right) j_{f M}^{\sigma} j_{f M}^{\tau *}
$$

$E_{M}$ and $E_{f}$ are the energies of initial and final nuclear states, $j_{f M}^{\sigma}=\left\langle f\left|j^{\sigma}\right| A, M\right\rangle, j_{f M}^{\tau *}=$ $\left\langle f\left|j^{\tau}\right| A, M\right\rangle^{*}, j^{\sigma}$ is the time $(\sigma=0)$ or space $(\sigma=x, y, z)$ component of the nuclear weak $\mathrm{NC}$ or $\mathrm{CC}$. 
In the general case when the lepton mass can not be neglected, we find the following expression for the inclusive cross section for CC processes by taking the three momentum $\vec{q}$ along the $z$ axis (i.e., the spin quantization axis) [32]:

$$
\left(\frac{d \sigma}{d \epsilon^{\prime} d \Omega}\right)_{\nu / \bar{\nu}}=\frac{G^{2}}{8 \pi^{2}} \frac{k^{\prime}}{\epsilon} F\left(Z, k^{\prime}\right)\left[v_{00} R_{00}+v_{z z} R_{z z}-v_{0 z} R_{0 z}+v_{x x+y y} R_{x x+y y} \mp v_{x y} R_{x y}\right]
$$

here $G$ is a constant, the last term has a negative sign for neutrino scattering and positive sign for anti neutrino scattering. $F\left(Z, k^{\prime}\right)$ is the Fermi function and accounts for the Coulomb distortion of the final lepton wave function in the charge-raising reaction,

$$
F\left(Z, k^{\prime}\right)=2(1+\gamma)\left(2 k^{\prime} r\right)^{2 \gamma-2} \exp (\pi y)\left|\frac{\Gamma(\gamma+i y)}{\Gamma(1+2 \gamma)}\right|^{2}
$$

$\gamma=\sqrt{1-(Z \alpha)^{2}}, \alpha$ is the fine structure constant, $Z$ is the atomic number, $\Gamma(z)$ is the Gamma function and $r$ is the radius of the target nucleus.

The $R_{i j}$ are nuclear response functions and contain the nuclear structure information. $v_{00}, v_{z z}, v_{o z}, v_{x x+y y}, v_{x y}$ are kinematical factors.

The response function are given by the following expressions:

$$
\begin{array}{r}
R_{00}(q, \omega)=\frac{1}{3} \sum_{M} \sum_{f} \delta\left(\omega+m_{A}-E_{f}\right)\left\langle f\left|j^{0}(\vec{q}, \omega)\right| A, M\right\rangle\left\langle f\left|j^{0}(\vec{q}, \omega)\right| A, M\right\rangle^{*}, \\
R_{z z}(q, \omega)=\frac{1}{3} \sum_{M} \sum_{f} \delta\left(\omega+m_{A}-E_{f}\right)\left\langle f\left|j^{z}(\vec{q}, \omega)\right| A, M\right\rangle\left\langle f\left|j^{z}(\vec{q}, \omega)\right| A, M\right\rangle^{*}, \\
R_{0 z}(q, \omega)=\frac{2}{3} \sum_{M} \sum_{f} \delta\left(\omega+m_{A}-E_{f}\right) R e\left[\left\langle f\left|j^{0}(\vec{q}, \omega)\right| A, M\right\rangle\left\langle f\left|j^{z}(\vec{q}, \omega)\right| A, M\right\rangle^{*}\right], \\
\left.\quad+\left\langle f\left|j^{y}(\vec{q}, \omega)\right| A, M\right\rangle\left\langle f\left|j^{y}(\vec{q}, \omega)\right| A, M\right\rangle^{*}\right], \\
R_{x x+y y}(q, \omega)=\frac{1}{3} \sum_{M} \sum_{f} \delta\left(\omega+m_{A}-E_{f}\right)\left[\left\langle f\left|j^{x}(\vec{q}, \omega)\right| A, M\right\rangle\left\langle f\left|j^{x}(\vec{q}, \omega)\right| A, M\right\rangle^{*}\right. \\
R_{x y}(q, \omega)=\frac{2}{3} \sum_{M} \sum_{f} \delta\left(\omega+m_{A}-E_{f}\right) \operatorname{Im}\left[\left\langle f\left|j^{x}(\vec{q}, \omega)\right| A, M\right\rangle\left\langle f\left|j^{y}(\vec{q}, \omega)\right| A, M\right\rangle^{*}\right],
\end{array}
$$

$m_{A}$ is the rest mass of the target nucleus $A$. In neutrino-nucleus scattering in addition to $R_{00}$ longitudinal and $R_{x x+y y}$ transverse response functions we have $R_{x y}$ interference response function and two additional response functions $\left(R_{z z}\right.$ and $\left.R_{0 z}\right)$.

The inclusive scattering cross section for $\mathrm{NC}$ interactions is the same as the one for CC interactions given in Eq. 39, in the limit where the lepton mass can be neglected and 
$Q^{2}=4 \epsilon \epsilon^{\prime} \sin ^{2} \theta / 2$. In this limit Eq. 39 reduces to the following expression:

$$
\begin{array}{r}
\left(\frac{d \sigma}{d \epsilon^{\prime} d \Omega}\right)_{\nu / \bar{\nu}}=\frac{G^{2}}{2 \pi^{2}} k^{\prime} \epsilon^{\prime} F\left(Z, k^{\prime}\right) \cos ^{2} \frac{\theta}{2}\left[R_{00}+\frac{\omega^{2}}{q^{2}} R_{z z}-\frac{\omega}{q} R_{0 z}+\left(\tan ^{2} \frac{\theta}{2}+\frac{Q^{2}}{2 q^{2}}\right) R_{x x+y y}\right. \\
\left.\mp \tan \frac{\theta}{2} \sqrt{\tan ^{2} \frac{\theta}{2}+\frac{Q^{2}}{q^{2}}} R_{x y}\right]
\end{array}
$$

$G$ is equal to Fermi constant $G_{\mathrm{F}}$ for the NC processes and is equal to $G_{\mathrm{F}} \cos \theta_{C}$ and $G_{\mathrm{F}}=$ $1.1803 \times 10^{-5} \mathrm{GeV}^{-2}, \cos \theta_{C}=0.97425$. As before the last term has a negative sign for neutrino scattering and positive sign for anti neutrino scattering.

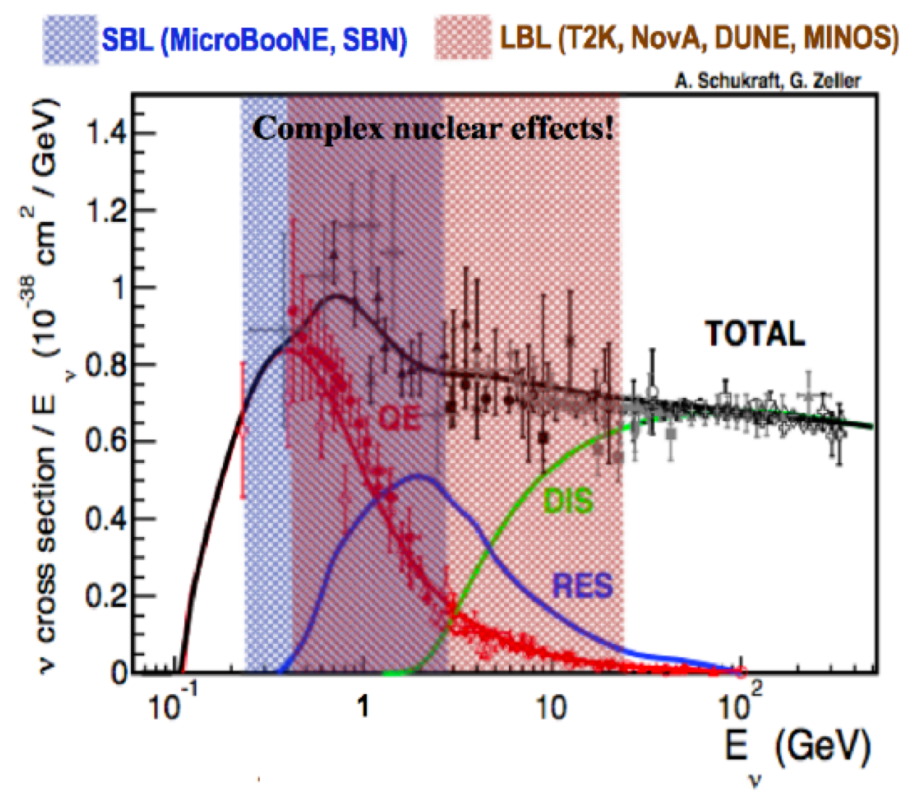

FIG. 17: Charged current neutrino-nucleus inclusive scattering cross section divided by neutrino energy as a function of neutrino energy. Contributions from different scattering processes are shown in different colors, and the total cross section is shown in black. The blue and red vertical bands show the energy range of the current short-baseline (SBL) and long-baseline (LBL) neutrino experiments [33].

Fig. 17 shows the charged-current neutrino total inclusive scattering cross section and contributions from different scattering processes as a function of neutrino energy.

Because of the axial vector component in the leptonic and hadronic weak currents, the $L_{\sigma \tau}$ and $W^{\sigma \tau}$ tensors in neutrino-nucleus scattering contain additional terms compared to those in electron-nucleus scattering. 
The inclusive electron-nucleus scattering cross section given by Eq. 32 has $1 / Q^{2}$ dependence coming from the propagator for electromagnetic interactions. This dependence is embedded in the Mott cross section. The inclusive neutrino-nucleus scattering cross section in Eq. 39 is almost independent of the kinematics for the $Q^{2}$ range of ongoing neutrino oscillation experiments, and the factor $k^{\prime} / \epsilon$ is of order 1 .

\subsubsection{OPEN QUESTIONS IN NEUTRINO-NUCLEUS SCATTERING}

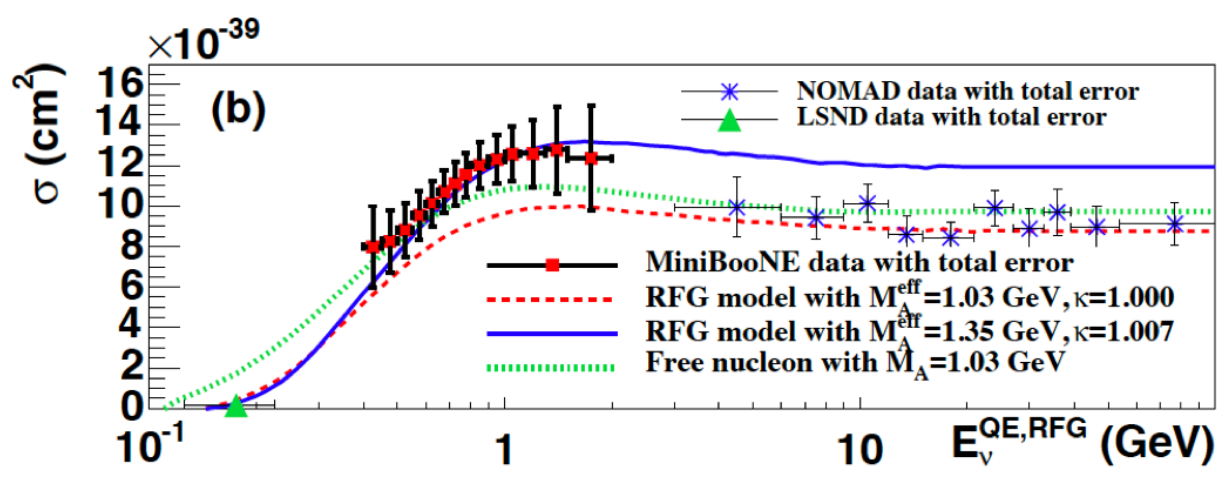

FIG. 18: Flux-unfolded MiniBooNE $\nu_{\mu}$ CCQE cross section per neutron as a function of reconstructed neutrino energy. Also shown are the results from NOMAD[34] and LSND[35] experiments. The data is compared with the predictions from the NUANCE simulation for a relativistic Fermi gas model with two different axial mass values and for scattering from free nucleons using the world average value of the axial mass (the $\kappa$ parameter influences the strength of the Pauli principle).

MiniBooNE has measured the QE scattering cross section for Charged Current QE and Neutral Current QE scattering events, see Fig. 18 [36]. The cross section is bigger than that expected from QE scattering in the Fermi-gas model. The NOMAD experiment on the other hand, the target for which consists of mainly carbon, found results consistent with the world average value of the axial mass obtained from a fit to old neutrino-nucleon, neutrinodeutron and electro-pion production data, which is $M_{A}=1.026 \mathrm{GeV}$. An axial mass value of $M_{A}=1.35 \mathrm{GeV}$ is needed describe the MiniBooNE data.

MiniBooNE finds more electron neutrino events than expected from neutrino oscillations and known backgrounds [37], [38]. This suggests that muon neutrinos oscillate into electron neutrinos in a shorter distances than expected.

Also the charged [39] and neutral [40] pion production cross section results for CC neutrino scattering in MinibooNE are noticeably higher than theory predictions [41]. 
The Liquid Scintillator Neutrino Detector (LSND) experiment at Los Alamos in the 1990s observed a different excess of $\overline{\nu_{e}}$ in $\overline{\nu_{\mu}} \rightarrow \overline{\nu_{e}}$ oscillation studies [42], that could be explained by addition of the fourth sterile neutrino with mass eigenstate $\nu_{4}$, that interacts only through gravity and is not included in the standard model. This result is unlikely.

Both MiniBooNE and LSND used a tank of mineral oil with scintillator doping as a detector. The observation of the significant excess of electron-like events in MiniBooNE could also be due to the fact that in Cherenkov counters the decay photons of neutral pions can be misidentified as electrons.

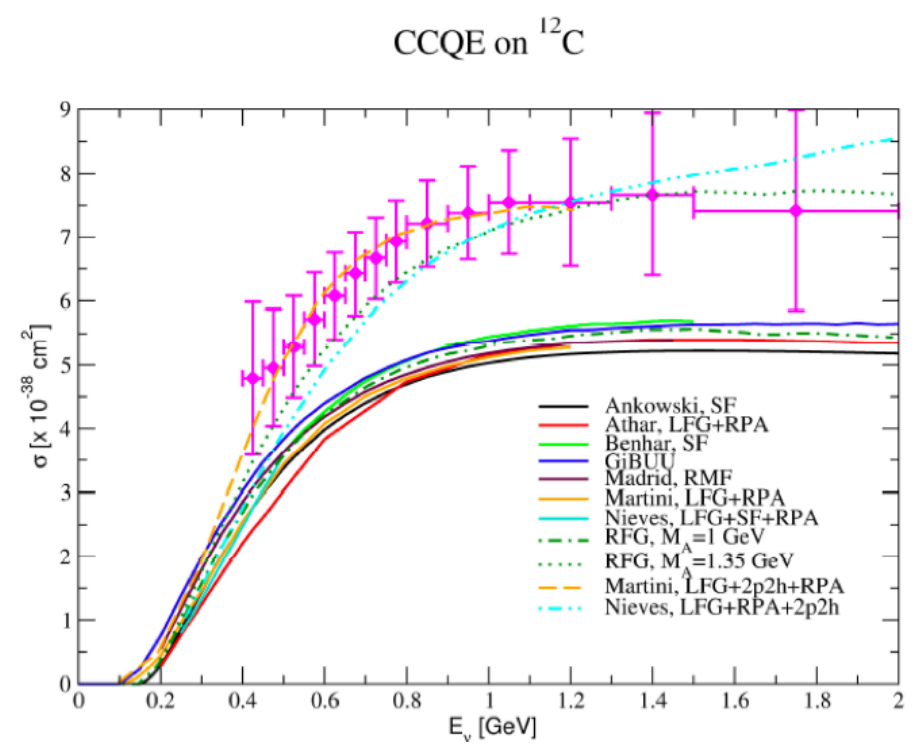

FIG. 19: CCQE cross section for a $\mathrm{CH}_{2}$ target obtained in the MiniBooNE experiment. The lower curves give various theoretical predictions for the true QE events obtained with an axial mass of $1.03 \mathrm{GeV}$; the dotted green curve gives shows the results for true QE events obtained with axial mass value $1.3 \mathrm{GeV}$. The orange and light blue dashed curves give predictions of models that take many body interactions into account with a smaller axial mass [43], [44].

This shows that the nuclear effects play an important role in the understanding of the neutrino-nucleus scattering mechanisms and in order to be able to describe the data, reaction mechanisms other than QE scattering should be also included in the model. These include:

- RPA An effective theory constructed to study the excitations of many-body systems (Long range correlations)

- $2 p-2 h$ interactions that include meson-exchange currents, short range correlations 
(SRC), pion production and reabsorption and any other process except rescattering, that leads to two nucleons in the final state,

- FSI final state interactions where the knocked out nucleon rescatters from other target nucleons when coming out of the target.

Studies done by Martini et al. [43] and Nieves et al. [44] show that the observed surplus in the MiniBooNE QE cross section can be described without increasing the axial mass value, by the addition of RPA excitations of the nucleus and $2 p 2 h$ excitations in the model, as shown in Fig. 19 [45].

\subsection{NEUTRINO INTERACTION AND ENERGY RECONSTRUCTION SYSTEMATICS}

Neutrino oscillation studies have entered the era of precise measurements, where instead of two neutrino oscillation approximation experiments, they consider three neutrino oscillations to determine all the parameters in neutrino oscillations including the $\mathrm{CP}$ violation phase $\delta_{\mathrm{CP}}$ and the mass hierarchy. To achieve these goals the oscillation probabilities need to be measured with an accuracy of $10 \%$ or better [46]. T2K and $\mathrm{NO} \nu \mathrm{A}$ experiments already started to contribute toward this and the DUNE and Hyper-K experiments are in the design stage.

Fig. 20 shows the $\nu_{e}$ appearance probability expected in DUNE for three different sets of values of $\delta_{C P}$ and $\theta_{13}$ [47]. The three curves can be distinguished from one another if the neutrino energy can be determined to better than $100 \mathrm{MeV}$.

Fig. 17 shows the energy range of these experiments includes contributions from different neutrino interaction processes, and nuclear effects are large in this region. Currently, the biggest known contribution to systematic uncertainties in long baseline neutrino oscillation experiments comes from the neutrino-nucleus interaction. The estimated cross section systematic uncertainty in $\nu_{\mu}$ disappearance searches is $5 \%$ for T2K [8] at much lower energies (see Table 3).

The current cross section systematic uncertainty for $\nu_{e}$ appearance searches at $\mathrm{NO} \nu \mathrm{A}$ is estimated to be $7 \%$ [48].

These uncertainties may be too big for the physics goals of future short and long-baseline experiments and they may be underestimated. Fig. 21 shows the $\delta_{\mathrm{CP}}$ sensitivity for $50 \%$ of likely $\delta_{\mathrm{CP}}$ values as a function of exposure $(($ kiloton $) \times$ beam power $(\mathrm{MW}) \times$ time $($ years $))$ for different sets of signal and background uncertainties and normal hierarchy. We can see that for current values of systematic uncertainties $5 \%$, it is possible to reach $5 \sigma$ sensitivity 


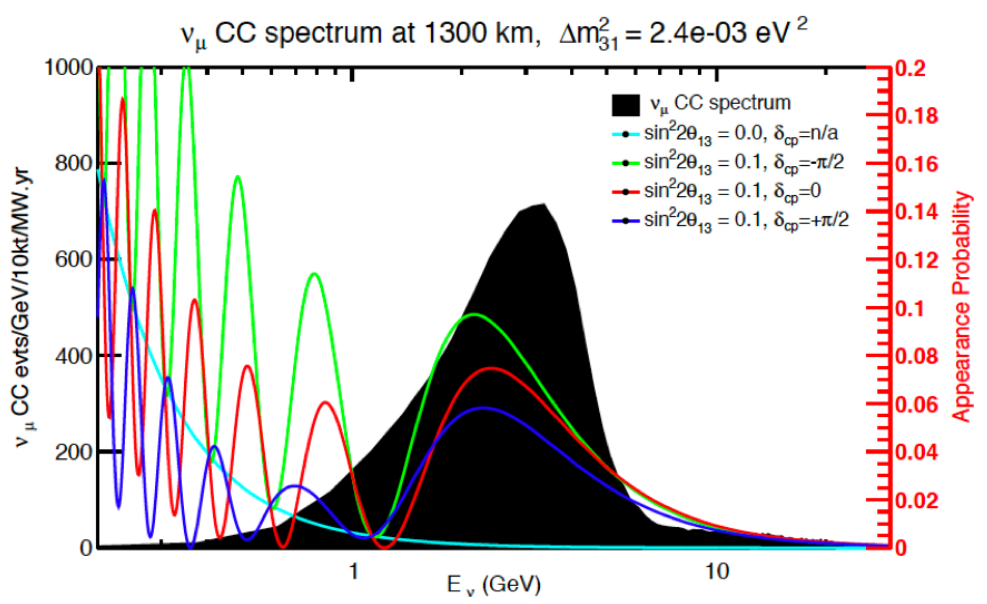

FIG. 20: The $\nu_{e}$ appearance probability in a $\nu_{\mu}$ beam at a distance of $1300 \mathrm{~km}$, calculated using standard oscillation mixing angles and normal hierarchy. The sensitivity of the signal to the different values of $\delta_{C P}$ and $\theta_{13}$ is shown by different colors, and the black solid distribution corresponds to $\nu_{\mu}$ unoscillated spectrum.

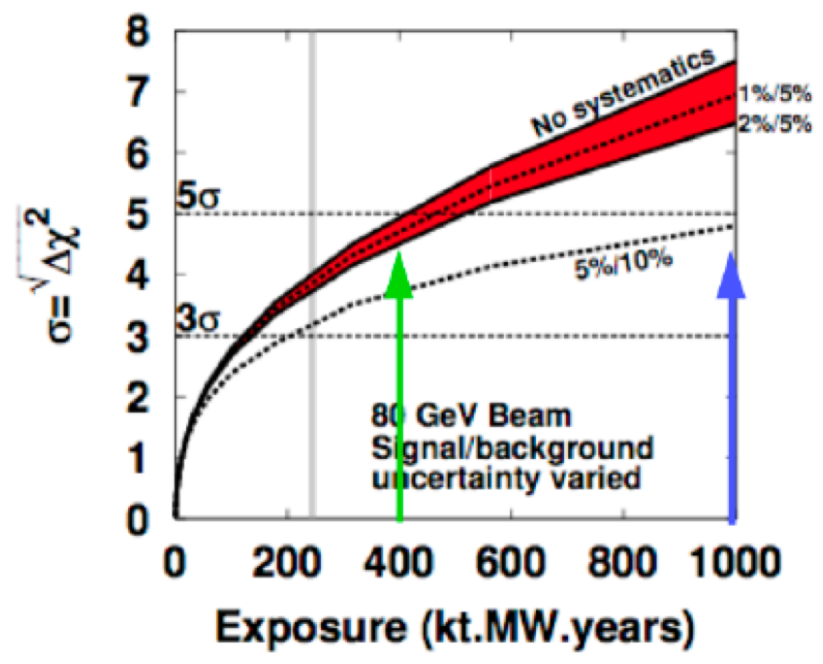

FIG. 21: $\delta_{\mathrm{CP}}$ sensitivity for $50 \%$ of possible $\delta_{\mathrm{CP}}$ values as a function of exposure (for a 12 MW and 34 kton detector) at DUNE experiment for normal hierarchy [33], [47]. The green arrow corresponds to 10 years of running and blue arrow corresponds to 25 years of running.

only with 25 years of running, while with $1 \%$ systematic uncertainties, it would be possible to reach the goal in only 10 years of running. 


\begin{tabular}{|ccc|}
\hline Uncertainties & $\nu_{\mu}$ disap. & $\nu_{e}$ app. \\
\hline \hline$\nu$ flux + xsec & $\pm 2.7 \%$ & $\pm 3.2 \%$ \\
\hline$\nu$ unconstrained xsec & $\pm 5.0 \%$ & $\pm 4.7 \%$ \\
\hline Far detector & $\pm 4.0 \%$ & $\pm 2.7 \%$ \\
\hline Total & $\pm 7.7 \%$ & $\pm 6.8 \%$ \\
\hline
\end{tabular}

TABLE 3: The table of uncertainties from different sources in T2K $\nu_{\mu}$ disappearance and $\nu_{e}$ appearance searches [8].

Neutrino event generators play a crucial role in neutrino interaction and oscillation studies, in particular in determination of the neutrino flux and description of the neutrino interaction physics and detector response. They are also used to connect the reconstructed quantities such as neutrino energy to true quantities as well as for systematic error evaluation.

The Independent Particle Model (IPM) assumes that the nucleons move in the average potential due to the rest of the nucleons. The Relativistic Fermi Gas model, that is a nuclear model based on IPM commonly used in neutrino event generators, is not sufficient to account for the complexity of the nuclear dynamics in neutrino nucleus interactions. Recent results from the Minerva experiment show that none of the nuclear models implemented in nuclear event generators accurately describes the measured $\nu_{\mu}$-nucleus inclusive scattering cross section on $\mathrm{C}, \mathrm{Fe}, \mathrm{Pb}$ and $\mathrm{CH}$ targets at neutrino energies $2-20 \mathrm{GeV}$ [49].

There is also a discrepancy between the CCQE neutrino-nucleus scattering cross section measurements by MiniBooNE and simulation results using relativistic Fermi gas model as discussed in section 1.5.6.

While theoretical models are being developed to describe neutrino-nucleus interactions from few hundred $\mathrm{MeV}$ to a few $\mathrm{GeV}$, the available electron-nucleus scattering data should be used to test the validity of these models, as the electromagnetic nuclear response has been studied carefully via inclusive, semi-inclusive and exclusive channels. The data used in this analysis has been collected with $\mathrm{C}, \mathrm{Fe}$, He targets, that are similar to those used in current and future neutrino oscillation experiments. Our goal is to use these data to test and improve the GENIE neutrino event generator performance, by running it in electron scattering mode (turning off the axial response).

We have used electron scattering data from the Jefferson Lab experimental Hall-B e2a 
experiment, to test neutrino energy reconstruction. We have selected events with an electron, a proton and no pions to enhance the QE scattering contribution. We then applied neutrino energy reconstruction methods to these events and compared energy reconstruction results from electron scattering data to neutrino event generator results running in electron scattering mode.

We have also used energy reconstruction results from data and energy reconstruction predictions of neutrino event generators running in neutrino mode, to reconstruct the $\nu_{\mu}$ energy spectrum at DUNE far detector. We show that the two results differ from each other and can lead to extraction of different oscillation parameters. 


\section{CHAPTER 2}

\section{EXPERIMENT}

\subsection{E2A EXPERIMENT}

\begin{tabular}{|l|l|l|l|}
\hline Target & length $[\mathrm{cm}]$ & Density $\left[\mathrm{g} / \mathrm{cm}^{3}\right]$ & Density $*$ length $\left[\mathrm{g} / \mathrm{cm}^{2}\right]$ \\
\hline${ }^{3} \mathrm{He}$ & 4.13 & 0.067 & 0.277 \\
${ }^{4} \mathrm{He}$ & $3.72-4.99$ & 0.125 & $0.465-0.624$ \\
${ }^{12} \mathrm{C}$ & 0.1 & 1.786 & 0.179 \\
${ }^{56} \mathrm{Fe}$ & 0.015 & 7.872 & 0.118 \\
$\mathrm{CH}_{2}$ & 0.07 & 1.392 & 0.097 \\
empty (4 types) & & & \\
\hline
\end{tabular}

TABLE 4: E2a target lengths and densities.

We have analyzed JLab (Thomas Jefferson National Accelerator Facility) Hall-B e2a experiment data, which ran from April 15 to May 27, 1999. We used the data collected at $4.461 \mathrm{GeV}$ and $2.261 \mathrm{GeV}$ beam energies with ${ }^{3} \mathrm{He},{ }^{4} \mathrm{He},{ }^{12} \mathrm{C},{ }^{56} \mathrm{Fe}$ targets and torus current equal to $2250 \mathrm{~A}$, and $1.161 \mathrm{GeV}$ with ${ }^{3} \mathrm{He},{ }^{12} \mathrm{C}$ targets and torus current equal to $750 \mathrm{~A}$. The beam current varied from 3 to $18 \mathrm{nA}$. The more detailed list of runs with corresponding beam currents can be found in [50]. This was done to test how well the neutrino beam energy reconstruction techniques describe the electron beam energy.

The experiment used the standard inclusive electron trigger, with Cherenkov Counters (CC) and Electromagnetic Calorimeter (EC) at 1.1 and $2.2 \mathrm{GeV}$ and EC only at $4.4 \mathrm{GeV}$. The e2a experiment used the CLAS (CEBAF Large Acceptance Spectrometer) spectrometer with " $4 \pi$ " acceptance to measure $A\left(e, e^{\prime} X\right)$ events, and has collected data in 4278 raw data files corresponding to 365 runs, $2.2 \times 10^{9}$ triggers and $13 \mathrm{mC}$ of beam charge. Table 6 shows the list of runs used in the current analysis. The data was skimmed for $\left(e, e^{\prime}\right) X$ and $\left(e, e^{\prime} p\right) X$ events. 


\begin{tabular}{|c|c|c|c|c|c|c|}
\hline Cell & $\begin{array}{l}\text { Time period } \\
(1999)\end{array}$ & Runs & Target & $\begin{array}{l}\text { Energy } \\
{[\mathrm{GeV}]}\end{array}$ & $\begin{array}{l}\text { Length } \\
{[\mathrm{cm}]}\end{array}$ & Windows \\
\hline 1 & April 15-May 7 & $17870-18206$ & ${ }^{4} \mathrm{He}$ & $2.2,4.4$ & 5.000 & $\begin{array}{l}\text { Mylar }\left(1.39 \mathrm{~g} / \mathrm{cm}^{3}\right) \\
\text { Aluminum }\left(2.70 \mathrm{~g} / \mathrm{cm}^{3}\right)\end{array}$ \\
\hline 2 & May 8-May 12 & $18221-18252$ & ${ }^{4} \mathrm{He}$ & 2.2 & 3.950 & Mylar, Aluminum \\
\hline 3 & May 12-May 15 & & ${ }^{3} \mathrm{He}$ & & 4.016 & Mylar, Aluminum \\
\hline 4 & May 15 -May 27 & $18308-18521$ & ${ }^{3} \mathrm{He}$ & $\begin{array}{l}1.1,2.2 \\
4.4\end{array}$ & 4.016 & $\begin{array}{l}\text { Copper }\left(8.96 \mathrm{~g} / \mathrm{cm}^{3}\right) \\
\text { Mylar, Aluminum }\end{array}$ \\
\hline
\end{tabular}

TABLE 5: E2a liquid target information.

The list of all the targets available in the e2a experiment data with corresponding lengths and densities is shown in Table 4 . The solid targets $\left({ }^{12} \mathrm{C},{ }^{56} \mathrm{Fe}\right.$ and $\left.\mathrm{CH}_{2}\right)$ were square plates of size $0.9 \times 0.9 \mathrm{~cm}^{2}$ and the cryotargets (liquid ${ }^{3} \mathrm{He},{ }^{4} \mathrm{He}$ ) had a cylindrical shape (with a diameter $\sim 2.8 \mathrm{~cm}$ ), with entrance and exit windows made of aluminum and a heat shield. A total of four different cryotarget cells have been used during the entire experiment run (see Table 5). While the solid target could be moved using the mechanical handler, the liquid target cell always stayed in the beamline.

\begin{tabular}{|l|l|l|l|l|l|}
\hline Energy & Torus current & ${ }^{3} \mathrm{He}$ & ${ }^{4} \mathrm{He}$ & ${ }^{12} \mathrm{C}$ & ${ }^{56} \mathrm{Fe}$ \\
\hline $1.1 \mathrm{GeV}$ & $750 \mathrm{~A}$ & $18318-18328$ & & $18284-18288$ & \\
\hline & & $18346-18437$ & $18176-18252$ & $18083-18134$ & 18158 \\
$2.2 \mathrm{GeV}$ & $2250 \mathrm{~A}$ & & & $18167-18171$ & $18163-18166$ \\
& & & & & $18258-18259$ \\
\hline & & $18443-18477$ & 17870,17871 & 17872 & 17962 \\
$4.4 \mathrm{GeV}$ & \multirow{2}{*}{$2250 \mathrm{~A}$} & $18520-18521$ & $17881-17886$ & $17901-17905$ & $18040-18044$ \\
& & & $17907-17991$ & $17997-18039$ & \\
& & & & 18061,18062 & \\
\hline
\end{tabular}

TABLE 6: List of E2a runs used in this analysis. 


\subsection{CONTINUOUS ELECTRON BEAM ACCELERATOR FACILITY}

The e2a experiment was carried out at the Thomas Jefferson National Accelerator Facility (TJNAF or JLab), which is located in Newport News, Virgina, USA. JLab is a user facility for scientists from different universities and physics research institutes worldwide.

The primary research at JLab is done using the CEBAF superconducting radio frequency (SRF) electron accelerator, that has been running since the early 1990s. The CEBAF accelerator supplied high quality continuous wave electron beam of up to $5.5 \mathrm{GeV}$ simultaneously to three experimental halls, called A, B and C (another hall named D has been constructed for the $12 \mathrm{GeV}$ upgrade). The schematic view of $6 \mathrm{GeV}$ JLab is shown in Fig. 22. The photo-electrons are produced at the injector by shining a laser on a gallium arsenide cathode. The $1.497 \mathrm{GHz}$ RF wave in the accelerating cavities supplies $499 \mathrm{MHz}$ beam to three halls at the same time. Every third bunch in the beam is supplied to a different hall, so each hall receives electron bunches every 2 ns. The bunches can be loaded with different electron densities to supply different currents to different halls.

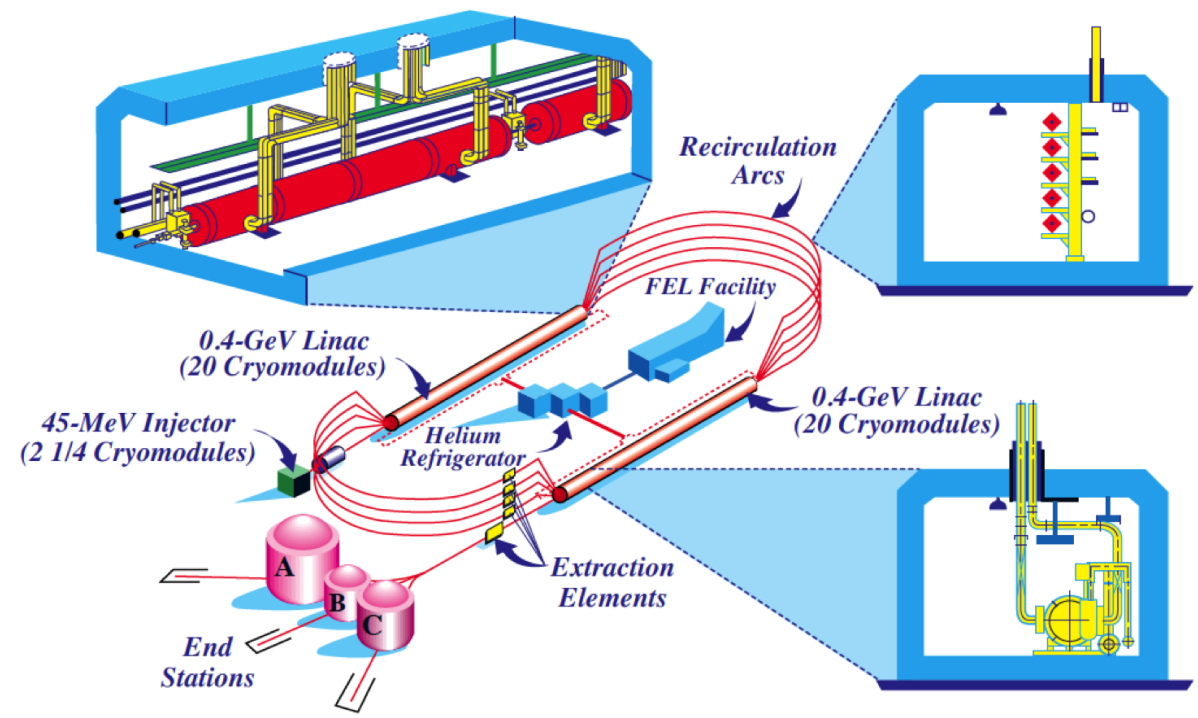

FIG. 22: The schematic view of JLab. The three blow up boxes correspond to: one of the LINAC cryomodules (top left); a cross section of the tunnel with five recirculating arcs (top right), and a cross section of a cryomodule (bottom right).

The electron beam produced at the injector is accelerated through the recirculating beamline, consisting of two parallel linacs that are connected with two $180^{\circ}$ arcs with curvature radii of $80 \mathrm{~m}$ as can be seen in Fig. 22. Each of the linacs accelerates the beam up to $550 \mathrm{MeV}$ after each pass, so the beam acquires an energy of up to $1.1 \mathrm{GeV}$ each time it 
completes a circle. The total energy that can be reached is $5.5 \mathrm{GeV}$ and the energy spread of the beam is $\Delta E / E<10^{-4}$. Each of the linacs consists of 20 cryomodules that each contain eight superconducting niobium cavities that accelerate the electrons. The cryomodules are cooled using liquid Helium at $2 \mathrm{~K}$. There are 2200 magnets used in the accelerator tunnel to keep the beam on track and focused.

After the beam is accelerated to the desired energy, it is directed to the three experimental halls that differ by detectors and physics programs. Hall A contains the left and right High Resolution Spectrometers (HRS) for electron and hadron high precision measurements. The angle between the spectrometers can be adjusted to accommodate the desired kinematics by moving the spectrometers. Hall $\mathrm{C}$ was equipped with two medium resolution magnetic spectrometers, High Momentum Spectrometer (HMS) and Short Orbit Spectrometer (SOS) that cover different momentum ranges. Experimental Hall B used the CEBAF Large Acceptance spectrometer (CLAS) that has " $4 \pi$ " coverage and is great for exclusive studies of many-particle final states. It is described in more detail below.

Hall B operates at lower beam currents (typically from 2-20 nA) than Halls A and C.

\subsection{CEBAF LARGE ACCEPTANCE SPECTROMETER (CLAS)}

The e2a experiment was carried out in JLab experimental Hall B using the CLAS spectrometer. As CLAS has almost full coverage and operates at the luminosities up to $10^{34} \mathrm{~cm}^{-2} \mathrm{sec}^{-1}$. The torus magnet, composed of iron-free super conducting coils arranged around the beam axis, divides CLAS into six independent sectors. The torus magnet is used for measurement of charged particle momenta by producing a toroidal magnetic field with maximum intensity of $2 \mathrm{~T}$. Its structure is nearly $5 \mathrm{~m}$ long and $2.5 \mathrm{~m}$ wide. There is no magnetic field at the target allowing operation with polarized targets.

The mini torus coil is much smaller and is located in the cavity of the main torus. It is used to eliminate low momentum (Moller) background electrons.

Charged particle tracks are reconstructed by the Drift Chambers (DC) to determine the charged particle momenta. The Cherenkov Counters (CC) are used for identification of electrons. The Electromagnetic Calorimeter (EC) and LAC (Large Angel Calorimeter) are used for electron identification and neutral particle detection. The Scintillation Counters (SC) allow charged particle Time Of Flight (TOF) measurements. The Faraday cup located at the end of the Hall B beam line measures the total accumulated beam charge. The 3D view of the CLAS spectrometer, with its components indicated by different colors is shown in Fig. 23. The polar angular coverage for $\mathrm{DC}$ is $8^{\circ}-140^{\circ}, 9^{\circ}-140^{\circ}$ for $\mathrm{SC}, 8^{\circ}-45^{\circ}$ for 


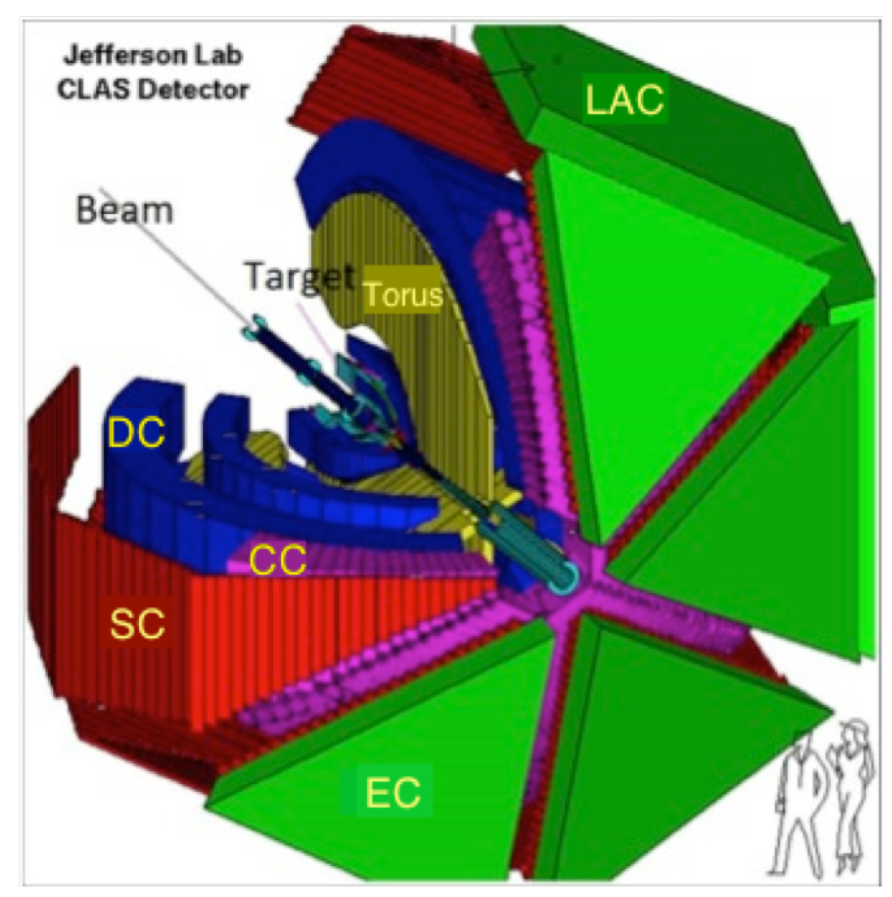

FIG. 23: The 3D view of CLAS.

EC and $45^{\circ}-75^{\circ}$ for LAC, which covers only two sectors of CLAS.

\subsubsection{DRIFT CHAMBERS}

The Drift Chambers (DC) are used for tracking and momentum measurements of charged particles and are described in detail in [51].

The DC modules are filled with a gas that is a 90\%/10\% mixture of Argon and $\mathrm{CO}_{2}$. When charged particles pass through drift chambers, they ionize the gas and the ions drift to cathode wires while the electrons drift to the anode wires, where the corresponding electrical signal is collected and used to determine the the shortest distance from the particle trajectory to the wires. As electrons get closer to the anode wires, the increased electric field causes electron-atom collisions to ionize the atoms. This "avalanch" increases the signal with a gain of $\sim 10^{4}$. The $\mathrm{CO}_{2}$ prevents secondary avalanches from occurring.

There are three radial regions of DC. Regions one and three are in low magnetic field regions (see Fig. 24), while region two is in the high magnetic field region. Each region is composed of two superlayers, each consisting of six layers of hexgonal drift cells as shown in Fig. 25. Only the most inner superlayer in region one has four layers of drift cells. Each hexagonal cell has a sense wire in the middle and field wires at the vertices. The wires in one 


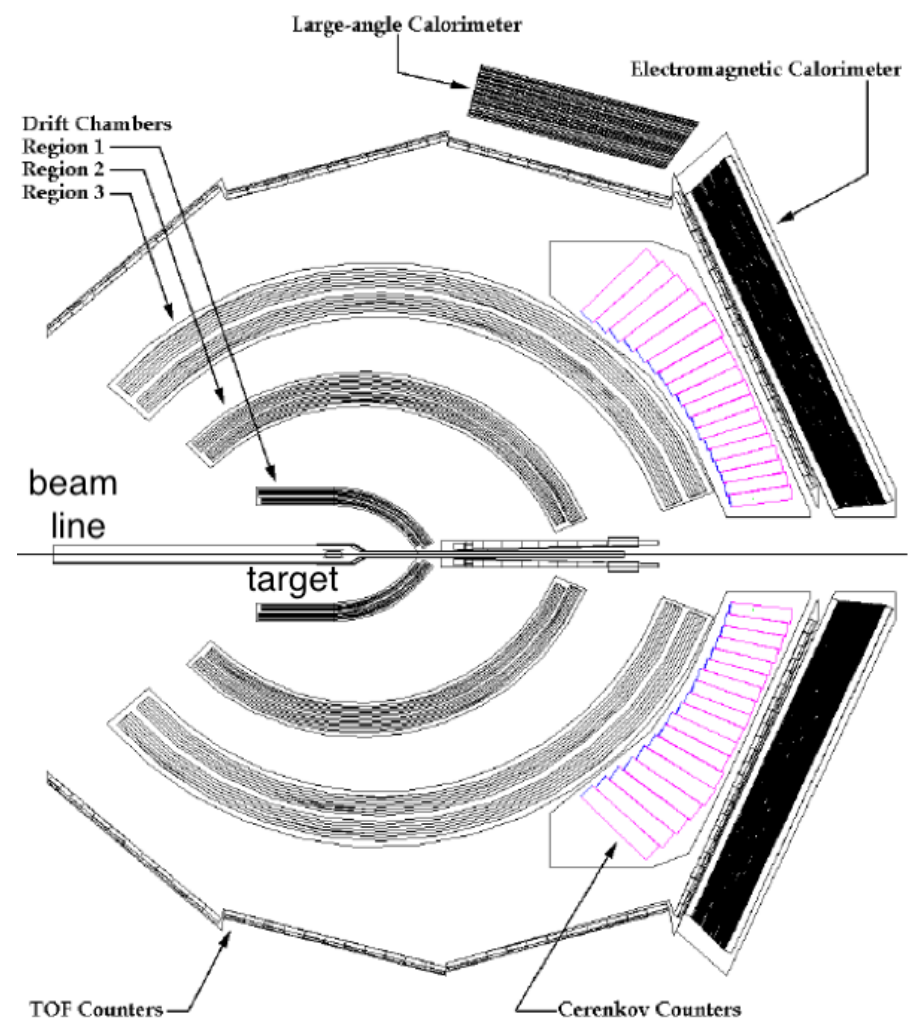

FIG. 24: The schematic view of CLAS.

superlayer of the region are parallel to the magnetic field and compose a $6^{\circ}$ angle with the wires in the other superlayer. The two superlayers allow to determine the azimuthal angle $\phi$ of the charged particle.

The position resolution is $\sim 400 \mu \mathrm{m}$, leading to few mrad for the angles and $\leq 1 \%$ for the reconstructed momentum.

\subsubsection{CHERENKOV COUNTERS}

The Cherenkov Counters (CC) [52] are used to separate electrons from pions and for the electron trigger. There are six counters, one in each sector (see Fig. 26). Each of the six counters is divided into 18 regions in $\theta$ (polar angle), from $\theta=8^{\circ}$ to $45^{\circ}$. Each region is composed of two separate modules, symmetric around the mid plane of the sector, called segments. Thus there are $36 \mathrm{CC}$ segments in each CLAS sector.

The gas used in the $\mathrm{CC}$ is perfluorobutane $\left(\mathrm{C}_{4} \mathrm{~F}_{10}\right)$ with a refractive index of $n=1.00153$, resulting in an energy detection threshold of $9.24 \mathrm{MeV}$ for electrons and $2.51 \mathrm{GeV}$ for pions.

When the particle velocity exceeds the speed of the light in the detector medium $(c / n$, 


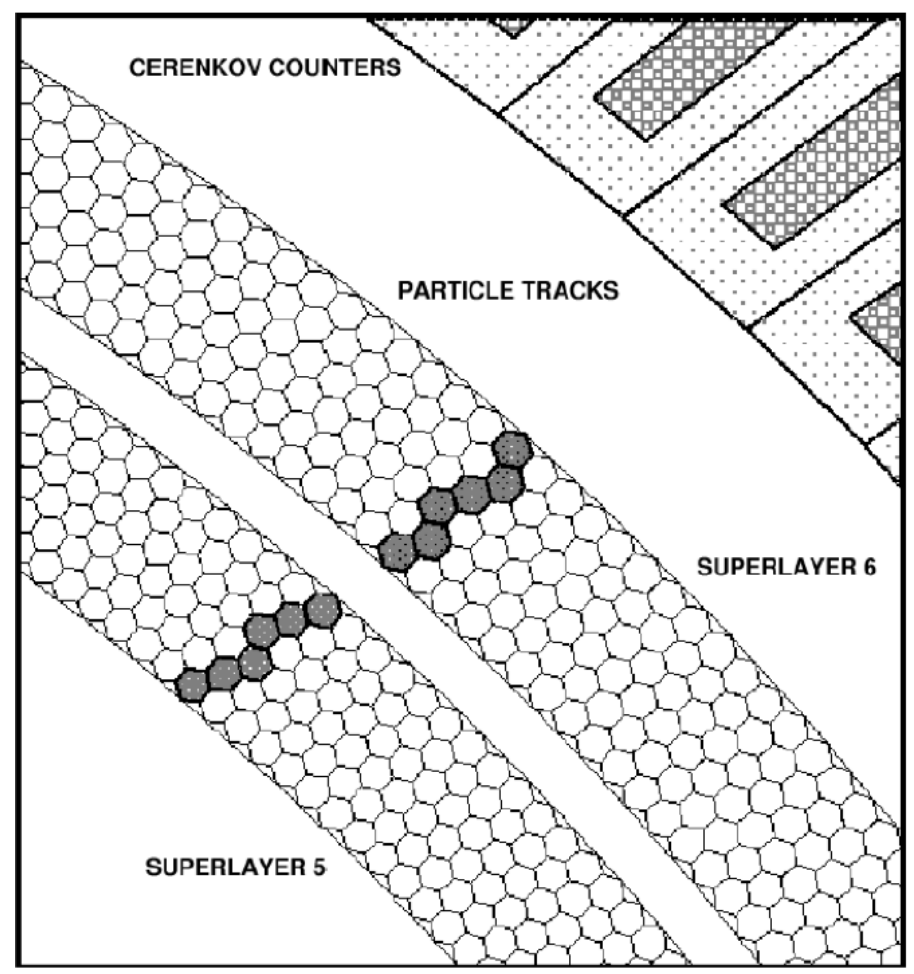

FIG. 25: A schematic view of two DC superlayers. The drift cells appear as hexagons, with the sense wire at the center and and the field wires at the vertices. The shaded cells represent a charged particle track.

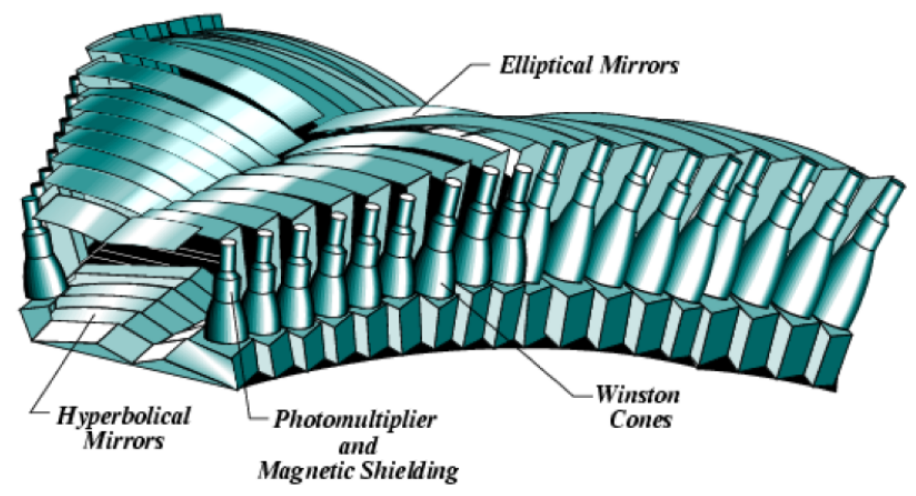

FIG. 26: A 3D view of CLAS Cherenkov Detector.

where $c$ is the speed of the light), it emits Cherenkov light. This light is then directed towards the light collecting Winston cone using elliptical, hyperbolic and cylindrical mirrors where it is focused into the photo-multiplier tube. 


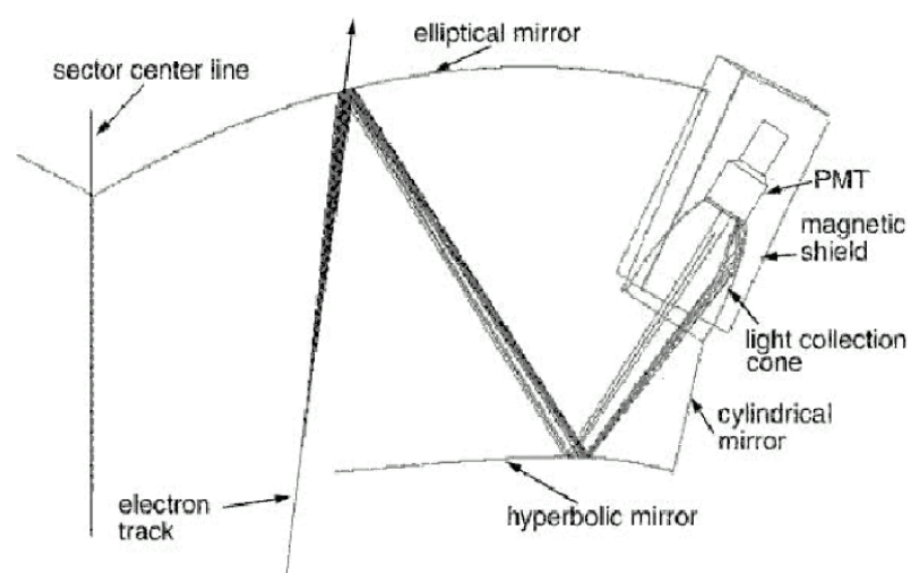

FIG. 27: A schematic view of a Cherenkov Counter segment.

The average signal of the $\mathrm{CC}$ is about 7 photoelectrons and the inefficiency is on the order of $10^{-3}$.

\subsubsection{TIME OF FLIGHT DETECTOR}

The Time Of Flight detectors (TOF) [53] are composed of scintillator "paddles" and are used for particle time of flight measurements. The time of flight from TOF $(t)$ and the tracking information from DC $(d)$ can be used to calculate the charged particle velocity $(v=d / t)$, which combined with the momentum measured from DC allows to identify the particle by calculating its mass:

$$
m=\frac{p \sqrt{1-\beta^{2}}}{\beta}
$$

There are 57 TOF "paddles" (BC-408) in each sector of CLAS. They are perpendicular to the beam direction and each cover about $2^{\circ}$ angular range. They span the angular range from $8^{\circ}$ to $142^{\circ}$ as shown in Fig. 28. The paddles in each sector are mounted in four panels that are combined together. The first 23 paddles that cover the angular range from $8^{\circ}$ to $45^{\circ}$ are called "forward paddles". The length of the paddles varies from $30 \mathrm{~cm}$ to $450 \mathrm{~cm}$, the width $15 \mathrm{~cm}$ or $22 \mathrm{~cm}$, and the thickness is $5.08 \mathrm{~cm}$.

Each of the paddles has a photo-multiplier tube mounted on each end. The last 18 paddles at large scattering angles were combined in pairs, with two scintillators having the same output on each side, forming a total of 48 logical counters per sector.

The average time resolution of the TOF for electrons is $163 \mathrm{ps}$. 


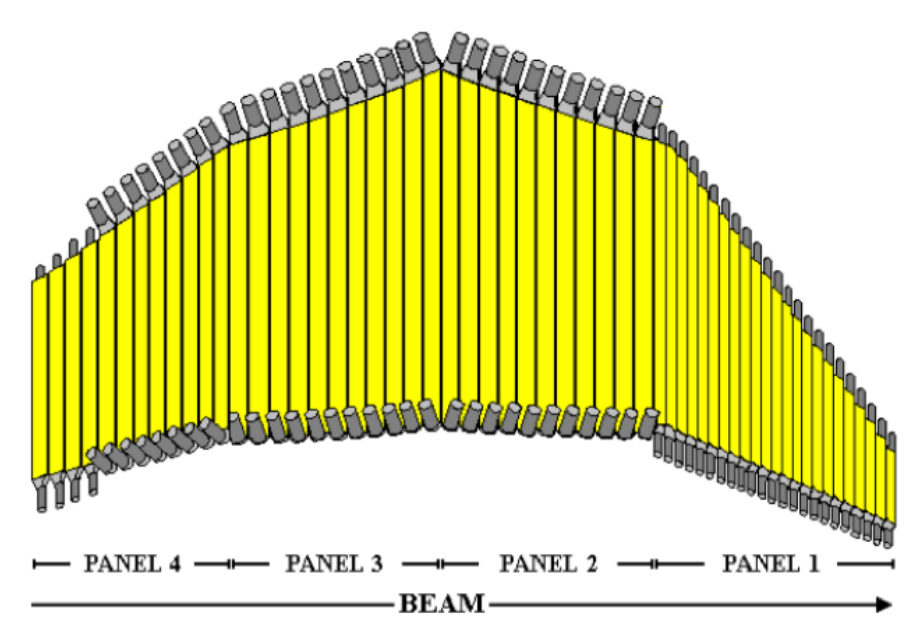

FIG. 28: Time of flight bars in each sector are equipped with four panels of scintillators.

\subsubsection{ELECTROMAGNETIC CALORIMETER}

The forward electromagnetic calorimeter (EC) [54] serves for electron identification and is used to trigger on electrons. It is also used for neutral particle detection with an efficiency of up to $60 \%$.

It has 6 modules, one in each CLAS sector, that each have the form of a triangular truncated pyramid with a projected vertex at the CLAS target point about $5 \mathrm{~m}$ away. This structure minimizes the leakage at the edges of the active volume. Each EC module has a thickness of 16 radiation lengths and contains 39 layers of a $10 \mathrm{~mm}$ thick BC412 scintillator strip followed by a $2.2 \mathrm{~mm}$ thick layer of lead. Each of the layers is triangular. The scintillator strips in each scintillator layer are parallel to one side of the triangle and are rotated by $120^{\circ}$ in each successive layer, providing three different views $u, v$ and $w$ (see Fig. 29) that provide stereo information about energy deposition. There are 13 layers in each view, with 5 layers grouped in the "inner layer" of EC and 8 layers in the "outer layer" to provide longitudinal sampling and improved particle identification. The total thickness of the scintillator material in a single EC module is $39 \mathrm{~cm}$ and the total thickness of the lead is $8.4 \mathrm{~cm}$. This ratio of scintillator to lead material results in around one third of the shower energy being deposited in the scintillators.

The energy resolution of the EC is

$$
\frac{\Delta E}{E}=0.003+\frac{0.093}{\sqrt{E[\mathrm{GeV}]}}
$$

The time resolution for electrons is about 200 ps and is 600 ps for neutrons. 


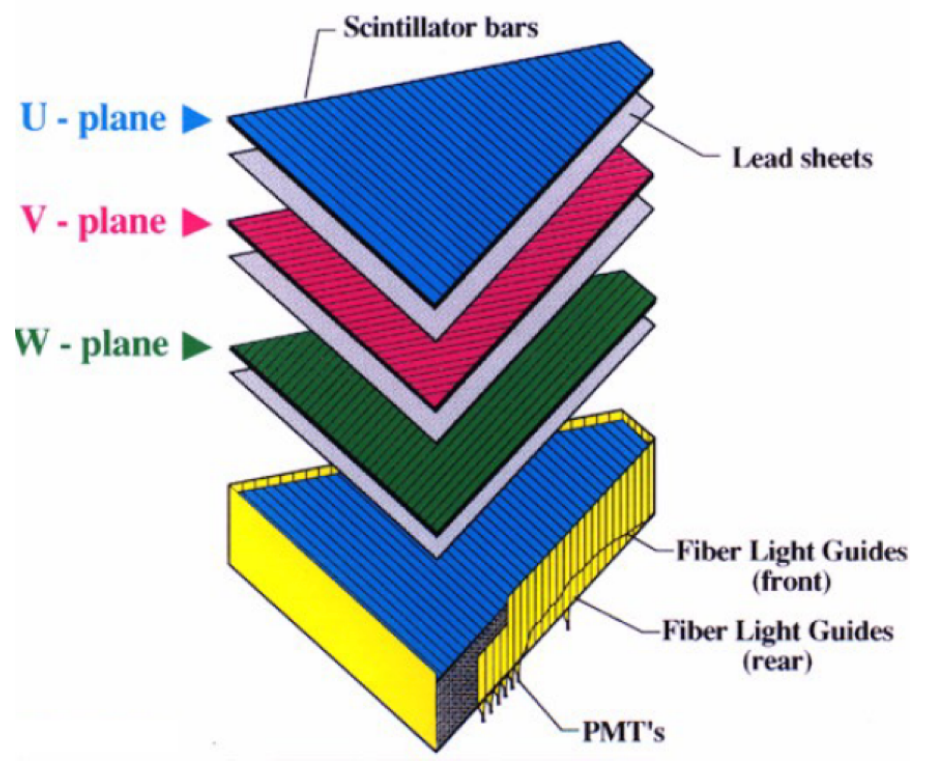

FIG. 29: The view of EC layers in single EC module. 


\section{CHAPTER 3}

\section{DATA PROCESSING}

\section{1 "COOKING"}

The e2a data was originally calibrated and processed in 1999.

We had to reprocess the raw e2a experiment data in order to include particle information from Large Angle Calorimeter (LAC).

To process the e2a experiment data we used the raw data that consists of digitized charge and time measurements recorded by the detector electronics during the experiment, and have converted these signals into particle information using calibration constants for the detector channels.

Originally the calibration constants were saved in "Maps", but after the MySQL databases were created, the existing general calibration constants of $6 \mathrm{GeV}$ CLAS have been copied from "Maps" to the database "calib.RunIndex".

We initially processed a small fraction of the data to study the LAC timing, that is described in detail in [55]. The LAC was found to be well calibrated with the calibration constants from the e1c experiment, run 17748, with a time resolution of about $\sigma_{\Delta t} \sim 0.26 \mathrm{~ns}$ for minimum ionizing particles, which corresponds to a momentum resolution of $\sigma_{\Delta p} \sim 2.2 \%$ for $p=1 \mathrm{GeV}$ and $\sigma_{\Delta p} \sim 4.5 \%$ for $p=1.5 \mathrm{GeV}$.

A new database "calib_user.RunIndexe2a" was created based on the existing database "calib.RunIndex" with the LAC calibration constants taken from run 17748. The e2a experiment data was then reprocessed using this new database.

We then discovered that the Time Of Flight detectors were not properly calibrated, and required recalibration. That is described in Section 3.2.

After we calibrated the TOF, we performed "pass2" processing of the e2a data. During data processing, particle trajectories and particle types are determined using the calibration constants from the database. We included all the good runs from the e2a run list that can be found via the following link https://userweb.jlab.org/ stepanya/e2/e2GoodRuns. list. I created a directory called "cooking" in the "clase2" home directory on the JLab machines, where I processed the data using the CLAS reconstruction program "user_ana" located in 
/e2_pass2/bin/LinuxRHEL6/user_ana. The e2_pass2.tcl steering file for user_ana is also located in the cooking directory. I used a shell script to generate .xml files for each of the raw data files to be submitted as a single job on the JLab batch farm. We saved the processed data in CLAS standard BOS, Root Tree and PAW Ntuple structure formats on JLab mss tapes (/mss/clas/e2a/production/pass2/v1/).

\subsection{RE-CALIBRATION OF TOF TIMING}

We studied LAC timing by looking at the LAC-TOF time difference for charged pions (the difference between the measured LAC and TOF arrival times and the expected travel time from TOF to LAC). We noticed that, depending on the TOF paddle, the resulting Gaussian distribution was centered at a different location. This indicated a TOF time mis-calibration.
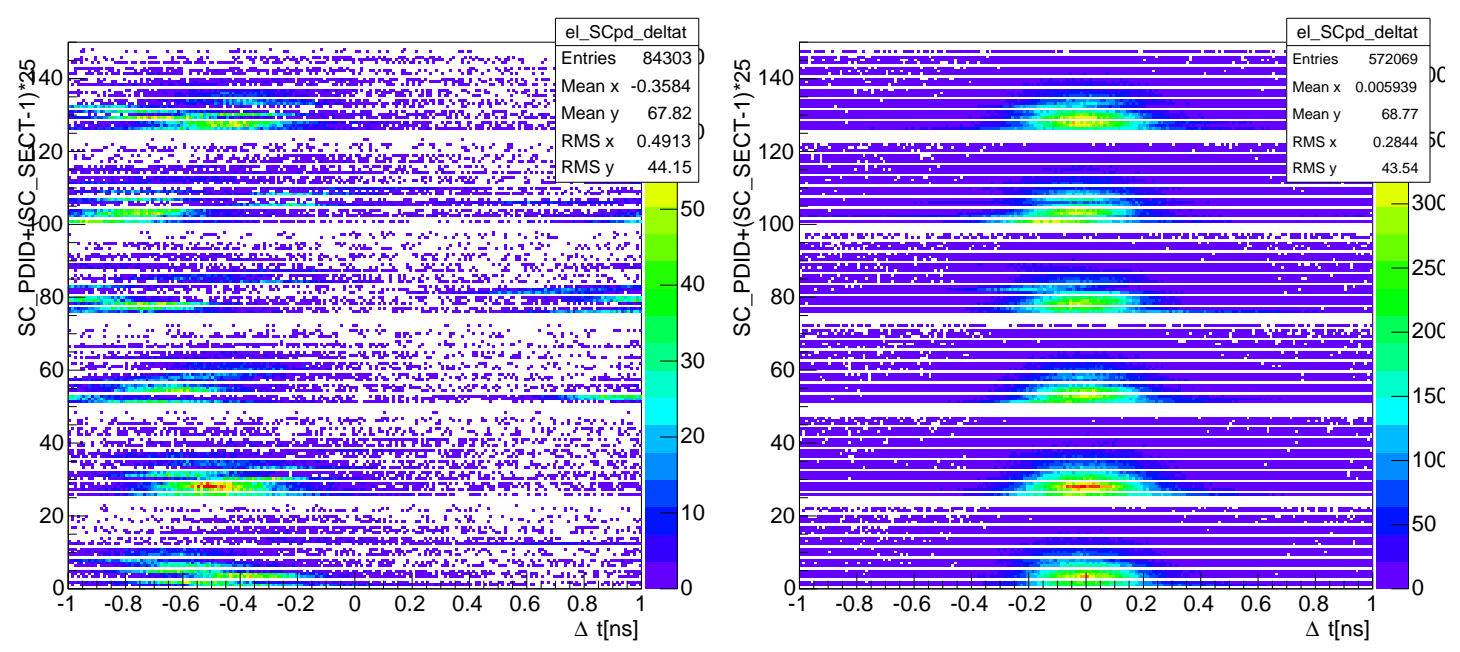

FIG. 30: The timing of forward paddles with respect to the RF time for electrons detected in the forward TOF paddles of different sectors, before (left) and after correction (right).

We used electrons to align the timing of the 22 forward TOF paddles. We then used an electron detected in one of the forward paddles with corrected timing in conjunction with a $\pi^{+}$from the same event detected in a paddle at larger angle in order to correct the timing of the larger angle paddles. We used positive pions as they are out bending and therefore reach the TOF paddles at larger scattering angles. We did this for each of the 6 sectors.

In order to correct the timing of the forward 22 TOF paddles, we looked at the $\Delta t$ difference between the $e^{-}$time from TOF $\left(t_{\mathrm{TOF}}^{e^{-}}\right)$, corrected for path length $d_{\mathrm{TOF}}^{e^{-}}$, the RF time $\left(t_{\mathrm{RF}}\right)$ and vertex $v_{Z}^{e^{-}}$, that is the distance between interaction vertex and z-point where 
the offset for $t_{\mathrm{RF}}$ was calibrated:

$$
\Delta t=t_{\mathrm{TOF}}^{e^{-}}-d_{\mathrm{TOF}}^{e^{-}} / c-v_{Z}^{e^{-}} / c-t_{\mathrm{RF}}
$$

here $c$ is the speed of the light and $v_{Z}^{e^{-}} / c$ is the $e^{-}$travel time inside the target before interaction.

The RF signal is delivered at constant frequency, and the time difference between two $\mathrm{RF}$ signals is a multiple of $R F_{\text {Structure }}=2.004 \mathrm{~ns}$, the time interval between two neighboring $e^{-}$beam bunches delivered to the target. We calculate $\Delta t$ for $e^{-}$detected in the same TOF paddle coming from different events (beam bunches). We then calculate the modulo of $\Delta t$ of $R F_{\text {Structure }}$. The histogram of these results for electrons detected in the same TOF paddle is a Gaussian distribution, and the mean is the offset to be used to align the timing of a particular forward TOF paddle with respect to the RF time (see Fig. 30). After correcting the timing of all forward TOF paddles with these offsets, the timing of different paddles differs only by multiples of $R F_{\text {Structure }}$.

After correcting the timing of the $e^{-}$and $\pi^{+}$particles from the same event detected in forward paddles with these offset factors $\left(\Delta t_{\text {corr }}\right)$, we have calculated the following time difference:

$$
\Delta t_{\text {Diff. }}=\left[\left(t_{\mathrm{TOF}}^{\pi^{+}}-\Delta t_{\mathrm{corr}}^{\pi^{+}}\right)-d_{\mathrm{TOF}}^{\pi^{+}} / v\right]-\left[\left(t_{\mathrm{TOF}}^{e^{-}}-\Delta t_{\mathrm{corr}}^{e^{-}}\right)-d_{\mathrm{TOF}}^{e^{-}} / c\right]
$$

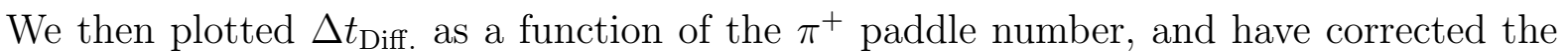
timing of the forward paddles from 1 to 22 , that correspond to $\Delta t_{\text {Diff. distributions that have }}$ their means shifted from 0 by multiples of $R F_{\text {Structure }}=2.004 \mathrm{~ns}$. We have then added these additional offsets to the ones obtained by electrons for each of the forward paddles to get the final timing correction for a given forward paddle.

Using the final corrections for forward paddles we again calculated $\Delta t_{\text {Diff. for positive }}$ pions detected in TOF logical paddles from 23-48. The $\Delta t_{\text {Diff. distributions for these paddles }}$ again describe Gaussian distributions, and the corresponding mean values are the corrections to be applied on the timing of these TOF paddles at larger scattering angles (see Fig. 31).

The final corrections for forward TOF paddles are those obtained with $e^{-}$and the ones for paddles from 23-48 are the ones obtained with $\pi^{+}$. 

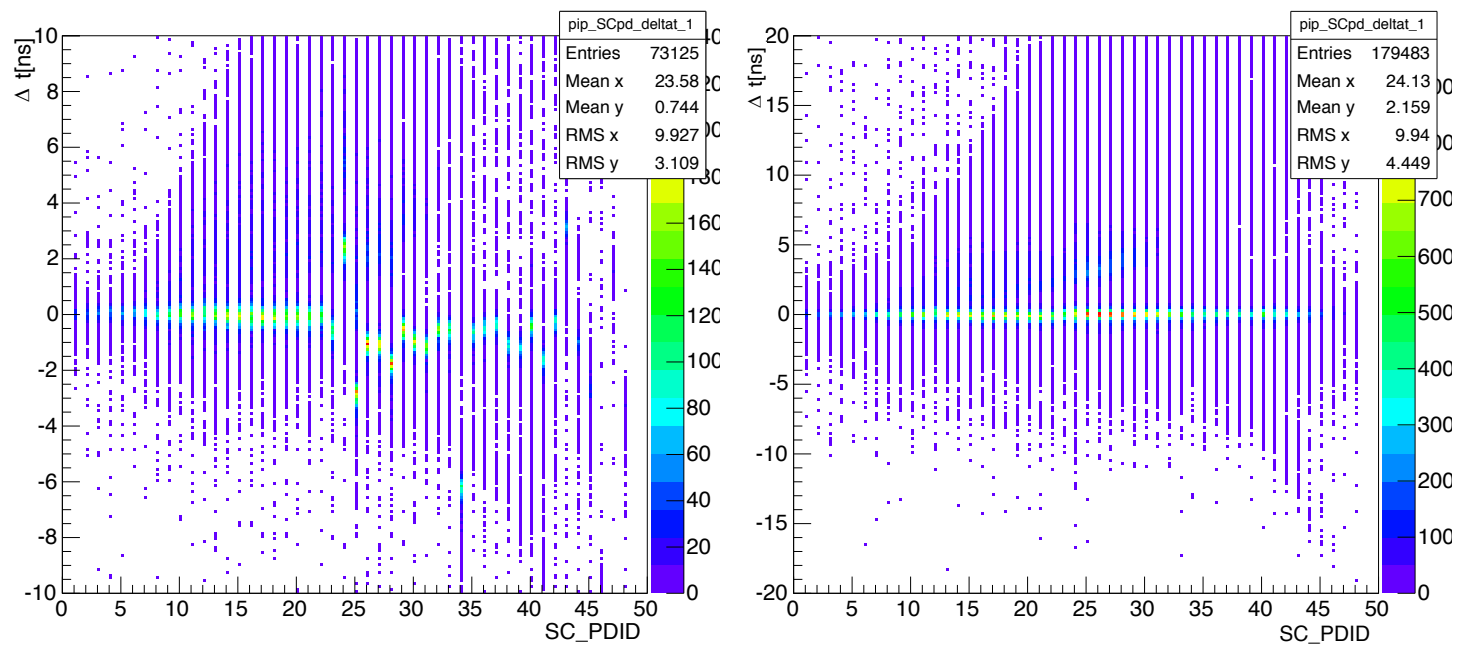

FIG. 31: The $\pi^{+}$and $e^{-}$TOF time difference plotted versus $\pi^{+}$TOF paddle number with $e^{-}$detected in forward TOF paddles, before (left) and after (right) correction. 


\section{CHAPTER 4}

\section{PARTICLE IDENTIFICATION}

This data was previously analyzed and published for other purposes. This analysis uses many of the previously approved cuts and corrections.

\subsection{ELECTRON IDENTIFICATION}

\subsection{GEV ANALYSIS}

The triggering particle used in the event reconstruction in CLAS electro-production data analysis is the electron, so it is crucial to have an accurate electron identification. This identification is done in few steps, by suppressing the charged pion contribution.

We are using the algorithm from [56] for electron identification that was already reviewed and approved and added extra cuts on $\mathrm{CC}$ variables.

In order to record the event in the initial data processing it was required that the event has at least one negatively charged track in the DC with a corresponding shower in the EC and a hit in the SC (Scintillator Counter) that geometrically matched the one in the EC.

This initial electron selection is very loose and further electron selection cuts need to be applied for proper identification.

We cut $3 \sigma$ around the electron band of the ratio of total energy deposited in the EC divided by momentum plotted as a function of momentum, to select events with $E_{\text {tot }} / p$ about the sampling fraction $(\sim 0.27)$, and to suppress the contribution of the accidental background, see Fig. 32. We take the total energy deposited in the entire EC or the sum of the total energies deposited in the inner and outer layers of the EC, whichever is larger.

The parameters of the fit to the sampling fraction band were stored as a function of electron momentum, and for each electron candidate the corresponding lower and upper limits of $E_{\text {tot }} / p$ were obtained which determined the acceptable region.

We also required that the energy deposited in the inner layer of the EC, $E_{\text {inner }}>55 \mathrm{MeV}$. This cut helps to separate electrons from minimum ionizing particles such as pions. Unlike the electrons, which shower inside the EC and thus deposit all their energy, pions deposit 


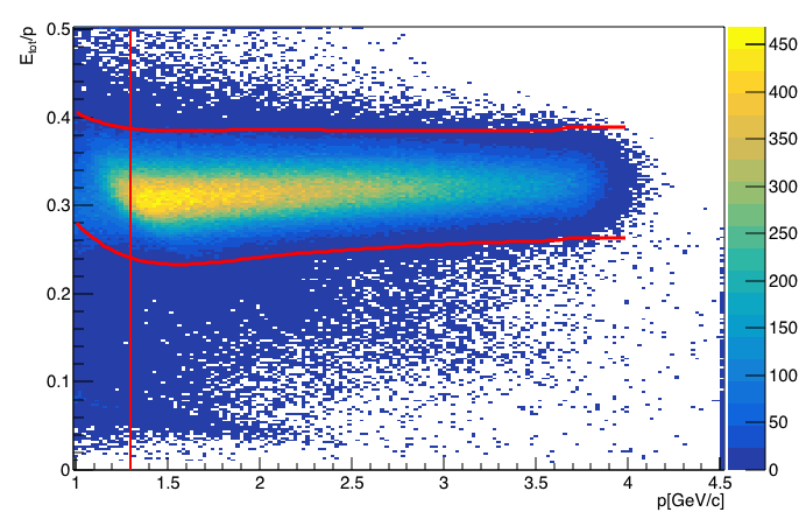

(a) ${ }^{3} \mathrm{He}$

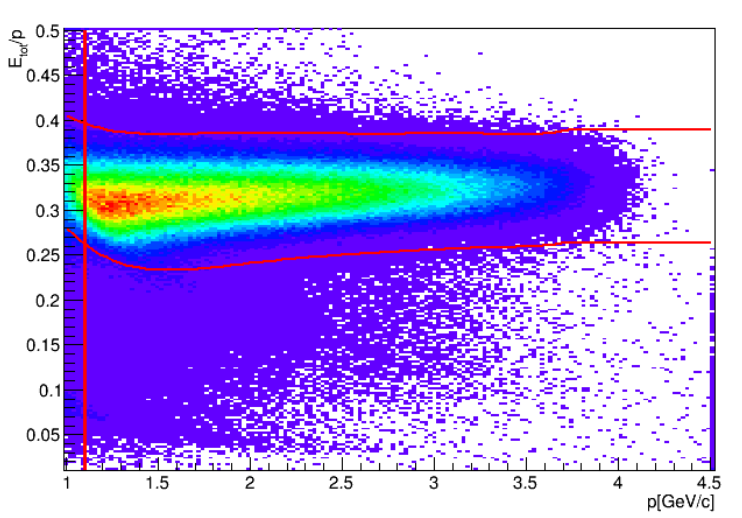

(b) ${ }^{56} \mathrm{Fe}$

FIG. 32: The ratio of the total energy deposited in the calorimeter over the momentum for the electron candidates. The vertical line corresponds to the cut on momentum and the two lines show the $3 \sigma$ cut around the electron band. The plot is made for the ${ }^{3} \mathrm{He}$ and ${ }^{56} \mathrm{Fe}$ targets at $4.4 \mathrm{GeV}$ after fiducial cuts were applied and requiring an associated $\mathrm{CC}$ signal.

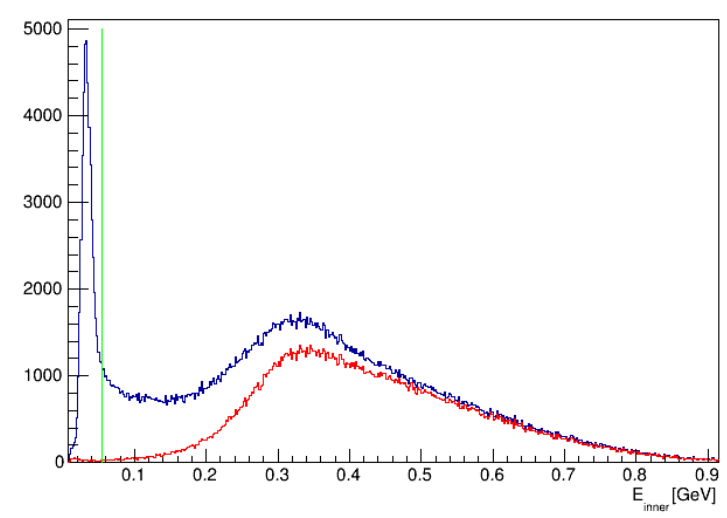

FIG. 33: The energy deposited in the inner layer of the calorimeter with a cut (green line) for the electron candidates. The results are shown for the ${ }^{3} \mathrm{He}$ target at $4.4 \mathrm{GeV}$, after fiducial cuts (blue) and after the other electron PID cuts and fiducial cuts (red).

the same energy in each scintillator layer of the EC. The $E_{\text {inner }}$ distributions after fiducial cuts only and after all other electron PID cuts in addition to fiducial cuts are shown in Fig. 33. Fiducial cuts select regions where the CLAS detection efficiency is constant, see section 


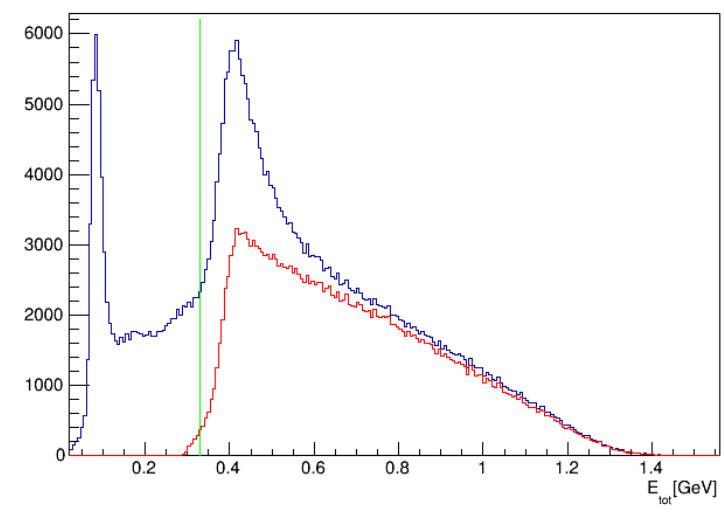

FIG. 34: The total energy deposited in the calorimeter for the electron candidates. The green line corresponds to the applied cut. The results are shown for the ${ }^{3} \mathrm{He}$ target at $4.4 \mathrm{GeV}$, after fiducial cuts (blue) and after the other electron PID cuts and fiducial cuts (red).

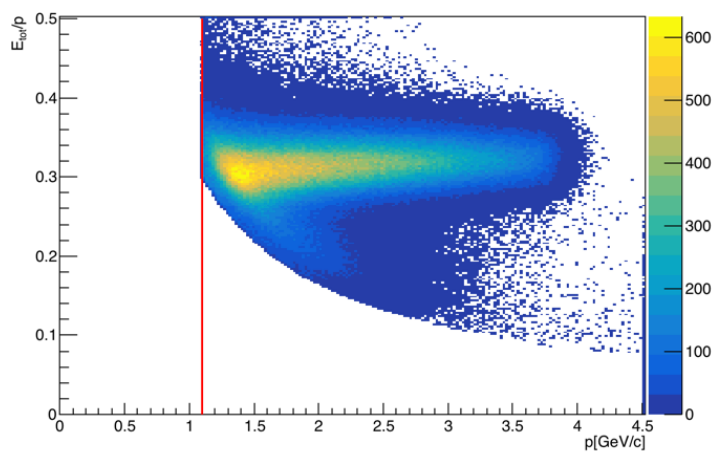

(a) Without CC cut

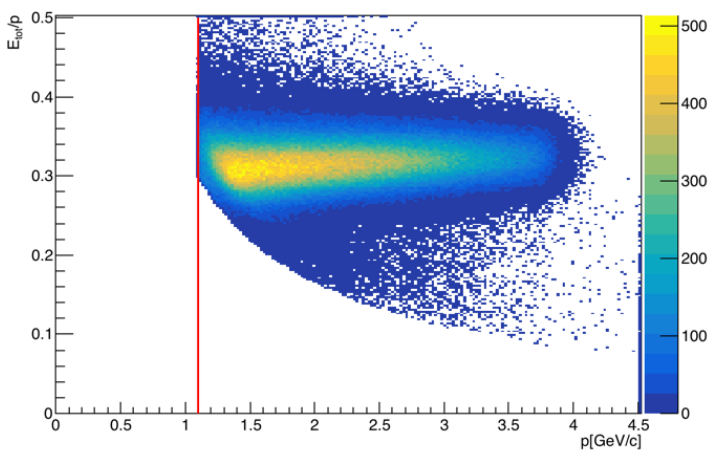

(b) With CC cut

FIG. 35: The ratio of the total energy deposited in the calorimeter over the momentum for the electron candidates for ${ }^{3} \mathrm{He}$ at $4.4 \mathrm{GeV}$. The cuts applied on the plots on the left side are fiducial cuts and all electron PID cuts except $3 \sigma$ cut around the electron band and the cut requiring there to be an associated signal in CC. The plot on the right side has the same cuts and an additional cut requiring to have an associated signal in CC.

4.7. The vertical green line shows the applied cut. The pion peak at $E_{\text {inner }} \sim 0.03 \mathrm{GeV}$ was fit with a Gaussian and the applied cut is about $3 \sigma$ away from the pion peak.

We also cut on the total energy deposited by an electron candidate to match the EC trigger threshold. We cut on $E_{\text {tot }}>0.33 \mathrm{GeV}$ and electron candidate momentum $p>1.1 \mathrm{GeV}$, 


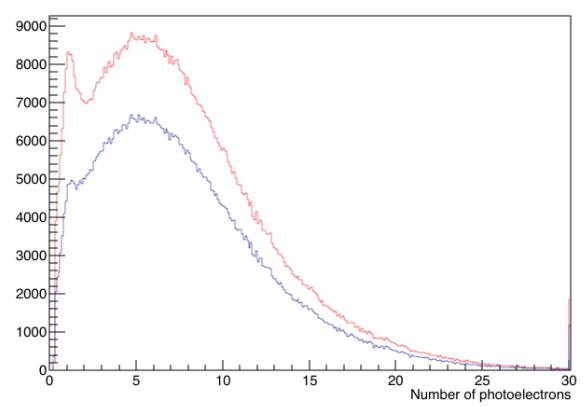

(a) ${ }^{3} \mathrm{He}$

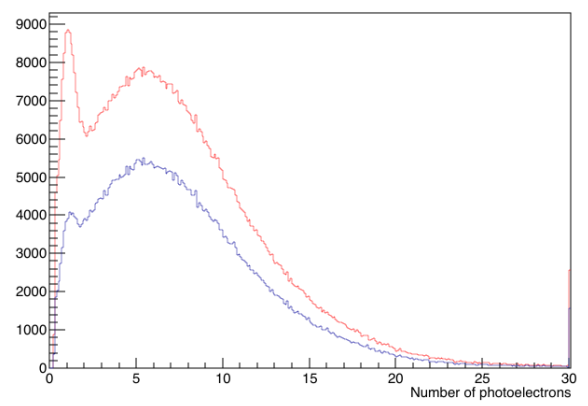

(c) ${ }^{12} \mathrm{C}$

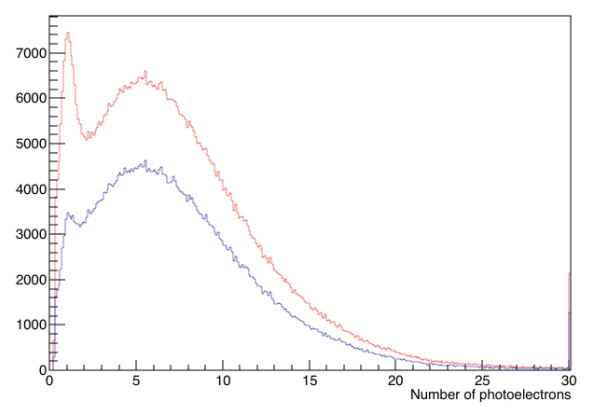

(b) ${ }^{4} \mathrm{He}$

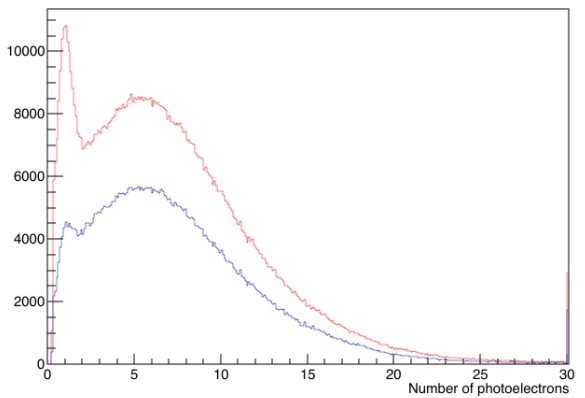

(d) ${ }^{56} \mathrm{Fe}$

FIG. 36: The distribution of the number of photo-electrons detected in the CC before electron PID and vertex cuts (red) and after applying all PID and vertex cuts (blue). The results are shown for all targets at $4.4 \mathrm{GeV}$ after fiducial cuts were applied.

identical to those in the previous CLAS approved analysis [56]. The $E_{\text {tot }}$ distributions after fiducial cuts and after the rest of the elecron PID cuts in addition to fiducial cuts are shown in Fig. 34. The green line shows the applied cut, there is a cut on the energy deposited in the inner layer to be greater than $55 \mathrm{MeV}$. The EC threshold was set higher for other targets compared to ${ }^{3} \mathrm{He}$. However the applied cut $E_{\text {tot }}>0.33 \mathrm{GeV}$ seems to work fine for all targets and it has been used in the previews approved analysis of e2a data so we chose to use the same value.

We initially did not apply cuts on any parameters measured by Cerenkov Counters (CC) in the $4 \mathrm{GeV}$ analysis based on [56]. We only required that the electron candidate had an associated signal in the CC. To eliminate a tail in the $E_{\text {tot }} / p$ vs $p$ plot at low momenta due to pion contamination in the EC as can be seen in Fig. 35.

The plots of the number of photo-electrons detected in the $\mathrm{CC}$ after the fiducial cuts, 


\begin{tabular}{lc} 
Sector & $\Delta t_{\mathrm{CC}}$ \\
\hline 1 & $-2 \mathrm{~ns}$ \\
2 & $-5 \mathrm{~ns}$ \\
3 & $-8 \mathrm{~ns}$ \\
4 & $-8 \mathrm{~ns}$ \\
5 & $-2 \mathrm{~ns}$ \\
6 & $2 \mathrm{~ns}$
\end{tabular}

TABLE 7: Cut values on the time difference between hits of the electron candidate in CC and SC, corrected for the distance traveled from CC to SC for different sectors of CLAS.

for electron candidates that have an associated signal in EC, SC and CC, before and after above listed PID cuts and electron vertex cuts are shown in Fig. 36. These plots suggest that after all the above mentioned electron PID cuts, there is still some hint of a pion peak at around one photo-electrons.

In order to further suppress the pion contamination, we have applied matching cuts between the hits of an electron candidate in the SC and CC following the electron identification procedure done in [57]. These cuts are new for e2a. We required that the time difference between hits of the electron candidate in the $\mathrm{CC}$ and SC, corrected for the distance traveled from $\mathrm{CC}$ to $\mathrm{SC}\left(\Delta t_{\mathrm{CC}}\right)$, was greater than a certain value.

$$
\Delta t_{\mathrm{CC}}=t_{\mathrm{CC}}-t_{\mathrm{SC}}-\frac{\left(d_{\mathrm{CC}}-d_{\mathrm{SC}}\right)}{c}
$$

The expression of $\Delta t_{\mathrm{CC}}$ is given in Eq. 47, where $t_{\mathrm{CC}}$ and $t_{\mathrm{SC}}$ are the times of electron candidate from SC and CC correspondingly, $d_{\mathrm{CC}}$ and $d_{\mathrm{SC}}$ are the path lengths of the electron candidate from production vertex to $\mathrm{CC}$ and SC. The cut value was determined for each sector separately, see Fig. 37. The multi-peak structure is due to the time offset between the photo-multipliers in CC segments. The corresponding cut values are shown in Table 7. We only cut the left tail of these distributions, as the right tail that is almost the same for red and blue distributions is probably due to the light doing multiple bounces inside the CC segment before getting detected.

The plots of the angle between a $\mathrm{CC}$ hit and the nearest SC hit with the corresponding cuts are shown in Fig. 38. The distribution of number of photo-electrons (Nphe) after different cuts on electron candidates is shown in Fig. 39. The red distribution is after the 
electron PID cuts on above mentioned EC variables with a requirement that there was an associated signal in $\mathrm{CC}$, while the green distribution also has cuts on $\Delta t_{\mathrm{CC}}$ and the angle between $\mathrm{CC}$ and $\mathrm{SC}$ hits. The purple line in addition to these cuts has a cut that removes the right tail in $\Delta t_{\mathrm{CC}}$ distributions shown in Fig. 37. It is clear that the last cut does not reduce the pion contamination and cuts away significant amounts of good electron candidates, so we should not use it. The cuts on $\Delta t_{\mathrm{CC}}$ and angle between $\mathrm{CC}$ and SC hits on the other hand suppress the pion peak leaving the rest of the distribution relatively unaffected.

\subsection{GEV ANALYSIS}

For the $2.2 \mathrm{GeV}$ analysis we have applied electron PID cuts similar to those at $4.4 \mathrm{GeV}$. The cut on CC is very helpful in separating electrons from pions, as at this energy all of the charged pions have their momentum below the $2.8 \mathrm{GeV} \mathrm{CC}$ detection threshold. We use the same two $\mathrm{CC}$ cuts at $2.2 \mathrm{GeV}$, the cut on the time difference between hits of the electron candidate in $\mathrm{CC}$ and $\mathrm{SC}$, corrected for the distance traveled from $\mathrm{CC}$ to $\mathrm{SC}$ for different sectors of CLAS, and the cut on the angle between a $\mathrm{CC}$ hit and the nearest $\mathrm{SC}$ hit of the electron candidates.

For the calorimeter, similar to the $4.461 \mathrm{GeV}$ analysis, we have applied a $3 \sigma$ cut around the electron band in the plot of the ratio of total energy deposited in the EC divided by momentum plotted as a function of momentum. The distribution with the corresponding cut is shown in Fig. 40. 


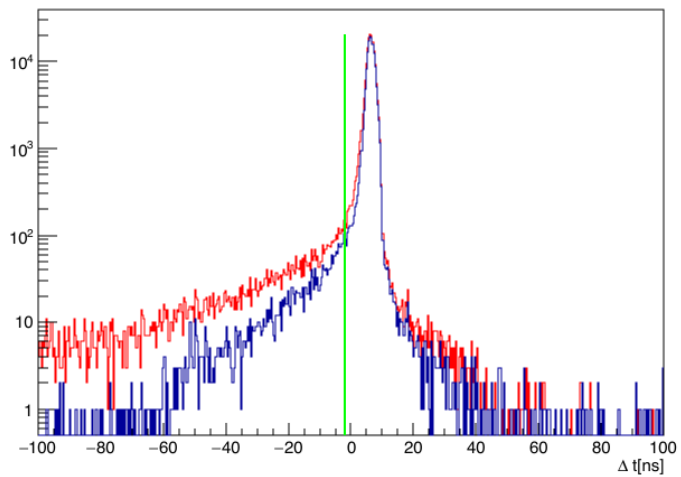

(a) Sector one.

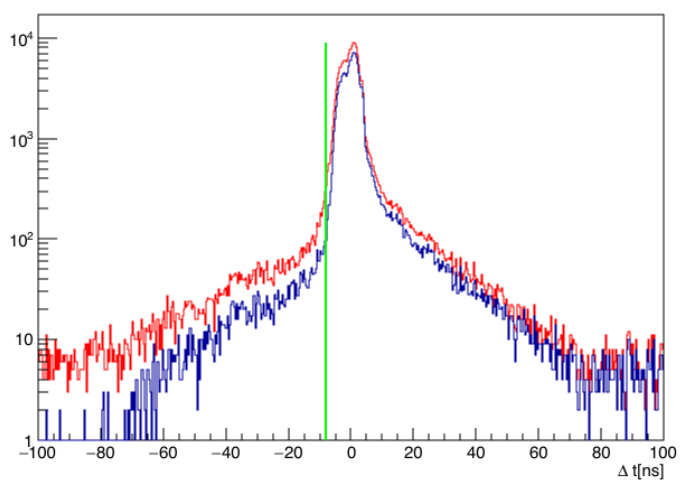

(c) Sector three.

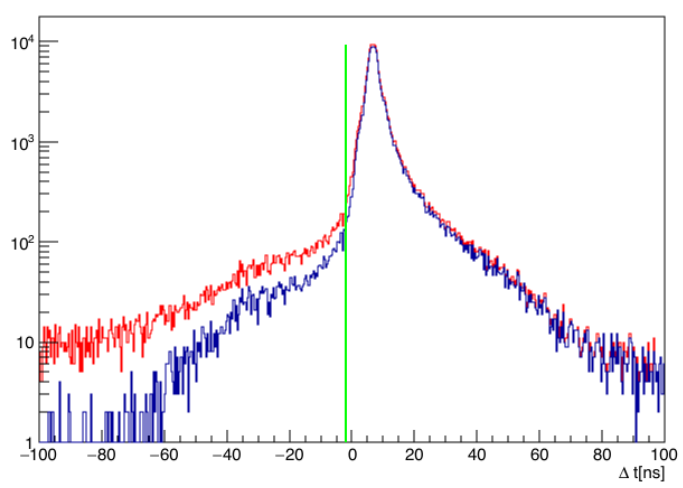

(e) Sector five.

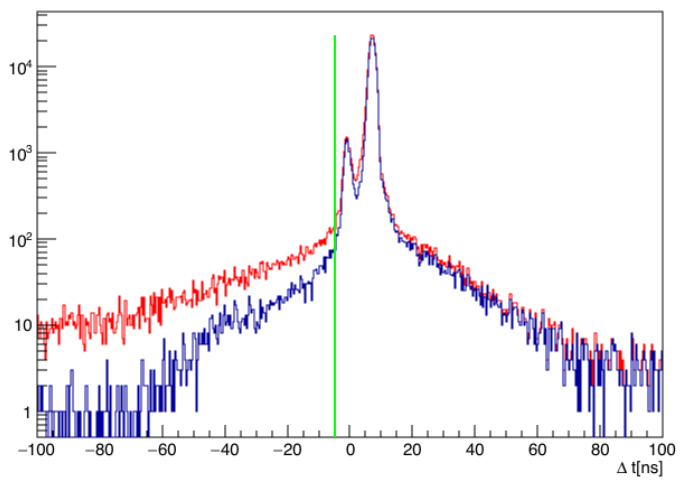

(b) Sector two.

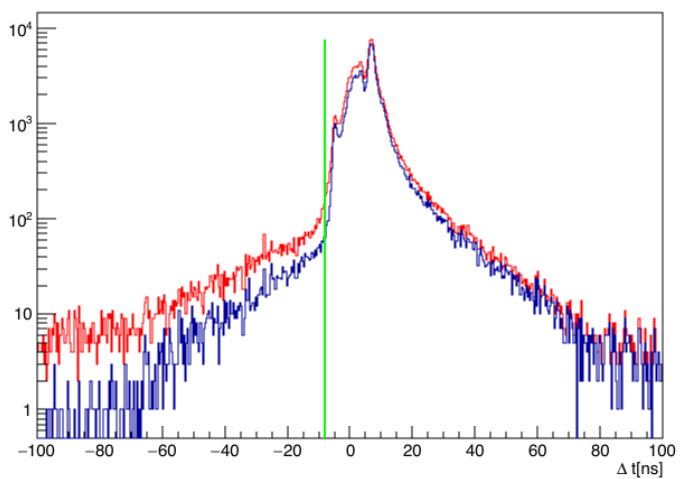

(d) Sector four.

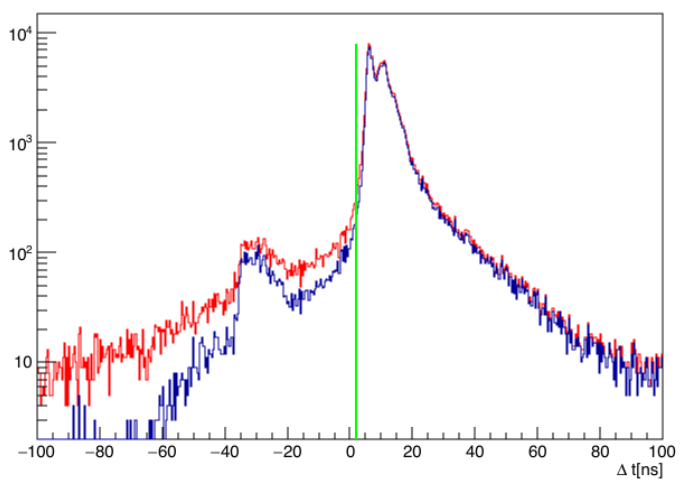

(f) Sector six.

FIG. 37: The time difference between hits of the electron candidate in CC and SC, corrected for the distance traveled from CC to SC before (red) and after (blue) a pion rejection cut (Nphe $>2.5$ at the CC). The green lines show the applied cut values. The results are shown for negative particles with associated signal in SC, EC and CC, for the ${ }^{3}$ He target at $4.4 \mathrm{GeV}$ after fiducial cuts were applied. 


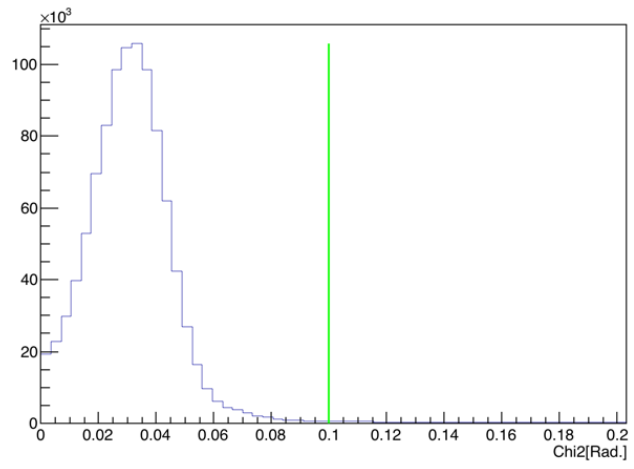

(a) ${ }^{3} \mathrm{He}$.

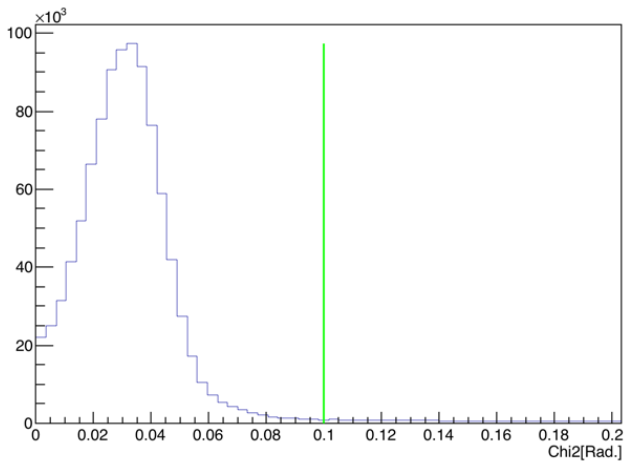

(b) ${ }^{56} \mathrm{Fe}$.

FIG. 38: The distribution of the angle between a CC hit and the nearest SC hit for negative particles with associated signal in SC, EC and CC. The green line corresponds to the applied cut value. The results are shown for ${ }^{3} \mathrm{He}$ and ${ }^{56} \mathrm{Fe}$ targets at $4.4 \mathrm{GeV}$ after fiducial cuts were applied.

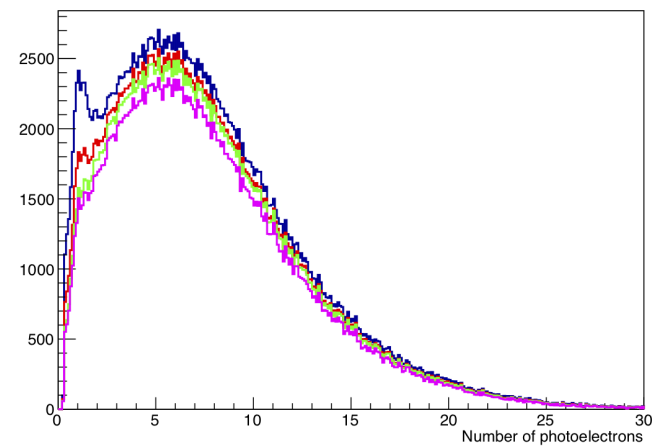

(a) ${ }^{3} \mathrm{He}$.

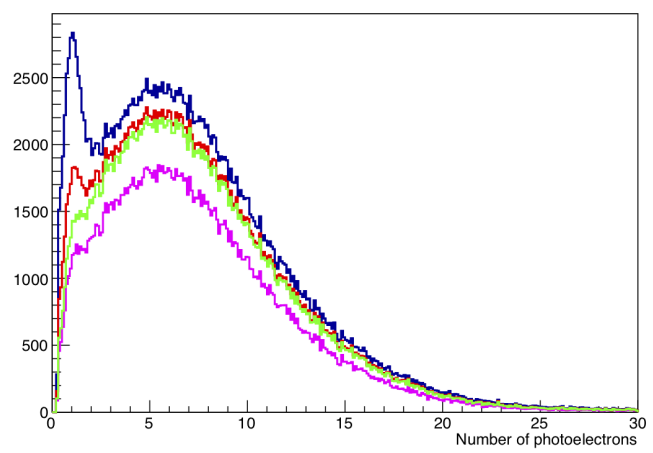

(b) ${ }^{56} \mathrm{Fe}$.

FIG. 39: The distribution of the number of photo-electrons detected in the CC for negative particles with associated SC,CC and EC signals before electron PID cuts (blue), after EC cuts (red), and after EC, $\Delta t_{\mathrm{CC}}$ and $\mathrm{CC}$ angle cuts (green). The result of also cutting the right-side-tail of the $\Delta t_{\mathrm{CC}}$ distributions shown in Fig. 37 (purple). The last cut removes good electrons without improving pion rejection, so we do not use it. The results are shown for ${ }^{3} \mathrm{He}$ and ${ }^{56} \mathrm{Fe}$ targets at $4.4 \mathrm{GeV}$, after fiducial cuts and electron vertex cuts were applied. 


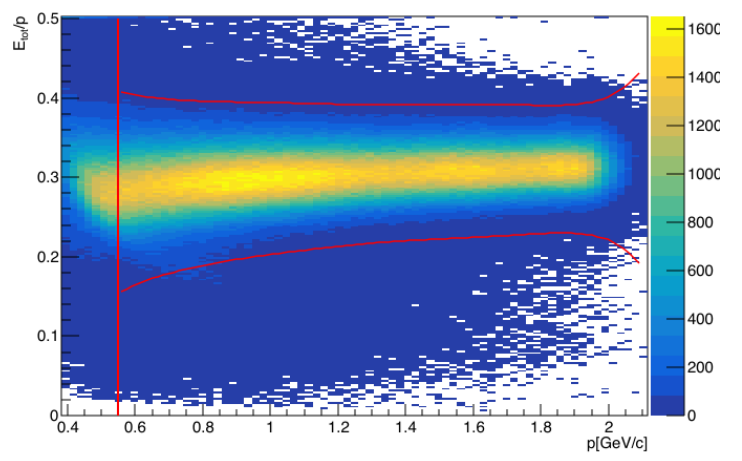

(a) ${ }^{3} \mathrm{He}$

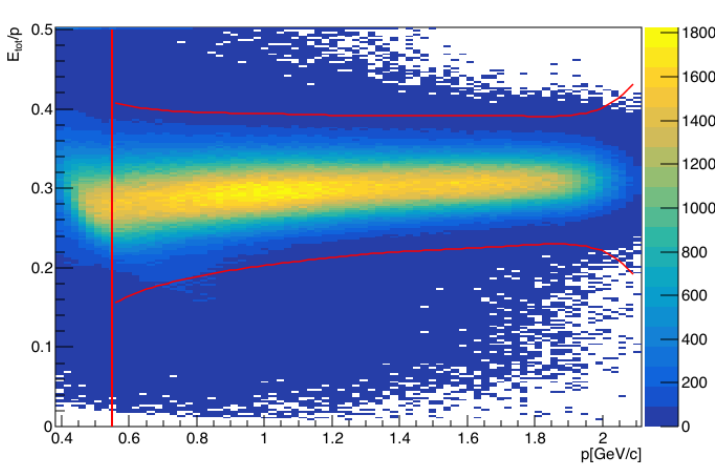

(b) ${ }^{56} \mathrm{Fe}$

FIG. 40: The ratio of the total energy deposited in the calorimeter over the momentum for the electron candidates. The vertical line shows the cut on momentum and the other two lines show the $3 \sigma$ cut around the electron band. The results are shown at $2.2 \mathrm{GeV}$, by looking at negative particles with EC, CC and SC status, after fiducial cuts were applied.

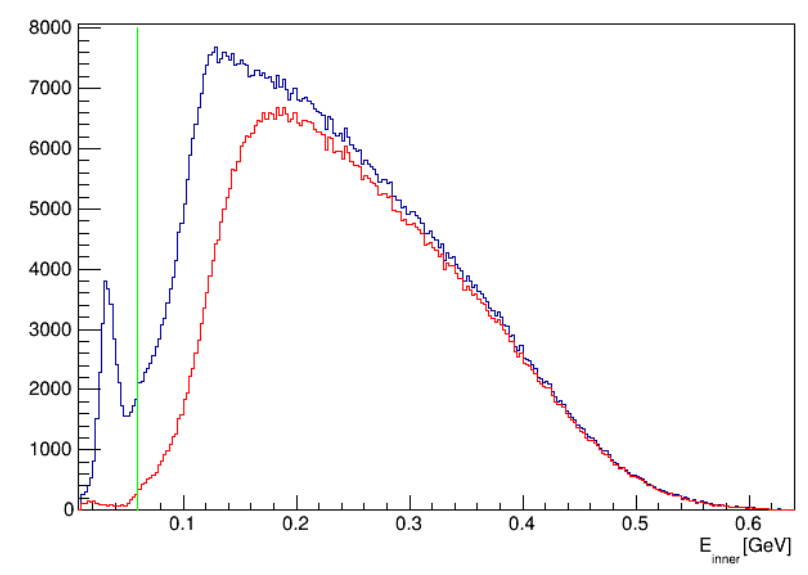

FIG. 41: The energy deposited in the inner layer of calorimeter with the cut (green line) for the electron candidates. The results are shown for the ${ }^{3} \mathrm{He}$ target at $2.2 \mathrm{GeV}$, after fiducial cuts (blue) and after the other electron PID cuts and fiducial cuts (red). 


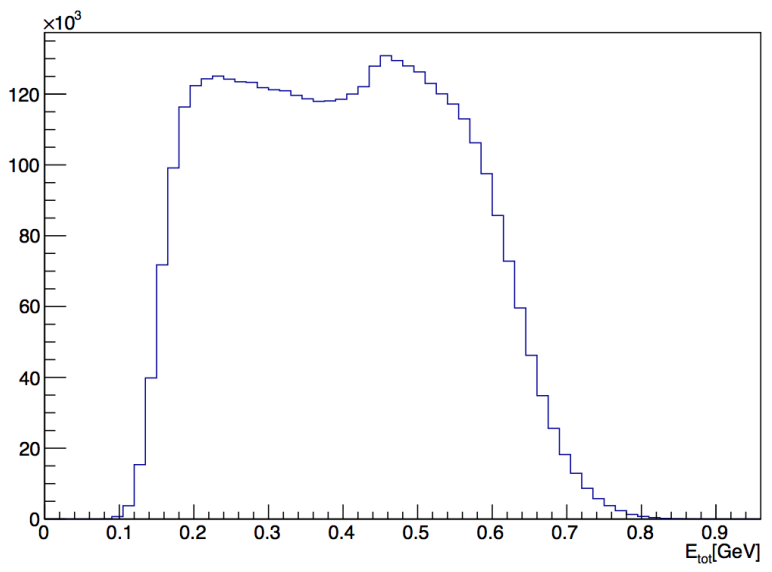

FIG. 42: The total energy deposited in the calorimeter for the electron candidates after all electron PID cuts. The results are shown for the ${ }^{3} \mathrm{He}$ target at $2.2 \mathrm{GeV}$.

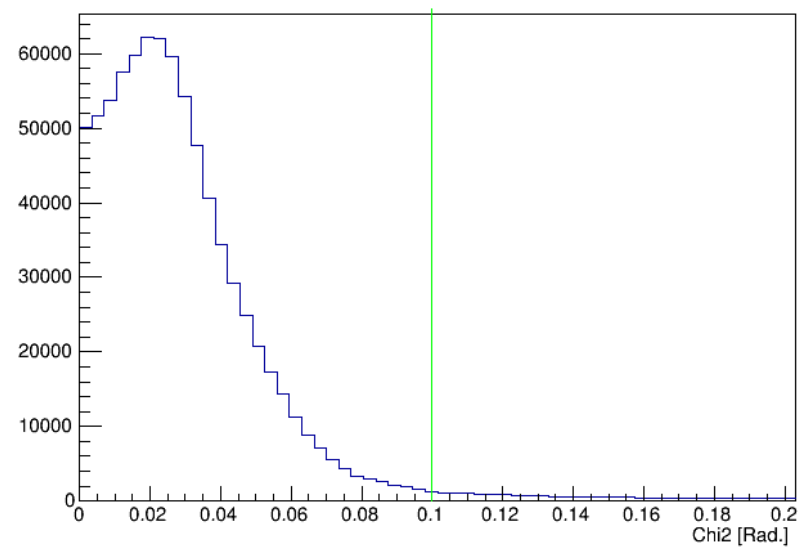

FIG. 43: The distribution of the angle between a $\mathrm{CC}$ hit and the nearest SC hit for negative particles. The green line corresponds to the applied cut value. The results are shown for the ${ }^{3} \mathrm{He}$ target at $2.2 \mathrm{GeV}$ after fiducial cuts were applied. 


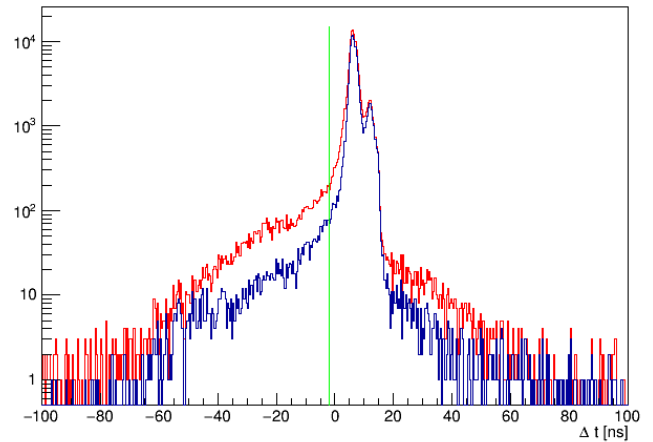

(a) Sector one.

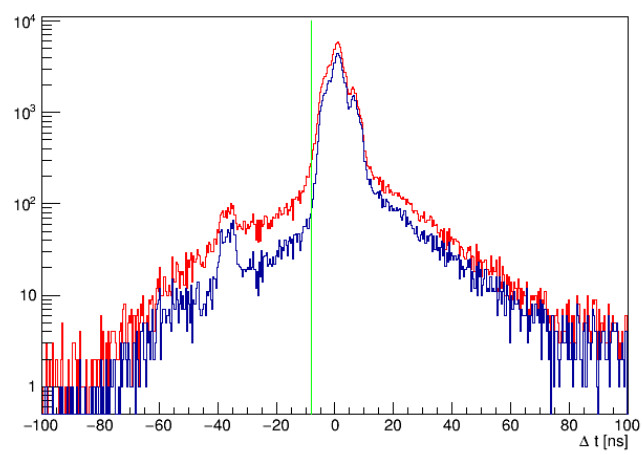

(c) Sector three.

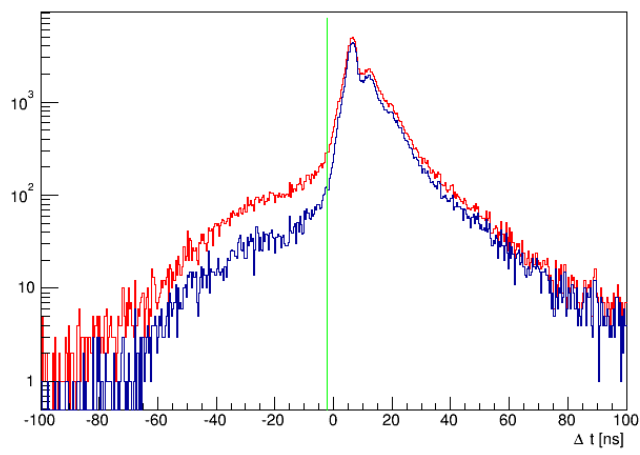

(e) Sector five.

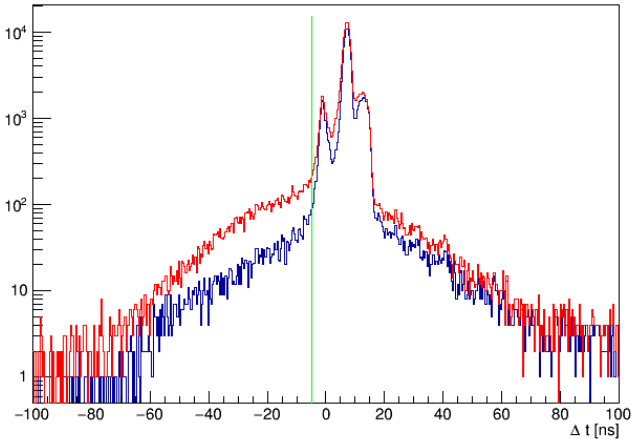

(b) Sector two.

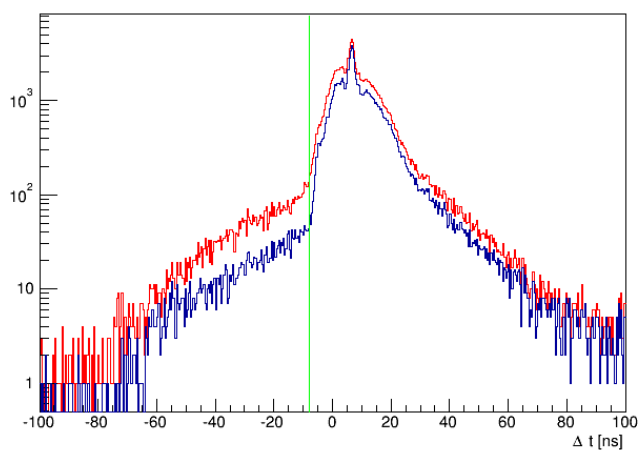

(d) Sector four.

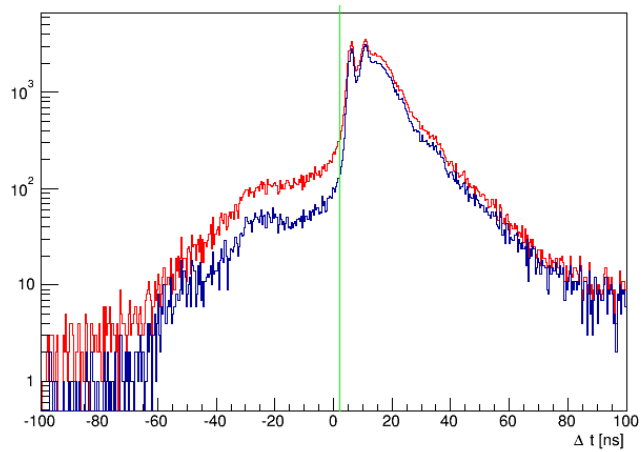

(f) Sector six.

FIG. 44: The time difference between hits of the electron candidate in CC and SC, corrected for the distance traveled from $\mathrm{CC}$ to $\mathrm{SC}$ before (red) and after (blue) a pion rejection cut (Nphe $>2.5$ at the CC). The green lines show the applied cut values. The results are shown for the ${ }^{3} \mathrm{He}$ target at $2.2 \mathrm{GeV}$ after fiducial cuts were applied.

We also cut on the energy deposited in the inner layer of the EC to be greater than $0.06 \mathrm{GeV}$. We have fit the pion peak with a Gaussian and found that the applied cut is nearly $3 \sigma$ away from the pion peak position. The distributions of the energy deposited in 
the inner layer of the EC with fiducial cuts and with the rest of the electron PID cuts in addition to fiducial cuts are shown in Fig. 41. The applied cut is indicated by the green line.

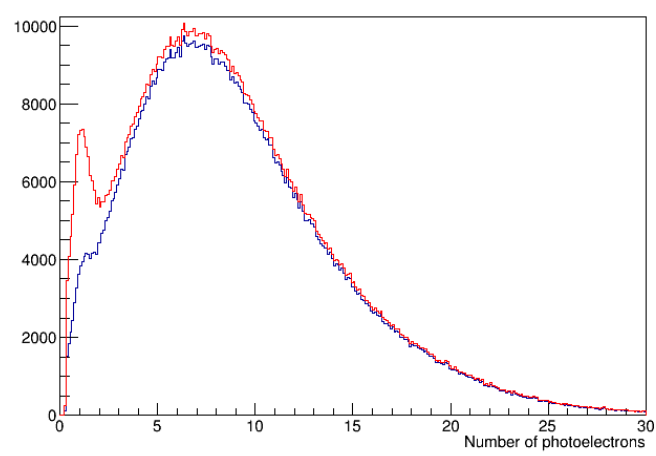

(a)

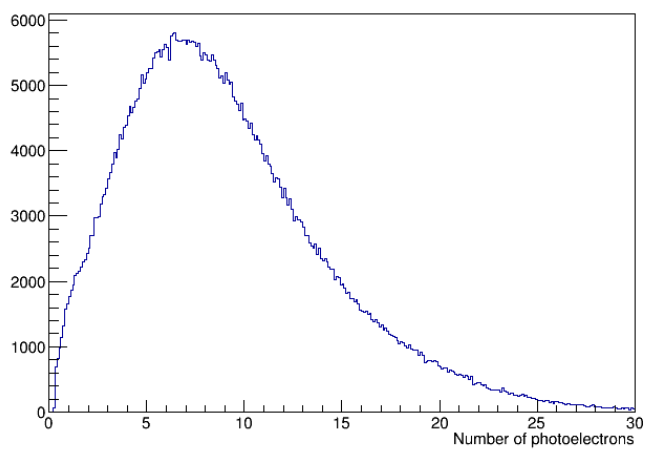

(b)

FIG. 45: The distribution of the number of photo-electrons detected in the CC before other $\mathrm{CC}$ cuts (red) and after other CC cuts (blue) (left plot). The plot on the right shows the same distribution after all electron PID cuts. The results are shown for the ${ }^{3} \mathrm{He}$ target at $2.2 \mathrm{GeV}$ after fiducial cuts were applied.

We did not apply a cut on the total energy deposited in the calorimeter because the hardware threshold was lower, however, we cut on the momentum of the electron candidate to be greater than $0.55 \mathrm{GeV} / \mathrm{c}$ (red vertical line in Fig. 40). The distribution of the total deposited energy in the calorimeter for the electron candidates after all the above mentioned cuts is shown in Fig. 42. It is clear from the plot that there is no need to apply an additional cut on $E_{\text {tot }}$. We also required the electron candidate momentum to be less than the beam energy to eliminate the few events that show up at higher momenta.

We again have applied the exact same cuts on the time difference between hits of the electron candidate in the $\mathrm{CC}$ and $\mathrm{SC}$, corrected for the distance traveled from $\mathrm{CC}$ to $\mathrm{SC}$ $\left(\Delta t_{\mathrm{CC}}\right)$ as at $4.4 \mathrm{GeV}$. The corresponding cut values are shown in Table 7 . We have also applied a cut on the angle between a CC hit and the nearest SC hit to be $\leq 0.1 \mathrm{rad}$, see Fig. 43. The plot of the number of photo-electrons detected by the $\mathrm{CC}$ for a given electron candidate after those two CC cuts is shown in Fig. 45a. The plot of the number of photoelectrons detected by the CC after all electron PID cuts is shown in Fig. 45b. It has the expected Poisson distribution form. It is clear that these cuts suppress the pion contribution at Nphe $<2.5$, and there is no need for an additional pion rejection cut Nphe $>2.5$. 


\subsection{GEV ANALYSIS}

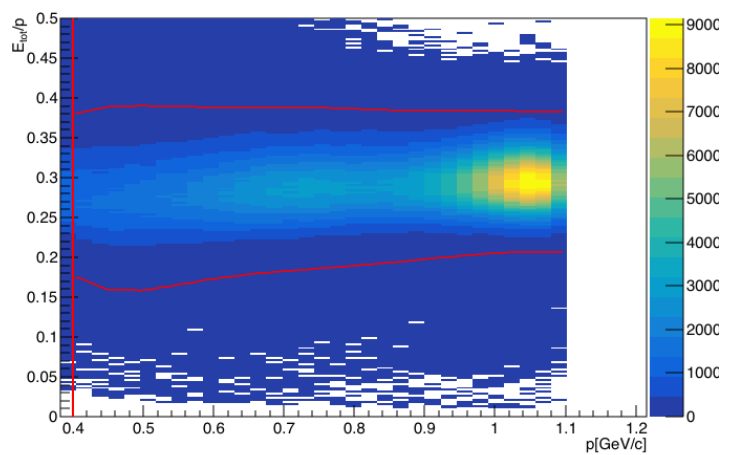

(a) ${ }^{3} \mathrm{He}$

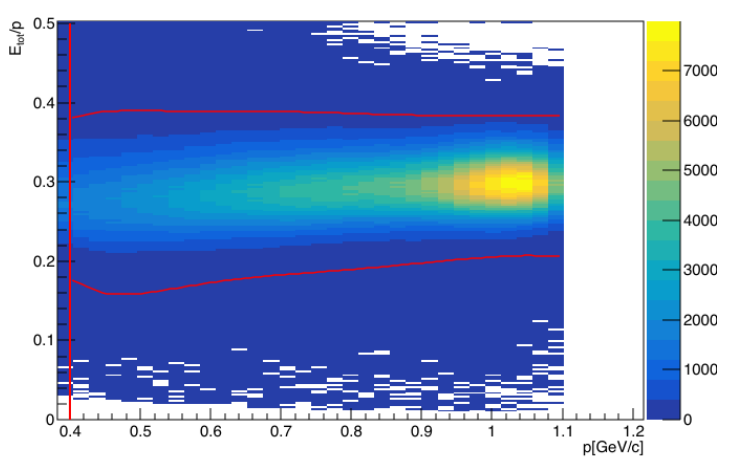

(b) ${ }^{12} \mathrm{C}$

FIG. 46: The ratio of the total energy deposited in the calorimeter over the momentum for the electron candidates. The vertical line shows the cut on momentum and the other two lines show the $3 \sigma$ cut around the electron band. The results are shown for all targets at 1.1 GeV, by looking at negative particles with EC, CC and SC status, after fiducial cuts were applied.

The electron PID cuts at $1.1 \mathrm{GeV}$ are similar to those at $2.2 \mathrm{GeV}$ and $4.4 \mathrm{GeV}$. We have again selected $3 \sigma$ region around the electron band in the plot of total energy deposited in the calorimeter divided by momentum plotted vs momentum as shown in Fig. 46 for ${ }^{12} \mathrm{C}$ and ${ }^{3} \mathrm{He}$. These are the targets that we have analyzed data for at $1.1 \mathrm{GeV}$.

We have also required the energy deposited in the inner layer of the calorimeter to be greater than $0.03 \mathrm{GeV}$ as shown in Fig. 47. We did not apply any cut on total energy deposited in the calorimeter. There is a cut requiring the momentum of the electron candidate to be greater than $0.4 \mathrm{GeV} / \mathrm{c}$ to get rid of the threshold effects.

The cuts on the time difference between hits of the electron candidate in the $\mathrm{CC}$ and $\mathrm{SC}$, corrected for the distance traveled from $\mathrm{CC}$ to $\mathrm{SC}\left(\Delta t_{\mathrm{CC}}\right)$ are the same as at 2.2 and $4.4 \mathrm{GeV}$ and are given in Table 7. The corresponding plots are shown in Fig. 48. We have also applied a cut on the angle between a $\mathrm{CC}$ hit and the nearest $\mathrm{SC}$ hit to be $\leq 0.1 \mathrm{Rad}$., see Fig. 49. The plots of the number of photo-electrons detected by the CC for a given electron candidate after those two $\mathrm{CC}$ cuts is shown in Fig. 50 (red). The plots of the number of photo-electrons detected by the CC after all electron PID cuts is shown in Fig. 50 (blue). The pion contribution at Nphe $<2.5$ is suppressed after all this cuts and there is no need 


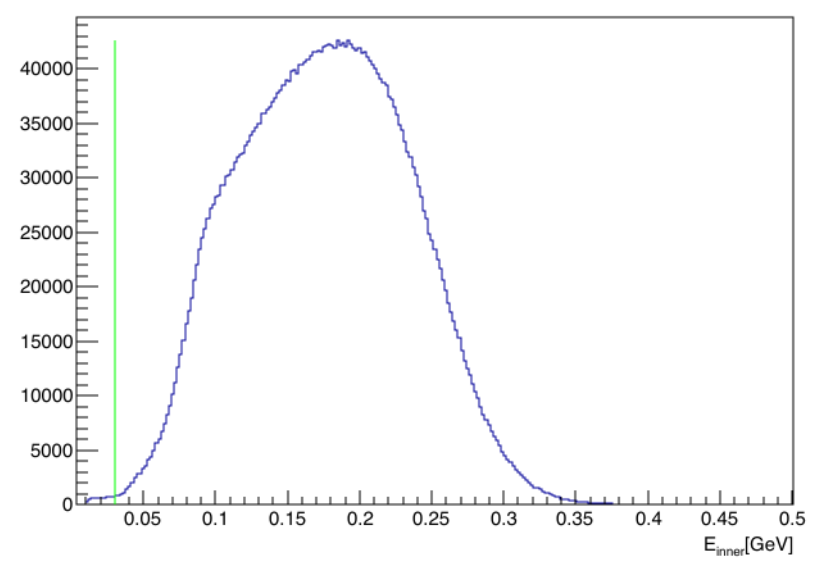

FIG. 47: The energy deposited in the inner layer of calorimeter with the cut (green line) for the electron candidates. The results are shown for the ${ }^{3} \mathrm{He}$ target at $1.1 \mathrm{GeV}$, after fiducial cuts.

for an additional pion rejection cut Nphe $>2.5$.

\subsection{PROTON IDENTIFICATION}

After selecting the electron, other charged particles in the event are identified by using the time of flight information from the SC and the momentum obtained from the Drift Chambers. Neutral particles are identified as the ones with a hit in the electromagnetic calorimeter and no associated charged track. For them the time information is used to separate neutrons and photons.

\subsection{GEV ANALYSIS}

Each particle is assigned a certain ID, by requiring that the difference $\left|\beta_{\text {measured }}-\beta_{\text {calculated }}\right|$ was minimal. This difference is between the particles' velocity obtained from the path length and the time information from the $\mathrm{SC}, \beta_{\text {measured, }}$ and the velocity obtained from

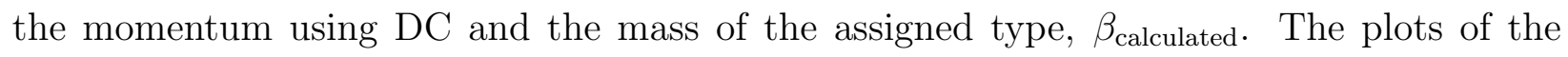
measured velocity as a function of particle momentum for positive particles and for particles with proton ID is shown in Fig. 51a and Fig. 51b, respectively. Different bands in the plot for positive particles correspond to positrons, pions, kaons, protons, deutrons and tritons.

The black vertical line in Fig. 51b shows the momentum threshold we have selected for 


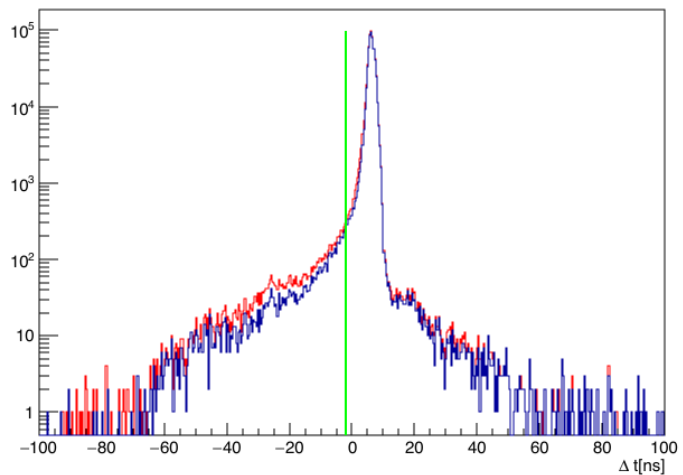

(a) Sector one.

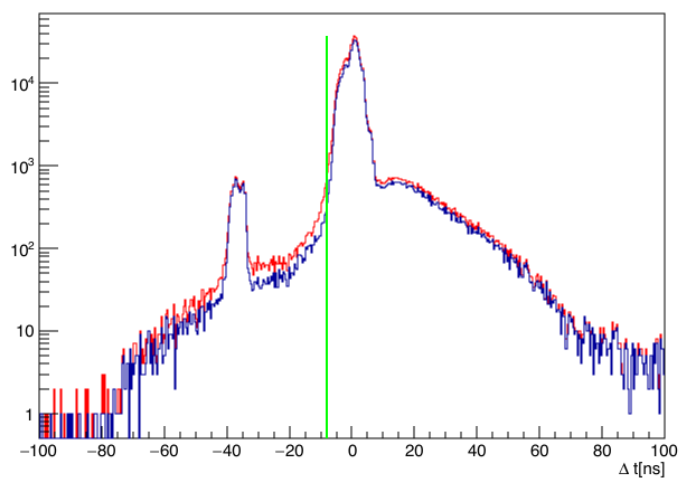

(c) Sector three.

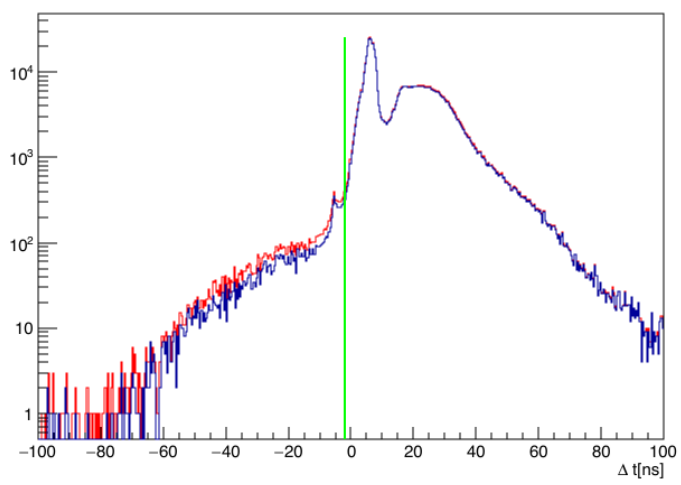

(e) Sector five.

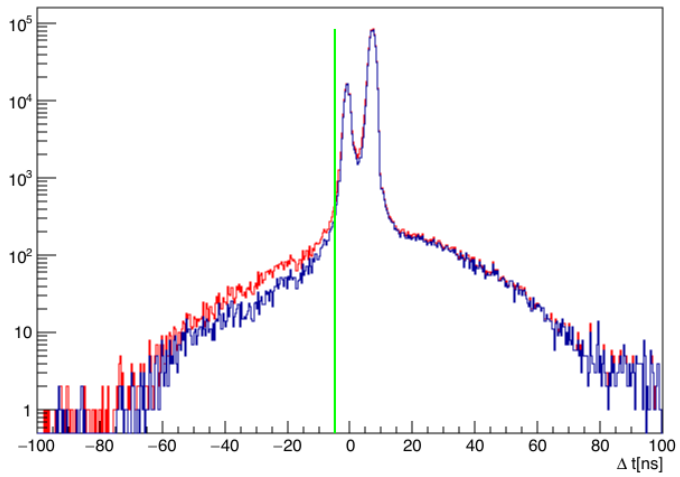

(b) Sector two.

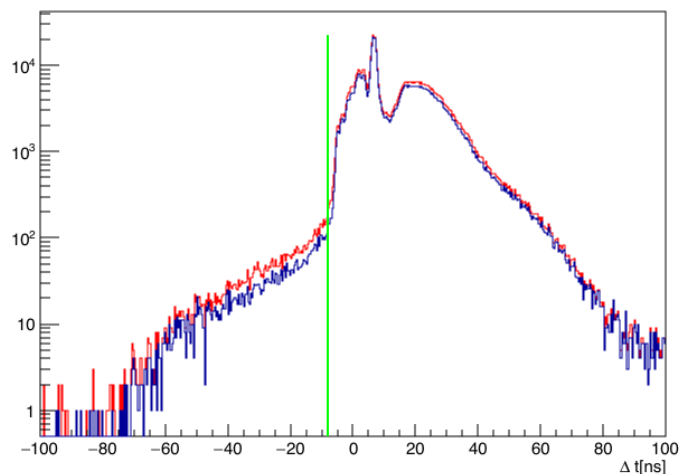

(d) Sector four.

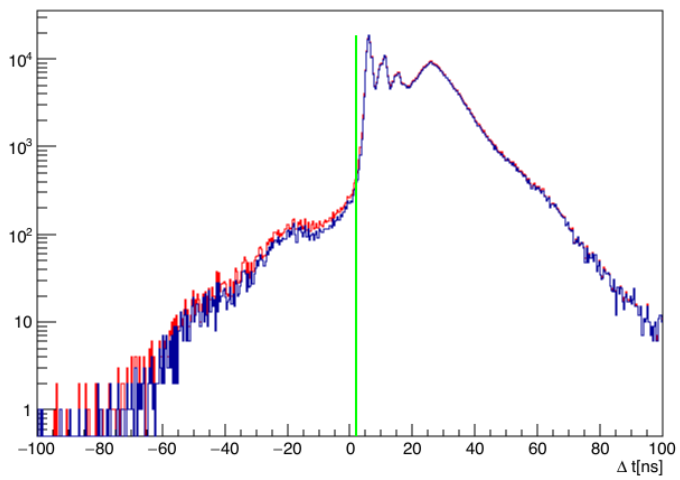

(f) Sector six.

FIG. 48: The time difference between hits of the electron candidate in CC and SC, corrected for the distance traveled from CC to SC before (red) and after (blue) a pion rejection cut (Nphe $>2.5$ at the $\mathrm{CC}$ ). The green lines show the applied cut values. The results are shown for the ${ }^{3} \mathrm{He}$ target at $1.1 \mathrm{GeV}$ after fiducial cuts were applied. 


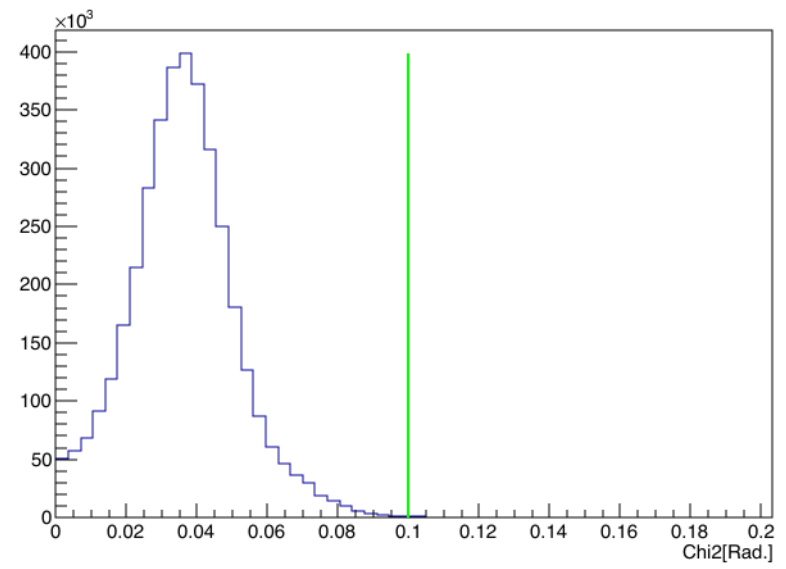

FIG. 49: The distribution of the angle between a CC hit and the nearest SC hit for negative particles. The green line corresponds to the applied cut value. The results are shown for the ${ }^{3} \mathrm{He}$ target at $1.1 \mathrm{GeV}$ after fiducial cuts were applied.

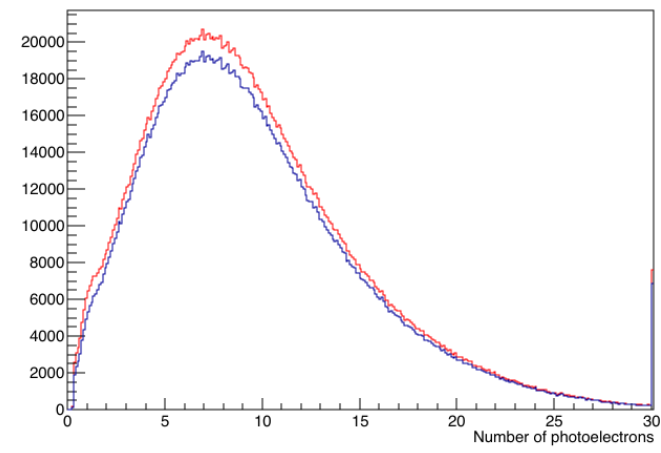

(a) ${ }^{3} \mathrm{He}$

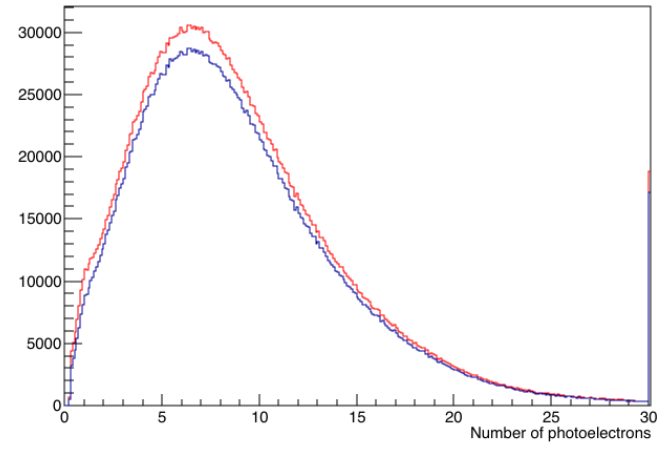

(b) ${ }^{12} \mathrm{C}$

FIG. 50: The distribution of the number of photo-electrons detected in the CC before EC cuts and other CC cuts (red) and after EC and the rest of CC cuts (blue). The results are shown for the ${ }^{3} \mathrm{He}$ and ${ }^{12} \mathrm{C}$ targets at $1.1 \mathrm{GeV}$ after fiducial and vertex cuts were applied.

protons, as below that the CLAS efficiency for proton detection is not well known. We apply this cut as part of proton PID at all energies (1.1 GeV, 2.2 GeV and 4.4 GeV).

We also checked the difference between the time of flight measured by the SC and the time of flight obtained from dividing the path length by the velocity obtained from the 


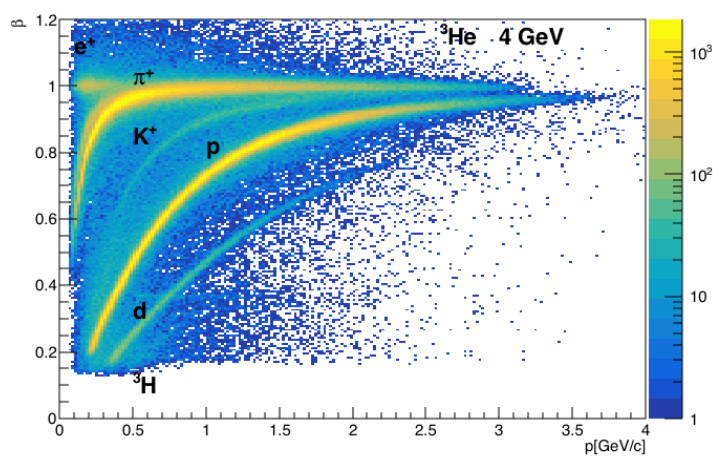

(a)

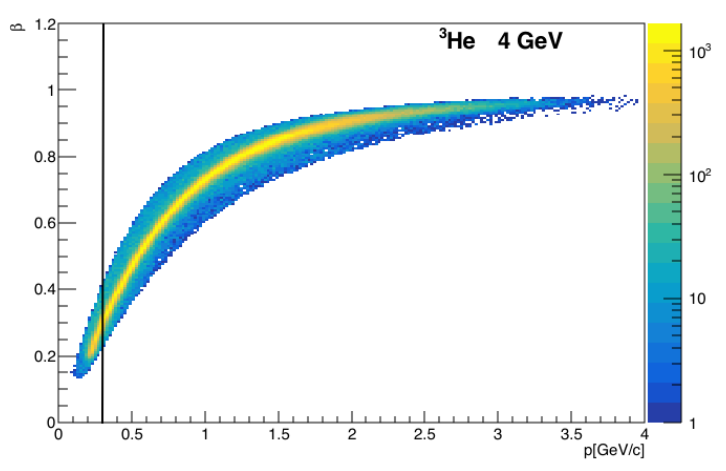

(b)

FIG. 51: a) The logarithmic scale plot of beta from the SC vs momentum from the DC for positively charged particles. b) The logarithmic scale plot of beta from the SC vs momentum from the DC for particles with proton ID, the black vertical line corresponds to the cut applied on proton momentum. The results are shown for the ${ }^{3} \mathrm{He}$ target at $4.4 \mathrm{GeV}$.

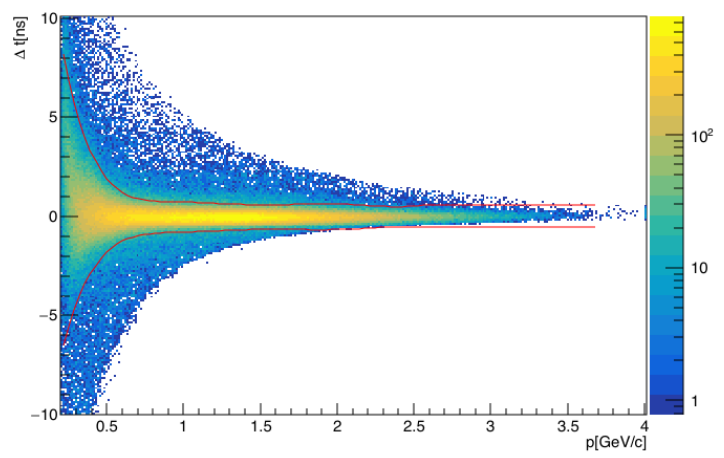

(a) ${ }^{3} \mathrm{He}$

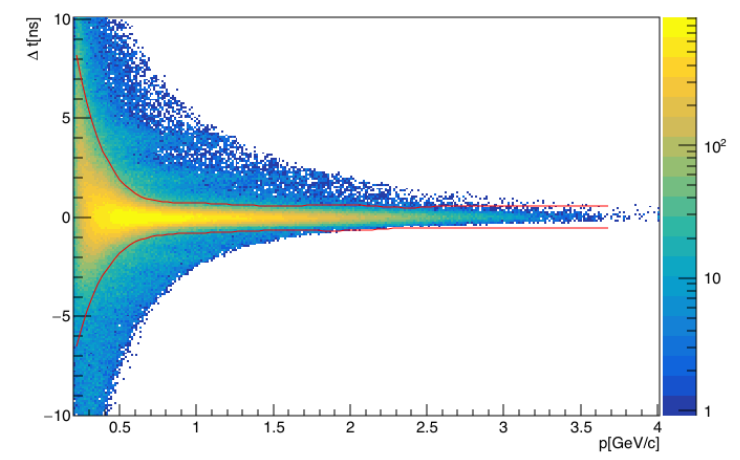

(b) ${ }^{56} \mathrm{Fe}$

FIG. 52: The logarithmic scale plot of the difference between the time of flight measured by the SC and the time of flight calculated using the path length and the velocity obtained from the momentum measured by the DC as a function of momentum for positive particles with proton ID. The red line shows the $3 \sigma$ PID cut. The horizontal lines at $\sim 2$ and $\sim 4$ ns correspond to particles from a different event. The results are shown for the ${ }^{3} \mathrm{He}$ and ${ }^{56} \mathrm{Fe}$ targets at $4.4 \mathrm{GeV}$. 


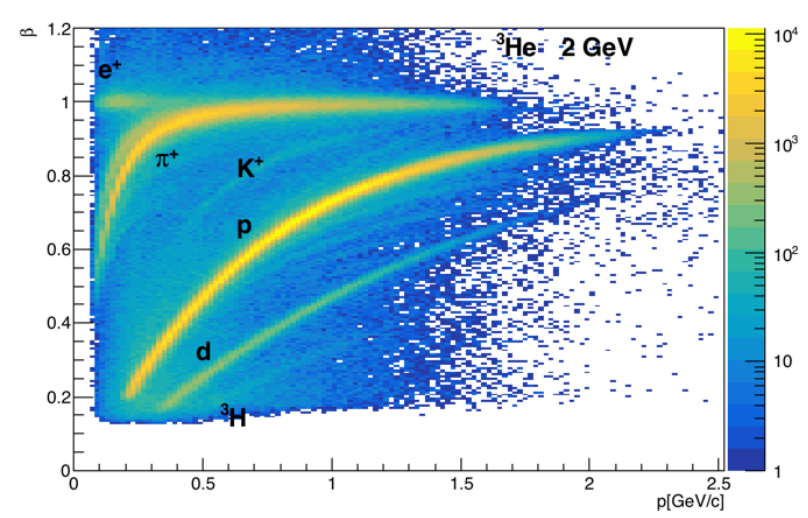

(a)

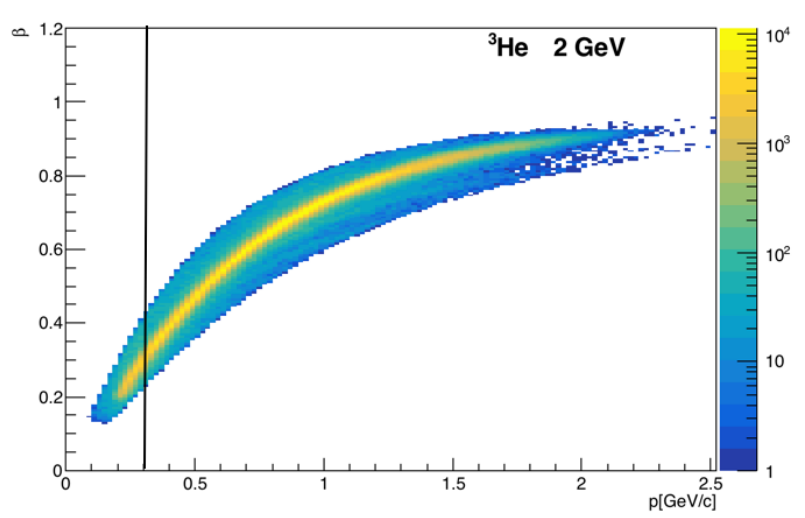

(b)

FIG. 53: a) The logarithmic scale plot of beta from the SC vs momentum from the DC for positively charged particles. b) The logarithmic scale plot of beta from the SC vs momentum from the DC for particles with proton ID, the black vertical line corresponds to the cut applied on proton momentum. The results are shown for the ${ }^{3} \mathrm{He}$ target at $2.2 \mathrm{GeV}$.

momentum measured by the DC for positive particles with proton ID. The difference $\Delta t$ is expressed by

$$
\Delta t=t_{\mathrm{SC}}-\frac{r_{\mathrm{SC}}}{\beta_{\mathrm{DC}} * c}-t_{\text {trigger }}
$$

where

$$
\beta_{\mathrm{DC}}=\frac{p}{\sqrt{p^{2}+m_{\mathrm{p}}^{2}}}
$$

where $t_{\mathrm{SC}}$ and $r_{\mathrm{SC}}$ are the time and path length information from the $\mathrm{SC}, p$ is the momentum from the DC, $t_{\text {trigger }}$ is the trigger time and $m_{\mathrm{p}}$ is the proton mass. We then applied a $3 \sigma$ cut around the $\Delta t$ (Eq. 48) band to select protons and to eliminate particles coming from different beam bunches. The $\Delta t$ distribution as a function of momentum for positive particles with positive status that have an associated signal in the SC and their ID equal to that of the proton is shown in Fig. 52. There are no other cuts applied on this plot (no vertex cuts, fiducial cuts, etc.). However when obtaining the functions (shown in red) for the $3 \sigma$ cut around the proton band in $\Delta t$ vs $p$ distribution, in addition to the above mentioned cuts we have applied fiducial cuts. 


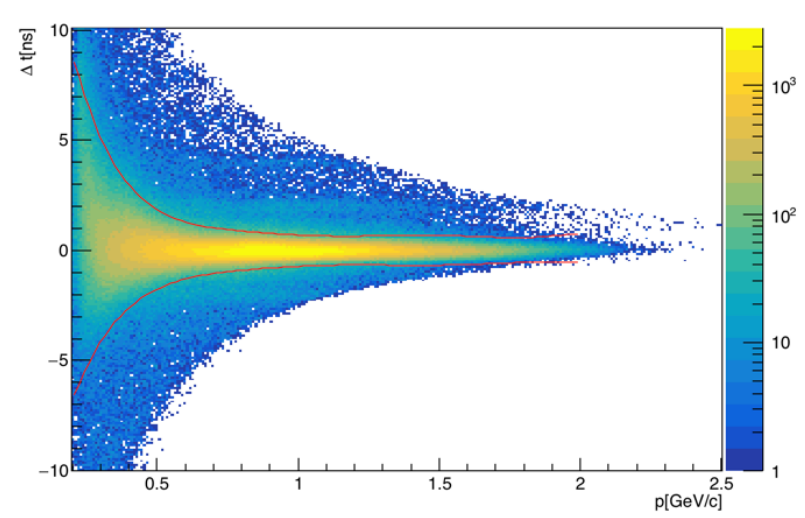

(a) ${ }^{3} \mathrm{He}$

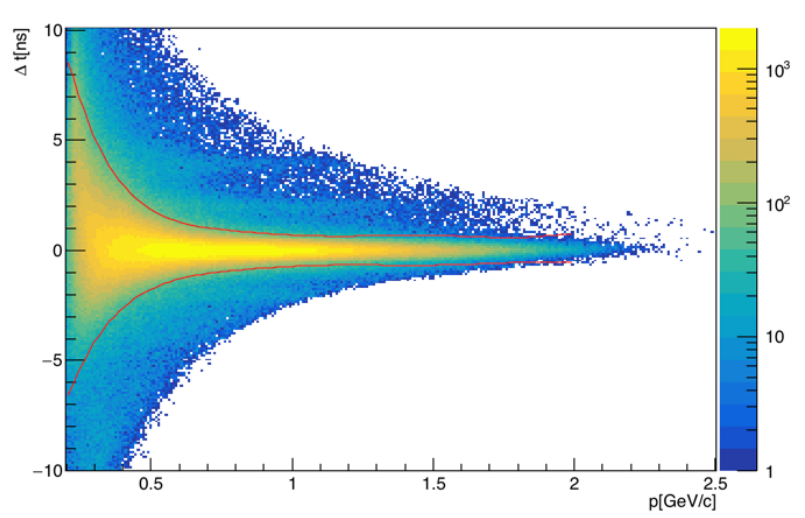

(b) ${ }^{56} \mathrm{Fe}$

FIG. 54: The logarithmic scale plot of the difference between the time of flight measured by the SC and the time of flight calculated using the path length and the velocity obtained from the momentum measured by the DC as a function of momentum for positive particles with proton ID. The red line shows the $3 \sigma$ PID cut. The horizontal lines at $\sim 2$ and $\sim 4$ ns correspond to particles from a different events. The results are shown for the ${ }^{3} \mathrm{He}$ and ${ }^{56} \mathrm{Fe}$ targets at the $2.2 \mathrm{GeV}$.

\subsection{GEV ANALYSIS}

An analysis similar to the one at $4.4 \mathrm{GeV}$ was done to determine the proton PID cuts for the $2.2 \mathrm{GeV}$ analysis. The plots of the velocity measured by the time of flight as a function of particle momentum for positive particles and for particles with proton ID are shown in Fig. 53a and Fig. 53b, respectively. Again it was concluded that using the proton ID to select proton candidates provides a good pion separation and a $3 \sigma$ cut needs to be applied around the proton band in the $\Delta t$ vs momentum distribution that is centered around 0 , to eliminate particles coming from different beam bunches, appearing as horizontal lines in Fig. 54. The plot was made for positive particles with positive status that have associated signal in SC and their ID equal to that of the proton. However when obtaining the functions (shown in red) for $3 \sigma$ cut around the proton band in $\Delta t$ vs $p$ distribution, in addition to the above mentioned cuts we have applied fiducial cuts. 


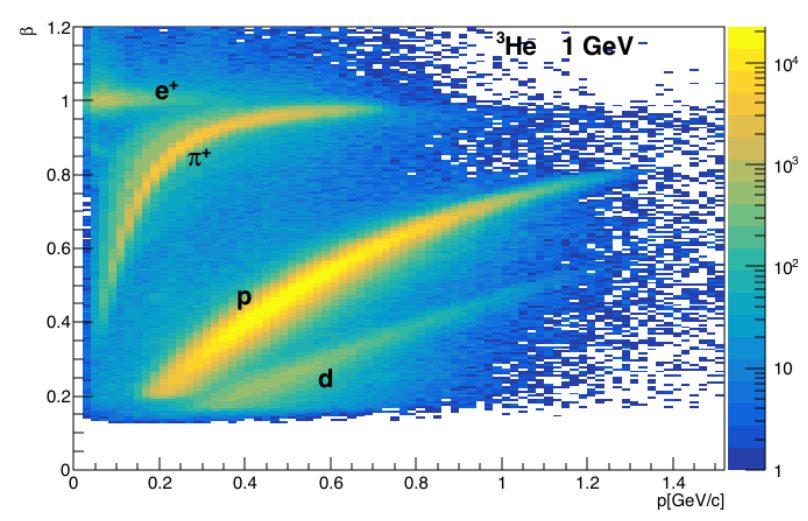

(a)

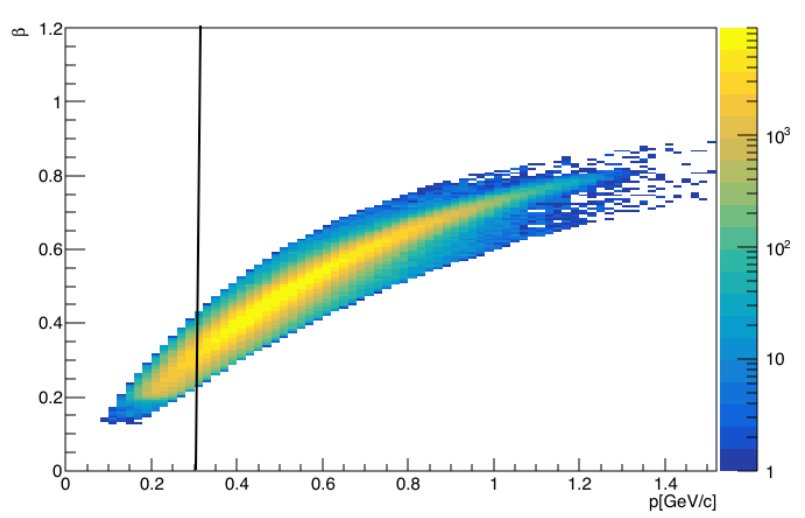

(b)

FIG. 55: a) The logarithmic scale plot of beta from the SC vs momentum from the DC for positively charged particles. b) The logarithmic scale plot of beta from the SC vs momentum from the DC for particles with proton ID, the black vertical line corresponds to the cut applied on proton momentum. The results are shown for the ${ }^{3} \mathrm{He}$ target at $1.1 \mathrm{GeV}$.

\subsection{GEV ANALYSIS}

The procedure for proton identification at $1.1 \mathrm{GeV}$ is the same as before. The plot of the velocity measured by the time of flight as a function of particle momentum for positive particles is shown in Fig. 55a. We can see in Fig. 56 that using proton ID we get a good separation of the proton band. To further improve the pion identification and get rid of the particles coming from different beam bunches we apply a $3 \sigma$ cut around the proton band in the $\Delta t$ vs momentum distribution that is centered around 0 as shown in Fig. 56. The plot was made for positive particles with positive status that have associated signal in SC and their ID equal to that of the proton. However when obtaining the functions (shown in red) for $3 \sigma$ cut around the proton band in $\Delta t$ vs $p$ distribution, in addition to the above mentioned cuts we have applied fiducial cuts. 


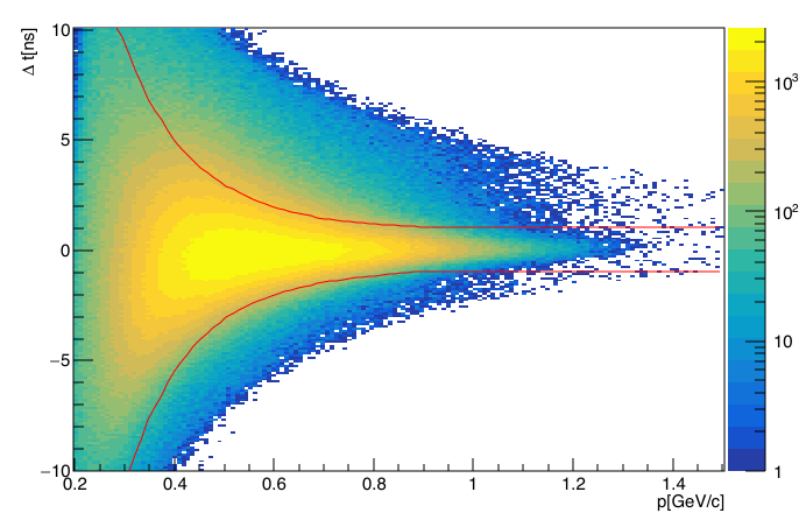

(a) ${ }^{3} \mathrm{He}$

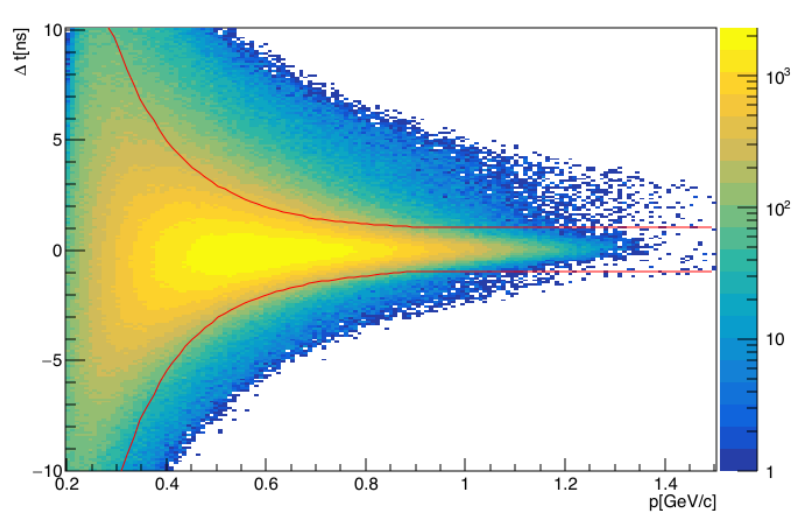

(b) ${ }^{12} \mathrm{C}$

FIG. 56: The logarithmic scale plot of the difference between the time of flight measured by the SC and the time of flight calculated using the path length and the velocity obtained from the momentum measured by the DC as a function of momentum for positive particles with proton ID. The red line shows the $3 \sigma$ PID cut. The horizontal lines at $\sim 2$ and $\sim 4$ ns correspond to particles from a different events. The results are shown for the ${ }^{3} \mathrm{He}$ and ${ }^{12} \mathrm{C}$ targets at the $1.1 \mathrm{GeV}$.

\subsection{CHARGED PION IDENTIFICATION}

\subsection{GEV ANALYSIS}

The charged pion selection is similar to the proton selection. We first looked at the charged particles that have an associated signal in SC and DC and positive status. For these particles we calculated $\Delta t$ similarly as for protons, but using the charged pion mass. Then we again selected a $3 \sigma$ region around the charged pion band that is centered around the $\Delta t=0$ line to select pions. The $\Delta t$ distribution as a function of momentum for $\pi^{+}$and $\pi^{-}$candidates at $4.4 \mathrm{GeV}$ are shown in Fig. 58 and Fig. 57 respectively. The plots were made for charged particles (positive and negative correspondingly) with positive status that have associated signal in SC and DC. However when obtaining the functions (shown in red) for $3 \sigma$ cut around the charged pion band in $\Delta t$ vs $p$ distribution, in addition to the above mentioned cuts we have required the ID of the charged pion candidate to be equal to that 


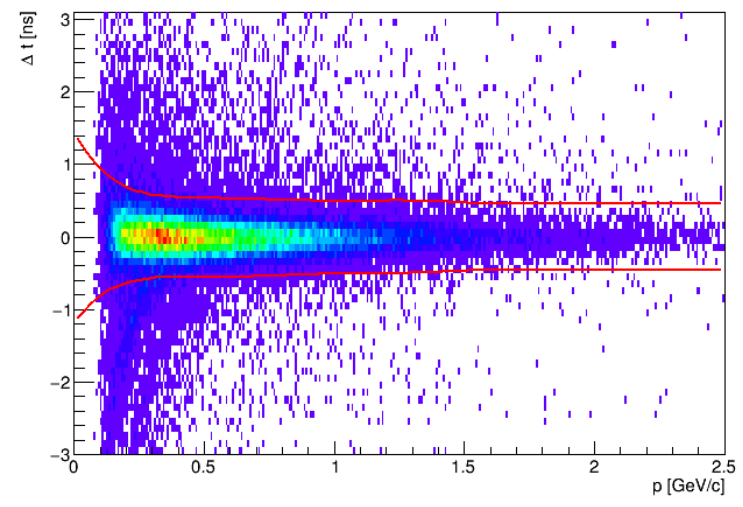

(a) ${ }^{3} \mathrm{He}\left(e, e^{\prime} p\right)$

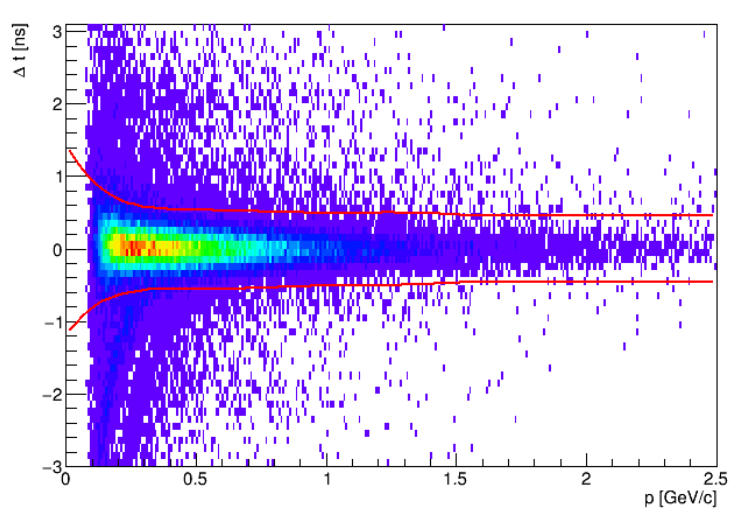

(b) ${ }^{56} \mathrm{Fe}\left(e, e^{\prime} p\right)$

FIG. 57: The plot of the difference between the time of flight measured by the SC and the time of flight calculated using the path length and the velocity obtained from the momentum measured by the DC as a function of momentum from DC for $\pi^{-}$candidates at $4.4 \mathrm{GeV}$. The red line shows the $3 \sigma$ PID cut.

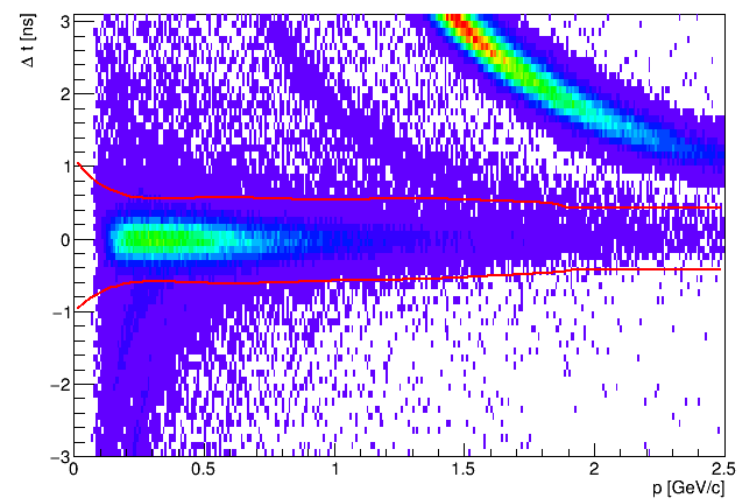

(a) ${ }^{3} \mathrm{He}\left(e, e^{\prime} p\right)$

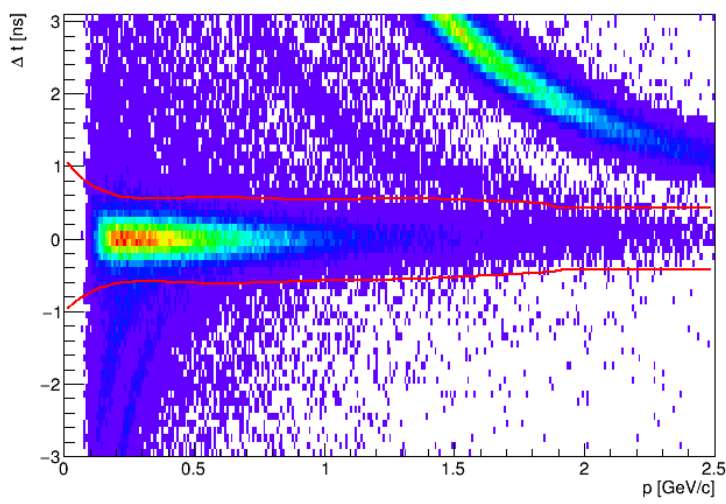

(b) ${ }^{56} \mathrm{Fe}\left(e, e^{\prime} p\right)$

FIG. 58: The plot of the difference between the time of flight measured by the SC and the time of flight calculated using the path length and the velocity obtained from the momentum measured by the DC as a function of momentum from DC for $\pi^{+}$candidates at $4.4 \mathrm{GeV}$. The red line shows the $3 \sigma$ PID cut. 
of the corresponding charged pion, and have also applied fiducial cuts.

We also require the momentum of the charged pion to be above $150 \mathrm{MeV} / \mathrm{c}$, as below that the efficiency of detection is not well known. We apply this cut at all three beam energies.

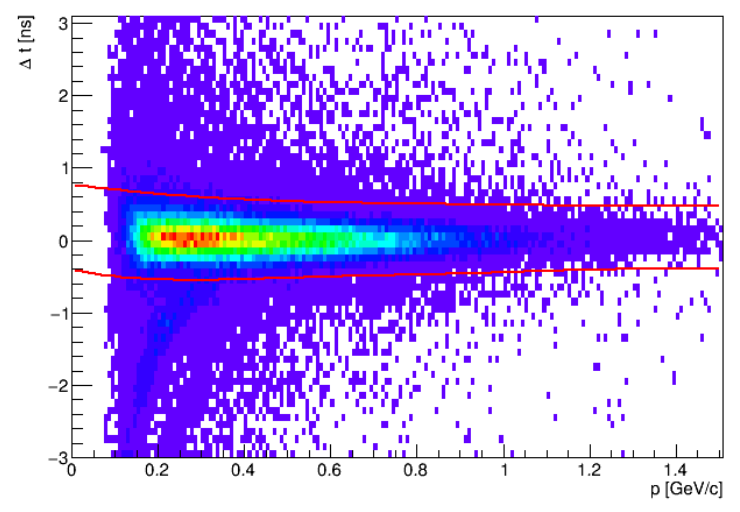

(a) ${ }^{3} \mathrm{He}(\mathrm{e}, \mathrm{e}$ 'p)

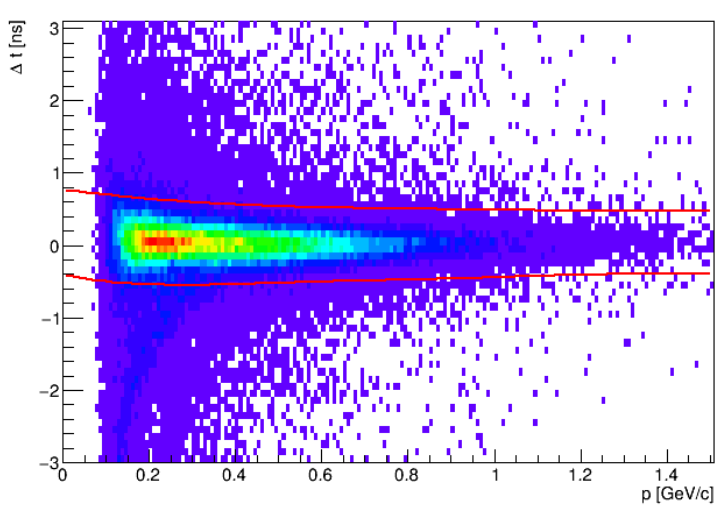

(b) ${ }^{56} \mathrm{Fe}(\mathrm{e}, \mathrm{e} ' \mathrm{p})$

FIG. 59: The plot of the difference between the time of flight measured by the SC and the time of flight calculated using the path length and the velocity obtained from the momentum measured by the DC as a function of momentum from DC for $\pi^{-}$candidates at $2.2 \mathrm{GeV}$. The red line shows the $3 \sigma$ PID cut.

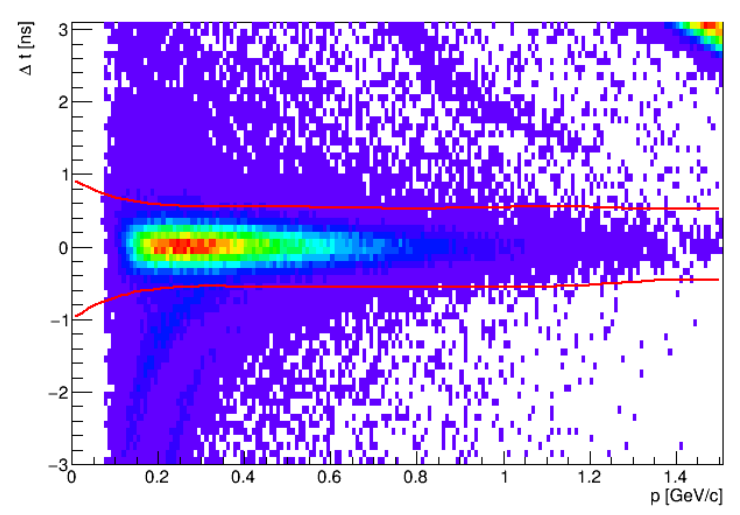

(a) ${ }^{3} \mathrm{He}(\mathrm{e}, \mathrm{e}$ 'p)

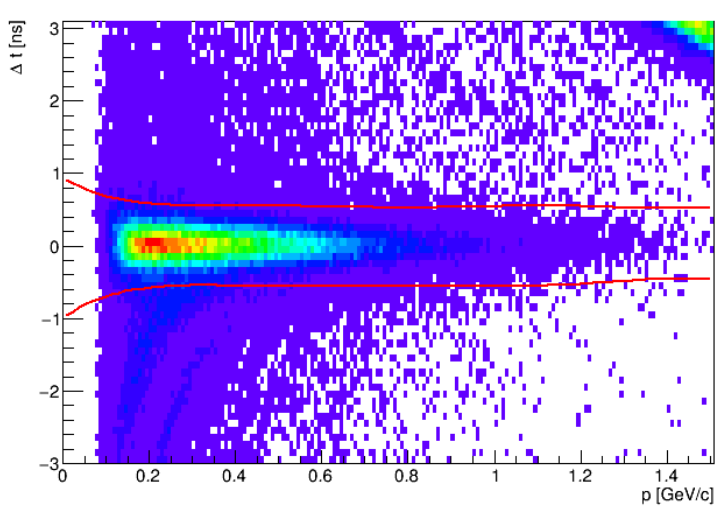

(b) ${ }^{56} \mathrm{Fe}(\mathrm{e}, \mathrm{e} \mathrm{p})$

FIG. 60: The plot of the difference between the time of flight measured by the SC and the time of flight calculated using the path length and the velocity obtained from the momentum measured by the DC as a function of momentum from DC for $\pi^{+}$candidates at $2.2 \mathrm{GeV}$. The red line shows the $3 \sigma$ PID cut. 


\subsection{GEV ANALYSIS}

The pion selection at $2.2 \mathrm{GeV}$ was done in the same way as for the $4.4 \mathrm{GeV}$ analysis. Again the plots in Fig. 60 and Fig. 59 were made for charged particles (positive and negative correspondingly) with positive status that have associated signal in SC and DC. However when obtaining the functions (shown in red) for $3 \sigma$ cut around the charged pion band in $\Delta t$ vs $p$ distribution, in addition to the above mentioned cuts we have required the ID of the charged pion candidate to be equal to that of the corresponding charged pion, and have also applied fiducial cuts.

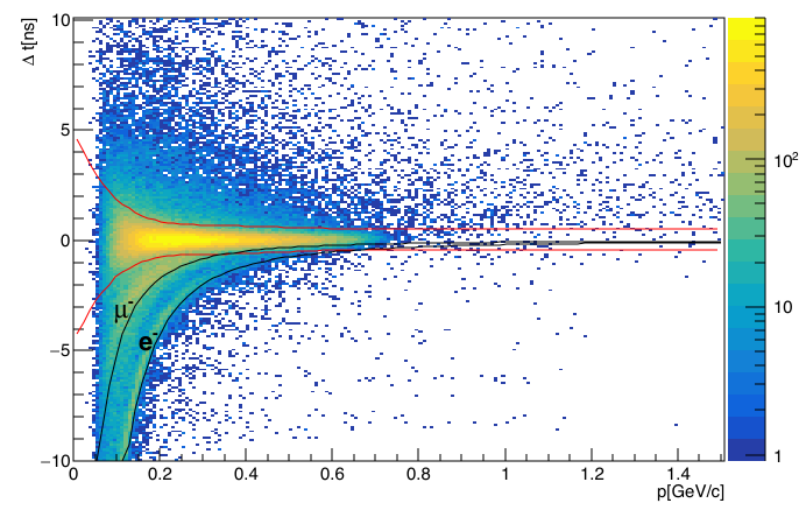

(a) ${ }^{3} \mathrm{He}(\mathrm{e}, \mathrm{e}$ 'p)

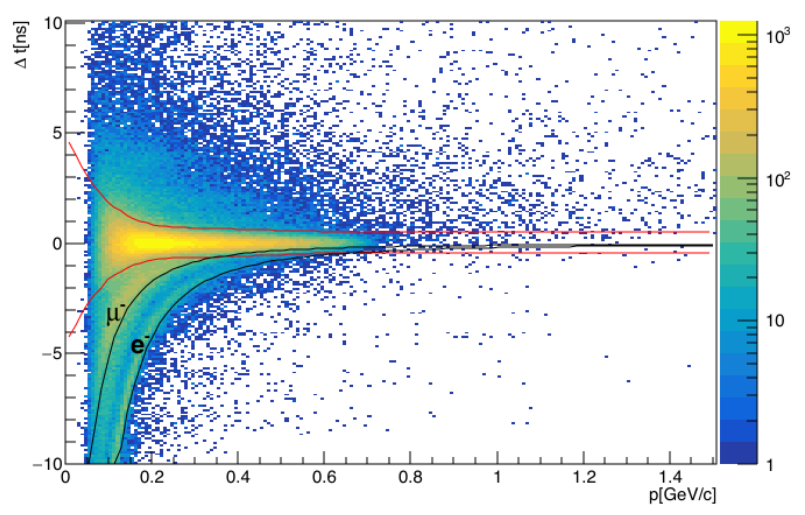

(b) ${ }^{12} \mathrm{C}(\mathrm{e}, \mathrm{e} \mathrm{p})$

FIG. 61: The plot of the difference between the time of flight measured by the SC and the time of flight calculated using the path length and the velocity obtained from the momentum measured by the DC as a function of momentum from DC for $\pi^{-}$candidates at $1.1 \mathrm{GeV}$. The red line shows the $3 \sigma$ PID cut.

\subsection{GEV ANALYSIS}

The pion selection at $1.1 \mathrm{GeV}$ was done in the same way as for the $2.2 \mathrm{GeV}$ and $4.4 \mathrm{GeV}$ analysis. We can see the $\Delta t$ vs $p$ distributions for charged particles (positive and negative correspondingly) with positive status that have associated signal in SC and DC in Fig. 62 and Fig. 61 . When obtaining the functions (shown in red) for $3 \sigma$ cut around the charged pion band in $\Delta t$ vs $p$ distribution, in addition to the above mentioned cuts we have required 


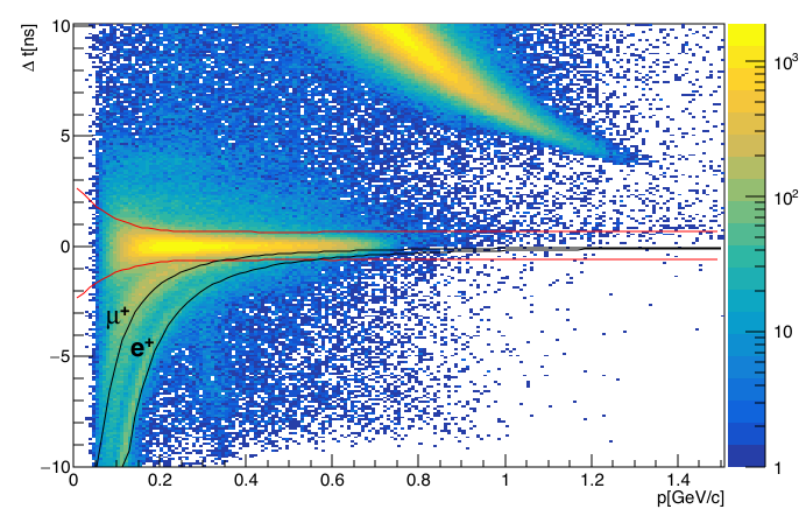

(a) ${ }^{3} \mathrm{He}(\mathrm{e}, \mathrm{e}$ 'p)

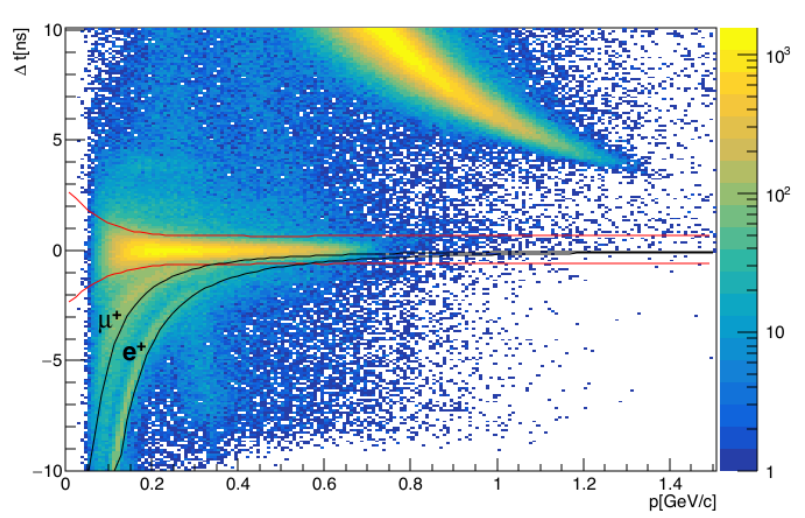

(b) ${ }^{12} \mathrm{C}(\mathrm{e}, \mathrm{e}, \mathrm{p})$

FIG. 62: The log scale plot of the difference between the time of flight measured by the SC and the time of flight calculated using the path length and the velocity obtained from the momentum measured by the DC as a function of momentum from DC for $\pi^{+}$candidates at $1.1 \mathrm{GeV}$. The red line shows the $3 \sigma$ PID cut.

the ID of the charged pion candidate to be equal to that of the corresponding charged pion, and have also applied fiducial cuts.

\subsection{PHOTON IDENTIFICATION}

\subsubsection{1, 2.2 AND 4.4 GEV ANALYSIS}

Photons were selected by choosing the neutral particles with positive status, that had been detected in the EC, did not have associated signals in the DC or SC. We have then applied a cut on the velocity of neutral particles to select photons. The plots of the beta distributions of neutral particles in EC are shown in Fig. 63 and Fig. 64. We have cut $2 \sigma$ to the left of the photon peak to select photons at 2.2 and $4.4 \mathrm{GeV}$, and we have cut $3 \sigma$ away from the peak at $1.1 \mathrm{GeV}$ as at this energy there is a clear separation between the neutron and photon peaks.

We have also required the energy of the photon to be above $0.3 \mathrm{GeV}$, as below that the detection efficiency of photon is not well known. The energy spectra of photon candidates 

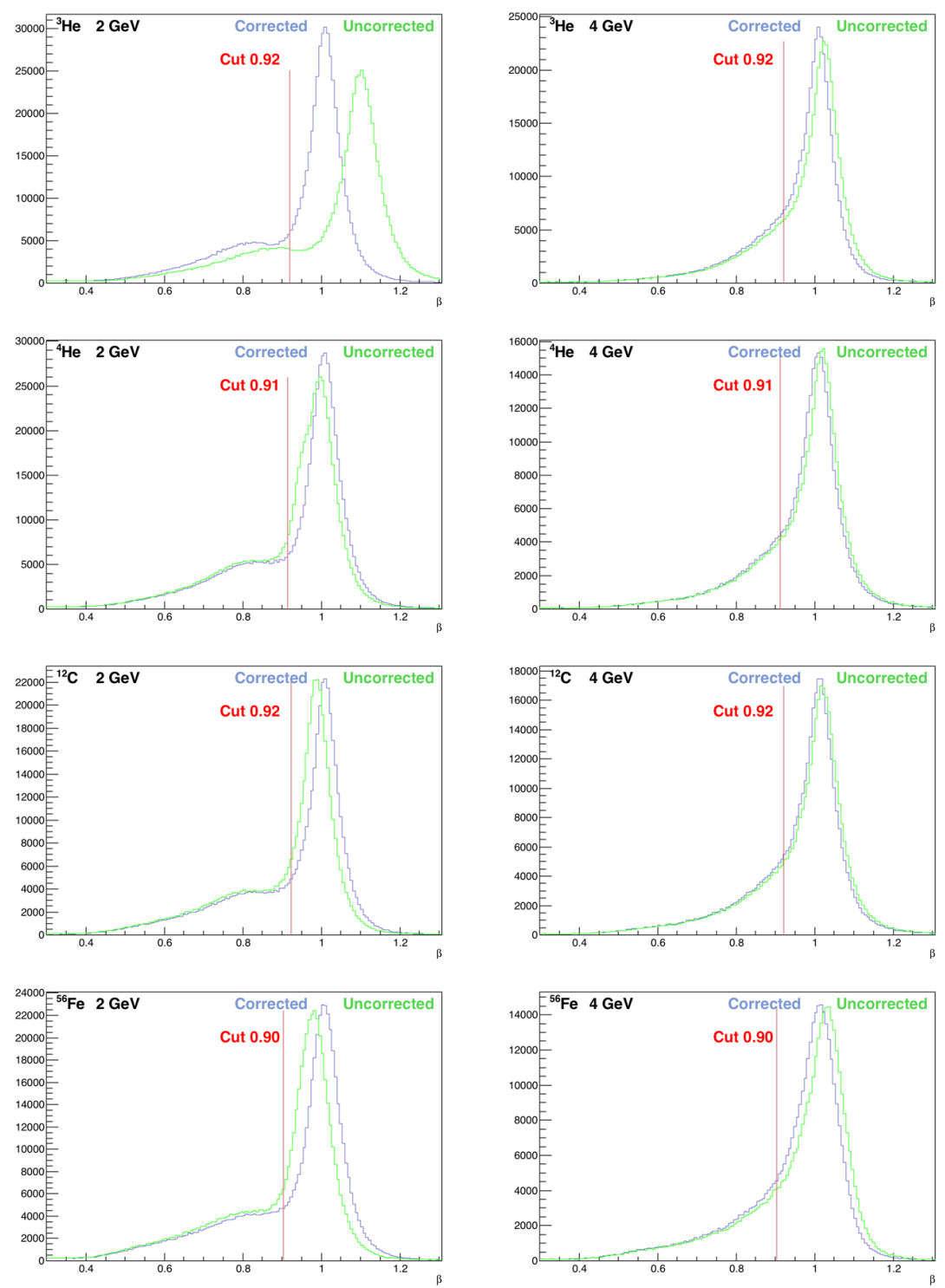

FIG. 63: The $\beta$ distributions of neutral particles in EC before and after corrections at 2.2 and $4.4 \mathrm{GeV}$ for all targets. The vertical line corresponds to the cut applied 2 sigma away from the peak for photon selection.

that have passed the photon PID ( $\beta$ cut) and fiducial cuts with the vertical line showing the applied cut, are shown in Fig 65.

We have noticed in Fig. 63 and Fig. 64 (blue distributions) that the photon peak is not centered at $\beta=1$. Also for ${ }^{4} \mathrm{He}$ at $2.2 \mathrm{GeV}$ the peak is asymmetric, so it is clear that the EC timing needed to be calibrated. To do so we have looked at the EC timing of the electrons 

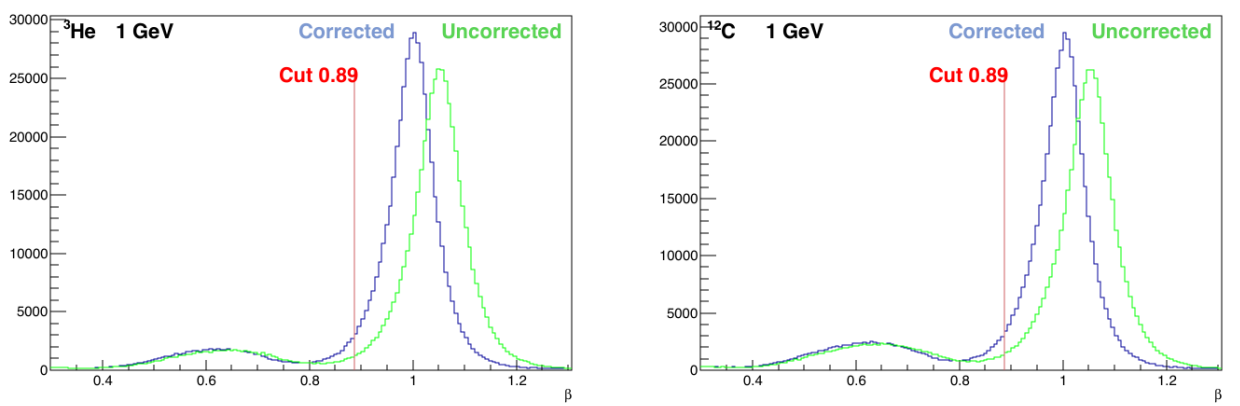

FIG. 64: The $\beta$ distributions of neutral particles in EC before and after corrections at $1.1 \mathrm{GeV}$ for all targets. The vertical line corresponds to the cut applied 3 sigma away from the peak for photon selection.
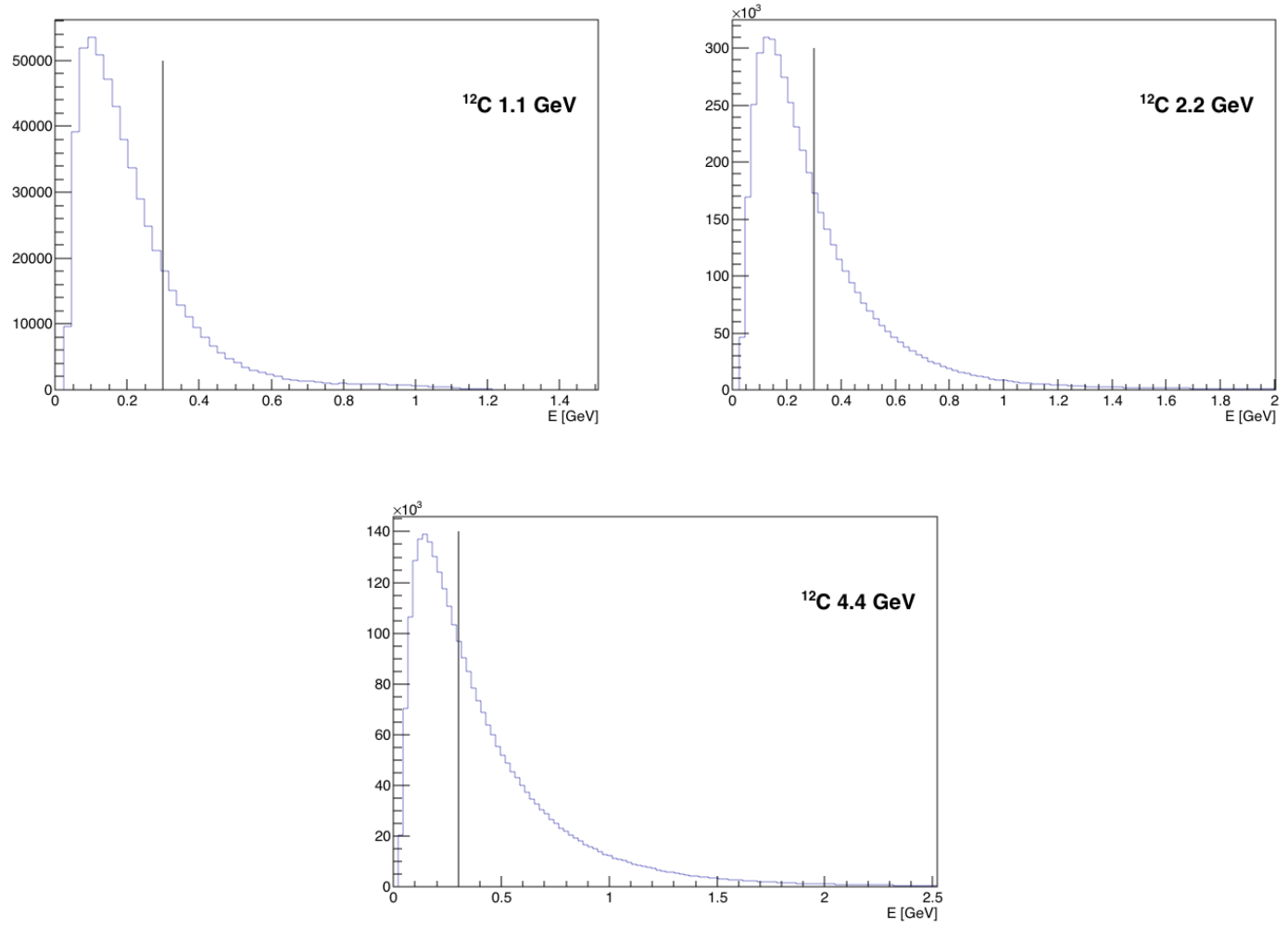

FIG. 65: The energy spectra of photon candidates at different beam energies for ${ }^{12} \mathrm{C}$. The vertical line corresponds to the cut applied on the energy of the photons. 

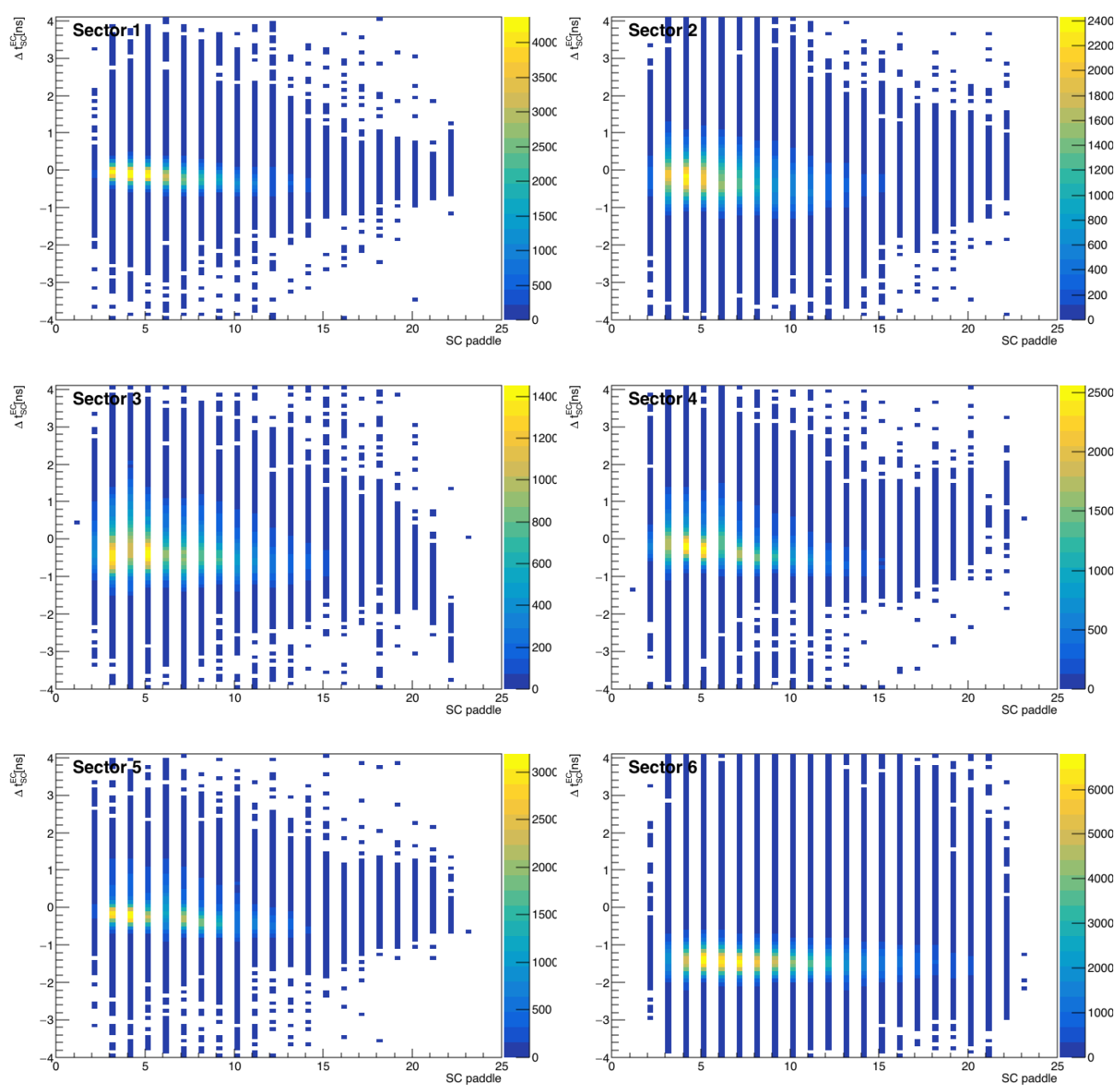

FIG. 66: The $\Delta t_{\mathrm{EC}}$ as a function of SC paddles in different CLAS sectors for electrons for ${ }^{3} \mathrm{He}$ at $4.4 \mathrm{GeV}$.

with respect to the SC timing for each of the CLAS sectors. We have then calibrated the EC timing for each of the sectors. To do so we have calculated $\Delta t_{\mathrm{EC}}$, that is given by the following expression:

$$
\Delta t_{\mathrm{EC}}=t_{\mathrm{EC}}-t_{\mathrm{SC}}-\frac{r_{\mathrm{EC}}-r_{\mathrm{SC}}}{c}
$$

We plotted $\Delta t_{\mathrm{EC}}$ as a function of $\mathrm{SC}$ paddle for different sectors to make sure that it is not SC dependent for a given sector, see Figs. 66, 67 and 68 . We then plotted $\Delta t_{\mathrm{EC}}$ for different sectors for a given target and beam energy and have fit the corresponding distribution with a Gaussian function. The mean of the fit is the EC timing to center the corresponding distribution at 0 . We then used these correction factors to correct $\Delta t_{\mathrm{EC}}$. The 

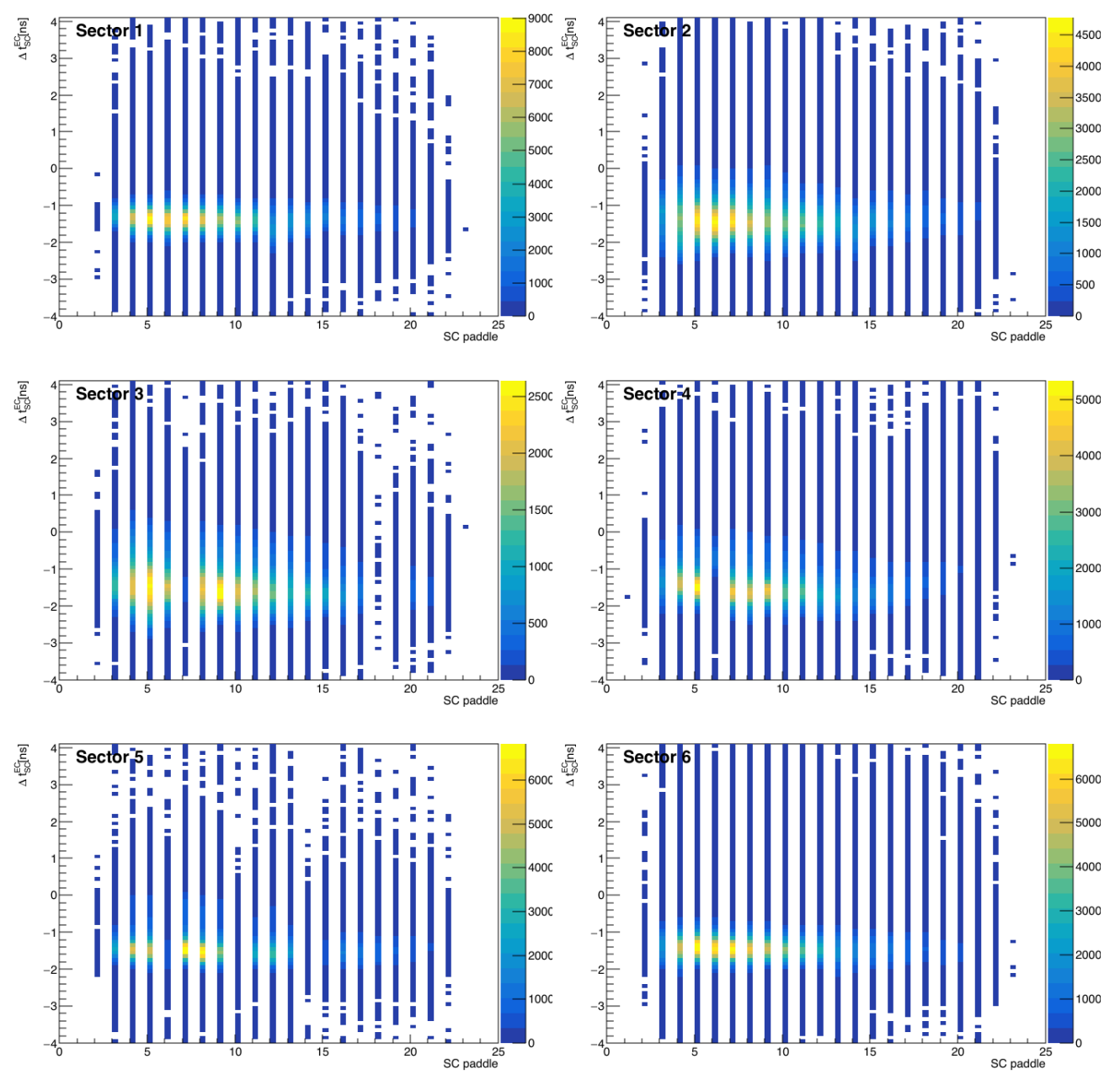

FIG. 67: The $\Delta t_{\mathrm{EC}}$ as a function of $\mathrm{SC}$ paddles in different CLAS sectors for electrons for ${ }^{3} \mathrm{He}$ at $2.2 \mathrm{GeV}$.

$\Delta t_{\mathrm{EC}}$ distributions are shown in Figs. 69, 70 and 71. The EC time resolution after the time calibration is about $0.33 \mathrm{~ns}$.

We then calculated the velocity of neutral particles detected in the EC using the corrected EC time and have cut $2 \sigma$ to the left of the photon peak to select photons. To calculate the corrected photon distribution we have used the following formula:

$$
\begin{gathered}
\beta_{\mathrm{EC}}=\frac{r_{\mathrm{EC}}}{t_{\mathrm{EC}}^{\text {corr }} c} \\
t_{\mathrm{EC}}^{\text {corr }}=t_{\mathrm{EC}}-\Delta t_{\mathrm{EC}}^{\text {offset }} \\
r_{\mathrm{EC}}=\sqrt{\left(x_{\mathrm{EC}}-x_{\mathrm{vert}}\right)^{2}+\left(y_{\mathrm{EC}}-y_{\mathrm{vert}}\right)^{2}+\left(z_{\mathrm{EC}}-z_{\mathrm{vert}}\right)^{2}}
\end{gathered}
$$



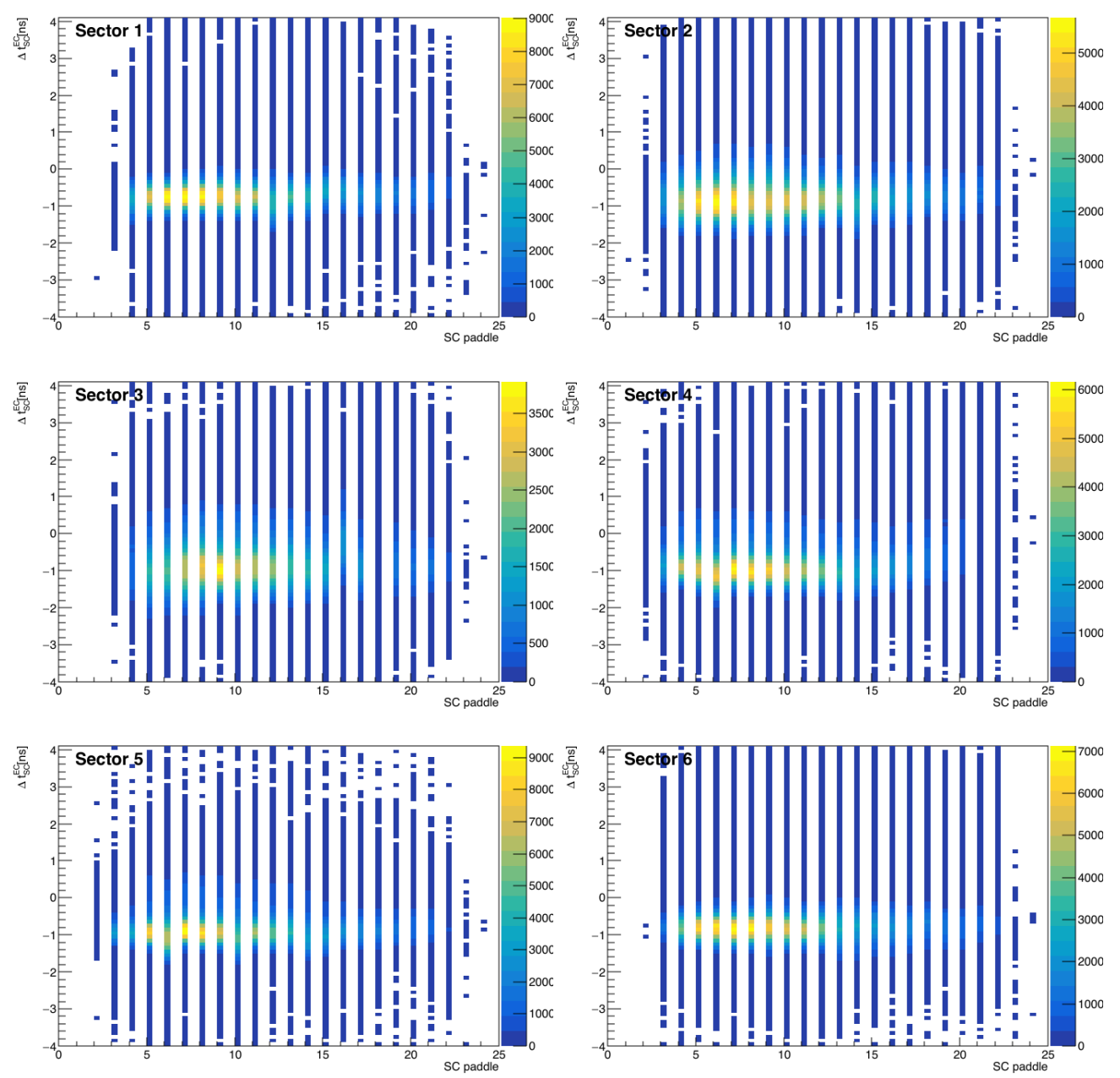

FIG. 68: The $\Delta t_{\mathrm{EC}}$ as a function of $\mathrm{SC}$ paddles in different CLAS sectors for electrons for ${ }^{3} \mathrm{He}$ at $1.1 \mathrm{GeV}$.

where $t_{\mathrm{EC}}$ is the electron time from $\mathrm{EC}, \Delta t_{\mathrm{EC}}^{\text {offset }}$ is the correction factor for $\mathrm{EC}$ timing, $x_{\mathrm{EC}}, y_{\mathrm{EC}}, z_{\mathrm{EC}}$ are the coordinates of the electron hit on $\mathrm{EC}$ and $x_{\text {vert }}, y_{\text {vert }}, z_{\text {vert }}$ are the electron vertex components.

The $\beta$ distributions of neutral particles before and after corrections with the corresponding cut value at 1.1, 2.2 and $4.4 \mathrm{GeV}$ are shown in Figs. 63 and 64. The correction improves the $\beta$ distributions, centering the photon peak at $\beta=1$. Also the photon peak in $\beta$ distribution of ${ }^{4} \mathrm{He}$ target at $2.2 \mathrm{GeV}$ becomes symmetric after the applied correction. The EC timing corrections and cuts applied on neutral particle velocity to select photons are summarized in Table 8. 

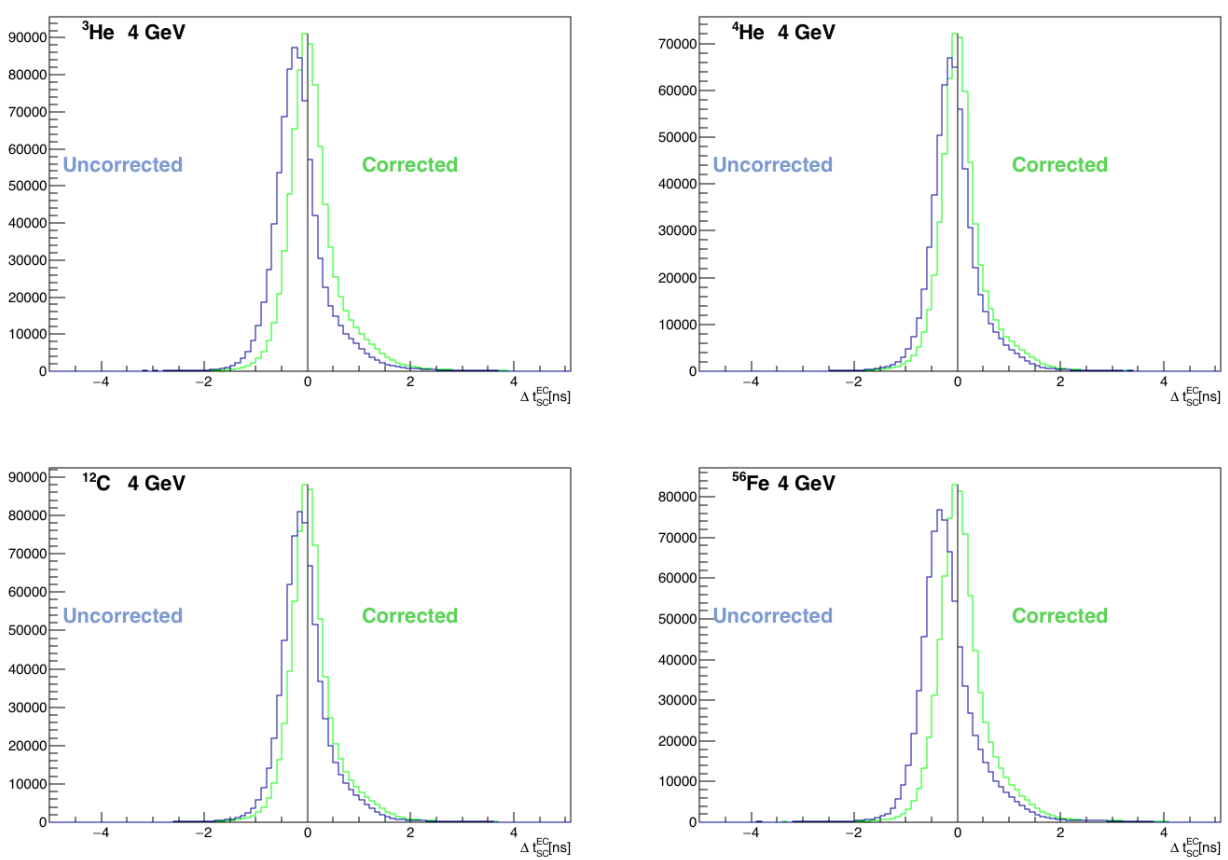

FIG. 69: The $\Delta t_{\mathrm{EC}}$ distributions before and after corrections plotted for all sectors together for electrons at $4.4 \mathrm{GeV}$ for all targets. The vertical line corresponds to $\Delta t=0$ and is made to guide the eye.

The energy and momentum thresholds for different particles are summarized in Table 9.

\subsection{PROTON ENERGY LOSS CORRECTION}

The proton energy loss correction function for all three beam energies is the same as the one used in [58] and the CLAS approved analysis [56].

Protons lose energy in the target and detector materials they pass through before being detected. While this energy loss is small for high momentum protons, it becomes significant at lower momenta and can be up to $50 \mathrm{MeV} / \mathrm{c}$.

The plot of the difference between reconstructed and generated proton momenta as a function of reconstructed momentum is shown in Fig. 72 [58]. The black vertical line shows the momentum threshold we have selected for protons, as below that the CLAS efficiency for proton detection is not know well. 


\begin{tabular}{lcccccccc}
\multirow{2}{*}{ Beam energy } & Target & Cuts on & \multicolumn{7}{c}{$\Delta t_{\text {EC }}^{\text {offset }}$ for different sectors } \\
& & $\beta_{\mathrm{EC}}$ & 1 & 2 & 3 & 4 & 5 & 6 \\
\hline \multirow{2}{*}{$1.1 \mathrm{GeV}$} & ${ }^{3} \mathrm{He}$ & 0.89 & -0.73 & -0.81 & -0.91 & -0.94 & -0.92 & -0.81 \\
& ${ }^{12} \mathrm{C}$ & 0.89 & -0.71 & -0.77 & -0.87 & -0.91 & -0.89 & -0.79 \\
\hline \multirow{3}{*}{$2.2 \mathrm{GeV}$} & ${ }^{3} \mathrm{He}$ & 0.93 & -1.37 & -1.42 & -1.55 & -1.53 & -1.49 & -1.44 \\
& ${ }^{4} \mathrm{He}$ & 0.92 & 0.72 & 0.27 & 0.16 & 0.21 & 0.22 & 0.21 \\
& ${ }^{12} \mathrm{C}$ & 0.92 & 0.50 & 0.39 & 0.29 & 0.29 & 0.32 & 0.33 \\
& ${ }^{56} \mathrm{Fe}$ & 0.90 & 0.75 & 0.49 & 0.37 & 0.39 & 0.43 & 0.44 \\
\hline \multirow{4}{*}{$4.4 \mathrm{GeV}$} & ${ }^{3} \mathrm{He}$ & 0.92 & -0.15 & -0.26 & -0.41 & -0.29 & -0.25 & -0.23 \\
& ${ }^{4} \mathrm{He}$ & 0.91 & -0.01 & -0.11 & -0.23 & -0.26 & -0.21 & -0.09 \\
& ${ }^{12} \mathrm{C}$ & 0.92 & -0.01 & -0.11 & -0.23 & -0.27 & -0.21 & -0.08 \\
& ${ }^{56} \mathrm{Fe}$ & 0.91 & -0.49 & -0.14 & -0.32 & -0.25 & -0.17 & -0.35
\end{tabular}

TABLE 8: The EC timing offsets for different sectors in ns and the cuts applied on the velocity of neutral particles to select photons for all targets at 2.2 and $4.4 \mathrm{GeV}$.

\begin{tabular}{|l|l|l|l|}
\hline Particle type & $1.1 \mathrm{GeV}$ & $2.2 \mathrm{GeV}$ & $4.4 \mathrm{GeV}$ \\
\hline$e^{-}$ & $p>0.4 \mathrm{GeV} / \mathrm{c}$ & $p>0.55 \mathrm{GeV} / \mathrm{c}$ & $p>1.1 \mathrm{GeV} / \mathrm{c}$ \\
$p$ & $p>0.3 \mathrm{GeV} / \mathrm{c}$ & $p>0.3 \mathrm{GeV} / \mathrm{c}$ & $p>0.3 \mathrm{GeV} / \mathrm{c}$ \\
$\pi^{-}$ & $p>0.15 \mathrm{GeV} / \mathrm{c}$ & $p>0.15 \mathrm{GeV} / \mathrm{c}$ & $p>0.15 \mathrm{GeV} / \mathrm{c}$ \\
$\pi^{-}$ & $p>0.15 \mathrm{GeV} / \mathrm{c}$ & $p>0.15 \mathrm{GeV} / \mathrm{c}$ & $p>0.15 \mathrm{GeV} / \mathrm{c}$ \\
photon & $E>0.3 \mathrm{GeV}$ & $E>0.3 \mathrm{GeV}$ & $E>0.3 \mathrm{GeV}$ \\
\hline
\end{tabular}

TABLE 9: The energy and momentum thresholds for different particles at all beam energies. 

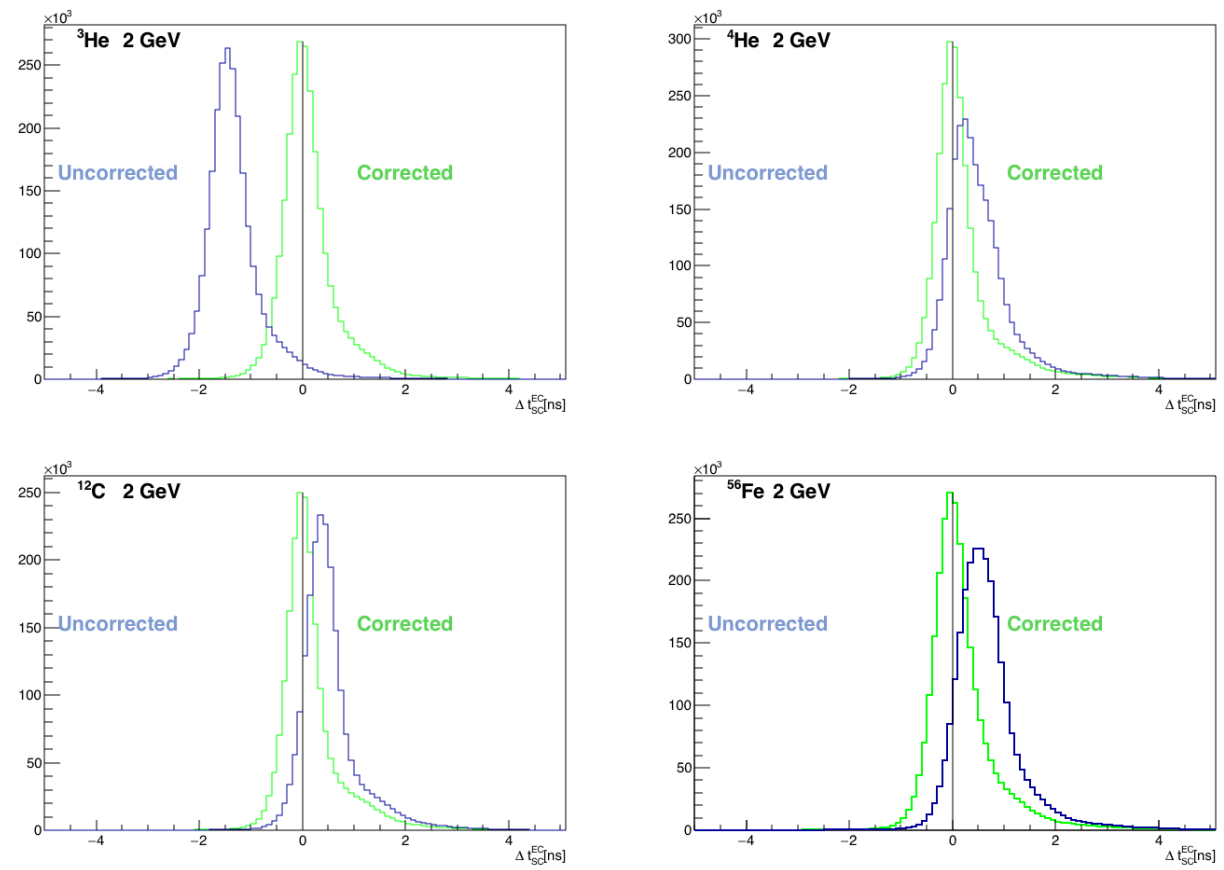

FIG. 70: The $\Delta t_{\mathrm{EC}}$ distributions before and after corrections plotted for all sectors together for electrons at $2.2 \mathrm{GeV}$ for all targets. The vertical line corresponds to $\Delta t=0$ and is made to guide the eye.
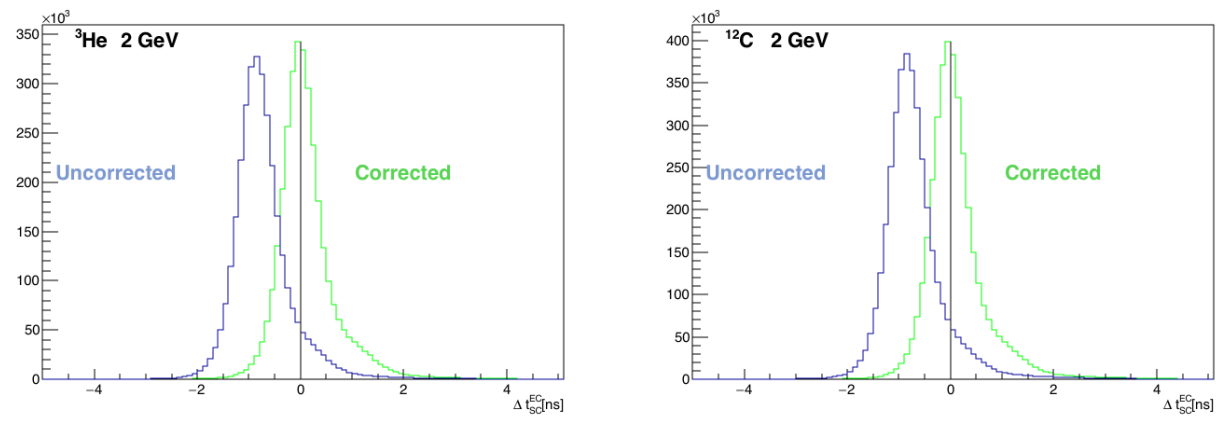

FIG. 71: The $\Delta t_{\mathrm{EC}}$ distributions before and after corrections plotted for all sectors together for electrons at $1.1 \mathrm{GeV}$ for all targets. The vertical line corresponds to $\Delta t=0$ and is made to guide the eye. 


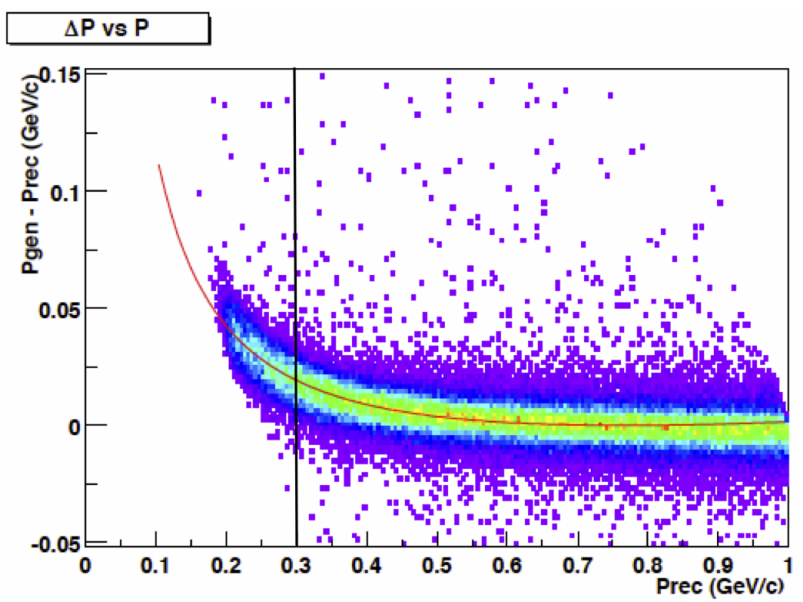

FIG. 72: The difference between reconstructed and generated proton momenta as a function of reconstructed momentum [58]. The results are shown for the ${ }^{3}$ He target and 2550A torus current setting at $2.2 \mathrm{GeV}$.
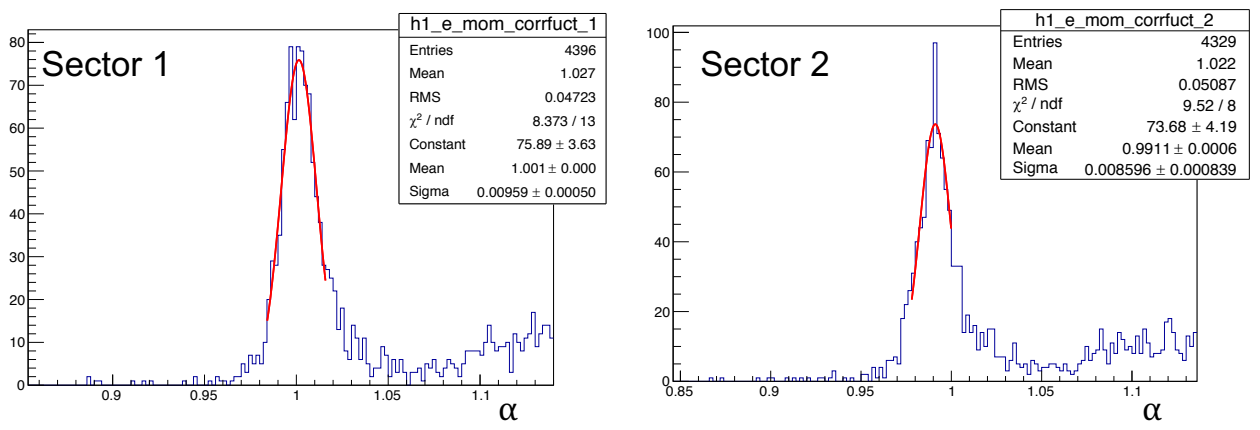

FIG. 73: The distributions of electron momentum corrections factors for sector 1 and 2 at $2.2 \mathrm{GeV}$, with a Gaussian fit, showing the location of the mean. The results include proton energy loss corrections. 


\subsection{ELECTRON MOMENTUM CORRECTIONS}

\subsection{GEV ANALYSIS}

The electron momentum measurement has errors due to different factors including inaccuracies in our knowledge of the torus magnetic field and in the location of the drift chambers. This can shift the measured electron momentum from its correct value by $1-2 \%$.

The data was reprocessed with a different torus magnetic field than was used in 1999, and the original electron momentum corrections were no longer valid.

After correcting the proton for energy loss, we plotted the ${ }^{3} \mathrm{He}\left(e, e^{\prime} p p\right) n$. The neutron missing mass was shifted for three beam energies, and thus electron momentum needs to be corrected.

The plots of missing mass as a function of electron $\phi$ angle at different energies (left plot in Figs. 74, 76 and 78) suggest that there is not much dependence on $\phi$ inside each sector, and a single multiplicative factor $\alpha$ can be used for each of the sectors at a given beam energy to correct the electron momentum. In order to find this factor, we calculated the missing mass $M M^{2}=\left(P_{{ }^{3} \mathrm{He}}+P_{\text {beam }}-P_{p_{1}}-P_{p_{2}}-P_{e}\right)^{2}$ for ${ }^{3} \mathrm{He}\left(e, e^{\prime} p p\right) n$ events in terms of the four momenta of the electron $P_{e}=\left(\alpha p_{e}, \alpha \vec{p}_{e}\right)$, two protons $P_{p_{1}}, P_{p_{2}}$, target $P_{3 \mathrm{He}}$ and beam electron $P_{\text {beam }}$ and solve it to find $\alpha$ for $M M^{2}=M_{n}^{2}$, where $M_{n}^{2}$ is the squared neutron mass. This results in the following expression for $\alpha$ :

$$
\alpha=\frac{-0.5\left(M_{n}^{2}-P^{2}\right)}{E p_{e}-\vec{P} \cdot \vec{p}_{e}}
$$

where $P=(E, \vec{P})=P_{3_{\mathrm{He}}}+P_{\text {beam }}-P_{p_{1}}-P_{p_{2}}$.

We calculated $\alpha$ for each target, see Fig. 73. We take the mean from the Gaussian fit as the value of the correction factor for a given sector. The $\alpha$ is the largest for sector 2 , We have used the same set of $\alpha$ values for all targets at each beam energy. As expected, the 2.2 and $4.4 \mathrm{GeV}$ analyses yield the same $\alpha$, as the magnetic field was the same at both energies, see Table 10 .

The missing mass distributions before and after corrections are shown in Figs. 74 and 75 for $4.4 \mathrm{GeV}$, Figs. 76 and 77 for $2.2 \mathrm{GeV}$ and Figs. 78 and 79 for $1.1 \mathrm{GeV}$.

We did not apply this correction to the proton momentum, as protons have smaller momenta than electrons, and the corresponding errors are smaller. 


\begin{tabular}{||c|c|c||}
\hline Sector & $1.1 \mathrm{GeV}$ & 2.2 and $4.4 \mathrm{GeV}$ \\
\hline \hline 1 & 1.007 & 1.001 \\
\hline 2 & 0.988 & 0.991 \\
\hline 3 & 1.008 & 1.005 \\
\hline 4 & 1.011 & 1.004 \\
\hline 5 & 1.014 & 1.006 \\
\hline 6 & 1.013 & 1.005 \\
\hline
\end{tabular}

TABLE 10: Electron momentum correction factors for different sectors at 1.1, 2.2 and $4.4 \mathrm{GeV}$ for all targets.

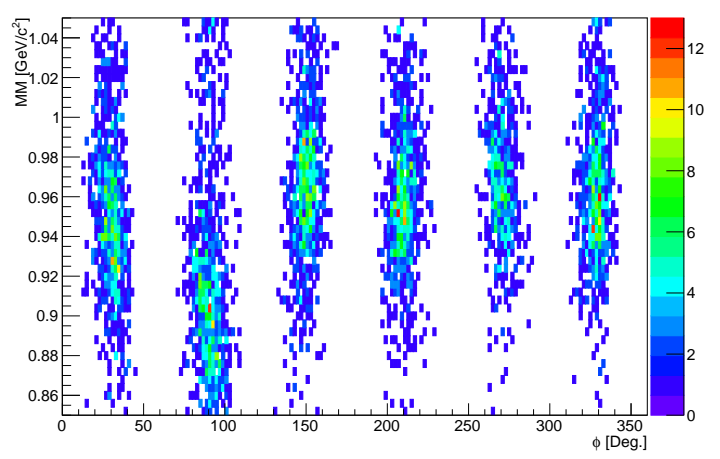

(a)

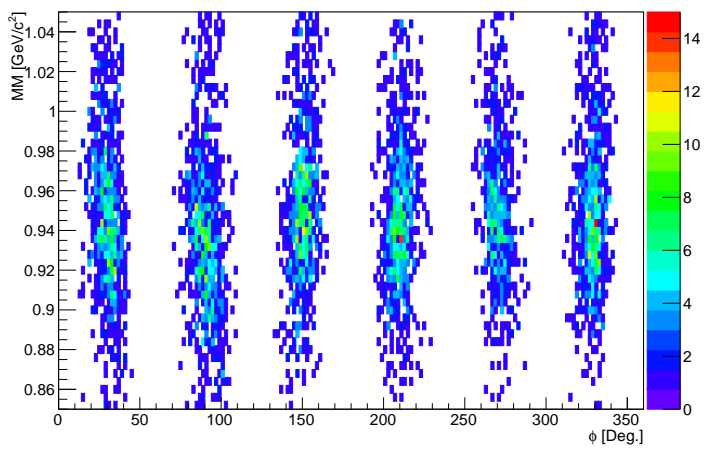

(b)

FIG. 74: The ${ }^{3} \mathrm{He}\left(e, e^{\prime} p p\right) n$ missing mass distributions as a function of electron $\phi$ angle a) without and b) with electron momentum corrections. The results are shown at $4.4 \mathrm{GeV}$ with proton energy loss corrections.

\subsection{ELECTRON FIDUCIAL CUTS}

Signals from different detectors are used for electron identification. Though the efficiencies of theses detectors are close to $100 \%$ around the middle of the sector, they might vary in some regions causing some systematic uncertainties in the physics analysis. This variations are due to the complicated structures of the detectors. 


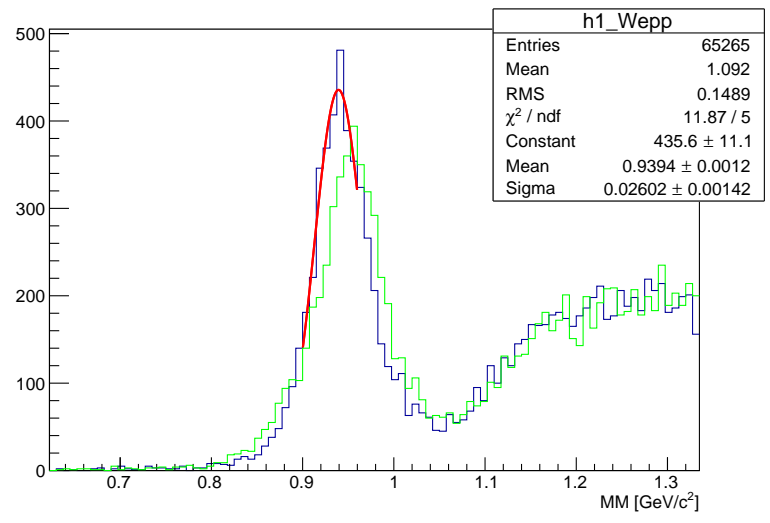

FIG. 75: The ${ }^{3} \mathrm{He}\left(e, e^{\prime} p p\right) n$ missing mass distributions before (green) and after (blue) electron momentum corrections at $4.4 \mathrm{GeV}$ with proton energy loss corrections. The Gaussian fit to the neutron peak is shown in red and the values of the fit parameters are given inside the box.

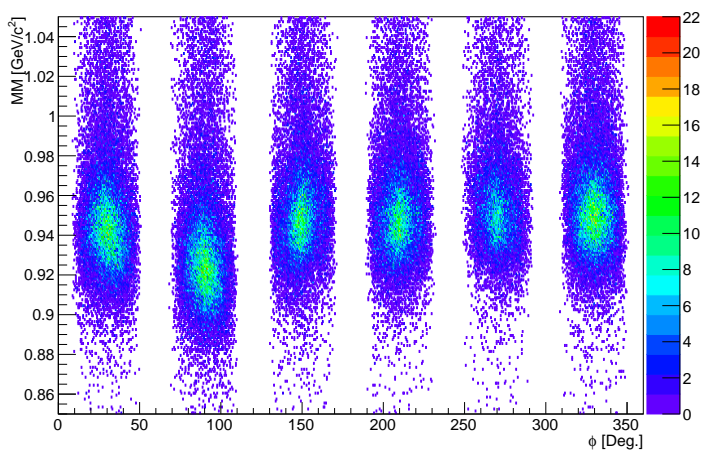

(a)

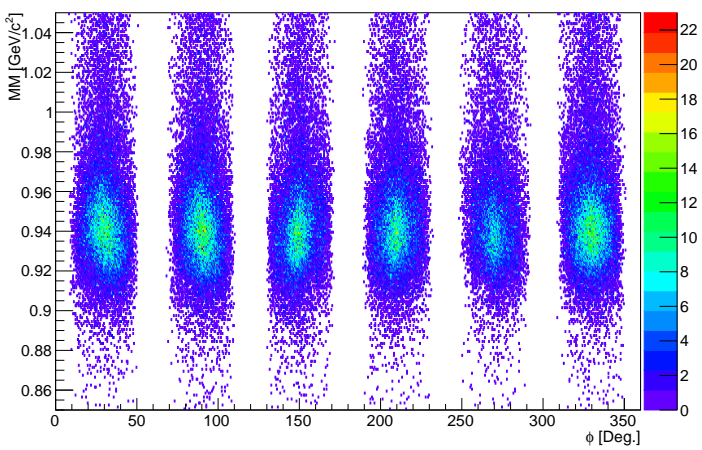

(b)

FIG. 76: The ${ }^{3} \mathrm{He}\left(e, e^{\prime} p p\right) n$ missing mass distributions as a function of electron $\phi$ angle a) without and b) with electron momentum corrections. The results are shown at $2.2 \mathrm{GeV}$ with proton energy loss corrections.

In order to select regions with uniform detector efficiencies (constant in $\phi$ ) a set of fiducial cuts were developed, which are different for different types of particles and different magnetic field settings.

We use the fiducial cuts previously approved for 2.2 [56] and $4.4 \mathrm{GeV}$ [56] which are 


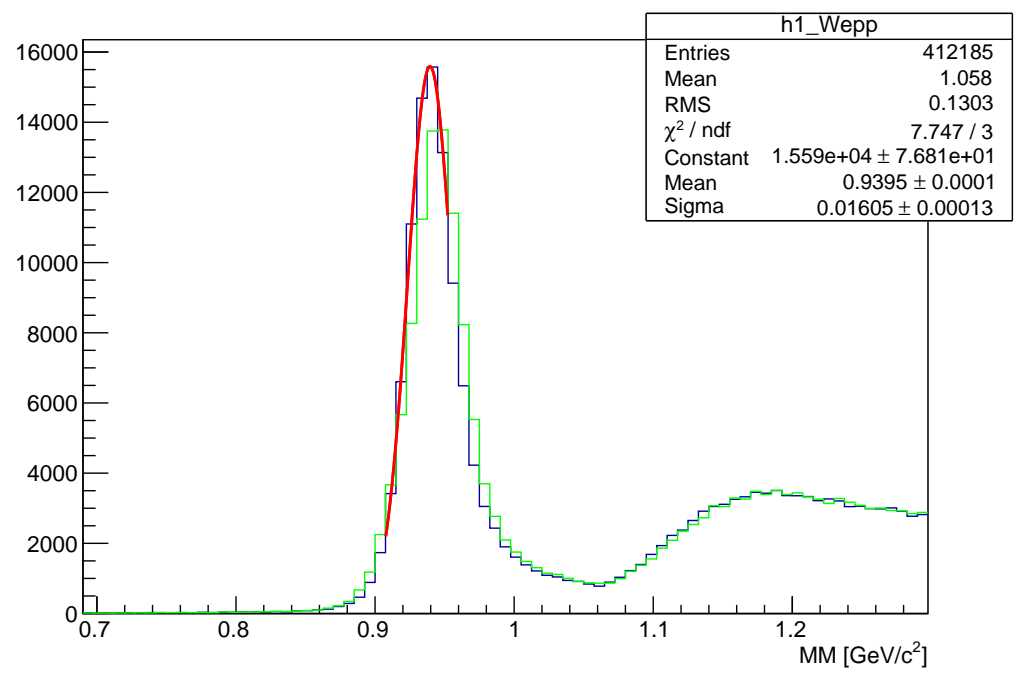

FIG. 77: The ${ }^{3} \mathrm{He}\left(e, e^{\prime} p p\right) n$ missing mass distributions before (green) and after (blue) electron momentum corrections at $2.2 \mathrm{GeV}$ with proton energy loss corrections. The Gaussian fit to the neutron peak is shown in red and the values of the fit parameters are given inside the box.

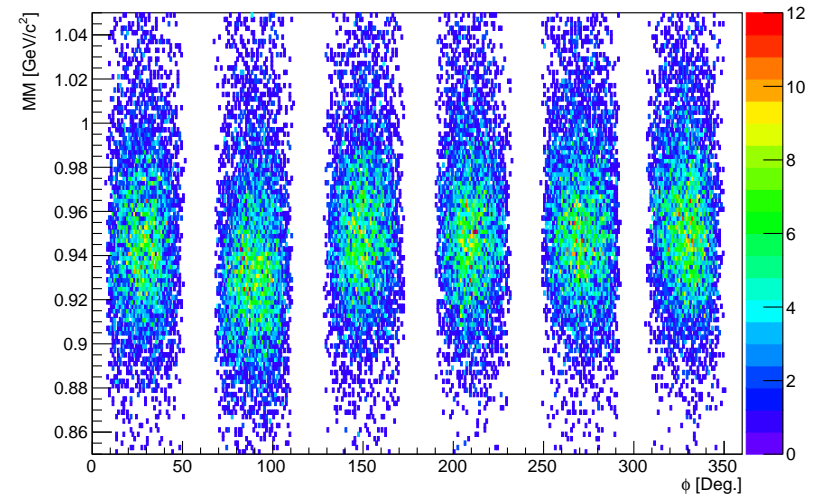

(a)

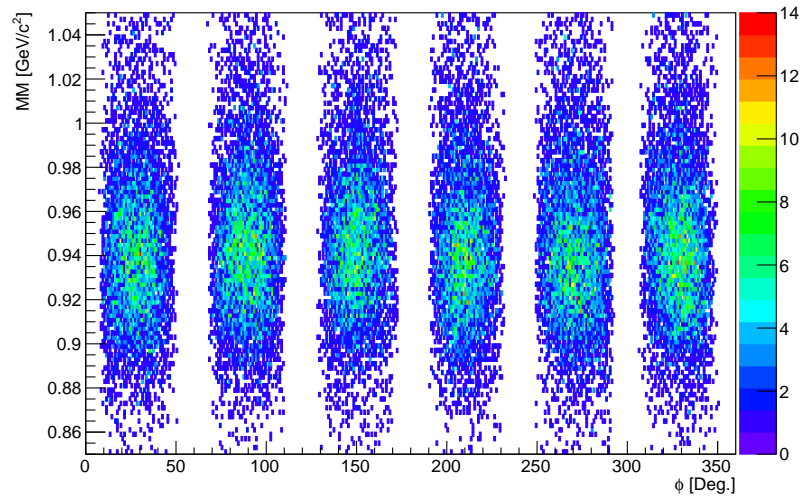

(b)

FIG. 78: The ${ }^{3} \mathrm{He}\left(e, e^{\prime} p p\right) n$ missing mass distributions as a function of electron $\phi$ angle a) without and b) with electron momentum corrections. The results are shown at $1.1 \mathrm{GeV}$ with proton energy loss corrections. 


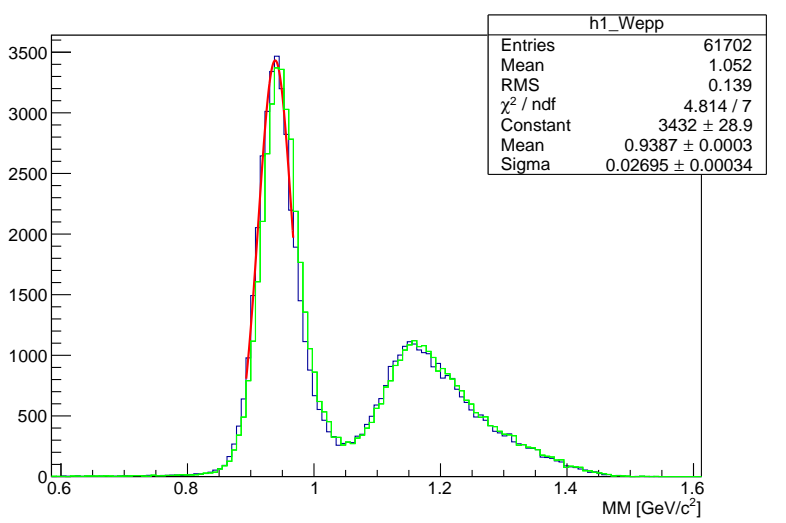

FIG. 79: The ${ }^{3} \mathrm{He}\left(e, e^{\prime} p p\right) n$ missing mass distributions before (green) and after (blue) electron momentum corrections at $1.1 \mathrm{GeV}$ with proton energy loss corrections. The Gaussian fit to the neutron peak is shown in red and the values of the fit parameters are given inside the box.

described in detail in [59] and [60].

\subsection{GEV ANALYSIS}

We first cut out the EC edges, to eliminate regions of smaller signal.

The $u, v, w$ cut regions for the $2 \mathrm{GeV}$ analysis were chosen individually for each sector, to eliminate the parts where the rising trends toward the edges end. As a result two or three strips were cut off. The $u, v, w$ cuts for different sectors are listed in Table 11. The plots for the $u, v, w$ distributions with corresponding cuts for sector 1 are shown in Fig. 80.

The electron $\theta$ vs $\phi$ angular distributions corresponding to different CLAS sectors were studied in $100 \mathrm{MeV} / \mathrm{c}$ momentum bins. Then the corresponding distributions were sliced in different $\theta$ bins, and the $\phi$ distributions for a given $\theta$ were fit with a trapezoidal function to determine the region where the acceptance is uniform. The two ends of the flat plateau region where parametrized as a function of momentum and scattering angle of the electron, to be later used to select electrons inside the plateau region.

We also eliminated regions of malfunctioning TOF counters. The entire $\theta$ range corresponding to each bad TOF scintillator was cut. Again the $\theta$ vs $\phi$ distribution of the electron detected in different sectors of CLAS was studied in different momentum bins and the lower and upper positions of the $\theta$ gaps were fit as a function of momentum. 


\begin{tabular}{lccc} 
Sector & $u$ & $v$ & $w$ \\
\hline 1 & $>60 \mathrm{~cm}$ & $<360 \mathrm{~cm}$ & $<400 \mathrm{~cm}$ \\
2 & $>55 \mathrm{~cm}$ & $<360 \mathrm{~cm}$ & $<400 \mathrm{~cm}$ \\
3 & $>50 \mathrm{~cm}$ & $<363 \mathrm{~cm}$ & $<400 \mathrm{~cm}$ \\
4 & $>52 \mathrm{~cm}$ & $<365 \mathrm{~cm}$ & $<396 \mathrm{~cm}$ \\
5 & $>60 \mathrm{~cm}$ & $<360 \mathrm{~cm}$ & $<398 \mathrm{~cm}$ \\
6 & $>50 \mathrm{~cm}$ & $<362 \mathrm{~cm}$ & $<398 \mathrm{~cm}$
\end{tabular}

TABLE 11: The table of cut values on the $u, v, w$ EC local coordinates of an electron for different sectors of CLAS, used to select the initial fiducial region used for the $2.2 \mathrm{GeV}$ analysis.

The electron $\theta$ vs $\phi$ distributions before and after fiducial cuts are shown for sector 1 for two electron momentum bins in Fig. 81 and the ones for sectors 3 and 4 for $p=2.5 \mathrm{GeV} / \mathrm{c}$ are shown in Fig. 82.

\subsection{GEV ANALYSIS}

The methods used to obtain the electron fiducial cuts for the $2.2 \mathrm{GeV}$ analysis are similar to the ones used in the $4.4 \mathrm{GeV}$ analysis and are again based on the CLAS approved analysis note [56]. The $u, v, w$ cuts for different sectors are the same as the ones listed in Table 11. The plots of the $u, v, w$ distributions with corresponding cuts for sector 5 are shown in Fig. 83.

The procedure of obtaining the fiducial cuts for $2.2 \mathrm{GeV}$ is described in detail in [60].

The plots of the electron $\theta$ vs $\phi$ distributions before and after fiducial cuts are shown for sector 5 and two different electron momentum bins in Fig. 84.

\subsection{GEV ANALYSIS}

We adopt the method for our fiducial cuts from [59], but a brief overview will be outlined in the following sections. The reasoning behind these cuts stems from the difficulty of understanding the efficiency of the CLAS detectors near the sector edges. In order to be able 


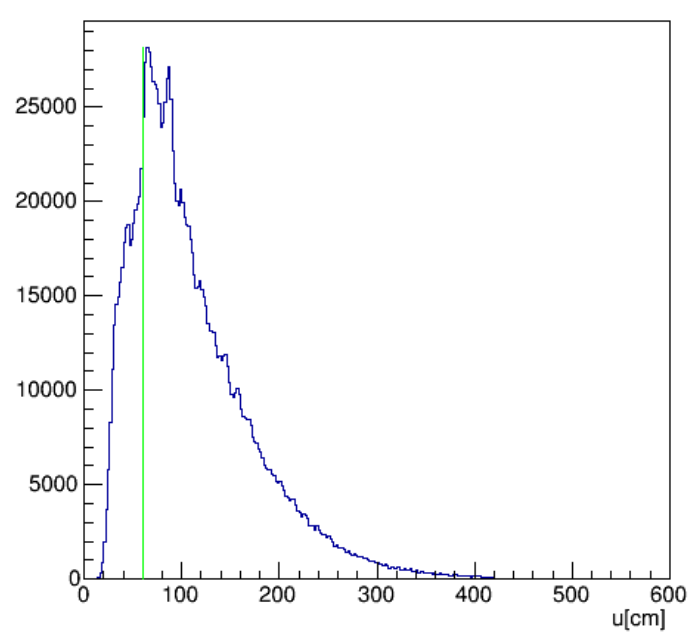

(a) u.

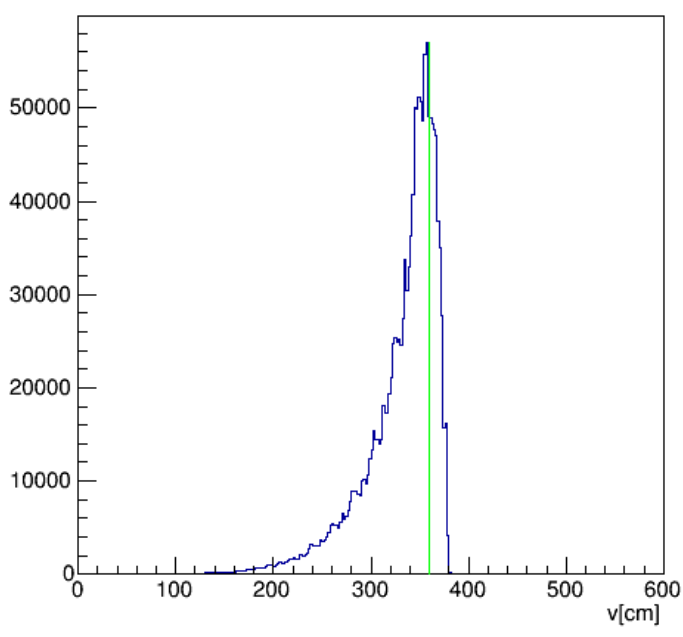

(b) v.

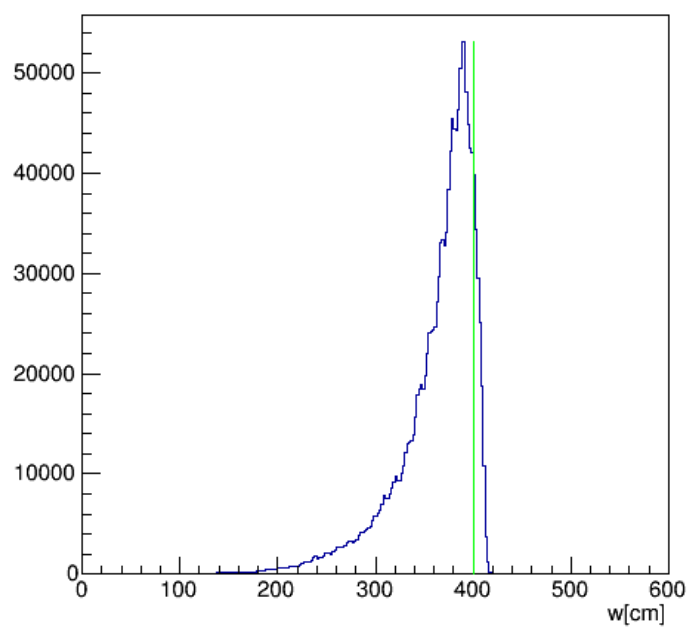

(c) w.

FIG. 80: The distributions for the electron EC local coordinates for sector 1. The green lines show the positions of the EC edge cuts. The results are shown for the ${ }^{3} \mathrm{He}$ target at $4.4 \mathrm{GeV}$.

to compare data to simulation and to make sure we are separating out detector effects from physics effects, we create fiducial boundaries that eliminate any particles detected outside of it. The fiducial cut defines a region in $(p, \theta, \phi)$ space where detection efficiency is constant in $\phi$ as a function of momentum and is created for each of the six sectors independently. 


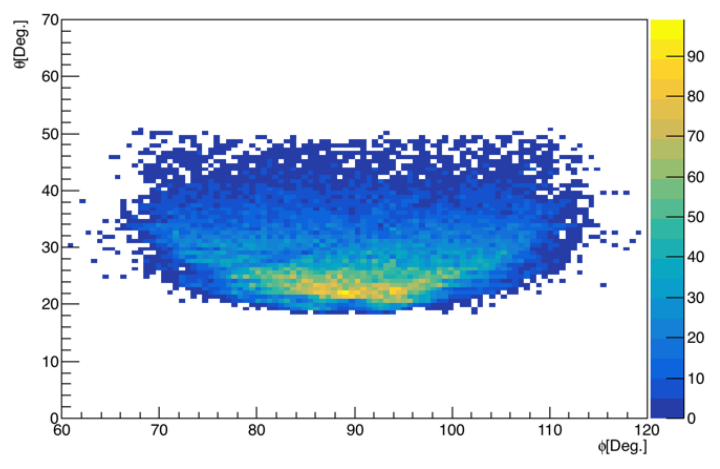

(a)

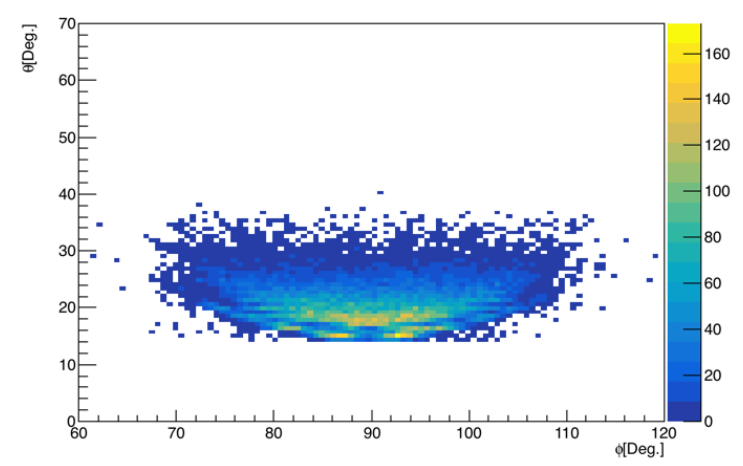

(c)

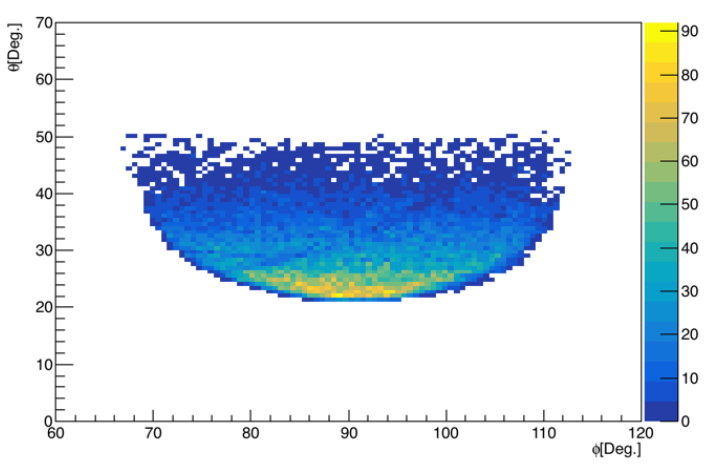

(b)

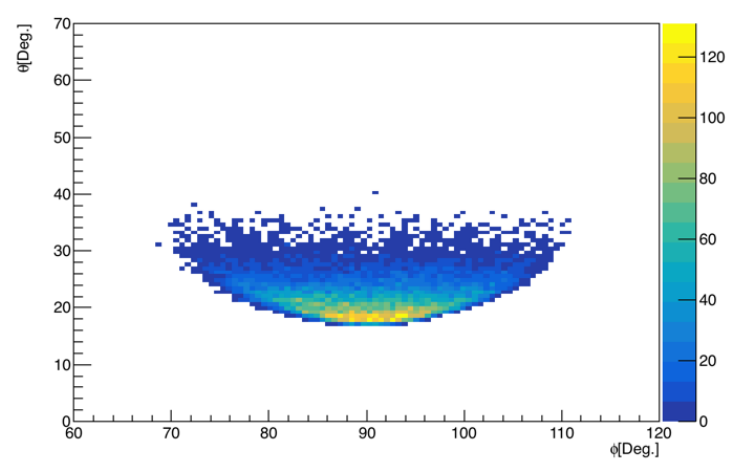

(d)

FIG. 81: The $\theta$ vs $\phi$ distributions for electron for two momentum bins in sector 1 , before and after fiducial cuts. a) and b) correspond to $p=1.4 \mathrm{GeV} / \mathrm{c}$ before and after fiducial cuts; c) and d) correspond to $p=2.5 \mathrm{GeV} / \mathrm{c}$ before and after fiducial cuts, respectively. The results are shown for the ${ }^{3} \mathrm{He}$ target at $4.4 \mathrm{GeV}$.

Any specificities regarding different particles will be noted in the appropriate section. The electron fiducial cuts are described in detail in Appendix C of Steve McLauchlan's thesis [61]. The fiducial cuts for the remaining particles and the bad scintillator cuts for all particles including electrons at $1 \mathrm{GeV}$ were created for this analysis.

The $u, v, w$ cuts for different sectors are the same as the ones listed in Table 11.

Defining Regions of Uniform Acceptance We binned the particles separately by sector and by momentum creating many plots of $\phi$ and $\theta$. All particles were binned from 0 to 1.1 $\mathrm{GeV} / \mathrm{c}$, electrons in steps of $50 \mathrm{MeV} / \mathrm{c}$ and the remaining particles in steps of $100 \mathrm{MeV} / \mathrm{c}$. For 


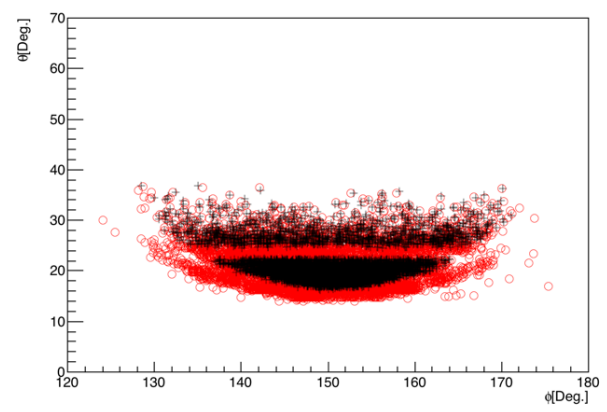

(a) Sector 3 .

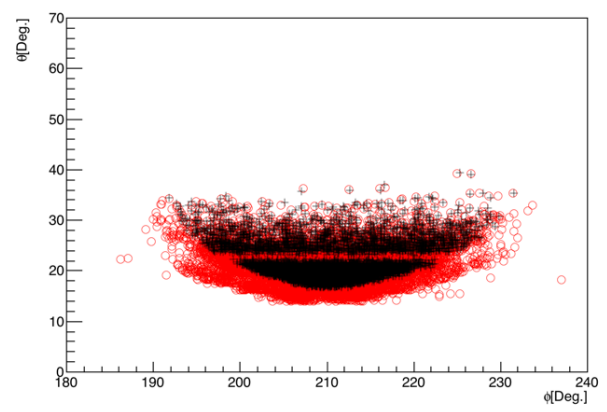

(b) Sector 4 .

FIG. 82: The $\theta$ vs $\phi$ distributions for electron for $p=2.5 \mathrm{GeV} / \mathrm{c}$ for different sectors, before (red) and after (black) fiducial cuts. The results are shown for the ${ }^{3} \mathrm{He}$ target at $4.4 \mathrm{GeV}$.

every degree in $\theta$ for electrons and every 2 degrees for the remaining particles, we plotted the $\phi$ distribution. We fit each projection with the trapezoid function seen in Eq. (55). Parameters $p_{1}$ and $p_{2}$ thus determine the upper and lower cut off values in $\phi$ for a specific bin of $\theta$ and momentum. Above $p_{3}$ and below $p_{0}$ the value of trapezoid function is zero. Examples of these fits are shown in Fig. 85.

$$
y= \begin{cases}0 & x \leq p_{0} \\ p_{4}\left(x-p_{0}\right) /\left(p_{1}-p_{0}\right) & p_{0}<x \leq p_{1} \\ p_{4} & p_{1}<x \leq p_{2} \\ p_{4}\left(x-p_{3}\right) /\left(p_{2}-p_{3}\right) & p_{2}<x \leq p_{3} \\ 0 & x>p_{3}\end{cases}
$$

Defining the Fiducial Function and Smoothing We plot $p_{1}$ and $p_{2}$ for all $\theta$ and fit each momentum bin using

$$
60(\text { sector }-1) \pm \phi(\theta)
$$

where the + and - signs correspond to the upper and lower boundaries, respectively, and where 


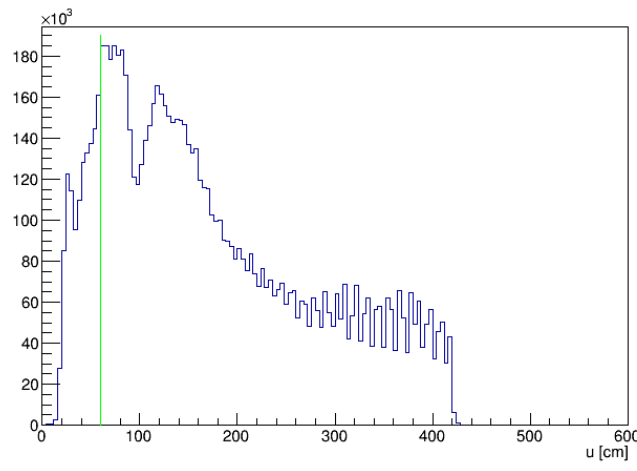

(a) u.

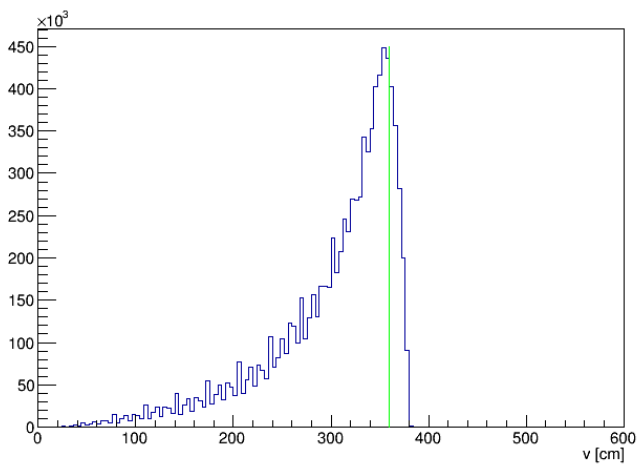

(b) v.

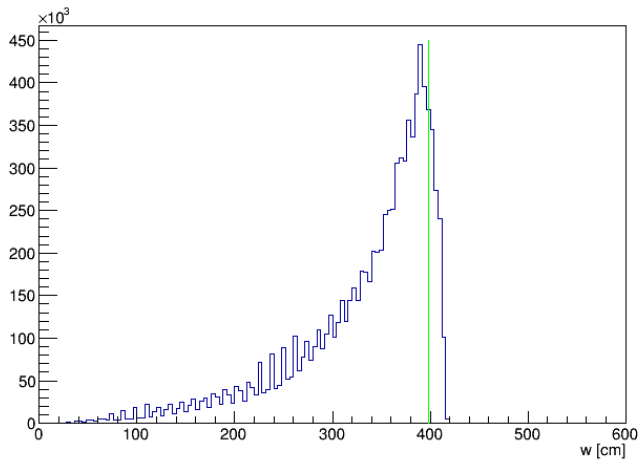

(c) w.

FIG. 83: The distributions of the electron EC local coordinates for sector 1. The green lines show the positions of the EC edge cuts. The results are shown for the ${ }^{3} \mathrm{He}$ target at $2.2 \mathrm{GeV}$.

$$
\phi(\theta)= \begin{cases}b\left(1-\left(\left(\theta-t_{0}\right) b / a+1\right)^{-1}\right) & t_{0}<\theta<t_{1} \\ 0 & \text { otherwise }\end{cases}
$$

Examples of these boundaries are seen in Fig. 86. Rather than using these discrete binned values, we then fit parameters $b, a$, and $t_{0}$ as polynomial functions of momentum in order to obtain a smooth, easily queried, and complete fiducial boundary. We choose to fit with a fifth order polynomial. Examples of this "smoothing" can be seen in Fig. 87.

Bad Scintillator Cut for Electrons Some time of flight detectors inefficient or dead. The detection efficiency in the theta range of these faulty paddles is difficult to understand and difficult to accurately reproduce in simulation. We treat these regions just like the 


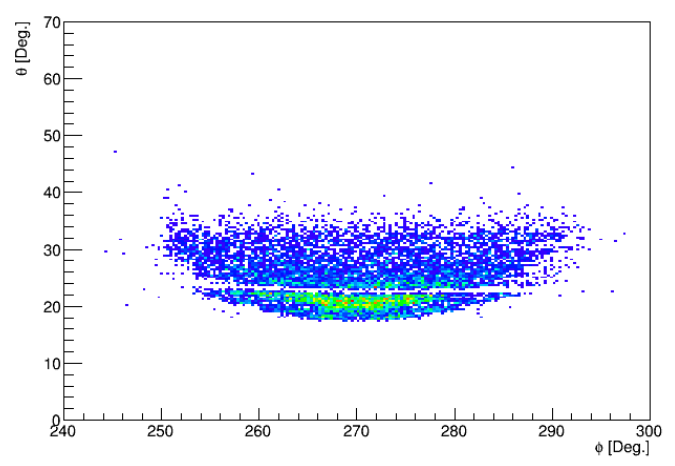

(a)

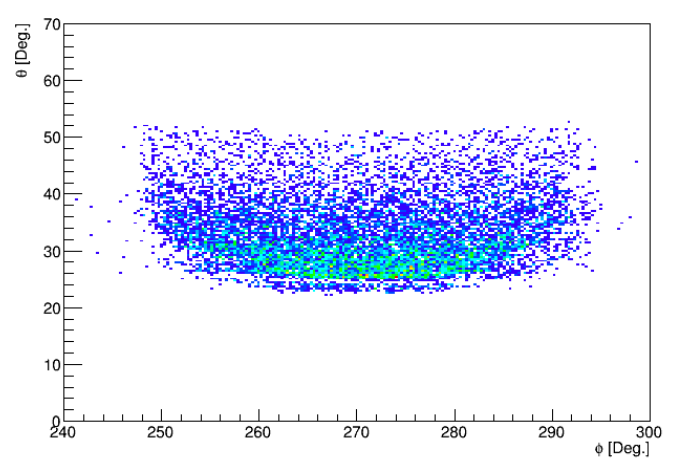

(c)

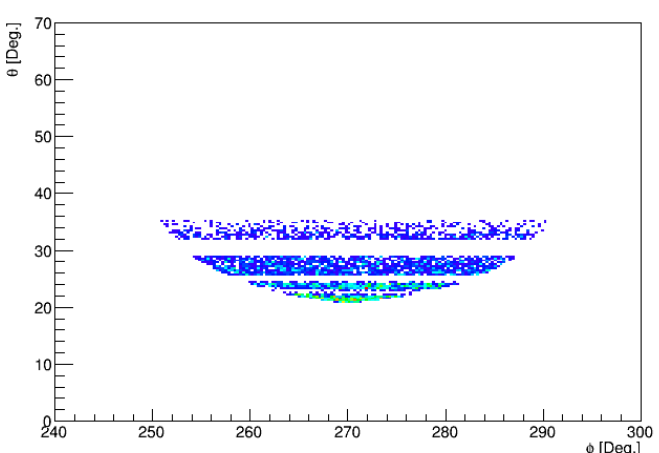

(b)

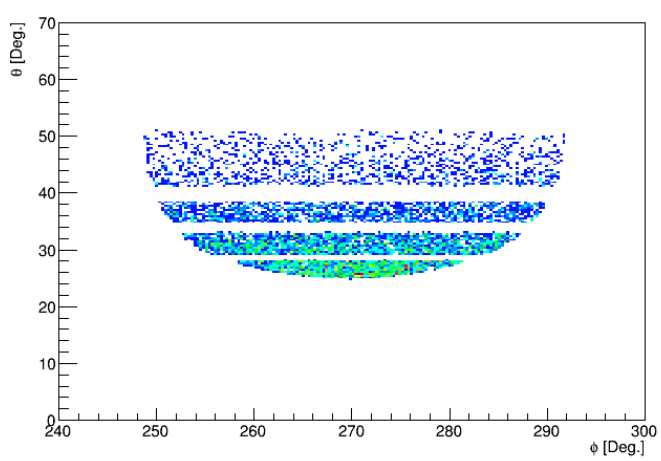

(d)

FIG. 84: The $\theta$ vs $\phi$ distributions of the electron for two momentum bins in sector 5 , before and after fiducial cuts. a) and b) correspond to $p=1.65 \mathrm{GeV} / \mathrm{c}$ before and after fiducial cuts; c) and d) correspond to $p=1 \mathrm{GeV} / \mathrm{c}$ before and after fiducial cuts, respectively. The results are shown for the ${ }^{3} \mathrm{He}$ target at $2.2 \mathrm{GeV}$.

regions close to the coils and cut them out in a similar fashion.

The work done in [60] finds the lower and upper bounds of each bad paddle in momentum steps of $50 \mathrm{MeV} / \mathrm{c}^{2}$. They then parameterize this as a function of momentum using a seventh order polynomial. We followed the same procedure to obtain the regions corresponding to bad paddles. We parameterized the lower and upper $\theta$ limits for a given bad TOF in each sector as a function of momentum using a function that is more well-behaved than a seventh order polynomial and that fits the momentum dependence as the momentum approaches 0 and infinity. Our functional form is 


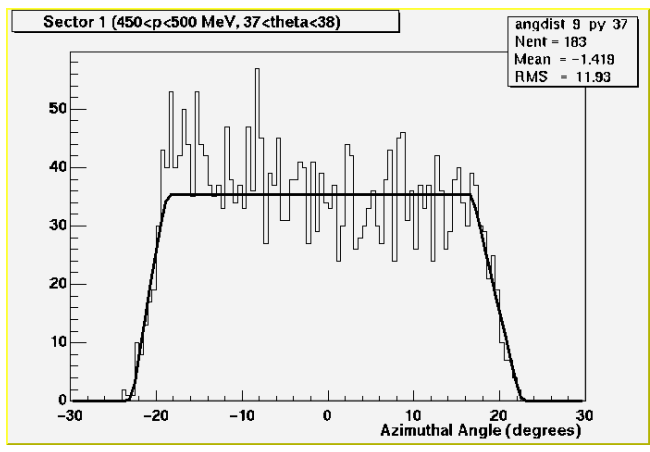

(a) Electrons located in sector 1 with momentum between 450 and $500 \mathrm{MeV} / \mathrm{c}$ and $\theta$ between 37 and 38 degrees

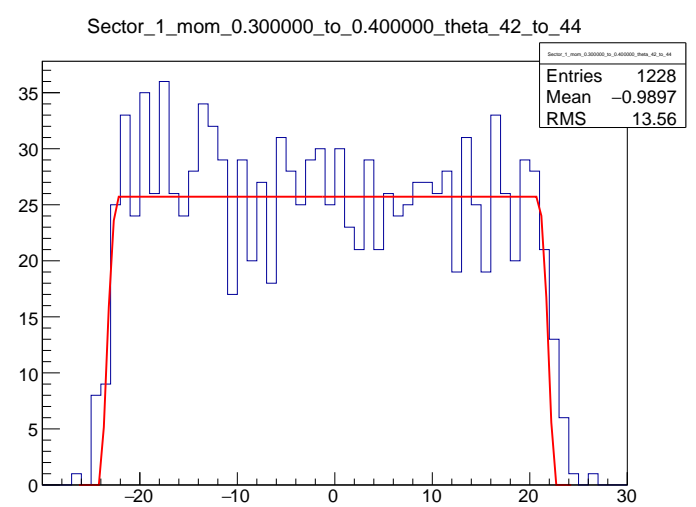

(c) $\pi^{-}$located in sector 1 with momentum between 300 and $400 \mathrm{MeV} / \mathrm{c}$ and $\theta$ between 42 and 44 degrees

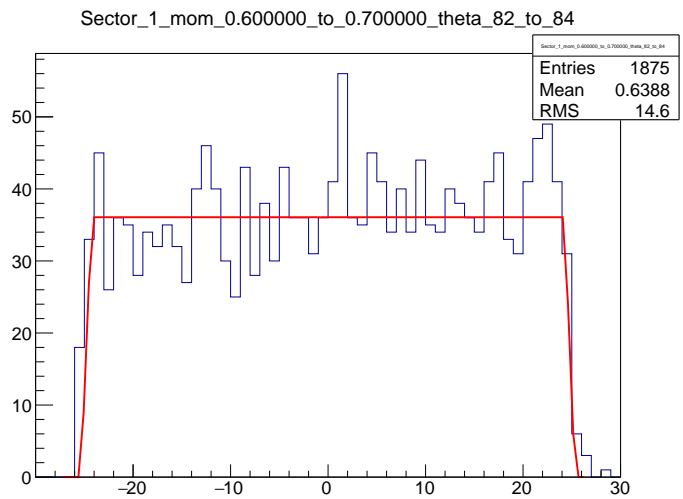

(b) Protons located in sector 1 with momentum between 600 and $700 \mathrm{MeV} / \mathrm{c}$ and $\theta$ between 82 and 84 degrees

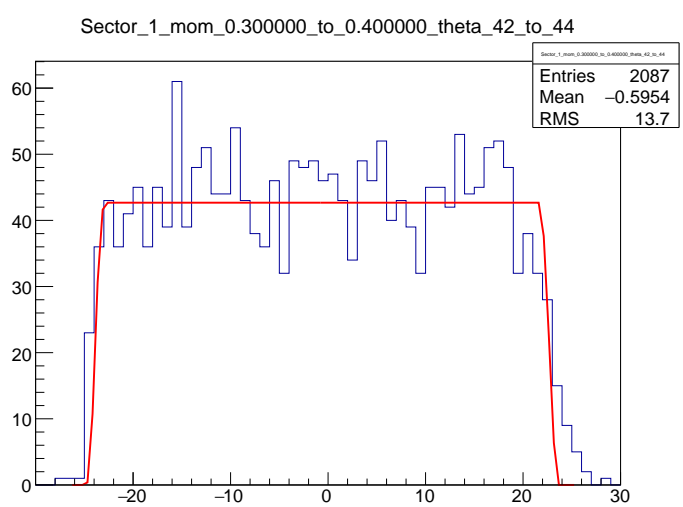

(d) $\pi^{+}$located in sector 1 with momentum between 300 and $400 \mathrm{MeV} / \mathrm{c}$ and $\theta$ between 42 and 44 degrees

FIG. 85: Example trapezoid fits

$$
p+\frac{1}{p}+\frac{1}{p^{2}}+\frac{1}{p^{3}}
$$

The electron $\theta$ vs $\phi$ distribution before and after fiducial cuts for different sectors of CLAS are shown in Fig. 88. If we compare the results at $1.1 \mathrm{GeV}$ to the ones at $2.2 \mathrm{GeV}$ for the same $p=1 \mathrm{GeV} / \mathrm{c}$ shown in Fig. 84, we can see that at $1.1 \mathrm{GeV}$ electrons avoid the bad TOF region seen by the electron at $2.2 \mathrm{GeV}$ analysis, as the torus current is nearly three times lower. Sectors 2, 3, 4 and 5 have bad TOF paddles [60]. 


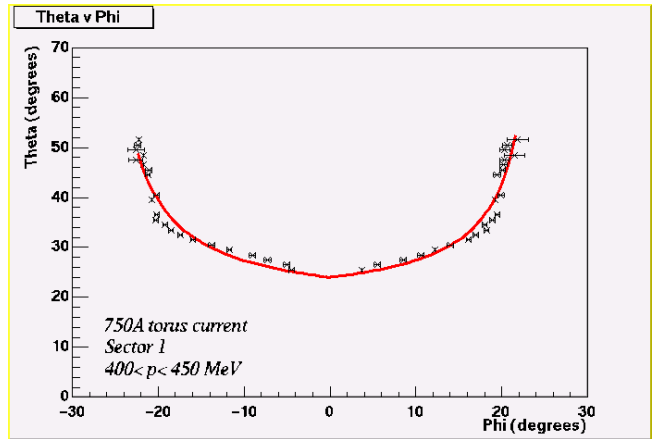

(a) Electrons located in sector 1 with momentum between 400 and $450 \mathrm{MeV} / \mathrm{c}$

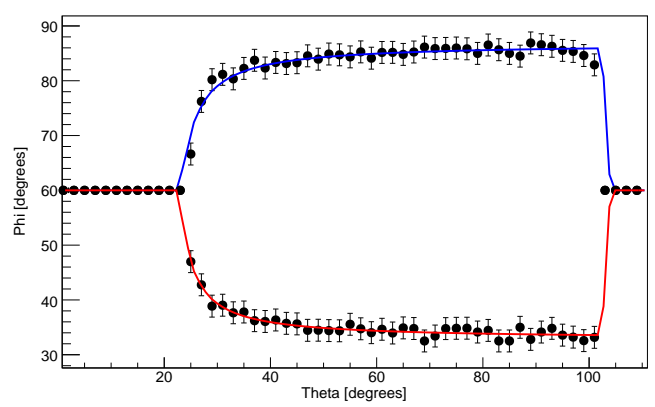

(c) $\pi^{-}$located in sector 2 with momentum between 300 and $400 \mathrm{MeV} / \mathrm{c}$

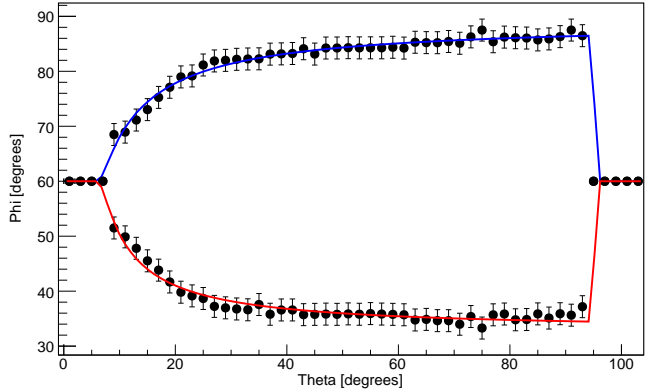

(b) Protons located in sector 2 with momentum between 700 and $800 \mathrm{MeV} / \mathrm{c}$

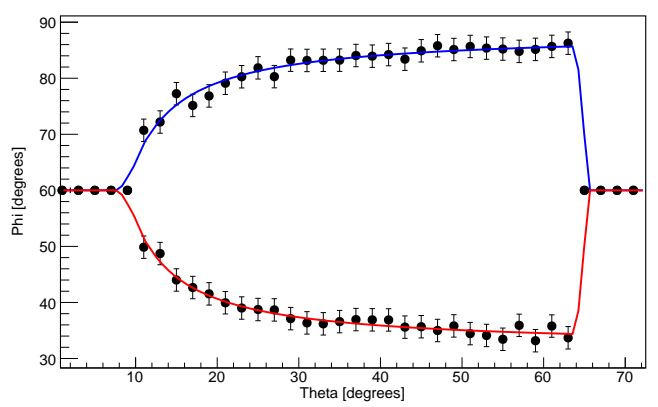

(d) $\pi^{+}$located in sector 2 with momentum between 500 and $600 \mathrm{MeV} / \mathrm{c}$

FIG. 86: Example fiducial boundary fits

The electron $\theta$ vs $\phi$ distribution before and after fiducial cuts for sectors 3 and 4 are shown in Fig. 88. 


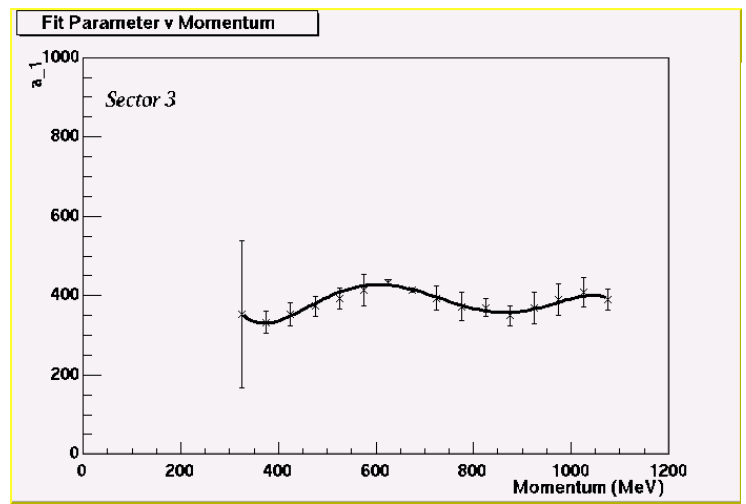

(a) Parameterizing the $a$ parameter for electrons in the lower half of sector 3

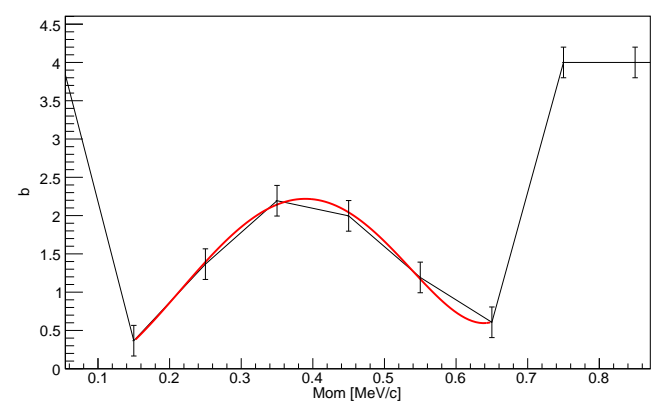

(c) Parameterizing the $b$ parameter for $\pi^{-}$ in the lower half of sector 2

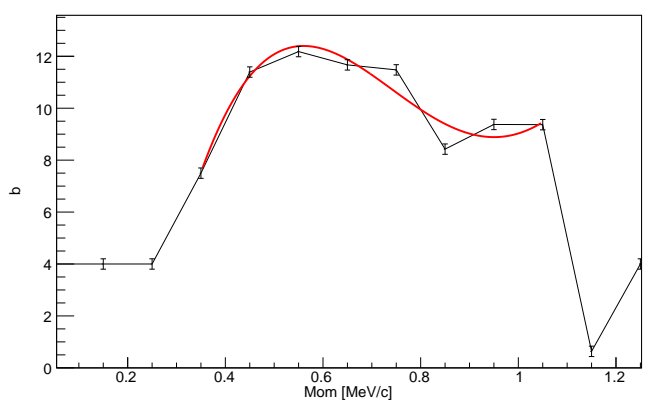

(b) Parameterizing the $b$ parameter for protons in the upper half of sector 1

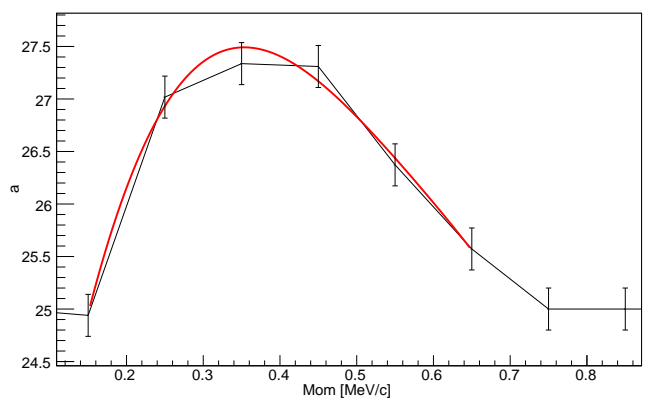

(d) Parameterizing the $a$ parameter for $\pi^{+}$ in the upper half of sector 3

FIG. 87: Example momentum fits

\subsection{PROTON FIDUCIAL CUTS}

\subsection{GEV ANALYSIS}

The analysis procedure to obtain the fiducial cuts for the proton is similar to that of the electron. Again the $\theta$ vs $\phi$ angular distributions were studied in different momentum bins as well as the information on $\theta$ gaps caused by bad scintillators was stored to select regions with flat efficiencies. The proton fiducial cuts for the $4.4 \mathrm{GeV}$ analysis are in the CLAS approved analysis note [56], and are described in detail in [62].

The $\theta$ vs $\phi$ distributions of the proton before and after fiducial cuts are shown for sector 

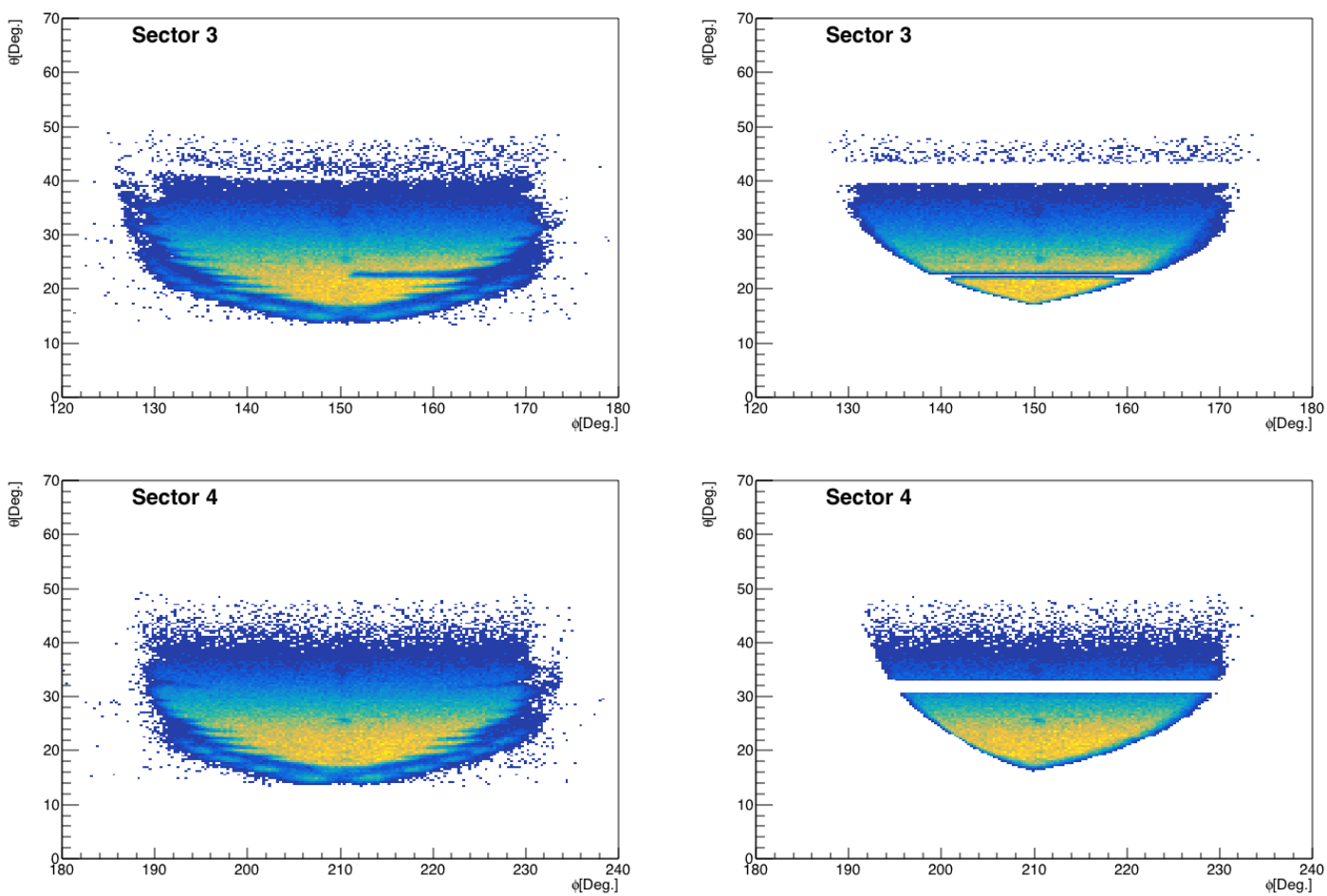

FIG. 88: The $\theta$ vs $\phi$ distributions for electrons with $p=1 \mathrm{GeV} / \mathrm{c}$ before (left plots) and after (right plots) fiducial cuts in sectors 3 and 4 . The results are shown for the ${ }^{3}$ He target at $1.1 \mathrm{GeV}$.

1 and momentum $p_{\mathrm{p}}=1 \mathrm{GeV} / \mathrm{c}$ in Fig. 89. We use the same fiducial cuts for all targets for given energy.

\subsection{GEV ANALYSIS}

The proton fiducial cuts for the $2.2 \mathrm{GeV}$ analysis are similar to the one at $4.4 \mathrm{GeV}$ and are the same as in the CLAS approved analysis note [56]. The detailed description of the analysis procedure can be found in [63].

The $\theta$ vs $\phi$ distributions of the proton before and after fiducial cuts are shown for sector 5 and momentum $p_{\mathrm{p}}=1 \mathrm{GeV} / \mathrm{c}$ in Fig. 90. 


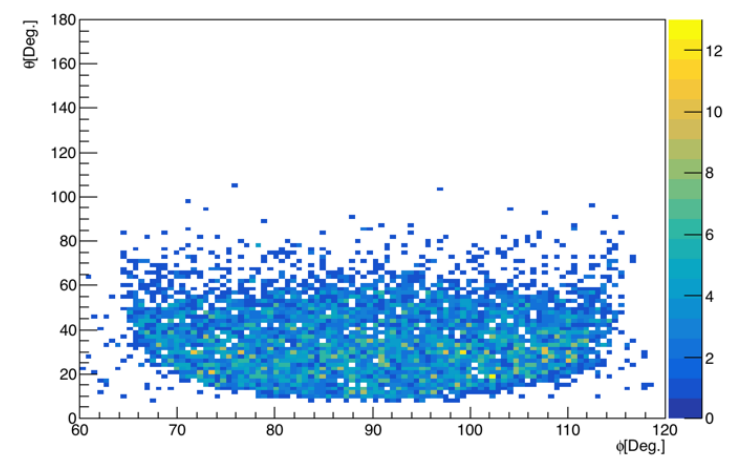

(a)

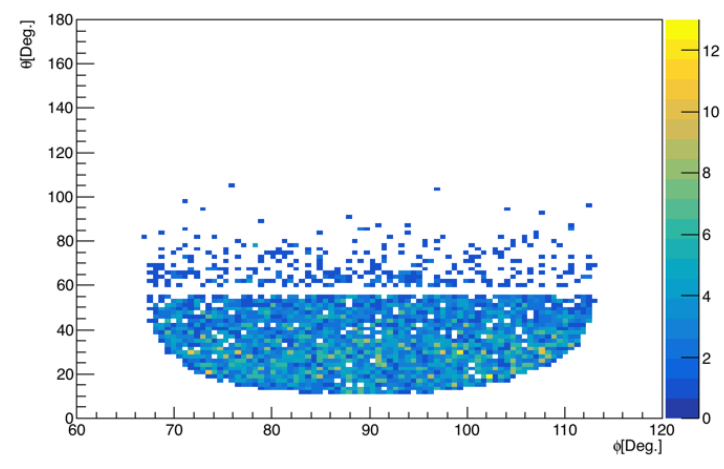

(b)

FIG. 89: The $\theta$ vs $\phi$ distributions for the proton in sector 2 , before and after fiducial cuts. a) and $\mathrm{b}$ ) are the distributions before and after fiducial cuts for proton momentum $p=1 \mathrm{GeV} / \mathrm{c}$, respectively. The results are shown for the ${ }^{3} \mathrm{He}$ target at $4.4 \mathrm{GeV}$.

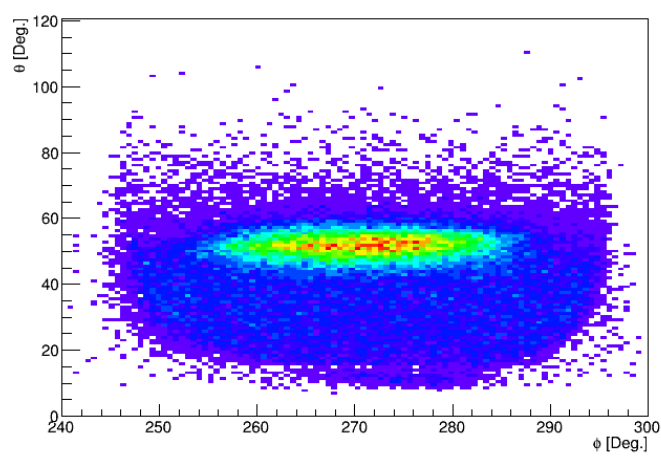

(a)

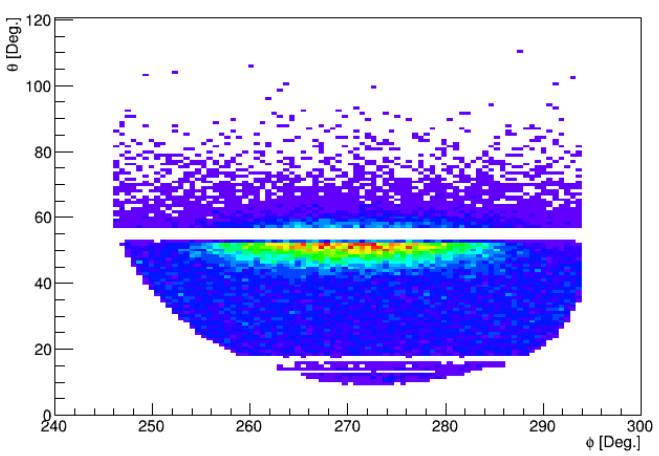

(b)

FIG. 90: The $\theta$ vs $\phi$ distributions for the proton in sector 5 , before and after fiducial cuts. a) and $\mathrm{b}$ ) are the distributions before and after fiducial cuts for proton momentum $p=1 \mathrm{GeV} / \mathrm{c}$, respectively. The results are shown for the ${ }^{3} \mathrm{He}$ target at $2.2 \mathrm{GeV}$.

\subsection{GEV ANALYSIS}

The opposite charge of the proton forces us to use different functions for the positions of the dead TOF paddles. Following the work done in [63], we have found the lower and upper bounds of each bad paddle in momentum steps of $50 \mathrm{MeV} / \mathrm{c}^{2}$. We have then used a function 


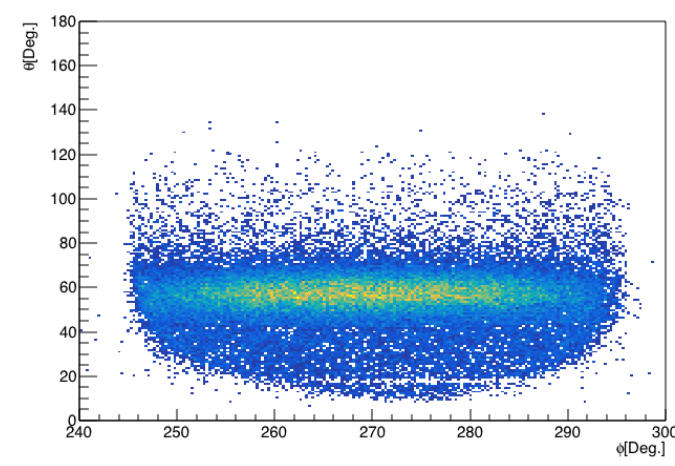

(a) Sector 5

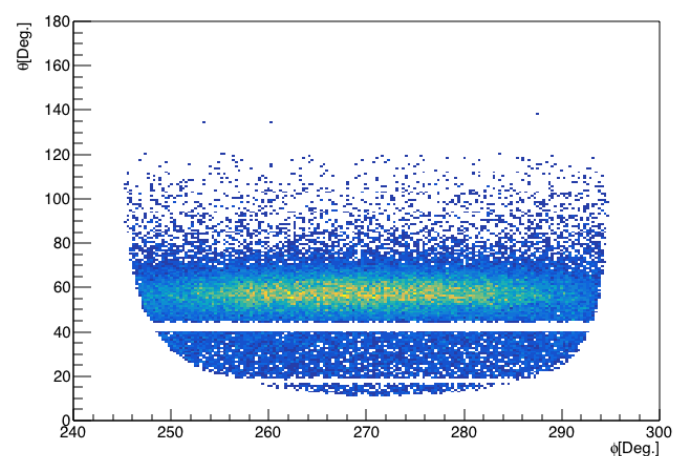

(b) Sector 5

FIG. 91: The $\theta$ vs $\phi$ distributions for protons with $p=0.6 \mathrm{GeV} / \mathrm{c}$ before (left plots) and after (right plots) fiducial cuts in sector 5. The results are shown for the ${ }^{3} \mathrm{He}$ target at $1.1 \mathrm{GeV}$.

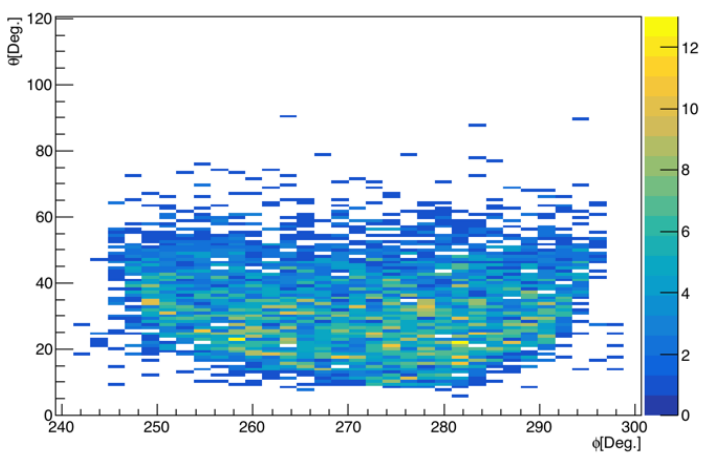

(a)

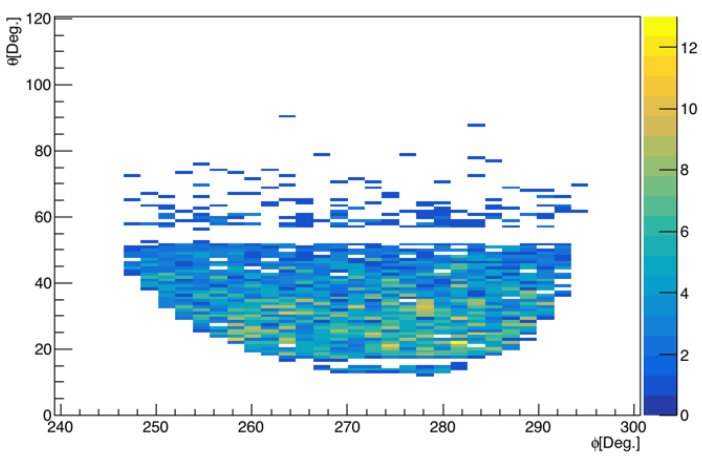

(b)

FIG. 92: The $\theta$ vs $\phi$ distributions for the $\pi^{+}$in sector 5 , before and after fiducial cuts. a) and $\mathrm{b}$ ) are the distributions before and after fiducial cuts for $\pi^{+}$momentum $p=1 \mathrm{GeV} / \mathrm{c}$, respectively. The results are shown for the ${ }^{3} \mathrm{He}$ target at $4.4 \mathrm{GeV}$.

of a form described by Eq. 4.7.3 to parametrize the lower and upper $\theta$ angle limits for a given bad TOF paddle region as a function of momentum. We have done this for protons detected in each of the CLAS sectors separately. We can see the $\theta$ vs $\phi$ angle distributions of protons before and after fiducial cuts in Fig. 91. There are bad TOF paddles in all six sectors. 


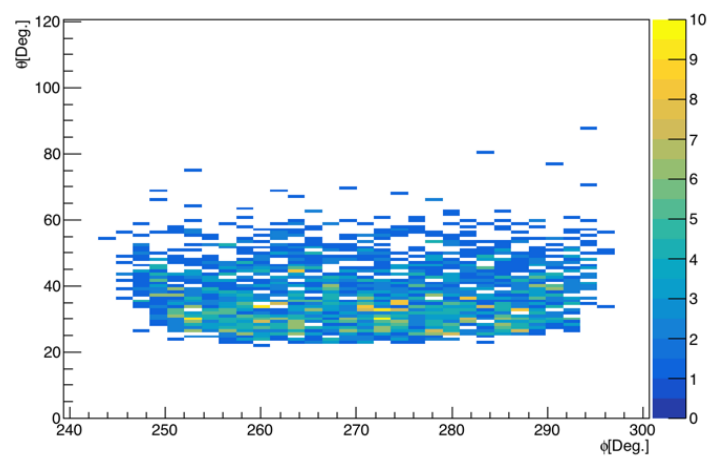

(a)

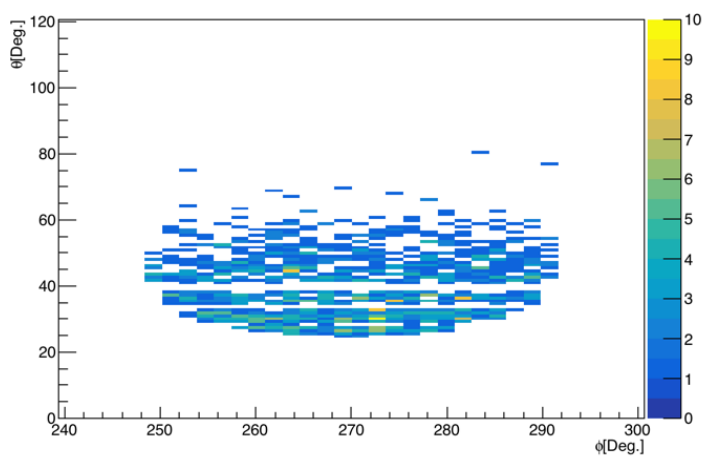

(b)

FIG. 93: The $\theta$ vs $\phi$ distributions for the $\pi^{-}$in sector 5 , before and after fiducial cuts. a) and b) are the distributions before and after fiducial cuts for $\pi^{-}$momentum $p=1 \mathrm{GeV} / \mathrm{c}$, respectively. The results are shown for the ${ }^{3} \mathrm{He}$ target at $4.4 \mathrm{GeV}$.

\subsection{PION FIDUCIAL CUTS}

We have used the proton fiducial cuts to determine the geometrical acceptance of CLAS for positive pions. The same way we have used electron fiducial cuts to determine the geometrical acceptance of CLAS for negative pions. This was done both at 2.2 and $4.4 \mathrm{GeV}$ analysis.

\subsection{AND 2.2 GEV ANALYSIS}

We have also used the same electron fiducial cuts obtained at $2.2 \mathrm{GeV}$ to estimate the geometrical acceptance for $\pi^{-}$both at 2.2 and $4.4 \mathrm{GeV}$ analysis. This is an acceptable approximation as the magnetic field was the same during both runs. We used the $2.2 \mathrm{GeV}$ $e^{-}$fiducial cuts since they extend to lower momentum than the $4.4 \mathrm{GeV}$ cuts.

The $2.2 \mathrm{GeV} e^{-}$fiducial cuts extend down to $350 \mathrm{MeV} / \mathrm{c}$. Therefore we have developed new fiducial cuts for $p<350 \mathrm{MeV} / \mathrm{c}$. We used the same procedure for obtaining the $\theta$ vs $\phi$ outline cuts and the $\theta$ gaps corresponding to malfunctioning TOF paddles in this low momentum region. We used $50 \mathrm{MeV} / \mathrm{c}$ momentum bins and the $2.2 \mathrm{GeV}^{12} \mathrm{C}$ data to obtain the cuts. The $\theta$ vs $\phi$ distributions before and after fiducial cuts for $\pi^{-}$with $p=0.25 \mathrm{GeV} / \mathrm{c}$ 


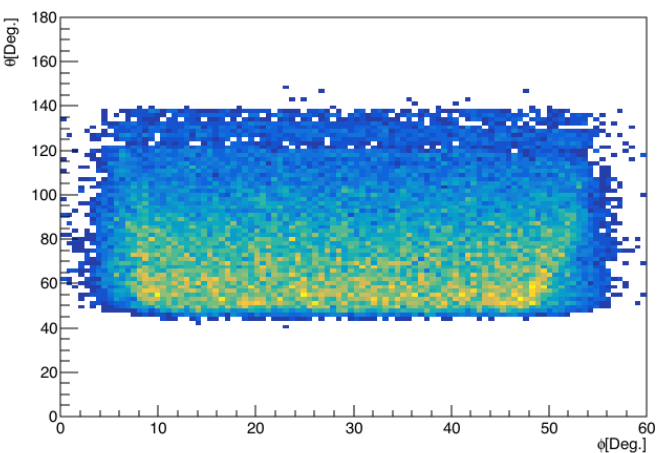

(a) Sector 1

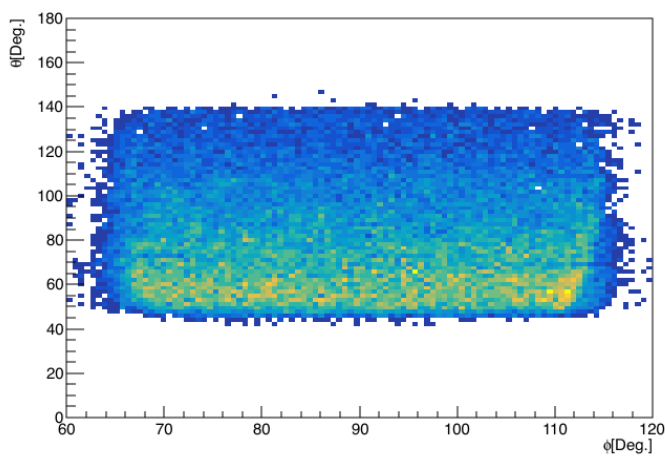

(c) Sector 2

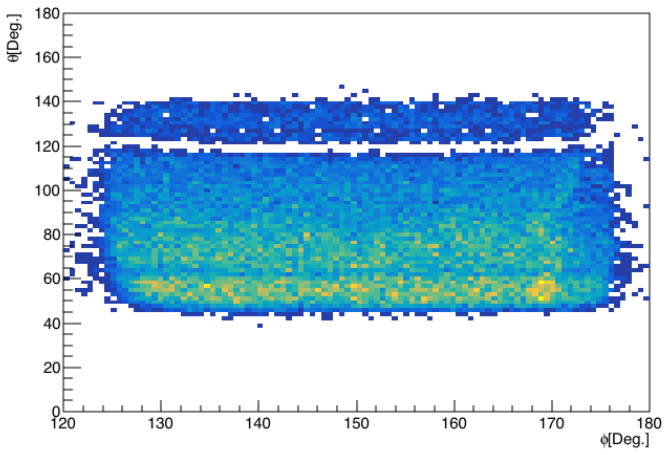

(e) Sector 3

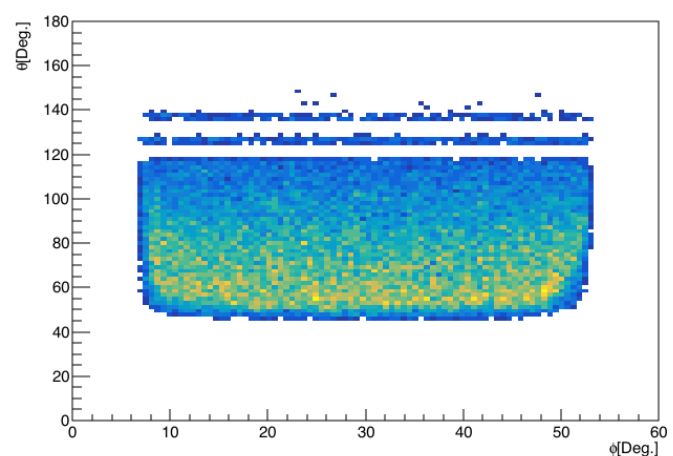

(b) Sector 1

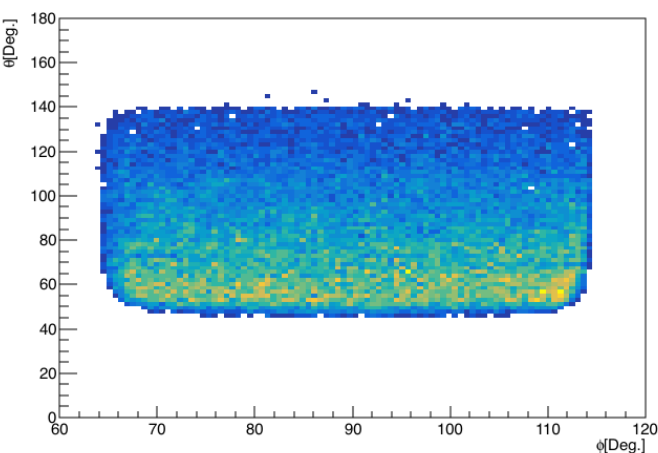

(d) Sector 2

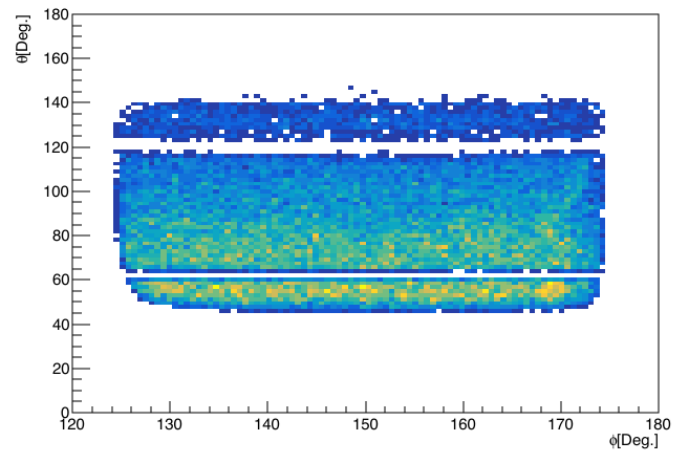

(f) Sector 3

FIG. 94: The $\theta$ vs $\phi$ distributions for $\pi^{-}$with $p=0.25 \mathrm{GeV} / \mathrm{c}$ before (left plots) and after (right plots) fiducial cuts in sector 1,2 and 3. The results are shown for the ${ }^{12} \mathrm{C}$ target at $2.2 \mathrm{GeV}$.

in sectors 1, 2 and 3 are shown in Fig. 94.

The $\theta$ vs $\phi$ distributions of the $\pi^{-}$before and after fiducial cuts are shown for sector 1 and momentum $p_{\mathrm{p}}=1.4 \mathrm{GeV} / \mathrm{c}$ in Fig. 93. 


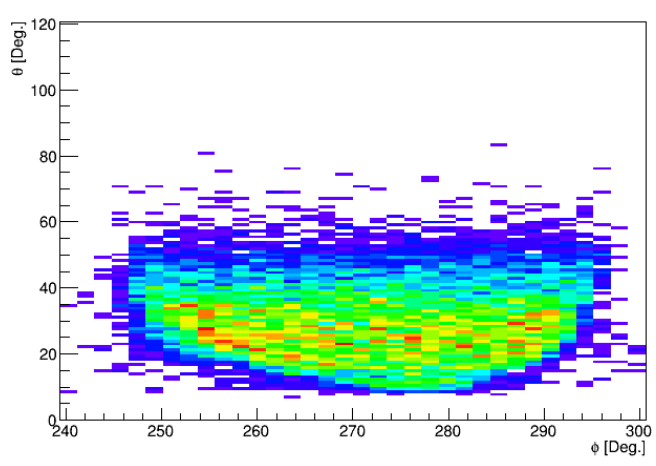

(a)

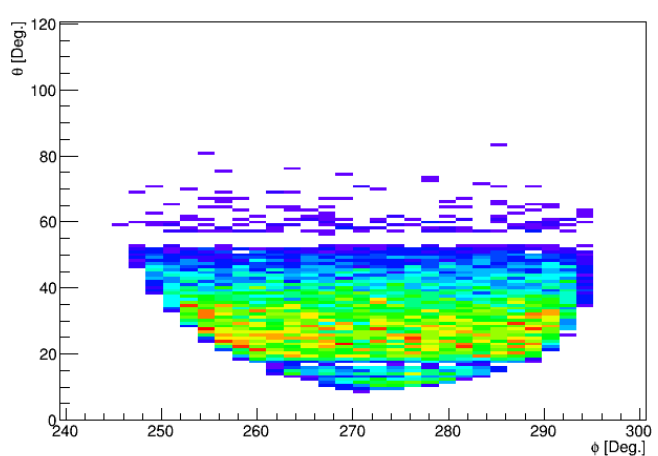

(b)

FIG. 95: The $\theta$ vs $\phi$ distributions for the $\pi^{+}$in sector 5 , before and after fiducial cuts. a) and $\mathrm{b}$ ) are the distributions before and after fiducial cuts for $\pi^{+}$momentum $p=1 \mathrm{GeV} / \mathrm{c}$, respectively. The results are shown for the ${ }^{3} \mathrm{He}$ target at $2.2 \mathrm{GeV}$.

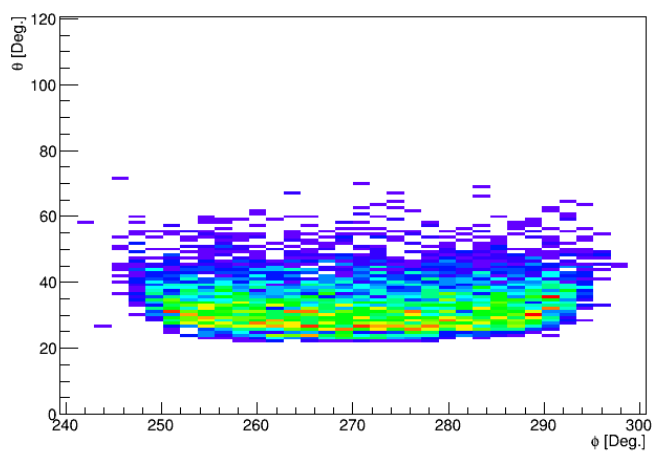

(a)

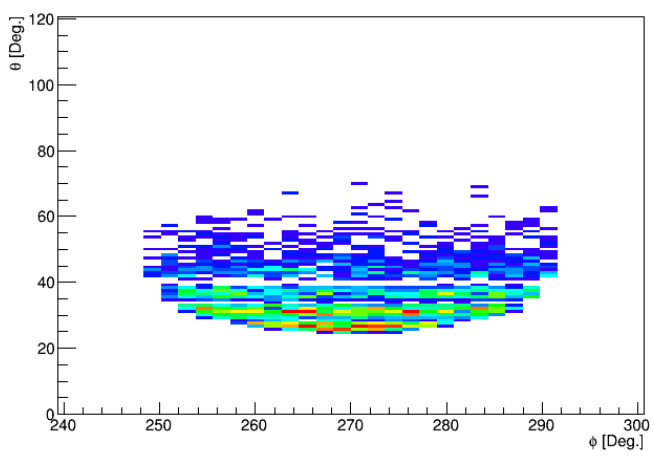

(b)

FIG. 96: The $\theta$ vs $\phi$ distributions for the $\pi^{-}$in sector 5, before and after fiducial cuts. a) and $\mathrm{b}$ ) are the distributions before and after fiducial cuts for $\pi^{-}$momentum $p=1 \mathrm{GeV} / \mathrm{c}$, respectively. The results are shown for the ${ }^{3} \mathrm{He}$ target at $2.2 \mathrm{GeV}$.

These fiducial cuts extend down to $p=0.1 \mathrm{GeV} / \mathrm{c} \pi^{-}$.

The $\theta$ vs $\phi$ distributions of the $\pi^{+}$before and after fiducial cuts are shown for sector 1 and momentum $p_{\mathrm{p}}=1 \mathrm{GeV} / \mathrm{c}$ in Fig. 92.

The $\theta$ vs $\phi$ distributions of the $\pi^{-}$at $2.2 \mathrm{GeV}$ before and after fiducial cuts are shown 
for sector 5 and momentum $p_{\mathrm{p}}=1 \mathrm{GeV}$ in Fig. 96. The $\theta$ vs $\phi$ distributions of the $\pi^{+}$at $2.2 \mathrm{GeV}$ before and after fiducial cuts are shown for sector 5 and momentum $p_{\mathrm{p}}=1 \mathrm{GeV}$ in Fig. 95.

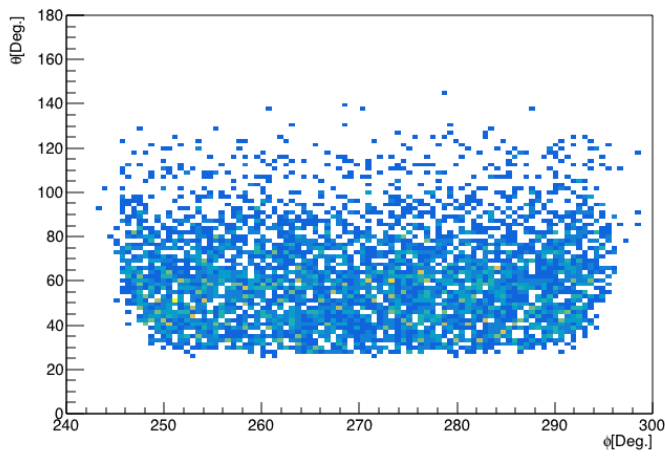

(a) Sector 5

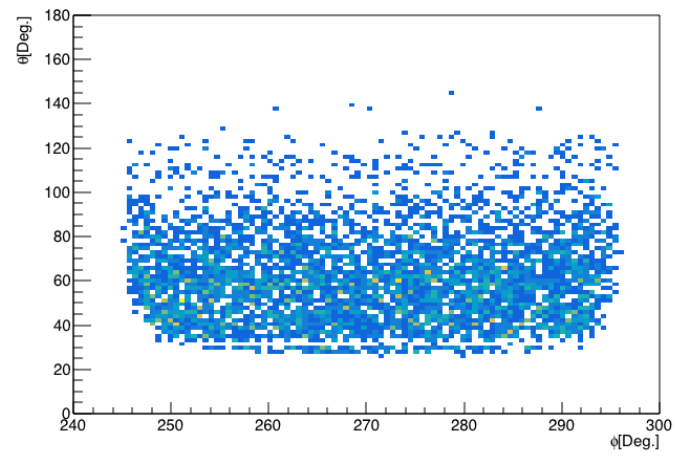

(b) Sector 5

FIG. 97: The $\theta$ vs $\phi$ distributions for $\pi^{-}$with $p=0.3 \mathrm{GeV} / \mathrm{c}$ before (left plots) and after (right plots) fiducial cuts in sector 5. The results are shown for the ${ }^{3} \mathrm{He}$ target at $1.1 \mathrm{GeV}$.

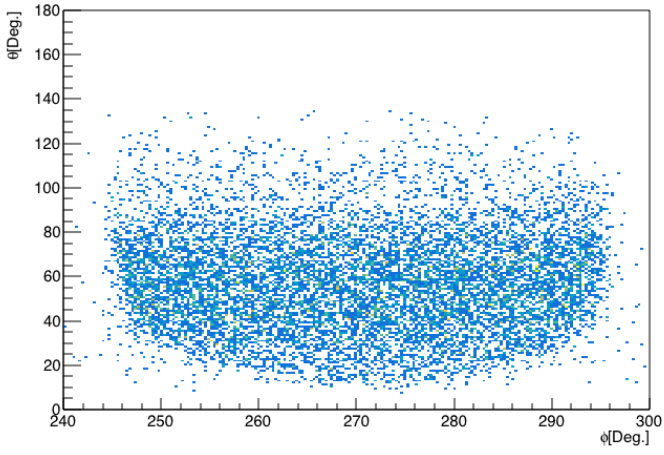

(a) Sector 5

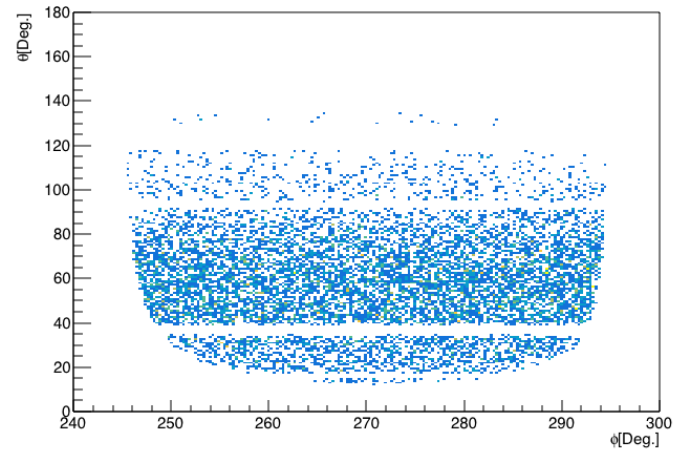

(b) Sector 5

FIG. 98: The $\theta$ vs $\phi$ distributions for $\pi^{+}$with $p=0.3 \mathrm{GeV} / \mathrm{c}$ before (left plots) and after (right plots) fiducial cuts in sector 5 . The results are shown for the ${ }^{3}$ He target at $1.1 \mathrm{GeV}$. 


\subsection{GEV ANALYSIS}

The positions of the $\pi^{+}$dead TOF paddles match that of the proton. We have looked at the proton and $\pi^{+}$angular distributions in different momentum bins in order to make sure that the bad TOF paddles are the same for both particles. We use the same bad TOF paddle cuts for protons and $\pi^{+}$.

The positions of the $\pi^{-}$dead TOF paddles match that of the electron. However there are some bad TOF paddles that affect only the $\pi^{-}$detection region, as they are located at larger scattering angles. We use the same bad TOF paddle cuts for electrons and $\pi^{-}$(including the bad TOF paddles at larger scattering angles).

The $\theta$ vs $\phi$ angle distributions of $\pi^{+}$and $\pi^{-}$before and after fiducial cuts that include $\theta$ vs $\phi$ outline cuts and removal of theta gaps corresponding to malfunctioning TOF paddles are shown in Fig. 97 and Fig. 98.

\subsection{PHOTON FIDUCIAL CUTS}

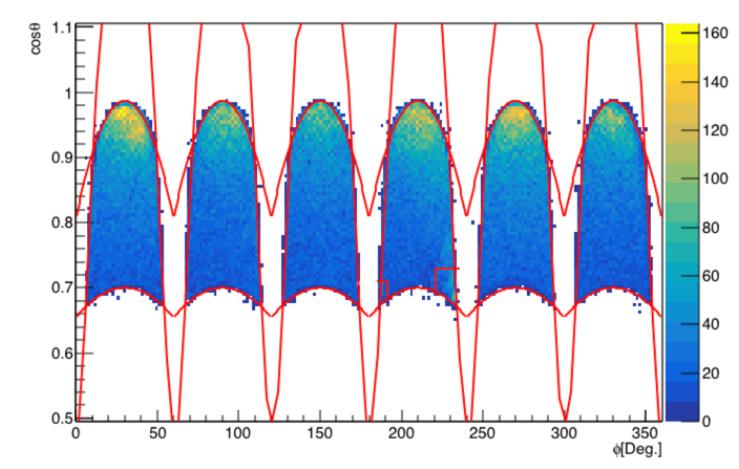

(a) $4.4 \mathrm{GeV}$.

FIG. 99: The $\cos \theta$ vs $\phi$ distributions for photons, after we have applied the electron $u, v$ and $w$ cuts on photons, with fiducial cut outline indicated by red for $4.4 \mathrm{GeV}$ analysis for ${ }^{3} \mathrm{He}$.

For neutral particles the geometrical acceptance is independent of momentum and is simply limited to the detection area of the electromagnetic calorimeter. To obtain the photon fiducial cuts we have plotted the $\cos \theta$ vs $\phi$ distributions for photons at $4.4 \mathrm{GeV}$ for ${ }^{3} \mathrm{He}$ after we have applied the electron $u, v$ and $w$ cuts on photons. We have used two first order polynomials to describe the outline of the sides of the sector and two second order 
polynomials to describe the top and bottom edges of the sector as shown in Fig. 99. We then used this outline to select the fiducial region for photons at all energies and for all targets. There are two hot spots in the bottom corners of the sector four, which we also cut out. The $\cos \theta$ vs $\phi$ distributions for photons with fiducial cut outlines indicated by red are shown in Fig. 100 .

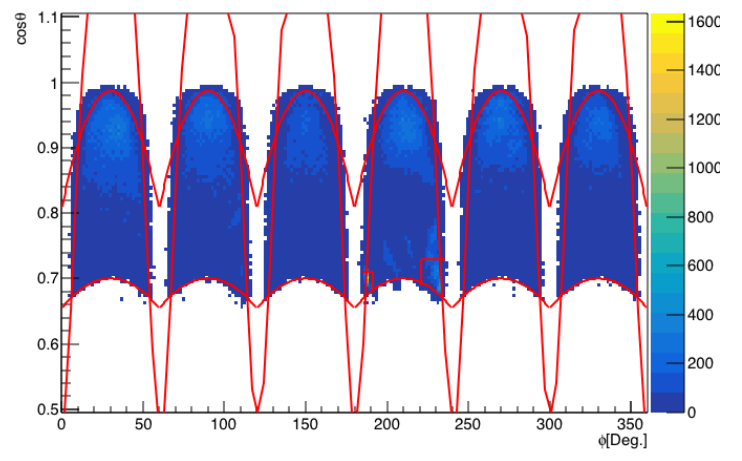

(a) $1.1 \mathrm{GeV}$.

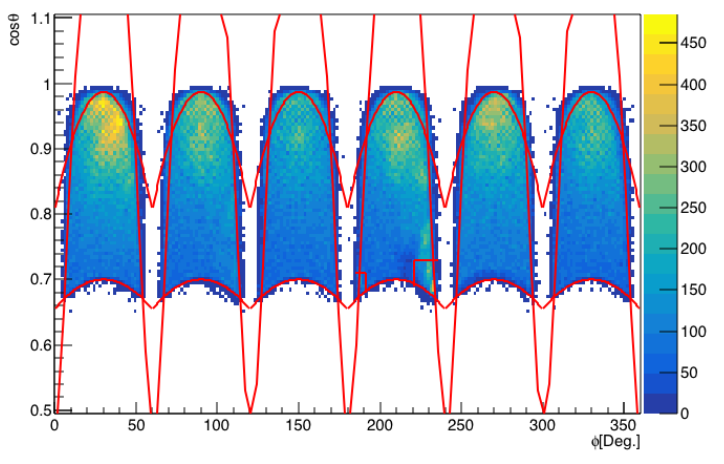

(b) $2.2 \mathrm{GeV}$.

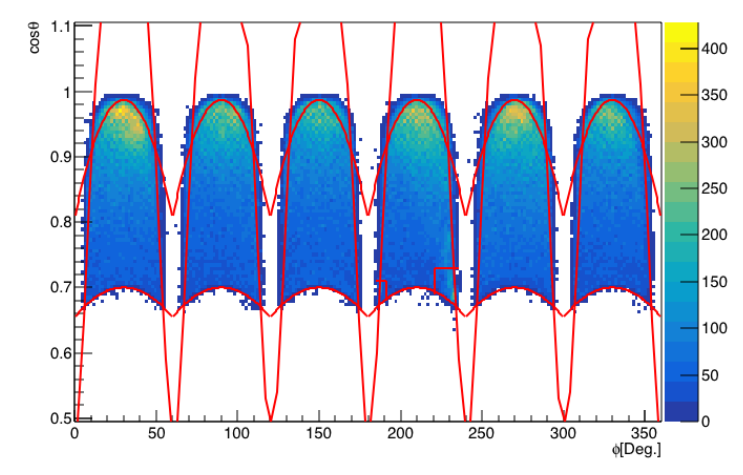

(c) $4.4 \mathrm{GeV}$.

FIG. 100: The $\cos \theta$ vs $\phi$ distributions for photons with fiducial cut outline indicated by red for $1.1 \mathrm{GeV}, 2.2 \mathrm{GeV}$ and $4.4 \mathrm{GeV}$ analysis for ${ }^{3} \mathrm{He}$.

\subsection{ACCEPTANCE MAPS}

For our analysis we also had to produce acceptance maps for different particles that we use in the analysis, to apply to GENIE neutrino event generator results, in later comparison of GENIE results in this analysis.

In several data mining applications, acceptance corrections must be calculated using 
CLAS simulations. As a way of streamlining this for e2a data mining analyses, we have produced e2a acceptance maps using CLAS simulations. These acceptance maps are estimates of the probability for CLAS to detect and identify a particle as a function of the particle's momentum vector. We have produced maps for each combination of particle type, target location, magnetic field setting, etc.

Acceptance maps save time by precomputing a large amount of simulated data, and distilling the results in a way that can be queried quickly. The CLAS simulation program GSIM and the reconstruction program RECSIS are both computationally expensive, and by running a large volume of simulated data once, while using the results for many applications makes acceptance maps an efficient choice. They also provide a convenient way for end users to study acceptance corrections without the risk of running the CLAS simulation incorrectly. 


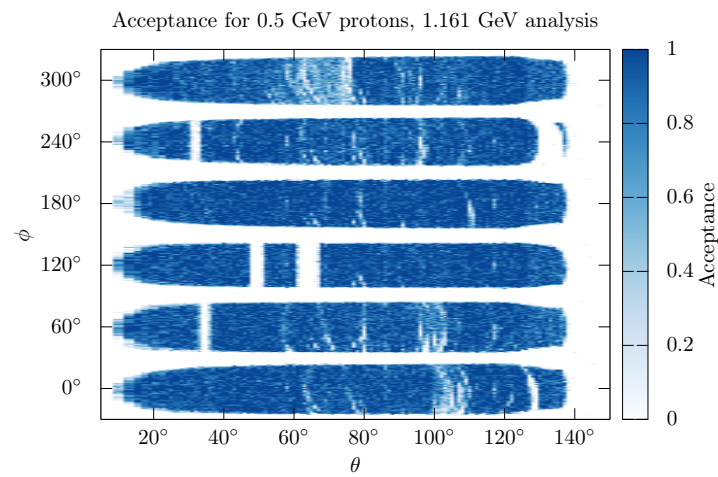

(a) For $0.5 \mathrm{GeV}$ protons at $1.1 \mathrm{GeV}$.

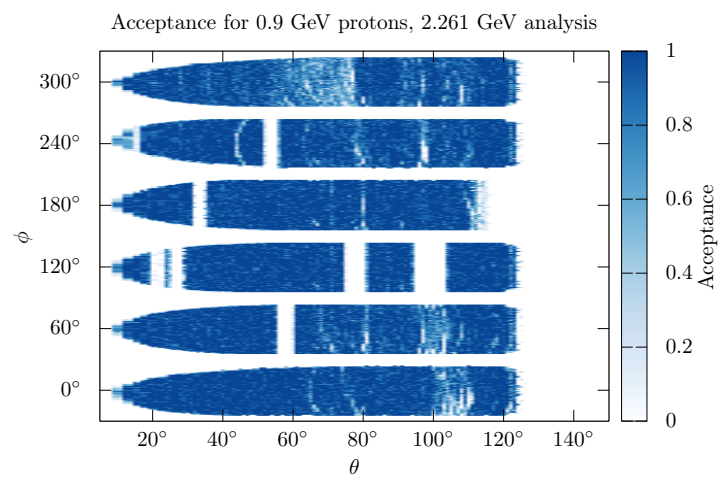

(c) For $0.9 \mathrm{GeV}$ protons at $2.2 \mathrm{GeV}$.

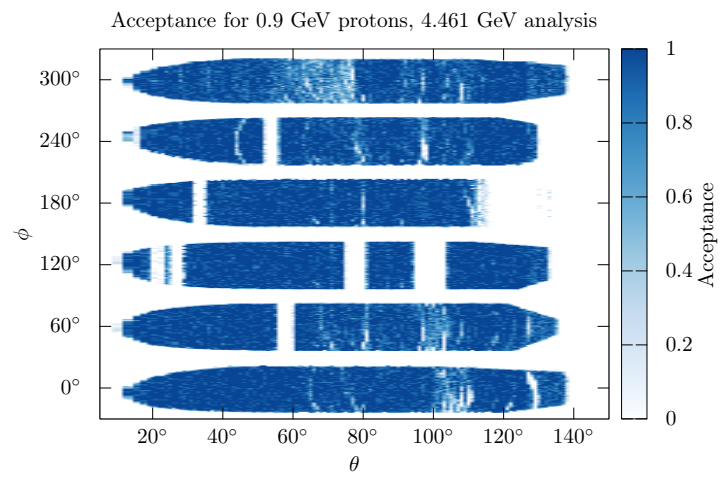

(e) For $0.9 \mathrm{GeV}$ protons at $4.4 \mathrm{GeV}$.

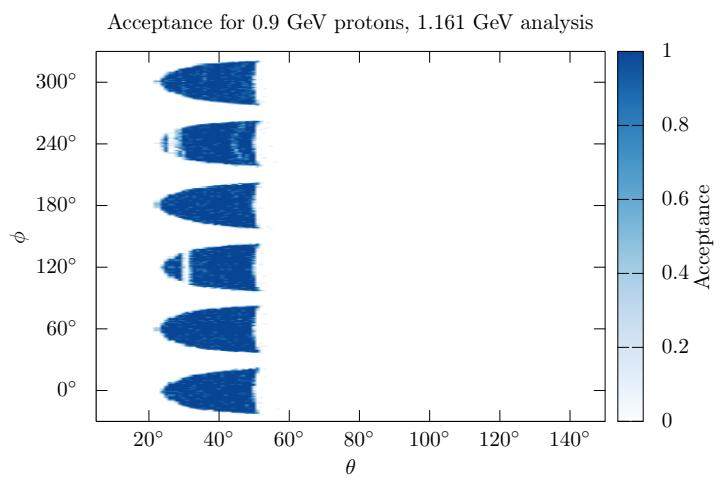

(b) For $0.9 \mathrm{GeV}$ protons at $1.1 \mathrm{GeV}$.

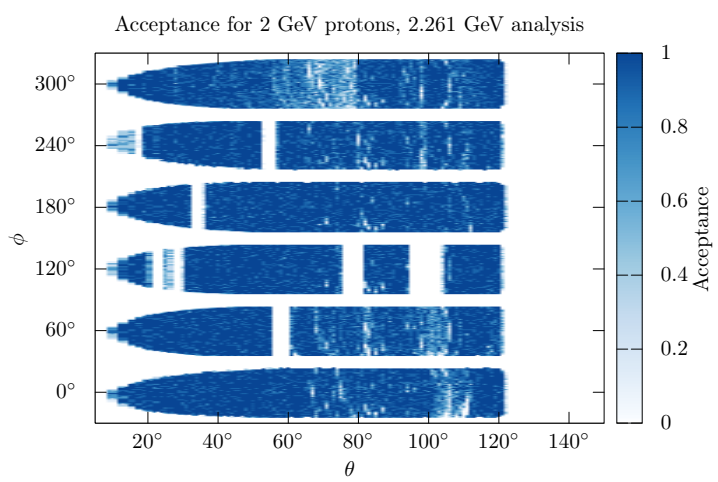

(d) For $2 \mathrm{GeV}$ protons at $2.2 \mathrm{GeV}$.

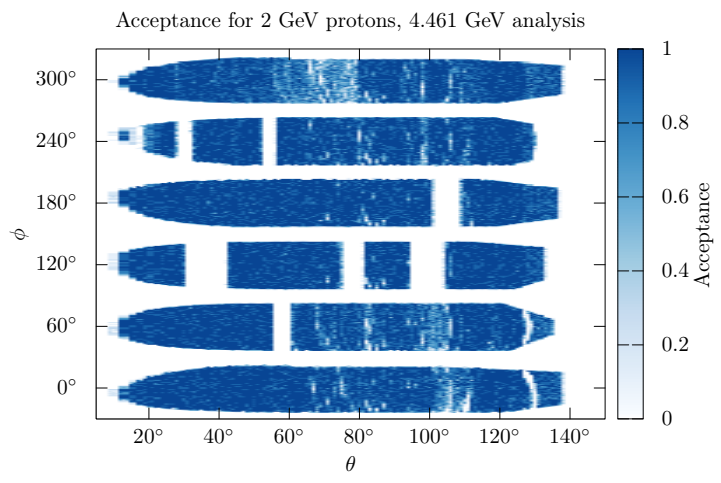

(f) For $2 \mathrm{GeV}$ protons at $4.4 \mathrm{GeV}$.

FIG. 101: The acceptances for different momenta of protons are shown as two-dimensional slices through $\theta, \phi$ space. The acceptance was calculated from the acceptance map corresponding to the solid foil target position, 750 A torus current at $1.1 \mathrm{GeV}$ and $2250 \mathrm{~A}$ torus current at 2.2 and $4.4 \mathrm{GeV}$, and PID and fiducial cuts from the $1.1 \mathrm{GeV}$ beam energy analysis (top plots), $2.2 \mathrm{GeV}$ (middle plots), and $4.4 \mathrm{GeV}$ beam energy analysis (bottom plots). 


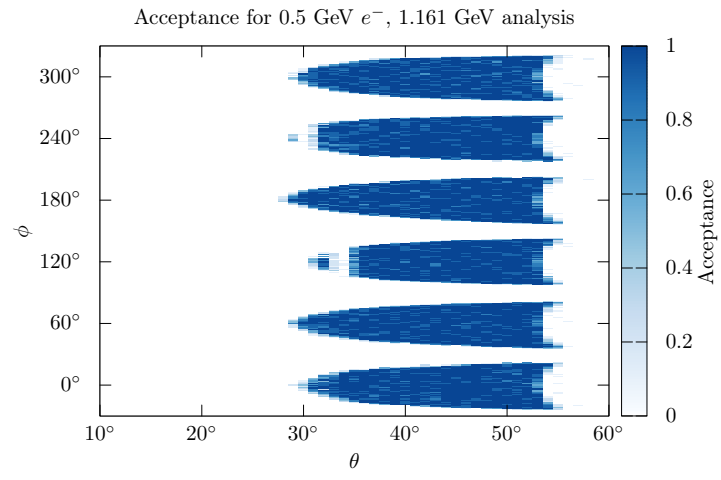

(a) For $0.5 \mathrm{GeV} e^{-}$at $1.1 \mathrm{GeV}$.

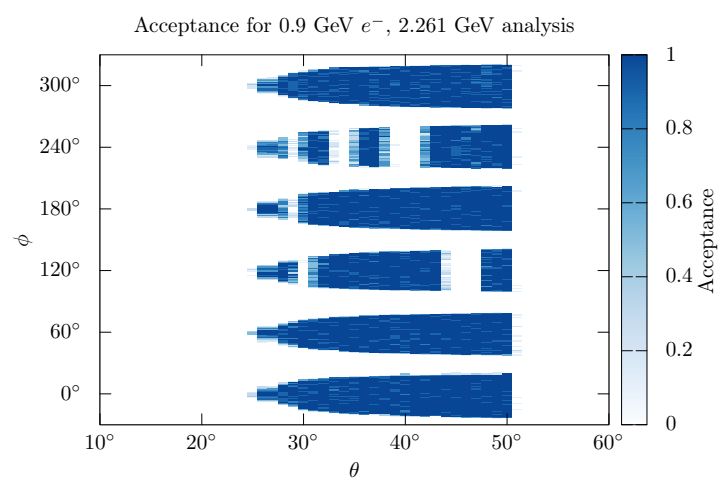

(c) For $0.9 \mathrm{GeV} e^{-}$at $2.2 \mathrm{GeV}$.

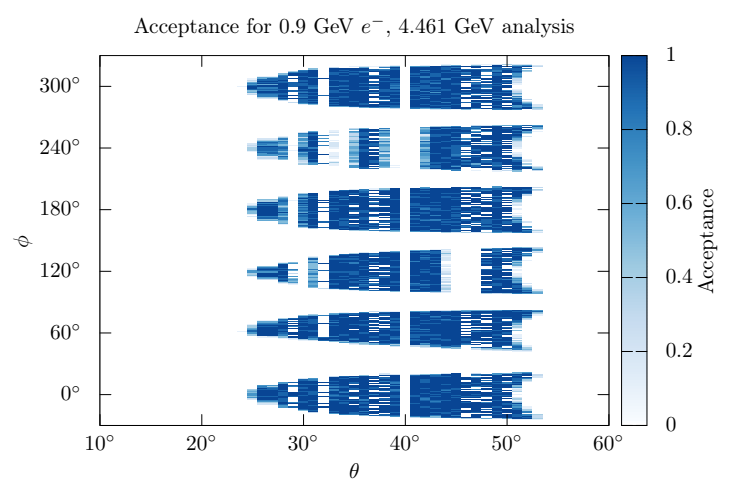

(e) For $0.9 \mathrm{GeV} e^{-}$at $4.4 \mathrm{GeV}$.

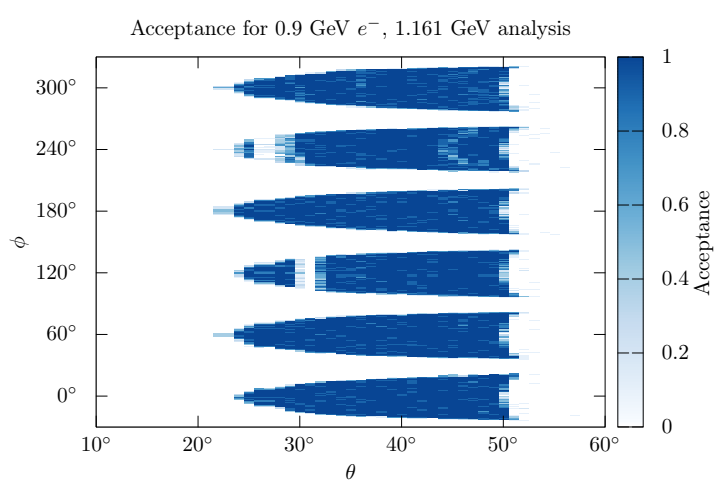

(b) For $0.9 \mathrm{GeV} e^{-}$at $1.1 \mathrm{GeV}$.

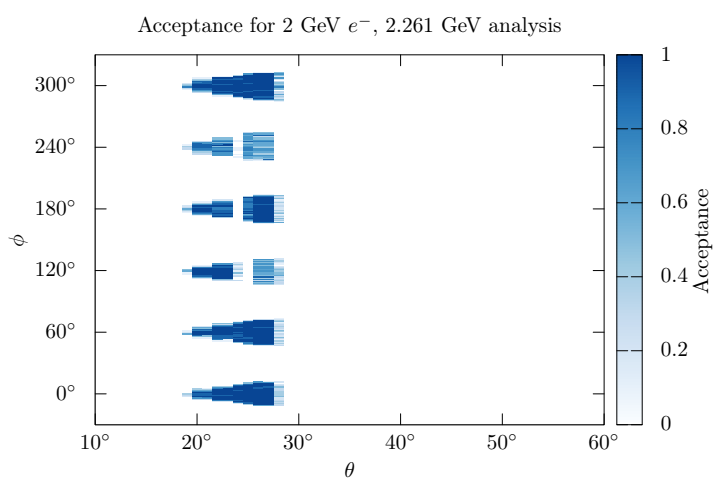

(d) For $2 \mathrm{GeV} e^{-}$at $2.2 \mathrm{GeV}$.

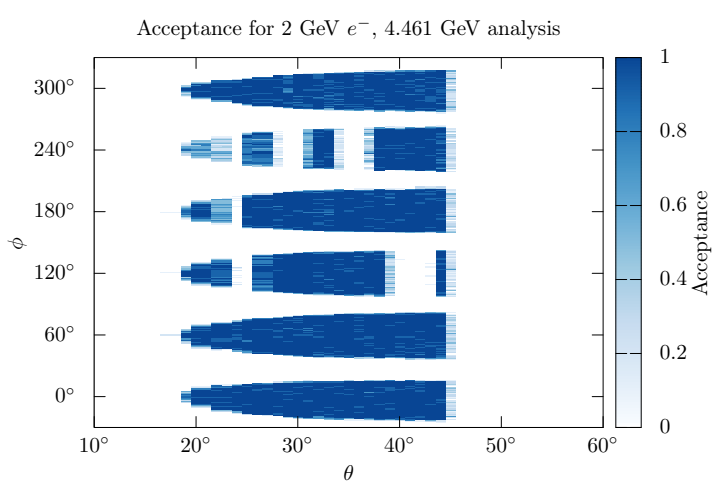

(f) For $2 \mathrm{GeV} e^{-}$at $4.4 \mathrm{GeV}$.

FIG. 102: The acceptances for different momenta of electrons are shown as two-dimensional slices through $\theta, \phi$ space. The acceptance was calculated from the acceptance map corresponding to the solid foil target position, 750 A torus current at $1.1 \mathrm{GeV}$ and $2250 \mathrm{~A}$ torus current at 2.2 and $4.4 \mathrm{GeV}$, and PID and fiducial cuts the $1.1 \mathrm{GeV}$ beam energy analysis (top plots), $2.2 \mathrm{GeV}$ (middle plots), and $4.4 \mathrm{GeV}$ beam energy analysis (bottom plots). 


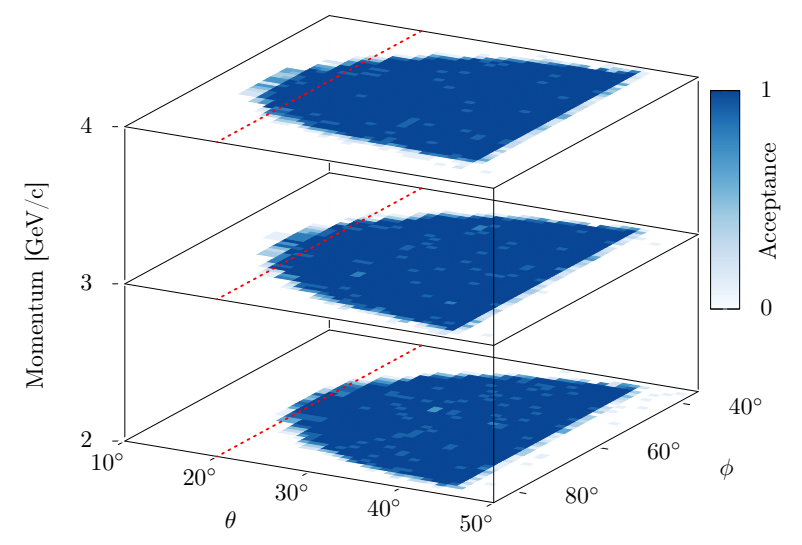

FIG. 103: Three momentum slices through a single sector of the electron acceptance are shown. The acceptance was calculated from the acceptance map corresponding to the solid foil target position, 2250 A torus current, and PID and fiducial cuts from the $4.461 \mathrm{GeV}$ beam energy analysis. Red dashed lines are shown at $20^{\circ}$ to illustrate how the detector covers more forward angles as the electron momentum increases, due to reduced in-bending.

We produced acceptance maps in the form of two 3D histograms, for which the axes represent particle momentum magnitude, $p$, polar angle $\cos \theta$, and azimuth $\phi$. One histogram contains the number of simulated events generated in each bin. The other histogram contains the number of events that were correctly tracked and reconstructed. The ratio of the two numbers is the CLAS efficiency, though technically this is a product of acceptance and efficiency. We provide both the number generated and the number accepted, rather than just the ratio, to allow end users to calculate and propagate errors according to the method of their choosing. An example of the CLAS acceptance for electrons in a single sector is shown in Fig. 103.

\subsubsection{CLAS Simulation Chain}

We produced acceptance maps from the results of CLAS simulations. In this section, we discuss how we ran these simulations. The CLAS simulation chain has several programs each serving a different purpose: the program GSIM propagates particle tracks through a Geant3 detector model, GPP produces simulated raw data from the simulated tracks, applies additional smearing to the simulated time and energy to match real data, applies efficiency 
maps of CLAS to the simulated data, and RECSIS is the general CLAS track reconstruction code. We also wrote a custom "Generator" program that produces a list of initial conditions for events for GSIM to simulate.

\section{Generator}

The Generator program produces a list of initial conditions for events to be simulated in GSIM. Since the maps are stored as a pair of histograms, one key condition is that there should be sufficient number of events generated in each bin. For this reason, the Generator iterates over each bin, and produces events with randomly drawn values of $p, \cos \theta$, and $\phi$, from flat distributions, as illustrated in figure 104. Our distributions range from 0 to $5 \mathrm{GeV}$, -1 to 1 , and $-30^{\circ}$ to $330^{\circ}$ in 100,200 , and 360 bins respectively.
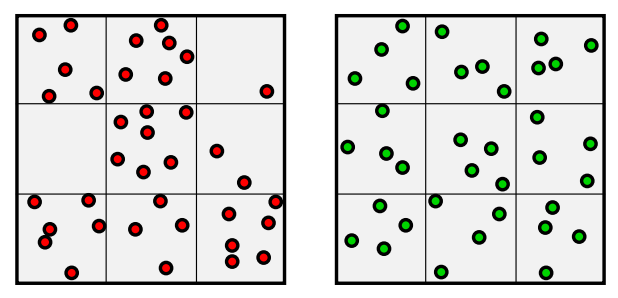

FIG. 104: Events were generated with a constant number per bin, as illustrated by the green points in the right cartoon. If events were generated randomly over the entire phase space, as shown by the red points in the left cartoon, some bins would be underpopulated, or even completely empty.

There is an added complication for the generation of maps for particles other than electrons. Since the CLAS reconstruction requires that each event have a reconstructed electron, it is necessary for the Generator to produce an electron in addition to the particle of interest. We chose to generate the electron in an area of high-acceptance, for efficiency, and to generate in a sector other than that of the particle of interest, to eliminate problems of close-track inefficiency. If, for any reason, this auxiliary electron were not reconstructed, this event would not be considered as a "generated event," regardless of how the particle of interest were reconstructed. A cartoon illustrating this approach for one sector is shown in figure 105 .

\section{GSIM}

GSIM is a Geant3-based simulation of the CLAS detector, which can determine the trajectories of particles through the CLAS magnetic fields while taking energy loss in material 


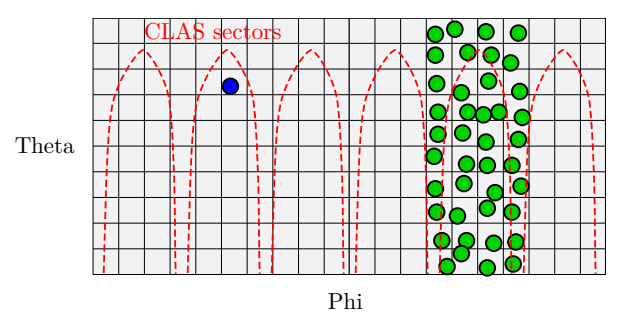

FIG. 105: For hadrons generated in sector 5 (illustrated by green dots), an accompanying electron was generated in the high-acceptance region of sector 2 (illustrated by the blue dot) to satisfy the simulation trigger requirements.

into account. GSIM has a number of configurable options, that are set by user-created configuration files called "ffread cards." A number of configurable options were important for the acceptance map simulations.

First, the various e2a targets could be chosen. In addition to selecting the various targets, offsets of a few millimeters were introduced so that the reconstructed simulated target positions matched those in data. The values used are listed in table 12 .

\begin{tabular}{||cc||}
\hline Target & Shift $[\mathrm{cm}]$ \\
\hline \hline Solid foils & -0.525 \\
\hline Helium-4 & -0.4 \\
\hline Helium-3 & -1.0 \\
\hline
\end{tabular}

TABLE 12: The simulated target positions were shifted from their default positions in order to better match the reconstructed target positions in data.

Second, the magnetic field strength can be set. The e2a runs with beam energies of 4.461 and $2.261 \mathrm{GeV}$ used $2250 \mathrm{~A}$ for the torus and $\approx 6000 \mathrm{~A}$ for the mini-torus, while runs at $1.161 \mathrm{GeV}$ used lower torus currents. Last, the number of simulated events had to be specified to match those produced by the generator.

\section{GPP}

GPP takes the trajectories from GSIM and produces simulated raw data, i.e. the ADC and TDC values for the various CLAS subdetectors. GPP takes into account the various detector elements that were inoperative during the e2a run by loading calibration constants 


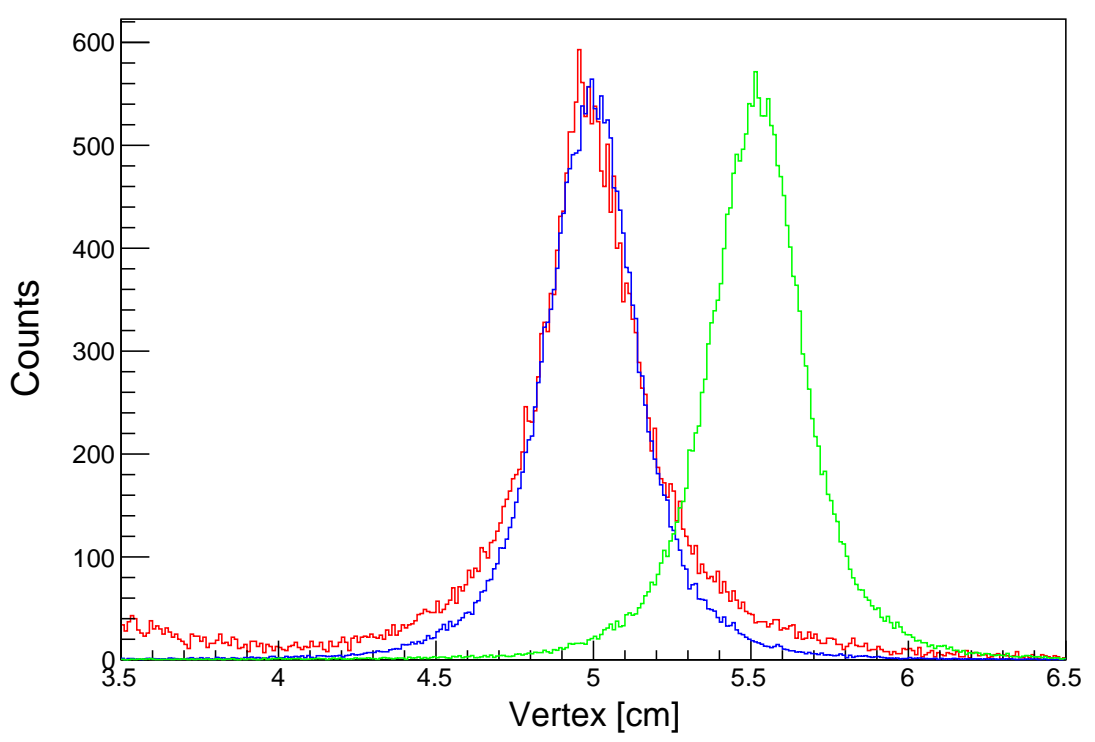

FIG. 106: Our vertex shift for the carbon foil target takes the original simulated vertex distribution in green and moves it to the blue distribution in order to match the position of the data in red.

from the corresponding e2a database. It also gives the user control over "resolution parameters." We tuned these resolution parameters so that the simulated data matched the real e2a data using two observables. First, the drift chamber resolution parameters, $(a, b, c)$, were tuned so that the vertex resolution in simulation matched that of data from the solid foil targets, taking care that the vertex resolution is heavily angle-dependent. The vertex resolution as a function of polar angle, $\theta$, is shown for both data and simulation in Fig. 107, for the final chosen values of $a, b$, and $c$. Once we established suitable drift chamber parameters, we looked at the proton mass-squared resolution when comparing momentum to time-of-flight.

We define mass-squared as

$$
M^{2}=\frac{p^{2}\left(1-\beta^{2}\right)}{\beta^{2}}
$$

where

$$
\beta=\frac{v}{c}=\frac{l}{c\left(T-T_{0}\right)}
$$

Here $l$ is the pathlength of the track from vertex to TOF and $T-T_{0}$ is the total time of 
travel.

We found that mass-squared resolution was dominated by the momentum resolution, and that we got the best agreement when setting $f$, the time-of-flight resolution parameter, to zero, as shown in Fig. 108. Table 13 shows the values of the resolution parameters.

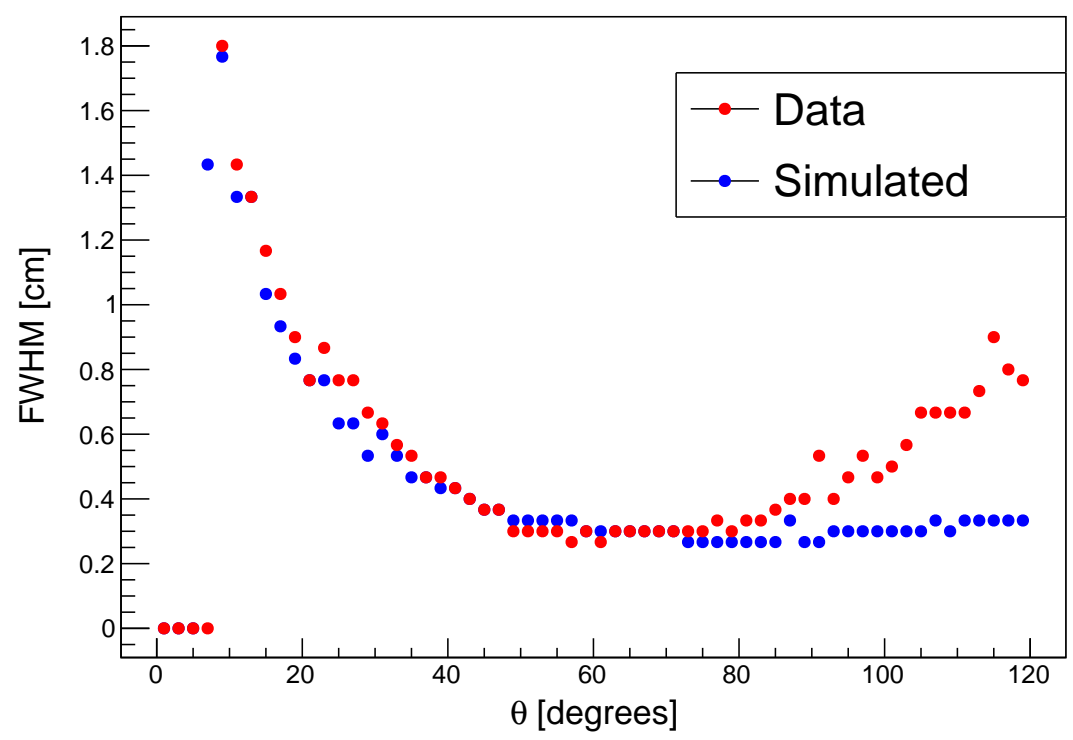

FIG. 107: The simulated vertex resolution as a function of scattering angle was tuned to match that in data.

\begin{tabular}{||clc||}
\hline Parameter & Effect & Value \\
\hline \hline$a$ & Inner drift chamber resolution & 4.0 \\
\hline$b$ & Middle drift chamber resolution & 2.0 \\
\hline$c$ & Outer drift chamber resolution & 2.0 \\
\hline$f$ & Time-of-flight resolution & 0.0 \\
\hline
\end{tabular}

TABLE 13: Final resolution parameters

\section{RECSIS}

RECSIS is the CLAS track reconstruction program. We used the same version of RECSIS to construct both the real and the simulated data. We did not modify any configurable settings other than selecting the proper torus magnetic field. 


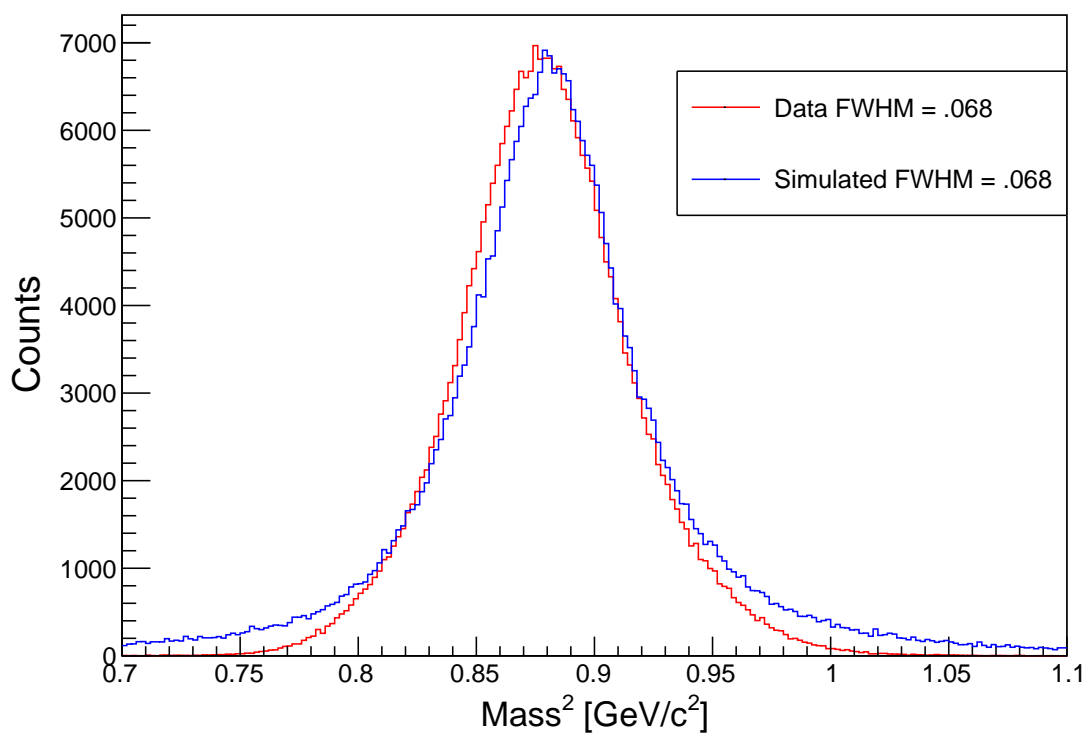

FIG. 108: The simulated mass-squared distribution for protons from Eq. 59 matched data best when we set $f$ to zero. The simulated distribution shown here has been scaled to show the shape agreement.

\subsubsection{Producing the Maps}

We stored the maps in the form of two three-dimensional histograms, one for generated events, and the other for accepted events. Examples are shown in figure 109. The generated histogram is filled before any cuts are applied, though hadron maps require a valid trigger electron. Since we choose a region of high acceptance for the electron, only $\approx 1-1.5 \%$ of generated events fail to meet this requirement.

To produce the accepted event histogram, we apply the same PID and fiducial cuts that are applied to data. If the simulated particle passes these cuts, the accepted event histogram is filled according to the generated quantities. 

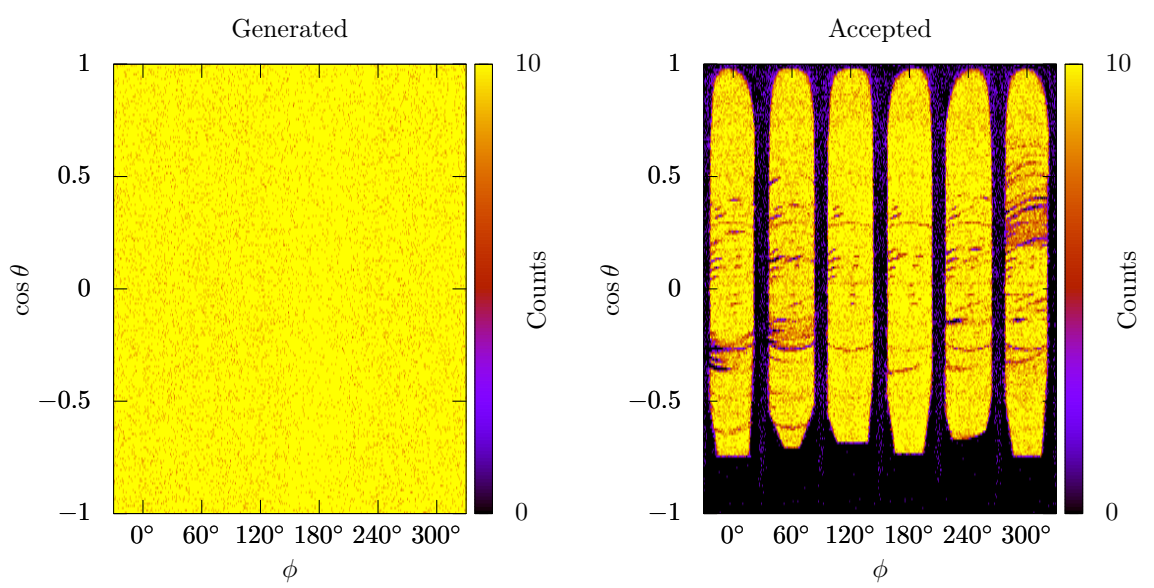

FIG. 109: Map histograms for $2 \mathrm{GeV} / c$ protons: the only reason there are not 10 generated events in every bin is because of the occasional loss of the trigger electron.

\subsection{Z-VERTEX CORRECTION AND CUTS OF PROTONS, ELECTRONS AND PIONS}

\subsection{GEV ANALYSIS}

There is an error in the determination of the Z-vertex position (position along the beamline of the beginning of the particle track) of the particles due to the CLAS tracking algorithm. We need to obtain a correct particle vertex since in our analysis we cut on the vertex to select particles originating from the same event.

The vertex correction for electrons and protons is the same for the same target. Different targets have different vertex corrections.

The CLAS tracking algorithm extrapolates the track of the particles from the drift chambers to the plane that is perpendicular to the mid plane of each sector and that includes the CLAS z-axis. The point of intersection with this plane is assumed to be the particle vertex. The error comes from the fact that the beam does not always pass through the $(0,0)$ point in the $(X, Y)$ plane as is assumed. Suppose the beam passes through the $\left(x_{\text {Beam }}, y_{\text {Beam }}\right)$ point in the $(X, Y)$ plane, described by angle $\phi_{\text {Beam }}$ and distance $d=\sqrt{x_{\text {Beam }}^{2}+y_{\text {Beam }}^{2}}$.

The particles with $\phi=\phi_{\text {Beam }}$ will have their vertex reconstructed upstream of the actual vertex and their $z$ vertex is equal to $z_{\text {up }}=z_{\operatorname{true}}+\frac{d}{\tan \theta}$ where $z_{\text {true }}$ is the true vertex of the 


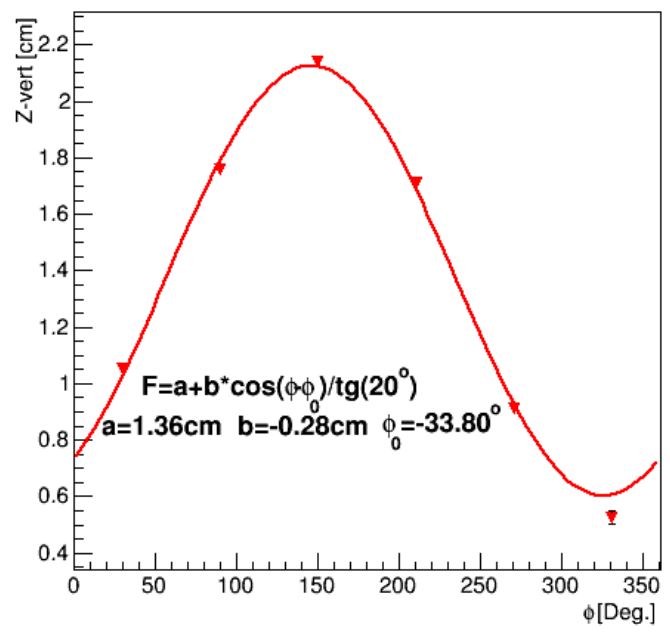

FIG. 110: The plot of the $z$ vertex as a function of the $\phi$ angle with the corresponding fit for the ${ }^{3} \mathrm{He}$ target at $4.4 \mathrm{GeV}$, for electrons at $\theta=20^{\circ}$.

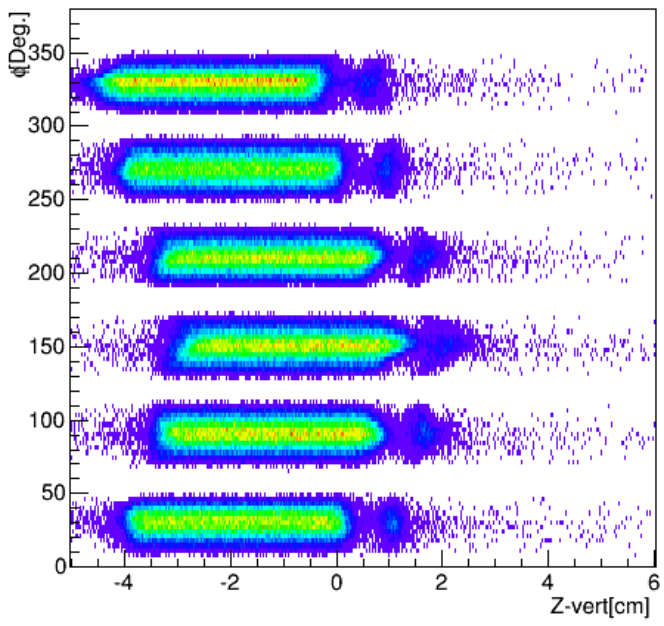

(a) Before correction.

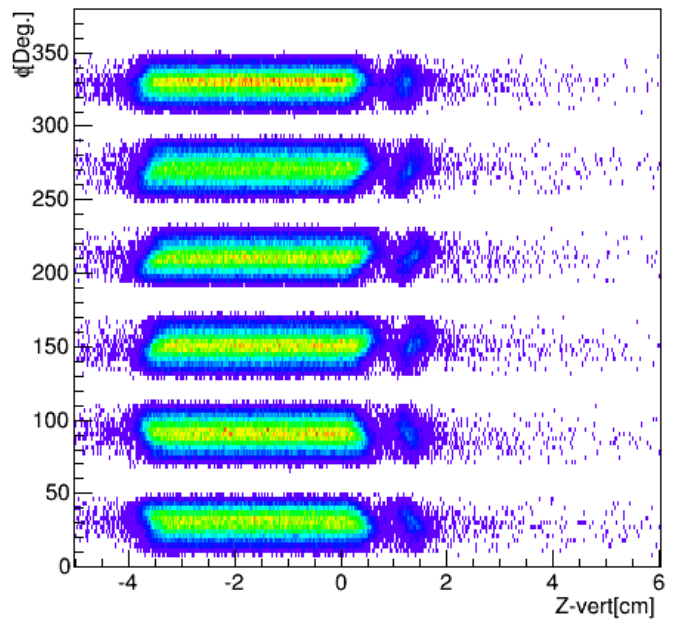

(b) After correction.

FIG. 111: The $\phi$ angle of the electrons plotted vs the $z$ component of the vertex in logarithmic scale. The results are shown for the ${ }^{3} \mathrm{He}$ target at $4.4 \mathrm{GeV}$. (a) Before the vertex correction, (b) After the vertex correction. 


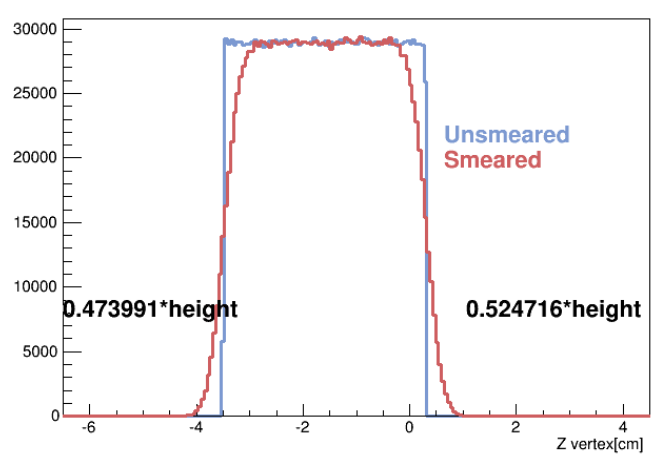

FIG. 112: Simulated $e^{-}$vertex distribution for 4th liquid target cell with and without smearing.

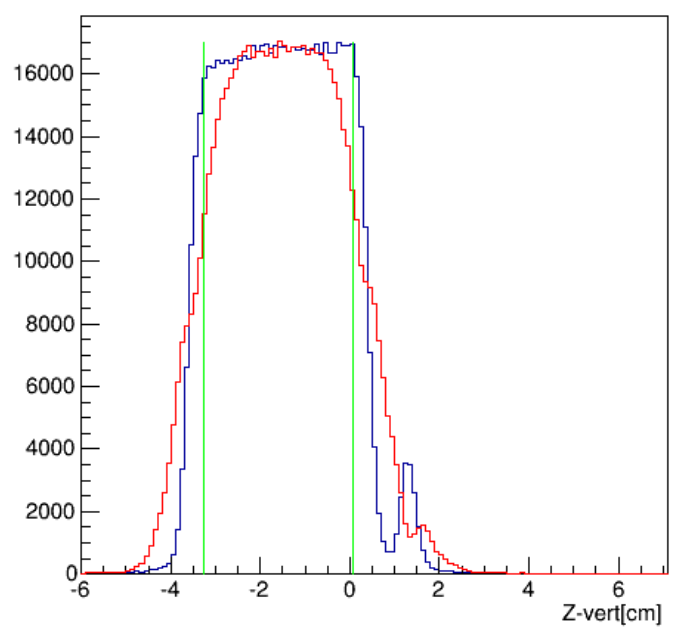

FIG. 113: The distributions of the electron $z$ vertex before (red) and after (blue) correction for ${ }^{3} \mathrm{He}$ at $4.4 \mathrm{GeV}$. The green lines correspond to the applied cut.

particle. Particles with $\phi=\phi_{\text {Beam }}+\pi$ will have their vertex reconstructed downstream of the actual vertex and equal to $z_{\text {down }}=z_{\text {true }}-\frac{d}{\tan \theta}$. The $\phi$ dependent expression for the reconstructed vertex of the particle is the following

$$
z=z_{\text {true }}+\frac{d}{\tan \theta} \times \cos \left(\phi-\phi_{\text {Beam }}\right)
$$

In order to determine the parameters $d$ and $\phi_{\text {Beam }}$, we selected electrons coming from the same point in the target, e.g., the heat shield, and plotted their measured Z-vertex position 


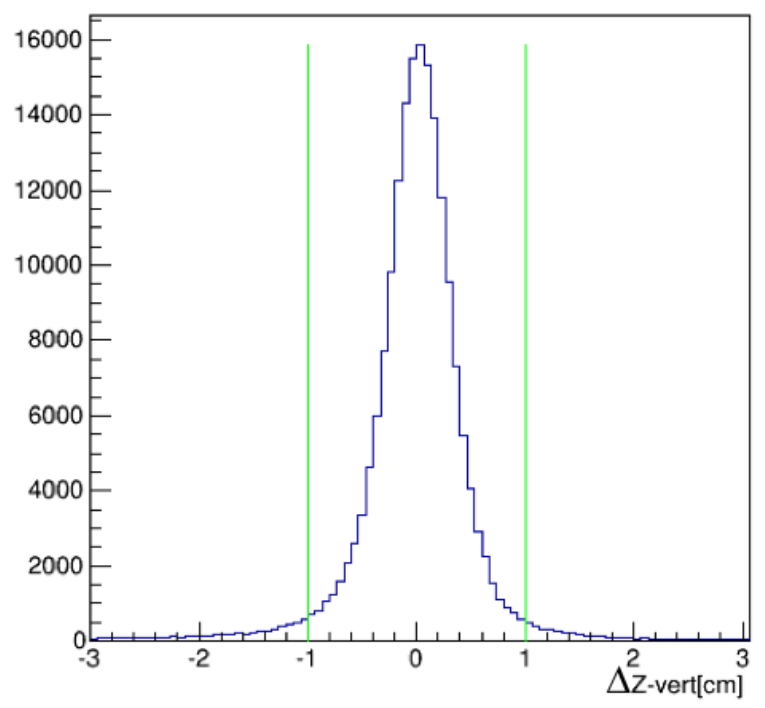

FIG. 114: The electron and proton $z$ vertex difference for ${ }^{3} \mathrm{He}$ at $4.4 \mathrm{GeV}$. The green lines correspond to the applied cut.

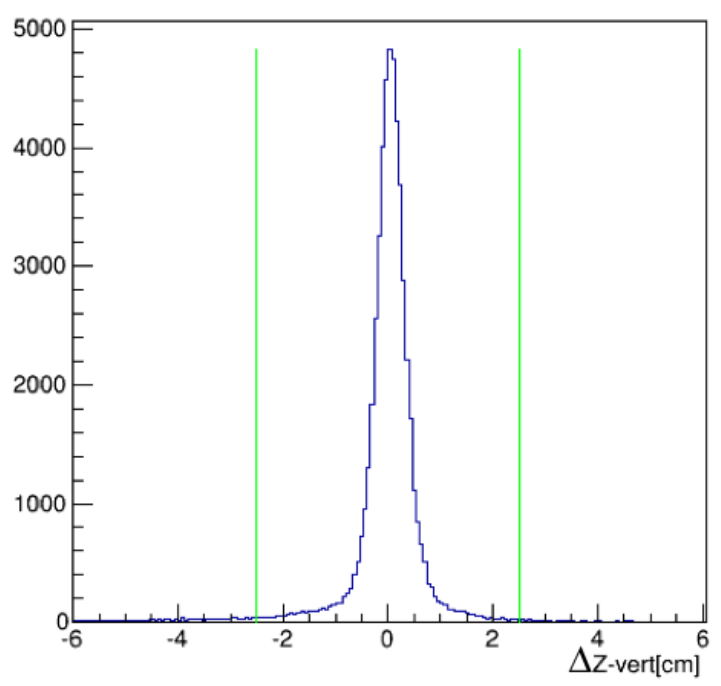

(a) $\pi^{-}$.

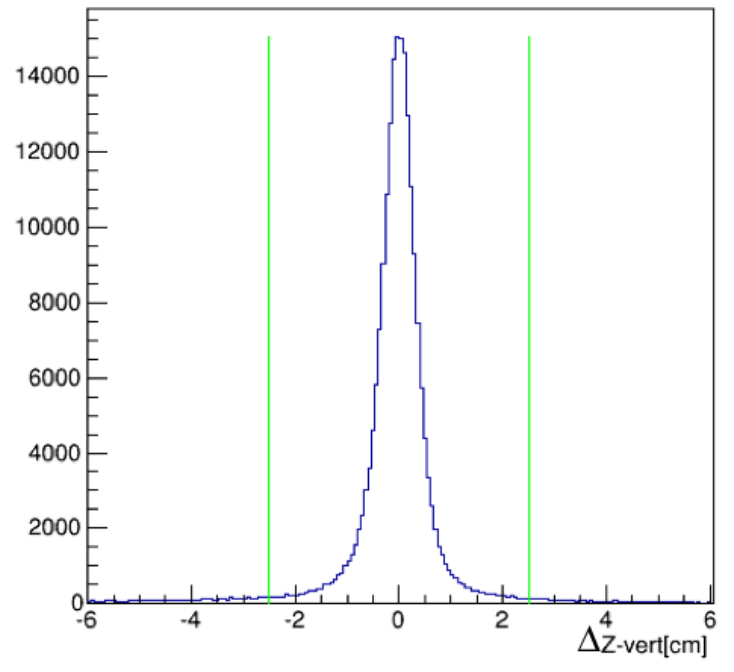

(b) $\pi^{+}$.

FIG. 115: The charged pion and proton $z$ vertex difference for ${ }^{3} \mathrm{He}$ at $4.4 \mathrm{GeV}$. The green lines correspond to the applied cut. 
vs $\phi$ (see Fig. 110). We then optimized $z_{\text {true }}, d$ and $\phi_{\text {Beam }}$ to best fit the points. We used $d$ and $\phi_{\text {Beam }}$ to calculate $\Delta z=z_{\text {true }}-z$ for all events. The $\phi$ angle vs the $z$ vertex distributions before and after vertex correction are shown in Fig. 111. We can see the target region on the left, which is a cryotarget cell full of liquid ${ }^{3} \mathrm{He}$, and there is small peak to the right of the target corresponding to the heat shield.

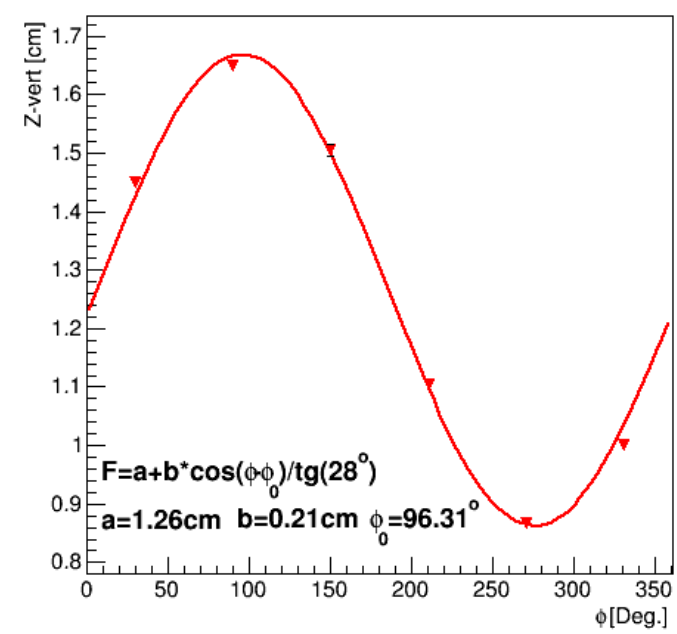

(a) Runs 18346-18382.

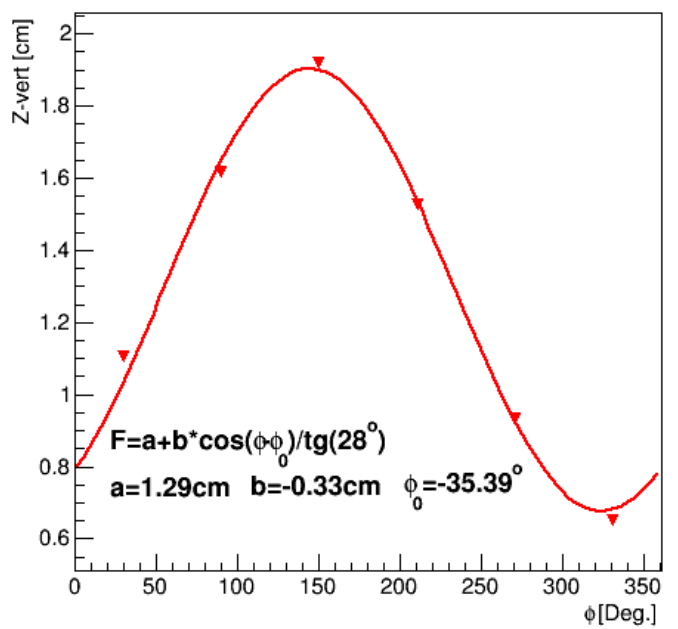

(b) Runs 18383-18437.

FIG. 116: The plot of the $z$ vertex as a function of the $\phi$ angle with corresponding fit for the ${ }^{3} \mathrm{He}$ target at $2.2 \mathrm{GeV}$, for electrons at $\theta=28^{\circ}$.

In order to select electrons coming from the target region we cut on the corrected electron vertex. We have done a small simulation to find out the location of the walls of the cryotarget cell in order to cut out the region in the electron vertex distribution corresponding to target walls, as there were a few different cryotarget cells used with different lengths and locations. We fit the peak corresponding to heat shield in the vertex distribution by a Gaussian function and have used the value of the $\sigma$ as the vertex resolution. We then uniformly generated a vertex distribution corresponding to the length of the 4 th target cell $(4.016 \mathrm{~cm})$ taken from empty target runs and smeared it with vertex resolution. By comparing the smeared and unsmeared electron vertex distributions, we found that the location of the walls are at the half-max points of the falling sides of the smeared vertex distribution (see Fig. 112). We then chose the cut region to be $2 \sigma$ inside from the target walls for cryotarget cells ${ }^{3} \mathrm{He},{ }^{4} \mathrm{He}$.

${ }^{3} \mathrm{He}$ data at $4.4 \mathrm{GeV}$ and ${ }^{4} \mathrm{He}$ data at $4.4 \mathrm{GeV}$ were corrected with a single vertex correction function each. 
The electron vertex distribution before and after the vertex correction with the corresponding cut is shown in Fig. 113.

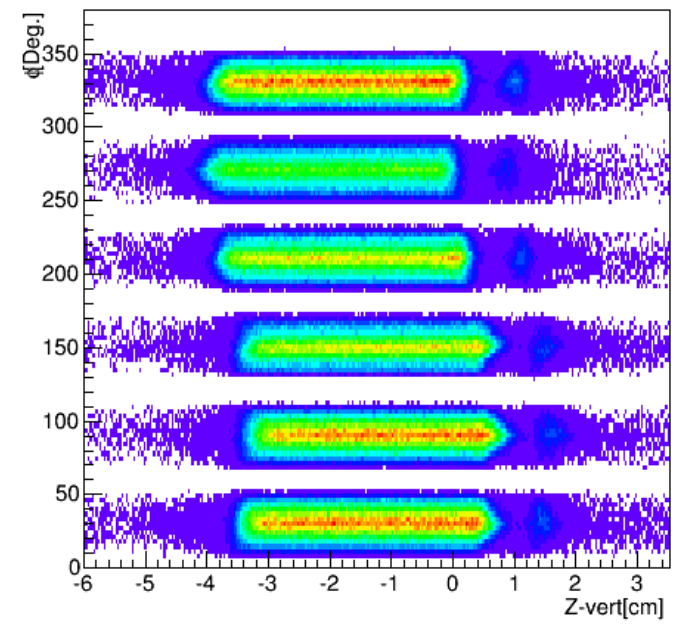

(a) Before correction.

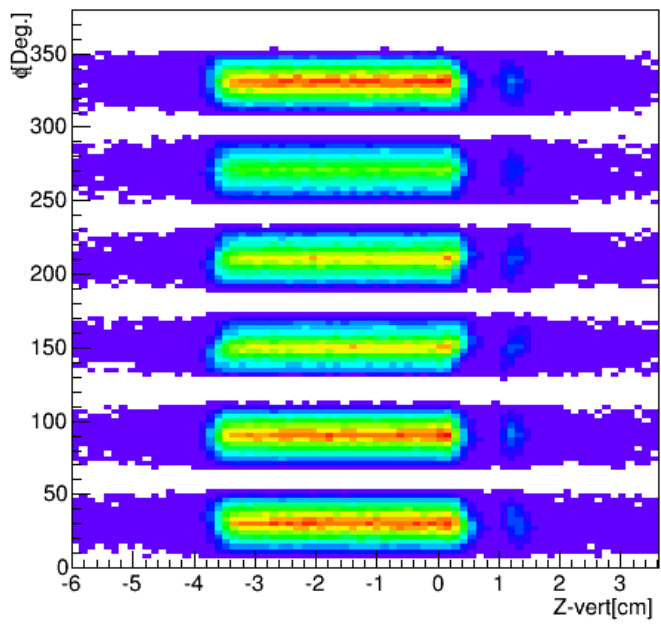

(b) After correction.

FIG. 117: The $\phi$ angle of the the electrons plotted vs the $z$ component of the vertex. The results are shown for the ${ }^{3} \mathrm{He}$ target at $2.2 \mathrm{GeV}$ for runs 18346-18382. (a) Before the vertex correction, (b) After the vertex correction.

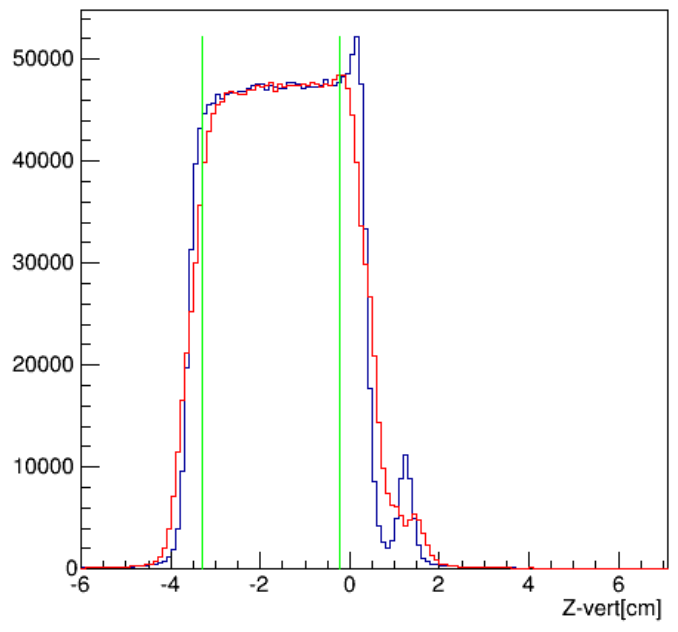

FIG. 118: The distributions of the electron $z$ vertex before (red) and after (blue) correction for ${ }^{3} \mathrm{He}$ at $2.2 \mathrm{GeV}$ for runs 18346 -18382. The green lines correspond to the applied cut.

We also cut on the difference of electron and proton vertices to make sure that they 
are coming from the same event. The plot of electron and proton vertex difference with a corresponding cut is shown in Fig. 114.

In a similar way we cut on the electron and charged pion vertex difference. We have used the same wide cut region $-2.5<z<2.5 \mathrm{~cm}$, at both 4.4 and $2.2 \mathrm{GeV}$ analysis, to make sure that the cut is wide enough to include all possible good pions, in order not to underestimate the number of undetected pions in later analysis. The plot of electron and charged pion vertex difference with a corresponding cut is shown in Fig. 115.

A more detailed description of the vertex correction procedure can be found in [64].

\subsection{GEV ANALYSIS}

The vertex corrections and cuts for the $2.2 \mathrm{GeV}$ analysis have been developed in a similar way as the ones for $4.4 \mathrm{GeV}$.

At $2.2 \mathrm{GeV}$ analysis there was a need to group the ${ }^{3} \mathrm{He}$ and ${ }^{4} \mathrm{He}$ data into two groups each and develop separate vertex corrections and cuts. The cuts are summarized in Table 14. The distributions for two run groups corresponding to runs 18346-18382 and runs 18383-18437 of $2.2 \mathrm{GeV}^{3} \mathrm{He}$ data are shown below.

The plots of the vertex correction functions for ${ }^{3} \mathrm{He}$ at $2.2 \mathrm{GeV}$ for two run groups are shown in Fig. 116. So depending on run number we use different parameters for the vertex correction functions.

The $\phi$ angle vs $z$ vertex distributions before and after the vertex correction for two run groups of $2.2 \mathrm{GeV}^{3} \mathrm{He}$ data are shown in Fig. 117 and Fig. 121.

The electron vertex distributions before and after the vertex correction with the corresponding cut are shown in Fig. 118 and Fig. 122. We can see in these figures that unlike for runs 18346-18382, there is a peak at the exit wall of the target for runs 18383-18437. As both run periods had the same target cell, we have concluded that probably the target cell or beam was slightly tilted during runs 18383-18437 and the beam has interacted with thicker parts of the exit window away from center. For this case we have fit the exit window peak with Gaussian function to find the location of the wall and have cut $2 \sigma$ inside from the wall to select electrons coming from the target region. The change in vertex distribution is in agreement with the records of the shift takers, made during this run period. In fact the vertex distribution changes after run 18382, and the records indicate that run 18383 was taken with new beam tuning.

The electron and proton vertex difference with a corresponding cut are shown in Fig. 119 


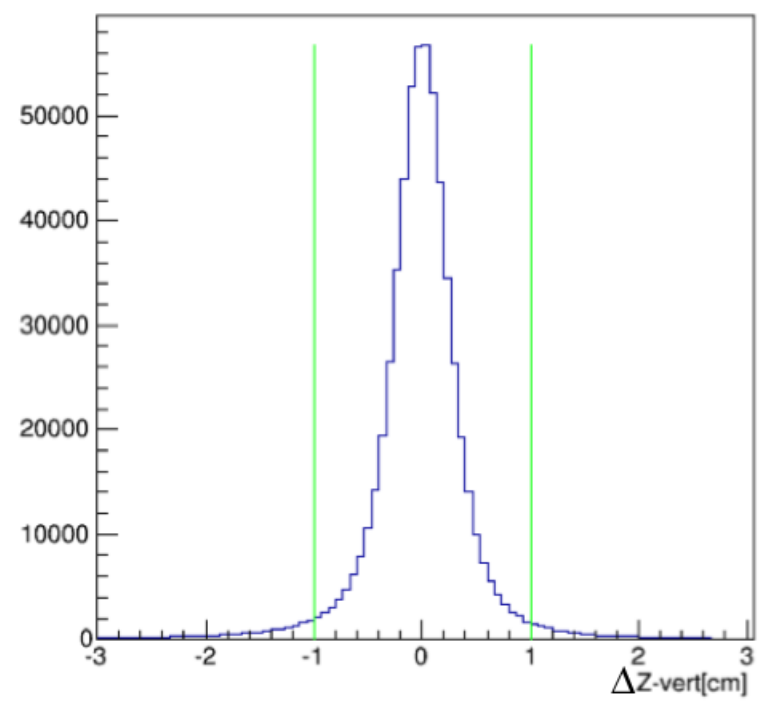

FIG. 119: The electron and proton $z$ vertex difference for ${ }^{3} \mathrm{He}$ at $2.2 \mathrm{GeV}$ for runs 18346 18382. The green lines correspond to the applied cut.

and Fig. 123 and the ones for the electron and charged pion vertex difference are shown in Fig. 120 and Fig. 124.

The vertex cuts for different targets and beam energies are listed in Table 14.

The vertex cuts and corrections for other targets at both energies are described in Appendix A.

\subsection{GEV ANALYSIS}

The vertex corrections and cuts for the $1.1 \mathrm{GeV}$ analysis have been developed in a similar way as the ones for $2.2 \mathrm{GeV}$ and $4.4 \mathrm{GeV}$.

The plots of the vertex correction functions for ${ }^{3} \mathrm{He}$ and ${ }^{12} \mathrm{C}$ targets at $1.1 \mathrm{GeV}$ are shown in Fig. 125.

The $\phi$ angle vs $z$ vertex distributions before and after the vertex correction for both targets at $1.1 \mathrm{GeV}$ are shown in Fig. 126. We also cut on the difference of electron and proton $z$ vertexes and the difference of electron and charged pion vertexes as shown in Figs. 128, 129 and 130 .

The vertex cuts are summarized in Table 14 . 


\begin{tabular}{c|c|c|c|c|} 
Target & Runs & $\begin{array}{c}\text { Beam energy } \\
{[\mathrm{GeV}]}\end{array}$ & $z$ vertex & $\Delta z_{e p}$ \\
\hline${ }^{3} \mathrm{He}$ & All & 1.1 & $-3.05<z<-0.18$ & $-1<\Delta z<1$ \\
${ }^{12} \mathrm{C}$ & All & 1.1 & $4.95<z<5.76$ & $-1<\Delta z<1$ \\
${ }^{3} \mathrm{He}$ & $18346-18382$ & 2.2 & $-3.29<z<-0.23$ & $-1<\Delta z<1$ \\
& $18383-18437$ & 2.2 & $-3.31<z<0.01$ & $-1<\Delta z<1$ \\
${ }^{4} \mathrm{He}$ & $18176-18206$ & 2.2 & $-2.53<z<1.73$ & $-1<\Delta z<1$ \\
${ }^{12} \mathrm{C}$ & $18221-18252$ & 2.2 & $-2.27<z<0.77$ & $-1<\Delta z<1$ \\
& All & 2.2 & $4.8<z<5.5$ & $-1<\Delta z<1$ \\
${ }^{56} \mathrm{Fe}$ & $18158-18166$ & 2.2 & $4.6<z<5.4$ & $-1<\Delta z<1$ \\
${ }^{3} \mathrm{He}$ & 18258,18259 & 2.2 & $5.2<z<6$ & $-1<\Delta z<1$ \\
${ }^{4} \mathrm{He}$ & All & 4.4 & $-3.27<z<0.07$ & $-1<\Delta z<1$ \\
${ }^{12} \mathrm{C}$ & All & 4.4 & $-2.51<z<1.71$ & $-1<\Delta z<1$ \\
${ }^{56} \mathrm{Fe}$ & All & 4.4 & $4.7<z<5.3$ & $-1<\Delta z<1$ \\
& All & 4.4 & $4.6<z<5.4$ & $-1<\Delta z<1$
\end{tabular}

TABLE 14: The cut values on the electron $z$ vertex, and the difference between the vertexes of the electron and the proton for different targets and beam energies. These cut values are used to select particles that are coming from the target region and correspond to the same event. 


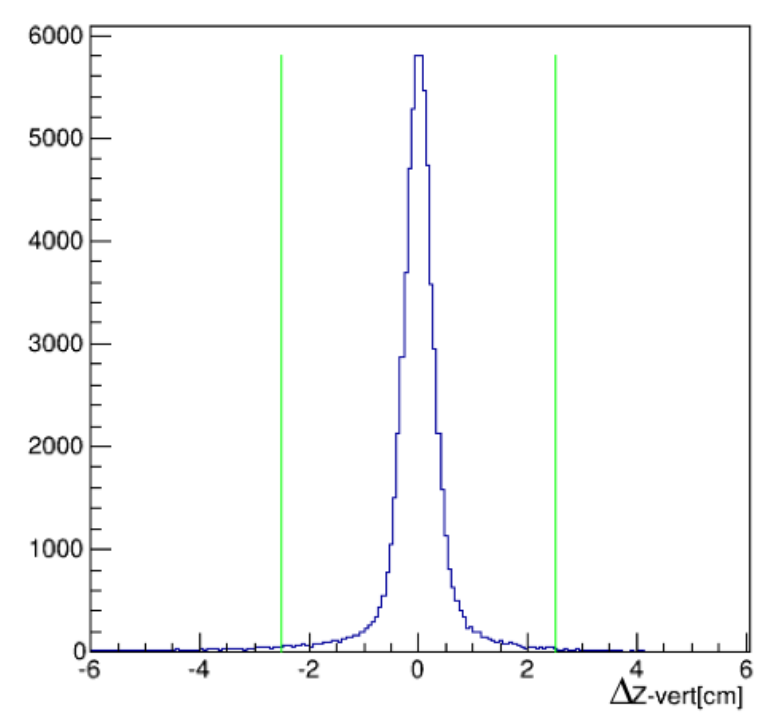

(a) $\pi^{-}$.

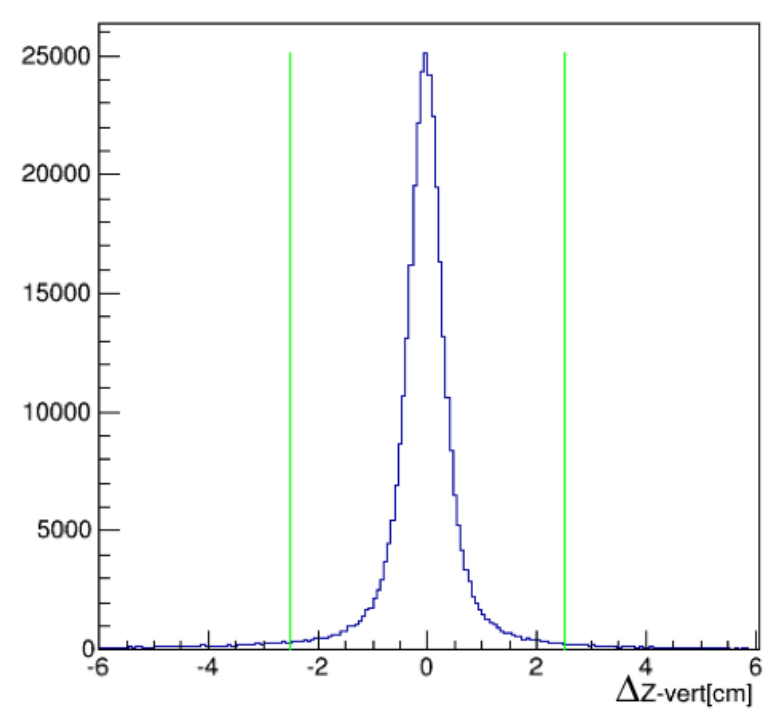

(b) $\pi^{+}$.

FIG. 120: The charged pion and proton $z$ vertex difference for ${ }^{3} \mathrm{He}$ at $2.2 \mathrm{GeV}$ for runs 18346-18382. The green lines correspond to the applied cut.

\subsubsection{Vertex cut studies}

We estimated the contamination from target walls in the liquid target analysis. We did this for the fourth cryotarget cell used during 1.1, 2.2 and $4.4 \mathrm{GeV}^{3} \mathrm{He}$ runs.

We first used only $1.1 \mathrm{GeV}{ }^{12} \mathrm{C}$ data (run 18294) taken with the empty fourth cryotarget cell very close to the $1.1 \mathrm{GeV}{ }^{3} \mathrm{He}$ runs taken with the same cell. We integrated the number of events in the ${ }^{12} \mathrm{C}$ target, the two target walls, and the heat shield (see Fig. 131a). The cryotarget entrance window has $2.6 \%$ as many counts as the ${ }^{12} \mathrm{C}$ target. We scaled this by the relative thickness of the ${ }^{3} \mathrm{He}$ and ${ }^{12} \mathrm{C}$ targets $\left(\rho^{3} \mathrm{He} * l^{3} \mathrm{He}\right) /\left(\rho^{12} \mathrm{C} * l_{12} \mathrm{C}\right)=$ $\left(0.277 \mathrm{~g} / \mathrm{cm}^{2}\right) /\left(0.179 \mathrm{~g} / \mathrm{cm}^{2}\right)=1.547$, to estimate that the target entrance window contributes about $1.7 \%$ of the events from the ${ }^{3} \mathrm{He}$ target. If we assume that the two windows are similar then the total number of events from both windows of the fourth cryotarget cell will be $2 * 1.7=3.4 \%$ of those from the ${ }^{3} \mathrm{He}$.

We then fit the peaks corresponding to target walls and the heat shield in the electron $\mathrm{z}$ vertex distribution of $1.1 \mathrm{GeV}^{12} \mathrm{C}$ data with a Gaussian function and used the corresponding 


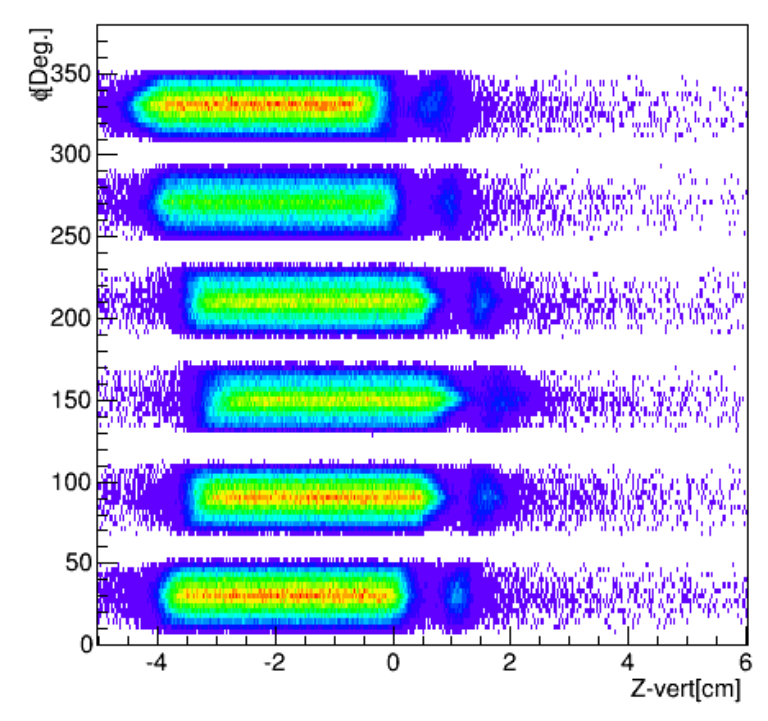

(a) Before correction.

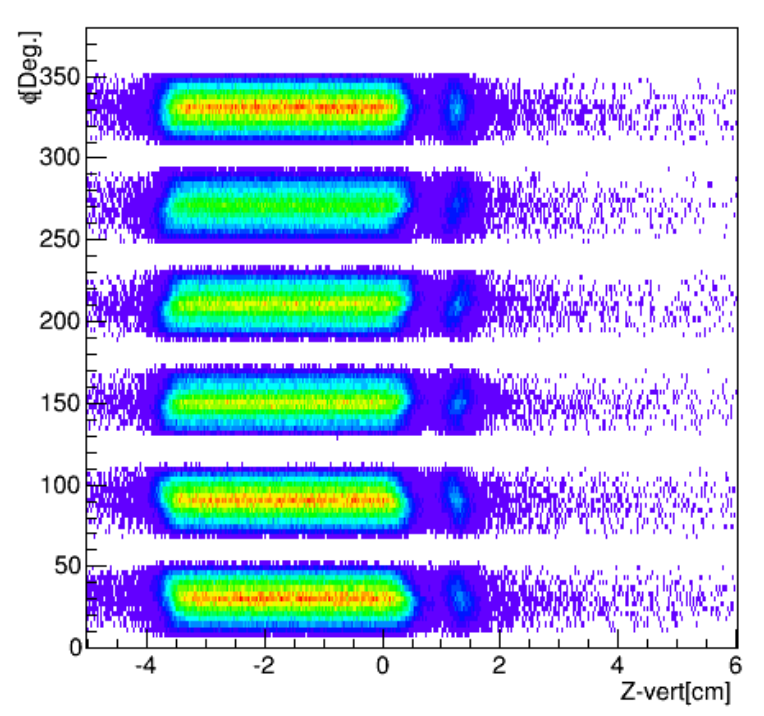

(b) After correction.

FIG. 121: The $\phi$ angle of the the electrons plotted vs the $z$ component of the vertex. The results are shown for the ${ }^{3} \mathrm{He}$ target at $2.2 \mathrm{GeV}$ for runs 18383-18437. (a) Before the vertex correction, (b) After the vertex correction.

heights, widths and locations to reconstruct the target walls in the electron vertex distribution of $1.1 \mathrm{GeV}^{3} \mathrm{He}$ data (run 18294). The corrected electron vertex distribution with the peaks is shown in Fig. 131b. The relative heights of the peaks for the cryotarget windows and the heat shield should be the same for ${ }^{12} \mathrm{C}$ and ${ }^{3} \mathrm{He}$. We re-calculated the ratio of the number of events from the target walls to the events from the target region and have found it to be $3.4 \%$, which is the same as the result obtained above using the ${ }^{12} \mathrm{C}$ target.

We estimated the contamination from the walls in the electron vertex region selected by vertex cuts for the $1.1 \mathrm{GeV}{ }^{3} \mathrm{He}$ data with the fourth liquid target cell $2 \sigma$ inside the target by cutting on the distributions in Fig. 131b. The events coming from target windows are only $0.1 \%$ of those from the target region. This is negligible.

Also to validate that $d$ and $\phi_{\text {beam }}$ are independent of $\theta$ to validate the explanation of Eq. 61 we found the parameters for the vertex correction function for ${ }^{3} \mathrm{He}$ at $4.4 \mathrm{GeV}$, by looking at the electron $\phi$ vs $z$ vertex distribution for $\theta$ angle values equal to 20,25 and $29^{\circ}$, and compared the three results. The $\theta$ angle distribution after the electron fiducial cuts is shown in Fig. 132. The values of $d$ and $\phi_{\text {beam }}$ are relatively close for these three cases, for $d$ 


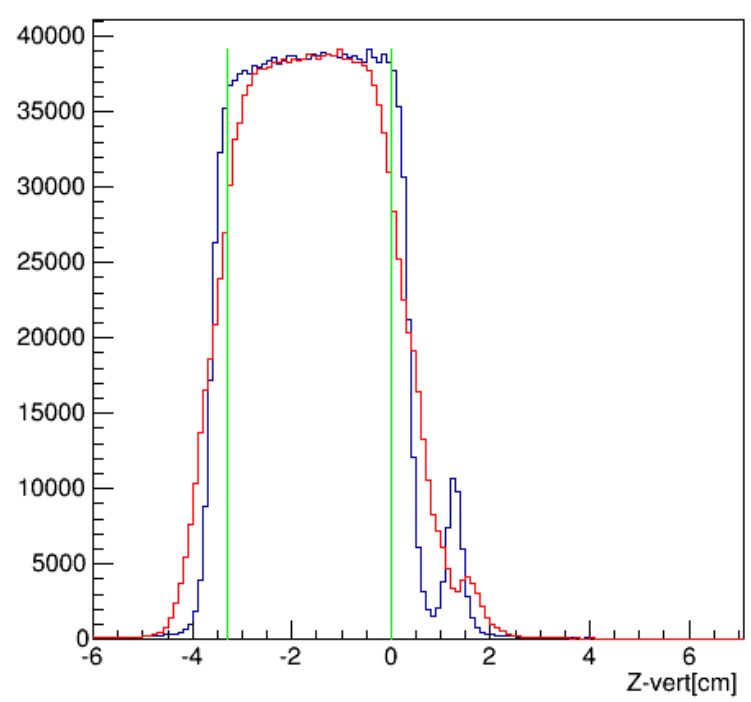

FIG. 122: The distributions of the electron $z$ vertex before (red) and after (blue) correction for ${ }^{3} \mathrm{He}$ at $2.2 \mathrm{GeV}$ for runs $18383-18437$. The green lines correspond to the applied cut.

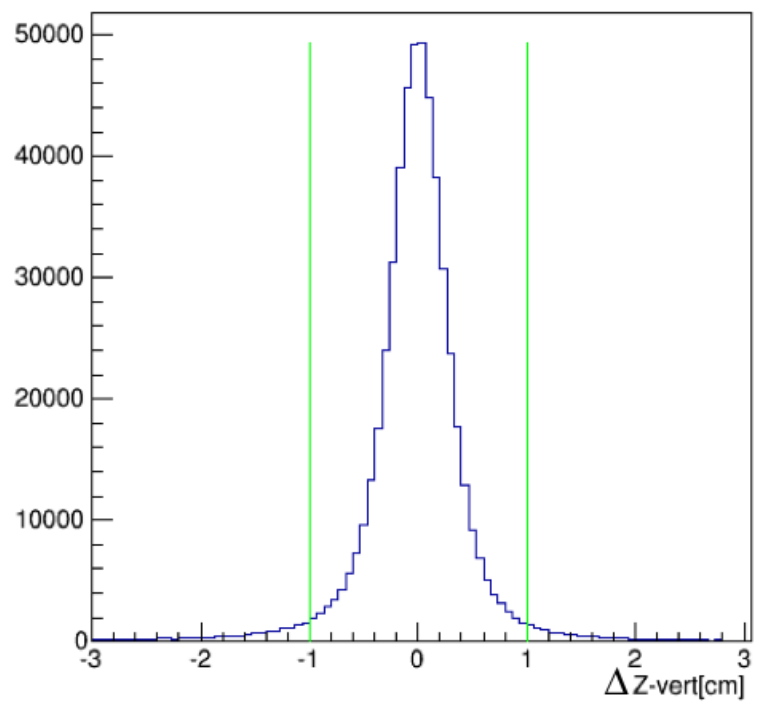

FIG. 123: The electron and proton $z$ vertex difference for ${ }^{3} \mathrm{He}$ at $2.2 \mathrm{GeV}$ for runs 18383 18437. The green lines correspond to the applied cut. 


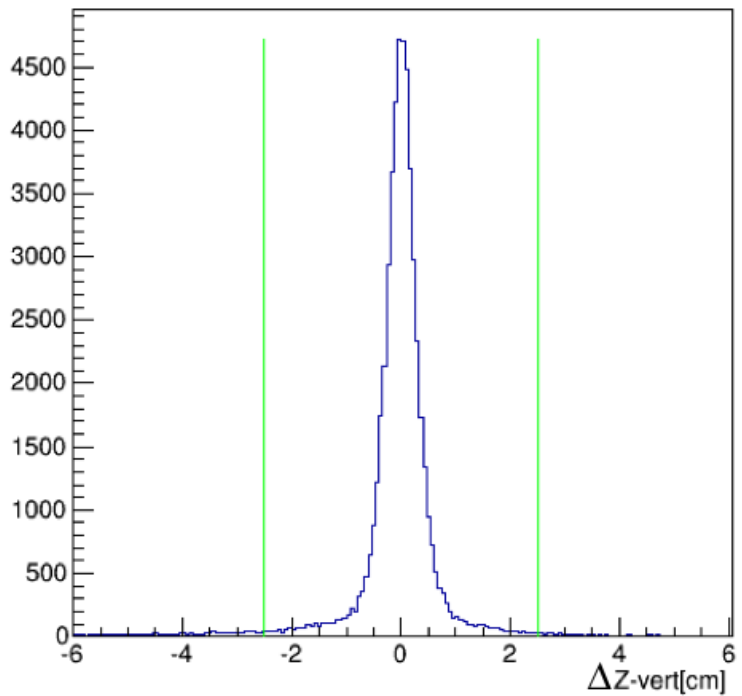

(a) $\pi^{-}$.

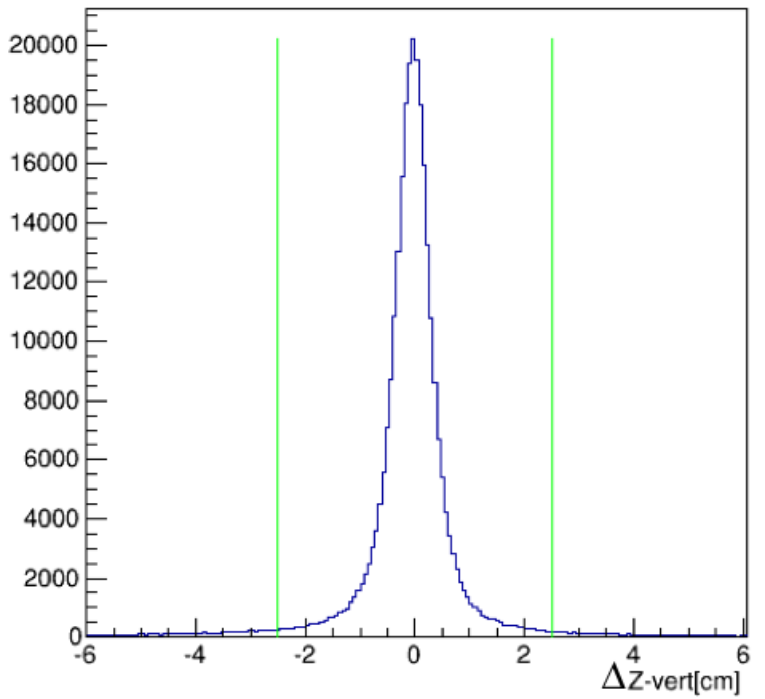

(b) $\pi^{+}$.

FIG. 124: The charged pion and proton $z$ vertex difference for ${ }^{3} \mathrm{He}$ at $2.2 \mathrm{GeV}$ for runs 18383-18437. The green lines correspond to the applied cut.

we get $0.9 \mathrm{~mm}$ difference at most, and for $\phi_{\text {beam }}$ the difference doesn't exceed $1^{\circ}$.

We have summarized the applied cuts and corrections for $2.2 \mathrm{GeV}$ and $4.4 \mathrm{GeV}$ beam energy analysis in Table 15. 


\begin{tabular}{|c|c|c|c|}
\hline Cut & Type & Source & Same parameters \\
\hline \multicolumn{4}{|c|}{$4.4 \mathrm{GeV}$} \\
\hline \multirow[t]{6}{*}{$e^{-} \mathrm{PID}$} & $E_{\text {tot }} / p$ vs $p$ & {$[56]$} & No \\
\hline & $p$ & {$[56]$} & Yes \\
\hline & $E_{\text {in }}$ & {$[56]$} & Yes \\
\hline & $E_{\text {tot }}$ & {$[56]$} & Yes \\
\hline & CC-SC time diff $(\Delta t)$ & new cut & No \\
\hline & angle betw. CC-SC hits & new cut & No \\
\hline$p$ PID & & different & No \\
\hline$e^{-}$momentum correction & & different & No \\
\hline p energy loss correction & & {$[56]$} & Yes (using the one for $2 \mathrm{GeV}$ ) \\
\hline \multirow[t]{2}{*}{$e^{-}$fiducial cuts } & EC edge cut & {$[58]$} & Yes \\
\hline & $\theta$ vs $\phi$ outline cut & {$[56]$} & Yes \\
\hline p fiducial cuts & $\theta$ vs $\phi$ outline cut & {$[56]$} & Yes \\
\hline vertex corrections & & different & No \\
\hline vertex cuts & & different & No \\
\hline \multicolumn{4}{|c|}{$2.2 \mathrm{GeV}$} \\
\hline \multirow[t]{6}{*}{$e^{-}$PID } & $E_{\mathrm{tot}} / p$ vs $p$ & {$[56]$} & No \\
\hline & $p$ & new cut & No \\
\hline & $E_{\text {in }}$ & {$[56]$} & No \\
\hline & $E_{\text {tot }}$ & {$[56]$} & No \\
\hline & CC-SC time diff $(\Delta t)$ & new cut & No \\
\hline & angle betw. CC-SC hits & new cut & No \\
\hline$p$ PID & & different & No \\
\hline$e^{-}$momentum correction & & different & No \\
\hline p energy loss correction & & {$[56]$} & Yes \\
\hline \multirow[t]{3}{*}{$e^{-}$fiducial cuts } & EC edge cut & {$[58]$} & Yes \\
\hline & $\theta$ vs $\phi$ outline cut & {$[56]$} & Yes \\
\hline & bad channel knock-out & {$[56]$} & Yes \\
\hline \multirow[t]{2}{*}{ p fiducial cuts } & $\theta$ vs $\phi$ outline cut & {$[56],[65]$} & Yes \\
\hline & bad channel knock-out & {$[56]$} & Yes \\
\hline vertex corrections & & different & No \\
\hline vertex cuts & & different & No \\
\hline
\end{tabular}

TABLE 15: The detailed table of the applied cuts and corrections. 


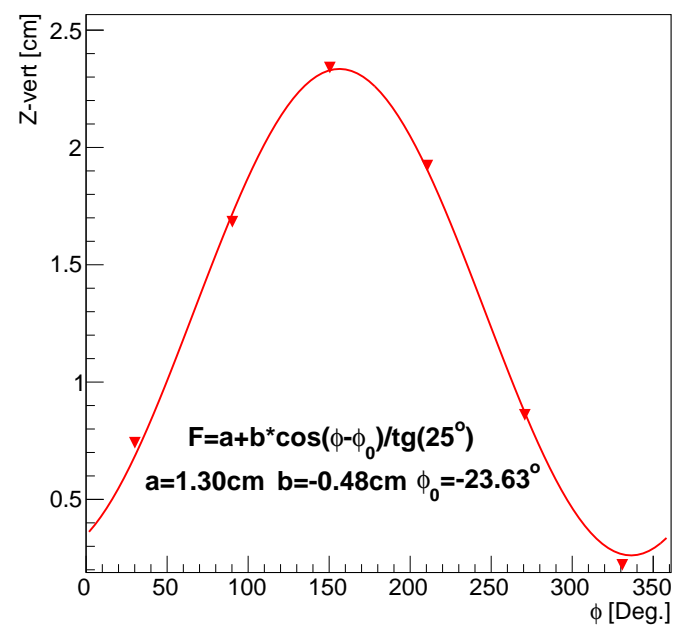

(a) ${ }^{3} \mathrm{He}$

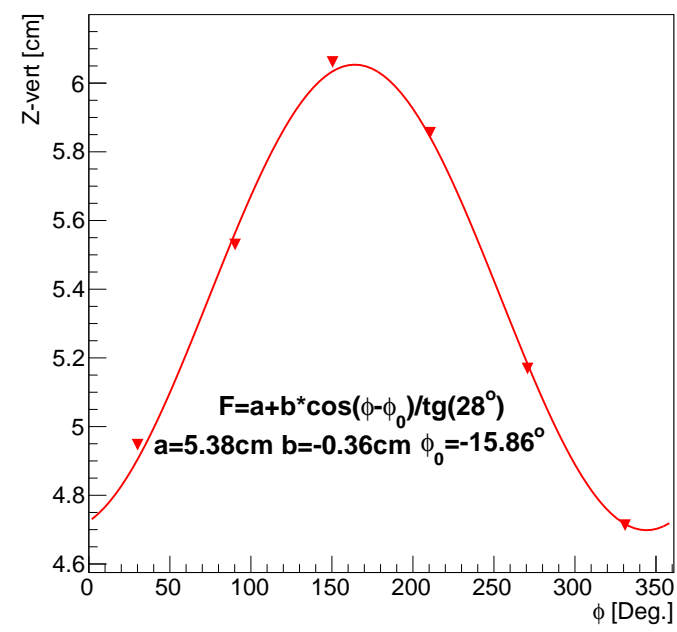

(b) ${ }^{12} \mathrm{C}$

FIG. 125: The plot of the $z$ vertex as a function of the $\phi$ angle with corresponding fit for ${ }^{3} \mathrm{He}$ and ${ }^{12} \mathrm{C}$ targets at $1.1 \mathrm{GeV}$, for electrons at $\theta=25^{\circ}$ and $\theta=28^{\circ}$ correspondingly. 


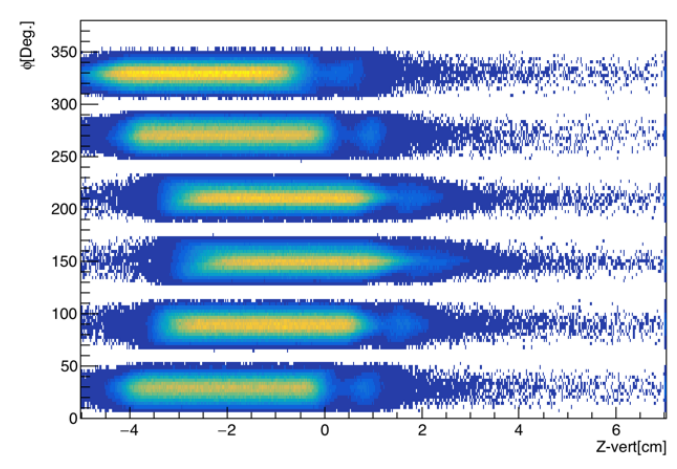

(a) ${ }^{3} \mathrm{He}$, before correction.

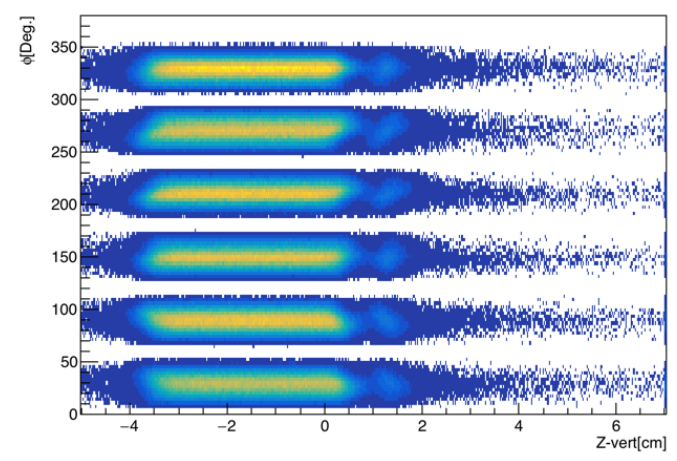

(c) ${ }^{3} \mathrm{He}$, after correction.

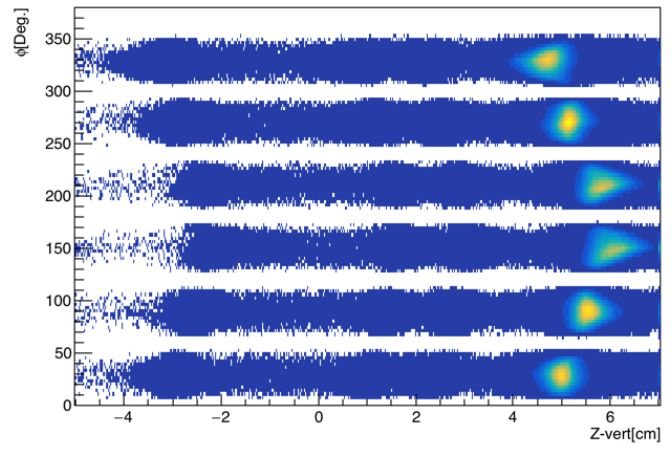

(b) ${ }^{12} \mathrm{C}$, before correction.

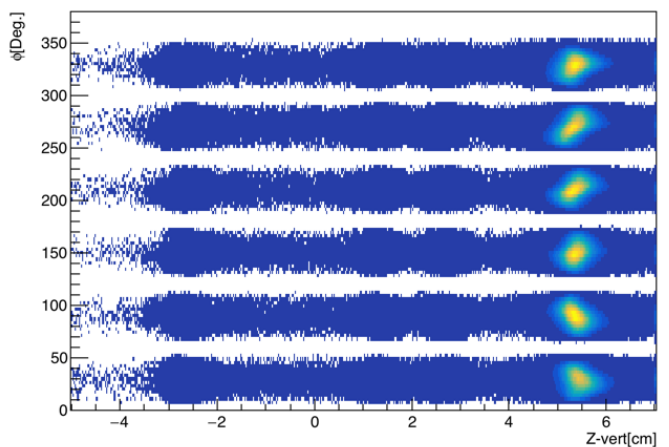

(d) ${ }^{12} \mathrm{C}$, after correction.

FIG. 126: The $\phi$ angle of the the electrons plotted vs the $z$ component of the vertex. The results are shown for the ${ }^{3} \mathrm{He}$ (plots on left) and ${ }^{12} \mathrm{C}$ (plots on right) target at $1.1 \mathrm{GeV}$ before (top plots) and after (bottom plots) vertex correction.

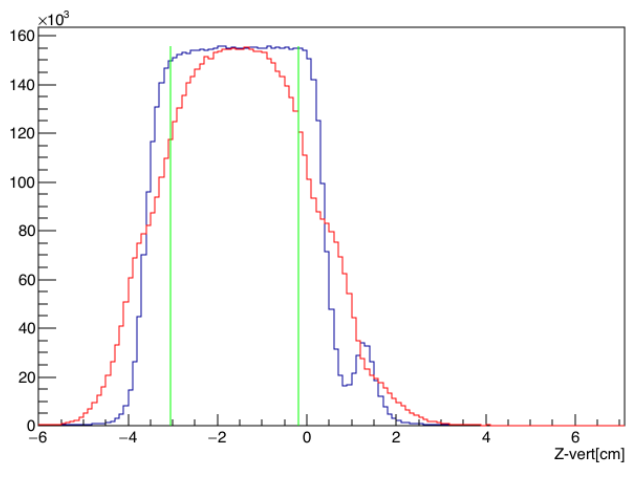

(a) ${ }^{3} \mathrm{He}$

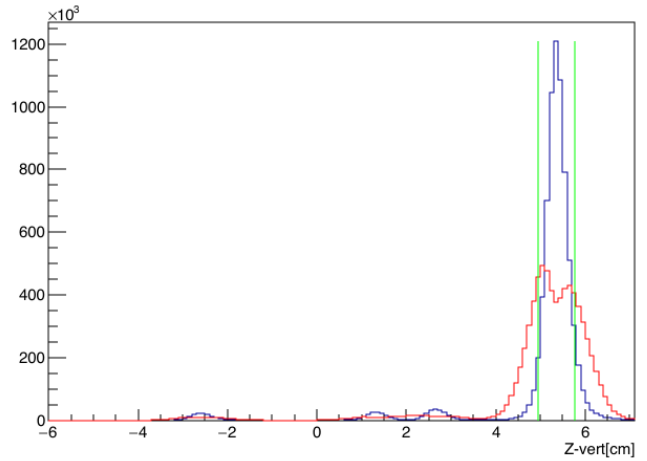

(b) ${ }^{12} \mathrm{C}$

FIG. 127: The distributions of the electron $z$ vertex before (red) and after (blue) correction for ${ }^{3} \mathrm{He}$ and ${ }^{12} \mathrm{C}$ at $1.1 \mathrm{GeV}$. The green lines correspond to the applied cuts. 


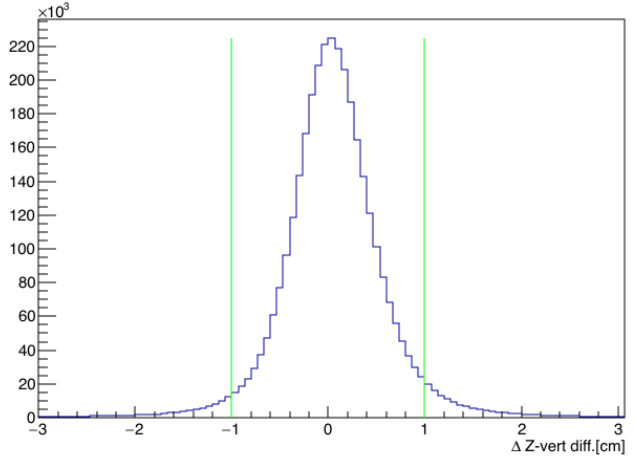

(a) ${ }^{3} \mathrm{He}$

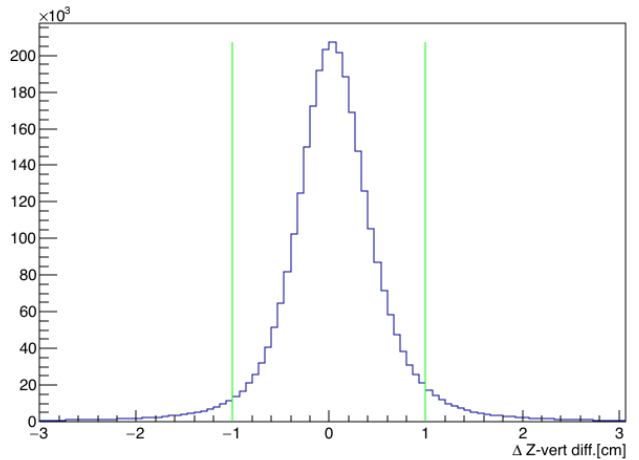

(b) ${ }^{12} \mathrm{C}$

FIG. 128: The electron and proton $z$ vertex difference for ${ }^{3} \mathrm{He}$ and ${ }^{12} \mathrm{C}$ at $1.1 \mathrm{GeV}$. The green lines correspond to the applied cut.

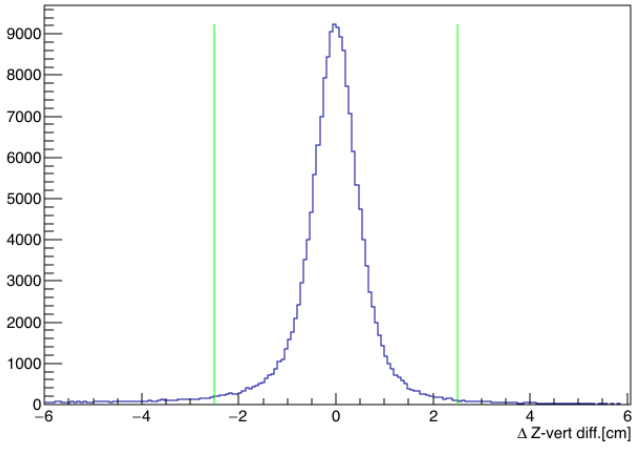

(a) $\pi^{-}$.

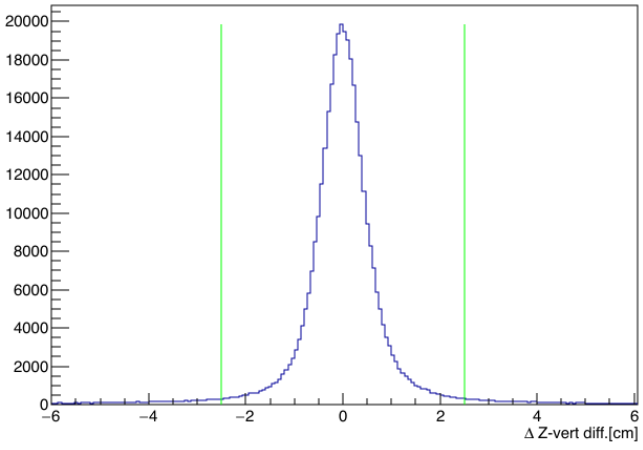

(b) $\pi^{+}$.

FIG. 129: The charged pion and proton $z$ vertex difference for ${ }^{3} \mathrm{He}$ at $1.1 \mathrm{GeV}$. The green lines correspond to the applied cut. 


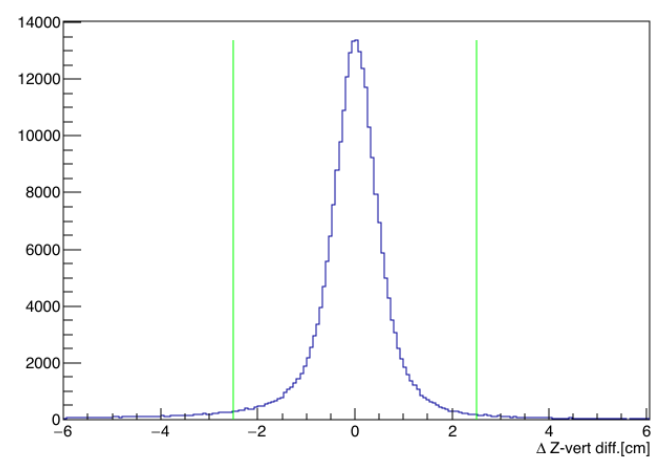

(a) $\pi^{-}$.

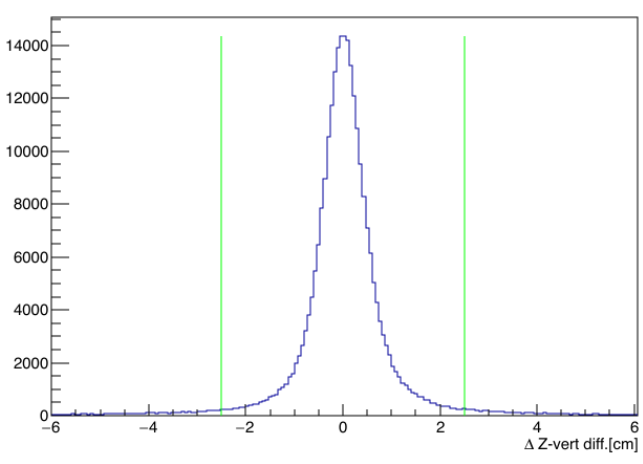

(b) $\pi^{+}$.

FIG. 130: The charged pion and proton $z$ vertex difference for ${ }^{12} \mathrm{C}$ at $1.1 \mathrm{GeV}$. The green lines correspond to the applied cut.

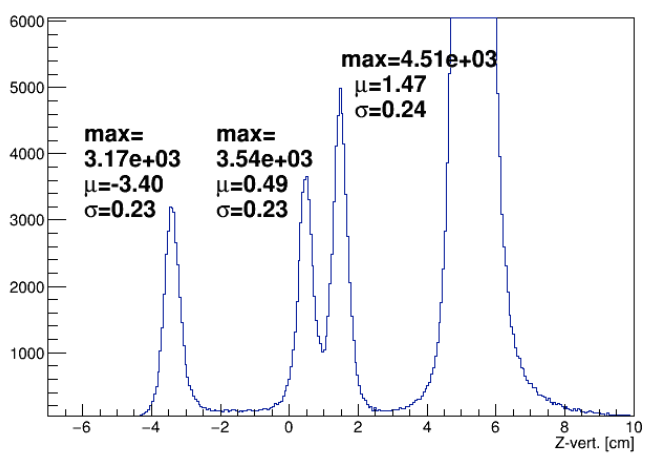

(a) ${ }^{12} \mathrm{C}$.

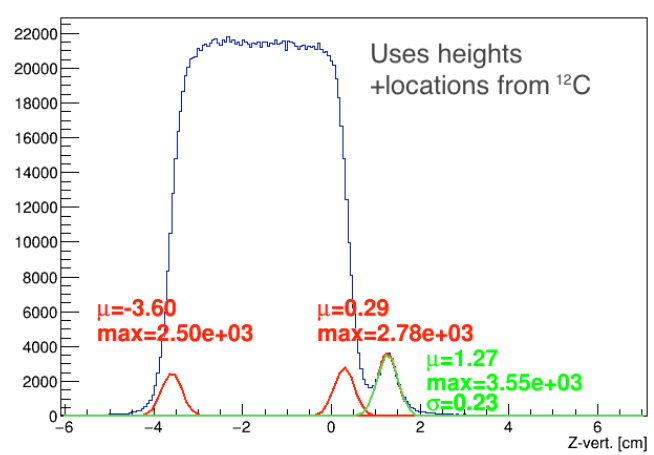

(b) ${ }^{3} \mathrm{He}$.

FIG. 131: The distributions of the corrected electron $z$ vertex for ${ }^{12} \mathrm{C}$ and ${ }^{3} \mathrm{He}$ at $1.1 \mathrm{GeV}$, corresponding to the run numbers 18294 and 18309. (a) The four peaks correspond to the target entrance and exit windows, the heat shield, and the ${ }^{12} \mathrm{C}$ target. 


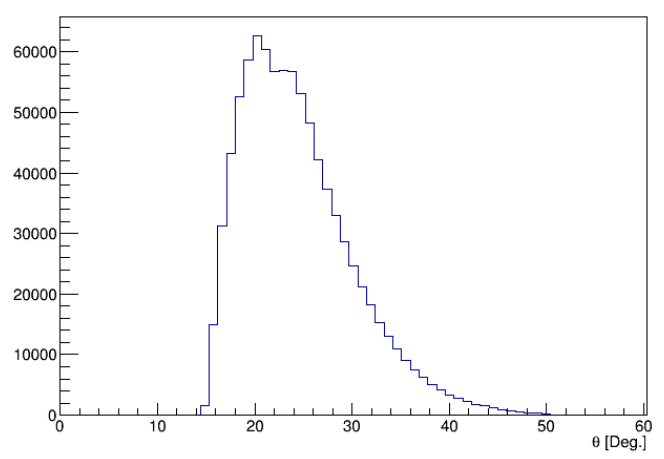

FIG. 132: The electron $\theta$ angle distribution ${ }^{3} \mathrm{He}$ at $4.4 \mathrm{GeV}$ after fiducial cuts.

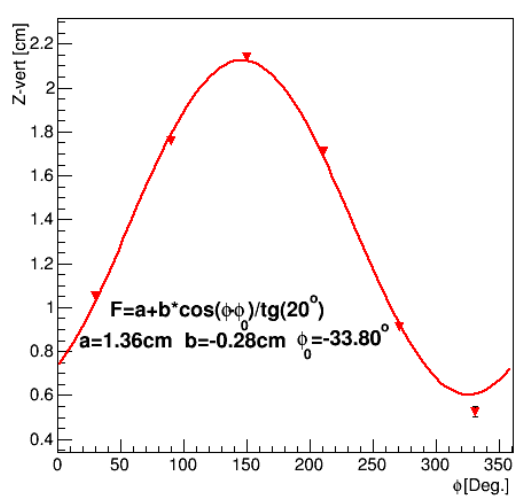

(a) $\theta=20^{\circ}$.

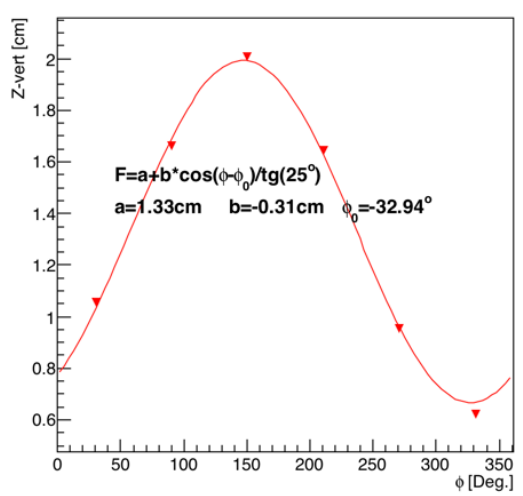

(b) $\theta=25^{\circ}$.

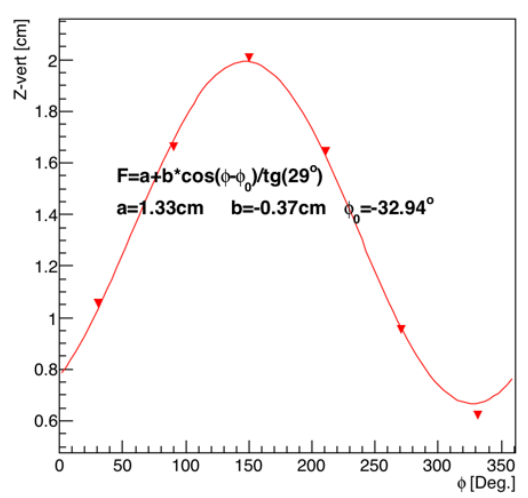

(c) $\theta=29^{\circ}$.

FIG. 133: The plot of the $z$ vertex as a function of the $\phi$ angle with the corresponding fit for the ${ }^{3} \mathrm{He}$ target at $4.4 \mathrm{GeV}$, for different values of $\theta_{e}$. 


\section{CHAPTER 5}

\section{PHYSICS ANALYSIS}

\subsection{ANALYSIS OF CLAS6 DATA}

Neutrino experiments typically extract a "QE data sample" in the CC QE Weak interaction by eliminating events with pions. This selection enhances the contribution of QE scattering events.

We need to use different QE event selection algorithms to suppress the contribution of non-QE interactions of electrons in the nucleus and to enhance the QE scattering spectrum. The QE energy reconstruction method will not work well with non-QE reaction mechanisms. Neutrino experiments have a limited ability to determine the reaction mechanism associated with the particular event.

If the electron scatters quasi-elastically on a nucleon inside the nucleus that is at rest and knocks it out, the initial energy of the electron can be reconstructed using only the information of the scattered electron via Eq. 1 as modified for electrons:

$$
E_{\mathrm{QE}}=\frac{2 M \epsilon-m_{\mathrm{e}}^{2}+2 M E_{\mathrm{e}}}{2\left(M-E_{\mathrm{e}}+\left|k_{\mathrm{e}}\right| \cos \theta_{e}\right)},
$$

where $M$ is the nucleon mass, $\epsilon$ is the single-nucleon separation energy and we take it to be equal $20 \mathrm{MeV}$ for all nuclei, $k_{\mathrm{e}}$ is its momentum, $E_{\mathrm{e}}$ is its energy and $\theta_{e}$ is the angle between the outgoing electron and the direction of the electron beam. This is the method used in neutrino experiments with water Cherenkov-detectors, which are sensitive only to the outgoing lepton.

Suppose now an electron scatters quasi-elastically on a nucleon inside the nucleus and knocks it out and no other reaction mechanisms take place. If we detect both the scattered electron and the knocked out nucleon and assume that the target nucleus is at rest and the residual A-1 nucleus stays intact, we will get the following relation from the energy conservation :

$$
E_{\text {calor }}+M_{\mathrm{A}}=E_{\mathrm{e}}+E_{\mathrm{p}}+E_{\mathrm{A}-1}
$$


where $E_{\text {calor }}$ is the energy of the incident electron, $M_{\mathrm{A}}$ is the mass of nucleus $A, E_{\mathrm{p}}$ is the energy of the detected proton and $E_{\mathrm{A}-1}=\sqrt{M_{\mathrm{A}-1}^{2}+p_{\mathrm{A}-1}^{2}}$ is the energy of the $A-1$ system, with $M_{\mathrm{A}-1}$ being its mass and $p_{\mathrm{A}-1}$ being its momentum which should be equal to the initial momentum of the knockout nucleon $\left(\vec{p}_{\text {init }}=-\vec{p}_{\mathrm{A}-1}\right)$ if there are no FSI. If we ignore the kinetic energy of the recoil system, which should be small then Eq. 63 reduces to

$$
E_{\text {calor }}=E_{\mathrm{e}}+T_{\mathrm{p}}+\epsilon
$$

where $T_{\mathrm{p}}$ is the kinetic energy of the knockout proton and $\epsilon$ is the average nucleon separation energy. We assume initially $\epsilon=E_{A}^{\text {Bind }}-E_{A-1}^{\text {Bind }}$, where $E_{A}^{\text {Bind }}$ and $E_{A}^{\text {Bind }}$ are the binding energies of $A$ and $A-1$ nuclei. Eq. 64 can be used to obtain the initial energy of the electron $\left(E_{\text {calor }}\right)$ via the energy sum method. This is the method used in calorimetric-type detectors, such as the ones in the experiments $\mathrm{NO} \nu \mathrm{A}, \mathrm{MINER} \nu \mathrm{VA}$ and MicroBooNE.

Momentum conservation gives:

$$
\vec{p}=\vec{p}_{\mathrm{e}}+\vec{p}_{\mathrm{p}}+\vec{p}_{\mathrm{A}-1}
$$

and can be used for $\mathrm{QE}$ event selection. $\vec{p}_{\mathrm{e}}, \vec{p}_{\mathrm{p}}$ and $\vec{p}_{\mathrm{A}-1}$ are the three momenta of the scattered electron, the knockout proton and the residual nucleus, respectively. If we now project Eq. 65 on the directions parallel (denoted by $z$ ) and perpendicular (denoted by $\perp$ ) to the incoming beam we will have:

$$
\begin{gathered}
P_{\mathrm{tot}}=p_{\mathrm{e}}^{z}+p_{\mathrm{p}}^{z}+p_{\mathrm{A}-1}^{z} \\
P_{\text {miss }}^{\perp}=p_{\mathrm{e}}^{\perp}+p_{\mathrm{p}}^{\perp} \cong p_{\text {init }}^{\perp}
\end{gathered}
$$

where we have used the fact that $\vec{p}_{\mathrm{A}-1}=-\vec{p}_{\text {init }}$, which is valid only for QE scattering. The perpendicular component of the momentum of the $A-1$ system should also be small and be less than or about the Fermi momentum (the momentum of the nucleon in the highest occupied state of the nuclear Fermi gas model that is $\approx 250 \mathrm{MeV} / \mathrm{c}$ ). This means that Eq. 66 can also be used to obtain the initial energy of the scattered electron from the detected final state particles. This avoids uncertainties coming from the determination of the binding energy in Eq. 64. However, Eq. 66 provides a less accurate description of the beam energy because of the initial momentum of the nucleons.

Eq. 67 can be used to select QE events, by calculating $P_{\text {miss }}^{\perp}$ and requiring that it was smaller than $p_{\text {Fermi }}$.

The dependence of the cross section on the kinematics of the scattering is different for neutrinos than electrons. In both cases the propagator goes like $\frac{1}{Q^{2}+M^{2}}$, where $Q^{2}$ is the 
four momentum transfer and $M^{2}$ is the mass of the exchanged boson ( $W^{ \pm}$and $Z^{0}$ bosons for the weak interaction and photon for the electromagnetic interactions). Thus, the propagator for neutrino scattering goes as $\frac{1}{M^{2}}$ since $m_{W}, m_{Z^{0}}>>Q^{2}$ (see Section 1.5 for more details). This means that the cross section goes as $\frac{1}{M^{4}}$ and thus is not sensitive to the kinematics.

For electron scattering the photon mass is zero and the Mott cross section will go as $\frac{1}{Q^{4}}$ :

$$
\left(d \sigma d \Omega_{e^{\prime}}\right)_{M}=\alpha^{2} \cos ^{2}\left(\theta_{e} / 2\right) /\left[4 E_{e}^{2} \sin ^{4}\left(\theta_{e} / 2\right)\right]
$$

In the analysis we weight each event by one over the Mott cross section to make the electron data more similar to neutrino data.

The electron interacts by a neutral current and the neutrino interactions considered here proceed via a charged current. Neutrino events where $\nu_{\mu} \rightarrow \mu^{-}$will have more protons and fewer neutrons in the final state than electron events, which in turn will have more protons and fewer neutrons in the final state than $\bar{\nu}_{\mu} \rightarrow \mu^{+}$.

We have applied the CLAS E2a standard cuts and corrections described in detail in the previous sections and we have obtained $A\left(e, e^{\prime}\right)$ and $A\left(e, e^{\prime} p\right)$ data samples for different targets and beam energies. For QE scattering we selected events without detected charged pions or photons, (to eliminate $\pi^{0}$, which decays into two photons). We also weighted each of the events with $1 / \sigma_{\text {Mott }}$ to make the electron scattering cross section more similar to the neutrino cross sections.

\begin{tabular}{ccccccc} 
Target & \multicolumn{2}{c}{$1.1 \mathrm{GeV}$} & \multicolumn{2}{c}{$2.2 \mathrm{GeV}$} & \multicolumn{2}{c}{$4.4 \mathrm{GeV}$} \\
& $\left(e, e^{\prime}\right)$ & $\left(e, e^{\prime} p\right)$ & $\left(e, e^{\prime}\right)$ & $\left(e, e^{\prime} p\right)$ & $\left(e, e^{\prime}\right)$ & $\left(e, e^{\prime} p\right)$ \\
\hline${ }^{3} \mathrm{He}$ & 8.3 & 3.8 & 24.9 & 10.0 & 2.8 & 0.9 \\
${ }^{4} \mathrm{He}$ & & & 39.9 & 14.5 & 5.3 & 1.7 \\
${ }^{12} \mathrm{C}$ & 7.9 & 2.6 & 31.1 & 11.6 & 3.6 & 1.2 \\
${ }^{56} \mathrm{Fe}$ & & & 1.7 & 0.6 & 0.3 & 0.1
\end{tabular}

TABLE 16: The number of good $\left(e, e^{\prime}\right)$ and $\left(e, e^{\prime} p\right)$ events in millions for different targets that satisfy $e$ and $p$ PID, vertex, fiducial and $W<2 \mathrm{GeV}$ invariant mass cuts for the $1.1 \mathrm{GeV}$, $2.2 \mathrm{GeV}$ and $4.4 \mathrm{GeV}$ analysis. The invariant mass cut is applied to eliminate deep inelastic scattering events.

The numbers of good $\left(e, e^{\prime}\right)$ and $\left(e, e^{\prime} p\right)$ events for different targets that satisfy $e$ and $p$ PID, vertex, fiducial and $W<2 \mathrm{GeV}$ invariant mass cuts are listed in Table 16 . We cut on 
the invariant mass $W=\sqrt{\left(m_{\mathrm{p}}+\nu\right)^{2}-\vec{q}^{2}}<2 \mathrm{GeV}$, where $m_{\mathrm{p}}$ is the proton mass, $\nu$ is the energy transfer and $\vec{q}$ is the three momentum transfer, to eliminate deep inelastic scattering events.

Our goal is to produce pionless $A\left(e, e^{\prime}\right)$ event samples and pionless $A\left(e, e^{\prime} p\right)$ samples with only one proton and to determine how well we can reconstruct the incident lepton energy using just the final state particles.

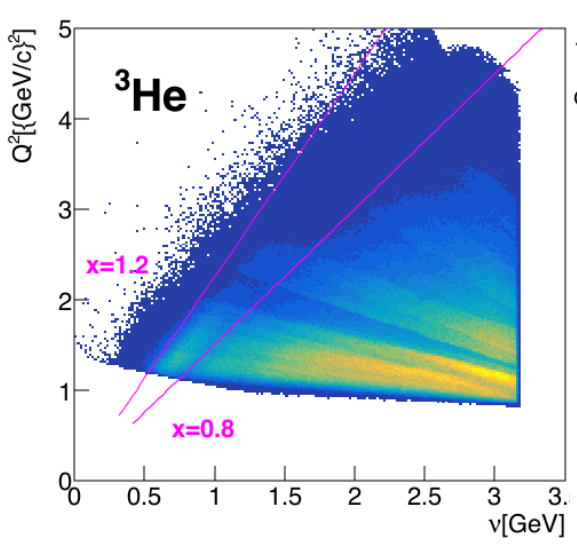

(a)

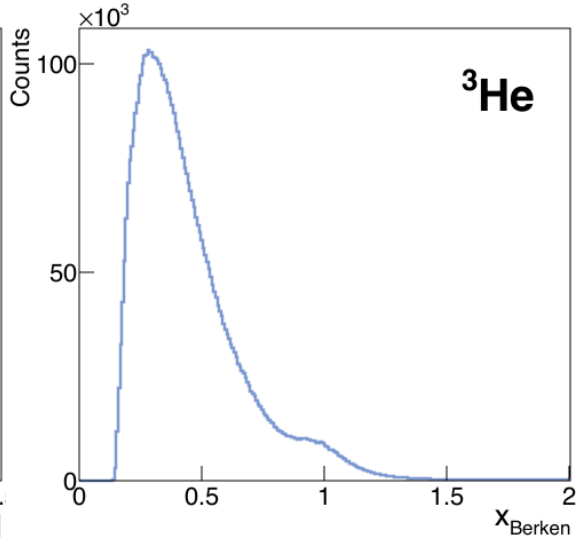

(b)

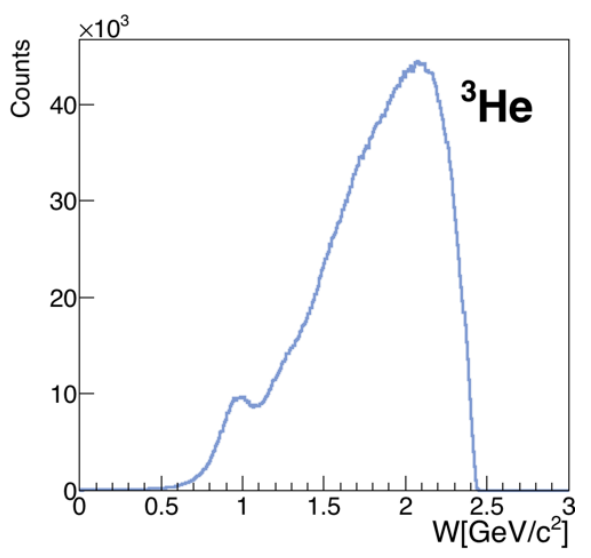

(c)

FIG. 134: The plots of (a) four momentum transfer $Q^{2}$ as a function of energy transfer $\nu$, (b) Bjorken $x\left(x_{\mathrm{B}}\right)$ and (c) Invariant mass of virtual photon and proton system $W(e p)$ for ${ }^{3} \mathrm{He}$ at $4.4 \mathrm{GeV}$. The plots are made after electron PID and Fiducial cuts.

We calculated $E_{\mathrm{QE}}$ (Eq. 62) and $E_{\text {calor }}$ to our electron scattering data. Plots for ${ }^{3} \mathrm{He}$ and ${ }^{56} \mathrm{Fe}$ are shown in this section. Plots for ${ }^{4} \mathrm{He}$ and ${ }^{12} \mathrm{C}$ are shown in the appendix $\mathrm{B}$.

This data is perfect for this type of analysis as the beam energy is close to that of the ongoing neutrino experiments (see Fig. 6), and the targets, especially ${ }^{12} \mathrm{C}(\mathrm{NO} \nu \mathrm{A})$ and ${ }^{56} \mathrm{Fe}$ 


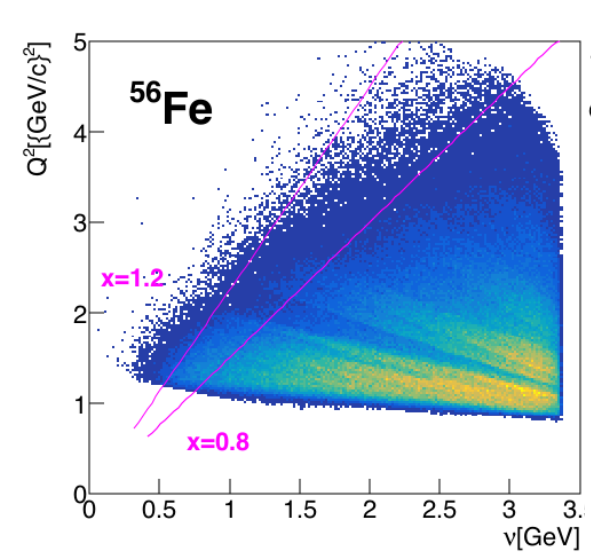

(a)

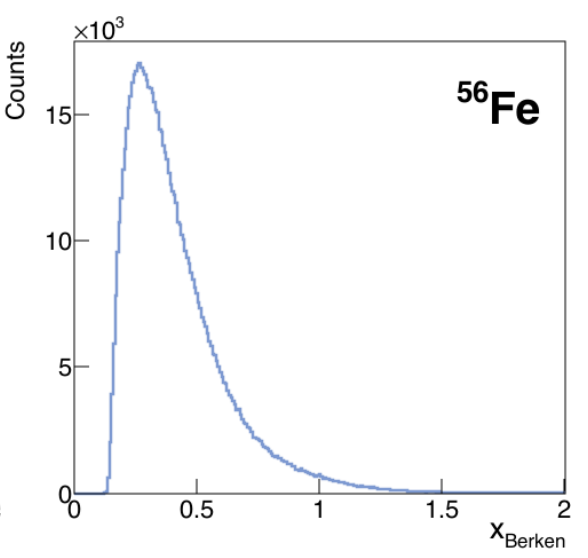

(b)

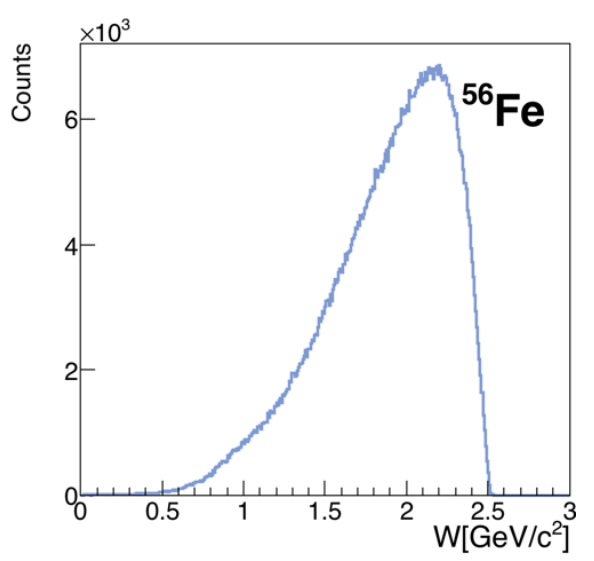

(c)

FIG. 135: The plots of (a) four momentum transfer $Q^{2}$ as a function of energy transfer $\nu$, (b) Bjorken $x\left(x_{\mathrm{B}}\right)$ and (c) Invariant mass of virtual photon and proton system $W(e p)$ for ${ }^{56} \mathrm{Fe}$ at $4.4 \mathrm{GeV}$. The plots are made after electron PID and Fiducial cuts.

$(\mathrm{MINER} \nu \mathrm{A})$ are similar to the ones used in these experiments.

\subsection{GEV $A\left(e, e^{\prime}\right)$ AND $A\left(e, e^{\prime} p\right)$ ANALYSIS}

We start by showing the kinematic distributions for the various event samples. The plots of four momentum transfer $\left(Q^{2}\right)$ as a function of energy transfer $\nu$, the distribution of $x_{\mathrm{B}}=Q^{2} / 2 M_{\mathrm{p}} \nu$, with $M_{\mathrm{p}}$ being the proton mass, as well as the invariant mass of the virtual photon-proton system for ${ }^{3} \mathrm{He}$ target at $4.4 \mathrm{GeV}$ are shown in Fig. 134. There is a small 
peak at $x_{\mathrm{B}} \sim 1$ corresponding to QE scattering events.

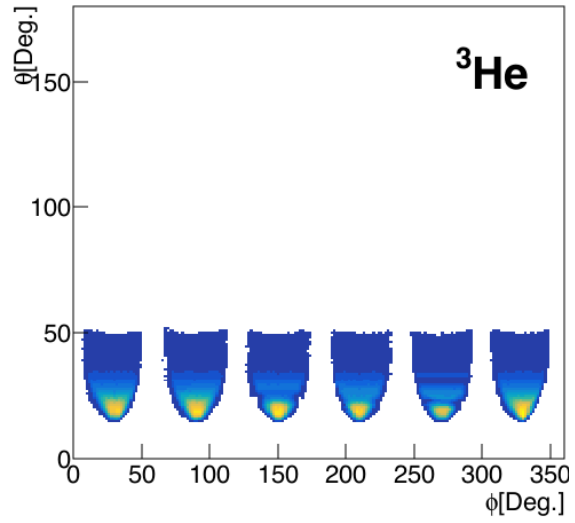

(a)

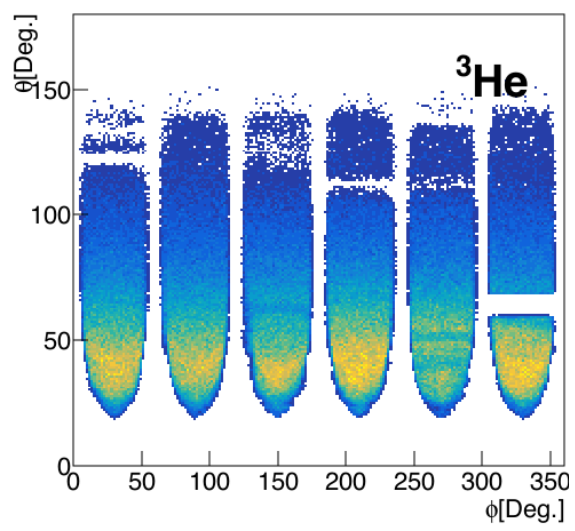

(c)

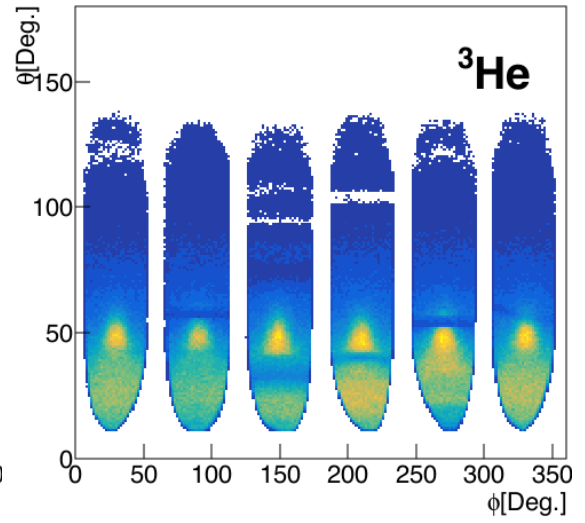

(b)

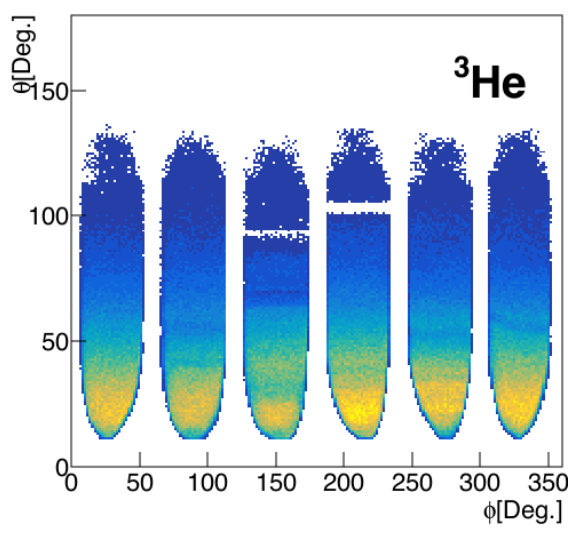

(d)

FIG. 136: The $\theta$ vs $\phi$ angular distribution of different particles for ${ }^{3} \mathrm{He}$ at $4.4 \mathrm{GeV}$. (a) electrons; (b) protons; (c) $\pi^{-}$; (d) $\pi^{+}$.

The invariant mass of the virtual photon-proton system for QE scattering is equal to the proton mass and appears as a small peak at the proton mass in the invariant mass distribution. The plots for ${ }^{56} \mathrm{Fe}$ are shown in Fig. 135. The small QE peak seen in ${ }^{3} \mathrm{He}$ is totally washed out for ${ }^{56} \mathrm{Fe}$.

In CLAS the electron detection is limited to $15^{\circ}<\theta<50^{\circ}$. The $\theta$ vs $\phi$ angular distribution of electrons is shown in top left of Fig. 136.

The CLAS angular coverage for charged pions is larger. However it is slightly different for negative pions compared to positive pions, since negative particles are bent towards the beam line and have a higher minimum $\theta$ (see Fig. 136). The superposition of proton and $\pi^{-}$angular distributions is shown in Fig. 137. The CLAS angular coverage for photons is 


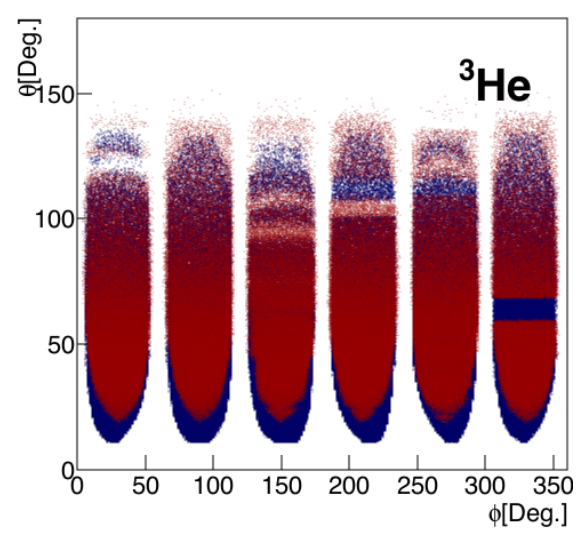

(a)

FIG. 137: The $\theta$ vs $\phi$ angular distribution of $\pi^{-}$and protons for ${ }^{3} \mathrm{He}$ at $4.4 \mathrm{GeV}$ plotted on top of each other.

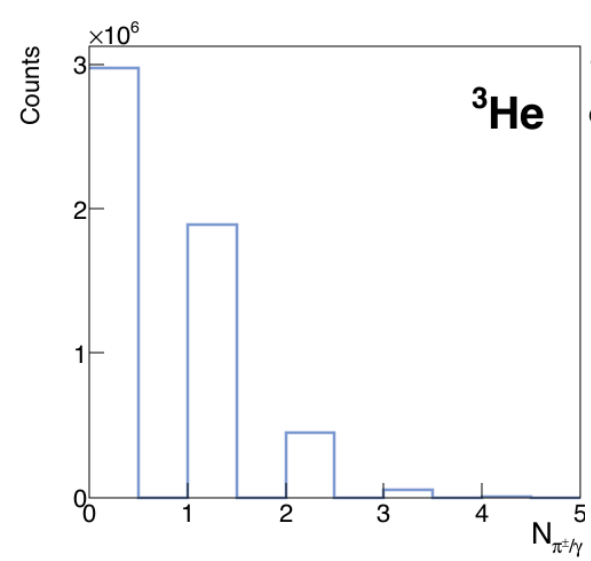

(a)

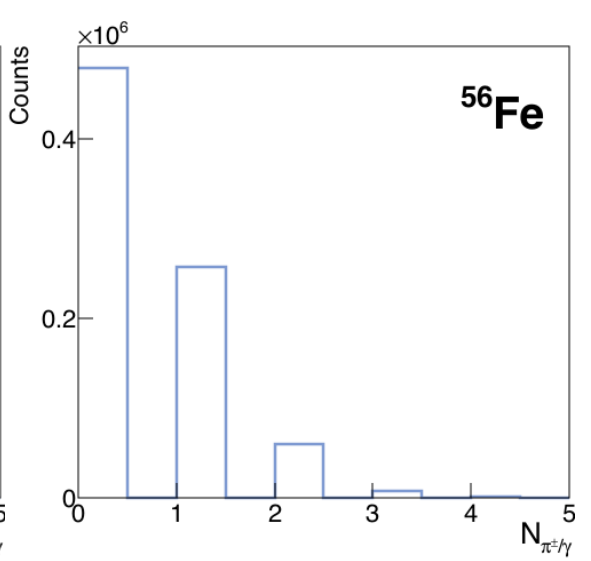

(b)

FIG. 138: Multiplicity distributions of $\pi^{ \pm} / \gamma$ for ${ }^{3} \mathrm{He}$ and ${ }^{56} \mathrm{Fe}$ targets at $4.4 \mathrm{GeV}$ (a) ${ }^{3} \mathrm{He}$; (b) ${ }^{56} \mathrm{Fe}$.

shown in 100.

To enhance the QE sample in our analysis we select events with 0 charged pions and 0 photons detected in EC, to reject $\pi^{0}$ that decay into photons. We refer to this cut as a pion rejection cut. Because of the CLAS geometrical acceptance, some of the charged pions and photons do not get detected. We estimate the contribution of events with undetected charged pions and photons and subtract this contribution from the event sample that has 


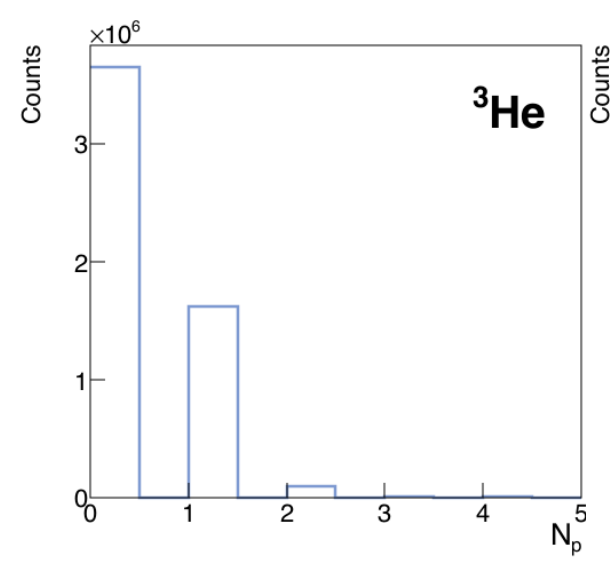

(a)

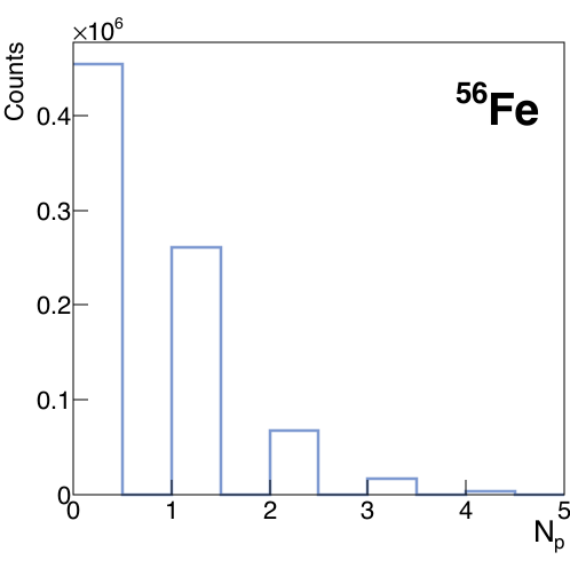

(b)

FIG. 139: Multiplicity distributions of protons for ${ }^{3} \mathrm{He}$ and ${ }^{56} \mathrm{Fe}$ targets at $4.4 \mathrm{GeV}$ (a) ${ }^{3} \mathrm{He}$; (b) ${ }^{56} \mathrm{Fe}$.

passed the pion rejection cut (see Section 5.1.1). Based on the multiplicity plots for charged pions and photons in Fig. 138, one can see that the zero or single $\pi^{ \pm} / \gamma$ events dominate the event sample. There are almost no events with more than three charged pions and photons. The multiplicity distributions for ${ }^{4} \mathrm{He}$ and ${ }^{12} \mathrm{C}$ are in between those of ${ }^{3} \mathrm{He}$ and ${ }^{56} \mathrm{Fe}$. The plots for $2.2 \mathrm{GeV}$ analysis are shown in section 5.1.2.

The angular distribution for protons is similar to positive pions (see Fig. 136). When looking at $A\left(e, e^{\prime} p\right)$ events with one detected proton, we will estimate the contributions of the events with a few protons, where some of the protons have not been detected, and subtract them. The proton multiplicity plots in Fig. 139 suggests that we can ignore contributions of events with five or more protons.

The $E_{\mathrm{QE}}$ distributions of $A\left(e, e^{\prime} p\right)$ events without any cuts, with pion rejection cuts and with an additional cut on $P_{\text {miss }}^{\perp}<200 \mathrm{GeV}$ are compared in Fig. 140. The vertical line shows the true beam energy.

As we apply the pion rejection cuts and add the cut on $P_{\text {miss }}^{\perp}$, we see that a larger fraction of events reconstructs to the correct beam, indicating that we are selecting $\mathrm{QE}$ effects. However, further analysis is required to understand the physics processes describing the events in the tail of the distribution to the left of the peak. For heavier targets, a smaller fraction of events reconstructs to the correct beam energy compared to light nuclei. 


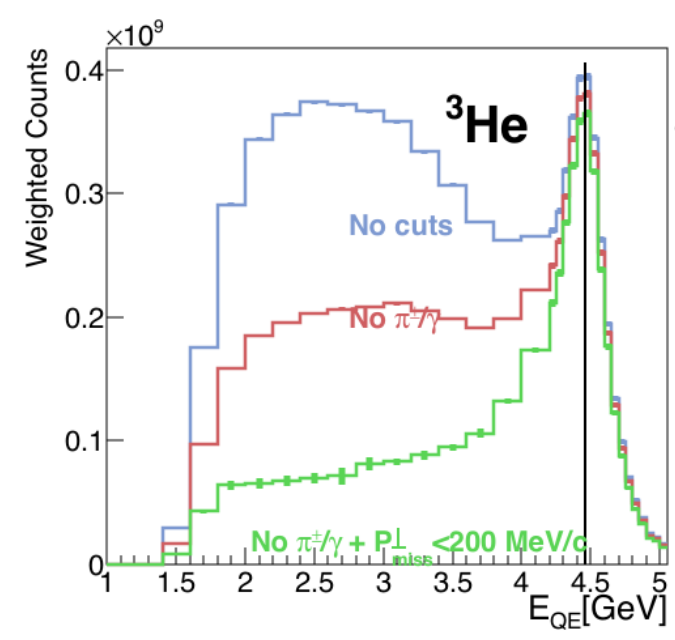

(a)

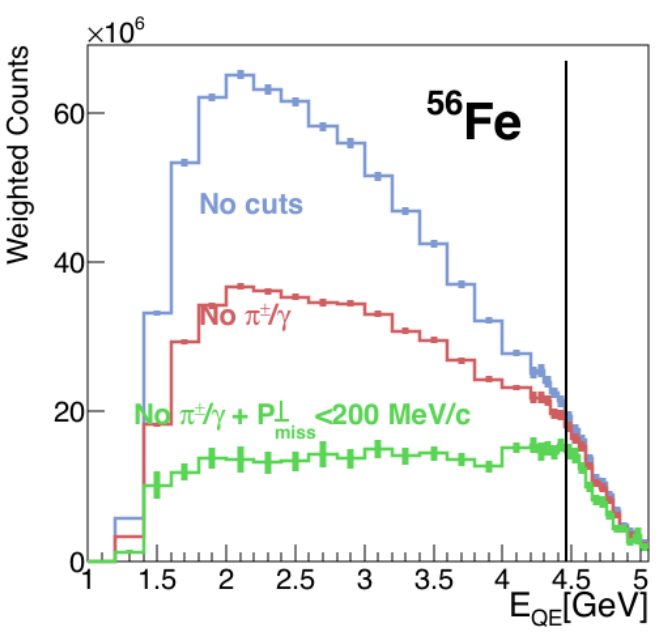

(b)

FIG. 140: Reconstructed energy $E_{\mathrm{QE}}$ of $A\left(e, e^{\prime} p\right)$ obtained using Eq. 62 with no cuts (blue), with pion rejection cut (red) and with cut on total perpendicular momentum of the scattered electron and the knockout nucleon system $P_{\text {miss }}^{\perp}<200 \mathrm{MeV}$ in addition to the pion rejection cut for ${ }^{3} \mathrm{He}$ and ${ }^{56} \mathrm{Fe}$ targets at $4.4 \mathrm{GeV}$ (a) ${ }^{3} \mathrm{He}$; (b) ${ }^{56} \mathrm{Fe}$.

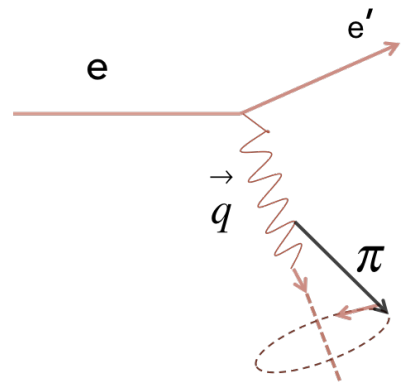

(a)

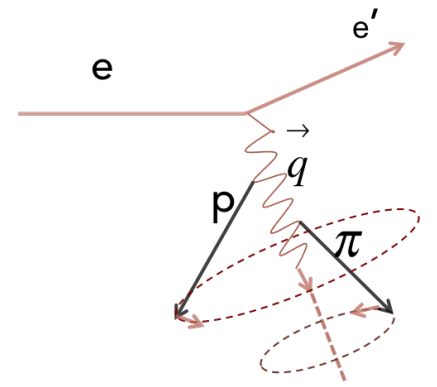

(b)

FIG. 141: The diagrams for the (a) $\pi$ rotation and the (b) $\pi$ and proton rotation around the three momentum transfer $(\vec{q})$ for the estimation of events with undetected $\pi$ in the $A\left(e, e^{\prime}\right)$ and $A\left(e, e^{\prime} p\right)$ analyses.

\section{Subtracting for undetected charged pions, photons and protons}

Non-QE interactions lead to multi-hadron final states. As we can see in Figs. 136 and 155, the geometrical acceptance of CLAS detector has some gaps. Some of the final state charged pions and photons miss the fiducial region and are not being detected. We need to 
estimate the amount of events with undetected $\pi$ and subtract this from the event sample that has passed the pion rejection cut (no charged pions and no photons) in order to obtain true $0 \pi^{ \pm} / \gamma A\left(e, e^{\prime}\right)$ and $A\left(e, e^{\prime} p\right)$ spectra. When describing the subtraction procedure for undetected charged pions and photons we will just refer to them as "pions" (e.g. $2 \pi$ denotes an event with two charged pions or one charged pion and one photon, or two photons).

We do not want to consider photons coming from electron radiation when subtracting for undetected photons, as these photons have small angles with the electron and high probability to get detected. In order to separate radiation and non-radiation photons we looked at the distribution of the angle between the electron and the photon plotted as a function of the difference between the electron and photon azimuthal angles $\phi$. The magnetic field does not affect the azimuthal angle of the electron, and this difference should always be small for radiation photons. We can see these distributions for different targets and beam energies in Fig. 142. The applied cut that encloses radiation photons is shown by red box.

To subtract the contribution of undetected pion events and to obtain a true $0 \pi$ spectrum in the $A\left(e, e^{\prime}\right)$ analyses, for each $A\left(e, e^{\prime} \pi\right)$ event with one detected pion and no detected radiated photons, we rotate the $\pi$ around the direction of the three momentum transfer $\vec{q}$ by a randomly generated angle $\phi$ within 0 to $2 \pi$, and find the probability of the $\pi$ being inside the acceptance region $P(1 \pi)=N_{\text {Detected }} / N_{\text {Rotations }}$, where $N_{\text {Detected }}$ is the number of rotation that lead to the pion being detected and $N_{\text {Rotations }}$ is the total number of rotations. This is used to estimate the corresponding number of undetected $\pi$ events $W=-(1-P(1 \pi)) / P(1 \pi)$ that contribute to $A\left(e, e^{\prime}\right)$ events, for each $A\left(e, e^{\prime} \pi\right)$ event with one detected $\pi$. The negative sign in front of the weight means that this contribution should be subtracted. We chose to rotate around $\vec{q}$ in order not to change the cross section of the reaction (see the left picture in Fig. 141). We will show later that the cross section has a small dependence on the angle between the hadronic and leptonic planes, but the associated error is small.

For each event with an electron and one detected pion there is a single contribution to the $A\left(e, e^{\prime}\right)$ event sample that has passed the pion rejection cut as shown in the one pion subtraction scheme in Fig. 143:

- $(1 \pi \rightarrow 0 \pi)$ This contribution is equal to $-(1-P(1 \pi)) / P(1 \pi)$.

We also need to consider events with two or more detected pions. Subtracting the contribution of undetected $2 \pi$ events is more complicated. $2 \pi$ events might appear as $1 \pi$ events or as $0 \pi$ events. Thus, for each event with two detected pions, we rotate the pions around $\vec{q}$ to estimate the contribution of events with undetected pions to the $0 \pi$ and $1 \pi$ events. This will reduce the number of $0 \pi$ events, but it will also reduce the number of $1 \pi$ events, which 
will in turn reduce the subtraction of $1 \pi$ events from the $0 \pi$ sample (see Fig. 143). Similarly, events with three pions can appear as $3 \pi, 2 \pi, 1 \pi$ or $0 \pi$ events. We use the detected $3 \pi$ events to estimate the number of $2 \pi, 1 \pi$ or $0 \pi$ events with one, two, or three undetected pions (see Fig. 143).

We have looked at the events with two detected charged $\pi_{1} \pi_{2}$, and have rotated those together around $\vec{q}$ for $\mathrm{N}$ times and have found the probabilities of detecting one $P\left(\pi_{1}\right)=$ $\left.N_{\pi_{1}} / N_{\pi_{1} \pi_{2}}, P\left(\pi_{2}\right)=N_{\pi_{2}} / N_{\pi_{1} \pi_{2}}\right)$ or zero pions $P(0 \pi)=N_{0 \pi} / N_{\pi_{1} \pi_{2}}$, where $N_{\pi_{1}}$ and $N_{\pi_{2}}$ are the number of times that only first and only second pion have been detected, $N_{0 \pi}$ is the number of times that none of the pions has been detected and $N_{\pi_{1} \pi_{2}}$ is the number of times that both of the pions have been detected. The contribution of each of these two pion events to 0 pion events is given by the weight $W=-P(0 \pi)$, and thus is subtracted from the event sample that has passed the pion rejection cut.

When subtracting for undetected pions by looking at one detected pion events above, we overestimate the undetected pion events since part of one detected pion events are due two pion events, where one of the pions did not get detected. Thus we subtract more than we are supposed to. To correct for this, for each two pion event we rotate each of the two pions separately and find the probabilities of detecting zero pions out of one $P\left(0 \pi_{1}\right)=$ $N_{0 \pi_{1}} / N_{\pi_{1}}, P\left(0 \pi_{2}\right)=N_{0 \pi_{2}} / N_{\pi_{2}}$, where $N_{0 \pi_{1}}$ and $N_{0 \pi_{2}}$ are the numbers of rotations that lead to the pion not getting detected and $N_{\pi_{1}}$ and $N_{\pi_{2}}$ are number of rotations that lead to the pion getting detected. We then add the corresponding contribution given by the product of the weights $P\left(\pi_{1}\right) * P\left(0 \pi_{1}\right)$ and $P\left(\pi_{2}\right) * P\left(0 \pi_{2}\right)$ to the event sample that has passed the pion rejection cut.

As a summary for each event with an electron and two detected pions there are two contribution to the $A\left(e, e^{\prime}\right)$ event sample that has passed the pion rejection cut as shown in the two pion subtraction scheme in Fig. 143:

- $(2 \pi \rightarrow 0 \pi)$ This contribution is equal to $-P(0 \pi)$, which is described above.

- $(2 \pi \rightarrow 1 \pi \rightarrow 0 \pi)$ This contribution is equal to $P\left(\pi_{1}\right) * P\left(0 \pi_{1}\right)+P\left(\pi_{2}\right) * P\left(0 \pi_{2}\right)$, which are again described above.

We use the same method to correct for undetected three pion events. The scheme of the subtraction for undetected one, two and three pion events is shown in Fig. 143, the sign in green shows whether the corresponding contribution should be added or subtracted from the $A\left(e, e^{\prime}\right)$ event sample that has passed the pion rejection cut. We have accounted for the contributions of up to 4 charged pion and/or photon events, however the undetected three 
charged pion and/or photon contribution is already negligible, as one would expect from the multiplicity plots in Fig. 138, so it is very clear that the results are converging nicely.

For each event with three detected pions there are four contribution to the $A\left(e, e^{\prime}\right)$ event sample that has passed the pion rejection cut as shown in three pion subtraction scheme in Fig. 143:

- $(3 \pi \rightarrow 0 \pi)$ This contribution is equal to $-N_{\text {none }} / N_{\text {all }}$, where $N_{\text {none }}$ is the number of rotations that leads to none of the three pions getting detected and $N_{\text {all }}$ is the number of rotations that leads to all of the three pions getting detected.

- $(3 \pi \rightarrow 1 \pi \rightarrow 0 \pi)$ This contribution is equal to $\sum_{i=1}^{3} \frac{N_{\pi_{i}}}{N_{\text {all }}} * \frac{N_{\pi_{i}}^{\text {undet }}}{N_{\text {rot }}}$, where $N_{\pi_{i}}$ is the number of simultaneous rotations of the three pions that leads to only $\pi_{i}$ getting detected, $N_{\text {all }}$ is the number of simultaneous rotations of the three pions that leads to all of the pions getting detected, $N_{\pi_{i}}^{\text {undet }}$ is the number of rotations of the $\pi_{i}$ only that leads to it not getting detected out of total $N_{\text {rot }}$ rotations.

- $(3 \pi \rightarrow 2 \pi \rightarrow 0 \pi)$ This contribution is equal to $\sum_{i=1}^{3} \sum_{j=i+1}^{3} \frac{N_{\pi_{i} \pi_{j}}^{3}}{N_{\pi_{1} \pi_{2} \pi_{3}}} * \frac{N_{\pi_{i} \pi_{j}}^{\text {none }}}{N_{\pi_{i} \pi_{j}}^{2}}$, where $N_{\pi_{i} \pi_{j}}^{3}$ is the number of simultaneous rotations of the three pions that leads to only $\pi_{i}$ and $\pi_{j}$ getting detected, $N_{\pi_{1} \pi_{2} \pi_{3}}$ is the number of simultaneous rotations of the three

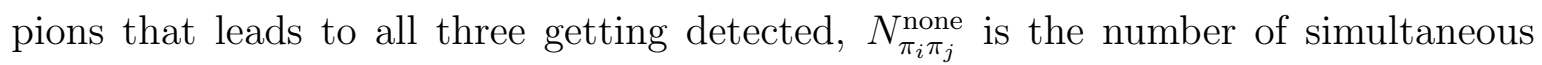
rotations of the two $\pi_{i}$ and $\pi_{j}$ pions that leads to none of them getting detected and $N_{\pi_{i} \pi_{j}}^{2}$ is the number of simultaneous rotations of the two $\pi_{i}, \pi_{j}$ pions that leads to both of the pions getting detected.

- $(3 \pi \rightarrow 2 \pi \rightarrow 1 \pi \rightarrow 0 \pi)$ This contribution is equal to:

$$
-\sum_{i=1}^{3} \sum_{j=i+1}^{3} \frac{N_{\pi_{i} \pi_{j}}^{3}}{N_{\pi_{1} \pi_{2} \pi_{3}}} \sum_{k=i, j}\left(\frac{N_{\pi_{i} \pi_{j}}^{\pi_{k}}}{N_{\pi_{i} \pi_{j}}^{2}} * \frac{N_{\pi_{k}}^{\text {undet }}}{N_{\text {rot }}}\right)
$$

where $N_{\pi_{i} \pi_{j}}^{3}$ and $N_{\pi_{1} \pi_{2} \pi_{3}}$ are defined above, $N_{\pi_{i} \pi_{j}}^{\pi_{k}}$ is the number of simultaneous rotations of the two $\pi_{i}$ and $\pi_{j}$ pions that leads to only one of them getting detected, $N_{\pi_{i} \pi_{j}}^{2}$ is the number of simultaneous rotations of the two $\pi_{i}$ and $\pi_{j}$ pions that leads to both of them getting detected and $N_{\pi_{k}}^{\text {undet }}$ is the number of rotations of the $\pi_{k}$ only that leads to it not getting detected out of total $N_{\text {rot }}$ rotations.

The plots of $E_{\mathrm{QE}}$ for detected (blue) and undetected (red) one pion events, as well as undetected two and three pion events are shown in Fig. 144. The different signs show 
whether the corresponding distribution is added or subtracted from the event sample that has passed the pion rejection cut. These plots suggest that we have nearly the same amount of detected and undetected one pion events, which is due to the $\sim 50 \%$ CLAS detector geometrical acceptance for charged pions.

The plots of $E_{\mathrm{QE}}$ for $A\left(e, e^{\prime}\right)$ events, with no cuts and with no photons or charged pions, before and after subtracting for undetected pions, are shown in the right side of Fig. 144. We can see that the cut and the subtraction reduce significantly the non-QE background. However, there are still a lot of events that correspond to reconstructed energy values much less than the true beam energy and thus these events are not QE. It is also evident from plots that subtraction does not affect the peak but reduces the tail as we would expect.

Now let us consider the effect of undetected pions and extra protons on the $A\left(e, e^{\prime} p\right)$ event sample. Here we need to correct for undetected few pion and few proton events to obtain the true one electron and one detected proton spectrum. The $2 \mathrm{D}$ multiplicity plot for ${ }^{56} \mathrm{Fe}$ at $4.4 \mathrm{GeV}$ is shown in Fig. 145. This data has the highest fraction of few proton and few pion events. The arrows show all the contributions that we have taken into account, 2 proton 0 pion, 3 proton 0 pion, 4 proton 0 pion, 1 proton 1 pion, 1 proton 2 pion, 1 proton 3 pion, 2 proton 1 pion, 2 proton 2 pion and 1 proton 3 pion events. The contribution of the rest of the few charged pion, photon and proton events to the $1 p 0 \pi$ sample is negligible. Each of these few pion and few proton events contributes to different event samples with fewer pions and protons, and we need to take all these contributions into account.

For example, to subtract the contribution of undetected $1 p 1 \pi$ events in the $A\left(e, e^{\prime} p\right)$ analyses, for each $A\left(e, e^{\prime} p \pi\right)$ event we rotate both the proton and $\pi$ around $\vec{q} N$ times by randomly generating $\phi$ within 0 to $2 \pi$ (see the right picture in Fig. 141), and find the probability of the proton being detected and the $\pi$ not being detected $P(1 p 0 \pi)=N_{p} / N_{p \pi}$, where $N_{p}$ is the number of times that proton was detected but pion was not and $N_{p \pi}$ is the number of times that both pion and proton were detected. Then we use this probability to estimate the corresponding number of undetected $\pi$ events $P(1 p 0 \pi)$ that contribute to $A\left(e, e^{\prime} p\right)$ events, for each $A\left(e, e^{\prime} p \pi\right)$ event with one detected $\pi$, and subtract it from the $A\left(e, e^{\prime} p\right)$ event sample that has passed the pion rejection cut.

To get rid of undetected extra protons in the $A\left(e, e^{\prime} p\right)$ analysis, for each event that has a detected electron, two detected protons and no detected photons, we again randomly rotate both of the protons around the direction of $\vec{q}$ for $N$ times. The total number of simulated events, where only one of the protons is inside the proton geometrical acceptance is $N_{p}$. The total number of simulated events with both protons inside the proton geometrical acceptance 
is $N_{p p}$. If we have one event with an electron and two detected protons, the corresponding number of events where one of the protons has not been detected will be $N_{p} / N_{p p}$.

The $4 p 0 \pi$ events can be mis-identified not only as $1 p 0 \pi$ events but also as $2 p 0 \pi, 3 p 0 \pi$ events. The scheme of the subtraction for undetected $4 p 0 \pi$ events is shown in Fig. 146 .

We correct for the rest of the few proton and few charged pion and photon contributions as shown in Fig. 145.

The plots of $E_{\mathrm{QE}}$ and $E_{\text {calor }}$ for $A\left(e, e^{\prime} p\right)$ with 0 pions (no photons and charged pions) before and after subtraction for undetected few pions and undetected few proton events for ${ }^{3} \mathrm{He}$ and ${ }^{56} \mathrm{Fe}$ targets are shown in Fig. 147. As we can see the subtraction has a small effect at the beam-energy peak, and mainly reduces the non-QE tail as one would expected. $1 p$ $1 \pi$ subtraction has the biggest contribution in the subtraction for undetected hadrons. The difference between the distributions corresponding to the subtraction for undetected $3 p 0 \pi$ (red) and $4 p 0 \pi$ (green) events is small, suggesting that the technique converges and there is no need to subtract for undetected hadron events with more than four protons. However even after subtraction, we are left with a significant number of background events that do not describe the true beam energy.

\section{Results}

In order to compare the results from the two energy reconstruction techniques, we plotted $E_{\mathrm{QE}}$ for $A\left(e, e^{\prime}\right)$ and $A\left(e, e^{\prime} p\right)$ events and $E_{\text {calor }}$ for $A\left(e, e^{\prime} p\right)$ events (see Fig. 148). The resolution of the reconstructed energy spectrum improves significantly when we also use information about the detected proton. There is a sharp peak evident in the distribution of $E_{\text {calor }}$ located nearly at the beam energy, and there is a tail extending to the left of the peak. Neglecting the kinetic energy of the recoil $A-1$ system does not affect the $E_{\text {calor }}$ distribution as its contribution should be at most a few $\mathrm{MeV}$.

The peak in $E_{\mathrm{QE}}$ is much broader and there is no clear separation between the peak and the background, especially for ${ }^{56} \mathrm{Fe}$. The width of the $E_{\mathrm{QE}}$ peak is caused by Fermi motion (Doppler broadening). The non-QE background increases for heavier nuclei. We can see that the tails to the left of the peak in the $A\left(e, e^{\prime} p\right) E_{\text {calor }}$ and $E_{\mathrm{QE}}$ distributions are the same for each of the targets. This is more evident in the $A\left(e, e^{\prime} p\right) E_{\text {calor }}$ vs $E_{\mathrm{QE}}$ distributions shown in Fig. 149. This shows that agreement between the two energy reconstruction methods does not necessarily mean that the energy is reconstructed correctly.

Fig. 150 shows $E_{\mathrm{QE}}$ (i.e., using only the electron information) for $\left(e, e^{\prime} p\right)$ events as a 
function of $P_{\text {miss }}^{\perp}$. The quasi-elastic scattering events appear as a distinct peak in the distribution with small $P_{\text {miss }}^{\perp}$ and $E_{\mathrm{QE}}$ close to the beam energy. The subtraction for undetected pions and protons reduces the non-QE background at higher values of $P_{\text {miss }}^{\perp}$. Most of the QE events are at $P_{\text {miss }}^{\perp}<200 \mathrm{MeV}$ as was expected for QE knock-out of mean field nucleons.

We have also plotted the $E_{\mathrm{QE}}$ and $E_{\text {calor }}$ distributions for three different regions of $P_{\text {miss }}^{\perp}$ (see Fig. 151). The vertical axis range is the same for $E_{\mathrm{QE}}$ and $E_{\text {calor }}$ distributions to better see the tails, and because of this the $E_{\text {calor }}$ peaks are cut off. The plots of $E_{\text {calor }}$ in the full range of vertical axis are shown in Fig. 152. We expect the region corresponding to $P_{\text {miss }}^{\perp}$ values from $0-200 \mathrm{MeV} / \mathrm{c}$ to be populated mainly with QE scattering from mean field nucleons. $200<P_{\text {miss }}^{\perp}<400 \mathrm{MeV} /$ c corresponds to a transition region between $\mathrm{QE}$ scattering off mean field nucleons and non-QE events, and the region corresponding to $P_{\text {miss }}^{\perp}$ values of $400 \mathrm{MeV} / \mathrm{c}$ and higher should be populated mainly with non-QE background events. As we go higher in $P_{\text {miss }}^{\perp}$ the contribution of non-QE electron scattering events increases as expected.

If the electron radiates a high-energy photon, it will cause the event to reconstruct to a lower beam energy. Either the incoming or the outgoing electron can radiate. However, any high energy photon radiated by the outgoing electron would be detected in the EC and would therefore be excluded from these events. Any high energy photon radiated by the incoming electron would change the reconstructed beam energy, but not $P_{\text {miss }}^{\perp}$. Therefore, the upper limit on the effects of electron radiation is given by the lower energy tail of the $E_{\text {calor }}$ distribution for $P_{\text {miss }}^{\perp}<200 \mathrm{MeV} /$ c. This is small. We will account for this effect in the event generators.

The peak in the $E_{\mathrm{QE}}$ distribution corresponding to $P_{\text {miss }}^{\perp}$ values from the range $0-$ $200 \mathrm{MeV} / \mathrm{c}$ and populated mostly with QE events is significantly broader compared to the one in $E_{\text {calor }}$ distribution corresponding to same $P_{\text {miss }}^{\perp}$ values for all targets. It is also evident that the non-QE background dominating at $P_{\text {miss }}^{\perp}$ values of $200 \mathrm{MeV} / \mathrm{c}$ and higher increases with respect to the peak at the beam energy as we go from ${ }^{3} \mathrm{He}$ to ${ }^{56} \mathrm{Fe}$ in both $E_{\mathrm{QE}}$ and $E_{\text {calor }}$ distributions. There is also no evident peak at beam energy for these high $P_{\text {miss }}^{\perp}$ values, which shows that the fraction of QE events in this region is small. Plots for the other targets can be found in Appendix B.1. 


\subsection{GEV $A\left(e, e^{\prime}\right)$ AND $A\left(e, e^{\prime} p\right)$ ANALYSIS}

The analysis procedure at $2.2 \mathrm{GeV}$ is identical to that for $4.4 \mathrm{GeV}$. We again applied CLAS E2a standard cuts and corrections described in detail in the previous sections and looked at the $A\left(e, e^{\prime}\right)$ and $A\left(e, e^{\prime} p\right)$ data samples.

The plots of $Q^{2}$ vs $\nu, x$ and $W$ at $2.2 \mathrm{GeV}$ are shown in Fig. 153. The QE peaks at $x_{\mathrm{B}} \sim 1$ and $W(e p) \sim m_{\text {proton }}$ for ${ }^{3} \mathrm{He}$ are much better separated from the rest of the distribution than at $4.4 \mathrm{GeV}$. The plots for ${ }^{56} \mathrm{Fe}$ are shown in Fig. 154. The yellow bands seen in the $Q^{2}$ vs $\nu$ plot are due to bad CLAS detectors. This structure is not seen in sector one, where there are no bad forward-angle detectors (see Fig. 154b).

The $\theta$ vs $\phi$ angular distributions of different particles at $2.2 \mathrm{GeV}$ are shown in Fig. 155. The superposition of proton and $\pi^{-}$angular distributions is shown in Fig. 156. The proton and charged pion plus photon multiplicity plots are shown in Figs. 158 and 157. We can see that as we go to heavier nuclei the ratio of few detected proton events to 1 detected proton events increases. This is expected as there are many more protons in heavier nuclei than light nuclei.

Proton and $\pi$ multiplicities are smaller at $E=2.2 \mathrm{GeV}$ than at $4.4 \mathrm{GeV}$. Thus the effects of subtractions for undetected few proton events and undetected few pion events are smaller at $2.2 \mathrm{GeV}$.

We again subtracted the undetected pions in the $A\left(e, e^{\prime}\right)$ analysis, to obtain the true 0 pion spectrum. The distributions of $E_{\mathrm{QE}}$ for detected and undetected one pion events as well as undetected two and three pion events for ${ }^{3} \mathrm{He}\left(e, e^{\prime} N \pi\right)$ are shown in Fig. 159a, and the ones for ${ }^{56} \mathrm{Fe}\left(e, e^{\prime} N \pi\right)$ in Fig. 159c. The $E_{\mathrm{QE}}$ distributions of zero pion spectrum before and after subtraction for undetected pions for ${ }^{3} \mathrm{He}\left(e, e^{\prime}\right)$ are shown in Fig. $159 \mathrm{~b}$ and the ones for ${ }^{56} \mathrm{Fe}\left(e, e^{\prime}\right)$ are shown in Fig. 159d.

In the $A\left(e, e^{\prime} p\right)$ analysis we have subtracted for undetected pions and undetected protons. The $E_{\mathrm{QE}}$ and $E_{\text {calor }}$ distributions of $A\left(e, e^{\prime} p\right) 0 \pi$ spectrum before and after subtraction for undetected pions and protons for ${ }^{3} \mathrm{He}$ (Fig. 160a, 160b) and ${ }^{56} \mathrm{Fe}$ (Fig. 160c, 160d) targets are shown in Fig. 160.

We have combined the obtained results for different targets in one plot. The $E_{\mathrm{QE}}$ distributions for $A\left(e, e^{\prime}\right)$ and $A\left(e, e^{\prime} p\right) 0 \pi$ spectrum and $E_{\text {calor }}$ distribution of $A\left(e, e^{\prime} p\right) 0 \pi$ spectrum

after subtraction for undetected pions and undetected protons are shown in Fig. 161. The energy reconstruction methods work better at $2.2 \mathrm{GeV}$ than at $4.4 \mathrm{GeV}$. However the peaks at beam energy of the $E_{\mathrm{QE}}$ distributions for $A\left(e, e^{\prime}\right)$ and $A\left(e, e^{\prime} p\right) 0 \pi$ spectrum are again wide 
and do not have a clear separation from the non-QE background. All energy reconstruction methods work the best for ${ }^{3} \mathrm{He}$.

The $E_{\mathrm{QE}}$ versus $E_{\text {calor }}$ distribution of $A\left(e, e^{\prime} p\right) 0 \pi$ spectrum are shown in Fig. 162.

Fig. 163 shows $E_{\mathrm{QE}}$ as a function of $P_{\text {miss }}^{\perp}$. The plots are shown for ${ }^{3} \mathrm{He}$ and ${ }^{56} \mathrm{Fe}$ targets before and after subtractions for undetected pions and protons.

We subtracted $E_{\text {calor }}$ and $E_{\mathrm{QE}} 0 \pi A\left(e, e^{\prime} p\right)$ distributions for three different regions of $P_{\text {miss }}^{\perp}$ are shown in Fig. 164. The full range plots of $E_{\text {calor }}$ for different targets are shown in Fig. 165. The peak at the beam energy for a given target is narrower at $2.2 \mathrm{GeV}$ than at $4.4 \mathrm{GeV}$. Again the peak at the beam energy in $E_{\text {calor }}$ for $0<P_{\text {miss }}^{\perp}<200 \mathrm{MeV} / \mathrm{c}$ is much narrower than the $E_{\mathrm{QE}}$ one. The background tail is the smallest for $E_{\text {calor }}$ for $0<P_{\text {miss }}^{\perp}<200 \mathrm{MeV} / \mathrm{c}$ for ${ }^{3} \mathrm{He}$ target at $2.2 \mathrm{GeV}$.

The plots for the other targets $\left({ }^{4} \mathrm{He}\right.$ and $\left.{ }^{12} \mathrm{C}\right)$ are shown in Appendix B.2.

\subsection{GEV $A\left(e, e^{\prime}\right)$ AND $A\left(e, e^{\prime} p\right)$ ANALYSIS}

The $1.1 \mathrm{GeV}$ analysis follows the same procedure as at 2.2 and $4.4 \mathrm{GeV}$. We have again applied CLAS E2a standard cuts and corrections described above in details and have looked at the $A\left(e, e^{\prime}\right)$ and $A\left(e, e^{\prime} p\right)$ data samples.

The distributions of different kinematic variables at $1.1 \mathrm{GeV}$ for ${ }^{3} \mathrm{He}$ and ${ }^{12} \mathrm{C}$ are shown in Figs.166 and 167. In all the distributions for ${ }^{3} \mathrm{He}$ we can clearly see two peak structure corresponding to QE scattering and $\Delta$ resonance regions.

The $\theta$ vs $\phi$ angular distributions of different particles at $1.1 \mathrm{GeV}$ are shown in Fig. 168. The superposition of proton and $\pi^{-}$angular distributions is shown in Fig. 169. The proton and charged pion plus photon multiplicity plots are shown in Figs. 171 and 170.

In order to obtain the zero pion $E_{\mathrm{QE}}$ spectrum in the $A\left(e, e^{\prime}\right)$ analysis we have subtracted for undetected few pion events. The distributions of $E_{\mathrm{QE}}$ for detected and undetected 1 pion events as well as undetected two, three and four pion events for ${ }^{3} \mathrm{He}\left(e, e^{\prime} N \pi\right)$ are shown in Fig. 172a, and the ones for ${ }^{12} \mathrm{C}\left(e, e^{\prime} N \pi\right)$ in Fig. 172c. The $E_{\mathrm{QE}}$ distributions of 0 pion spectrum before and after subtraction for undetected pions for ${ }^{3} \mathrm{He}\left(e, e^{\prime}\right)$ are shown in Fig. $172 \mathrm{~b}$ and the ones for ${ }^{12} \mathrm{C}\left(e, e^{\prime}\right)$ are shown in Fig. $172 \mathrm{~d}$.

In the $A\left(e, e^{\prime} p\right)$ analysis we subtract for undetected few proton and few pion events to obtain one proton and zero pion sample. The $E_{\mathrm{QE}}$ and $E_{\text {calor }}$ distributions of $A\left(e, e^{\prime} p\right) 0 \pi$ spectrum before and after subtraction for undetected pions and protons for ${ }^{3} \mathrm{He}$ (Fig. 173a, 173b) and ${ }^{12} \mathrm{C}$ (Fig. 173c, 173d) targets are shown in Fig. 173. 
In order to compare the results from different energy reconstruction methods we have again combined the $E_{\mathrm{QE}}$ distributions for $A\left(e, e^{\prime}\right)$ and $A\left(e, e^{\prime} p\right) 0 \pi$ spectrum and $E_{\text {calor }}$ distribution of $A\left(e, e^{\prime} p\right) 0 \pi$ spectrum after subtraction for undetected pions and undetected protons are shown in Fig. 174. The energy reconstruction methods work the best at $1.1 \mathrm{GeV}$ for ${ }^{3} \mathrm{He}$. The $E_{\mathrm{QE}}$ versus $E_{\text {calor }}$ distribution of $A\left(e, e^{\prime} p\right) 0 \pi$ spectrum for both targets is shown in Fig. 175.

Fig. 176 shows $E_{\mathrm{QE}}$ as a function of $P_{\text {miss }}^{\perp}$. The plots are shown for ${ }^{3} \mathrm{He}$ and ${ }^{12} \mathrm{C}$ targets before and after subtractions for undetected pions and protons.

The plots of $E_{\text {calor }}$ and $E_{\mathrm{QE}}$ for $0 \pi A\left(e, e^{\prime} p\right)$ events after subtraction for undetected pions and undetected protons for three different regions of $P_{\text {miss }}^{\perp}$ are shown in Fig. 177. Again the peak at the beam energy in $E_{\text {calor }}$ for $0<P_{\text {miss }}^{\perp}<200 \mathrm{MeV} / \mathrm{c}$ is much narrower than the $E_{\mathrm{QE}}$ one, for all targets. The background tail is the smallest for $E_{\text {calor }}$ for $0<P_{\text {miss }}^{\perp}<200 \mathrm{MeV} / \mathrm{c}$ for ${ }^{3} \mathrm{He}$ target at $1.1 \mathrm{GeV}$.

\subsection{COMPARISON OF 1.1, 2.2 AND 4.4 GEV $A\left(e, e^{\prime}\right)$ AND $A\left(e, e^{\prime} p\right)$ ANALYSIS}

We have combined the energy reconstruction results for different targets at 2.2 and 4.4 GeV in Fig. 178, and for all three beam energies in Fig. 179.

Because we use approximate values for single nucleon removal energies the QE peaks in reconstructed energy spectra are not centered at the exact beam energies $(1.161 \mathrm{GeV}$, $2.261 \mathrm{GeV}$ and $4.461 \mathrm{GeV}$ ). The single nucleon removal energy $\epsilon$ is equal to the difference between the binding energies of $A$ and $A-1$ nuclei $\epsilon(A)=E_{\text {Bind }}^{\mathrm{A}}-E_{\text {Bind }}^{\mathrm{A}-1}\left(5 \mathrm{MeV}\right.$ for ${ }^{3} \mathrm{He}$, $\epsilon\left({ }^{4} \mathrm{He}\right)=20 \mathrm{MeV}, \epsilon\left({ }^{12} \mathrm{C}\right)=16 \mathrm{MeV}$ and $\left.\epsilon\left({ }^{56} \mathrm{Fe}\right)=10 \mathrm{MeV}\right)$. We added a constant shift to the reconstructed energy values to shift the corresponding distributions to the correct energy. We corrected the binding energy with the same shift values in $E_{\mathrm{QE}}\left(e, e^{\prime} p\right), E_{\text {calor }}\left(e, e^{\prime} p\right)$ and $E_{\mathrm{QE}}\left(e, e^{\prime}\right)$. These are about a few $\mathrm{MeV}$ and are the same for each target at all beam energies. We have obtained the shift values by fitting the QE peak in $E_{\text {calor }}\left(e, e^{\prime} p\right)$ energy spectra for small $P_{\text {miss }^{\perp}}$ values from $0-200 \mathrm{MeV} / \mathrm{c}$ using $2.2 \mathrm{GeV}$ data. The shift values are shown in Table 17. These shifts center the QE peaks at the beam energy and do not significantly affect events at the background tail corresponding to reconstructed energies that are off by few hundred $\mathrm{MeV}$ from the beam energy. The shift values obtained by fitting the QE peak in $E_{\text {calor }}\left(e, e^{\prime} p\right)$ distributions at different energies are consistent with each other. As an example we get $5 \mathrm{MeV}$ shift for ${ }^{12} \mathrm{C}$ at all three beam energies, which is probably due to knockout of nucleons from different shells ( ${ }^{1} p$ and more deeply bound ${ }^{1} s$ shells). 


\begin{tabular}{l|c|}
\hline Target & Offsets \\
\hline${ }^{3} \mathrm{He}$ & $4 \mathrm{MeV}$ \\
${ }^{4} \mathrm{He}$ & $5 \mathrm{MeV}$ \\
${ }^{12} \mathrm{C}$ & $5 \mathrm{MeV}$ \\
${ }^{56} \mathrm{Fe}$ & $11 \mathrm{MeV}$
\end{tabular}

TABLE 17: Offsets to correct the binding energies in reconstructed energy calculations for 1.1, 2.2 and $4.4 \mathrm{GeV}$ analysis in $\mathrm{MeV}$. The offsets have been obtained using $2.2 \mathrm{GeV}$ data.

It is evident from these figures that both energy reconstruction methods work better at the lower beam energy $(1.1 \mathrm{GeV})$. The peak in the corresponding distributions at the beam energy is narrower, and the background tail to the left of the peak is smaller. There is not an evident peak in the $E_{\mathrm{QE}}$ distributions of $A\left(e, e^{\prime}\right)$ and $A\left(e, e^{\prime} p\right)$ events for ${ }^{12} \mathrm{C}$ and ${ }^{56} \mathrm{Fe}$ targets at $4.4 \mathrm{GeV}$.

The non-QE background tail increases with respect to the QE peak as we go to heavier nuclei.

The beam energy dependence of the energy reconstruction is more obvious by looking at the energy feed down $\left(\left(E_{\text {rec }}-E_{\text {true }}\right) / E_{\text {true }}\right)$ spectra shown in Figs. 180 and 181. At higher beam energies the ratio of the events at the tail to the ones at the peak increases.

We used the energy feed down spectra for our data at different energies from $E_{\text {calor }}$ method to reconstruct the true neutrino flux at DUNE far detector simulated by GENIE. We then reconstructed the same flux by using the energy feed down (the fraction of the total energy reconstructed in the detector) obtained using GENIE, and show that we get two different energy spectra that correspond to different sets of values of oscillation parameters. The analysis procedure is described in more details in Section 6.3.

We calculated the fraction of the events that have their energy reconstructed within different ranges $(5 \%, 10 \%$ and $20 \%$ ) around the true value of the beam energy for different energy reconstruction methods (see Tables 18, 19 and 20). Though $E_{\text {calor }}$ provides a better description of the beam energy than $E_{\mathrm{QE}}$, still $66 \%\left({ }^{3} \mathrm{He}\right.$ at $\left.1.1 \mathrm{GeV}\right)$ or less of the events have their energy reconstructed within $5 \%$ of the beam energy. This may result in big systematic uncertainties in neutrino experiments. The neutrino experiments that are able to detect both the outgoing lepton and the nucleon (calorimetric-detector based neutrino experiments) can improve the energy reconstruction with the $P_{\text {miss }}^{\perp}$ QE selection cut mentioned above. Energy 
reconstruction from only the outgoing lepton gives a poor description of the neutrino beam energy even at low energies with light targets (only $44 \%$ of events have their beam energy reconstructed within $\pm 5 \%$ of the beam energy for ${ }^{3} \mathrm{He}$ at $1.1 \mathrm{GeV}$ ).

\begin{tabular}{l|c|c|c|c|c|c|} 
& \multicolumn{2}{|c|}{$1.1 \mathrm{GeV}$} & \multicolumn{2}{|c|}{$2.2 \mathrm{GeV}$} & \multicolumn{2}{c|}{$4.4 \mathrm{GeV}$} \\
\hline Target & $E_{\mathrm{QE}}\left(e, e^{\prime}\right)$ & $E_{\text {calor }}\left(e, e^{\prime} p\right)$ & $E_{\mathrm{QE}}\left(e, e^{\prime}\right)$ & $E_{\text {calor }}\left(e, e^{\prime} p\right)$ & $E_{\mathrm{QE}}\left(e, e^{\prime}\right)$ & $E_{\text {calor }}\left(e, e^{\prime} p\right)$ \\
\hline${ }^{3} \mathrm{He}$ & 0.47 & 0.72 & 0.32 & 0.54 & 0.25 & 0.44 \\
${ }^{4} \mathrm{He}$ & & & 0.24 & 0.47 & 0.18 & 0.36 \\
${ }^{12} \mathrm{C}$ & \multirow{2}{*}{0.33} & 0.55 & 0.20 & 0.39 & 0.21 & 0.28 \\
${ }^{56} \mathrm{Fe}$ & & & 0.16 & 0.26 & 0.16 & 0.16
\end{tabular}

TABLE 18: Fraction of events reconstructed within 5\% of beam energy at 1.1, 2.2 and 4.4 GeV analysis.

\begin{tabular}{l|c|c|c|c|c|c|} 
& \multicolumn{2}{|c|}{$1.1 \mathrm{GeV}$} & \multicolumn{2}{c|}{$2.2 \mathrm{GeV}$} & \multicolumn{2}{c|}{$4.4 \mathrm{GeV}$} \\
\hline Target & $E_{\mathrm{QE}}\left(e, e^{\prime}\right)$ & $E_{\text {calor }}\left(e, e^{\prime} p\right)$ & $E_{\mathrm{QE}}\left(e, e^{\prime}\right)$ & $E_{\text {calor }}\left(e, e^{\prime} p\right)$ & $E_{\mathrm{QE}}\left(e, e^{\prime}\right)$ & $E_{\text {calor }}\left(e, e^{\prime} p\right)$ \\
\hline${ }^{3} \mathrm{He}$ & 0.63 & 0.79 & 0.47 & 0.64 & 0.35 & 0.52 \\
${ }^{4} \mathrm{He}$ & & & 0.42 & 0.57 & 0.37 & 0.43 \\
${ }^{12} \mathrm{C}$ & \multirow{3}{*}{0.49} & 0.68 & 0.38 & 0.50 & 0.31 & 0.35 \\
${ }^{56} \mathrm{Fe}$ & & & 0.32 & 0.37 & 0.25 & 0.21
\end{tabular}

TABLE 19: Fraction of events reconstructed within $10 \%$ of beam energy at 1.1, 2.2 and $4.4 \mathrm{GeV}$ analysis.

You can see the $5 \%$ region within particular energy value on the plot of oscillation signal from T2K experiment from before in Fig. 182. The uncertainty in the determination of the energy has two sources:

1. Resolution of the peak at the beam energy.

2. The tail to the left of the peak

The first contribution is negligible when energy is reconstructed using $E_{\text {calor }}$. 


\begin{tabular}{l|c|c|c|c|c|c|} 
& \multicolumn{2}{|c|}{$1.1 \mathrm{GeV}$} & \multicolumn{2}{c|}{$2.2 \mathrm{GeV}$} & \multicolumn{2}{c|}{$4.4 \mathrm{GeV}$} \\
\hline Target & $E_{\mathrm{QE}}\left(e, e^{\prime}\right)$ & $E_{\text {calor }}\left(e, e^{\prime} p\right)$ & $E_{\mathrm{QE}}\left(e, e^{\prime}\right)$ & $E_{\text {calor }}\left(e, e^{\prime} p\right)$ & $E_{\mathrm{QE}}\left(e, e^{\prime}\right)$ & $E_{\text {calor }}\left(e, e^{\prime} p\right)$ \\
\hline${ }^{3} \mathrm{He}$ & 0.75 & 0.88 & 0.60 & 0.73 & 0.55 & 0.69 \\
${ }^{4} \mathrm{He}$ & & & 0.56 & 0.68 & 0.51 & 0.61 \\
${ }^{12} \mathrm{C}$ & 0.70 & 0.81 & 0.59 & 0.62 & 0.45 & 0.52 \\
${ }^{56} \mathrm{Fe}$ & & & 0.52 & 0.49 & 0.39 & 0.36
\end{tabular}

TABLE 20: Fraction of events reconstructed within $20 \%$ of beam energy at 1.1, 2.2 and 4.4 GeV analysis. 


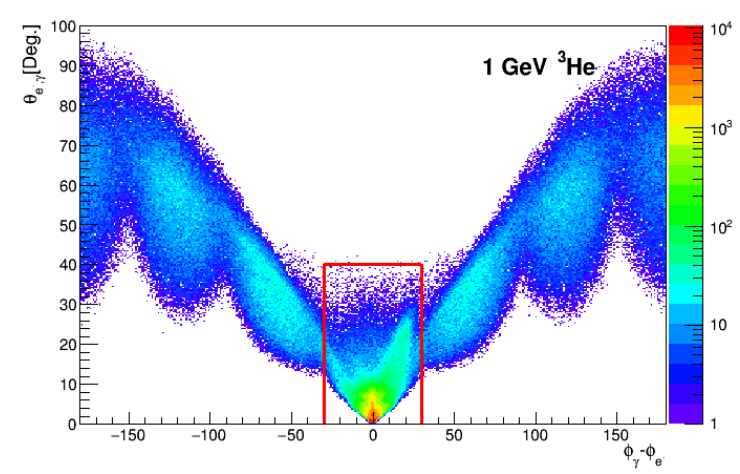

(a) $1.1 \mathrm{GeV},{ }^{3} \mathrm{He}$

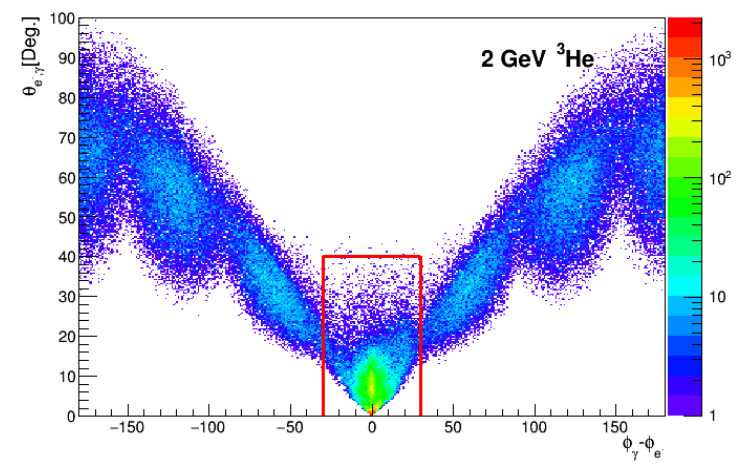

(c) $2.2 \mathrm{GeV},{ }^{3} \mathrm{He}$

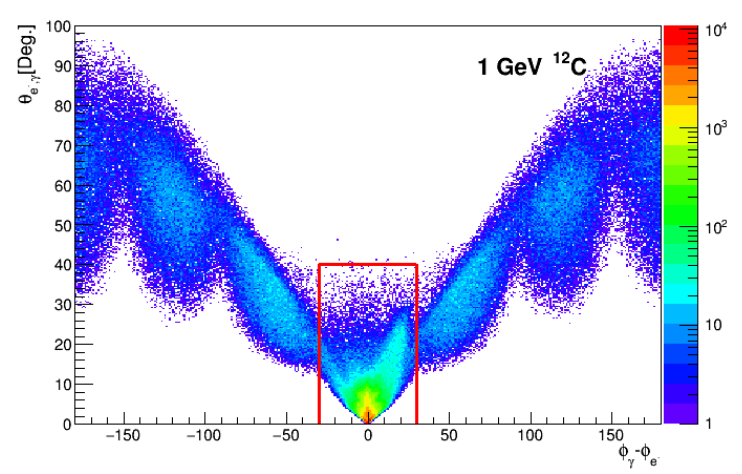

(b) $1.1 \mathrm{GeV},{ }^{12} \mathrm{C}$

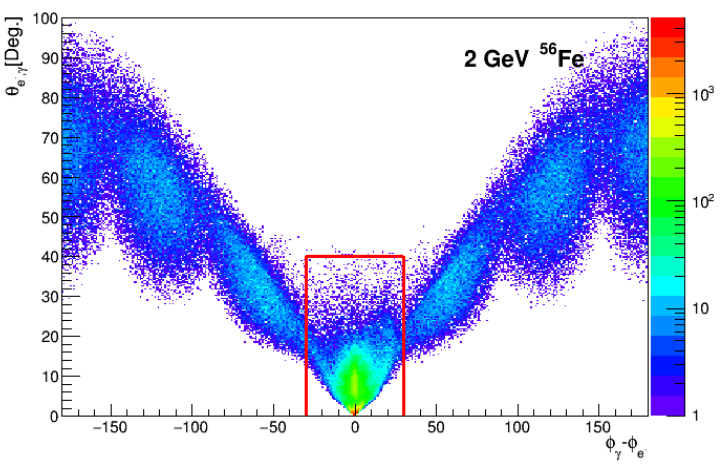

(d) $2.2 \mathrm{GeV},{ }^{56} \mathrm{Fe}$

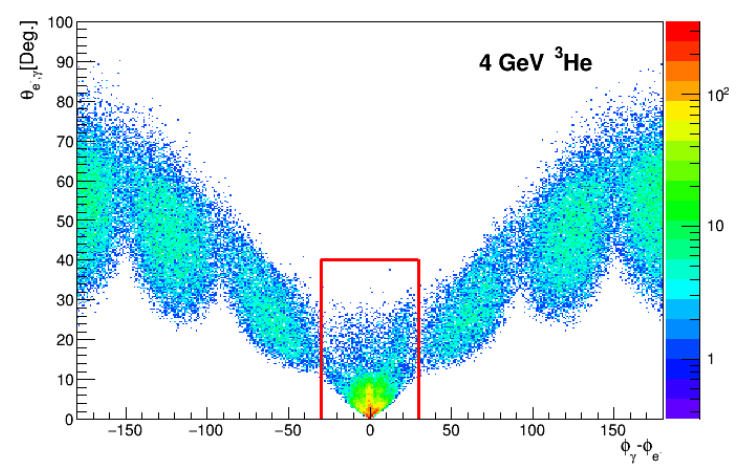

(e) $4.4 \mathrm{GeV},{ }^{3} \mathrm{He}$

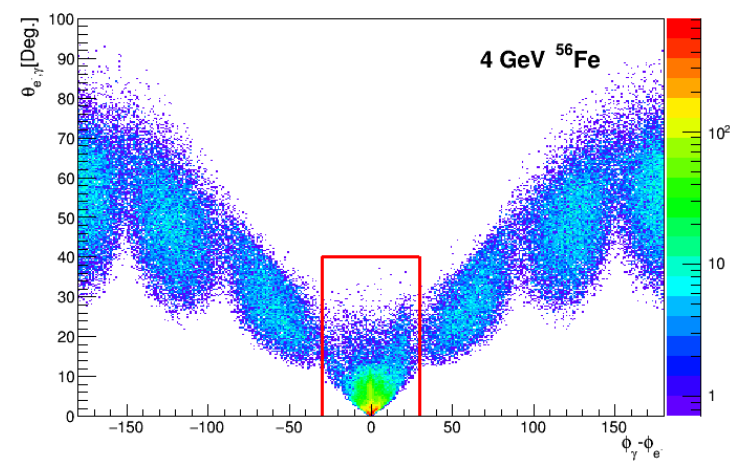

(f) $4.4 \mathrm{GeV},{ }^{56} \mathrm{Fe}$

FIG. 142: The number of events (in log scale) as a function of angle between the electron and the photon versus the difference of their azimuthal angles for different targets for three different energies of the beam. The red box represents the cut used to select radiation photons in logarithmic scale. 


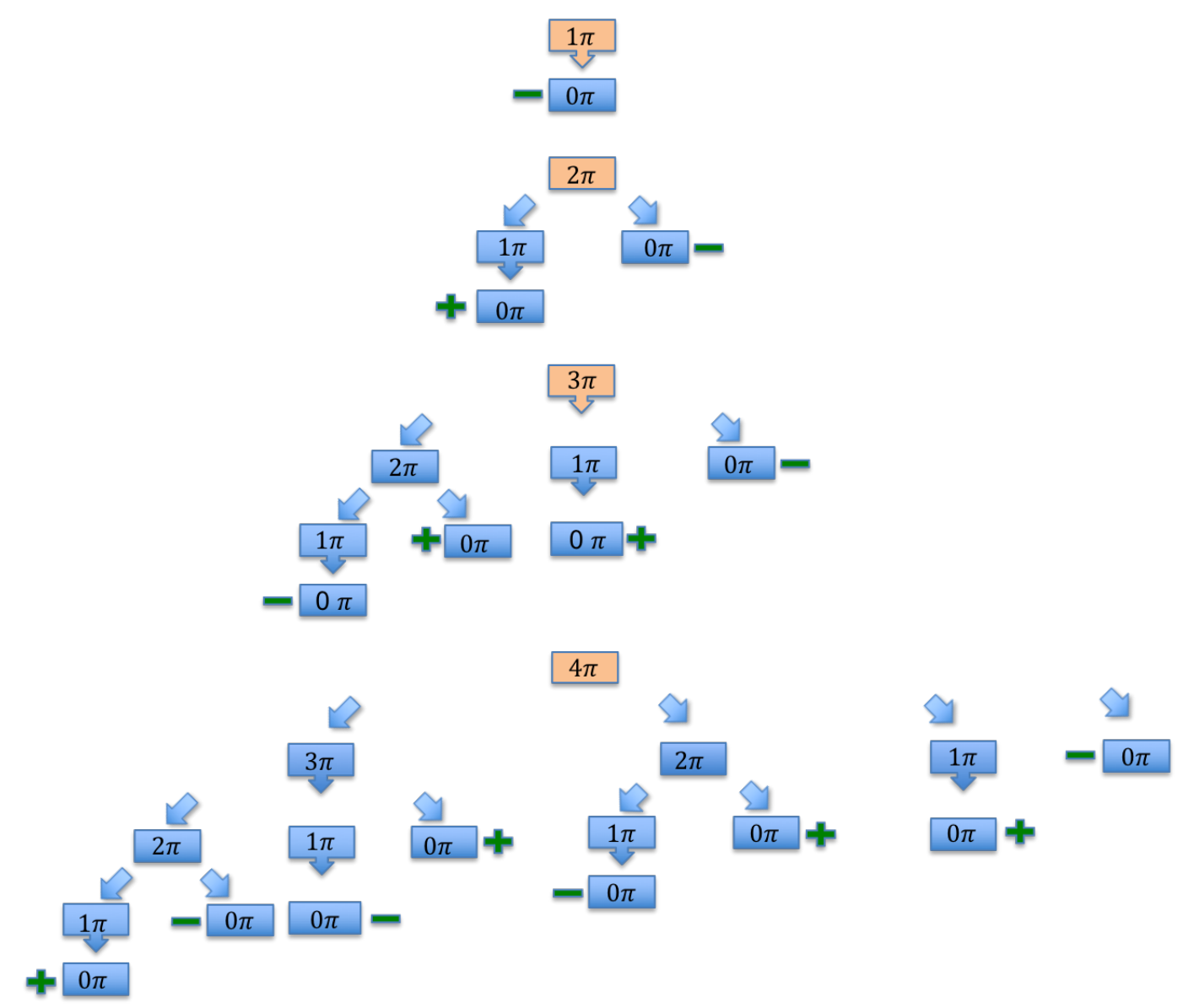

FIG. 143: The scheme of the subtraction for undetected few charged pion and photon events in $A\left(e, e^{\prime}\right)$ analysis. The green signs $(+$ or -$)$ show weather the resulting events are added or subtracted from our results. 


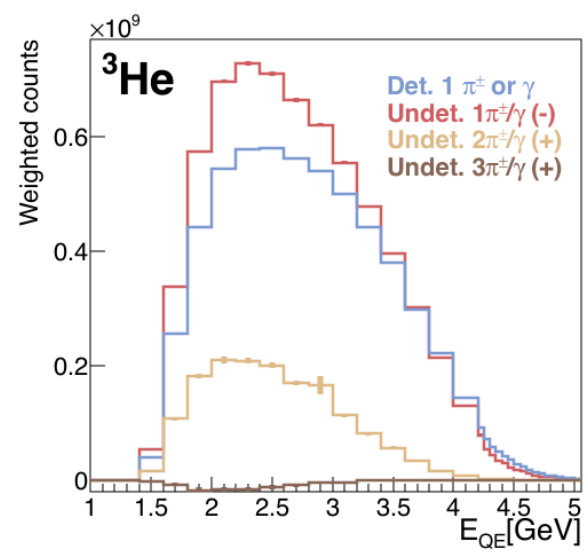

(a)

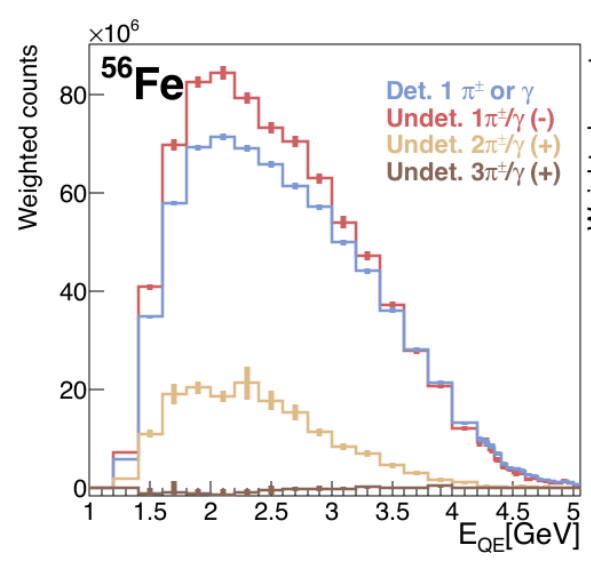

(c)

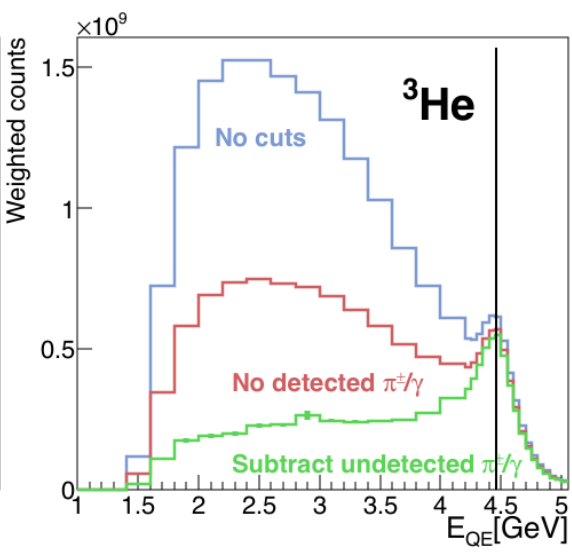

(b)

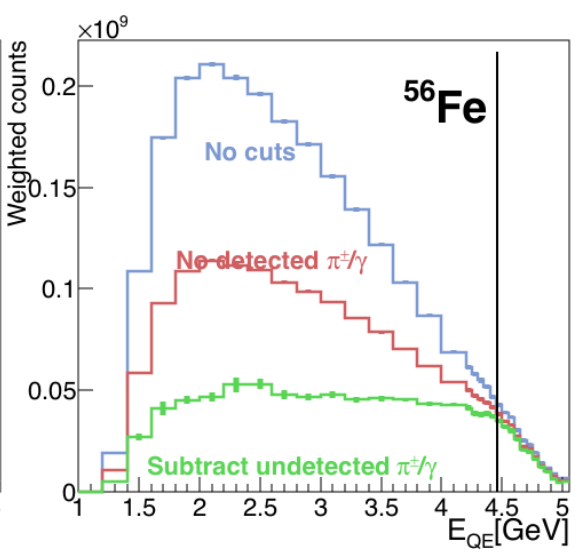

(d)

FIG. 144: $E_{\mathrm{QE}}$ obtained using Eq. 62, for detected (blue) and undetected (red) one pion events, as well as undetected two and three pion events for (a) ${ }^{3} \mathrm{He}\left(e, e^{\prime} N \pi\right) ;(\mathrm{c}){ }^{56} \mathrm{Fe}\left(e, e^{\prime} N \pi\right)$ at $4.4 \mathrm{GeV}$. Reconstructed energy $E_{\mathrm{QE}}$ of $A\left(e, e^{\prime}\right) 0 \pi$ spectrum before (red) and after (green) subtraction for undetected pions for (b) ${ }^{3} \mathrm{He}$ and (d) ${ }^{56} \mathrm{Fe}$ targets at $4.4 \mathrm{GeV}$. 


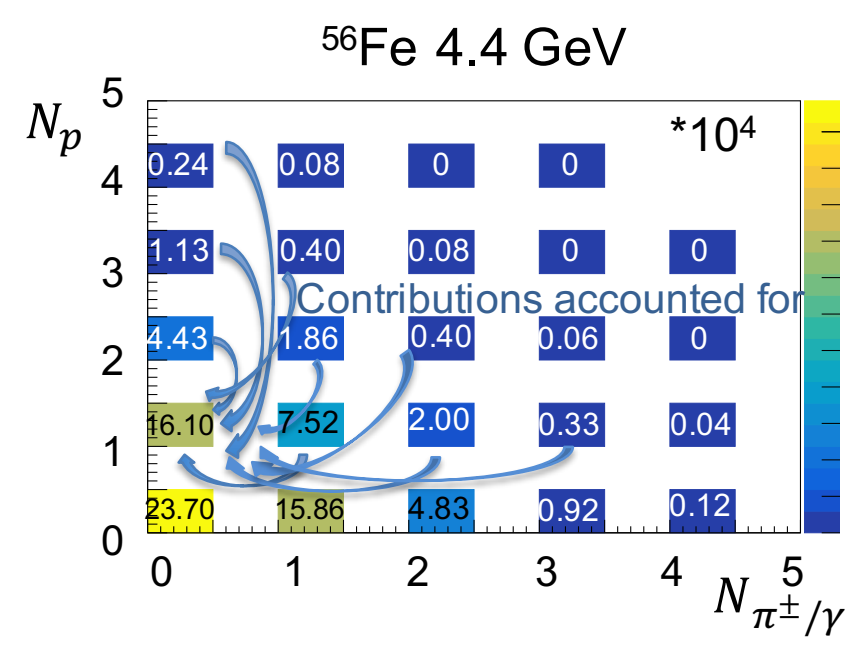

FIG. 145: The plot of the number of protons as a function of the number of charged pions and photons for ${ }^{56} \mathrm{Fe}$ at $4.4 \mathrm{GeV}$.

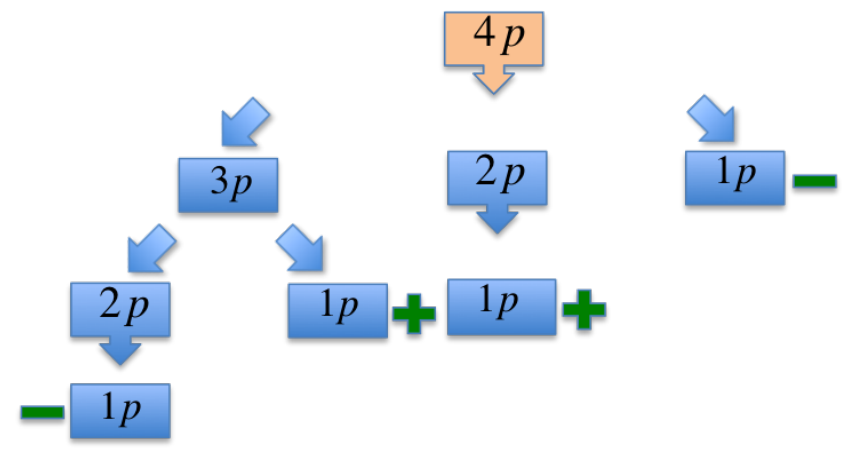

FIG. 146: The scheme of the subtraction for undetected four proton events in $A\left(e, e^{\prime} p\right)$ analysis. 


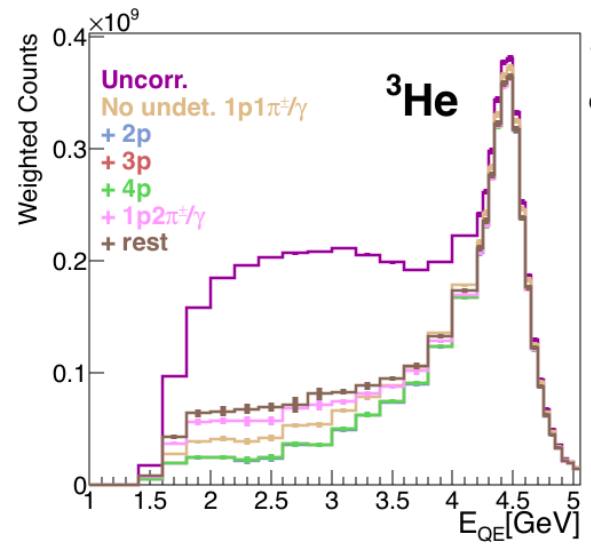

(a)

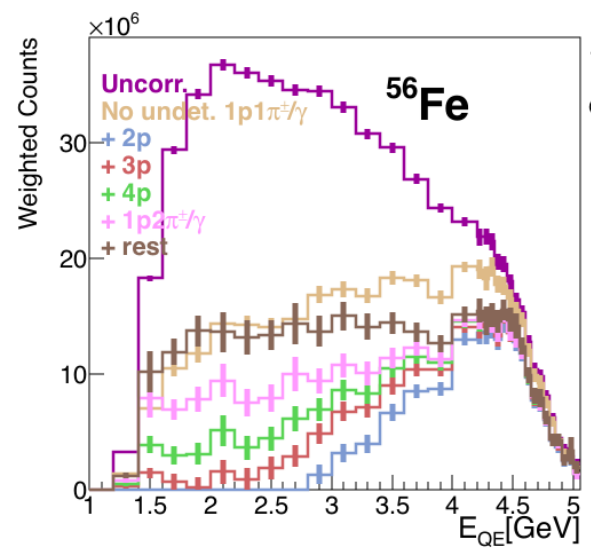

(c)

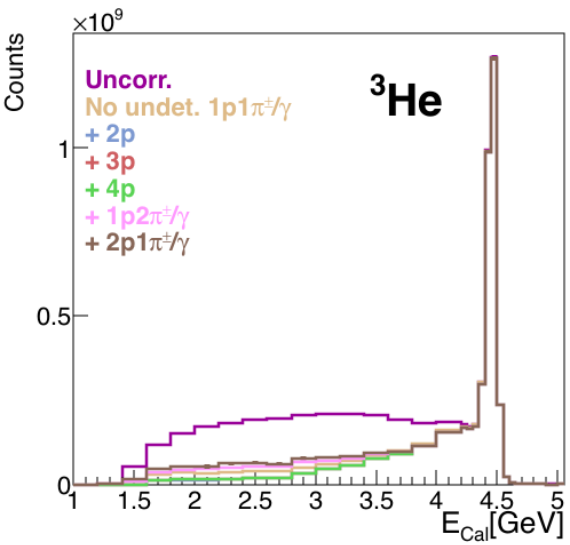

(b)

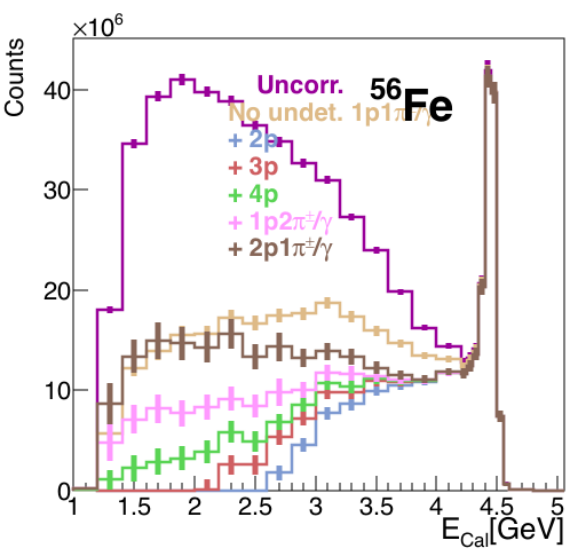

(d)

FIG. 147: $E_{\mathrm{QE}}$ (plots on the left) and $E_{\text {calor }}$ (plots on the right) distributions of $A\left(e, e^{\prime} p\right) 0 \pi$ spectrum before and after subtraction for undetected pions and undetected protons (green) for (a), (b) ${ }^{3} \mathrm{He}$ and (c),(d) ${ }^{56} \mathrm{Fe}$ targets at $4.4 \mathrm{GeV}$. 


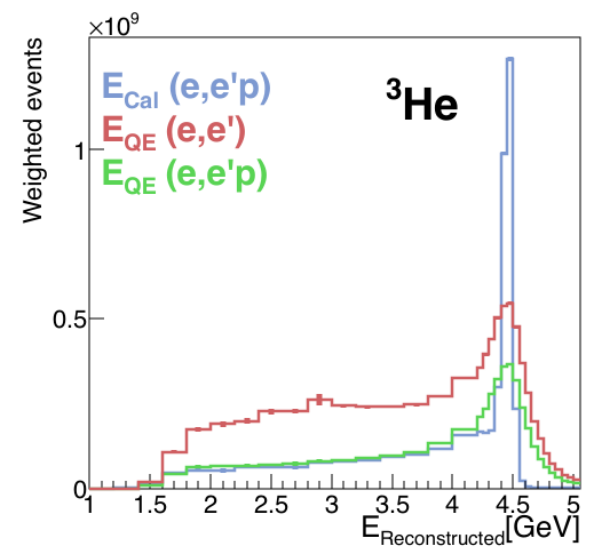

(a)

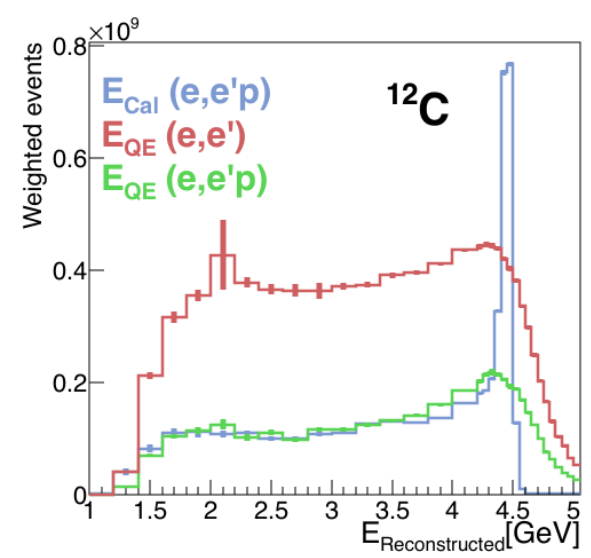

(c)

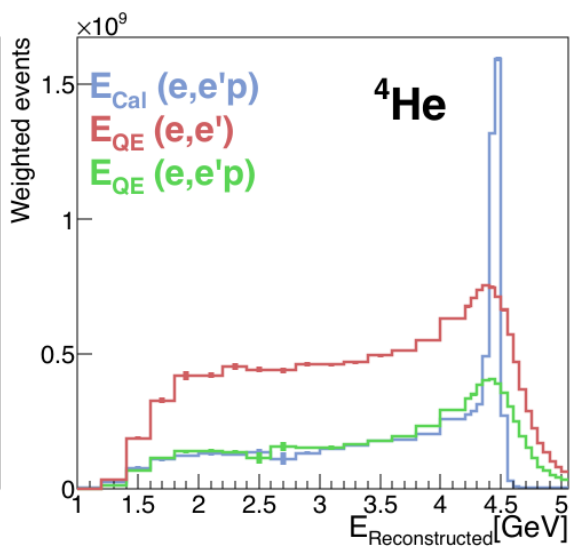

(b)

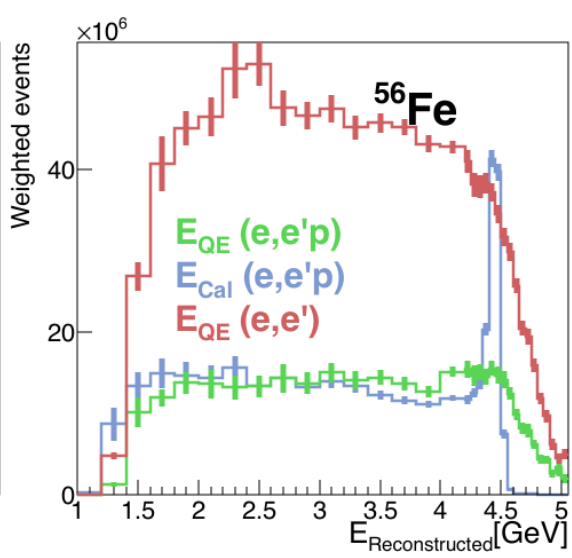

(d)

FIG. 148: $E_{\mathrm{QE}}$ distributions of $A\left(e, e^{\prime}\right)$ (red) and $A\left(e, e^{\prime} p\right)$ (green) $0 \pi$ spectrum and $E_{\text {calor }}$ distribution of $A\left(e, e^{\prime} p\right)$ (blue $0 \pi$ spectrum after subtraction for undetected pions and undetected protons for (a) ${ }^{3} \mathrm{He}$, (b) ${ }^{4} \mathrm{He},(\mathrm{c}){ }^{12} \mathrm{C}$ and (d) ${ }^{56} \mathrm{Fe}$ targets at $4.4 \mathrm{GeV}$. 

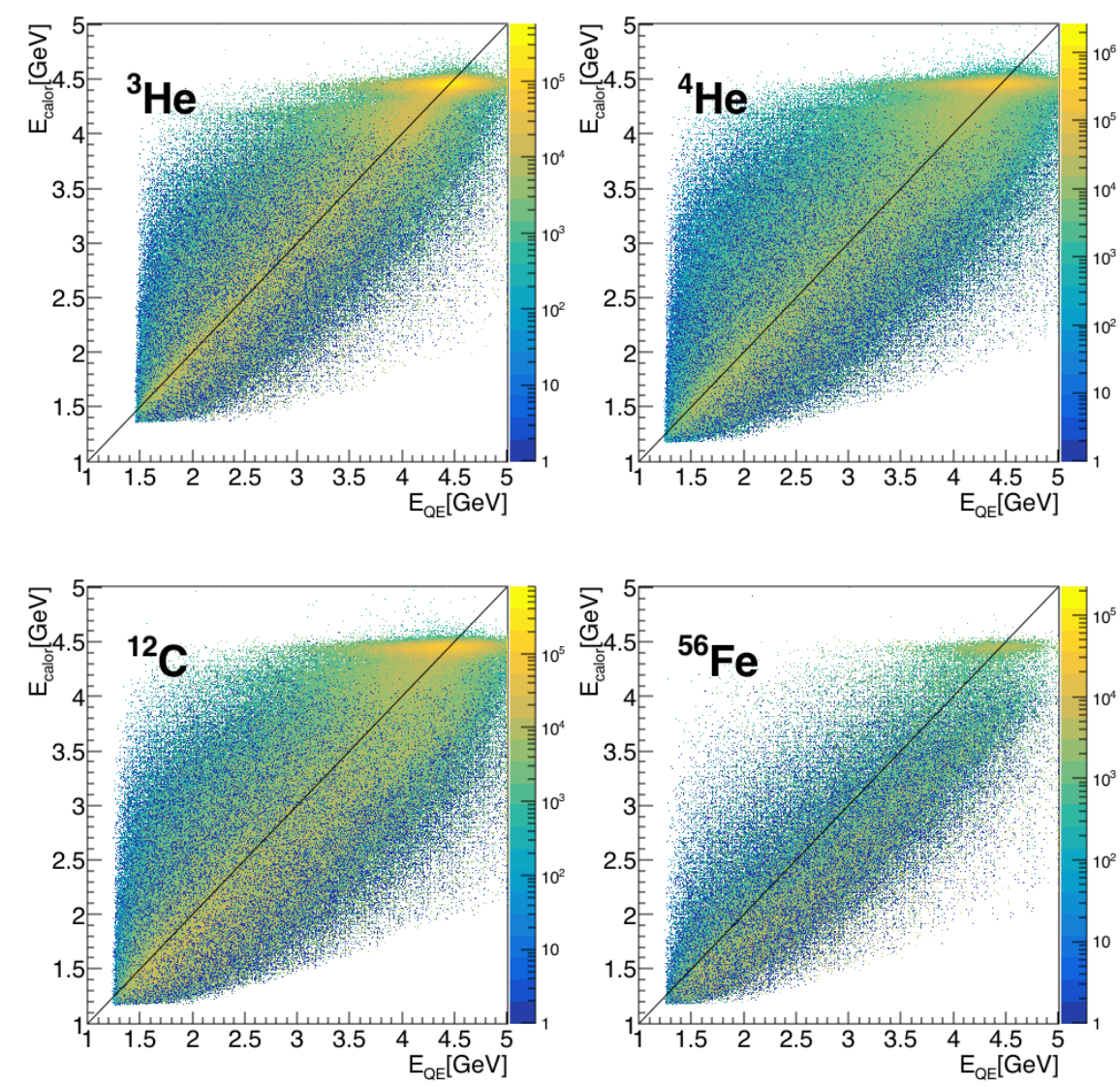

FIG. 149: $E_{\text {calor }}$ versus $E_{\mathrm{QE}}$ distributions of $A\left(e, e^{\prime} p\right) 0 \pi$ spectrum for different targets at $4.4 \mathrm{GeV}$. 


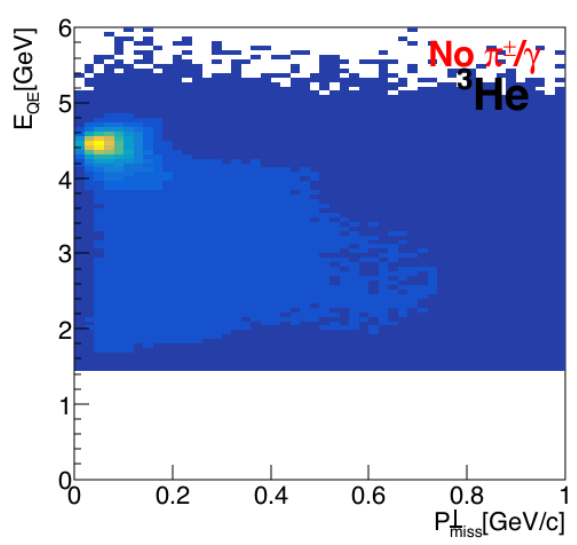

(a)

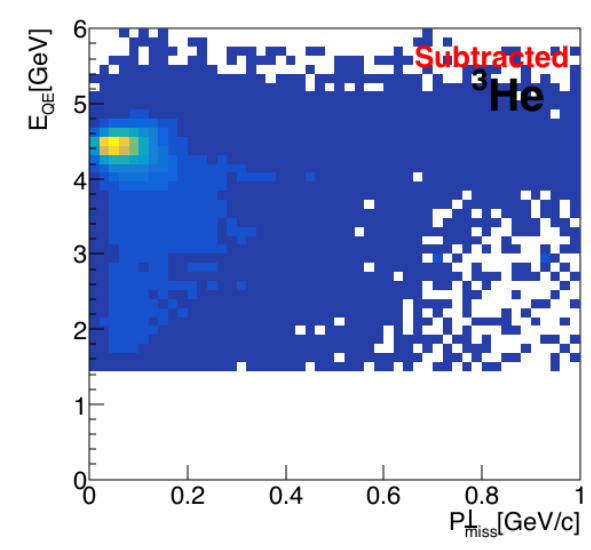

(c)

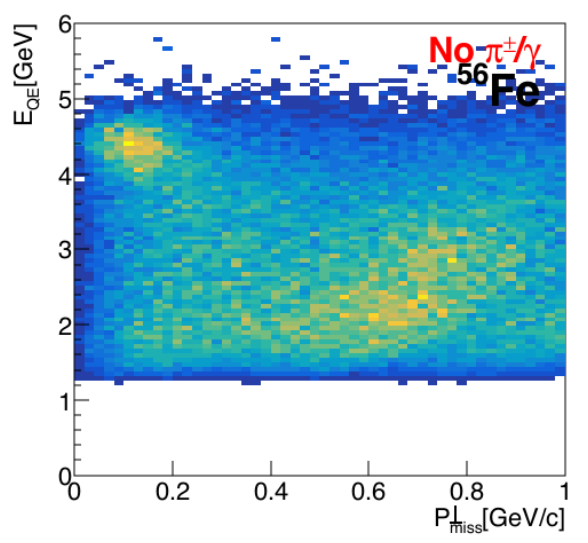

(b)

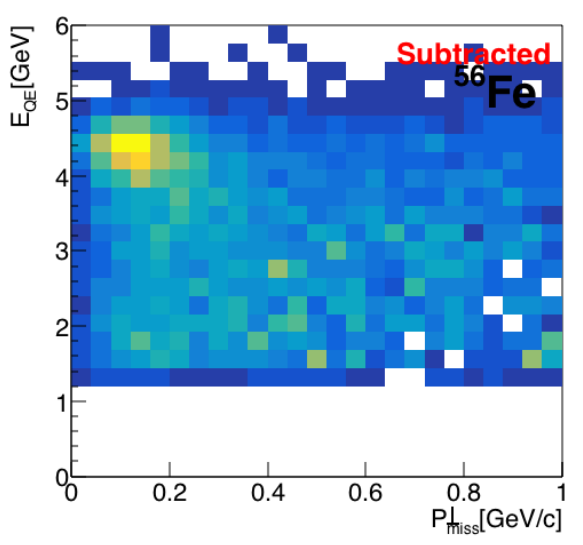

(d)

FIG. 150: Reconstructed energy $E_{\mathrm{QE}}$ obtained using Eq. 62 as a function of the total perpendicular momentum of the scattered electron and the knockout nucleon for ${ }^{3} \mathrm{He}$ and ${ }^{56} \mathrm{Fe}$ targets at $4.4 \mathrm{GeV}$ before (top plots) and after (bottom plots) subtracting for undetected pions and protons. (a),(c) ${ }^{3} \mathrm{He}$; (b), (d) ${ }^{56} \mathrm{Fe}$. 


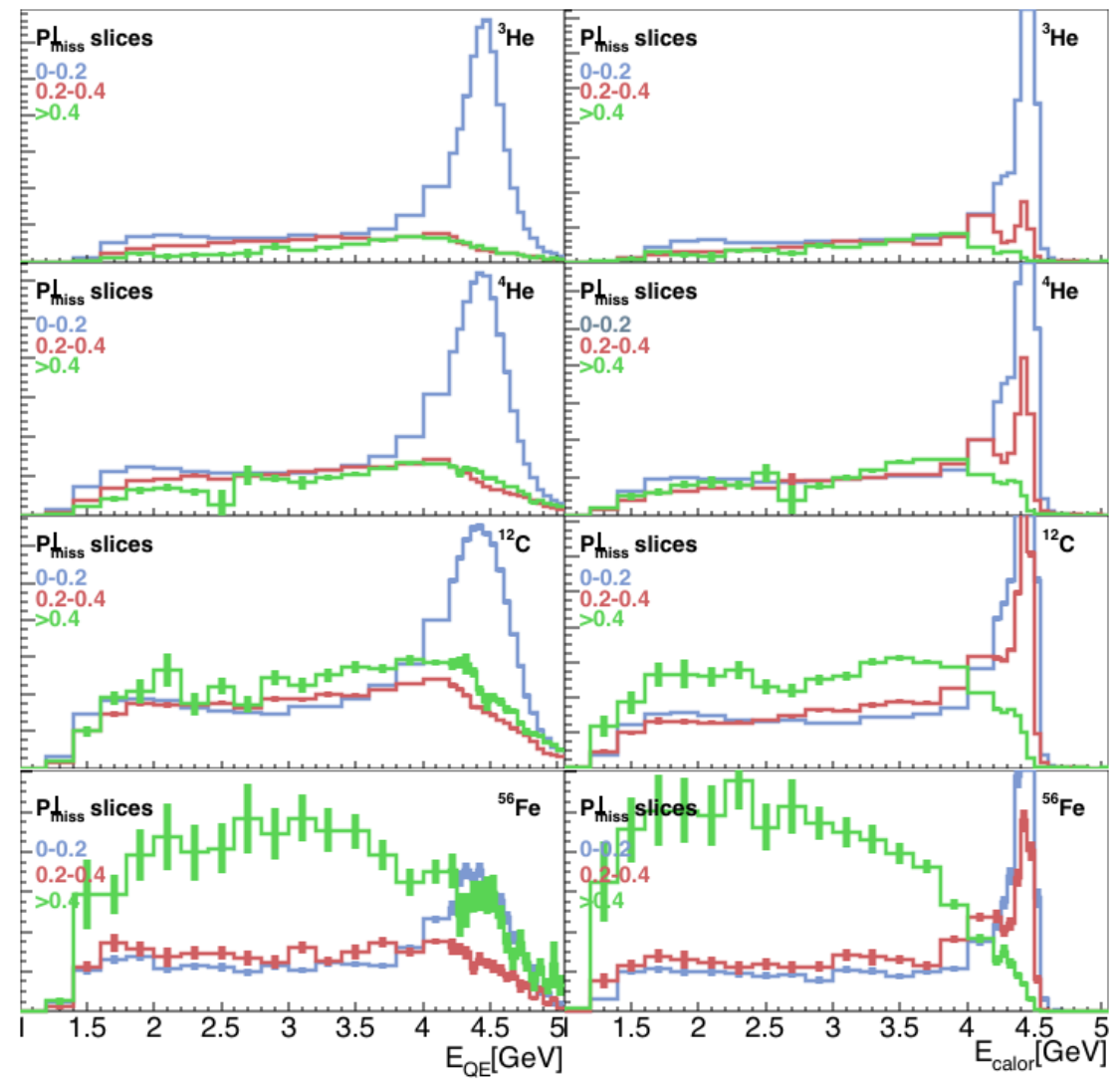

FIG. 151: $E_{\text {calor }}$ (right) and $E_{\mathrm{QE}}$ (left) distributions of $A\left(e, e^{\prime} p\right) 0 \pi$ spectrum after subtraction for undetected pions and undetected protons for three different regions of $P_{\text {miss }}^{\perp}$ $\left(0-200 \mathrm{MeV} / \mathrm{c}\right.$ (blue), $200-400 \mathrm{MeV} / \mathrm{c}$ (red) and $400 \mathrm{MeV} / \mathrm{c}$ (green) and higher) for ${ }^{3} \mathrm{He}$, ${ }^{4} \mathrm{He},{ }^{12} \mathrm{C}$ and ${ }^{56} \mathrm{Fe}$ targets at $4.4 \mathrm{GeV}$. Left and right side plots have the same vertical scale. 


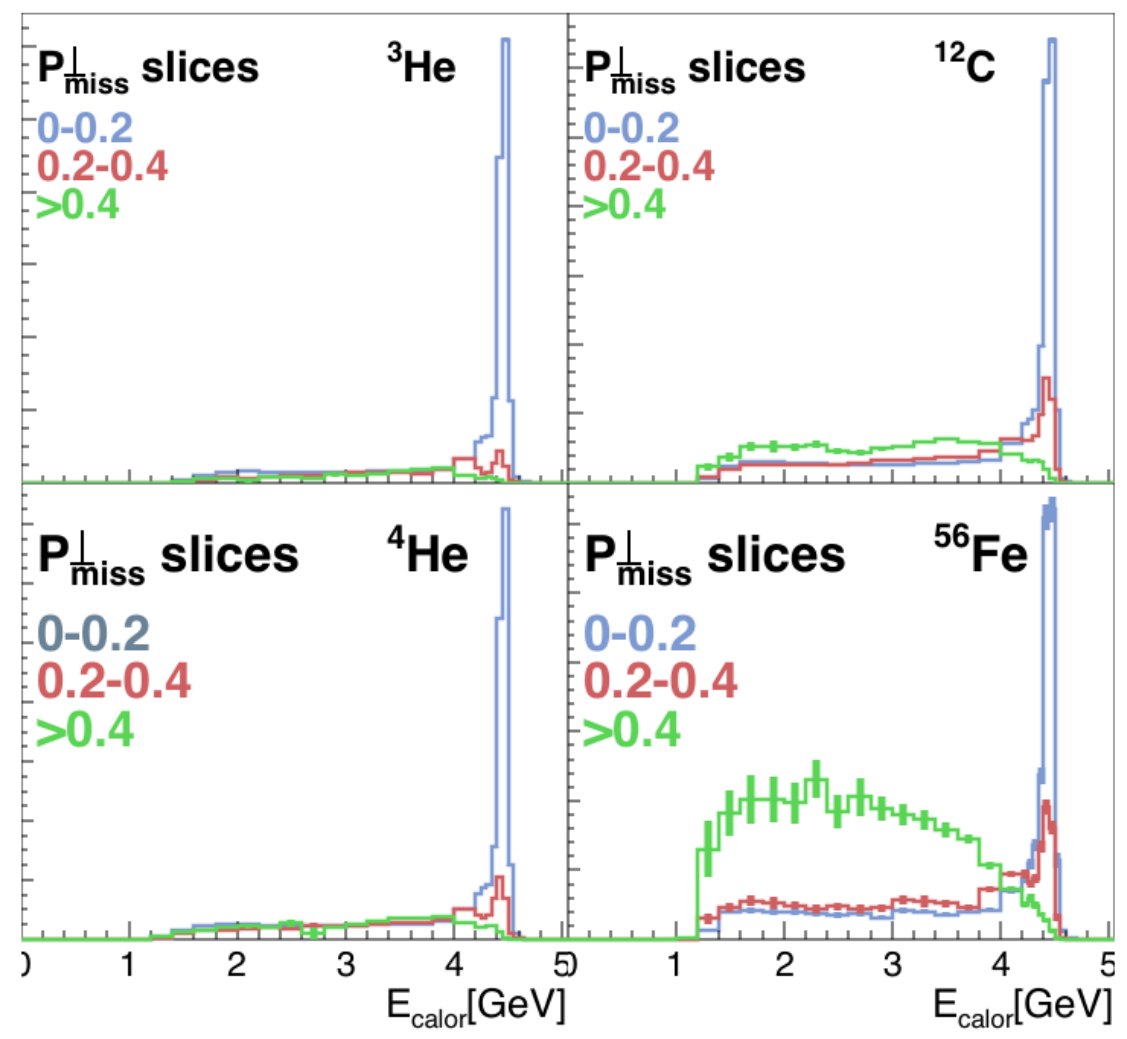

FIG. 152: $E_{\text {calor }}$ distributions of $A\left(e, e^{\prime} p\right) 0 \pi$ spectrum after subtraction for undetected pions and undetected protons for three different regions of $P_{\text {miss }}^{\perp}(0-200 \mathrm{MeV} / \mathrm{c}, 200-400 \mathrm{MeV} / \mathrm{c}$ and $400 \mathrm{MeV} / \mathrm{c}$ and higher) for ${ }^{3} \mathrm{He},{ }^{4} \mathrm{He},{ }^{12} \mathrm{C}$ and ${ }^{56} \mathrm{Fe}$ targets at $4.4 \mathrm{GeV}$ in full range. 


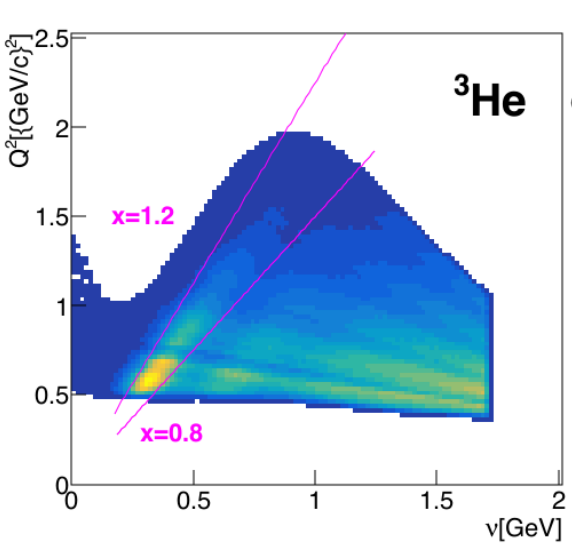

(a)

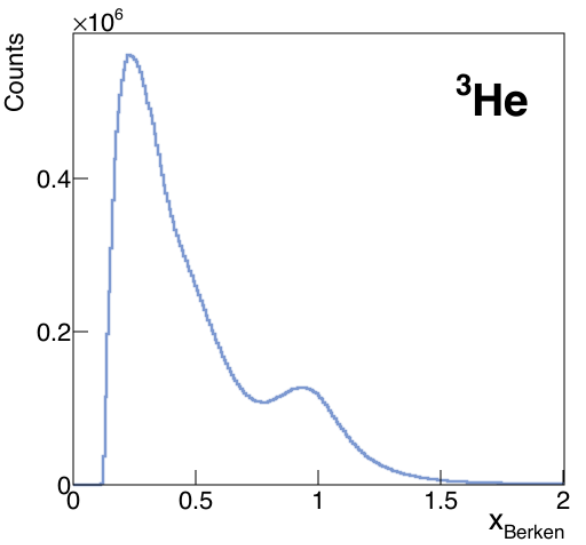

(b)

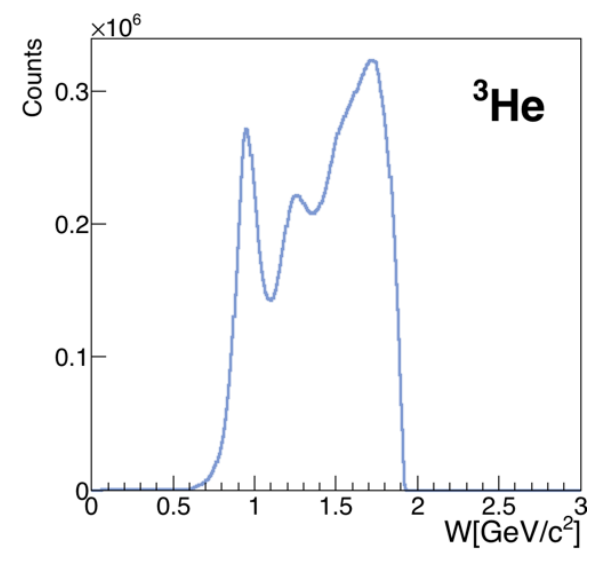

(c)

FIG. 153: The plots of (a) four momentum transfer $Q^{2}$ as a function of energy transfer $\nu$, (b) Bjorken $x$ variable $\left(x_{\mathrm{B}}\right)$ and (c) Invariant mass of electron and proton system $W(e p)$ for ${ }^{3} \mathrm{He}$ at $2.2 \mathrm{GeV}$. 


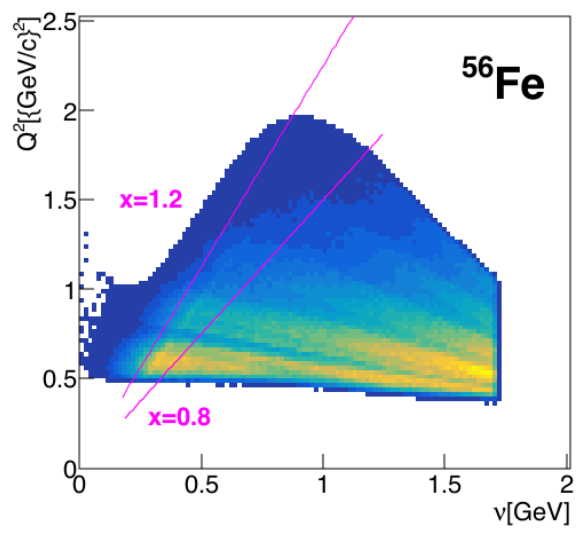

(a)

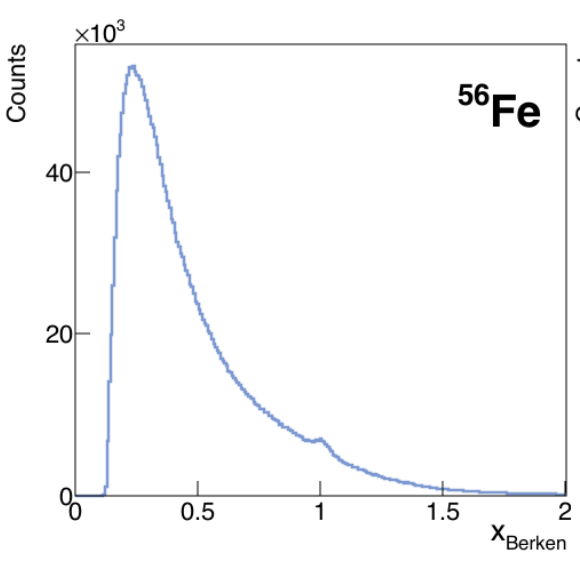

(c)

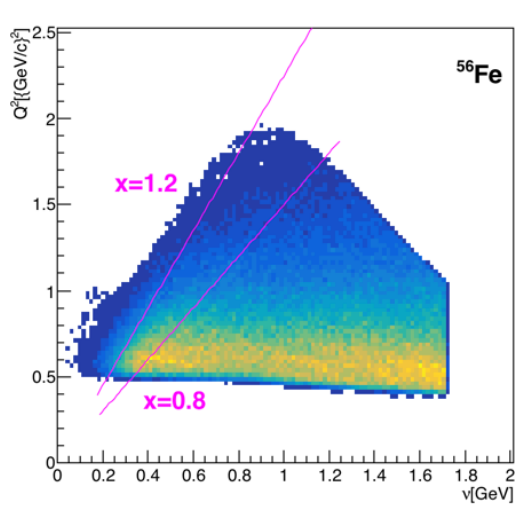

(b)

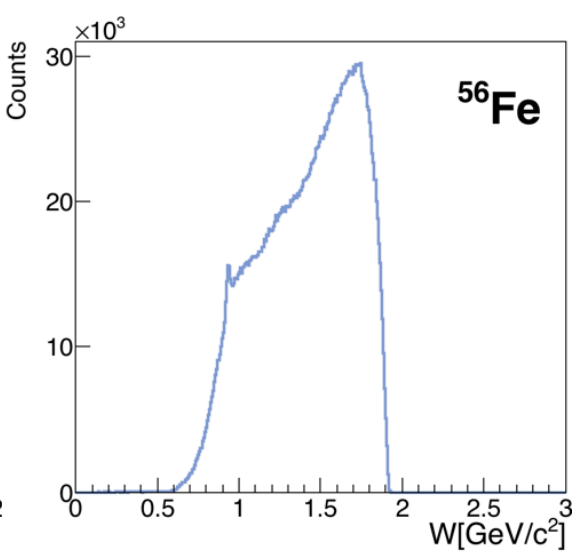

(d)

FIG. 154: The plots of (a) four momentum transfer $Q^{2}$ as a function of energy transfer $\nu$, (b) four momentum transfer $Q^{2}$ as a function of energy transfer $\nu$ for only sector 1, (c) Bjorken $x$ variable $\left(x_{\mathrm{B}}\right)$ and $(\mathrm{d})$ Invariant mass of electron and proton system $W(e p)$ for ${ }^{56} \mathrm{Fe}$ at $2.2 \mathrm{GeV}$. 


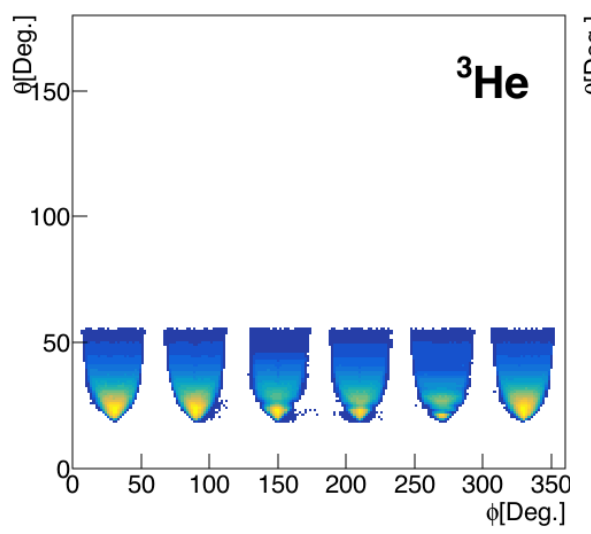

(a)

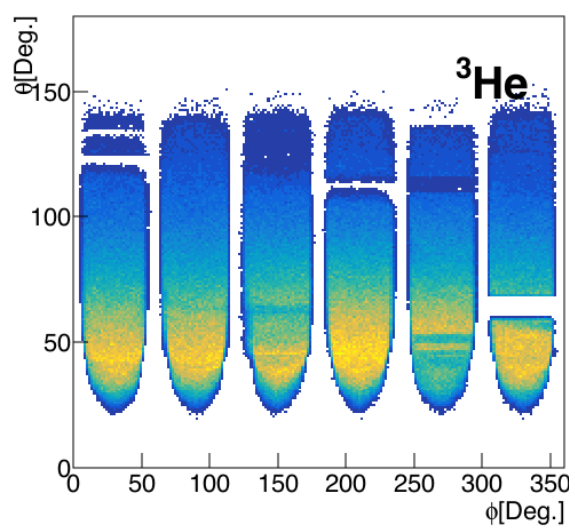

(c)

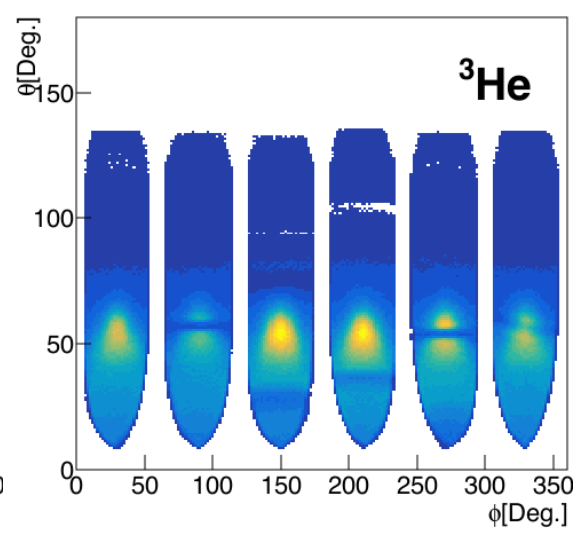

(b)

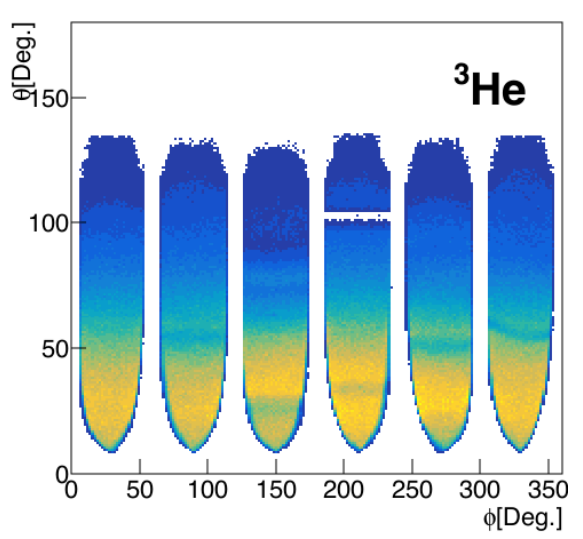

(d)

FIG. 155: The $\theta$ vs $\phi$ angular distribution of different particles for ${ }^{3} \mathrm{He}$ at $2.2 \mathrm{GeV}$. electrons; (b) protons; (c) $\pi^{-}$; (d) $\pi^{+}$. 


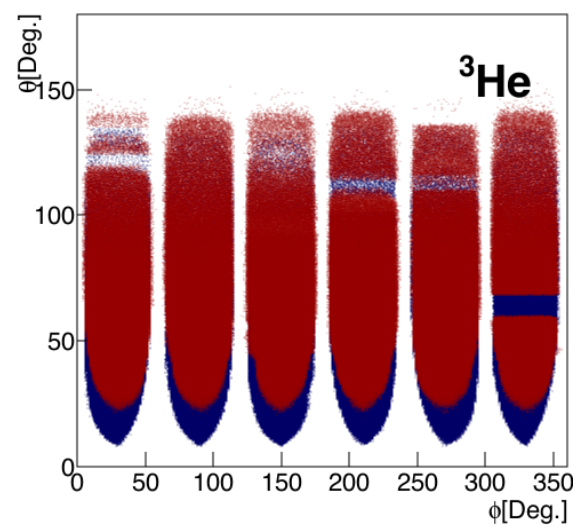

(a)

FIG. 156: The $\theta$ vs $\phi$ angular distribution of $\pi^{-}$and protons for ${ }^{3} \mathrm{He}$ at $2.2 \mathrm{GeV}$ plotted on top of each other.

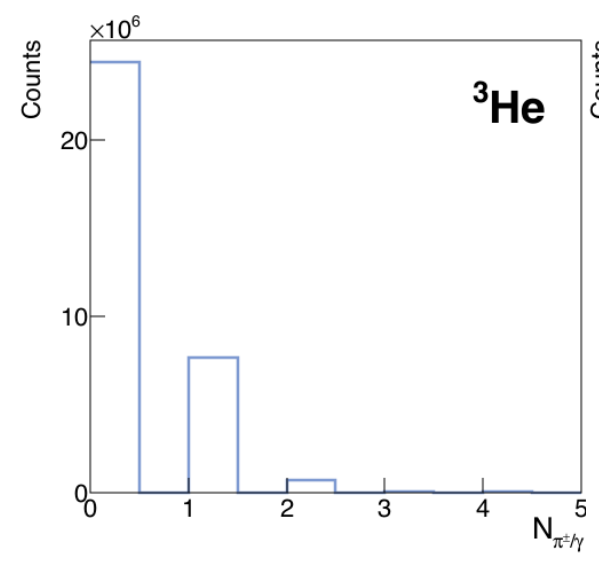

(a)

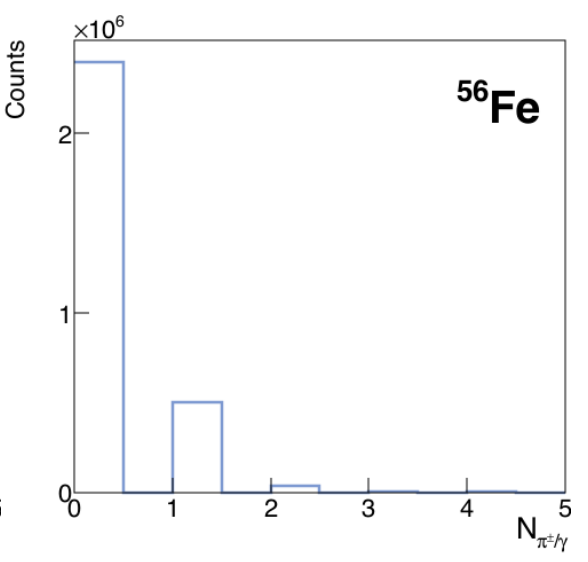

(b)

FIG. 157: Multiplicity distributions of $\pi^{ \pm} / \gamma$ for ${ }^{3} \mathrm{He}$ and ${ }^{56} \mathrm{Fe}$ targets at $2.2 \mathrm{GeV}$ (a) ${ }^{3} \mathrm{He}$; (b) ${ }^{56} \mathrm{Fe}$. 


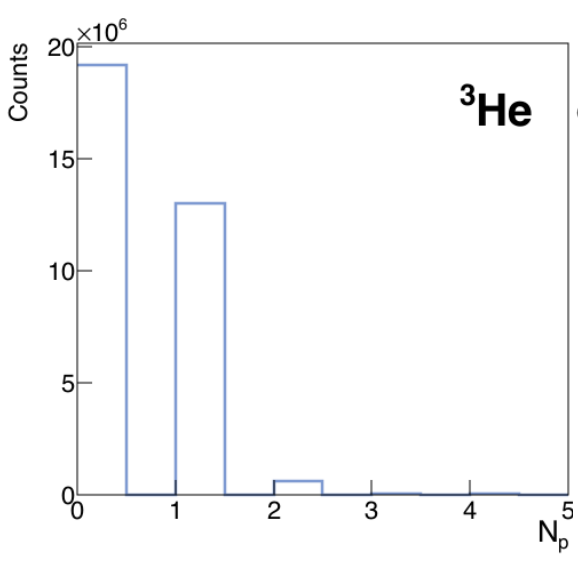

(a)

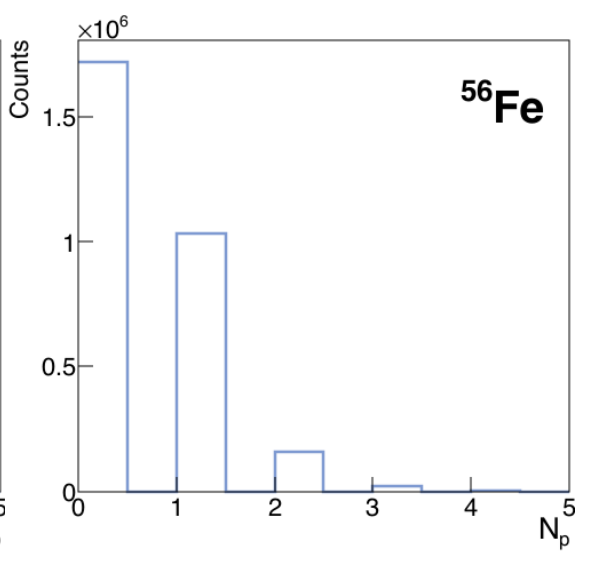

(b)

FIG. 158: Multiplicity distributions of protons for ${ }^{3} \mathrm{He}$ and ${ }^{56} \mathrm{Fe}$ targets at $2.2 \mathrm{GeV}$ (a) ${ }^{3} \mathrm{He}$; (b) ${ }^{56} \mathrm{Fe}$. 


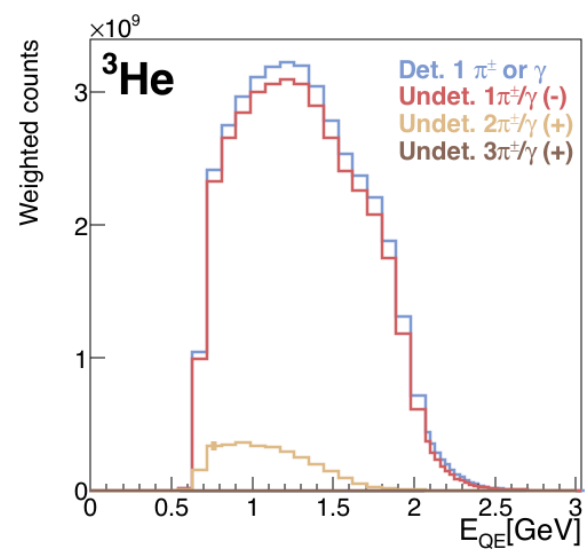

(a)

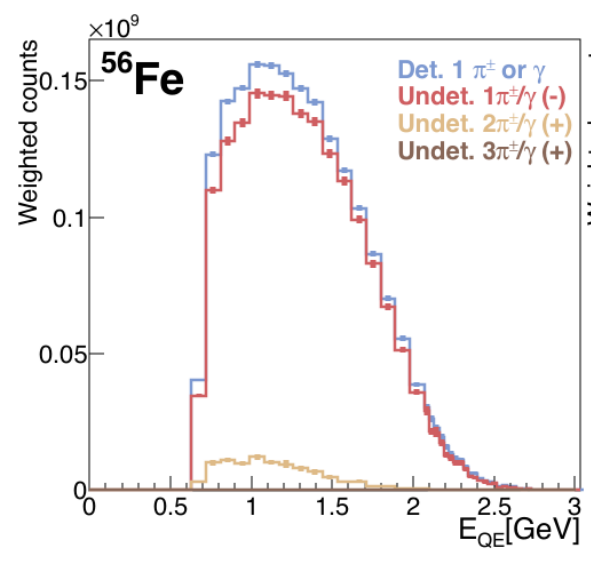

(c)

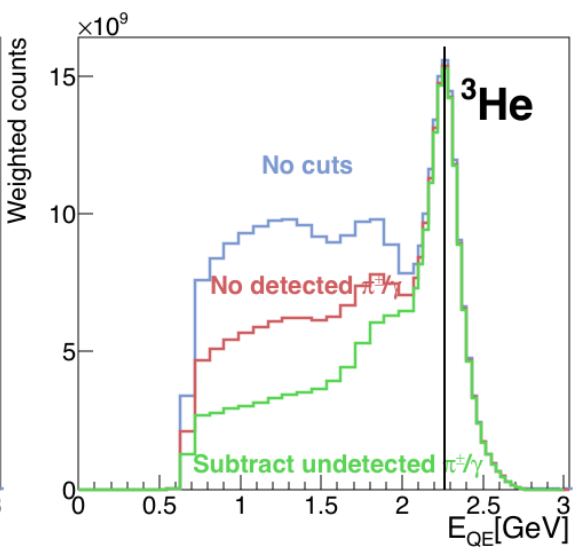

(b)

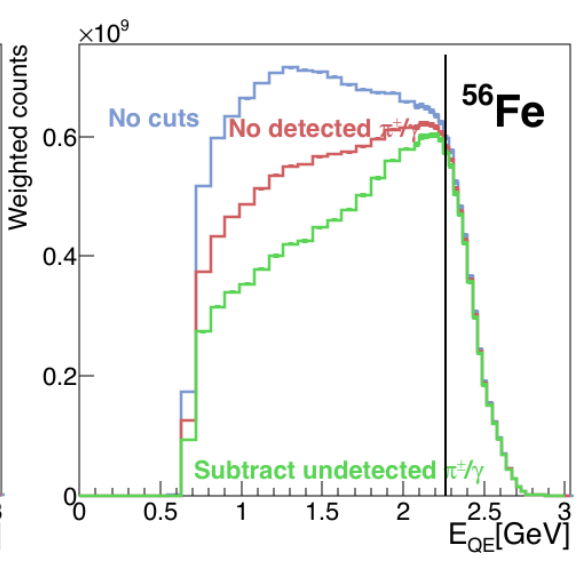

(d)

FIG. 159: $E_{\mathrm{QE}}$ obtained using Eq. 62, for detected (blue) and undetected (red) 1 pion events as well as undetected 2 and 3 pion events for (a) ${ }^{3} \mathrm{He}\left(e, e^{\prime} N \pi\right)$; (c) ${ }^{56} \mathrm{Fe}\left(e, e^{\prime} N \pi\right)$ at $2.2 \mathrm{GeV}$. Reconstructed energy $E_{\mathrm{QE}}$ of $A\left(e, e^{\prime}\right) 0 \pi$ spectrum before (red) and after (green) subtraction for undetected pions for (b) ${ }^{3} \mathrm{He}$ and (d) ${ }^{56} \mathrm{Fe}$ targets at $2.2 \mathrm{GeV}$. 


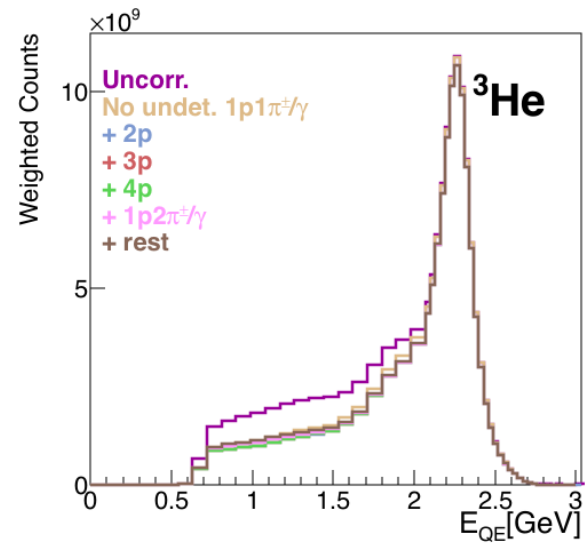

(a)

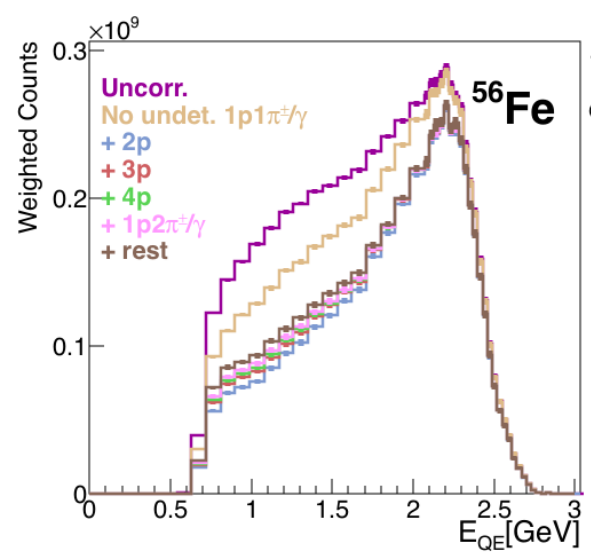

(c)

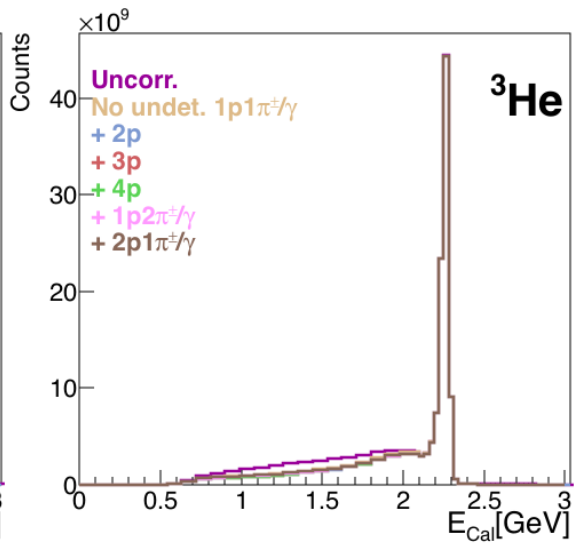

(b)

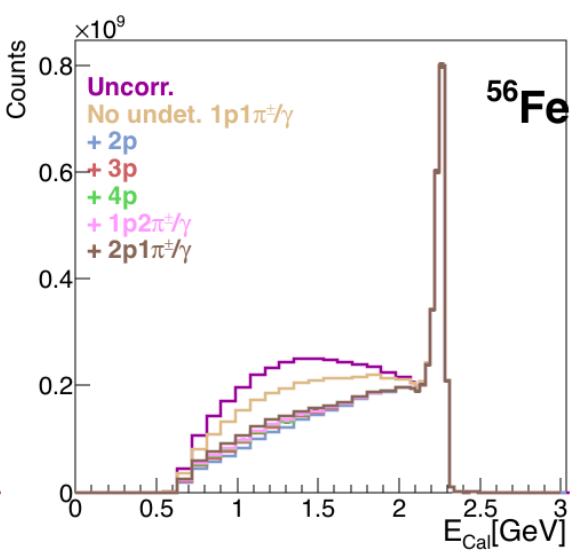

(d)

FIG. 160: $E_{\mathrm{QE}}$ (plots on the left) and $E_{\text {calor }}$ (plots on the right) distributions of $A\left(e, e^{\prime} p\right) 0 \pi$ spectrum before and after subtraction for undetected pions and undetected protons (green) for (a), (b) ${ }^{3} \mathrm{He}$ and (c),(d) ${ }^{56} \mathrm{Fe}$ targets at $2.2 \mathrm{GeV}$. 


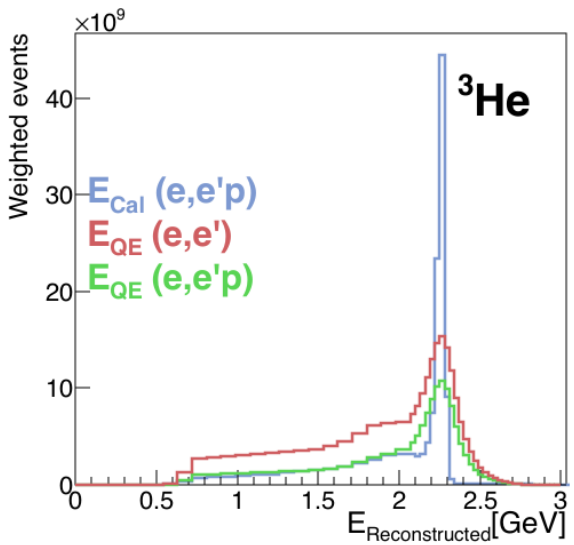

(a)

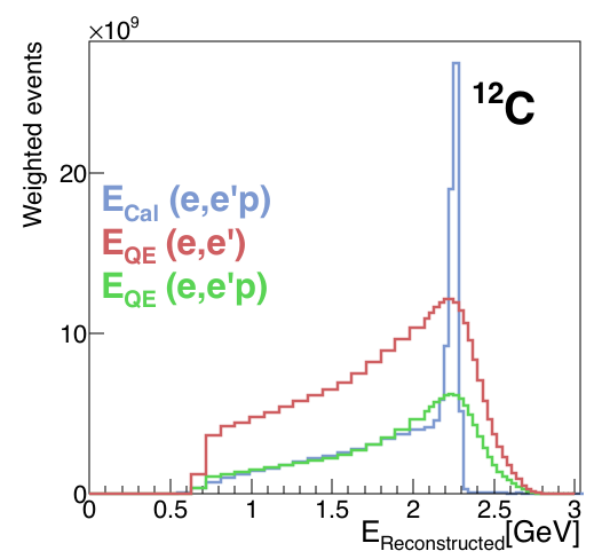

(c)

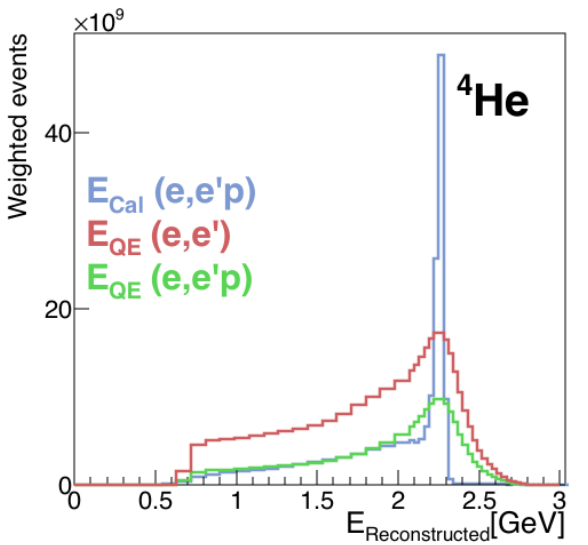

(b)

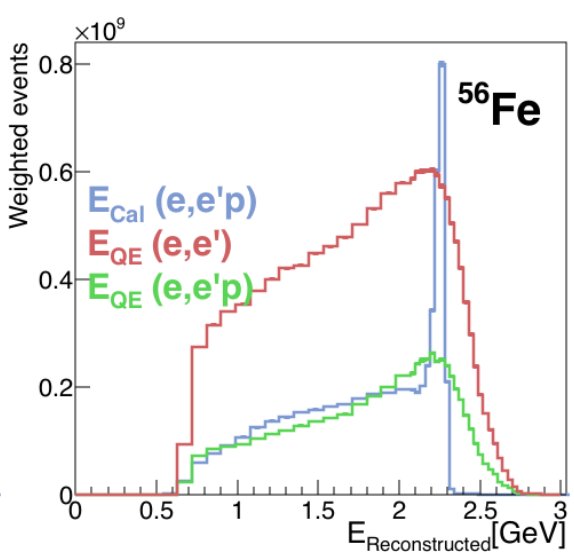

(d)

FIG. 161: $E_{\mathrm{QE}}$ distributions of $A\left(e, e^{\prime}\right)$ (red) and $A\left(e, e^{\prime} p\right)$ (green) $0 \pi$ spectrum and $E_{\text {calor }}$ distribution of $A\left(e, e^{\prime} p\right)$ (blue) $0 \pi$ spectrum after subtraction for undetected pions and undetected protons for (a) ${ }^{3} \mathrm{He}$, (b) ${ }^{4} \mathrm{He}$, (c) ${ }^{12} \mathrm{C}$ and (d) ${ }^{56} \mathrm{Fe}$ targets at $2.2 \mathrm{GeV}$. 

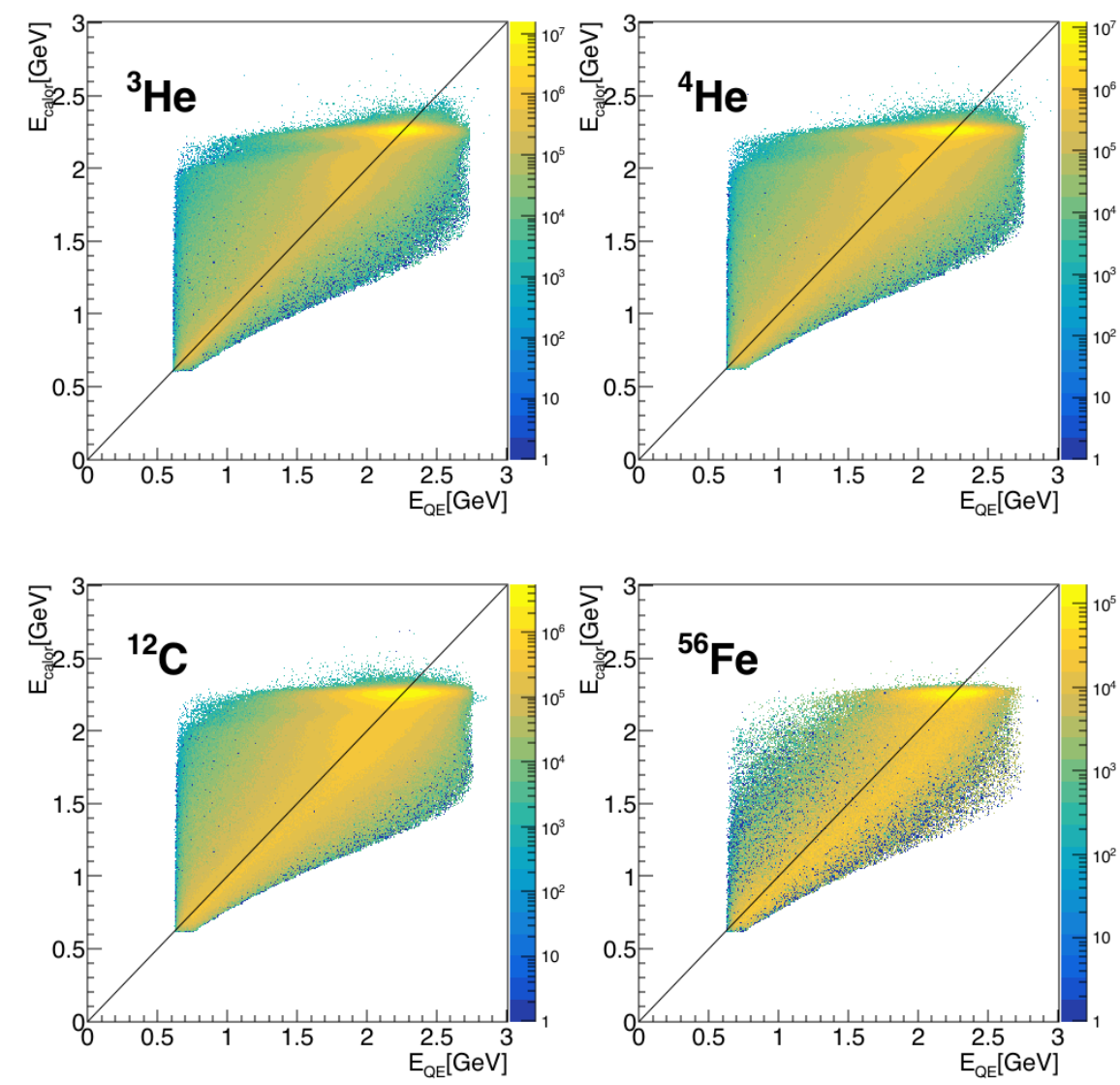

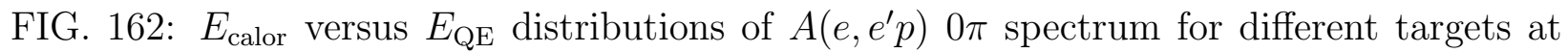
$2.2 \mathrm{GeV}$. 


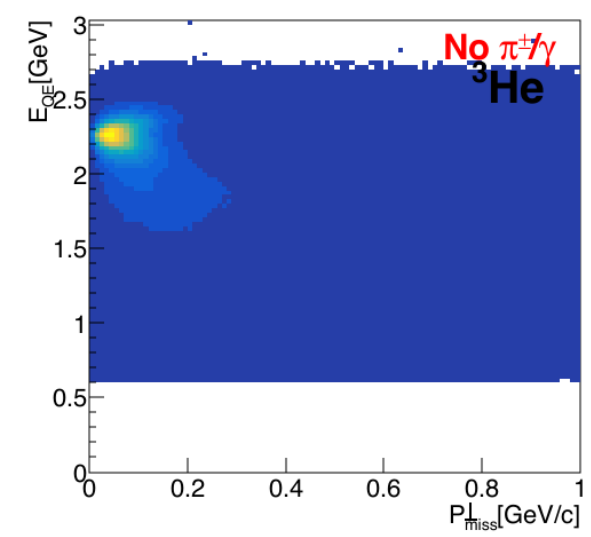

(a)

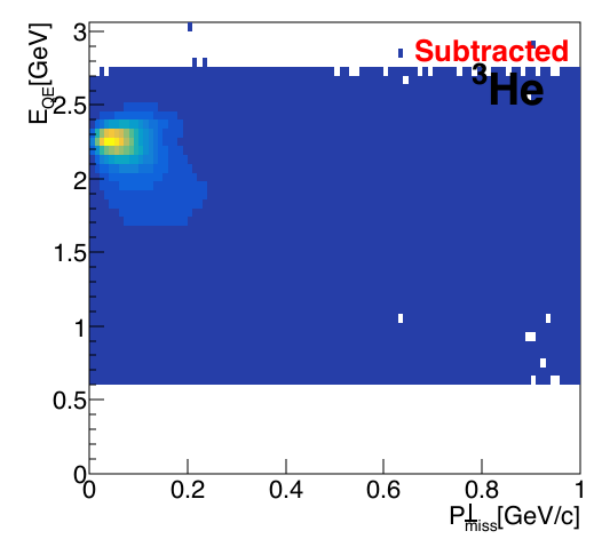

(c)

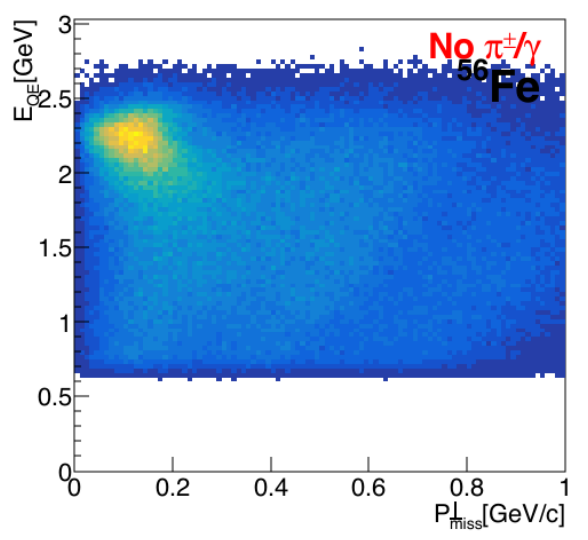

(b)

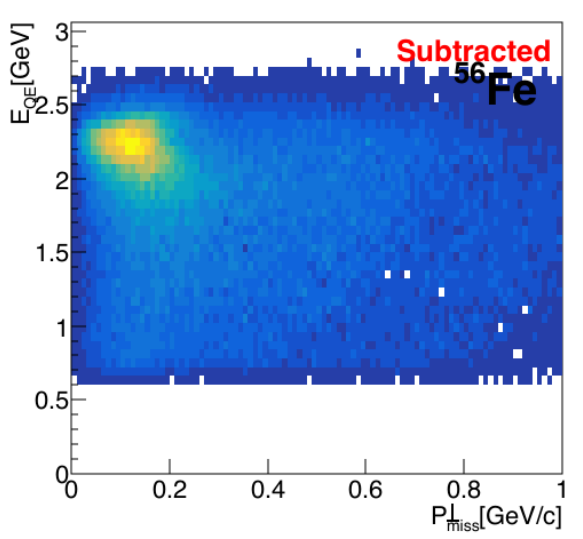

(d)

FIG. 163: Reconstructed energy $E_{\mathrm{QE}}$ obtained using Eq. 62 as a function of the total perpendicular momentum of the scattered electron and the knockout nucleon system for ${ }^{3} \mathrm{He}$ and ${ }^{56} \mathrm{Fe}$ targets at $2.2 \mathrm{GeV}$ before (top plots) and after (bottom plots) subtracting for undetected pions and protons. (a),(c) ${ }^{3} \mathrm{He}$; (b),(d) ${ }^{56} \mathrm{Fe}$. 


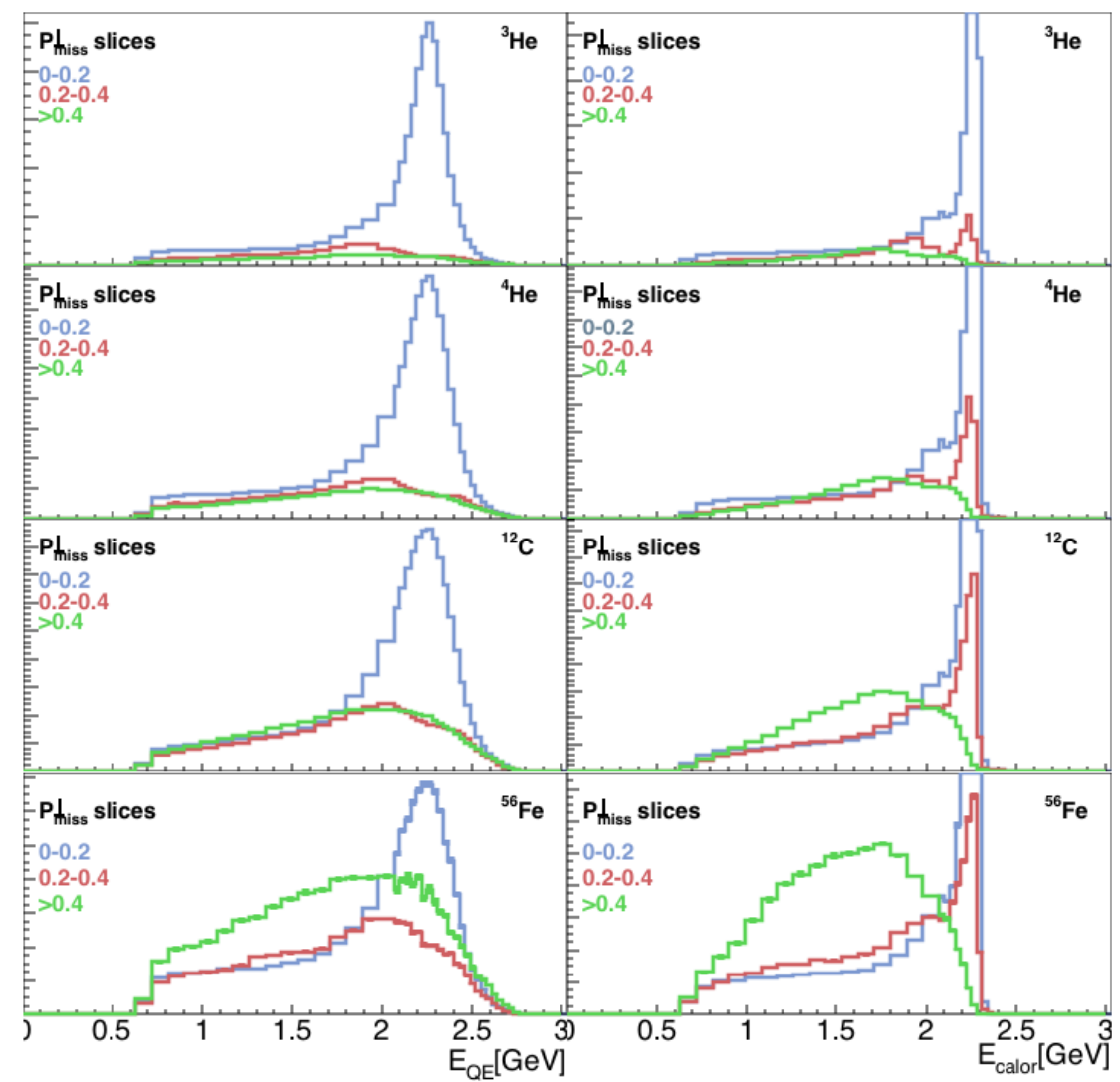

FIG. 164: $E_{\text {calor }}$ (right) and $E_{\mathrm{QE}}$ (left) distributions of $A\left(e, e^{\prime} p\right) 0 \pi$ spectrum after subtraction for undetected pions and undetected protons for three different regions of $P_{\text {miss }}^{\perp}$ $\left(0-200 \mathrm{MeV} / \mathrm{c}\right.$ (blue), $200-400 \mathrm{MeV} / \mathrm{c}$ (red) and $400 \mathrm{MeV} / \mathrm{c}$ and higher (green)) for ${ }^{3} \mathrm{He}$, ${ }^{4} \mathrm{He},{ }^{12} \mathrm{C}$ and ${ }^{56} \mathrm{Fe}$ targets at $2.2 \mathrm{GeV}$. The left and right plots have the same vertical scale. 


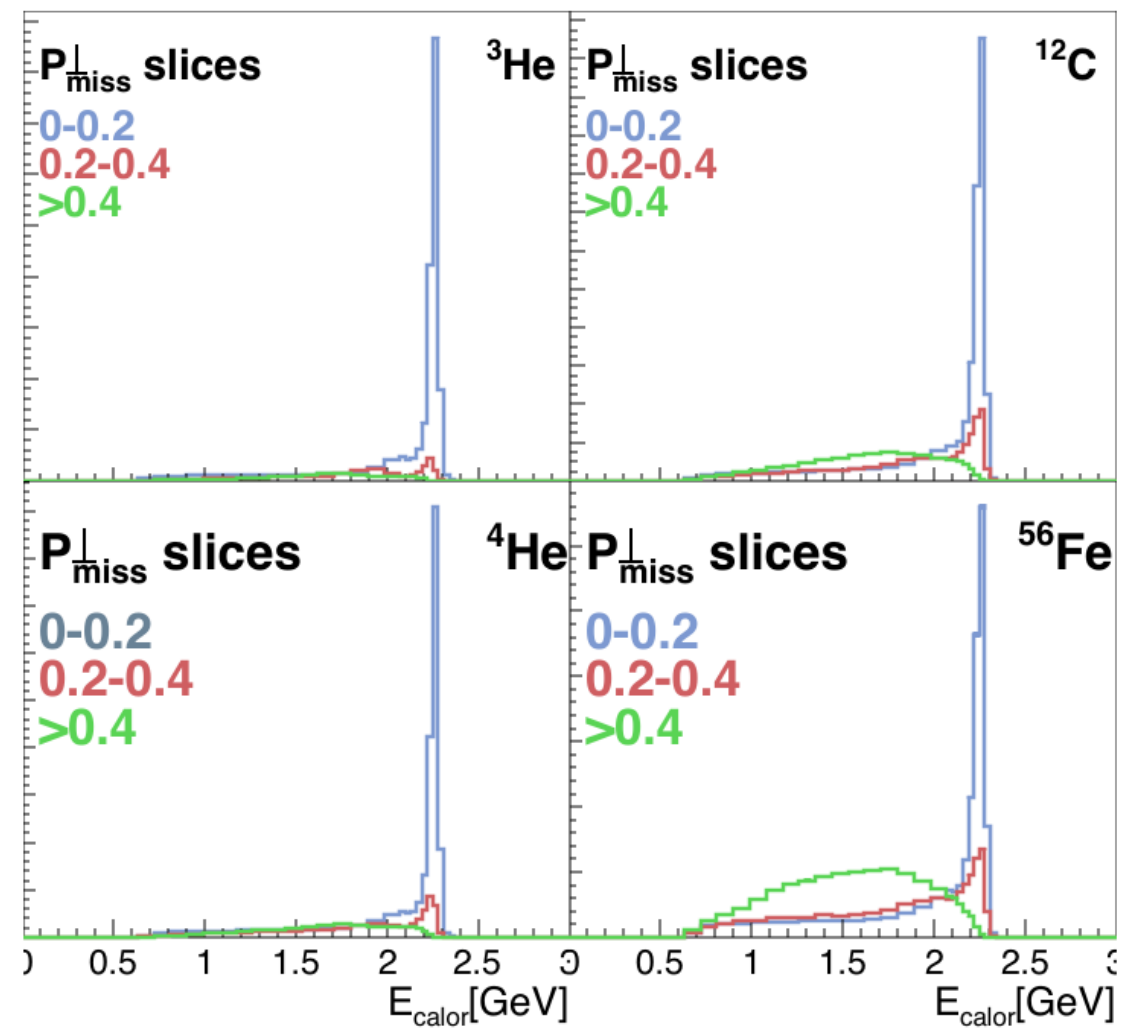

FIG. 165: $E_{\text {calor }}$ distributions of $A\left(e, e^{\prime} p\right) 0 \pi$ spectrum after subtraction for undetected pions and undetected protons for three different regions of $P_{\text {miss }}^{\perp}(0-200 \mathrm{MeV} / \mathrm{c}, 200-400 \mathrm{MeV} / \mathrm{c}$ and $400 \mathrm{MeV} / \mathrm{c}$ and higher) for ${ }^{3} \mathrm{He},{ }^{4} \mathrm{He},{ }^{12} \mathrm{C}$ and ${ }^{56} \mathrm{Fe}$ targets at $2.2 \mathrm{GeV}$ in full range. 


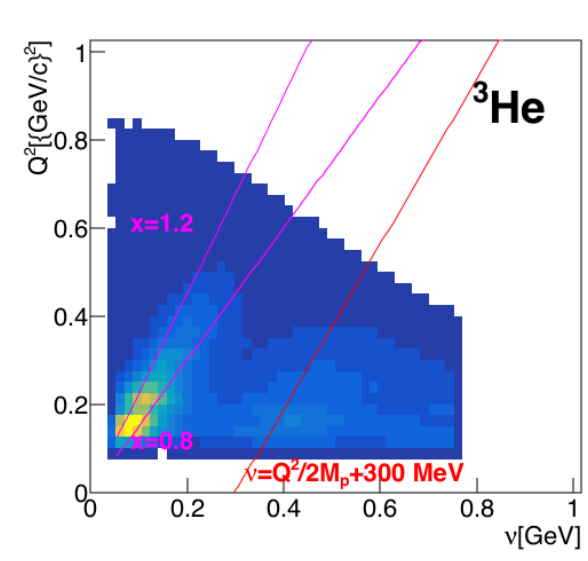

(a)

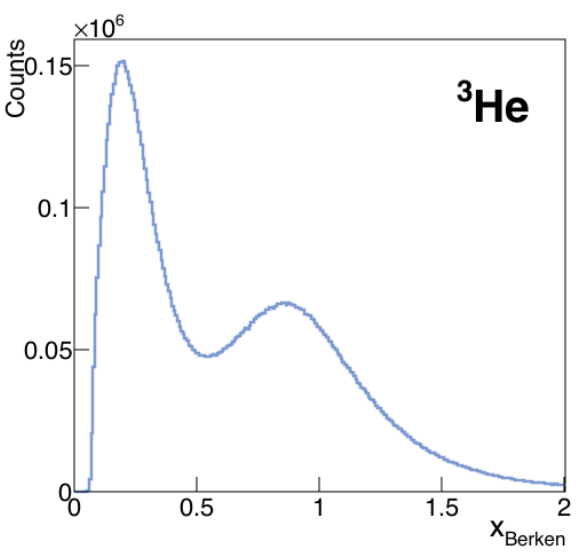

(b)

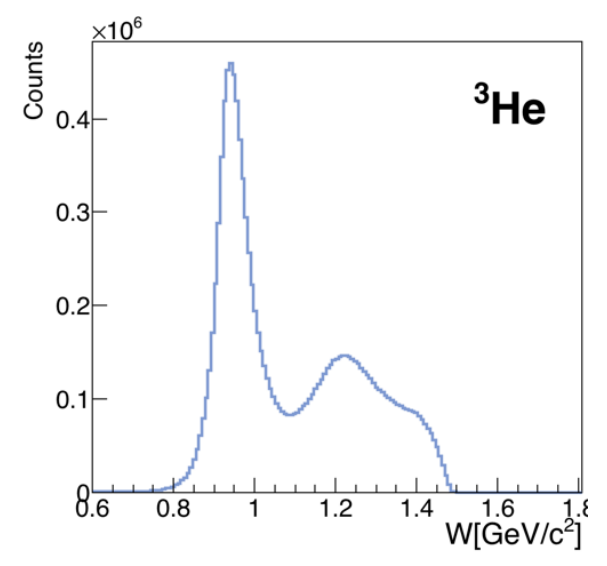

(c)

FIG. 166: The plots of (a) four momentum transfer $Q^{2}$ as a function of energy transfer $\nu$, the red line corresponds to $\nu=Q^{2} / 2 M_{p}+300 \mathrm{MeV}$ and shows where the location of $\Delta$ resonance peak should be.(b) Bjorken $x$ variable $\left(x_{\mathrm{B}}\right)$ and (c) Invariant mass of electron and proton system $W(e p)$ for ${ }^{3} \mathrm{He}$ at $1.1 \mathrm{GeV}$. 


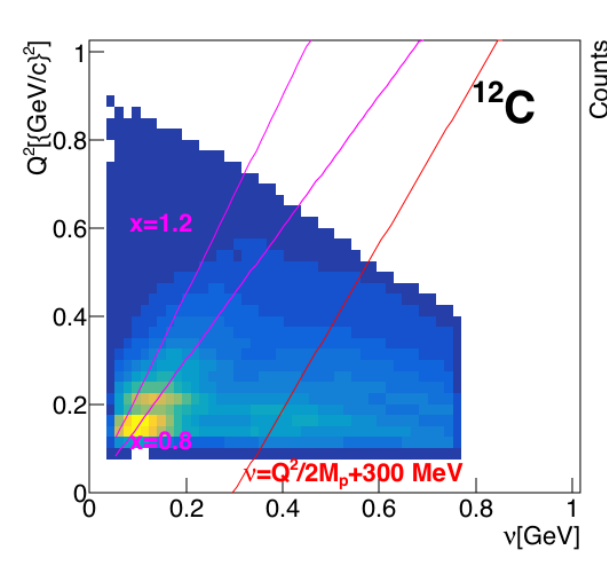

(a)

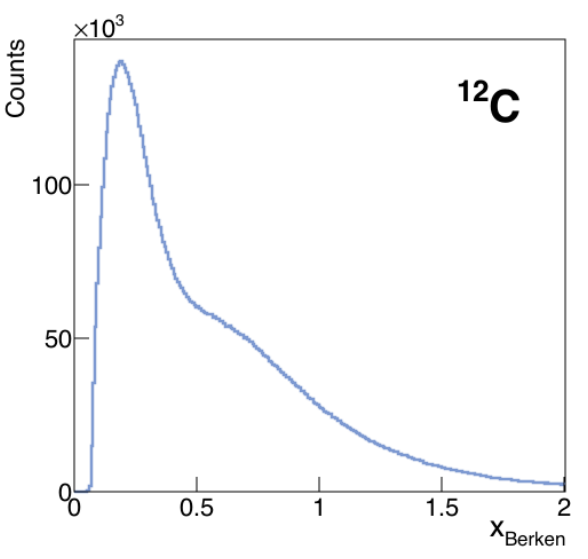

(b)

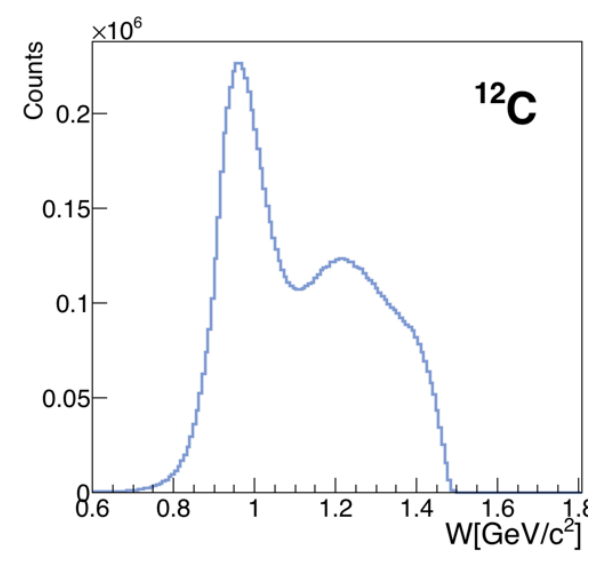

(c)

FIG. 167: The plots of (a) four momentum transfer $Q^{2}$ as a function of energy transfer $\nu$, the red line corresponds to $\nu=Q^{2} / 2 M_{p}+300 \mathrm{MeV}$ and shows where the location of $\Delta$ resonance peak should be. (b) Bjorken $x$ variable $\left(x_{\mathrm{B}}\right)$ and (c) Invariant mass of electron and proton system $W(e p)$ for ${ }^{12} \mathrm{C}$ at $1.1 \mathrm{GeV}$. 


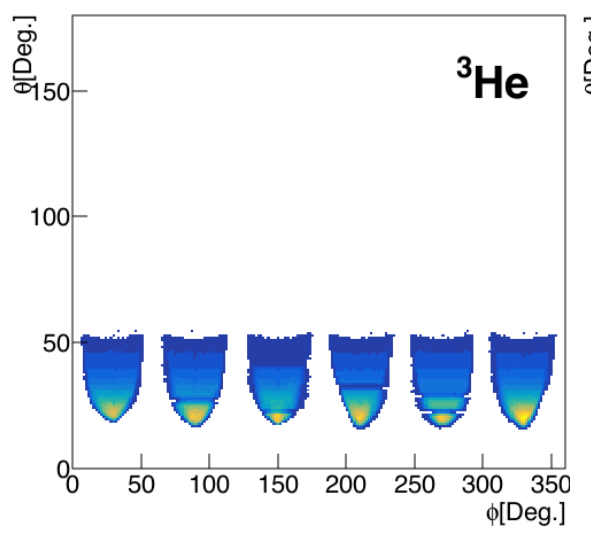

(a)

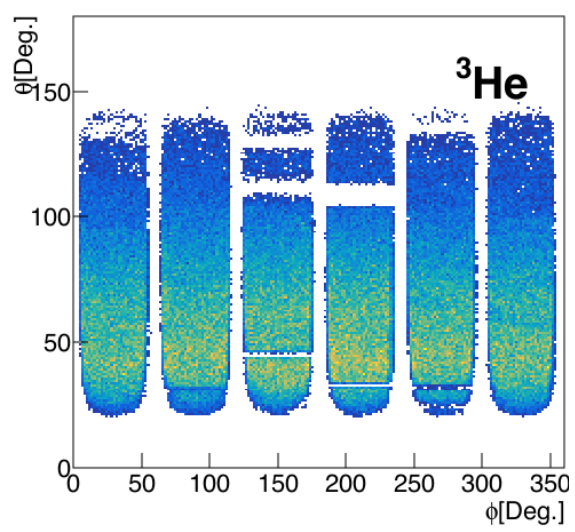

(c)

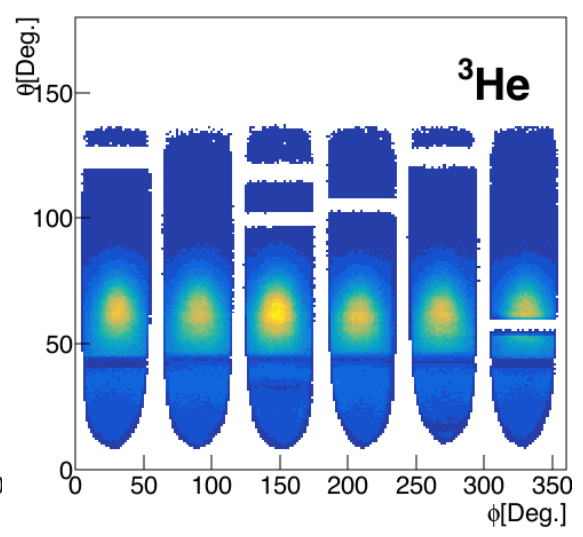

(b)

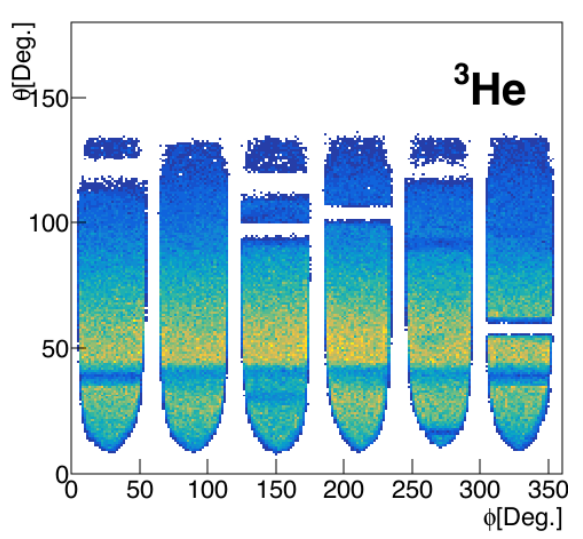

(d)

FIG. 168: The $\theta$ vs $\phi$ angular distribution of different particles for ${ }^{3} \mathrm{He}$ at $1.1 \mathrm{GeV}$. electrons; (b) protons; (c) $\pi^{-}$; (d) $\pi^{+}$. 


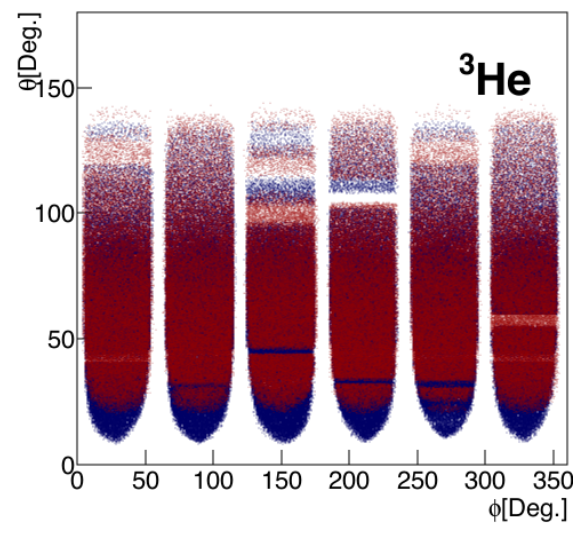

(a)

FIG. 169: The $\theta$ vs $\phi$ angular distribution of $\pi^{-}$and protons for ${ }^{3} \mathrm{He}$ at $1.1 \mathrm{GeV}$ plotted on top of each other.

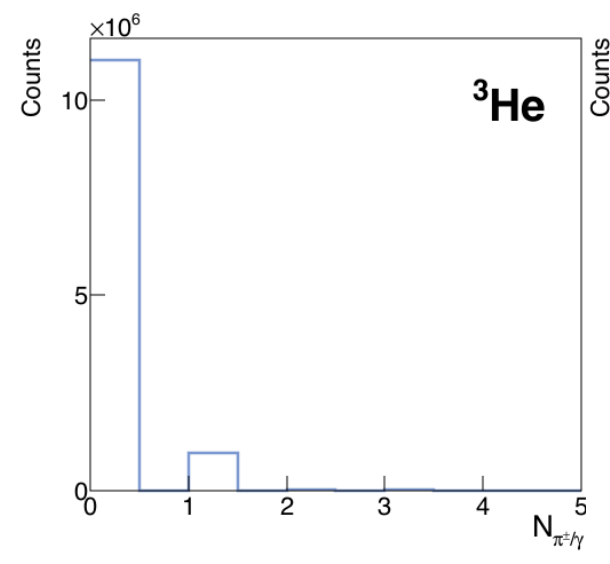

(a)

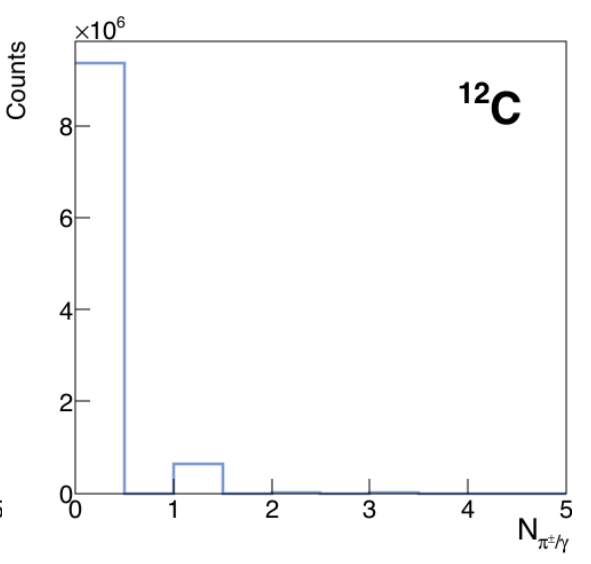

(b)

FIG. 170: Multiplicity distributions of $\pi^{ \pm} \gamma$ for ${ }^{3} \mathrm{He}$ and ${ }^{12} \mathrm{C}$ targets at $1.1 \mathrm{GeV}$ (a) ${ }^{3} \mathrm{He}$; (b) ${ }^{12} \mathrm{C}$. 


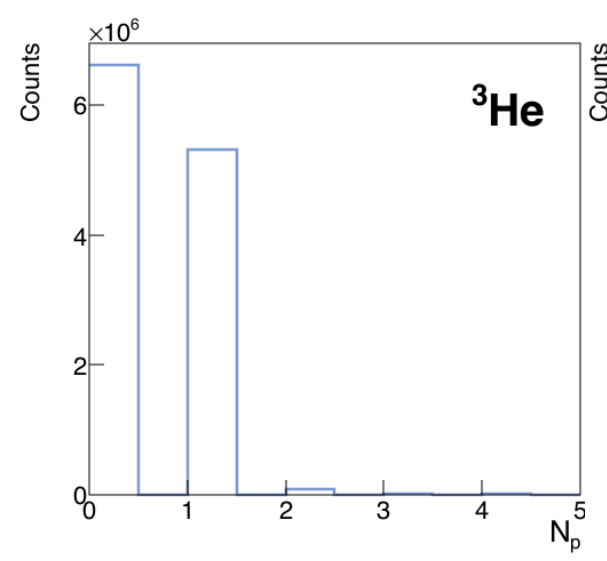

(a)

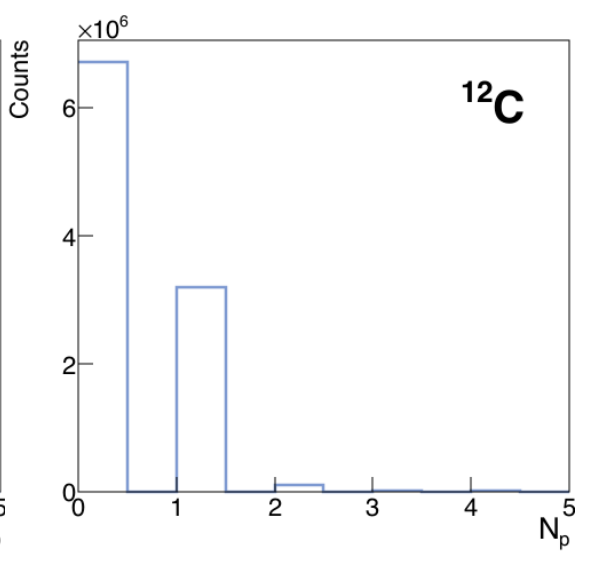

(b)

FIG. 171: Multiplicity distributions of protons for ${ }^{3} \mathrm{He}$ and ${ }^{56} \mathrm{Fe}$ targets at $1.1 \mathrm{GeV}$ (a) ${ }^{3} \mathrm{He}$; (b) ${ }^{12} \mathrm{C}$. 


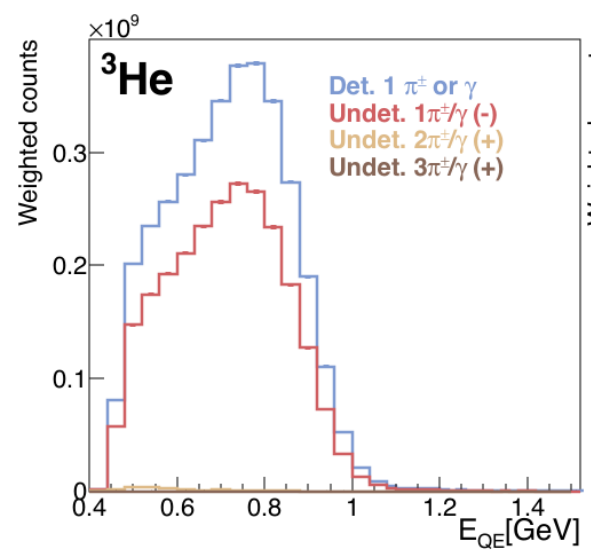

(a)

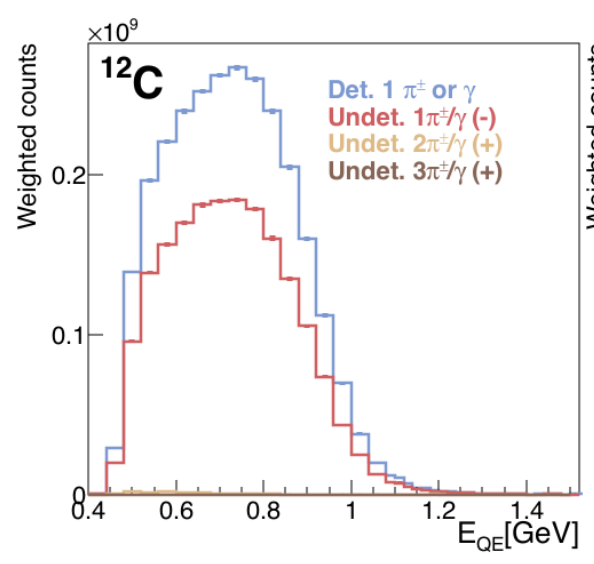

(c)

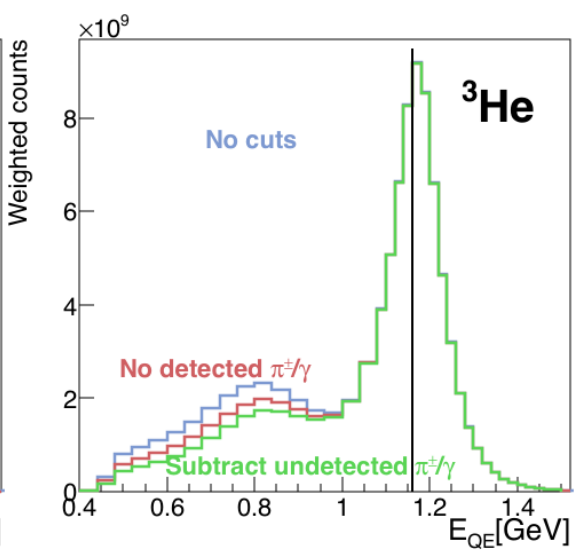

(b)

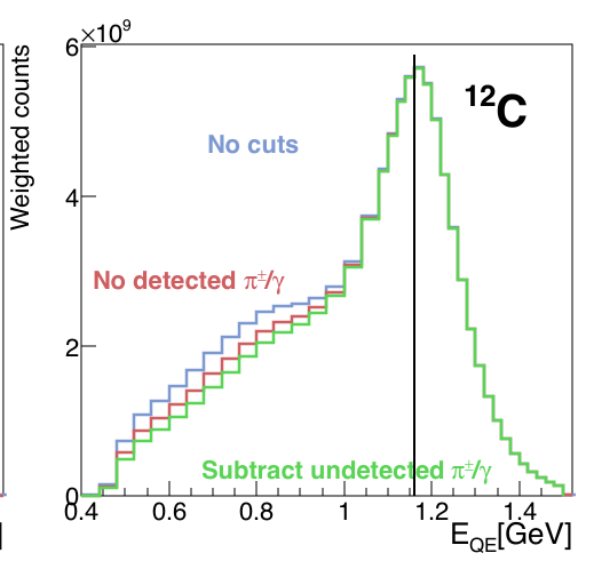

(d)

FIG. 172: $E_{\mathrm{QE}}$ obtained using Eq. 62, for detected (blue) and undetected (red) 1 pion events as well as undetected 2 and 3 pion events for (a) ${ }^{3} \mathrm{He}\left(e, e^{\prime} N \pi\right) ;(\mathrm{c}){ }^{12} \mathrm{C}\left(e, e^{\prime} N \pi\right)$ at $1.1 \mathrm{GeV}$. Reconstructed energy $E_{\mathrm{QE}}$ of $A\left(e, e^{\prime}\right) 0 \pi$ spectrum before (red) and after (green) subtraction for undetected pions for (b) ${ }^{3} \mathrm{He}$ and $(\mathrm{d}){ }^{12} \mathrm{C}$ targets at $1.1 \mathrm{GeV}$. 


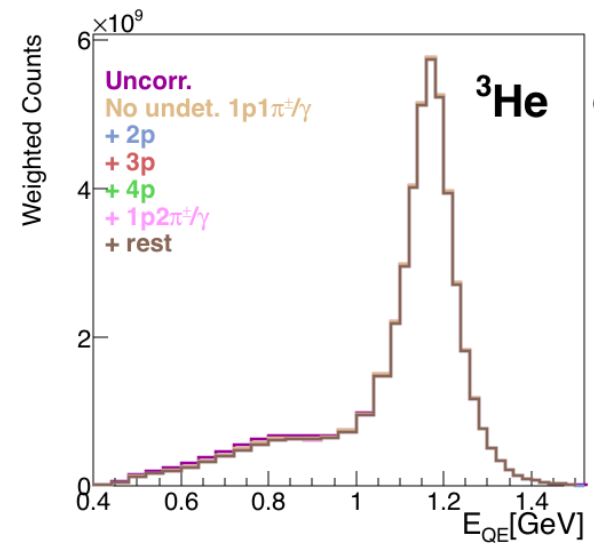

(a)

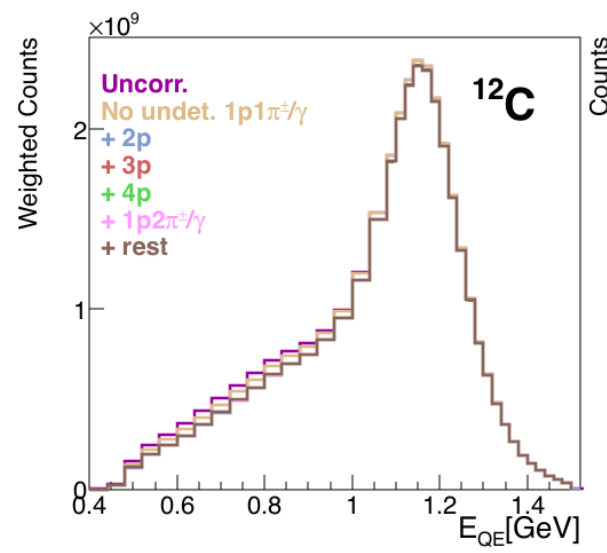

(c)

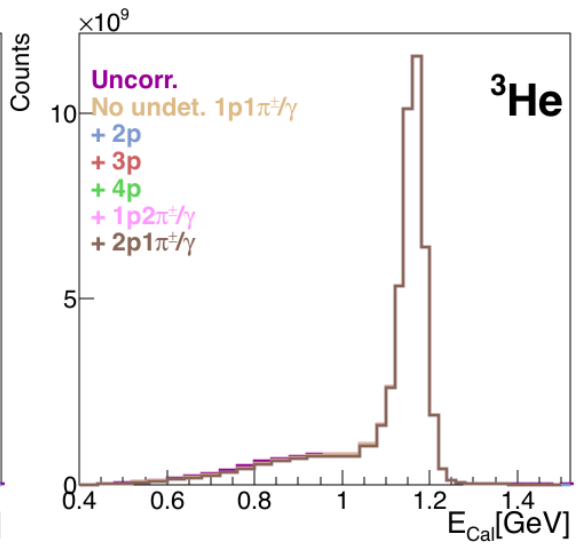

(b)

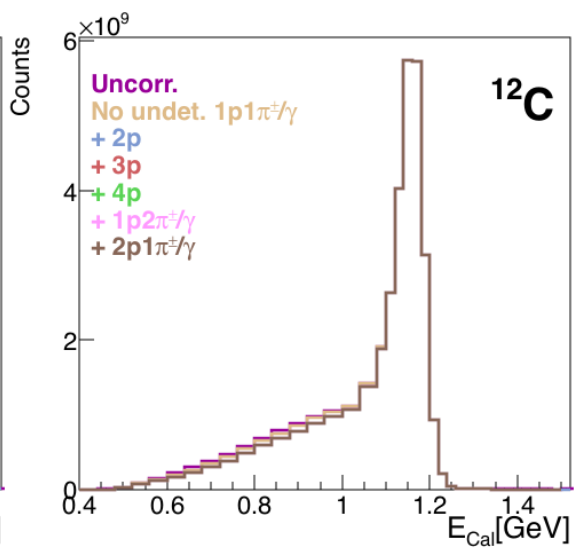

(d)

FIG. 173: $E_{\mathrm{QE}}$ (plots on the left) and $E_{\text {calor }}$ (plots on the right) distributions of $A\left(e, e^{\prime} p\right) 0 \pi$ spectrum before and after subtraction for undetected pions and undetected protons (green) for (a), (b) ${ }^{3} \mathrm{He}$ and (c),(d) ${ }^{12} \mathrm{C}$ targets at $1.1 \mathrm{GeV}$. 


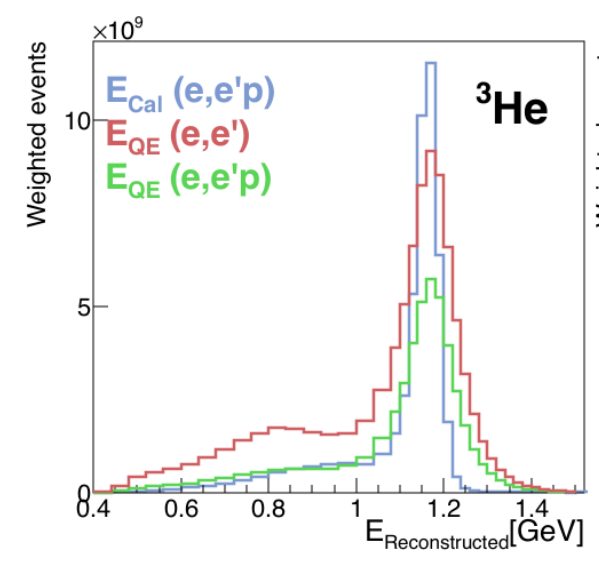

(a)

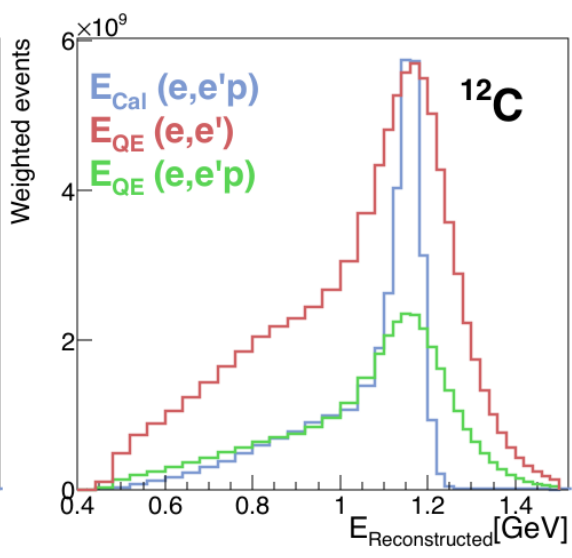

(b)

FIG. 174: $E_{\mathrm{QE}}$ distributions of $A\left(e, e^{\prime}\right)$ (red) and $A\left(e, e^{\prime} p\right)$ (green) $0 \pi$ spectrum and $E_{\text {calor }}$ distribution of $A\left(e, e^{\prime} p\right)$ (blue) $0 \pi$ spectrum after subtraction for undetected pions and undetected protons for (a) ${ }^{3} \mathrm{He}$, (b) ${ }^{12} \mathrm{C}$ targets at $1.1 \mathrm{GeV}$.
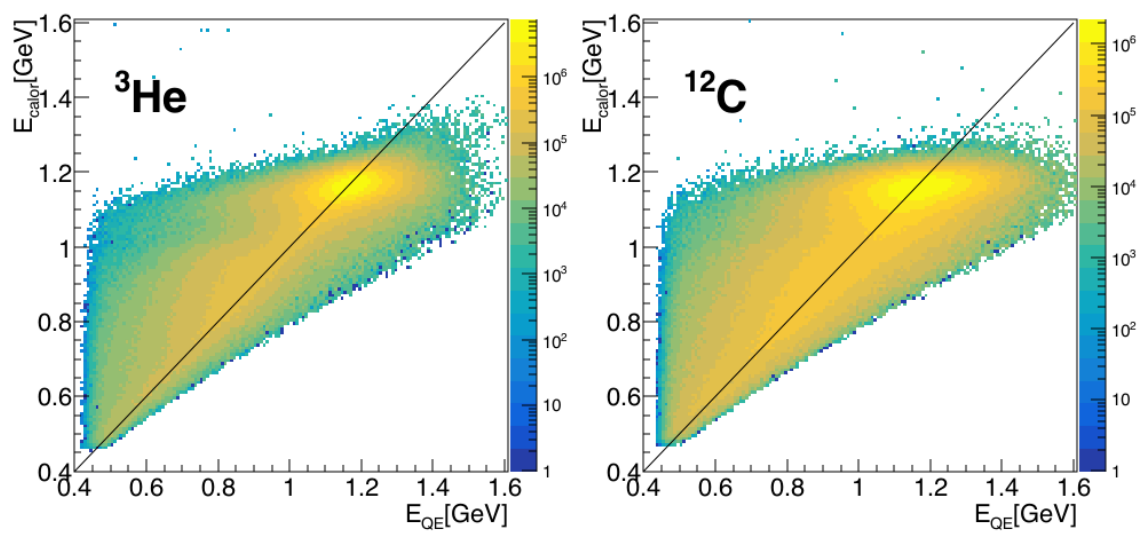

FIG. 175: $E_{\text {calor }}$ versus $E_{\mathrm{QE}}$ distributions of $A\left(e, e^{\prime} p\right) 0 \pi$ spectrum for different targets at $1.1 \mathrm{GeV}$. 


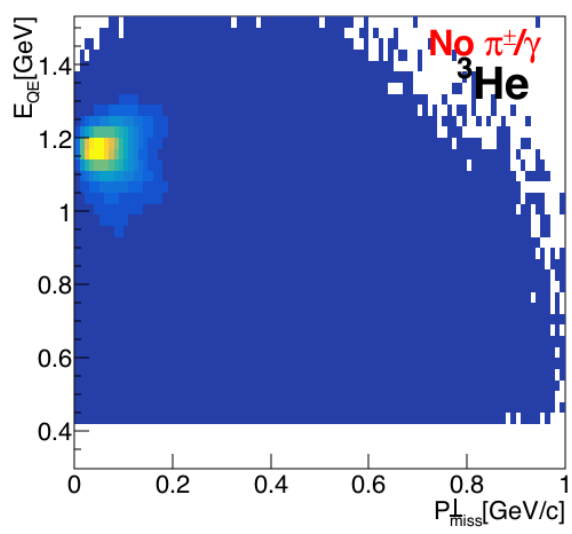

(a)

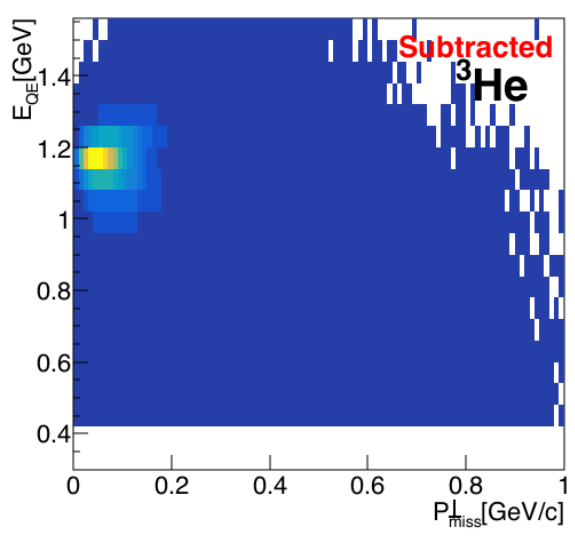

(c)

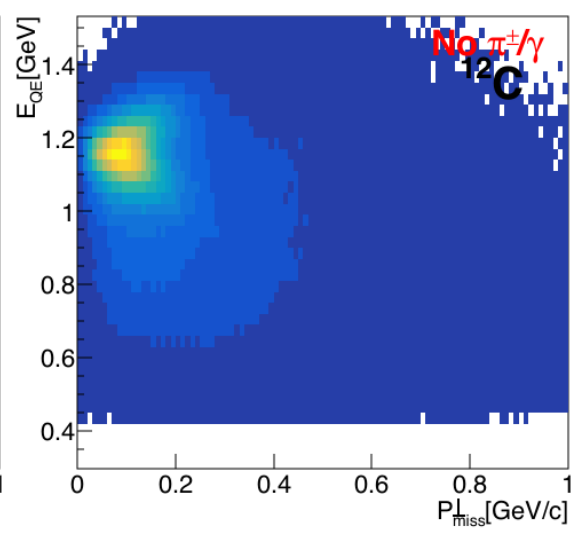

(b)

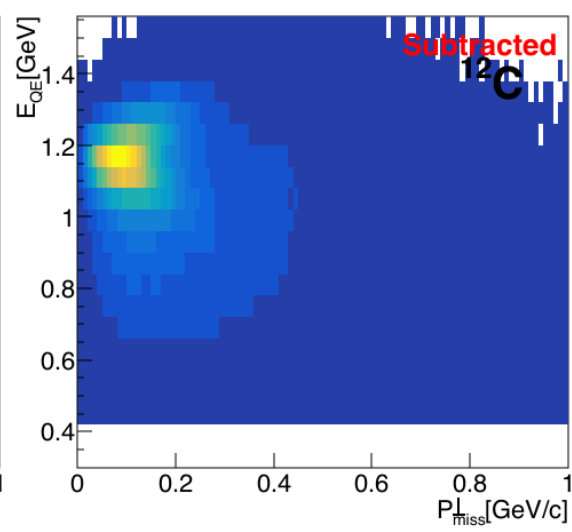

(d)

FIG. 176: Reconstructed energy $E_{\mathrm{QE}}$ obtained using Eq. 62 as a function of the total perpendicular momentum of the scattered electron and the knockout nucleon system for ${ }^{3} \mathrm{He}$ and ${ }^{12} \mathrm{C}$ targets at $1.1 \mathrm{GeV}$ before (top plots) and after (bottom plots) subtracting for undetected pions and protons. (a),(c) ${ }^{3} \mathrm{He}$; (b), (d) ${ }^{12} \mathrm{C}$. 


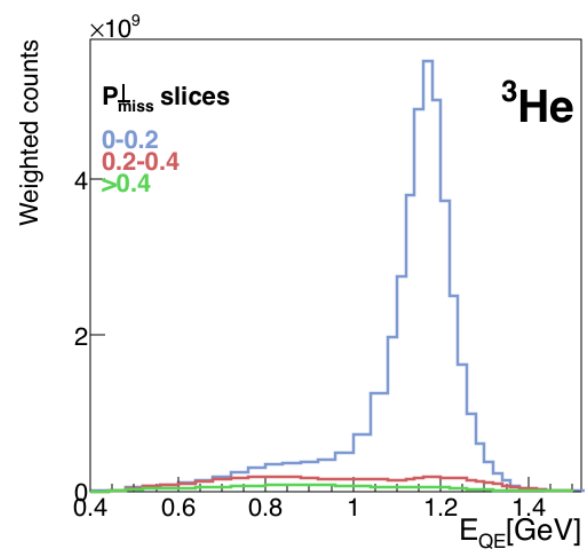

(a)

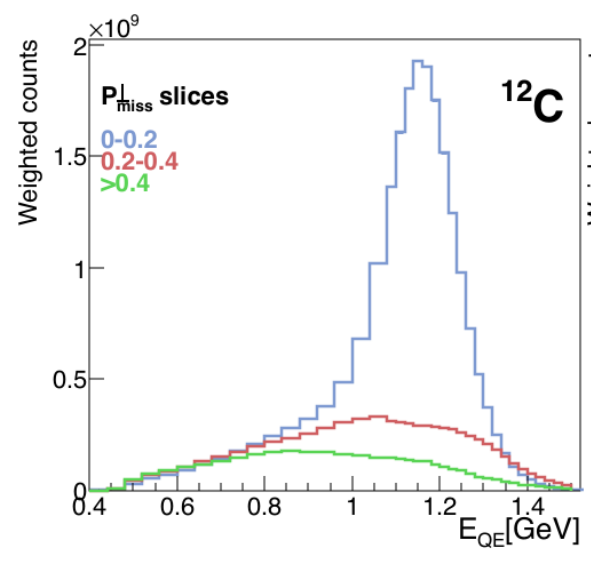

(c)

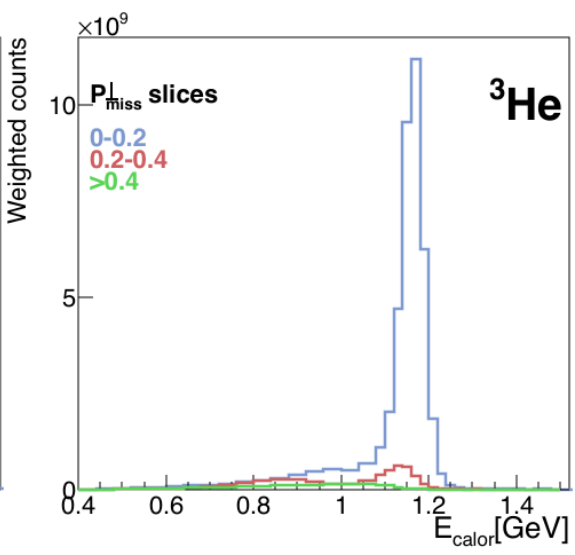

(b)

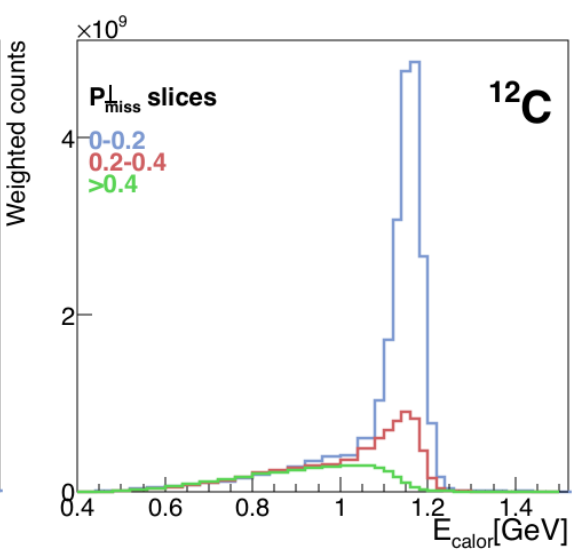

(d)

FIG. 177: $E_{\text {calor }}$ (right) and $E_{\mathrm{QE}}$ (left) distributions of $A\left(e, e^{\prime} p\right) 0 \pi$ spectrum after subtraction for undetected pions and undetected protons for three different regions of $P_{\text {miss }}^{\perp}$ (0 - $200 \mathrm{MeV} / \mathrm{c}$ (blue), $200-400 \mathrm{MeV} / \mathrm{c}$ (red) and $400 \mathrm{MeV} / \mathrm{c}$ and higher (green)) for ${ }^{3} \mathrm{He}$ and ${ }^{12} \mathrm{C}$ targets at $1.1 \mathrm{GeV}$. 

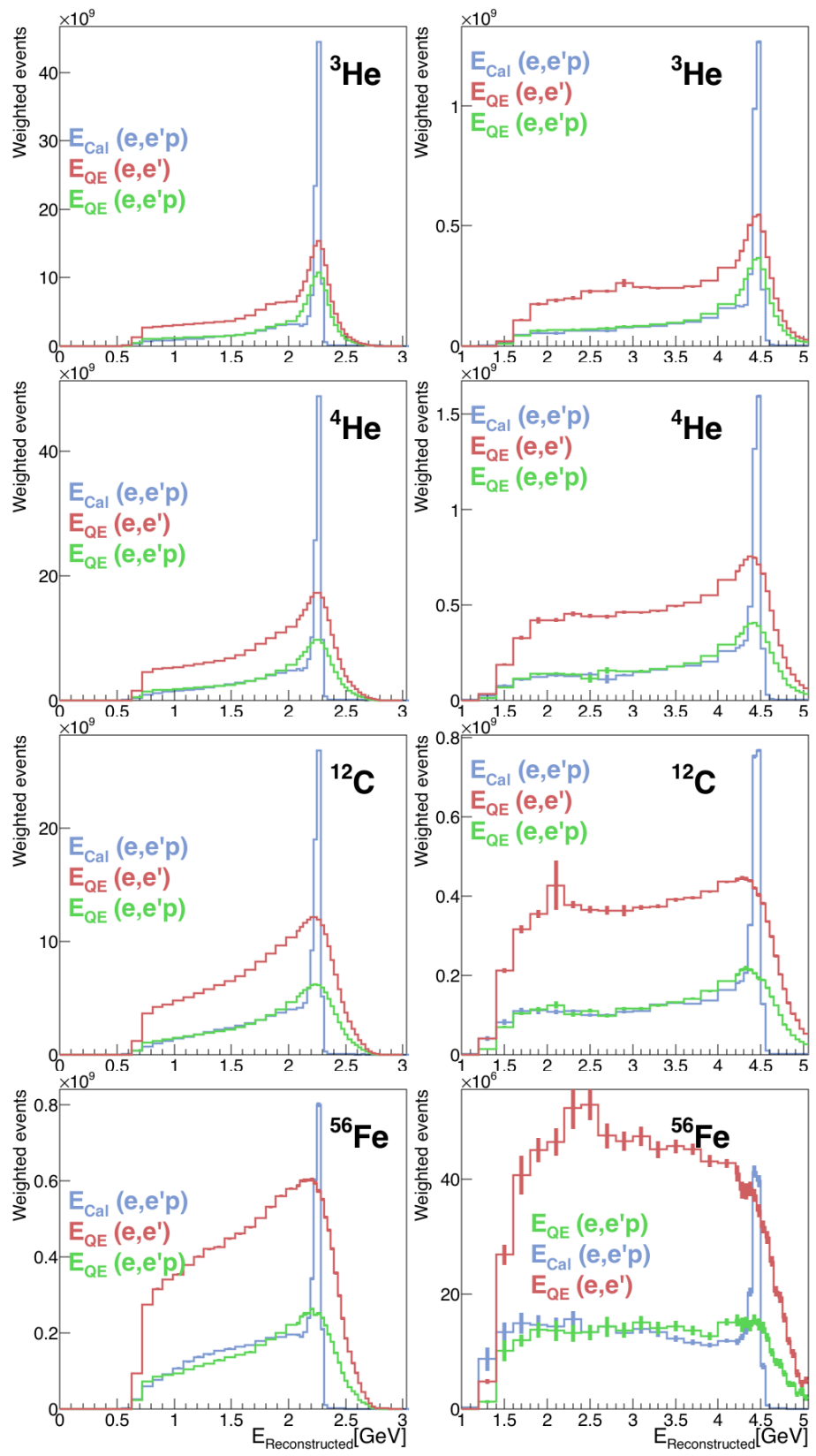

FIG. 178: $E_{\mathrm{QE}}$ distributions of $A\left(e, e^{\prime}\right)$ (red) and $A\left(e, e^{\prime} p\right)$ (green) $0 \pi$ spectrum and $E_{\text {calor }}$ distribution of $A\left(e, e^{\prime} p\right)$ (blue) $0 \pi$ spectrum after subtraction for undetected pions and undetected protons for ${ }^{3} \mathrm{He},{ }^{4} \mathrm{He},{ }^{12} \mathrm{C}$ and ${ }^{56} \mathrm{Fe}$ targets at $2.2 \mathrm{GeV}$ (plots on the left) and at $4.4 \mathrm{GeV}$ (plots on the right). 

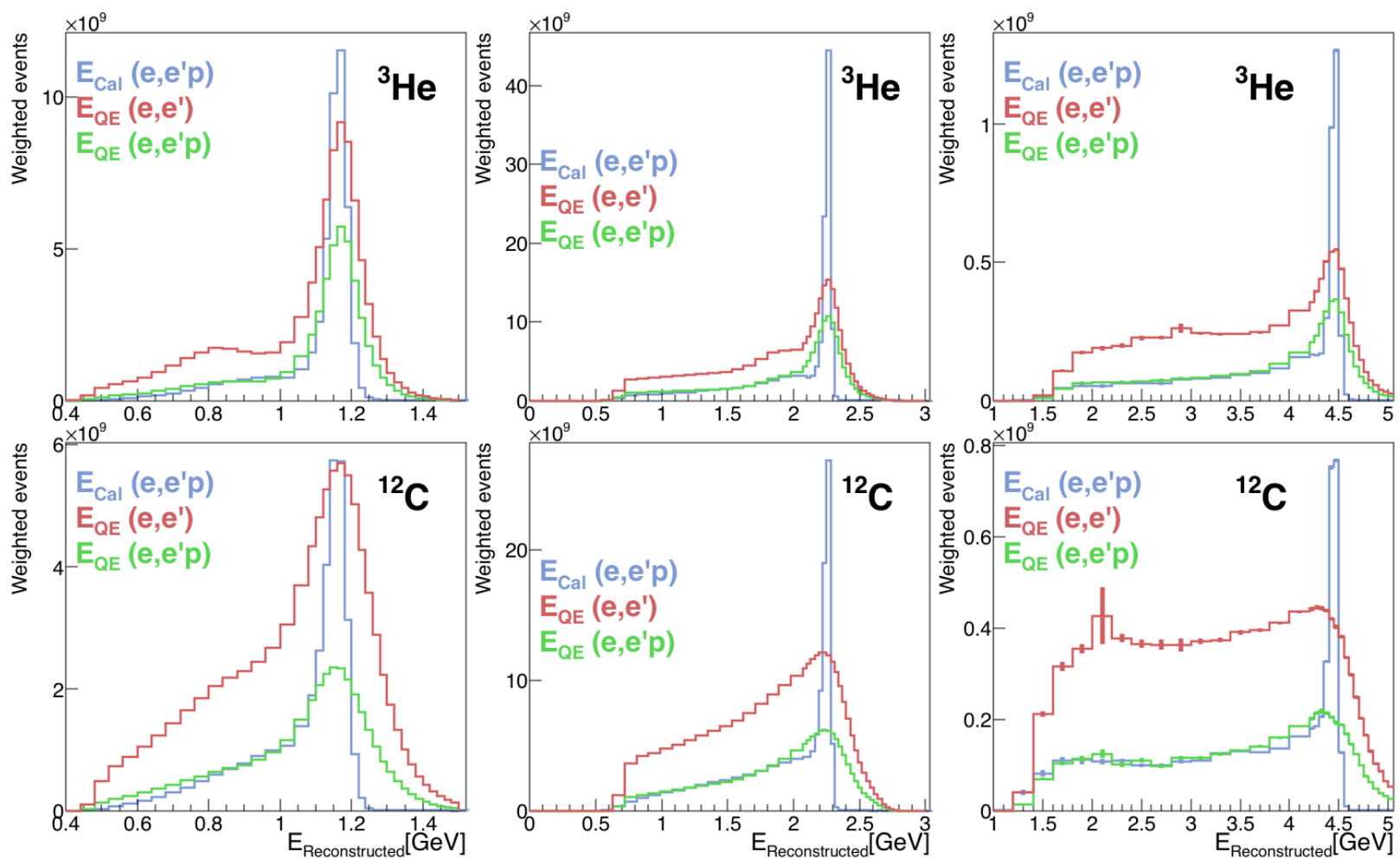

FIG. 179: $E_{\mathrm{QE}}$ distributions of $A\left(e, e^{\prime}\right)$ (red) and $A\left(e, e^{\prime} p\right)$ (green) $0 \pi$ spectrum and $E_{\text {calor }}$ distribution of $A\left(e, e^{\prime} p\right)$ (blue) $0 \pi$ spectrum after subtraction for undetected pions and undetected protons for ${ }^{3} \mathrm{He},{ }^{12} \mathrm{C}$ targets at $1.1 \mathrm{GeV}$ (plots on the left), $2.2 \mathrm{GeV}$ (plots in the middle) and at $4.4 \mathrm{GeV}$ (plots on the right). 

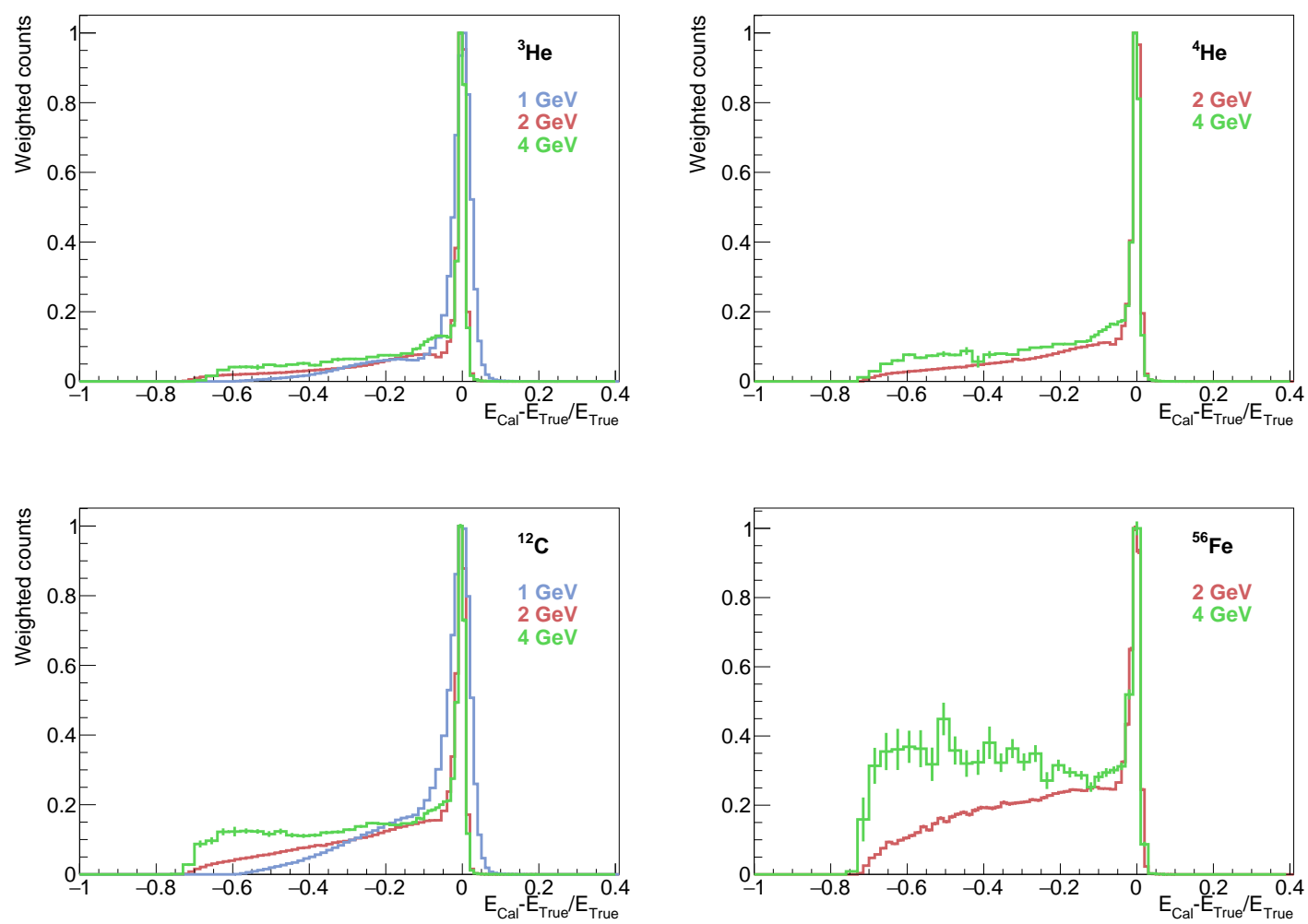

FIG. 180: Fractional energy reconstruction "feed down" for $E_{\text {calor }}$ method and $A(e, e p)$ spectrum, for all targets at $1.1 \mathrm{GeV}, 2.2 \mathrm{GeV}$ and $4.4 \mathrm{GeV}$. 

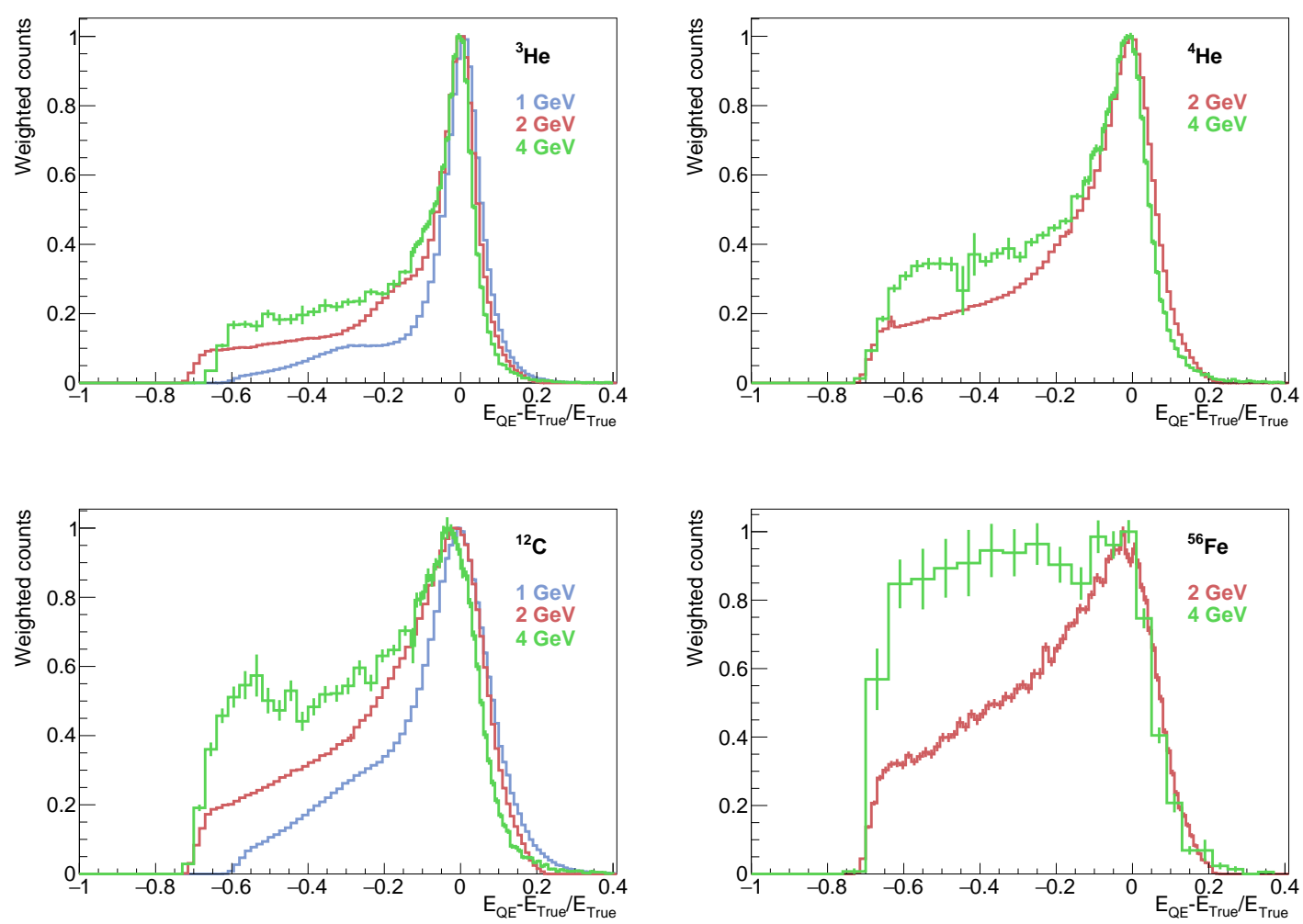

FIG. 181: Fractional energy reconstruction "feed down" for $E_{\mathrm{QE}}$ method and $A\left(e, e^{\prime} p\right)$ spectrum, for all targets at $1.1 \mathrm{GeV}, 2.2 \mathrm{GeV}$ and $4.4 \mathrm{GeV}$.

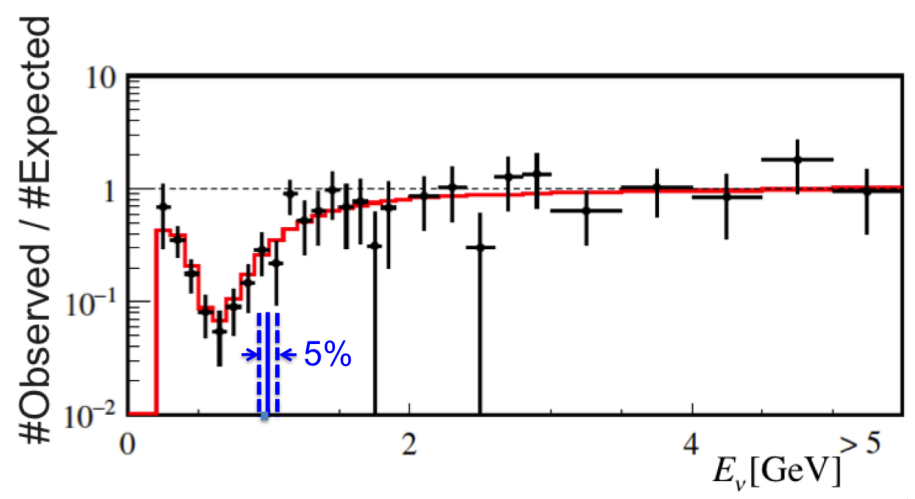

FIG. 182: The neutrino oscillation signal as function of neutrino energy from the T2K experiment with $5 \%$ error region. [8]. 


\subsection{SOURCES OF UNCERTAINTY}

The uncertainty sources in our analysis are:

1. Statistical uncertainty is shown by error bars on histograms.

2. Uncertainty of the subtraction of undetected pions, photons and protons.

(a) Uncertainty due to angular dependence of pion and photon cross sections.

(b) Statistical uncertainty due to number of $\left(e, e^{\prime} \pi\right)$ events used to determine undetected pion contribution. This is included in the statistical uncertainties shown on histograms.

(c) Statistical uncertainty due to rotating $\left(e, e^{\prime} \pi\right)$ events to determine undetected pion contribution. We rotate $\left(e, e^{\prime} \pi\right)$ events enough times to reduce this uncertainty below $1 \%$.

(d) Systematic uncertainty due to imperfect description of the geometrical acceptance.

(e) Systematic uncertainty due to photon identification cut (missing photons and neutron contamination).

\subsubsection{STATISTICAL UNCERTAINTY DUE TO THE NUMBER OF ROTA- TIONS}

The statistical uncertainty due to the number of rotations contributes to the uncertainty of the obtained weights used for subtraction for undetected hadrons. This can be described by the uncertainty of probability in binomial distribution given by

$$
\begin{gathered}
\sigma=\frac{n}{N} \sqrt{\frac{1}{n}-\frac{1}{N}} \\
\lim _{n \rightarrow 0} \sigma=\frac{\sqrt{n}}{N} \\
\lim _{n \rightarrow N} \sigma=\frac{\sqrt{N-n}}{N}
\end{gathered}
$$

where $N$ is the total number of trials and $n$ is the number of successes, and is kept small with sufficient number of rotation (is not included in uncertainty calculation). 


\subsubsection{SYSTEMATIC UNCERTAINTY DUE TO THE ANGULAR DEPEN- DENCE OF THE PION CROSS SECTION}

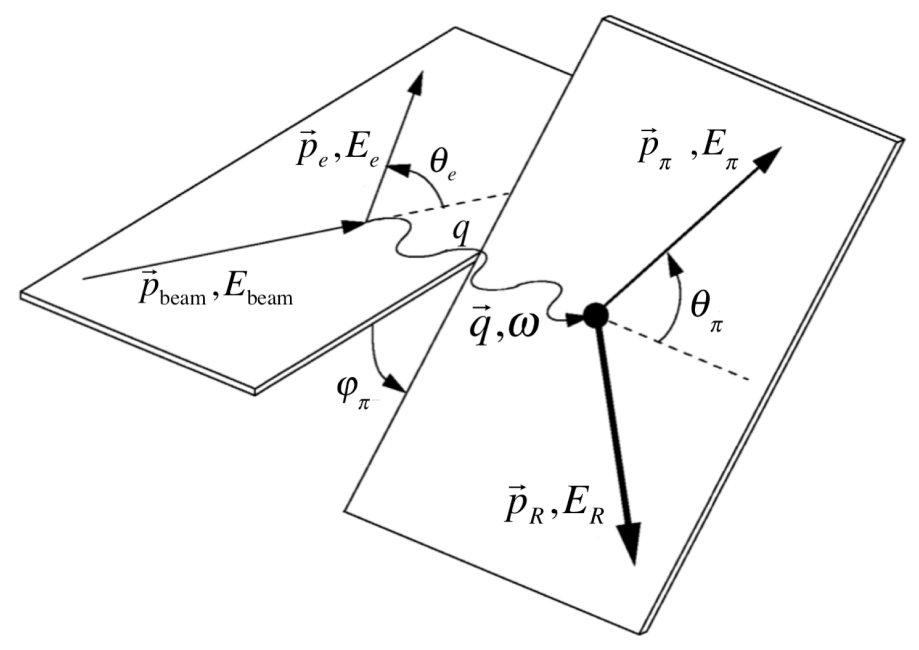

FIG. 183: The scheme of single pion electro-production.

We have studied the systematic uncertainty due to the dependence of the pion electroproduction cross section on the $\phi_{\pi}$ angle between the leptonic $\left(\vec{p}_{e}, \vec{p}_{e^{\prime}}\right)$ and hadronic $\left(\vec{q}, \vec{p}_{\pi}\right)$ planes (see Fig. 183), which we ignored in the subtraction of undetected one pion events in the inclusive scattering analysis. We assumed that the rotation around the three momentum transfer $\vec{q}$ does not change the cross section. $\vec{p}$ is the three momenta of the corresponding particles. We see below that this dependence has a small effect on the reconstructed energy spectra.

The cross section for unpolarized pion electroproduction on a single nucleon can be written [66];

$$
\frac{d^{4} \sigma}{d W d Q^{2} d \Omega_{\pi}^{*}}=J \Gamma_{\nu} \frac{d^{2} \sigma}{d \Omega_{\pi}^{*}}
$$

where $W=\sqrt{M^{2}+2 M \nu-Q^{2}}$ is the invariant mass of the virtual photon-nucleon system, $Q^{2}, \nu$ are the four-momentum transfer and the energy transfer and $M$ is the nucleon mass, the Jacobian $J=\partial\left(Q^{2}, W\right) / \partial\left(E^{\prime}, \cos \theta_{e}, \phi_{e}\right)$ relates the differential volume element $d Q^{2} d W$ to the measured electron kinematics $d E_{f} d \cos \theta_{e} d \phi_{e}$, where $\theta_{e}$ and $\phi_{e}$ are the scattering and azimuthal angles of the electron, $E_{f}$ and $E_{i}$ are the initial and final energies of the electron and $\Gamma_{\nu}$ virtual photon flux is given by the following expression;

$$
\Gamma_{\nu}=\frac{\alpha}{2 \pi^{2}} \frac{E_{f}}{E_{i}} \frac{k_{\gamma}}{Q^{2}} \frac{1}{1-\epsilon}
$$


with $\alpha$ being the fine structure constant, $k_{\gamma}$ being the "photon equivalent energy";

$$
k_{\gamma}=\frac{W^{2}-M^{2}}{2 M}
$$

and the virtual photon polarization is the following;

$$
\epsilon=\frac{1}{1+2\left(1+\frac{\nu^{2}}{Q^{2}} \tan ^{2} \frac{\theta_{e}}{2}\right)}
$$

The schematic view of the single pion production is shown in Fig. 183. In the single photon exchange approximation the virtual photon cross section $\frac{d \sigma}{d \Omega_{\pi}^{*}}$ for exclusive $\pi^{0} p$ electroproduction can be written in terms of experimental exclusive structure functions $\sigma_{\mathrm{T}}+\epsilon \sigma_{\mathrm{L}}, \sigma_{\mathrm{LT}}$ and $\sigma_{\mathrm{TT}}$ as shown in Eq. 77 [67]. Each of these functions depends implicitly on $\left(W, Q^{2}, \theta_{\pi}\right)$ and is described by different products of reaction amplitudes and their complex conjugate values.

$$
\begin{gathered}
\frac{d \sigma}{d \Omega_{\pi}^{*}}\left(W, Q^{2}, \theta_{\pi}, \phi_{\pi}\right)=A+B \cos \phi_{\pi}+C \cos 2 \phi_{\pi} \\
A=\left(\sigma_{\mathrm{T}}+\epsilon \sigma_{\mathrm{L}}\right) \frac{p_{\pi}^{*}}{k_{\gamma}^{*}} \\
B=\sigma_{\mathrm{LT}} \frac{p_{\pi}^{*}}{k_{\gamma}^{*}} \sin \theta_{\pi} \sqrt{2 \epsilon(\epsilon+1)} \\
C=\sigma_{\mathrm{TT}} \frac{p_{\pi}^{*}}{k_{\gamma}^{*}} \sin ^{2} \theta_{\pi} \epsilon
\end{gathered}
$$

where $p_{\pi}^{*}, \theta_{\pi}$ and $\phi_{\pi}$ are the magnitude of the three momentum, polar and azimuthal angles of the $\pi^{0}$ in the CM-frame, and $k_{\gamma}^{*}=k_{\gamma} M / W . \phi_{\pi}$ is shown in Fig. 183 and is the azimuthal angle between the hadronic and leptonic planes and $\theta_{\pi}$ is the angle between the direction of the pion and the virtual photon. These expressions are taken from $\pi^{0} p$ electroproduction studies in [67], but they can also be used for charged pion production studies, like in our case.

When subtracting for undetected one pion events in inclusive analysis we have assumed that the second and third terms on the right side of the Eq. 77 are negligible compared to the first term, and so ignored the dependence of the cross section on $\phi_{\pi}$. To check if this is valid we have estimated the number of undetected one charged pion events with and without the $\phi_{\pi}$ dependence of the cross section, and have found that both lead to similar results.

For this study we have used the values of exclusive structure functions $\sigma_{\mathrm{T}}+\epsilon \sigma_{\mathrm{L}}, \sigma_{\mathrm{TT}}$ and $\sigma_{\mathrm{LT}}$ from [67] shown for $\cos \theta_{\pi}=0.1$ and $0.4 \leq Q^{2} \leq 1 \mathrm{GeV}^{2}$. We have used the largest 


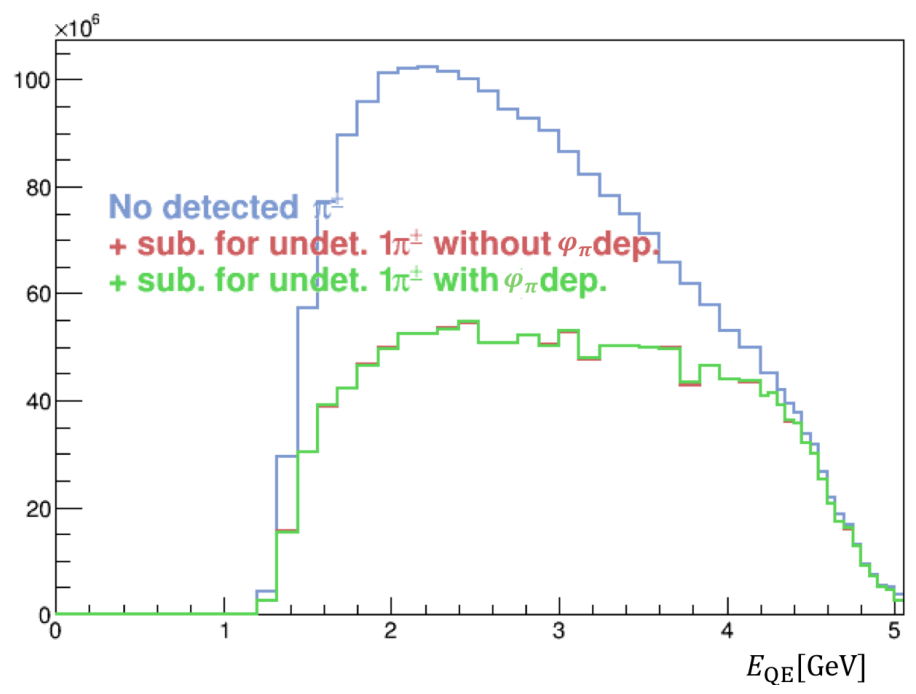

FIG. 184: $E_{\mathrm{QE}}$ zero pi spectrum for $A\left(e, e^{\prime}\right)$ subtracted for undetected one charged pion events with (green) and without (red) including the $\phi_{\pi}$ dependence of the cross section at $4.4 \mathrm{GeV}$ for ${ }^{56} \mathrm{Fe}$ target.

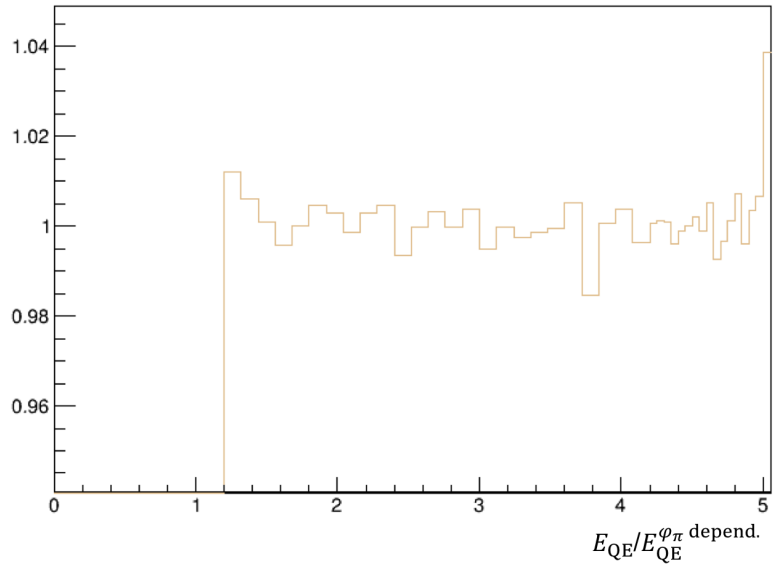

FIG. 185: The ratio of the $E_{\mathrm{QE}} 0$ pi spectrum for $A\left(e, e^{\prime}\right)$ subtracted for undetected 1 charged pion events without accounting for $\phi_{\pi}$ dependence of the cross section over the one subtracted for undetected 1 charged pion events with accounting for $\phi_{\pi}$ dependence of the cross section at $4.4 \mathrm{GeV}$ for ${ }^{56} \mathrm{Fe}$ target.

provided absolute values $\sigma_{\mathrm{T}}+\epsilon \sigma_{\mathrm{L}}=30 \mu \mathrm{b}, \sigma_{\mathrm{TT}}=-10 \mu \mathrm{b}$ and $\sigma_{\mathrm{LT}}=-2 \mu \mathrm{b}$ corresponding to $Q^{2}=0.45 \mathrm{GeV}^{2}$. 
In the $A\left(e, e^{\prime}\right)$ inclusive analysis, we looked at the events with one detected charged pion and rotated the pion many times around $\vec{q}$ to find the weight for undetected pion events, which is given by the following expression;

$$
W=\frac{\sum_{i=1}^{N_{\text {Undet }}} 1}{\sum_{i=1}^{N_{\text {Det }}} 1}
$$

if we ignore the dependence on $\phi_{\pi}$ (ignore the contributions of the terms with $B$ and $C$ ), and is given by;

$$
W=\frac{\sum_{i=1}^{N_{\text {Undet }}} 1+(B / A) \cos \phi_{\pi}+(C / A) \cos 2 \phi_{\pi}}{\sum_{i=1}^{N_{\text {Det }}} 1+(B / A) \cos \phi_{\pi}+(C / A) \cos 2 \phi_{\pi}}
$$

if we take those into account, where $N_{\text {Det }}$ and $N_{\text {Undet }}$ are the number of rotations that led to the pion getting detected and undetected correspondingly. We have then compared the $E_{\mathrm{QE}}$ distributions of $A\left(e, e^{\prime}\right) 0 \pi$ events, subtracted for undetected pions in Fig. 184. We can see that the difference between the two distributions is small. The ratio of these two spectra is shown in Fig. 185 and suggests that the difference is $<1 \%$. This means that our assumption is valid. We have done the comparison at $4.4 \mathrm{GeV}$ for ${ }^{56} \mathrm{Fe}$ as in this case the effect of the subtraction for the undetected pions is the biggest and any difference between the two distributions should be largest.

\subsubsection{SYSTEMATIC UNCERTAINTY DUE TO PHOTON IDENTIFICATION CUT}

In order to separate photons from neutrons, we cut on the neutral particle velocity. The velocity distribution of neutral particles has a peak at $\beta=1$ corresponding to photons and another at lower velocity corresponding to neutrons. To select photons we cut $2 \sigma$ to the left of the photon peak and select the region above it. At $1.161 \mathrm{GeV}$ analysis we cut $3 \sigma$ away from the photon peak as the neutron and photon peaks are well separated at this energy. The photon selection cuts for all the targets and beam energies are listed in Table.8.

We have studied the sensitivity of our results on the location of this cut. We have obtained the reconstructed energy spectra by increasing the original cut by 0.01 which is about $0.25 \sigma$ change. We have then obtained the same results by decreasing the original cut by 0.01 . The systematic uncertainty due to cut location is simply the RMS of these two results and the 

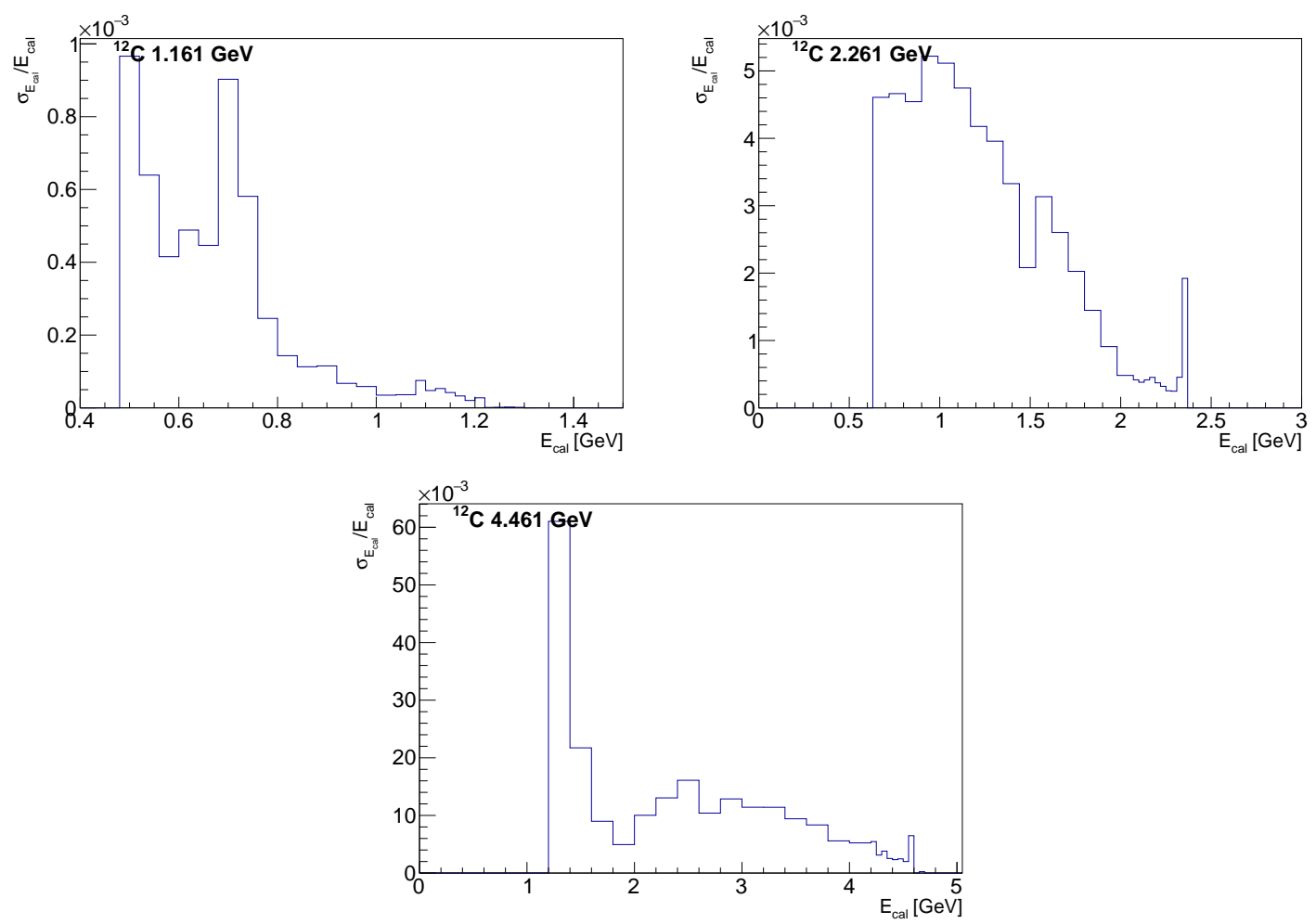

FIG. 186: The fractional systematic uncertainty due to photon PID cut vs $E_{\text {calor }}\left(e, e^{\prime} p\right)$ for ${ }^{12} \mathrm{C}$ at different energies.

result obtained using the original cut (thus we have three different results). To calculate the RMS for each energy bin in the reconstructed energy spectrum, we have calculated the mean bin content for each energy bin in the reconstructed energy spectrum. The mean bin content for bin $i$ is equal to $x_{\text {mean }}^{i}=\left(\sum_{k=1}^{3} x_{k}^{i}\right) / 3$, where $x_{k}^{i}$ is the bin content of the $i$ th bin of the $k$ th result. We have then calculated the RMS for each energy bin using the following formula:

$$
x_{\mathrm{RMS}}^{i}=\sqrt{\frac{\sum_{k=1}^{3}\left(x_{k}^{i}-x_{\text {mean }}^{i}\right)^{2}}{3}}
$$

The ratios of $\sigma_{E_{\text {cal }}}$ the photon ID systematic uncertainty distributions to the $E_{\text {cal }}$ reconstructed energy spectra are shown in Fig. 186. The systematic uncertainty due to photon identification is less than $0.1 \%$ at $1.1 \mathrm{GeV}$, less than $0.5 \%$ at $2.2 \mathrm{GeV}$ and less than $2 \%$ at $4.4 \mathrm{GeV}$.

At $2.2 \mathrm{GeV}$ and $4.4 \mathrm{GeV}$ analysis we include $95 \%$ of the photons, while at $1.1 \mathrm{GeV}$ we include $99.7 \%$ of the photons. Unlike at $1.1 \mathrm{GeV}$, there is some overlap between the neutron 
and photon peaks in the neutral particle velocity distribution at $2.2 \mathrm{GeV}$ and $4.4 \mathrm{GeV}$. Because of this there is some neutron contamination in the selected photon sample. To estimate the neutron contamination, we have looked at the velocity $(\beta)$ distribution of neutral particles that have deposited energy only in the outer layer of EC. This should include mainly neutrons, as photons should also have an associated energy deposition in the inner layer of EC. We then scale this distribution to match the left shoulder of the velocity distribution of all neutral particles as shown in Fig. 187. There are still some photons present in the green
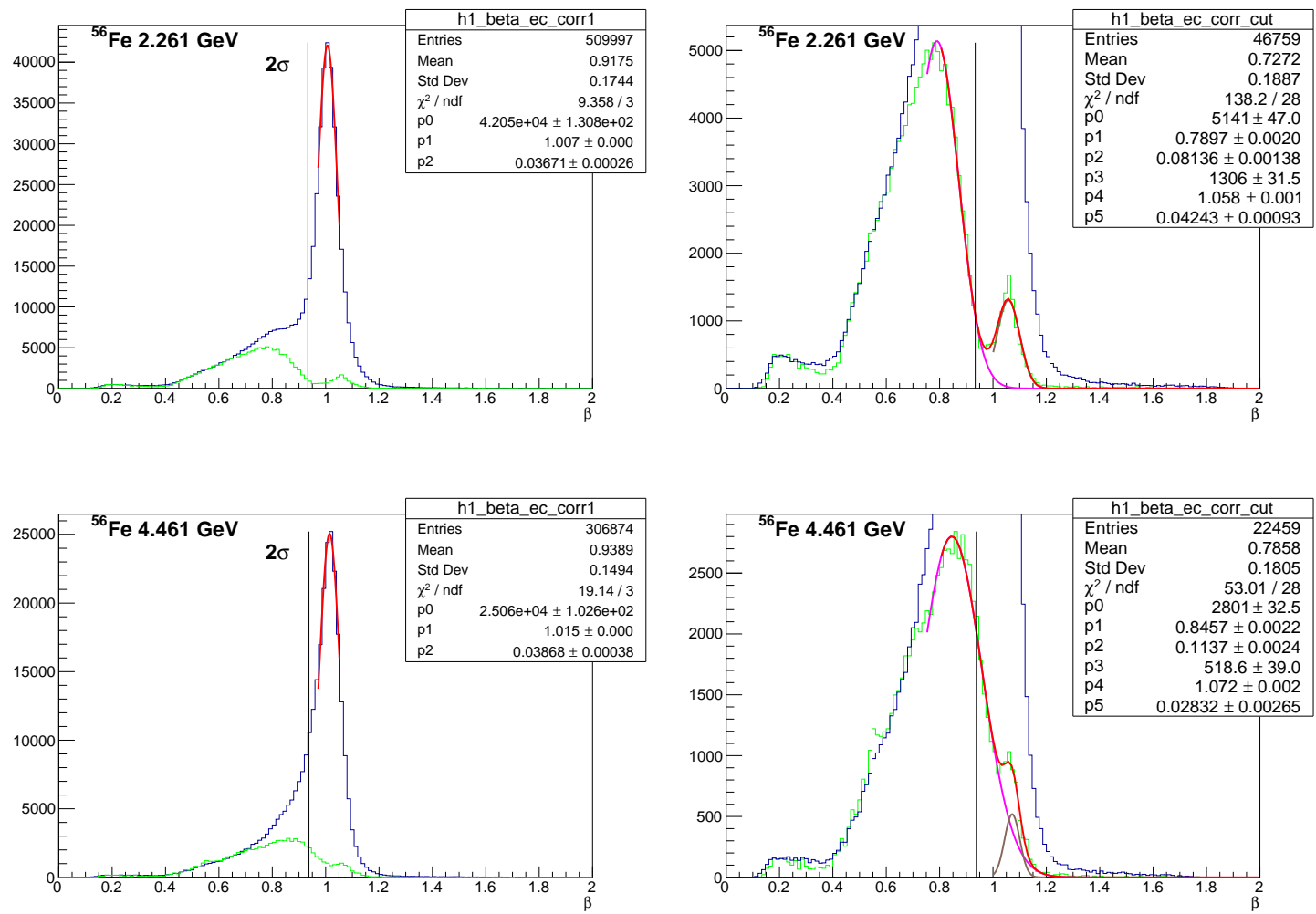

FIG. 187: The $\beta$ distribution of all neutral particles (blue) and the ones that have deposited energy only in outer layer of EC (green) for $2.2 \mathrm{GeV}$ and $4.4 \mathrm{GeV}$ analysis for ${ }^{56} \mathrm{Fe}$ target. The zoomed version of the same plots are shown on the right side. The right shoulder of the green distribution is well described by a fit function that is a sum of two Gaussian function shown in pink and brown. The vertical line represents the photon selection cut.

distribution, so we have used a fitting function that is a sum of two Gaussian functions to fit the right shoulder of the distribution that extends to the right side of the photon selection cut. To estimate the neutron contamination in this region we divide the integral of the first Gaussian function shown in pink over the integral of the blue distribution in this region. 
We have found that the neutron contamination in the selected photon sample is $0.94 \%$ at $2.2 \mathrm{GeV}$ and is $6.1 \%$ at $4.4 \mathrm{GeV}$ for the ${ }^{56} \mathrm{Fe}$ target. The contamination for lighter targets should be smaller. Because we are excluding 2.5\% percent of photons by cutting $2 \sigma$ away from the photon peak, we select $2.5 \%-1 \%=1.5 \%$ less neutral particles at $2.2 \mathrm{GeV}$ analysis and $6 \%-2.5 \%=3.5 \%$ more neutral particles at $4.4 \mathrm{GeV}$.

In addition to the $\beta$ cut, we require the energy of the photons to be greater than $0.3 \mathrm{GeV}$. This additional PID cut applied on photon reduces the estimated photon contamination. Therefore we add a $3 \%$ systematic uncertainty to the photon subtraction.

\subsubsection{EFFECT OF FIDUCIAL CUTS ON UNDETECTED PARTICLE SUB- TRACTION}

If we could obtain the ideal fiducial cuts that describe perfectly the geometrical acceptance with flat detection efficiency for different particles, our energy reconstruction results would be independent of how tight or wide the geometrical acceptance is. This is however hard to achieve in real analysis. We have estimated the sensitivity of our results to the change in geometrical acceptance. We have obtained the reconstructed energy spectra after changing the original fiducial cuts for charged pions and photons in different CLAS sectors. We have moved the left and right sides of $\theta$ vs $\phi$ distribution outline inwards in $\phi$ by $3^{\circ}$. This decreases the geometrical acceptance in each sector by $6^{\circ}$ in $\phi$. This decreases the number of detected pions and increases the number of undetected pions. We compared the reconstructed energy spectra obtained with original fiducial cuts to the ones obtained with modified tighter fiducial cuts. The plots for $E_{\text {calor }}$ energy reconstruction methods are shown on the left side of Fig. 188.

The systematic uncertainty due to the change in geometrical acceptance is the RMS of these two results obtained using the original and modified geometrical acceptances. The systematic uncertainty is calculated the same way as in the previous section.

The ratio of $\sigma_{E_{\text {cal }}}$ systematic uncertainty distributions over the $E_{\text {cal }}$ reconstructed energy spectra are shown on the right side of Fig. 188. This uncertainty is the biggest at $4.4 \mathrm{GeV}$ and is less than $4 \%$. At $2.2 \mathrm{GeV}$ it is less than $1.2 \%$ and at $1.1 \mathrm{GeV}$ it is less than $0.8 \%$.

The statistical uncertainties due to total available statistics and $\left(e, e^{\prime} \pi\right)$ statistics are shown by error bars on the reconstructed energy distributions. The uncertainties from different sources are summarized in Table 21. 

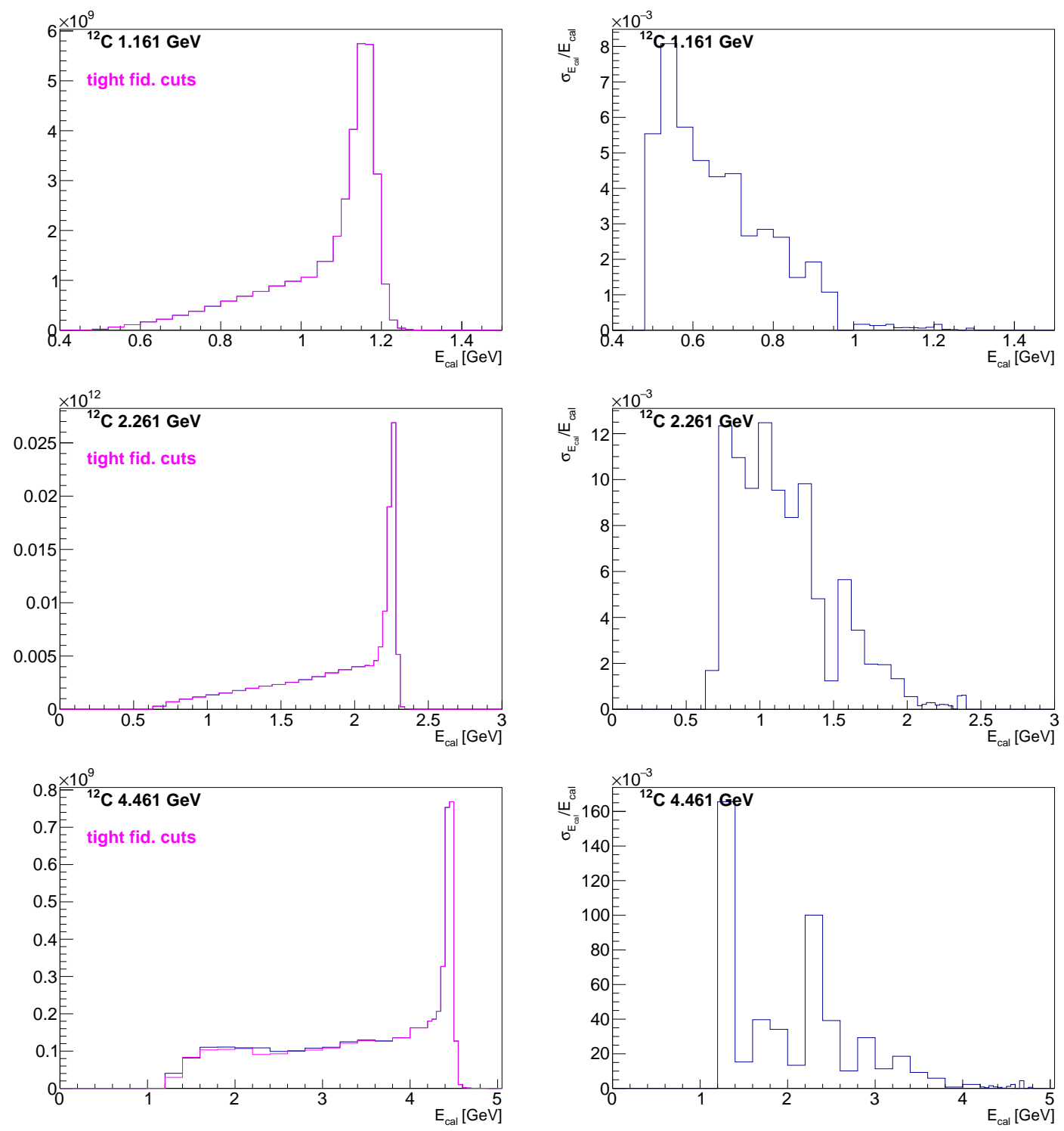

FIG. 188: The $E_{\text {calor }}$ subtraction uncertainty due to fiducial cuts. (left) $E_{\text {calor }}\left(e, e^{\prime} p\right)$ spectra with original (blue) and modified (pink) geometrical acceptance for ${ }^{12} \mathrm{C}$ at different energies. The different fiducial cuts are applied to the subtracted particles (pions and photons) only; (right) the relative difference between the two results. 


\begin{tabular}{|l|c|c|c|c|}
\hline \multirow{2}{*}{$\mathrm{E}[\mathrm{GeV}]$} & \multicolumn{4}{|c|}{ Uncertainty due to } \\
\cline { 2 - 5 } & $\phi$ dep. & $\#\left(e, e^{\prime} \pi\right)$ rot. & Imperf. accept. & $\gamma$ ID cut \\
\hline 1.1 & $1 \%$ & $1 \%$ & $0.8 \%$ & $0.1 \%$ \\
\hline 2.2 & $1 \%$ & $1 \%$ & $1.2 \%$ & $0.5 \%$ \\
\hline 4.4 & $1 \%$ & $1 \%$ & $4 \%$ & $2 \%$ \\
\hline
\end{tabular}

TABLE 21: The summary of statistical and systematic uncertainty sources in our analysis at different beam energies. " $\phi$ dep." corresponds to uncertainty due to the angular dependence of the pion and photon cross sections, "\# $\left(e, e^{\prime} \pi\right)$ rot." denotes statistical uncertainty due to rotation of $\left(e, e^{\prime} \pi\right)$ events to determine the undetected pion contribution, "imperf. accept." denotes the systematic uncertainty due to imperfect description of the geometrical acceptance which is used to subtract undetected pions, and $\gamma$ ID cut corresponds to systematic uncertainty due to the photon identification cut. 


\section{CHAPTER 6}

\section{RESULTS AND DISCUSSIONS}

\subsection{SUMMARY OF DATA ANALYSIS RESULTS}

The neutrino beams from accelerators are not mono-energetic. Accelerator-based oscillation experiments detect neutrinos by measuring charged particles (electrons, muons, protons, etc) produced by the weak interaction of neutrinos with atomic nuclei. The extraction of neutrino oscillation parameters relies on reconstructing the incident energy of the interacting neutrino from the measured momenta and angles of these charged particles. This requires a detailed understanding of the neutrino-nucleus interaction cross-section for various interaction channels, different atomic nuclei, and a wide range of neutrino energies.

However, none of these energy reconstruction techniques have been tested experimentally using beams of known energy. We exploited the similarity of electron-nucleus and neutrinonucleus interactions to measure how well we can reconstruct the known incident electron energy from particles detected in electron-nucleus collisions in a large acceptance detector. We found that contrary to expectation, only a small fraction of events are reconstructed to the correct incident energy.

In this analysis we present tests of energy feed-down spectra for electron scattering from nuclei. Electrons are also leptons and interact similarly to neutrinos. Electrons interact with nuclei via a vector current and neutrinos interact via a vector plus axial-vector current. The nuclear ground state is the same in both cases and many of the nuclear reaction effects are similar.

We measured electron scattering from ${ }^{3} \mathrm{He},{ }^{4} \mathrm{He}, \mathrm{C}$, and $\mathrm{Fe}$ at energies of 1.1, 2.2 and $4.4 \mathrm{GeV}$, detecting the scattered and knocked out particles over a wide range of angles and momenta in the CLAS6 spectrometer at the Thomas Jefferson National Accelerator Facility (Jefferson Lab). These incident energies are comparable to typical accelerator-based neutrino maximum incident energies from 1 to about $6 \mathrm{GeV}$. The carbon is similar to the scintillator in experiments such as Miner $\nu$ a and to the oxygen in water-based Cherenkov detectors such as Kamiokande. The iron is similar to the argon in the liquid argon time projection chambers of MicroBoone and DUNE. 

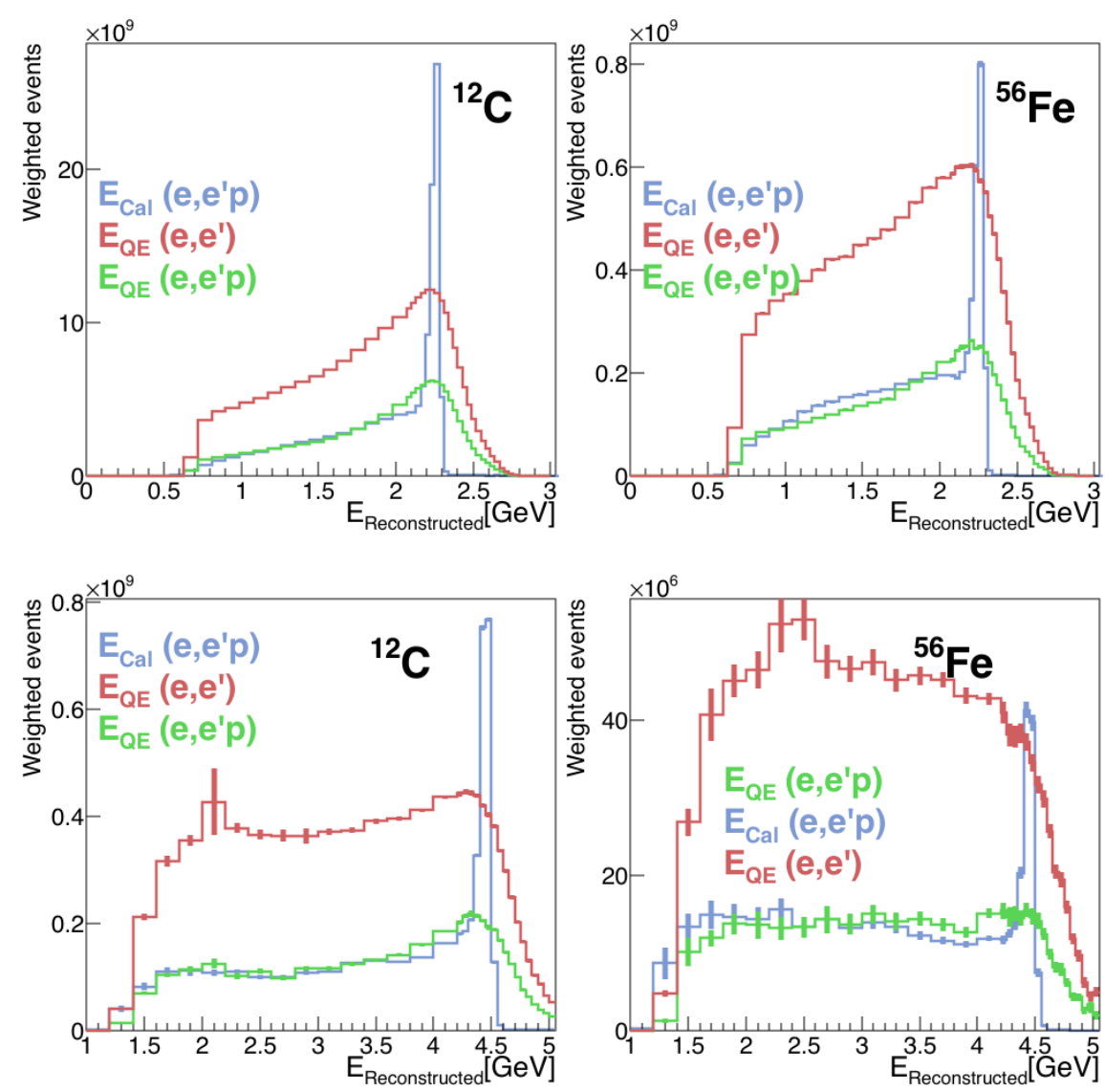

FIG. 189: $E_{\mathrm{QE}}$ distributions of $A\left(e, e^{\prime}\right)$ (red) and $A\left(e, e^{\prime} p\right)$ (green) $0 \pi$ spectrum and $E_{\text {calor }}$ distribution of $A\left(e, e^{\prime} p\right)$ (blue) $0 \pi$ spectrum after subtraction for undetected pions and undetected protons for ${ }^{12} \mathrm{C}$ and ${ }^{56} \mathrm{Fe}$ targets at 2.2 (top plots) and $4.4 \mathrm{GeV}$ (bottom plots).

We analyzed our electron-scattering data similarly to many neutrino analyses, by requiring that there are zero pions and photons (from $\pi^{0}$ decay) in the final state. Because the CLAS6 geometrical acceptance is incomplete (50\%), we also estimated the contribution of events with undetected pions or photons and subtracted it from our data set. This gave us an $\left(e, e^{\prime}\right) X 0 \pi$ data set where there were any number of detected or undetected protons and neutrons. Similarly, we looked at events with one detected proton and no charged pions. We estimated the contribution from events with undetected pions or photons or extra protons and subtracted it from our data set. This gave us an $\left(e, e^{\prime} p\right) X 1 p 0 \pi$ data set where there were any number of detected or undetected neutrons. 


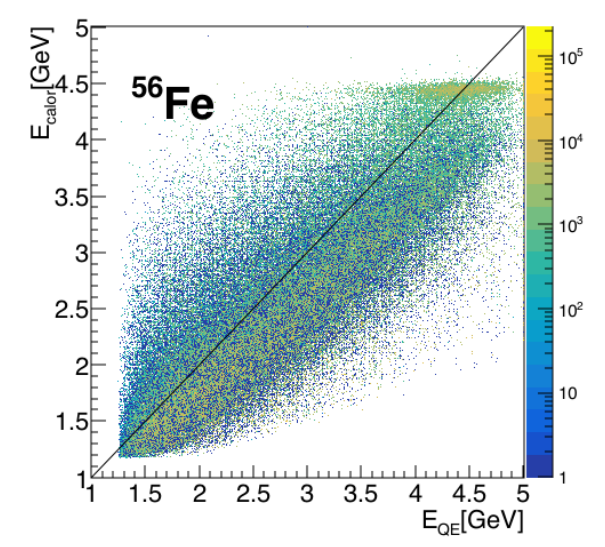

FIG. 190: $E_{\text {calor }}$ versus $E_{\mathrm{QE}}$ distributions of $A\left(e, e^{\prime} p\right) 0 \pi$ spectrum for ${ }^{56} \mathrm{Fe}$ at $4.4 \mathrm{GeV}$.

There are two general approaches for reconstructing $E_{\nu}$, first using just the outgoing lepton and assuming that it scattered quasielastically from a stationary nucleon in the nucleus:

$$
E_{\mathrm{QE}}=\frac{2 M \epsilon-m_{1}^{2}+2 M E_{1}}{2\left(M-E_{1}+\left|k_{1}\right| \cos \theta_{1}\right)}
$$

where $M$ is the nucleon mass, $\epsilon$ is the single-nucleon separation energy, $m_{1}$ is the mass of the outgoing lepton, $k_{1}$ is its momentum, $E_{1}$ is its energy and $\theta_{1}$ is the angle between the outgoing lepton and the direction of the neutrino beam. Second, by summing the energies of all detected particles:

$$
E_{\text {calor }}=E_{1}+T_{\mathrm{p}}+\epsilon
$$

where $T_{\mathrm{p}}$ is the kinetic energy of the nucleon. The first method is typically used for Cherenkov detectors and the second is used for ionization and scintillation detectors.

The reconstructed incident electron energies for 2.2 and $4.4 \mathrm{GeV}$ electrons incident on $\mathrm{C}$ and Fe are shown in Fig. 189. The calorimetric reconstruction $E_{\text {calor }}$ gives a very narrow peak at the actual beam energy for $\left(e, e^{\prime} p\right)$ events, but there is a very large "tail" of events which reconstruct to much lower beam energies. This tail increases significantly with both energy and target mass $A$. The quasielastic reconstruction gives a much broader peak at the actual beam energy (doppler broadened by the fermi motion of the nucleons), but the same tail of lower energy events for the same $\left(e, e^{\prime} p\right)$ data set. The $\left(e, e^{\prime}\right)$ data set, with any number of detected or undetected protons and neutrons, has a larger tail than the $\left(e, e^{\prime} p\right)$ data set, with only one proton.

While the peak of the reconstructed energy distribution is broader for the quasielastic reconstruction method than for the calorimetric reconstruction method, the low energy tails 


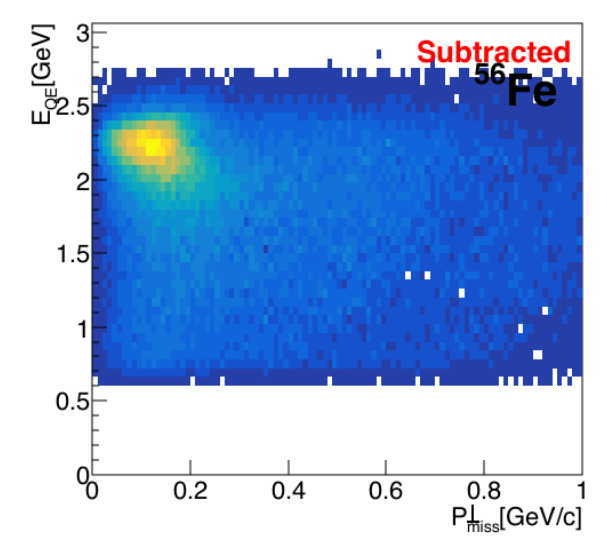

FIG. 191: Reconstructed energy $E_{\mathrm{QE}}$ as a function of $P_{\text {miss }}^{\perp}$ for ${ }^{56} \mathrm{Fe}$ at $2.2 \mathrm{GeV}$.

are almost identical. The two energy reconstruction methods give remarkably similar results and therefore consistency between the two methods does not indicate accuracy, see Fig. 190.

We next considered the missing transverse momentum of the reaction $P_{\text {miss }}^{\perp}$ that is the sum of the electron and proton perpendicular (with respect to beam direction) momenta. We expect that purely quasielastic events where the electron scattered from a bound proton and both the electron and proton were detected will have small $P_{\text {miss }}^{\perp}$ (i.e., consistent with a single-nucleon momemtum distribution) and will reconstruct to the correct incident energy, but that events where other nucleons were knocked out of the nucleus will have larger $P_{\text {miss }}^{\perp}$ and will not reconstruct to the correct incident energy. Fig. 191 shows reconstructed energy distribution as a function of $P_{\text {miss }}^{\perp}$. There is a large blob for events with small $P_{\text {miss }}^{\perp}$ which reconstruct to the correct incident energy and a relatively flat background elsewhere. Thus neutrino experiments should be able to dramatically reduce the background of incorrectenergy events by cutting on $P_{\text {miss }}^{\perp}$.

However this energy reconstruction is not a problem if it is well modeled by neutrino event generator.

We next compare data and event generator results. 


\subsection{GENIE TO DATA COMPARISONS}

Because the energy reconstruction is not perfect, it is simulated using neutrino event generators such as GENIE to model the neutrino-nucleus interactions and using detector simulations to model the particle detection. The resulting reconstructed energy spectrum for a particular energy incident neutrino is referred to as the "energy feed-down spectrum". As long as the simulated feed-down spectrum matches reality, the neutrino oscillation parameters can be accurately reconstructed [4].

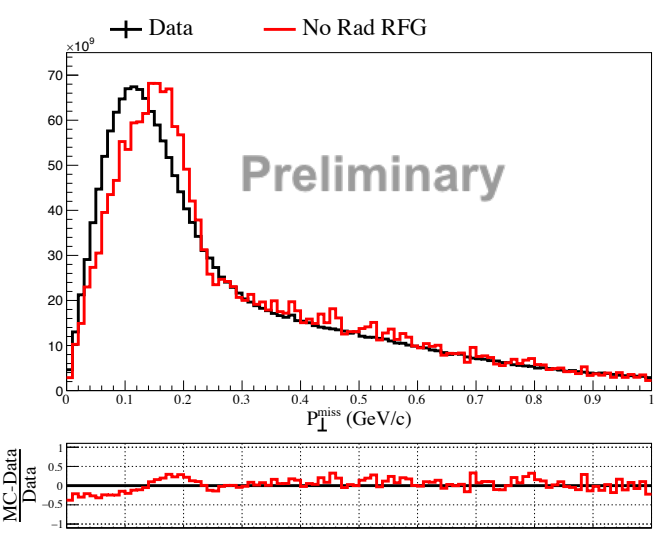

(a) $2.2 \mathrm{GeV}$

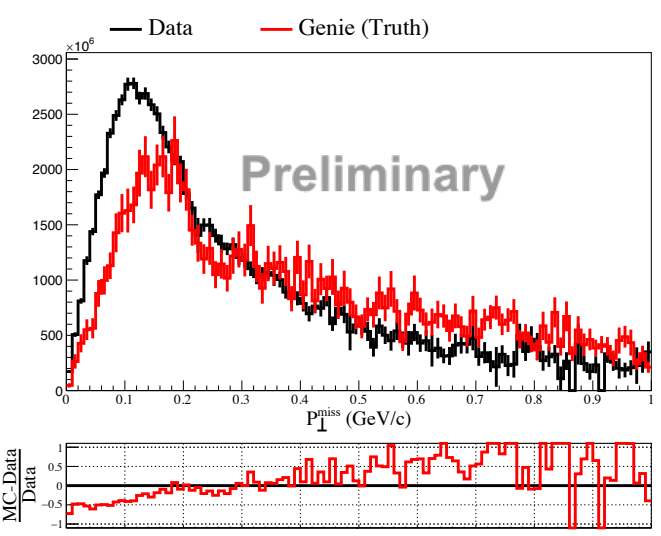

(b) $4.4 \mathrm{GeV}$

FIG. 192: The distribution of $P_{\text {miss }}^{\perp}$ at $2.2 \mathrm{GeV}$ and $4.4 \mathrm{GeV}$ from data (in black) and GENIE (in red) for ${ }^{12} \mathrm{C}$.

GENIE is one of the most popular neutrino event generators used in neutrino experiments, especially those carried out at Fermilab, US. It is Root-based, has been developed entirely in $\mathrm{C}++$ and was first released in 2007. The most up to date version is v3.0.6.

We have used the electron version of the latest release of GENIE (e-GENIE), that is updated to be consistent with the neutrino version and to better describe existing inclusive electron scattering data [68]. We generated events with e-GENIE and used the measured CLAS acceptance and resolution to determine which particles were detected and to smear their measured momenta. We then used the generated events to reconstruct the incident electron energy in the same way as the data.

We did not correct our data for radiative effects, since that would require an accurate knowledge of the electron-nucleus scattering cross section over a very wide range. Instead we are going to correct GENIE for initial electron radiation and veto events where the photon from final state radiation was detected in CLAS. Electron radiation is currently 

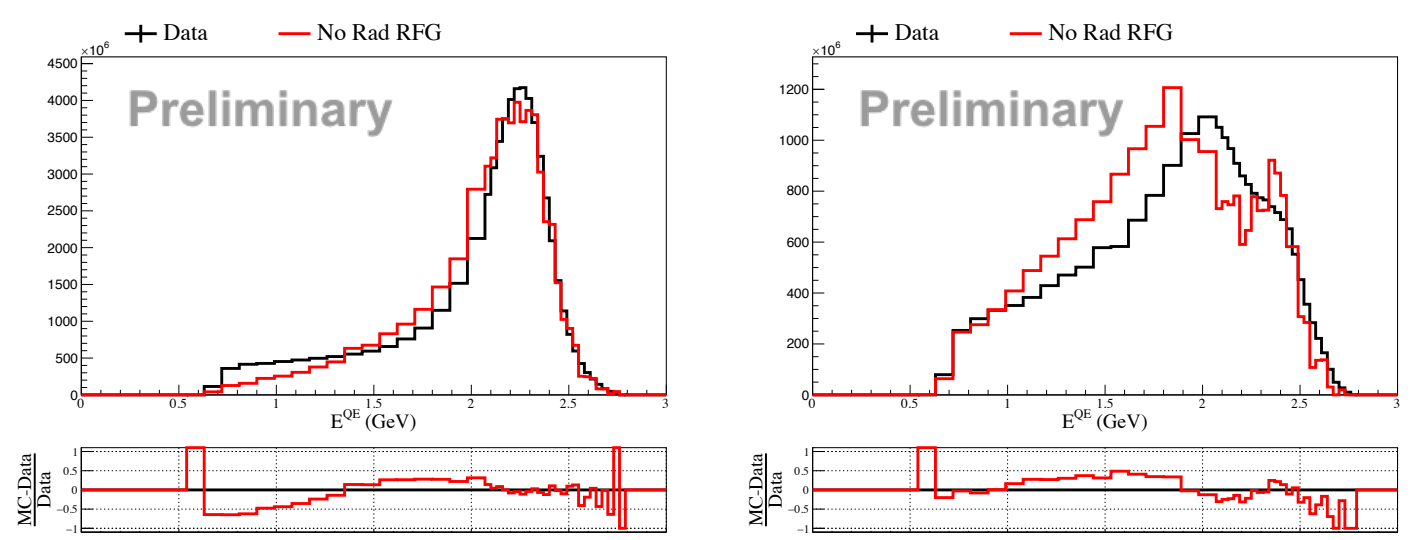

(a) $P_{\text {miss }}^{\perp}=0-200 \mathrm{MeV} / \mathrm{c}$

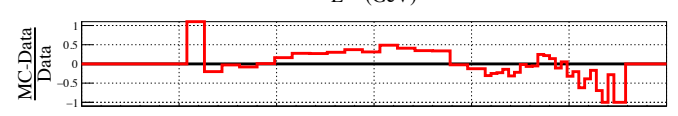

(b) $P_{\text {miss }}^{\perp}=200-400 \mathrm{MeV} / \mathrm{c}$

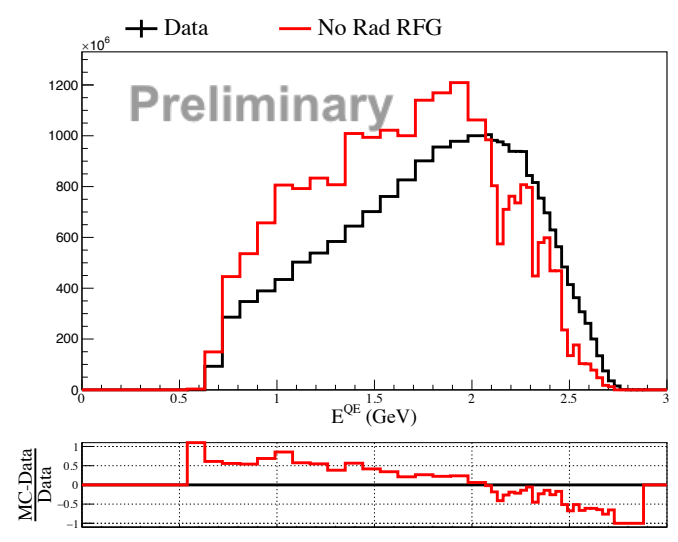

(c) $P_{\text {miss }}^{\perp}=400 \mathrm{MeV} / \mathrm{c}$ and higher

FIG. 193: The $E_{\mathrm{QE}}$ distributions of ${ }^{12} \mathrm{C}\left(e, e^{\prime} p\right) 0 \pi$ spectrum for different regions of $P_{\text {miss }}^{\perp}$ $(0-200 \mathrm{MeV} / \mathrm{c}, 200-400 \mathrm{MeV} / \mathrm{c}$ and $400 \mathrm{MeV} / \mathrm{c}$ and higher) at $2.2 \mathrm{GeV}$ from data (black) and GENIE (red).

being implemented in GENIE.

All the results from data shown here are subtracted for undetected pions and protons.

We apply the acceptance maps described earlier to the GENIE results, in order to account for CLAS efficiency and geometrical acceptance. For comparison we have normalized the plots from GENIE and data to the same integral.

Based on the charged particle momentum resolution given in [69], we have smeared the momenta of the electrons, charged pions and protons by $0.5 \%, 0.7 \%$ and $1 \%$ correspondingly. We did not smear the azimuthal and scattering angles. The exact smearing is not important as this is not a high-resolution experiment. 
All the plots have the following cuts $Q^{2}>0.8(\mathrm{GeV} / \mathrm{c})^{2}$ at $4.4 \mathrm{GeV}, Q^{2}>0.4(\mathrm{GeV} / \mathrm{c})^{2}$ at $2.2 \mathrm{GeV}$ and $W(e, p)<2 \mathrm{GeV} / \mathrm{c}^{2}$. The $W(e, p)<2 \mathrm{GeV} / \mathrm{c}^{2}$ cut on invariant mass mainly eliminates the contribution of deep inelastic scattering events. The $Q^{2}$ cut is made to make the simulation more efficient (simulate data faster).

Fig. 192 shows the $P_{\text {miss }}^{\perp}$ spectrum compared to the e-GENIE result for ${ }^{12} \mathrm{C}\left(e, e^{\prime} p\right)$ at $2.2 \mathrm{GeV}$ and $4.4 \mathrm{GeV}$. For both beam energies there is a discrepancy at low $P_{\text {miss }}^{\perp}$ where we should mostly have QE scattering events. e-GENIE reproduces well the proportion of events with large $P_{\text {miss }}^{\perp}$ at $2.2 \mathrm{GeV}$ but there is a discrepancy between the results from data and GENIE at $4.4 \mathrm{GeV}$.

The $E_{\mathrm{QE}}$ distributions for different regions of $P_{\mathrm{miss}}^{\perp}$ from data and GENIE for ${ }^{12} \mathrm{C}\left(e, e^{\prime} p\right)$ at $2.2 \mathrm{GeV}$ are shown in Fig. 193. The energy reconstruction works the best for $P_{\text {miss }}^{\perp}$ values from $0-200 \mathrm{MeV} / \mathrm{c}$ for both data and GENIE. However GENIE predicts better energy reconstruction with smaller tail than data. At higher values of $P_{\text {miss }}^{\perp}$ GENIE predicts a bigger background tail. Also the results from GENIE for $P_{\text {miss }}^{\perp}>200 \mathrm{MeV} / \mathrm{c}$ exhibit a double peak structure not present in data. This could be due to $\Delta$ resonance excitation processes, where the pion from the decay of $\Delta$ is absorbed leading to an emission of other nucleons. While the pion in this process can be off shell, it is possible that GENIE requires the pion to be on-shell, and the physical mass of the pion causes the gap in the reconstructed energy spectrum. This failure of the event generator to describe the reconstructed energy spectrum of the data could cause errors for unfolding the actual incident energy spectrum.

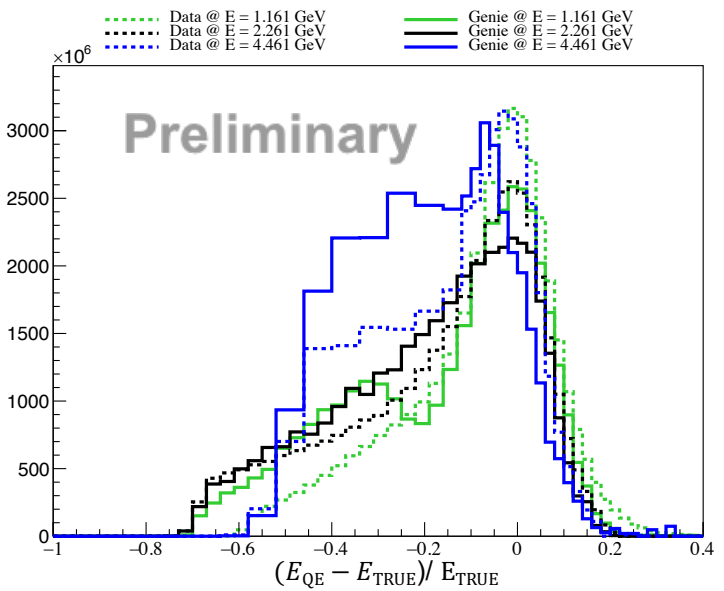

(a) ${ }^{12} \mathrm{C}\left(e, e^{\prime} p\right)$

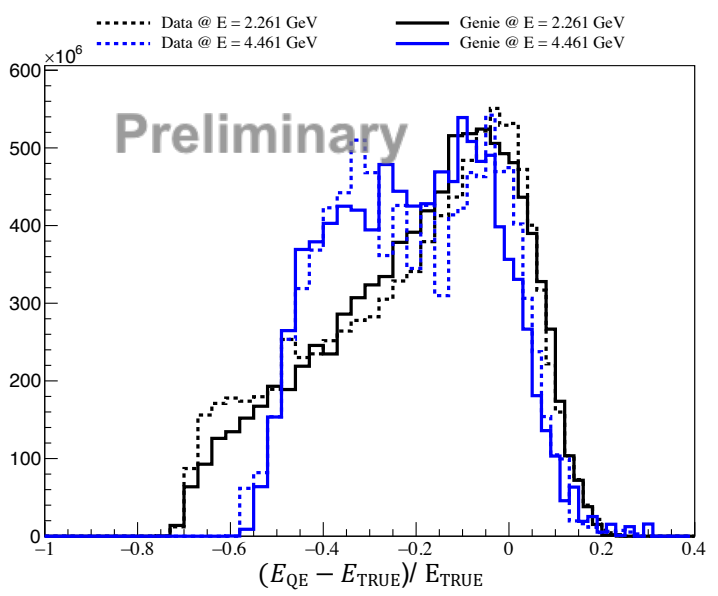

(b) ${ }^{56} \mathrm{Fe}\left(e, e^{\prime} p\right)$

FIG. 194: The energy feed-down spectra for $E_{\mathrm{QE}}$ method from data (points) and GENIE (line), for ${ }^{12} \mathrm{C}\left(e, e^{\prime} p\right)$ and ${ }^{56} \mathrm{Fe}\left(e, e^{\prime} p\right)$ at all beam energies. 


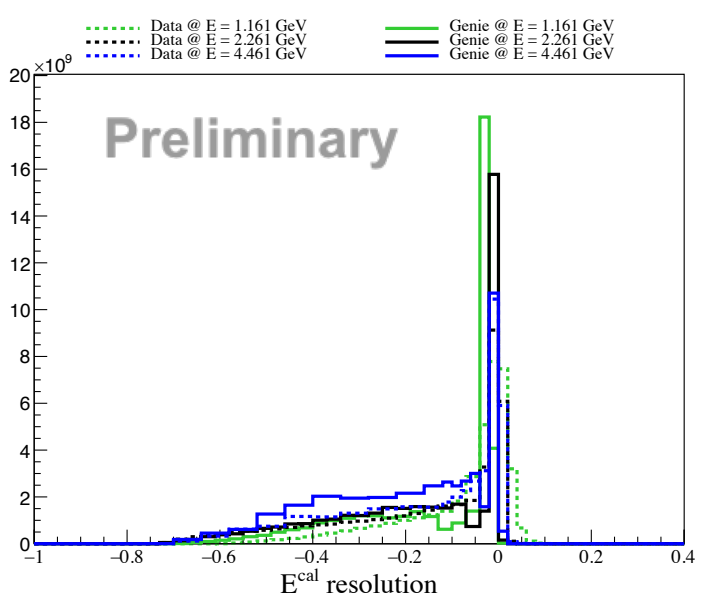

(a) ${ }^{12} \mathrm{C}\left(e, e^{\prime} p\right)$

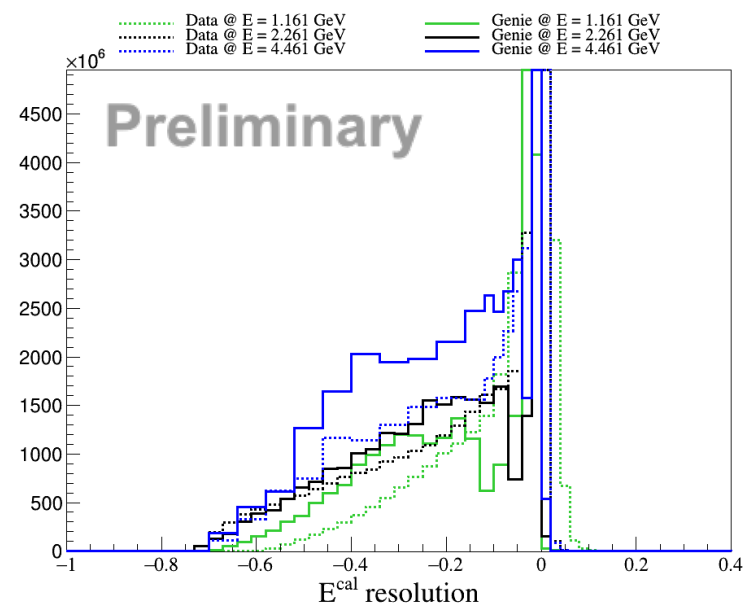

(c) ${ }^{12} \mathrm{C}\left(e, e^{\prime} p\right)$, zoomed

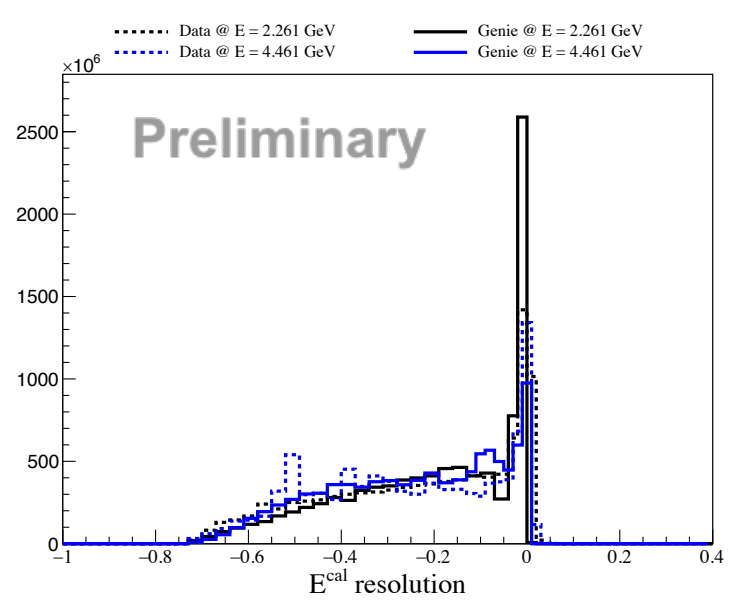

(b) ${ }^{56} \mathrm{Fe}\left(e, e^{\prime} p\right)$

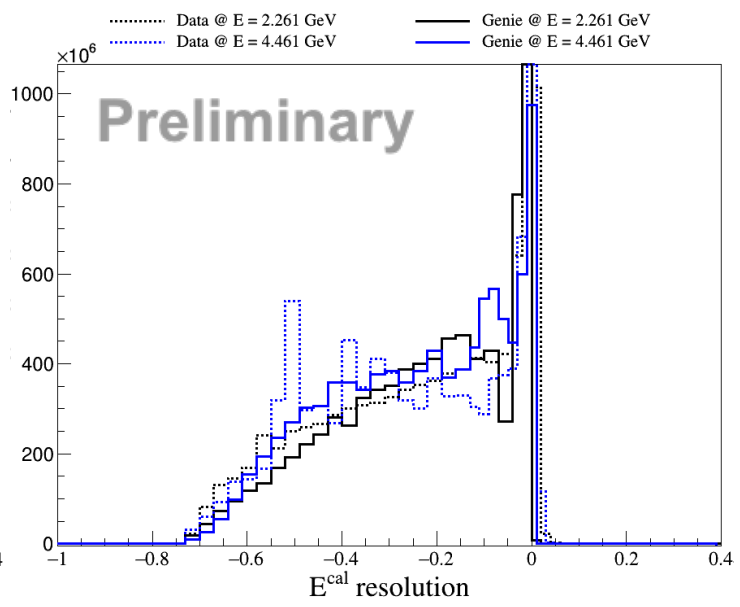

(d) ${ }^{56} \mathrm{Fe}\left(e, e^{\prime} p\right)$, zoomed

FIG. 195: The energy feed-down spectra for $E_{\text {calor }}$ method from data (points) and GENIE (line), for ${ }^{12} \mathrm{C}\left(e, e^{\prime} p\right)$ and ${ }^{56} \mathrm{Fe}\left(e, e^{\prime} p\right)$ at all beam energies.

We created energy feed-down spectra, $\left(E_{\mathrm{QE}}-E_{\mathrm{TRUE}}\right) / E_{\mathrm{TRUE}}$, to compare results at different beam energies. The energy feed-down spectra from data and GENIE for $E_{\mathrm{QE}}$ and $E_{\text {cal }}$ energy reconstruction methods are shown in Figs. 194 and 195. As we can see, for a particular target and beam energy, the energy feed-down spectra from GENIE and data disagree for ${ }^{12} \mathrm{C}$ and look surprisingly good for ${ }^{56} \mathrm{Fe}$. The energy feed down gets worse with increased beam energy and is also worse with increased atomic number for both data and GENIE. 


\subsection{PROJECTED IMPACT ON NEUTRINO UNCERTAINTIES}

This failure of the generator to accurately describe the data can have a big impact on the ability to reconstruct neutrino oscillation parameters. We looked at the case of the DUNE detector, which will use ${ }^{40} \mathrm{Ar}$ as its target and detector material.

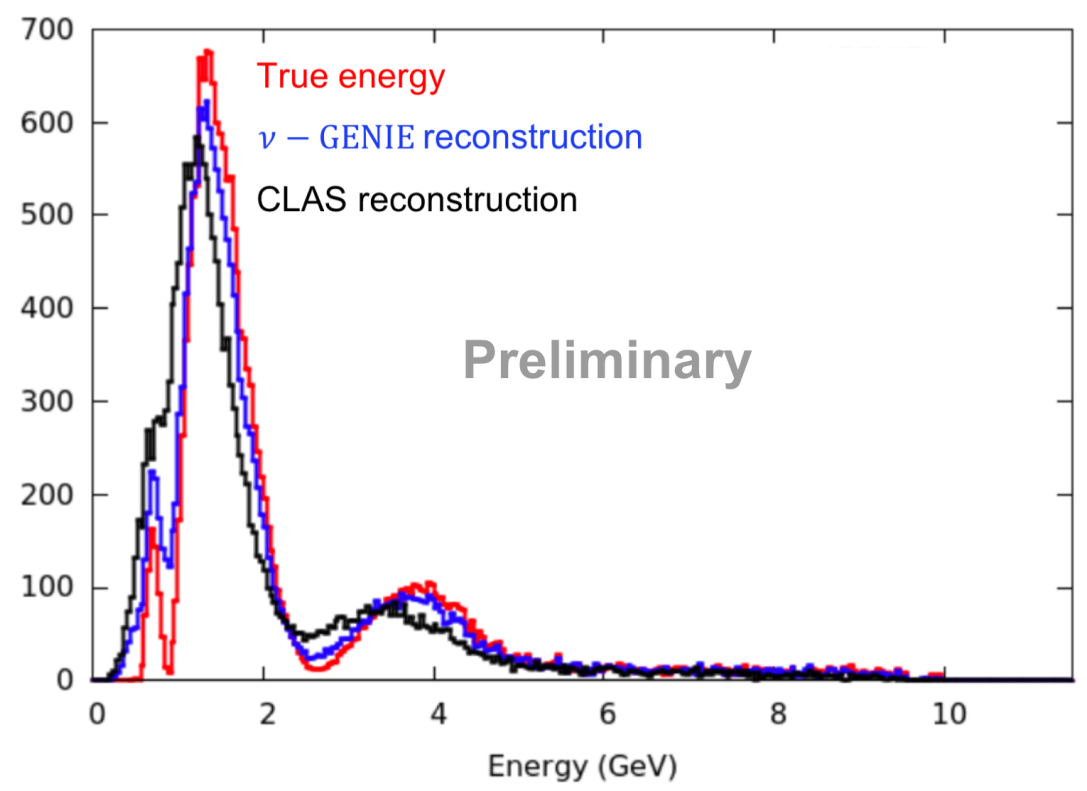

FIG. 196: The reconstructed incident neutrino energy spectra for the DUNE far detector with oscillation included, simulated with $\nu$-GENIE and reconstructed with (black) fractional energy reconstruction ("feed down") from ${ }^{12} \mathrm{C}$ zero-pion $\left(e, e^{\prime} p\right)$ data and (blue) using the calorimetric energy reconstruction in $\nu$-GENIE.

We created energy feed down spectra (plotting $\left.\Delta E=\left(E_{\text {reco }}-E_{\mathrm{TRUE}}\right) / E_{\mathrm{TRUE}}\right)$ for ${ }^{12} \mathrm{C}\left(e, e^{\prime} p\right)$ events for $1.1,2.2$ and $4.4 \mathrm{GeV}$ electron beams and interpolated linearly between the energies to get $\Delta E$ spectra at all incident energies from our data. We took the expected DUNE Far Detector neutrino flux and simulated the events using $\nu$-GENIE for zero-pion $\left(\nu_{\mu}, \mu^{-} p\right)$ events from interactions with ${ }^{12} \mathrm{C}$. We required the proton momentum $p_{\mathrm{p}}>0.3 \mathrm{GeV} / \mathrm{c}, Q^{2}>0.5(\mathrm{GeV} / \mathrm{c})^{2}$ and $W<2 \mathrm{GeV} / \mathrm{c}^{2}$.

We then reconstructed the neutrino energy spectrum using the calorimetric $\Delta E$ spectra obtained from electron scattering data and from $\nu$-GENIE.

Fig. 196 shows the reconstructed incident energy spectrum at the Far Detector of DUNE. There is a big difference between the reconstructed spectra from data and from $\nu$-GENIE. Both data and $\nu$-GENIE reconstructed spectra are different from the true energy spectrum 
shown in red. This difference could produce dramatically different neutrino oscillation parameters.

To summarize:

- This analysis demonstrates the first use of electron data to test neutrino energy reconstruction algorithms. The results obtained using $E_{\mathrm{QE}}$ and $E_{\text {calor }}$ energy reconstruction methods are improved by a transverse momentum cut to better select QE events.

- The $E_{\text {calor }}$ method gives a better description of the beam energy, however only $16-55 \%$ of events reconstruct to within $5 \%$ of the beam energy. The reconstruction works better for lighter nuclei and lower energies.

- The background tail is the same for both reconstruction methods. Agreement between the results from the two methods does not imply that the energy has been reconstructed correctly.

- This type of analysis has excited tremendous interest in the neutrino community and can have serious implications for neutrino oscillation measurements.

- We will publish experimental results showing the fraction of zero-pion events that reconstruct to the beam energy for the two methods in order to help the neutrino physicists understand their energy reconstruction. We are also going to publish data vs neutrino Monte Carlo comparison results and the potential impact of discrepancy between the results from data and GENIE neutrino event generator prediction running in electron scattering mode on the neutrino oscillation analysis of DUNE experiment.

- This analysis is part of the ongoing Electrons for Neutrinos (e $4 \nu$ ) project. We will measure more targets and energies and identify regions with good and bad energy reconstruction GENIE modeling. An experiment titled "Electrons for Neutrinos" and proposal number "PR12-17-006" has been approved with a scientific rating of "A" by PAC 46 . 


\section{BIBLIOGRAPHY}

[1] L. Lapikas, "Quasi-elastic electron scattering off nuclei," Nucl. Phys., vol. A553, pp. 297c-308c, 1993.

[2] R. S. et al., "Momentum distributions in A= 3 and 4 nuclei," Nucl.Phys., vol. A449, pp. 219-242, 1986.

[3] A.Nikolakopoulos et al., "Mean-field approach to reconstructed neutrino energy distributions in accelerator-based experiments," Phys. Rev. C, vol. 98, p. 054603, 2018.

[4] P.Coloma et al., "Neutrino-nucleus interaction models and their impact on oscillation analyses," Phys.ReV.D, vol. 89, p. 073015, 2014.

[5] S. Boyd, "Neutrino oscillations." https://warwick.ac.uk/fac/sci/physics/staff/ academic/boyd/warwick_week/neutrino_physics/lec_oscillations.pdf, May 2016.

[6] D. Scully, Neutrino Induced Coherent Pion Production. PhD thesis, University of Warwick, 2013.

[7] "Neutrino masses, mixing, and oscillations." http://pdg.lbl.gov/2018/reviews/ rpp2018-rev-neutrino-mixing.pdf.

[8] K.Abe et al., "Measurements of neutrino oscillation in appearance and disappearance channels by the T2K experiment with $6.6 \times 10^{20}$ protons on target," Phys. Rev. D, vol. 91, p. 072010, 2015.

[9] P.Adamson et al., "The NuMI neutrino beam," Nucl.Instrum.Meth., vol. A806, pp. 279306, 2016.

[10] "J-PARC neutrino experiment facility." https://j-parc.jp/Neutrino/en/ nu-facility.html.

[11] T.Leitner et al., "Neutrino-nucleus scattering reexamined: Quasielastic scattering and pion production entanglement and implications for neutrino energy reconstruction," Phys. Rev. C, vol. 81, p. 064614, 2010.

[12] "Minerva experiment." https://minerva.fnal.gov/. 
[13] "MINOS experiment." https://www-numi.fnal.gov/.

[14] "Nova experiment." https://novaexperiment.fnal.gov/.

[15] "ArgoNeuT experiment." http://t962.fnal.gov/.

[16] "MicroBooNE experiment." https://microboone.fnal.gov/.

[17] V.Papadimitriou, "Design of the LBNF beamline." https://arxiv.org/pdf/1704. 04471 .pdf, 2017.

[18] "DUNE experiment." http://www.dunescience.org/.

[19] "Hyper-Kamiokande experiment." http://www.hyperk.org/.

[20] A. Furmanski et al., "Neutrino energy reconstruction from one muon and one proton events," Phys. Rev. C, vol. 95, p. 065501, 2017.

[21] M. Martini et al., "Neutrino energy reconstruction problems and neutrino oscillations." https://arxiv.org/pdf/1202.4745.pdf, 2012.

[22] S. Braibant, Particles and fundamental interactions. Springer, 2011.

[23] D. Griffiths, Introduction to elementary particles. WILEY-VCH, 2008.

[24] M.Tanabashi et al., "The review of particle physics," Phys. Rev. D, vol. 98, p. 030001, 2018.

[25] O.Benhar et al., "Neutrino-nucleus interactions and the determination of oscillation paramters," Physics Reports, vol. 700, pp. 1-47, 2017.

[26] J. Morrison et al., "Quasielestic ${ }^{12} \mathrm{C}\left(e, e^{\prime} p\right)$ reaction at high momentum transfer.," Phys. Rev. C, vol. 59, pp. 221-232, 1999.

[27] H. Baghaei et al., "Electroexcitation of the $\delta$ resonance in the $\left(e, e^{\prime} p\right)$ reaction.," Phys. Rev. C, vol. 39, pp. 177-180, 1989.

[28] S. D. Penn, An Examination of two-nucleon correlations in ${ }^{12} \mathrm{C}\left(e, e^{\prime} p\right)$ and $\left(e, e^{\prime} d\right)$. $\mathrm{PhD}$ thesis, 1985.

[29] L. Weinstein et al., "Quasielestic Reaction Mechanism Studied Using the Reaction ${ }^{12} \mathrm{C}\left(e, e^{\prime} p\right) ., "$ Phys. Rev. Letters, vol. 64, pp. 1646-1649, 1990. 
[30] R. Lourie et al., "Reaction ${ }^{12} \mathrm{C}\left(e, e^{\prime} p\right)$ in the Dip Region.," Phys. Rev. Letters, vol. 56, pp. 2364-2367, 1986.

[31] R. Schiavilla, Private comunication.

[32] G.Shen et al., "Inclusive neutrino scattering off deuteron from threshold to GeV energies," Phys. Rev. C, vol. 86, p. 035503, 2012.

[33] G. Sowjanya, "Neutrino cross section future." https://arxiv.org/pdf/1602.05299. pdf, 2016.

[34] V.Lyubushkin et al., "A study of quasi-elastic muon neutrino and antineutrino scattering in the NOMAD experiment," Eur. Phys. J., vol. C63, pp. 355-381, 2009.

[35] L.Auerbach et al., "Measurements of charged current reactions of muon neutrinos on ${ }^{12}$ C," Phys. Rev. C, vol. 66, p. 015501, 2002.

[36] A.A.Aguilar-Arevalo et al., "First measurement of the muon neutrino charged current quasielastic double differential cross section," Phys. Rev. D, vol. 81, p. 092005, 2010.

[37] A.A.Aguilar-Arevalo et al., "Significant excess of electron like events in the MiniBooNE short-baseline neutrino experiment," Phys. Rev. Lett., vol. 121, p. 221801, 2018.

[38] J. Kopp, "The plot thickens for a fourth neutrino," APS Physics, vol. 11, p. 122, 2018.

[39] A.A.Aguilar-Arevalo et al., "Measurement of neutrino induced charged current charged pion production cross sections on mineral oil at $E \sim 1 \mathrm{GeV}, "$ Phys. Rev. D, vol. 83, p. 052007, 2011.

[40] A.A.Aguilar-Arevalo et al., "Measurement of $\nu_{\mu}$ - induced charged current neutral pion production cross sections on mineral oil at $e_{\nu} \in 0.5-2.0 \mathrm{GeV}, "$ Phys. Rev. D, vol. 83, p. 052009, 2011.

[41] O.Lalakulich et al., "Pion production in the MiniBooNE experiment," Phys. Rev. C, vol. 87, p. 014602, 2013.

[42] G. B. M. et al., "Neutrino Oscillation Results from LSND," Nucl. Phys. B, vol. 66, pp. 382-386, 1998.

[43] M.Martini et al., "Unified approach for nucleon knock-out coherent and incoherent pion production in neutrino interactions with nuclei," Phys. Rev. C, vol. 80, p. 065501, 2009. 
[44] J.Nieves et al., "Inclusive charged-current neutrino-nucleus reactions," Phys. Rev. C, vol. 83, p. 045501, 2011.

[45] U. Mosel, "Neutrino interactions with nucleons and nuclei: Importance for long-baseline experiments." https://arxiv.org/pdf/1602.00696.pdf, 2016.

[46] A. Friedland and S. W. Li, "Understanding the energy resolution of liquid argon neutrino detectors." https://arxiv.org/pdf/1811.06159.pdf, 2019.

[47] C.Adams et al., "The long-baseline experiment: Exploring fundamental symmetries of the universe." https://arxiv.org/pdf/1307.7335.pdf, 2014.

[48] M. Muether NuInt, 2015.

[49] B. Tice et al., "Measurement of Ratios of $\nu_{\mu}$ Charged-Current Cross Sections on C, Fe, and $\mathrm{Pb}$ to $\mathrm{CH}$ at Neutrino Energies 2-20 GeV," Phys. Rev. Letters, vol. 112, p. 231801, 2014.

[50] S. Stepanyan, "e2a run list." https://userweb.jlab.org/ stepanya/e2/e2CalRuns . html, 1999.

[51] M. Mestayer et al., "The CLAS drift chamber system," Nucl. Instrum. Meth. A, vol. 449, pp. 81-111, 2000.

[52] G.Adams et al., "The CLAS Cherenkov detector," Nucl. Instrum. Meth. A, vol. 465, pp. 414-427, 2001.

[53] E. Smith et al., "The time-of-flight system for CLAS," Nucl. Instrum. Meth. A, vol. 432, pp. 265-298, 1999.

[54] M.Amarian et al., "The CLAS forward electromagnetic calorimeter," Nucl. Instrum. Meth. A, vol. 460, pp. 239-265, 2001.

[55] M.Khachatryan et al., "Time calibration studies of the Large Angle Calorimeter (LAC)," CLAS-NOTE 2016-001 JLAB, 2016.

[56] R. Niyazov, Measurement of Correlated Pair Momentum Distributions on ${ }^{3} H e\left(e, e^{\prime} p p\right) n$ with CLAS. PhD thesis, Old Dominion University, 2003.

[57] M.Osipenko et al., "Measurememnt of deuteron structure function $F_{2}$ in the resonance region and evaluation of its moments," Phys.Rev.C, vol. 73, p. 045205, 2006. 
[58] B. Zhang, Searching for Short Range Correlations Using (e,e'NN) Reactions. PhD thesis, MIT, 2003.

[59] D. Protopopescu, "Fiducial cuts for electrons in the CLAS/E2 data at 4.4 GeV," CLASNOTE 2000-007 JLAB, 2000.

[60] B. Zhang, "Electron fiducial cuts." https://www.jlab.org/Hall-B/secure/e2/bzh/ efiducialcut.html\#edge, 2003.

[61] S. McLauchlan, $\Delta$ electroproduction in ${ }^{12} C . \mathrm{PhD}$ thesis, University of Glasgow, January 2003. Appendix C.

[62] L. W. Rustam Niyazov, "Fiducial cut for positive hadrons in CLAS/E2 data at 4.4 GeV," CLAS-NOTE 2001-013 JLAB, 2001.

[63] B. Zhang, "Positive hadron fiducial cuts." https://www.jlab.org/Hall-B/secure/ e2/bzh/pfiducialcut.html, 2003.

[64] S. S. Nathan Baltzell, "Beam XY-position at the target for clas/eg6," CLAS-NOTE 2013-007 JLAB, 2013.

[65] D. Protopopescu, "Measurements of $\mathrm{A}_{1 \mathrm{t}}$ ' assymetries in $\left(e, e^{\prime} p\right)$ reactions on ${ }^{4} \mathrm{He}$ and ${ }^{12} \mathrm{C}, "$ CLAS Analysis Paper, 2003.

[66] F. X.Lee et al., "Quasifree pion electroproduction from nuclei in the $\delta$ region," Phys. Rev. C, vol. 55, p. 318, 1997.

[67] N. Markov et al., "Exclusive $\pi^{0} p$ electroproductions off protons in resonance region at photon virtualities $0.4 \leq Q^{2} \leq 1 \mathrm{GeV}^{2}$." https://arxiv.org/pdf/1202.4745.pdf, 2019.

[68] A. Papadopoulou et al., "Leveraging electron scattering data to constrain neutrino simulation tools," In preparation.

[69] B. A. M. et al., "The CEBAF large acceptance spectrometer (CLAS)," Nucl. Instrum. Methods, vol. 503, p. 513, 2003. 


\title{
APPENDIX A
}

\section{Z-VERTEX CORRECTION AND CUTS OF PROTONS, PIONS}

\author{
AND ELECTRONS FOR ${ }^{4} \mathrm{He},{ }^{12} \mathrm{C}$ AND ${ }^{56} \mathrm{Fe}$ TARGETS FOR
}

\subsection{GEV AND 4.4 GEV BEAM ENERGIES.}

\section{A.1 4.4 GEV ANALYSIS}

We have obtained individual vertex correction functions for each of the targets. We have then used this correction function to correct the proton and electron vertexes. To get idea about the quality of applied corrections we have looked at the vertex distributions before and after vertex corrections explained in details in current analysis.

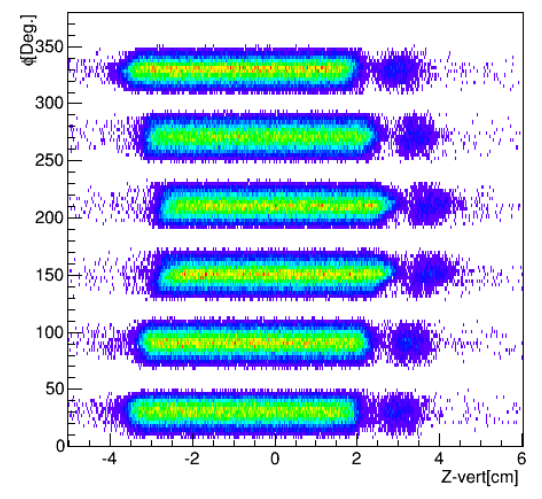

(a) Before correction.

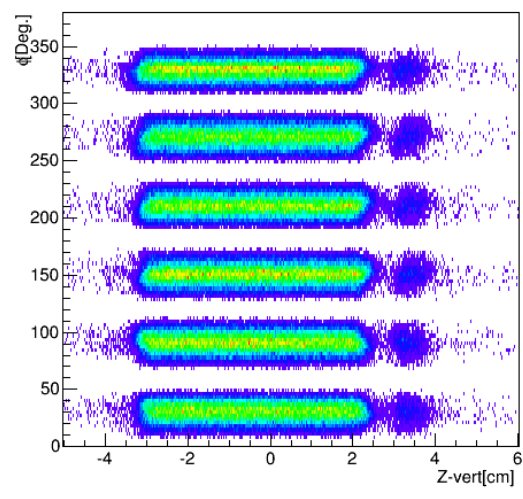

(b) After correction.

FIG. 197: The $\phi$ angle of the electrons plotted vs z component of vertex in logarithmic scale, before and after corrections. The results are shown for the ${ }^{4} \mathrm{He}$ target at $4.4 \mathrm{GeV}$.

The vertex cuts for different target and beam energy analysis can be found in Table 14 .

The plots of electron $\phi$ azimuthal angle versus z vertex for ${ }^{4} \mathrm{He}$ at $4.4 \mathrm{GeV}$ before and after corrections are shown in Fig. 197. We can see that after applied correction the target 


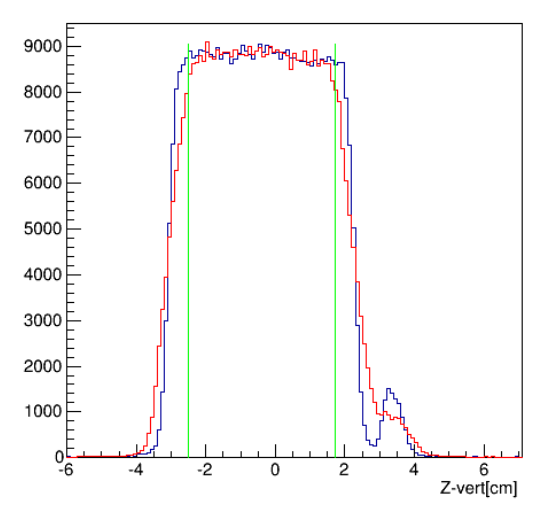

(a)

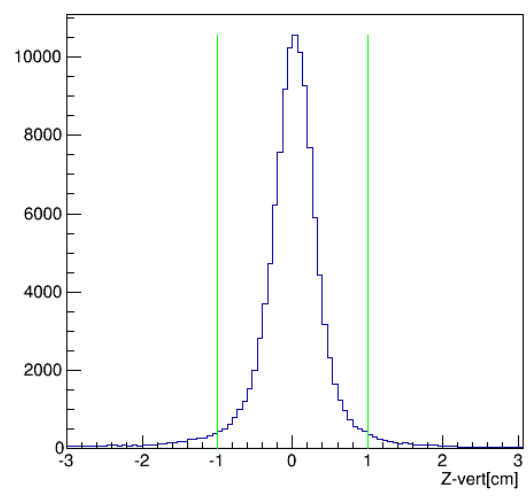

(b)

FIG. 198: a) The distributions of electron $z$ vertex before (red) and after (blue) correction. b) The distribution of electron and proton $z$ vertex differences. The green lines correspond to the applied cut. Both plots are made for ${ }^{4} \mathrm{He}$ at $4.4 \mathrm{GeV}$.

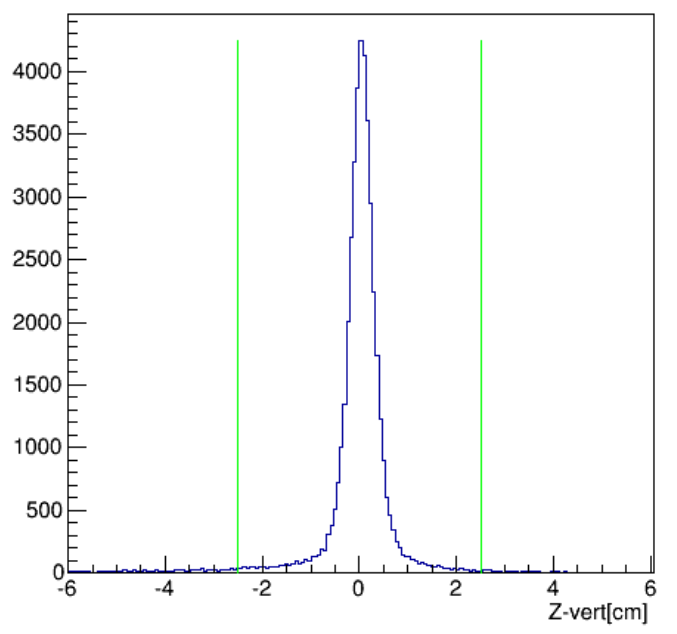

(a) $\pi^{-}$.

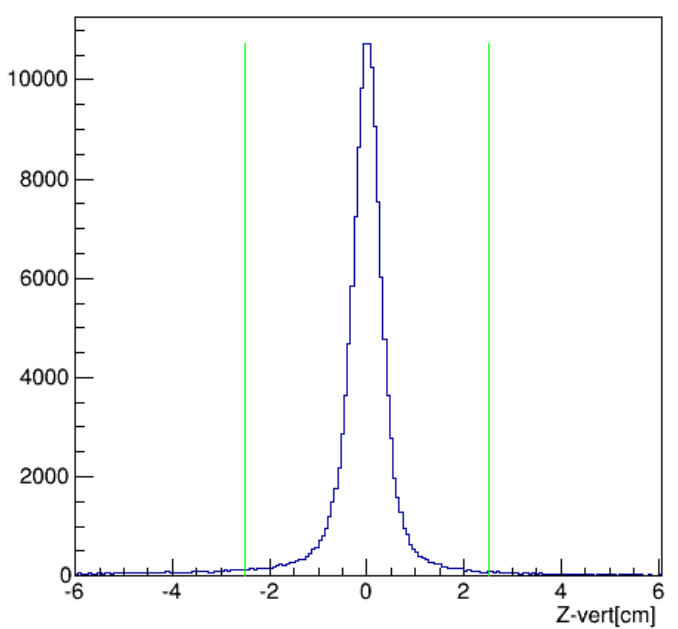

(b) $\pi^{+}$.

FIG. 199: The charged pion and proton $z$ vertex difference for ${ }^{4} \mathrm{He}$ at $4.4 \mathrm{GeV}$. The green lines correspond to the applied cut.

region, which represents a cryotarget cell full of liquid ${ }^{4} \mathrm{He}$, and the heat shield region to the right of it are nicely aligned for electrons detected in different CLAS sectors. We can also see 


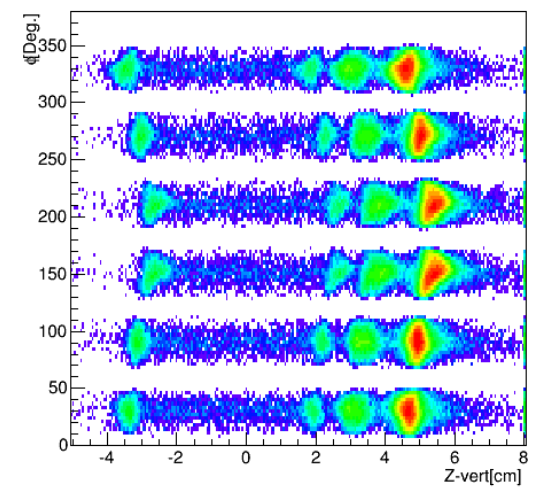

(a) Before correction.

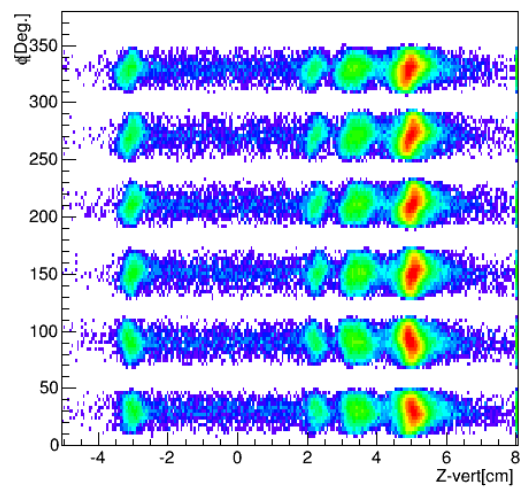

(b) After correction.

FIG. 200: The $\phi$ angle of the electrons plotted vs z component of vertex in logarithmic scale, before and after corrections. The results are shown for the ${ }^{12} \mathrm{C}$ target at $4.4 \mathrm{GeV}$.

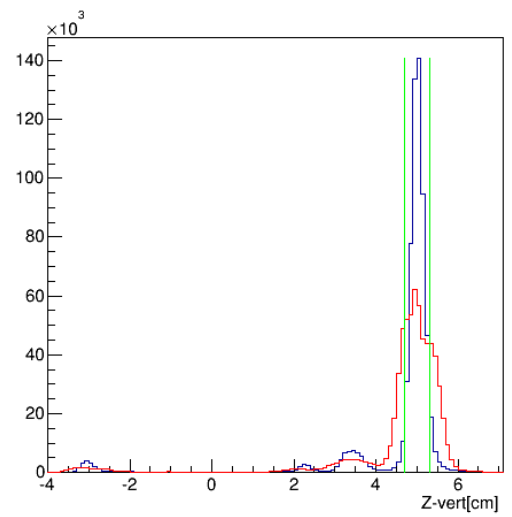

(a)

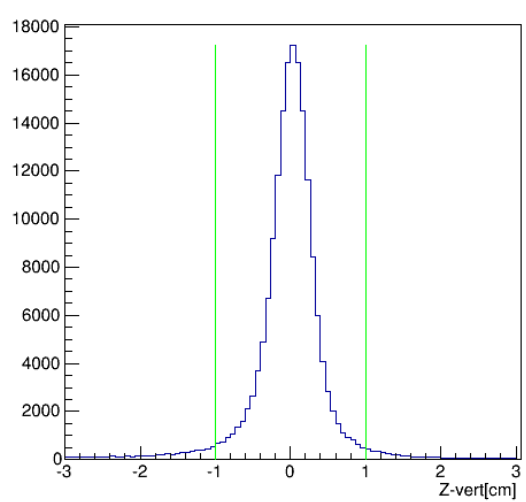

(b)

FIG. 201: a) The distributions of electron $z$ vertex before (red) and after (blue) correction. b) The distribution of electron and proton $z$ vertex differences. The green lines correspond to the applied cut. Both plots are made for ${ }^{12} \mathrm{C}$ at $4.4 \mathrm{GeV}$.

an improvement in electron vertex distribution shown in the left of Fig. 198, by comparing the distributions before and after vertex corrections and looking at the difference between electron and proton vertexes on the right side of the figure.

In Fig. 200 we can see the plots of electron $\phi$ azimuthal angle as a function of $\mathrm{z}$ vertex for 


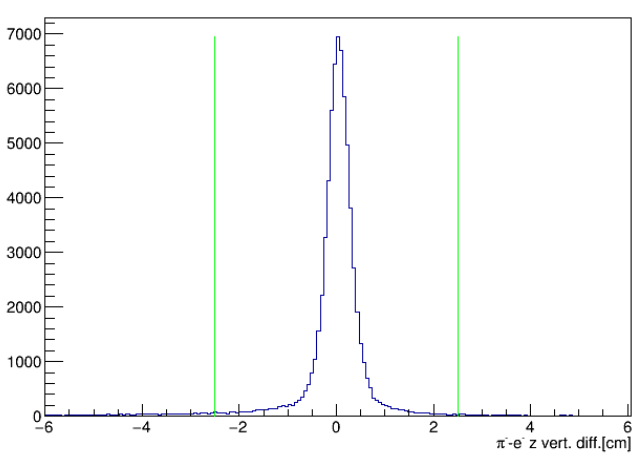

(a) $\pi^{-}$.

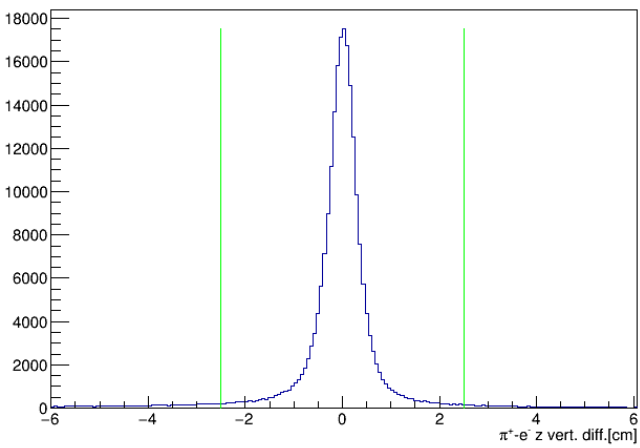

(b) $\pi^{+}$.

FIG. 202: The charged pion and proton $z$ vertex difference for ${ }^{12} \mathrm{C}$ at $4.4 \mathrm{GeV}$. The green lines correspond to the applied cut.

${ }^{12} \mathrm{C}$ at $4.4 \mathrm{GeV}$ target. The carbon target was placed downstream of the empty cryotarget cell. Again it looks like the vertex correction works fine. The same can be seen in the electron vertex distribution plots before and after the correction and the difference between the electron and proton vertexes, see Fig. 201.

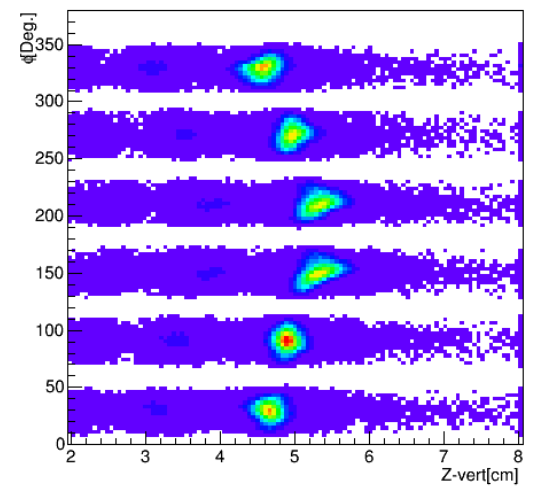

(a) Before correction.

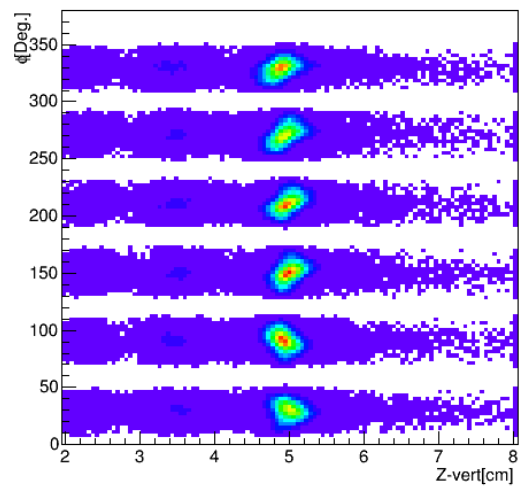

(b) After correction.

FIG. 203: The $\phi$ angle of the electrons plotted vs z component of vertex in logarithmic scale, before and after corrections. The results are shown for the ${ }^{56} \mathrm{Fe}$ target at $4.4 \mathrm{GeV}$.

The second solid target used in this analysis is the iron. The plots of electron $\phi$ azimuthal angle as a function of $\mathrm{z}$ vertex for ${ }^{56} \mathrm{Fe}$ at $4.4 \mathrm{GeV}$ target before and after corrections are shown in Fig. 203. The empty cryotarget cell was also present during the ${ }^{56} \mathrm{Fe}$ at $4.4 \mathrm{GeV}$ 


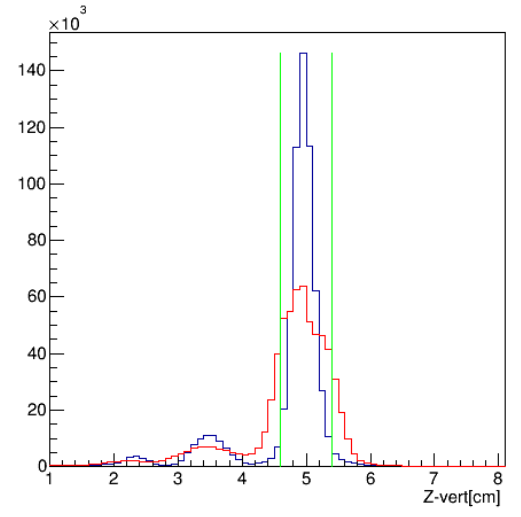

(a)

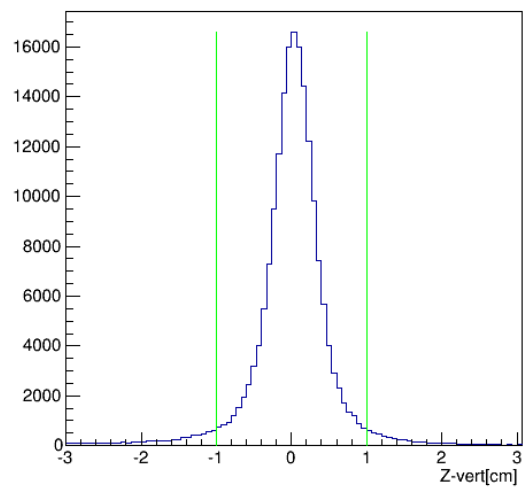

(b)

FIG. 204: a) The distributions of electron $z$ vertex before (red) and after (blue) correction. b) The distribution of electron and proton $z$ vertex differences. The green lines correspond to the applied cut. Both plots are made for ${ }^{56} \mathrm{Fe}$ at $4.4 \mathrm{GeV}$.

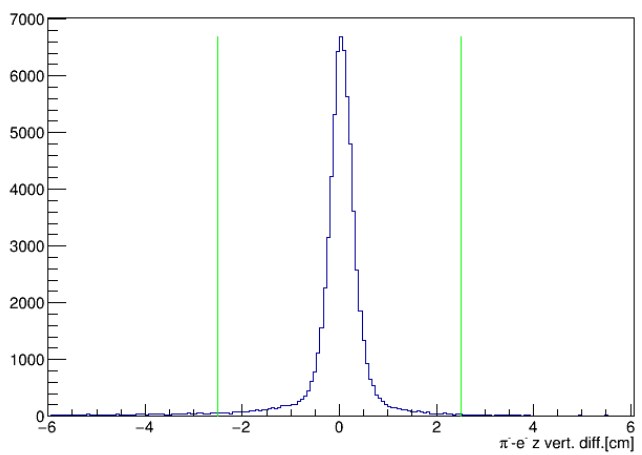

(a) $\pi^{-}$.

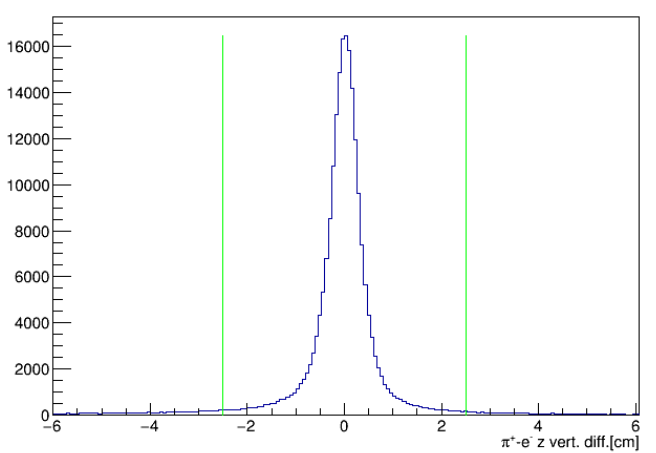

(b) $\pi^{+}$.

FIG. 205: The charged pion and proton $z$ vertex difference for ${ }^{56} \mathrm{Fe}$ at $4.4 \mathrm{GeV}$. The green lines correspond to the applied cut.

and so the very left peak in the electron vertex distribution in the right side of Fig. 204 corresponds to the exit window of the liquid target cell, the small peak next to it corresponds to the heat shield and the large peak to the right of the heat shield corresponds to the solid target.

The difference between $z$ vertexes of an electron and a charged pion for different targets 
at $4.4 \mathrm{GeV}$ are shown in Figs. 199, 202 and 205.

\section{A.2 2.2 GEV ANALYSIS}

The ${ }^{4} \mathrm{He}$ data was grouped into two groups with separate vertex corrections and cuts. The first group includes runs 18176-18206 and the second one runs 18221-18252 of 2.2 GeV ${ }^{4}$ He data.

The corresponding plots for ${ }^{4} \mathrm{He},{ }^{12} \mathrm{C}$ and ${ }^{56} \mathrm{Fe}$ at $2.2 \mathrm{GeV}$ are shown in Figs. 206-219.

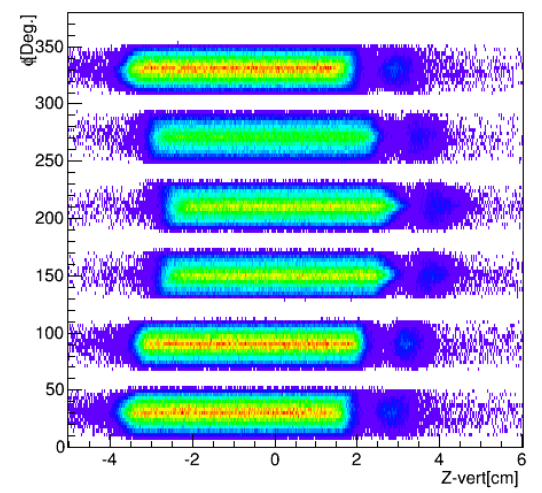

(a) Before correction.

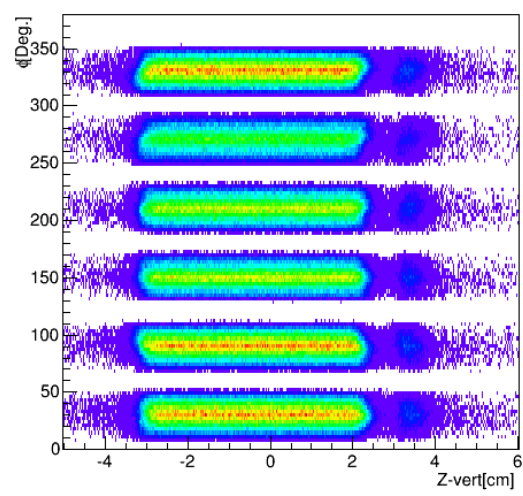

(b) After correction.

FIG. 206: The $\phi$ angle of the electrons plotted vs z component of vertex in logarithmic scale, before and after corrections. The results are shown for the ${ }^{4} \mathrm{He}$ target at $2.2 \mathrm{GeV}$ for runs 18176-18206.

Nearly half of the data with ${ }^{56} \mathrm{Fe}$ target at $2.2 \mathrm{GeV}$ was taken with presumably an exploded liquid target cell (referred to here as the 2nd run group). The $\phi$ azimuthal angle versus z vertex for these runs is shown in Fig. 218. In the left plot corresponding to the distribution before the vertex correction, we can see that the peak corresponding to the solid target region (very right side) is sufficiently isolated from the rest of the distribution to be selected and used in the analysis. We obtained a separate correction function for this part of ${ }^{56} \mathrm{Fe}$ data (runs 18258 and 18259) and corrected the $z$ vertexes of electrons and protons. The right plot shows the distribution of $\phi$ azimuthal angle as a function of $z$ vertex after vertex correction.

The plots of electron $z$ vertex distributions for these runs, before and after correction and the difference between $z$ vertexes of an electron and a proton are shown in the left and 


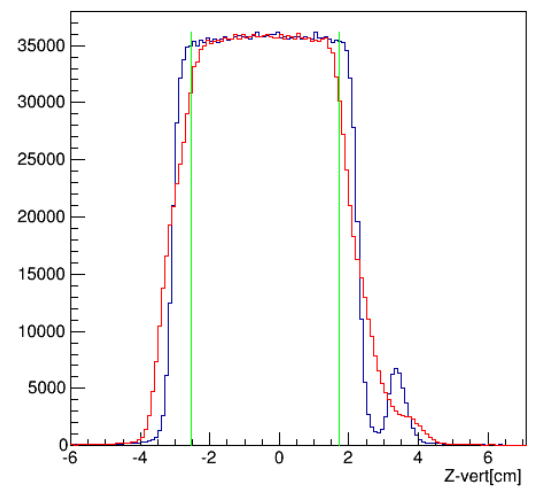

(a)

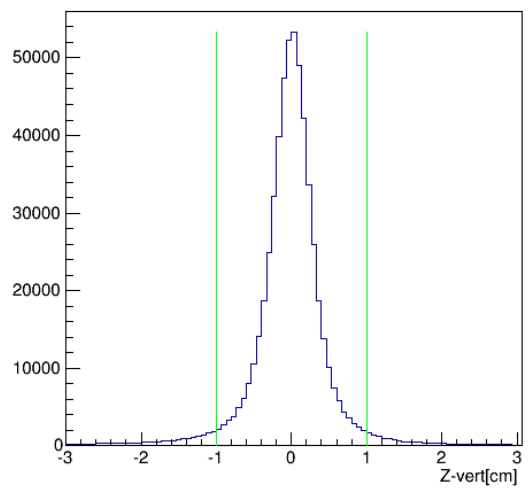

(b)

FIG. 207: a) The distributions of electron $z$ vertex before (red) and after (blue) correction. b) The distribution of electron and proton $z$ vertex differences. The green lines correspond to the applied cut. Both plots are made for ${ }^{4} \mathrm{He}$ at $2.2 \mathrm{GeV}$ for runs $18176-18206$.

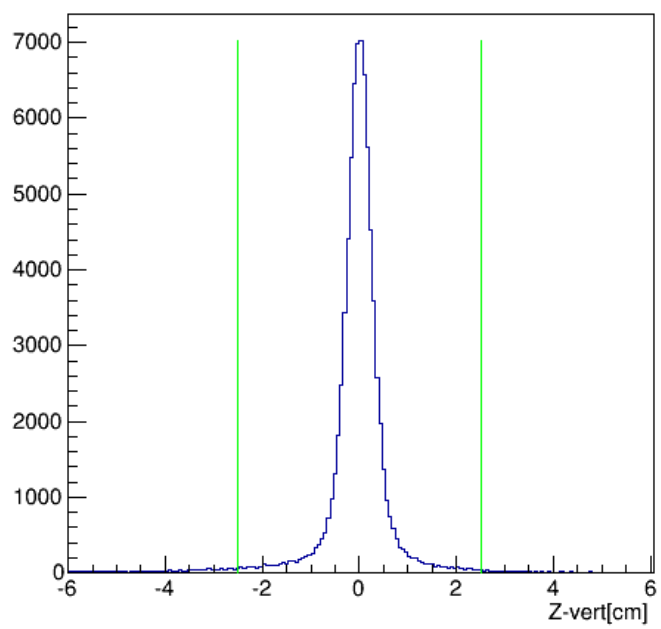

(a) $\pi^{-}$.

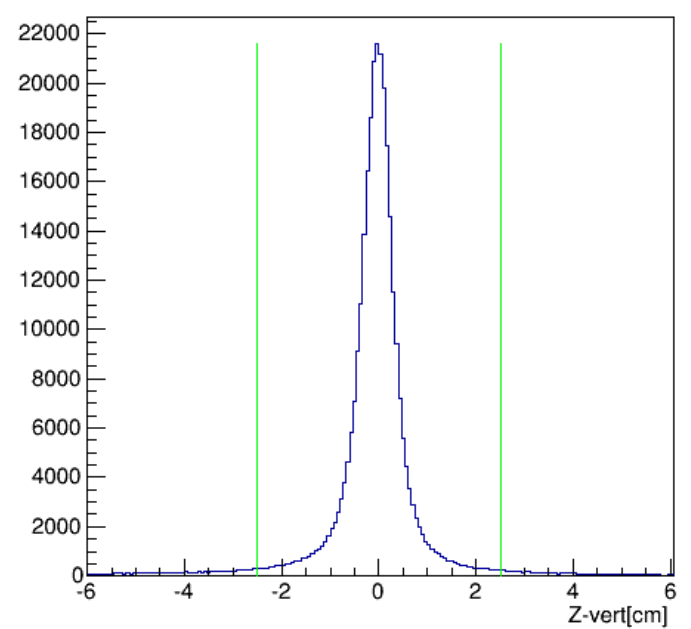

(b) $\pi^{+}$.

FIG. 208: The charged pion and proton $z$ vertex difference for ${ }^{4} \mathrm{He}$ at $2.2 \mathrm{GeV}$ for runs 18176-18206. The green lines correspond to the applied cut.

right sides of Fig. 219 respectively. The green lines correspond to the applied cuts.

The difference between $z$ vertexes of an electron and charged pions for different targets 


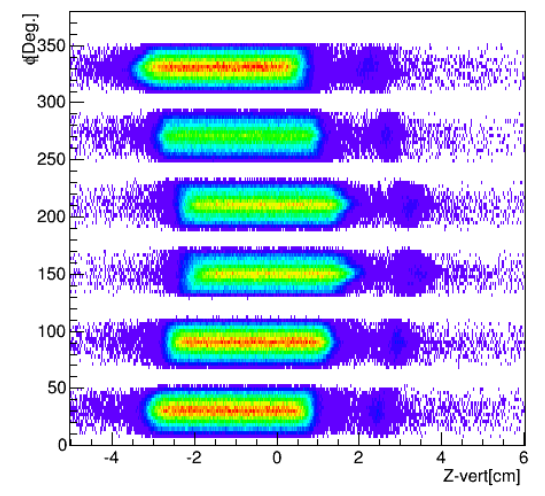

(a) Before correction.

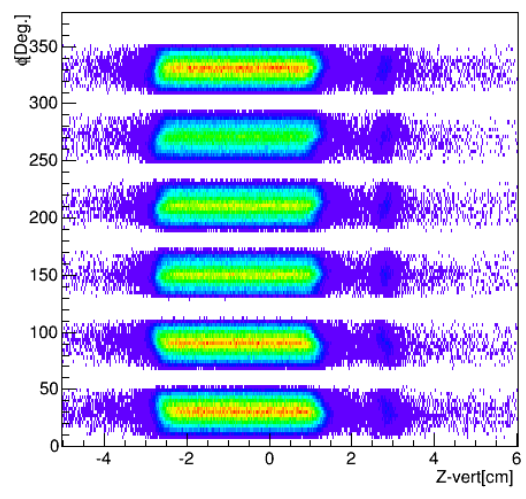

(b) After correction.

FIG. 209: The $\phi$ angle of the electrons plotted vs z component of vertex in logarithmic scale, before and after corrections. The results are shown for the ${ }^{4} \mathrm{He}$ target at $2.2 \mathrm{GeV}$ for runs 18221-18252.

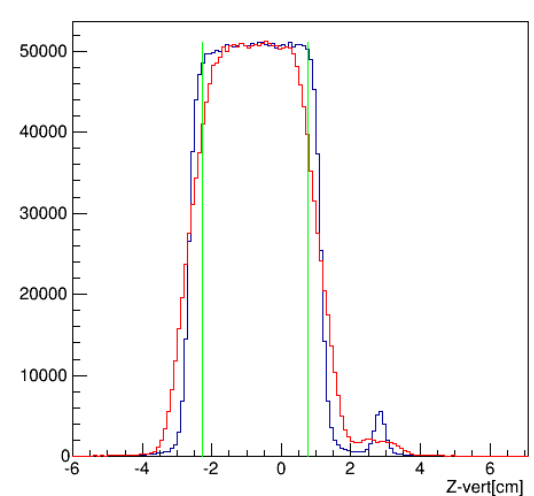

(a)

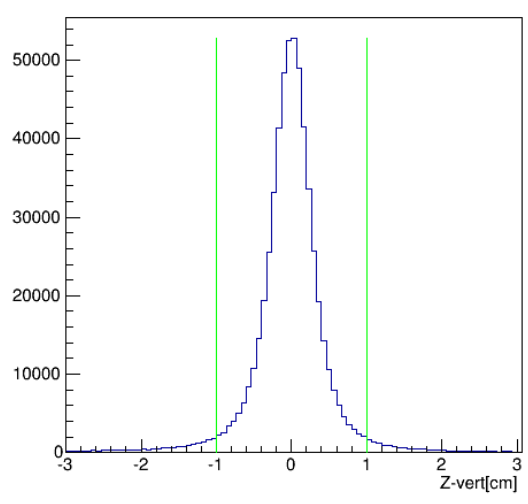

(b)

FIG. 210: a) The distributions of electron $z$ vertex before (red) and after (blue) correction. b) The distribution of electron and proton $z$ vertex differences. The green lines correspond to the applied cut. Both plots are made for ${ }^{4} \mathrm{He}$ at $2.2 \mathrm{GeV}$ for runs $18221-18252$.

at $2.2 \mathrm{GeV}$ are shown in Figs. 208, 211, 214, 217 and 220. 


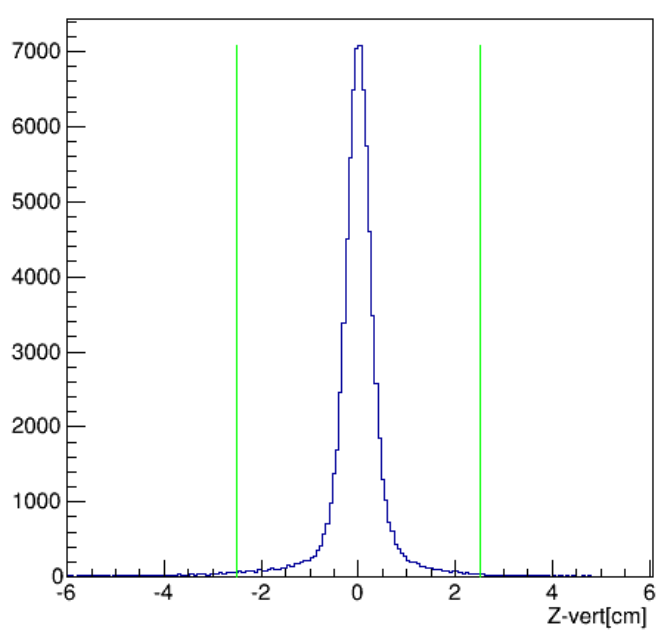

(a) $\pi^{-}$.

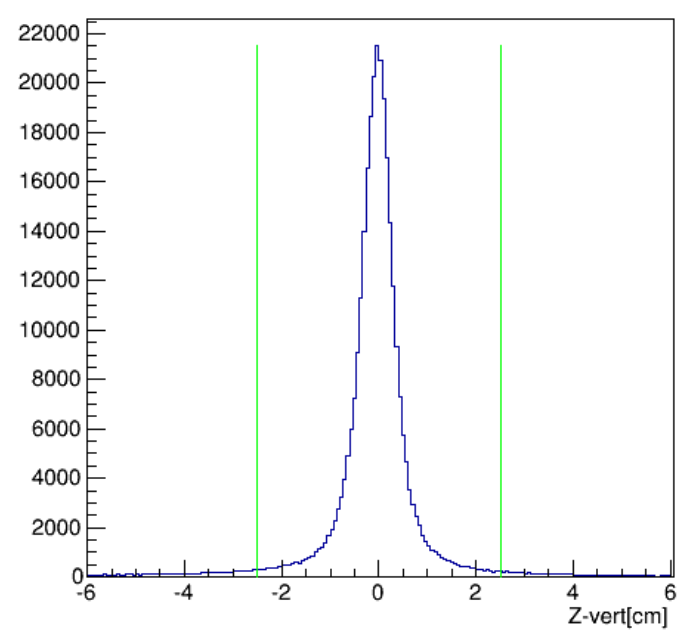

(b) $\pi^{+}$.

FIG. 211: The charged pion and proton $z$ vertex difference for ${ }^{4} \mathrm{He}$ at $2.2 \mathrm{GeV}$ for runs 18221-18252. The green lines correspond to the applied cut.

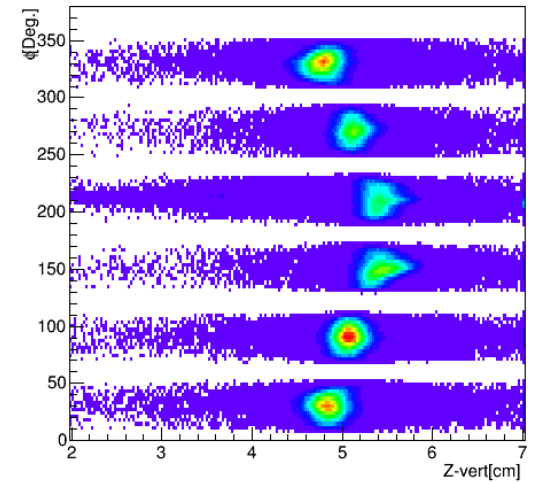

(a) Before correction.

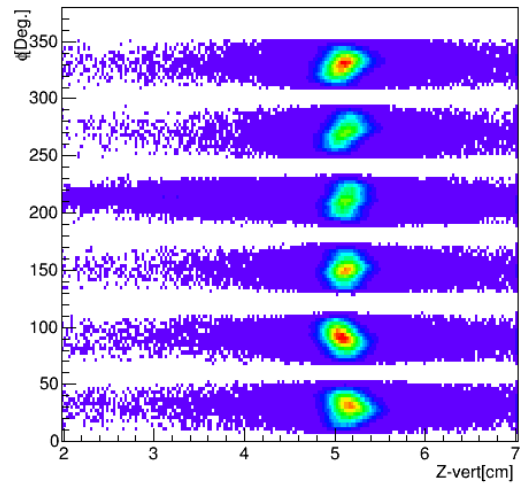

(b) After correction.

FIG. 212: The $\phi$ angle of the electrons plotted vs z component of vertex in logarithmic scale, before and after corrections. The results are shown for the ${ }^{12} \mathrm{C}$ target at $2.2 \mathrm{GeV}$. 


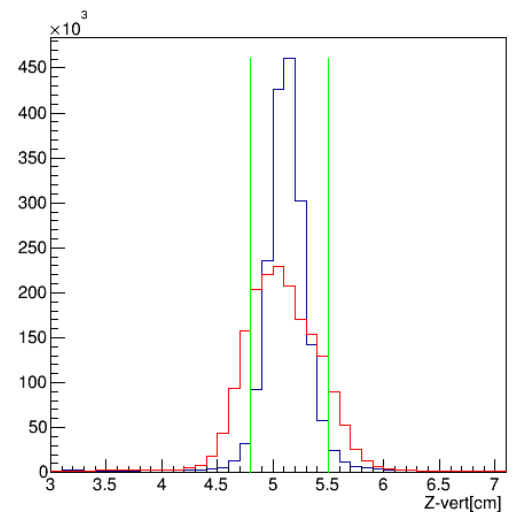

(a)

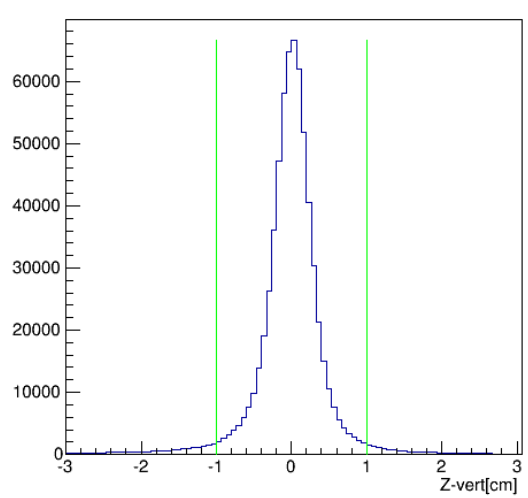

(b)

FIG. 213: a) The distributions of electron $z$ vertex before (red) and after (blue) correction. b) The distribution of electron and proton $z$ vertex differences. The green lines correspond to the applied cut. Both plots are made for ${ }^{12} \mathrm{C}$ at $2.2 \mathrm{GeV}$.

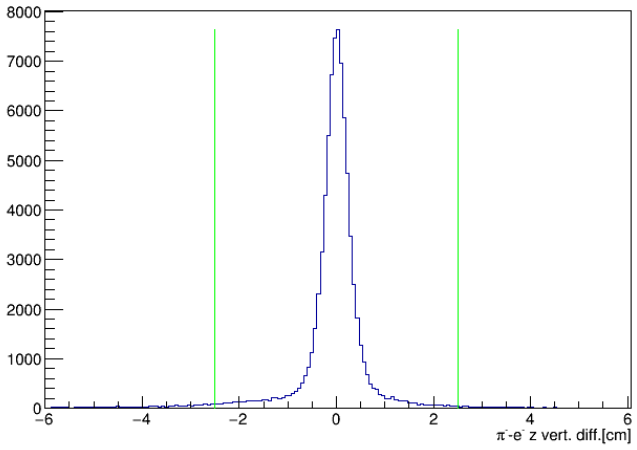

(a) $\pi^{-}$.

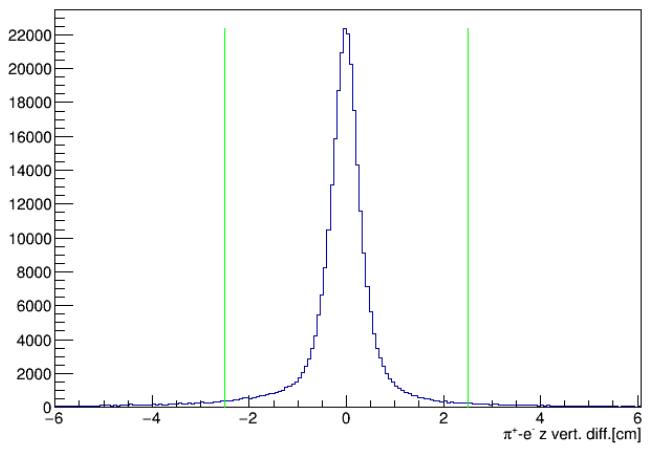

(b) $\pi^{+}$.

FIG. 214: The charged pion and proton $z$ vertex difference for ${ }^{12} \mathrm{C}$ at $2.2 \mathrm{GeV}$. The green lines correspond to the applied cut. 


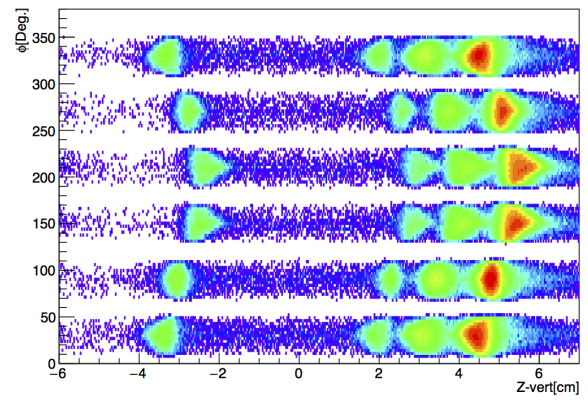

(a) Before correction.

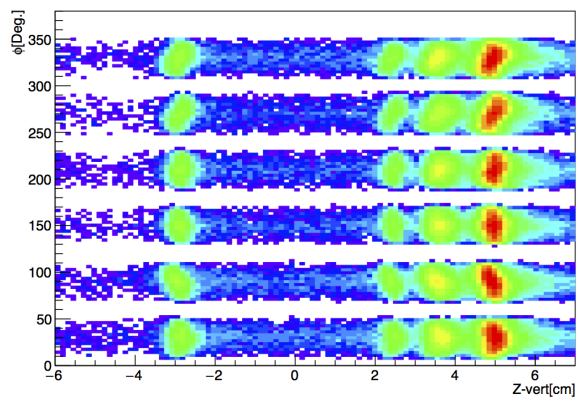

(b) After correction.

FIG. 215: The $\phi$ angle of the electrons plotted vs z component of vertex in logarithmic scale, before and after corrections. The results are shown for the ${ }^{56} \mathrm{Fe}$ target at $2.2 \mathrm{GeV}$ (1nd run group).

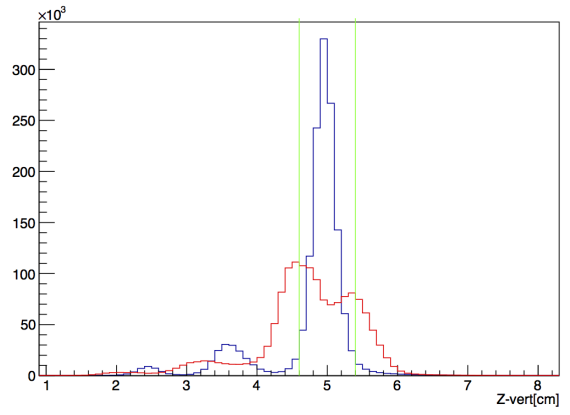

(a)

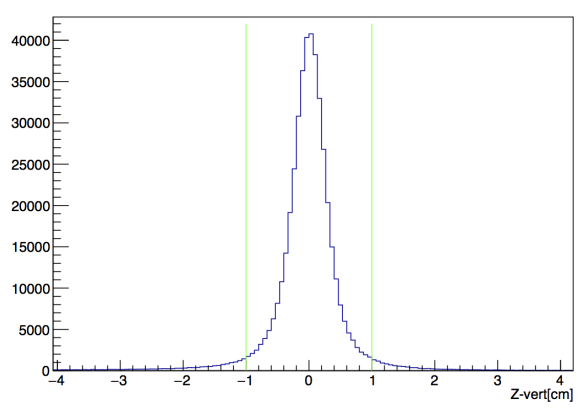

(b)

FIG. 216: a) The distributions of electron $z$ vertex before (red) and after (blue) correction. b) The distribution of electron and proton $z$ vertex differences. The green lines correspond to the applied cut. Both plots are made for ${ }^{56} \mathrm{Fe}$ at $2.2 \mathrm{GeV}$ (1nd run group). 


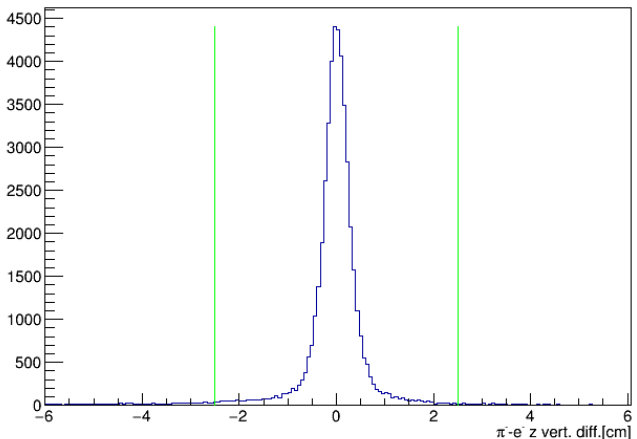

(a) $\pi^{-}$.

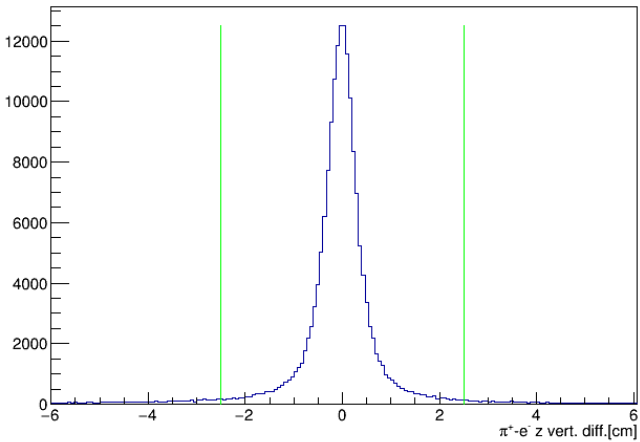

(b) $\pi^{+}$.

FIG. 217: The charged pion and proton $z$ vertex difference for ${ }^{56} \mathrm{Fe}$ at $2.2 \mathrm{GeV}$ for 1 st run group. The green lines correspond to the applied cut.

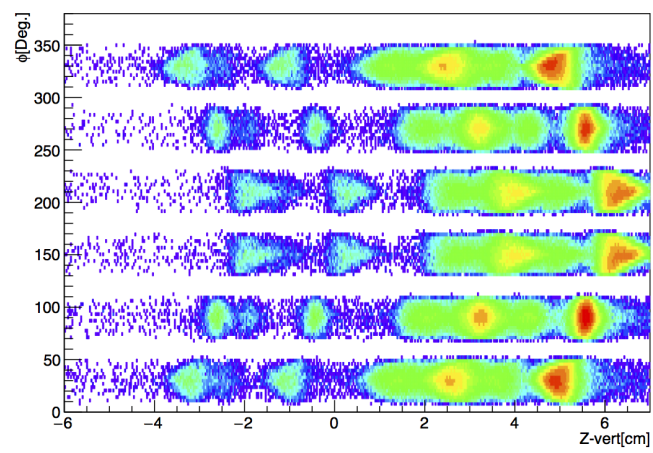

(a) Before correction.

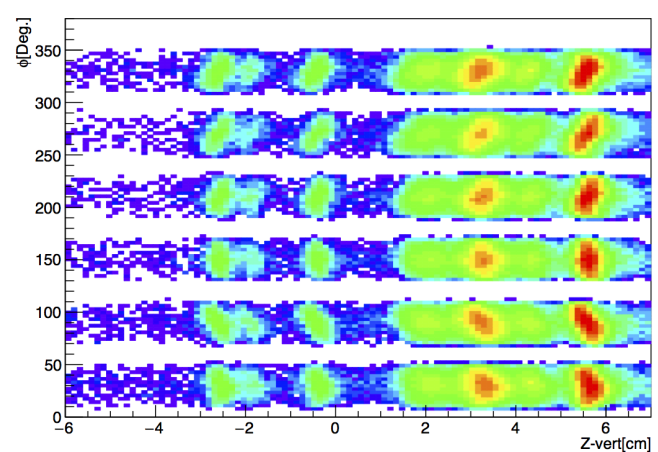

(b) After correction.

FIG. 218: The $\phi$ angle of the electrons plotted vs z component of vertex in logarithmic scale, before and after corrections. The results are shown for the ${ }^{56} \mathrm{Fe}$ target at $2.2 \mathrm{GeV}$ for runs with exploded liquid target cell (2nd run group). 


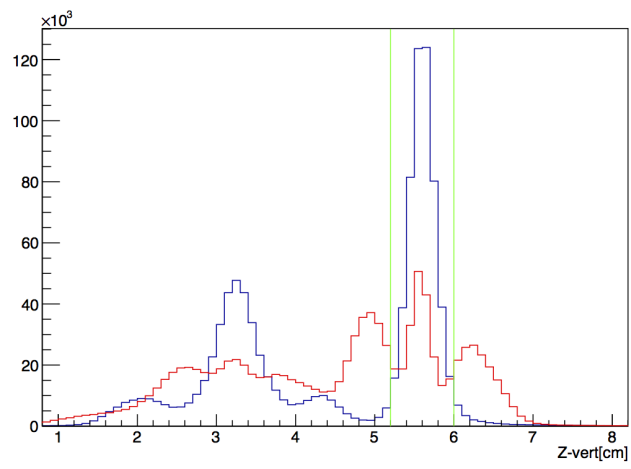

(a)

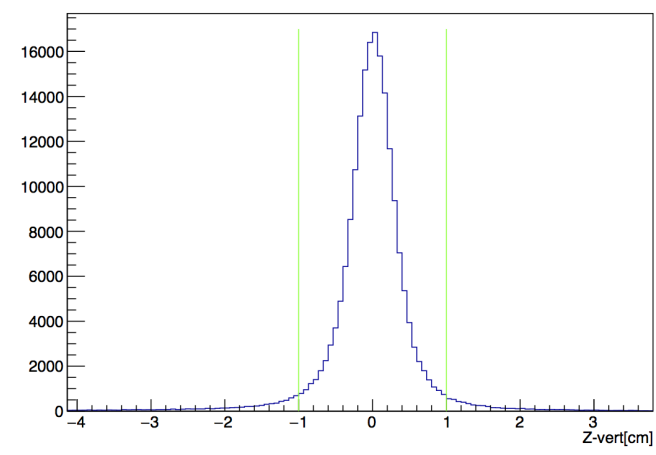

(b)

FIG. 219: a) The distributions of electron $z$ vertex before (red) and after (blue) correction. b) The distribution of electron and proton $z$ vertex differences. The green lines correspond to the applied cut. Both plots are made for ${ }^{56} \mathrm{Fe}$ at $2.2 \mathrm{GeV}$ for runs with exploded liquid target cell (2nd run group).

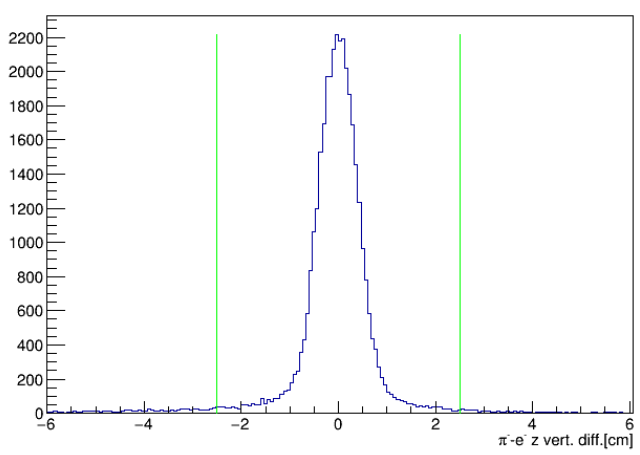

(a) $\pi^{-}$.

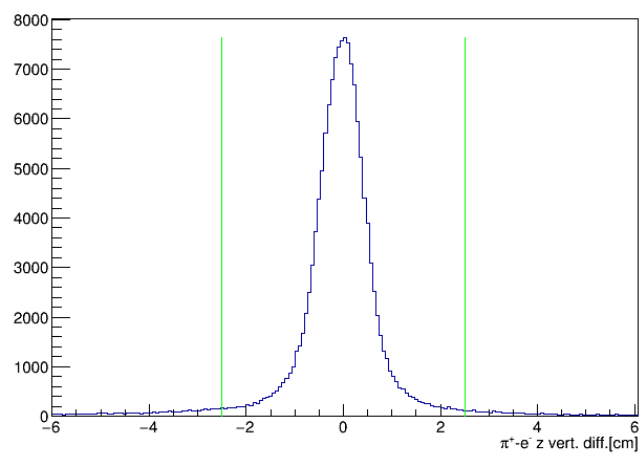

(b) $\pi^{+}$.

FIG. 220: The charged pion and proton $z$ vertex difference for ${ }^{56} \mathrm{Fe}$ at $2.2 \mathrm{GeV}$ for runs with exploded liquid target cell. The green lines correspond to the applied cut. 


\section{APPENDIX B}

THE $A\left(e, e^{\prime}\right)$ AND $A\left(e, e^{\prime} p\right)$ ANALYSIS PLOTS FOR ${ }^{4} \mathrm{He}$ AND ${ }^{12} \mathrm{C}$ TARGETS FOR 2.2 GEV AND 4.4 GEV BEAM ENERGIES.

B.1 4.4 GEV $A\left(e, e^{\prime}\right)$ AND $A\left(e, e^{\prime} p\right)$ ANALYSIS

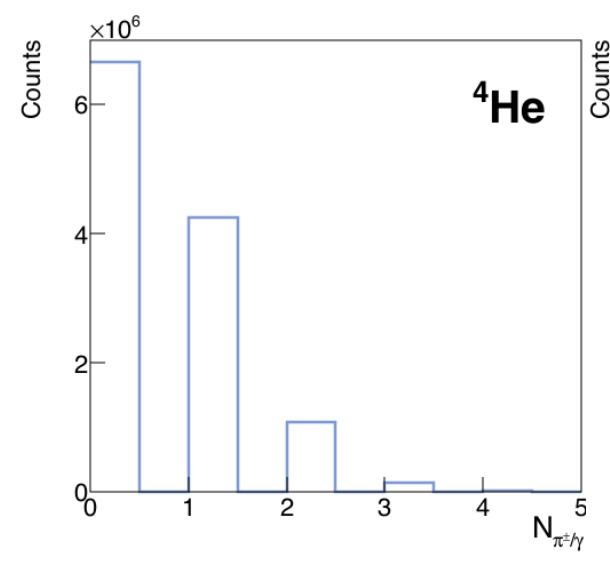

(a)

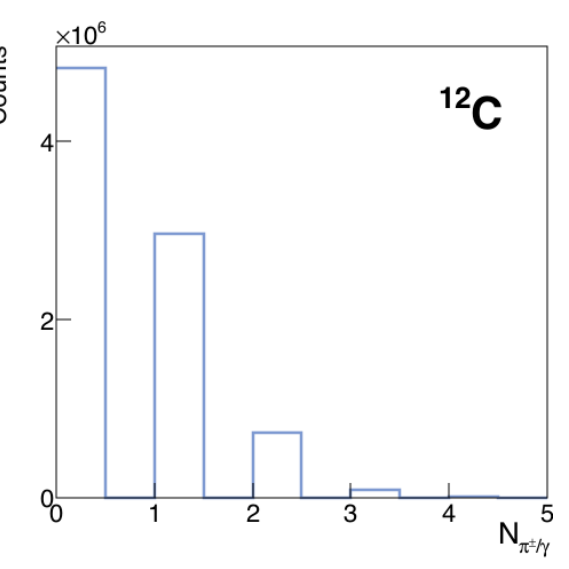

(b)

FIG. 221: Multiplicity distributions of $\pi^{ \pm}$for ${ }^{4} \mathrm{He}$ and ${ }^{12} \mathrm{C}$ targets at $4.4 \mathrm{GeV}$ (a) ${ }^{4} \mathrm{He}$; (b) ${ }^{12} \mathrm{C}$.

The multiplicity plots of protons and charged pions for ${ }^{4} \mathrm{He}$ and ${ }^{12} \mathrm{C}$ targets are shown in Figs. 222 and 221 correspondingly.

We can see the plot of $E_{\mathrm{QE}}$ as a function of $P_{\text {miss }}^{\perp}$ in Fig. 223. The plots are shown for ${ }^{4} \mathrm{He}$ and ${ }^{12} \mathrm{C}$ targets before and after subtraction for undetected pions and protons.

The distributions of $E_{\mathrm{QE}}$ for detected and undetected 1 pion events for ${ }^{4} \mathrm{He}\left(e, e^{\prime} N \pi\right)$ and ${ }^{12} \mathrm{C}\left(e, e^{\prime} N \pi\right)$ are shown in Fig. 224. The $E_{\mathrm{QE}}$ distributions of 0 pion spectrum before and after subtraction for undetected pions for ${ }^{4} \mathrm{He}\left(e, e^{\prime}\right)$ and ${ }^{12} \mathrm{C}\left(e, e^{\prime}\right)$ are shown in Fig. 224.

Fig. 225 shows $E_{\mathrm{QE}}$ and $E_{\text {calor }}$ distributions of $A\left(e, e^{\prime} p\right) 0 \pi$ spectrum before and after subtraction for undetected pions and undetected protons for ${ }^{4} \mathrm{He}$ and ${ }^{12} \mathrm{C}$ targets. 


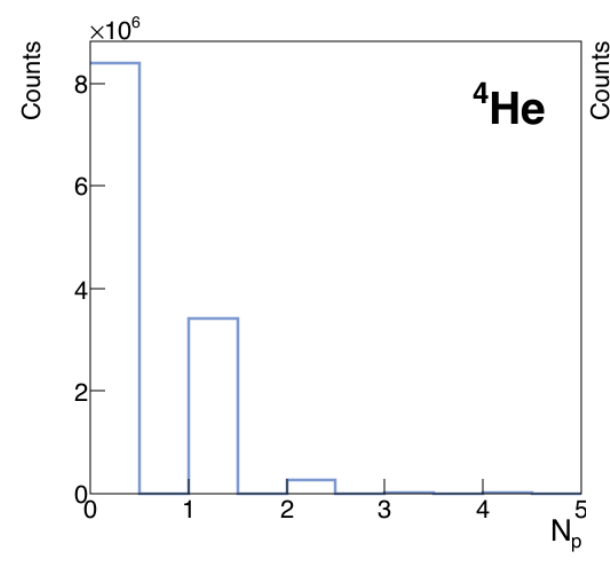

(a)

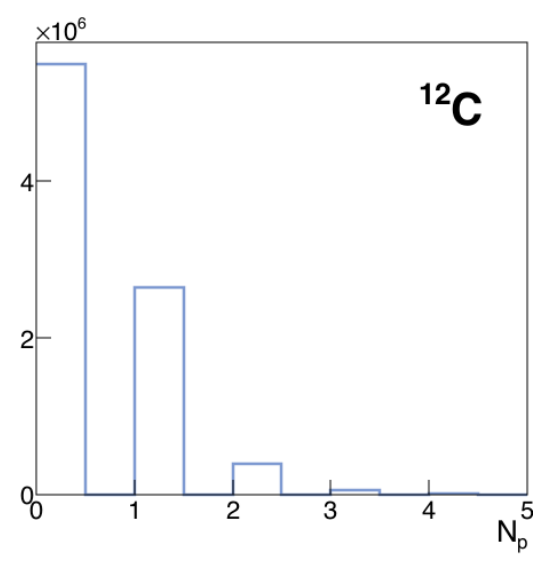

(b)

FIG. 222: Multiplicity distributions of protons for ${ }^{4} \mathrm{He}$ and ${ }^{12} \mathrm{C}$ targets at $4.4 \mathrm{GeV}$ (a) ${ }^{4} \mathrm{He}$; (b) ${ }^{12} \mathrm{C}$.

\section{B.2 2.2 GEV $A\left(e, e^{\prime}\right)$ AND $A\left(e, e^{\prime} p\right)$ ANALYSIS}

The proton and charged pion multiplicity plots are shown in Figs. 227 and 226 correspondingly.

We can see the plot of $E_{\mathrm{QE}}$ as a function of $P_{\text {miss }}^{\perp}$ in Fig. 228. The plots are shown for ${ }^{4} \mathrm{He}$ and ${ }^{12} \mathrm{C}$ targets before and after subtracting for undetected pions and protons.

The distributions of $E_{\mathrm{QE}}$ for detected and undetected 1 pion events for ${ }^{4} \operatorname{He}\left(e, e^{\prime} \pi^{ \pm}\right)$are shown in Fig. 229a, and the ones for ${ }^{12} \mathrm{C}\left(e, e^{\prime} \pi^{ \pm}\right)$in Fig. 229c. The $E_{\mathrm{QE}}$ distributions of 0 pion spectrum before and after subtraction for undetected pions for ${ }^{4} \mathrm{He}\left(e, e^{\prime}\right)$ are shown in Fig. $229 \mathrm{~b}$ and the ones for ${ }^{12} \mathrm{C}\left(e, e^{\prime}\right)$ are shown in Fig. $229 \mathrm{~d}$.

In Fig. 230 we can see $E_{\mathrm{QE}}$ (plots on the left) and $E_{\text {calor }}$ (plots on the right) distributions of $A\left(e, e^{\prime} p\right) 0 \pi$ spectrum before and after subtraction for undetected pions and protons for ${ }^{4}$ He (Figs. 230a, 230b) and ${ }^{12} \mathrm{C}$ (Figs. 230c, 230d) targets. 


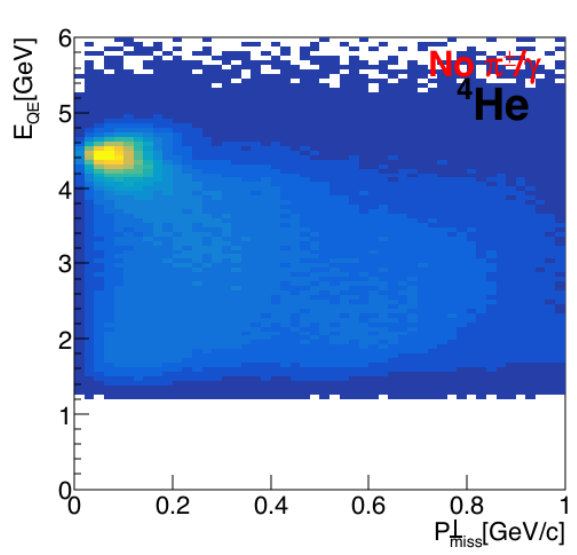

(a)

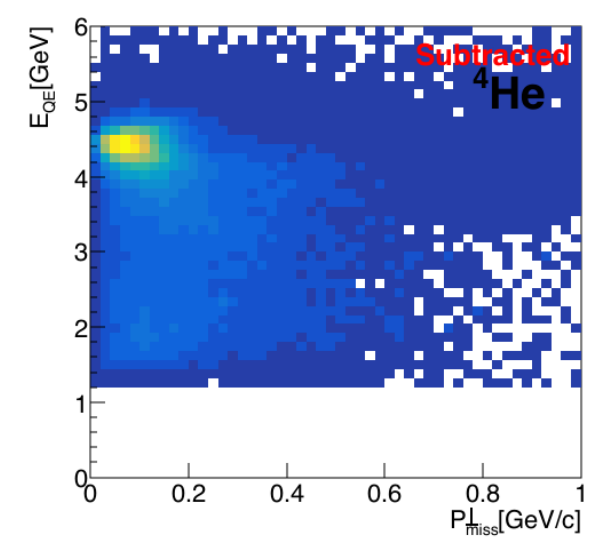

(c)

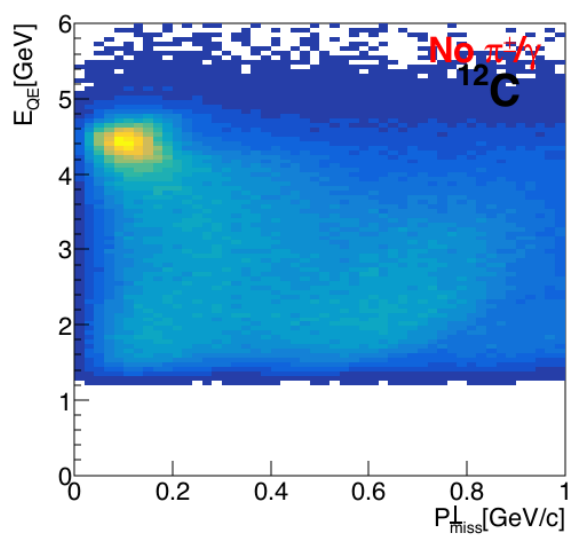

(b)

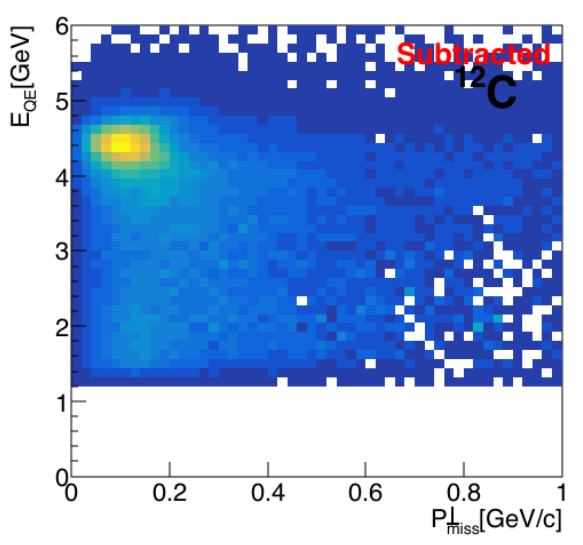

(d)

FIG. 223: Reconstructed energy $E_{\mathrm{QE}}$ obtained using Eq. 62 as a function of the total perpendicular momentum of the scattered electron and the knockout nucleon system for ${ }^{4} \mathrm{He}$ and ${ }^{12} \mathrm{C}$ targets at $4.4 \mathrm{GeV}$ before (top plots) and after (bottom plots) subtraction for undetected pions and protons. (a),(c) ${ }^{4} \mathrm{He}$; (b), (d) ${ }^{12} \mathrm{C}$. 


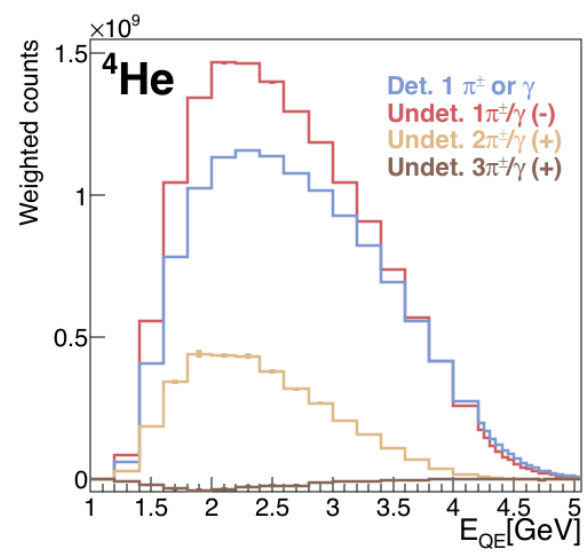

(a)

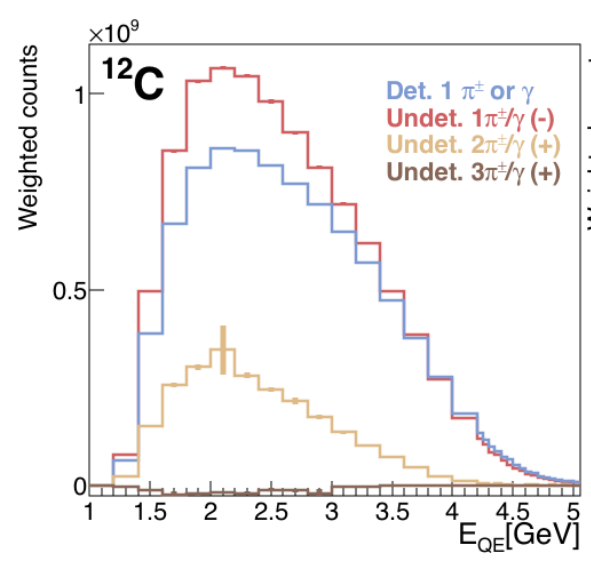

(c)

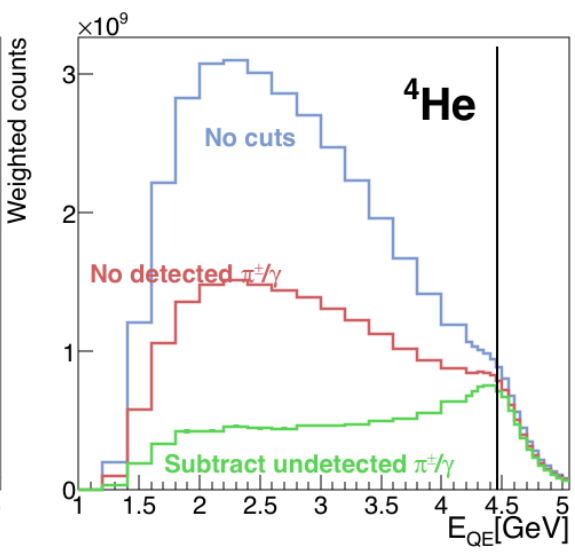

(b)

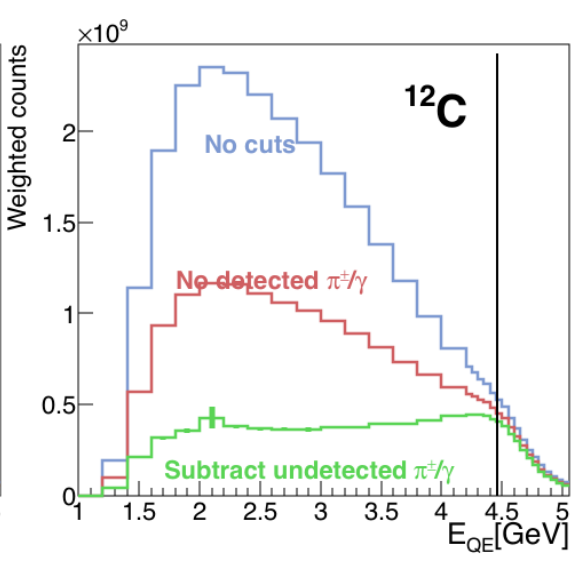

(d)

FIG. 224: The $E_{\mathrm{QE}}$ obtained using Eq. 62, for detected (blue) and undetected 1 pion events (red) as well as undetected 3 and 2 pion events for (a) ${ }^{4} \mathrm{He}\left(e, e^{\prime} N \pi\right)$; (c) ${ }^{12} \mathrm{C}\left(e, e^{\prime} N \pi\right)$ at $4.4 \mathrm{GeV}$. Reconstructed energy $E_{\mathrm{QE}}$ of $A\left(e, e^{\prime}\right) 0 \pi$ spectrum before (red) and after (green) subtraction for undetected pions for (b) ${ }^{4} \mathrm{He}$ and (d) ${ }^{12} \mathrm{C}$ targets at $4.4 \mathrm{GeV}$. 


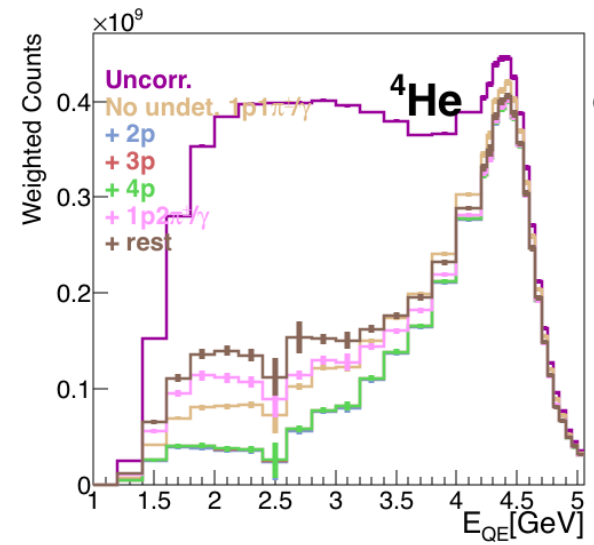

(a)

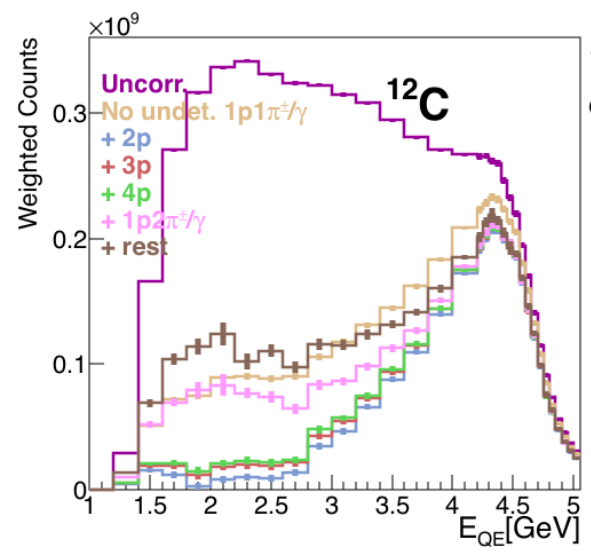

(c)

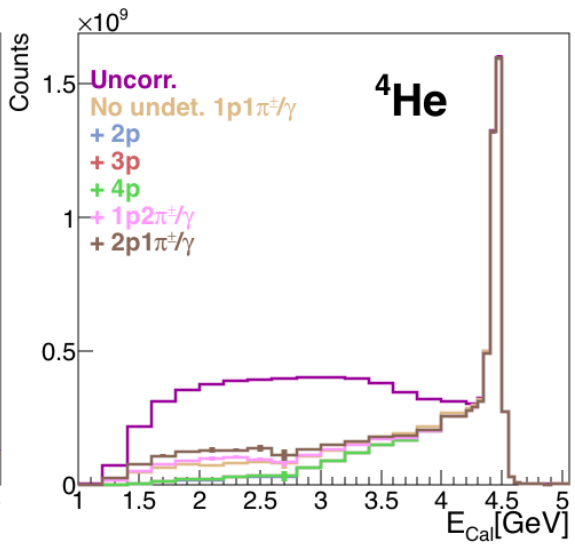

(b)

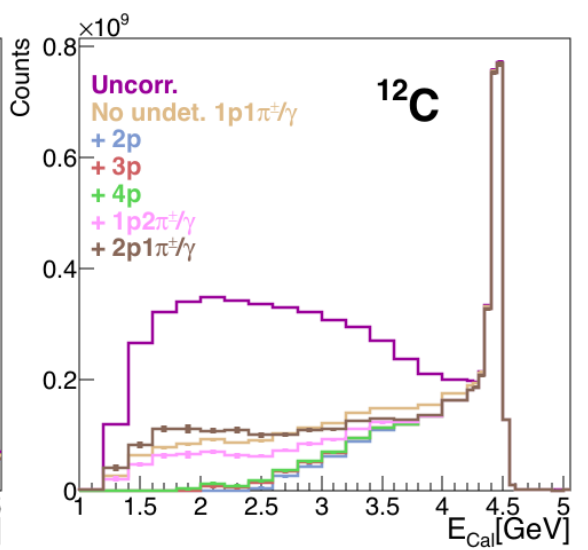

(d)

FIG. 225: $E_{\mathrm{QE}}$ (plots on the left) and $E_{\text {calor }}$ (plots on the right) distributions of $A\left(e, e^{\prime} p\right) 0 \pi$ spectrum before subtraction for undetected pions and after subtraction for undetected pions and undetected protons (green) for (a), (b) ${ }^{4} \mathrm{He}$ and (c),(d) ${ }^{12} \mathrm{C}$ targets at $4.4 \mathrm{GeV}$. 


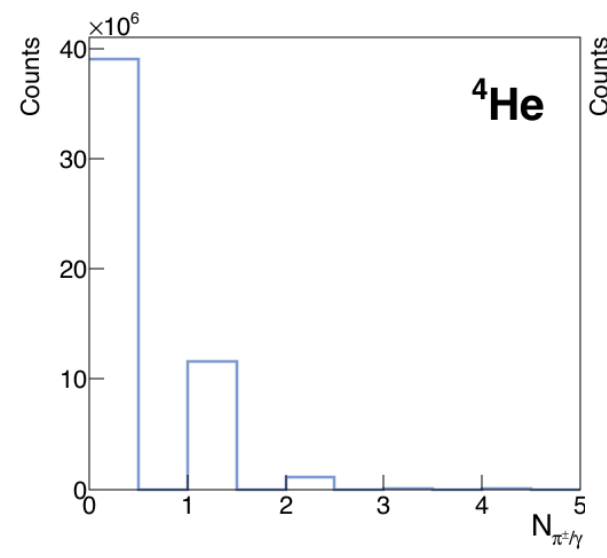

(a)

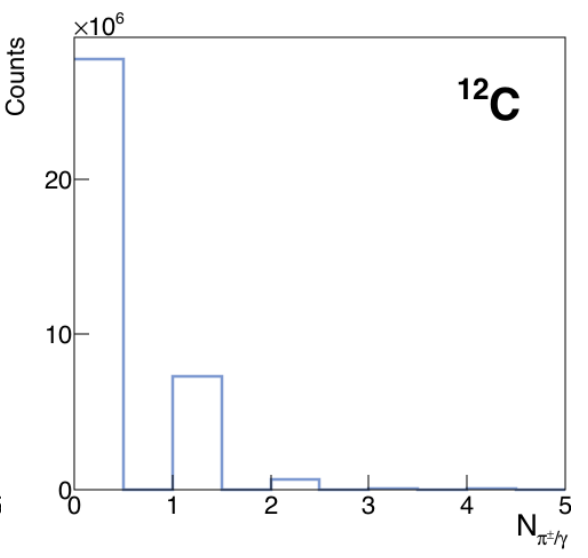

(b)

FIG. 226: Multiplicity distributions of $\pi^{ \pm}$for ${ }^{4} \mathrm{He}$ and ${ }^{12} \mathrm{C}$ targets at $2.2 \mathrm{GeV}$ (a) ${ }^{4} \mathrm{He}$; (b) ${ }^{12} \mathrm{C}$.

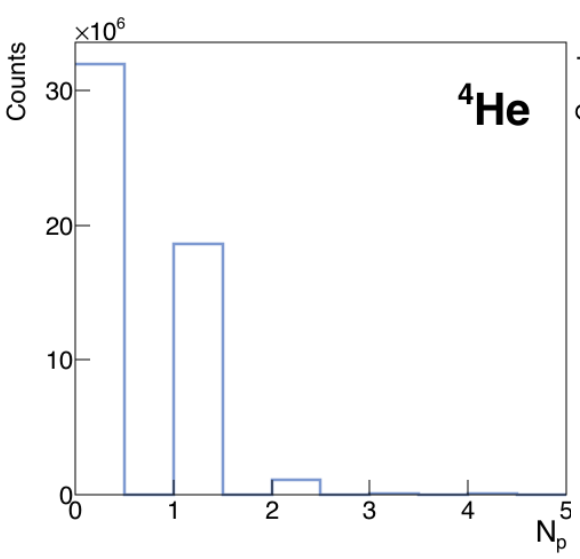

(a)

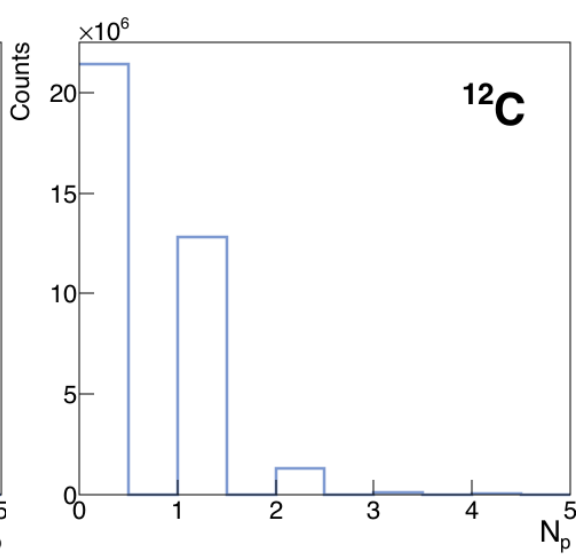

(b)

FIG. 227: Multiplicity distributions of protons for ${ }^{4} \mathrm{He}$ and ${ }^{12} \mathrm{C}$ targets at $2.2 \mathrm{GeV}$ (a) ${ }^{4} \mathrm{He}$; (b) ${ }^{12} \mathrm{C}$. 


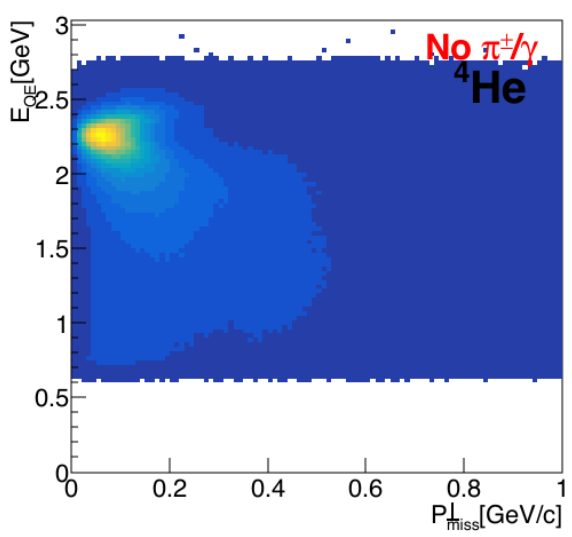

(a)

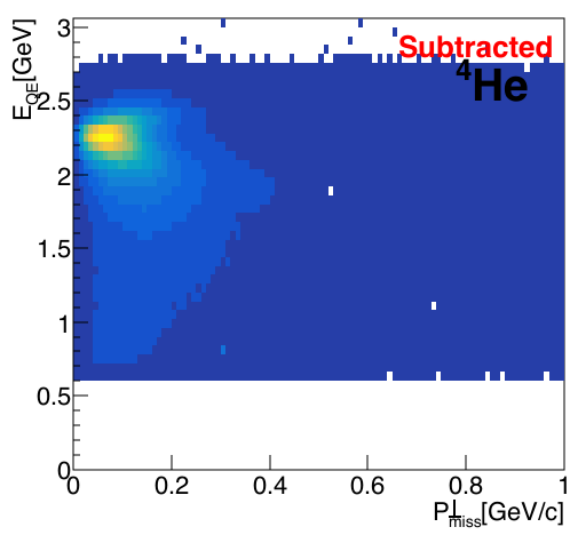

(c)

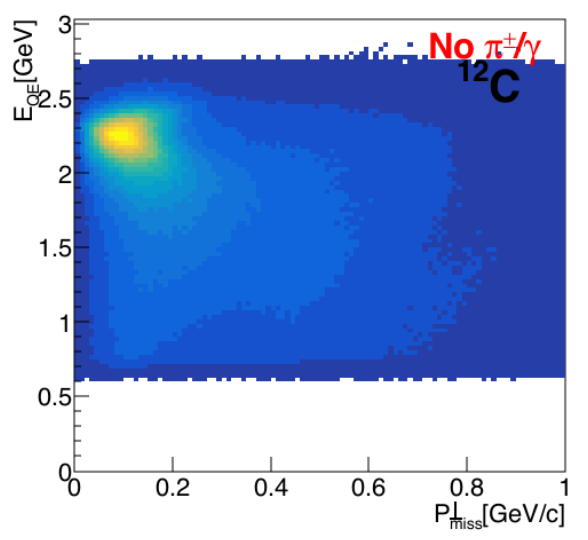

(b)

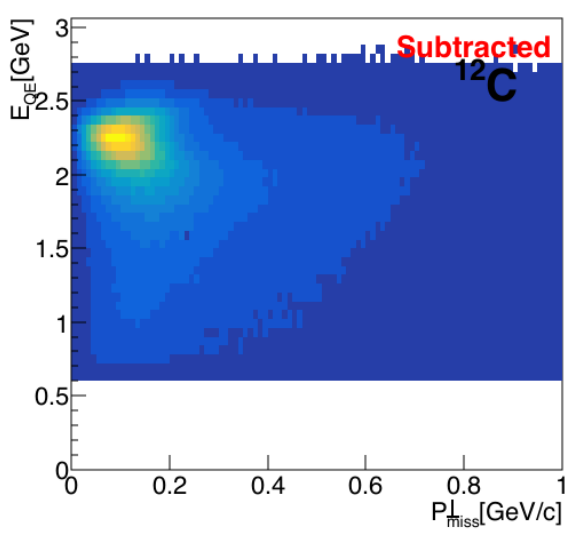

(d)

FIG. 228: Reconstructed energy $E_{\mathrm{QE}}$ obtained using Eq. 62 as a function of the total perpendicular momentum of the scattered electron and the knockout nucleon system for ${ }^{4} \mathrm{He}$ and ${ }^{12} \mathrm{C}$ targets at $2.2 \mathrm{GeV}$ before (top plots) and after (bottom plots) subtracting for undetected pions and protons. (a),(c) ${ }^{4} \mathrm{He}$; (b), (d) ${ }^{12} \mathrm{C}$. 


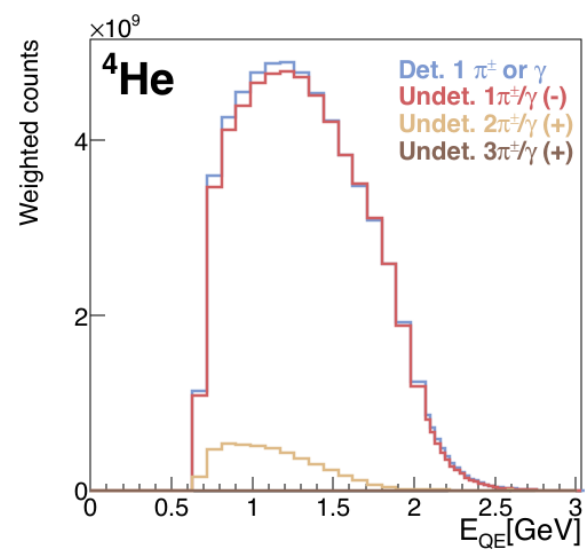

(a)

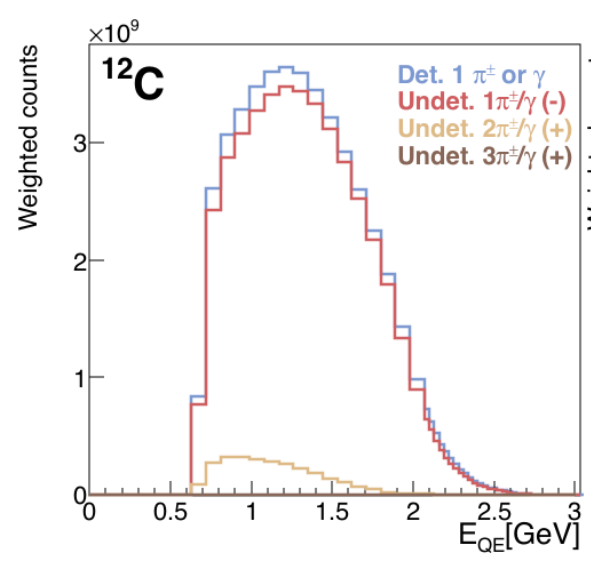

(c)

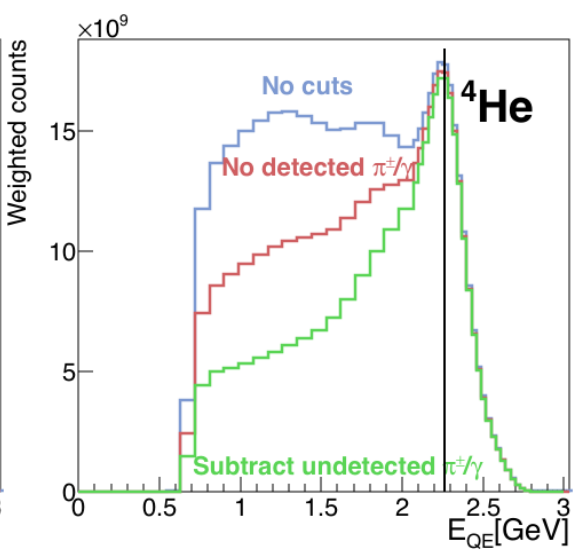

(b)

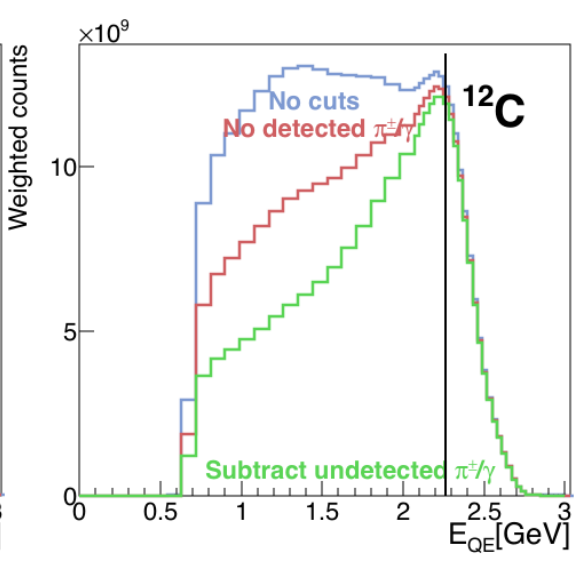

(d)

FIG. 229: The $E_{\mathrm{QE}}$ obtained using Eq. 62, for detected (blue) and undetected (red) 1 pion events as well as undetected 2 and 3 pion events for (a) ${ }^{4} \mathrm{He}\left(e, e^{\prime} N \pi^{ \pm}\right)$; (c) ${ }^{12} \mathrm{C}\left(e, e^{\prime} N \pi^{ \pm}\right)$at $2.2 \mathrm{GeV}$. Reconstructed energy $E_{\mathrm{QE}}$ of $A\left(e, e^{\prime}\right) 0 \pi$ spectrum before (red) and after (green) subtraction for undetected pions for (b) ${ }^{4} \mathrm{He}$ and (d) ${ }^{12} \mathrm{C}$ targets at $2.2 \mathrm{GeV}$. 


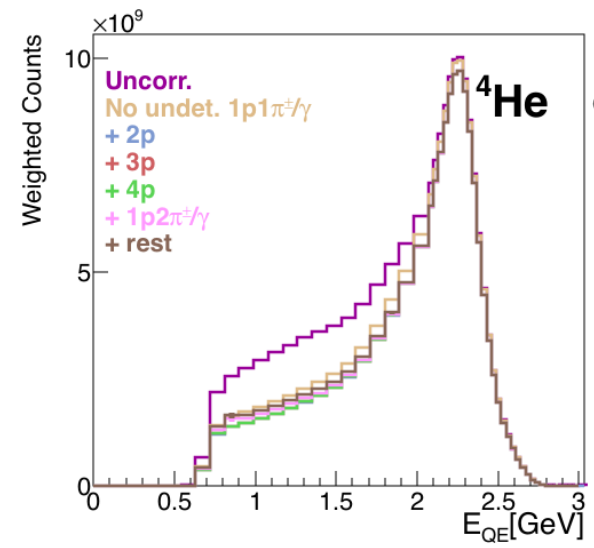

(a)

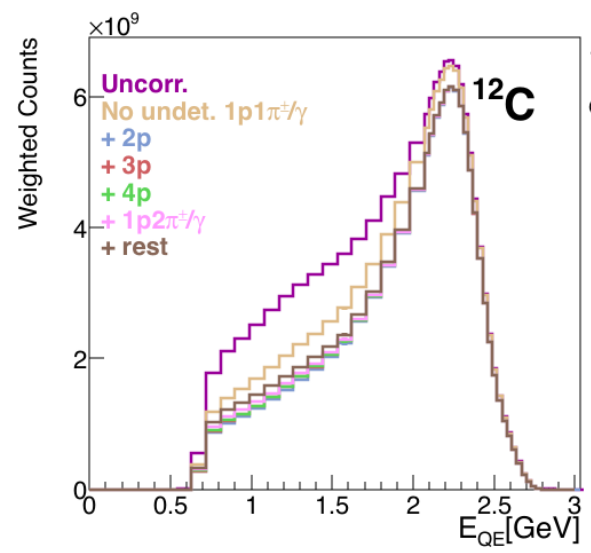

(c)

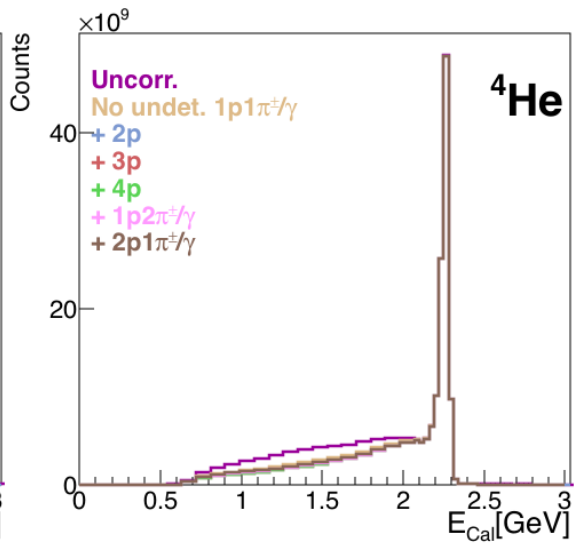

(b)

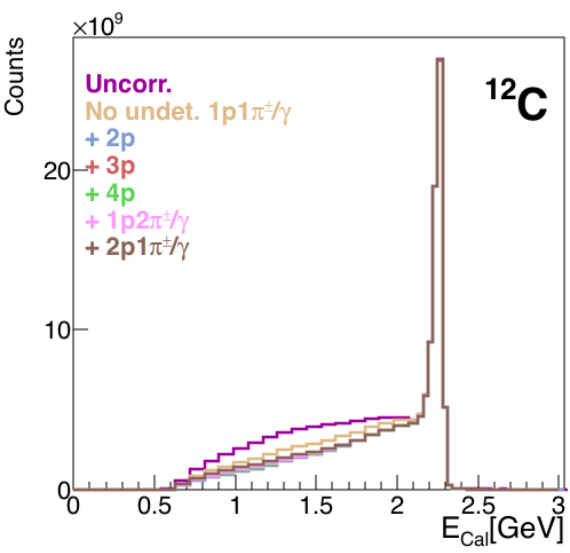

(d)

FIG. 230: $E_{\mathrm{QE}}$ (plots on the left) and $E_{\text {calor }}$ (plots on the right) distributions of $A\left(e, e^{\prime} p\right) 0 \pi$ spectrum before subtraction for undetected pions and after subtraction for undetected pions and undetected protons (green) for (a), (b) ${ }^{4} \mathrm{He}$ and (c),(d) ${ }^{12} \mathrm{C}$ targets at $2.2 \mathrm{GeV}$. 


\section{VITA}

Mariana Khachatryan

Department of Physics

Old Dominion University

Norfolk, VA 23529

\section{Education}

Ph.D. Department of Physics, Old Dominion University, Norfolk, VA, Dec 2019.

M.S. Department of Physics, Old Dominion University, Norfolk, VA, Dec 2015.

M.S. in Nuclear Physics, Yerevan State University, Yerevan, Armenia, May 2013.

B.S. in Physics, Yerevan State University, Yerevan, Armenia, May 2011.

\section{Honors \& Awards}

First place prize; Annual Jefferson Lab User Group Meeting Poster Competition; June 19th, 2018.

Jefferson Science Associates Graduate Fellowship award; Jefferson Science Associates; 20182019.

\section{Invited presentations}

JLab Users Organization Meeting: Constraining neutrino-nucleus interactions with electron scattering data; June 24, 2019, JLAB, VA, USA.

Photonuclear reactions: Validation of neutrino energy estimation using electron scattering data; August 2018, Gordon Research Conference; Holderness, NH.

\section{Other presentations}

NPWG: Validation of neutrino energy estimation using electron scattering data; CLAS collaboration meeting; VA, USA; (February 2016, March 2017, June 2017, March 2018, July 2018, March 2019, June 2019).

Fifth Joint Meeting of the Nuclear Physics Divisions of the APS and the JPS: Study of neutrino energy reconstruction using electron scattering; October 23-27, 2018, Waikoloa, Hawaii.

JLAB CLAS Nuclear Data Mining Group meeting: Validation of neutrino energy estimation using electron scattering data; August 2018, Cambridge, MA.

APS meeting: Validation of neutrino energy estimation using electron scattering data; April 17, 2018, Columbus, OH, USA.

JLAB CLAS Nuclear Data Mining Group meeting: Comparing proton and neutron momentum distributions in ${ }^{3} \mathrm{He}$ using the Large Angle Calorimeter (LAC) and using Forward Electromagnetic Calorimeter (EC); October 2017, Cambridge, MA, July 2015, Buffalo, NY. Odu Physics Department Nuclear Group Seminar: Comparing proton and neutron momentum distributions in ${ }^{3} \mathrm{He}$ using the Large Angle Calorimeter (LAC); February 2015, March 2016, Old Dominion University, Norfolk, VA, USA.

\section{Summer Student Programs}

Hampton University Graduate Program; Summer 2012 and Summer 2013, JLab, VA, USA.

\section{Publications}

R. Cruz-Torrez et al. "Comparing proton momentum distributions in $A=2$ and 3 nuclei via ${ }^{2} \mathrm{H}^{3} \mathrm{H}$ and ${ }^{3} \mathrm{He}\left(e, e^{\prime} p\right)$ measurements". Phys.Lett. B797 (2019) 134890.

Typeset using $\mathrm{AT}_{\mathrm{E}} \mathrm{X}$. 